\title{
WATER-QUALITY, WATER-LEVEL, AND LAKE-BOTTOM-SEDIMENT DATA COLLECTED FROM THE DEFENSE FUEL SUPPLY POINT AND ADJACENT PROPERTIES, HANAHAN, SOUTH CAROLINA, 1990-96
}

By Matthew D. Petkewich, Don A. Vroblesky, J. Frederick Robertson, and Paul M. Bradley

U.S. GEOLOGICAL SURVEY

Open-File Report 96-488

Prepared in cooperation with the U.S. DEPARTMENT OF DEFENSE, DEFENSE LOGISTICS AGENCY

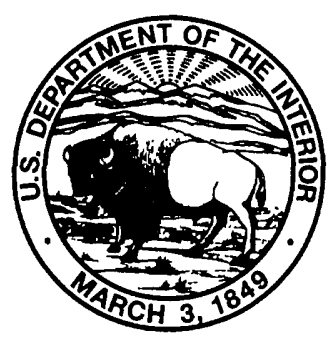




\section{U.S. DEPARTMENT OF THE INTERIOR BRUCE BABBITT, Secretary}

U.S. GEOLOGICAL SURVEY

Gordon P. Eaton, Director

For additional information write to:

Copies of this report can be purchased from:

District Chief

U.S. Geological Survey

Stephenson Center-Suite 129

720 Gracern Road

Columbia, SC 29210-7651
U.S. Geological Survey

Branch of Information Services

Box 25286

Denver, CO 80225-0286 


\section{CONTENTS}

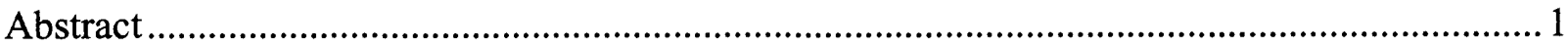

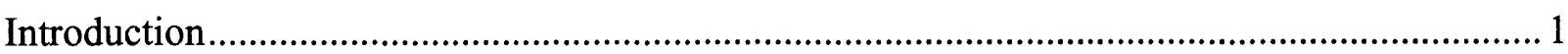

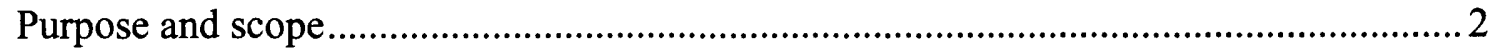

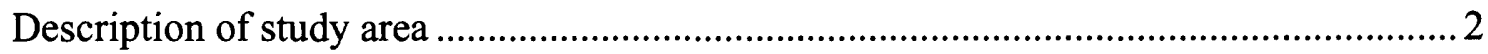

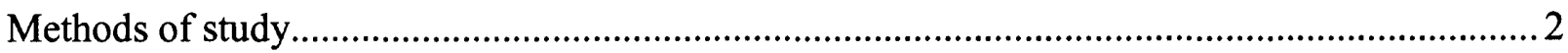

Collection of sediment samples for lithologic description .......................................... 3

Monitoring-well installation and development .................................................... 3

Water-quality sample collection and analysis .......................................................... 5

Water-level measurements ............................................................................... 8

Lake-bottom-sediment collection and analysis.......................................................... 8

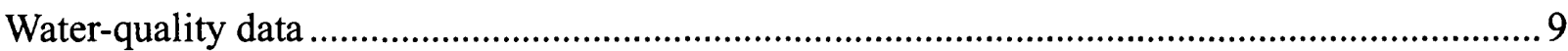

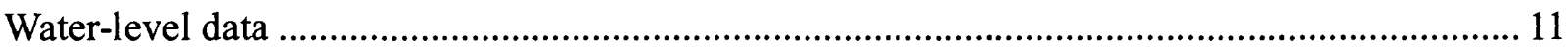

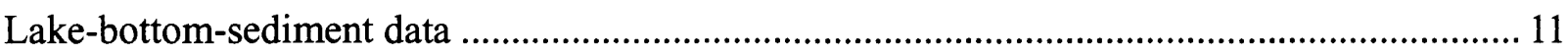

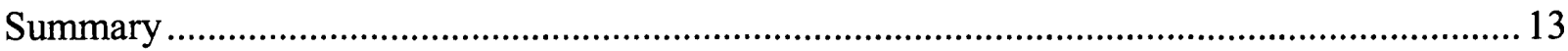

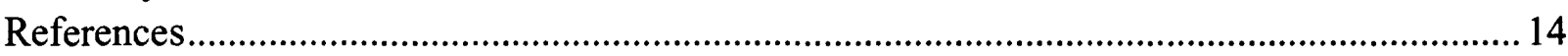

Appendix--Lithologic descriptions of sediment recovered from boreholes at the

Defense Fuel Supply Point property, Hanahan, S.C .................................................. 278

\section{ILLUSTRATIONS}

Plate 1. Map showing locations of observation wells, boreholes, surface-water sampling sites, and lake-bottom-sediment sampling sites at the Defense Fuel Supply Point and adjacent properties, Hanahan, South Carolina [In pocket]

Figure 1. Graphs showing specific conductance in wells MWGS-33A and MWGS-33B and wells MWGS-34A and MWGS-34B at the Defense Fuel Supply Point, Hanahan, S.C., February 5, 1992 to December 5, 1995

2. Hydrograph of wells W-103, W-107, and W-108 at the Defense Fuel

Supply Point, Hanahan, S.C., February 27, 1991 to December 5, 1995 


\section{TABLES}

Page

Table 1. Construction data for monitoring wells installed by the U.S.

Geological Survey at the Defense Fuel Supply Point,

Hanahan, S.C.

2. Construction data for privately owned wells and monitoring wells installed during previous investigations in the vicinity of the Defense Fuel Supply Point, Hanahan, S.C.

3. Analytical detection limits for volatile-organic, extractable-organic, and metal analyses determined for lake-bottom-sediment samples collected from Gold Cup Springs Lake, Hanahan, S.C., February 8, 1994 25

4. Water-quality constituents and properties measured in the field at the Defense Fuel Supply Point and adjacent properties, Hanahan, S.C., between December 1990 and January 1996

5. Organic water-chemistry data collected at the Defense Fuel Supply Point and adjacent properties, Hanahan, S.C., between December 1990 and January 1996 89

6. Inorganic water-chemistry data collected at the Defense Fuel Supply Point and adjacent properties, Hanahan, S.C., between December 1990 and January 1996

7. Tentative identification and estimated concentrations of volatile- and extractable-organic compounds using comparison of mass spectral data collected at the Defense Fuel Supply Point and adjacent properties, Hanahan, S.C.

8. Water-level data collected at the Defense Fuel Supply Point and adjacent properties, Hanahan, S.C., between April 1991 and September 1995 205

9. Analytical results for volatile organics, extractable organics, and metals detected in lake-bottom-sediment samples collected from Gold Cup Springs Lake, Hanahan, S.C., February 8, 1994 


\begin{tabular}{lll}
\hline Multiply & By & \multicolumn{1}{c}{ To obtain } \\
\hline & Length & \\
inch (in.) & 2.54 & centimeter \\
foot (ft) & 0.3048 & meter \\
& & \\
& Volume & liter \\
gallon (gal) & 3.785 & \\
& & \\
& Flow & liter per minute \\
gallon per minute (gal/min) & 3.785 & \\
& & \\
\hline
\end{tabular}

Sea level: In this report "sea level" refers to the National Geodetic Vertical Datum of 1929 -- a geodetic datum derived from a general adjustment of the first-order level nets of the United States and Canada, formerly called Sea Level Datum of 1929.

Temperature: In this report, temperature is expressed in degrees Celsius $\left({ }^{\circ} \mathrm{C}\right)$, which can be corverted to degrees Fahrenheit $\left({ }^{\circ} \mathrm{F}\right)$ by the following equation:

$$
{ }^{\circ} \mathrm{F}=1.8 \times\left({ }^{\circ} \mathrm{C}\right)+32
$$

Chemical concentration: $\ln$ this report, chemical concentration is expressed in micrograms per kilogram $(\mu \mathrm{g} / \mathrm{kg})$, micrograms per liter $(\mu \mathrm{g} / \mathrm{L})$, micromolar $(\mu \mathrm{M})$, milligrams per kilogram $(\mathrm{mg} / \mathrm{kg})$, milligrams per liter $(\mathrm{mg} / \mathrm{L})$, or nanomolar $(\mathrm{nM})$ where molar $(\mathrm{M})$ is equivalent to moles per liter.

Specific electrical conductance is given in microsiemens per centimeter at 25 degrees Celsius $\left(\mu \mathrm{S} / \mathrm{cm}\right.$ at $\left.25^{\circ} \mathrm{C}\right)$. 


\section{CONVERSION FACTORS, ABBREVIATIONS, VERTICAL DATUM, AND ACRONYMS--Continued}

\section{ADDITIONAL ABBREVIATIONS}

L, liter $\mu \mathrm{m}$, micrometer $\mathrm{mL}$, milliliter

$>$, greater than

$<$, less than

\section{ACRONYMS}

BTEX, benzene, toluene, ethylbenzene, and xylenes

DFSP, Defense Fuel Supply Point

DLA, Defense Logistics Agency

DIC, dissolved inorganic carbon

ID, inside diameter

OD, outside diameter

PVC, polyvinyl chloride

SS, stainless steel

TOC, total organic carbon

$\mathrm{TPH}$, total petroleum hydrocarbons

USEPA, U. S. Environmental Protection Agency

USGS, U. S. Geological Survey

The use of trade, product, industry, or firm names in this report is for identification or location purposes only, and does not constitute endorsement of products by the U. S. Government, nor impute responsibility for any present or potential effects on the natural resources. 


\title{
WATER-QUALITY, WATER-LEVEL, AND LAKE-BOTTOM-SEDIMENT DATA COLLECTED FROM THE DEFENSE FUEL SUPPLY POINT AND ADJACENT PROPERTIES, HANAHAN, SOUTH CAROLINA, 1990-96
}

\author{
By Matthew D. Petkewich, Don A. Vroblesky, J. Frederick Robertson, \\ and Paul M. Bradley
}

\begin{abstract}
A 9-year scientific investigation to determine the potential for bioremediation of ground-water contamination and to monitor the effectiveness of an engineered bioremediation system located at the Defense Fuel Supply Point and adjacent properties in Hanahan, S.C., has culminated in the collection of abundant waterquality and water-level data. This report presents the analytical results of the study that monitored the changes in surface- and ground-water quality and water-table elevations in the study area from December 1990 to January 1996. This report also presents analytical results of lake-bottom sediments collected in the study area.
\end{abstract}

\section{INTRODUCTION}

In 1975, a tank located at a Defense Fuel Supply Point (DFSP), referred to hereinafter as the facility, near Hanahan, S.C., leaked a reported 83,000 gals of JP-4 jet fuel (pl. 1). The leak contaminated water and sediment in the shallow surficial aquifer beneath the facility with petroleum hydrocarbons, in particular, benzene, toluene, ethylbenzene, and xylenes (collectively termed BTEX) (U.S. Army Environmental Hygiene Agency, 1975).

Investigations by the U.S. Geological Survey (USGS) began at the facility in 1987 ir cooperation with the U.S. Defense Logistics Agency (DLA), through the U.S. Navy, Southerr Division Naval Facilities Engineering Command. The USGS was asked to determine the potential for in situ bioremediation of the ground-water contamination at the study site. The study, which was completed in collaboration with DLA and its contractors, included the design. construction, and operation of an engineered bioremediation system that utilized both microbiological and hydraulic contaminant-depletion technologies. Although the study area included the facility and surrounding areas, the investigation concentrated on the northern part of the facility and the ground-water-contamination plumes extending northward into Gold Cup Springs subdivision (pl. 1). This investigation culminated in the collection of abundant waterquality and water-level data. 


\section{Purpose and Scope}

This report presents data collected between December 1990 and January 1996 that documented the changes in surface- and ground-water quality and water-table elevations in the study area. This report also presents analytical results of contaminants present in lake-bottom sediments collected from Gold Cup Springs Lake. The scope of work included drilling to obtain lithologic descriptions of surficial aquifer sediments and to install monitoring wells in this aquifer; collection of surface- and ground-water samples to delineate contaminated areas and to monitor temporal changes in the distribution of the contaminant plumes; measurement of water levels in wells and streams to determine the direction of ground-water flow; and collection of lake-bottom sediments to determine if fuel-related contaminants were present.

\section{Description of Study Area}

The facility is surrounded by private properties on the northern, western, and southern borders and the Charleston Naval Weapons Station to the east (pl. 1). The study area is underlain by unconsolidated Pleistocene sediments composed of quartz sand and silt with discontinuc'ss layers of clay, clayey sand, and sandy clay. The base of the Pleistocene sediments in the area ranges from depths of 11 - to 33 - $\mathrm{ft}$ below land surface. Layers of dense calcarenite and calcilutite are approximately 270 - $\mathrm{ft}$ thick beneath the Pleistocene sediments at the facility, which vertically isolates the surficial aquifer from deeper water-bearing units.

The Pleistocene sediments function as an unconfined water-bearing zone and are referred to as the surficial aquifer. However, clay lenses within these sediments produce locally confired flow conditions. Recharge to the surficial aquifer is primarily from rainfall infiltration. Groundwater discharge at the site is by flow to nearby drains and streams, and by evapotranspiration. The predominant direction of ground-water flow beneath the facility is to the northwest (Vroblesky and Chapelle, 1994; Vroblesky and others, in press).

The engineered bioremediation system at the facility consisted of infiltration galleries and an extraction system. Three infiltration galleries were located north of the fuel storage tanks on the facility (pl. 1) and were designed to inject uncontaminated water and nutrients into the contaminated aquifer to enhance bioremediation. The extraction system consisted of 17 wells that were located along the western, northern, and northeastern perimeter of the facility and c ne located in Gold Cup Springs subdivision (pl. 1), and was designed to remove contaminated water from the surficial aquifer.

\section{METHODS OF STUDY}

Standard methods typically were used in this investigation to obtain water-quality, waterlevel, and lake-bottom-sediment-quality data. Specific methods used during monitoring-well installation and development, water-level measurements, and collection and analysis of waterquality and lake-bottom-sediment-quality data are discussed in the following sections. 


\section{Collection of Sediment Samples for Lithologic Description}

Sediment borings were made at the facility to collect subsurface-sediment samples for lithologic description and for the installation of monitoring wells (pl. 1). Sediment samples for lithologic description were collected from 19 boreholes between July 1990 and June 1994. Most of the holes were bored using hollow-stem augers [5.5-, 8.5-, and 10.25-in. outside diameters (OD)], and sediment samples were collected with a split-spoon sampler [1.375-in. inside diameter (ID)] using standard methods (American Society for Testing and Materials, 1994). Sediment samples from borehole DV-4 were collected with a coring hand auger.

Lithologic descriptions of sediments collected from the 19 boreholes are presented in the appendix. Continuous subsurface-sediment sample collection began at depths varying from 0 - to 10 - $\mathrm{ft}$ below land surface. Estimates of grain size, color, and composition were based on visual examination of the sediments. Grain-size classifications were based on the Wentworth Scale (1922).

\section{Monitoring-Well Installation and Development}

Eighty-five monitoring wells and piezometers were installed at or near the facility by the USGS or under the direction of the USGS (pl. 1). All wells were installed in the surficial aquifer from June 1990 to June 1994. Generally, these wells were installed at locations and depths intended to provide necessary geochemical and water-level data where previously unobtained, thereby, complementing existing monitoring wells installed during previous investigations. Construction data for wells installed by the USGS are listed in table 1 (at end of report). Water-level and geochemical data also were collected from 34 monitoring wells installed during previous investigations in the vicinity of the facility, as well as 4 privately owned domestic wells located in Gold Cup Springs subdivision. Available well-construction data for these wells are presented in table 2 (at end of report).

Monitoring wells installed by the USGS were identified by the prefix MWGS followed by the numbers 5 and 20 through 63 . Well pairs and clusters were further identified alphabetically to indicate relative depths, with the letter A representing the shallowest well of the pair or cluster; B identifying the deepest well of a pair, or the second most shallow of a cluster; $\mathrm{C}$ identifying the third most shallow well of a cluster, and so forth. For example, MWGS-27A is the shallowest well of the MWGS-27 cluster, and MWGS-27C is the deepest, with MWGS-27B at an intermediate depth. Well MWGS-05A, however, was installed adjacent to a previously existing well, MW-5, and is the deepest of the pair. Piezometers installed by the USGS were identified by the prefix WT followed by sequential numbers 1 through 11 .

Specific wells and groups of wells were installed for various reasons, and not necessarily limited to filling in spatial data gaps. Wells MWGS-42 through MWGS-63 were installed in the Tank 1 basin for use as test galleries for experimental purposes. The WT-series wells were installed primarily for gathering water-level data. Well pairs and clusters were installed to assess the vertical distribution of contaminants. 
All MWGS-series wells were installed using hollow-stem augers. All WT-series piezometers were installed using a coring hand auger. Wells and piezometers were installed by USGS personnel or under the direction of the USGS, except for well clusters MWGS-23, MWGS-24, MWGS-25, MWGS-26, MWGS-27, and MWGS-28, which were installed by a U.S. Environmental Protection Agency (USEPA) drill crew in cooperation with the USGS. Well clusters MWGS-23, MWGS-24, MWGS-25, and MWGS-26 were constructed with 0.25-in. OD stainless-steel (SS) tubing, with each cluster installed in a single, respective borehole. Screened intervals for these wells were constructed of $0.2-\mathrm{ft}$ lengths of 60 mesh SS screen. Well clusters MWGS-27 and MWGS-28 were constructed with 1-in. ID polyvinyl chloride (PVC) flush-threaded pipe with slotted-screen sections cut to desired lengths in the field. Wells MWGS-35 through MWGS-39 were constructed with 4-in. ID flush-threaded PVC monitor pipe and slotted screen (0.010-in. slots). All other MWGS- and WT-series wells were constructed with 2-in. ID flush-threaded PVC monitor pipe and slotted screen (0.010-in. slots). In most cases, the screened section of shallower wells was placed to bracket the water table, unless the depth to the water table was too shallow to allow such placement. The annular space around the screened sections of all wells was filled with clean filter sand to various heights, but typically about 2-ft above the top of the screens. A bentonite seal was placed above the filter sand, and the remaining annular space was grouted with cement to about $1-\mathrm{ft}$ below land surface. Wells were finished at land surface with a concrete pad and protective cover. Because of their intended use as temporary wells, wells MWGS-42 through MWGS-63 were sealed to land surface with bentonite and were not finished with protective covers.

Monitoring wells were developed either by bailing with dedicated Teflon bailers or pumping until the withdrawn water was clear and assumed to be representative of formation water. The amount of water required to be withdrawn varied from well to well. All withdrawn development water was contained in wastewater holding tanks located on the facility.

Extraction wells EW-01 through EW-17 (pl. 1) were constructed with 30-ft lengths of 6-in. ID PVC slotted-screen sections (slot size 0.010 in.), placed to bracket the entire saturated thickness of the surficial aquifer. Because of the shallower depth to the bottom of the surficial aquifer, extraction well EW-18 was installed with a $25-\mathrm{ft}$ screened section. The annular spere from the bottom of the borehole to not less than $1-\mathrm{ft}$ above the top of the screened interval was filled with filter sand. A 1-ft thick bentonite seal was placed above the sand pack, and the remaining annular space above the bentonite seal was grouted to land surface. Each wellhead was surrounded by a floored concrete vault, the bottom of which was approximately 2 - $\mathrm{ft}$ below land surface.

A 0.5 -horsepower submersible pump, capable of providing flow rates of about $10 \mathrm{gal} / \mathrm{min}$, was installed in each extraction well. The pumps were outfitted with automatic level cont"ol switches to deactivate and reactivate pump operation (cycling), thereby preventing the pump from operating while not completely submerged in water. All extraction wells were connected to a common discharge pipe, through which all extracted ground water was directed to a splitter pit on the eastern side of the facility. By use of control valves in the splitter pit, all of the extracted water could be directed offsite for treatment. 


\section{Water-Quality Sample Collection and Analysis}

Ninety-one wells and 13 surface-water sites located on and adjacent to the facility were sampled intermittently during this investigation from December 1990 to January 1996. Between 49 and 79 wells were sampled on a quarterly basis from January 1992 to July 1995 . The actual number of surface-water sites sampled each quarter varied somewhat, but typically, all 13 sites were sampled. Extraction wells were sampled on a monthly basis between March 1992 and September 1995, depending on their operational status. The infiltration gallery port, IG-2, was sampled on a monthly basis between September 1993 and September 1995.

All monitoring wells were purged of casing water prior to collecting ground-water samples. At least three casing volumes of water were removed from each well with either a Teflon bailer, a peristaltic pump with silicon tubing, or a 1.8-in. OD SS submersible pump with a rubber hose, except for wells that were bailed dry prior to removing three casing volumes. Because the extraction wells were continuously pumped, these wells could be sampled without any additional purging. Each well was sampled immediately following bailing. All purge water was contained in wastewater holding tanks located on the facility.

Designated Teflon bailers eliminated the need for extensive cleaning between wells. Although designated bailers were used to collect ground-water samples from most monitoring wells, a common bailer used at a few wells was decontaminated prior to sampling each well using a detergent solution and rinsing with deionized water. Before sampling with the peristaltic pump, the silicon tubing used in conjunction with the pump was decontaminated by pumping approximately $1 \mathrm{~L}$ of deionized water through the system prior to sampling each well. The outside of the silicon tubing was rinsed with deionized water. The submersible pump and hose were decontaminated by pumping at least 25 gal of a detergent solution through the system and then rinsing with approximately 25 gal of water. The outside of the hose and pump were scrubbed with the detergent solution and then rinsed with water. Additionally, the filter stands and membrane filters used to collect filtered samples were rinsed thoroughly with deionized water, followed by ground water from the well to be sampled, prior to collecting samples at each well.

Dissolved oxygen, ferrous iron, and total-sulfide concentrations were measured in the field. After well purging, the dissolved oxygen concentration in water from each well was determined by Winkler titration (Hach Company, 1992). Ferrous iron was analyzed using the Hach colorimeter/FerroZine method (Stookey, 1970). Total-sulfide concentration, as hydrogen sulfide $\left(\mathrm{H}_{2} \mathrm{~S}\right)$ or acid-soluble metallic sulfides, was determined in the field using a colorimetric method (Hach Company, 1992). Particulate matter in the samples was allowed to settle and then the clear supernatant analyzed. The results represent the approximate concentrations of dissolved $\mathrm{H}_{2} \mathrm{~S}$.

After collecting the dissolved oxygen sample, specific conductance, $\mathrm{pH}$, and water temperature were measured using techniques described by Wood (1976). Specific conductance was measured with a Yellow Springs Instrument model 33 SCT meter. The pH was measured using a digital $\mathrm{pH}$ meter equipped with a combination temperature-compensated $\mathrm{pH}$ electrode. Water temperature was measured to the nearest $0.1{ }^{\circ} \mathrm{C}$ with the temperature probe of the $\mathrm{pH}$ meter or to the nearest $0.5^{\circ} \mathrm{C}$ with a mercury-filled glass thermometer. Specific conductance in the surficial aquifer also was measured by continuous specific conductance recorders (recorded at 15minute intervals) in four 2-in. ID wells (MWGS-33A, MWGS-33B, MWGS-34A, and MWGS34B) located on the facility (pl. 1). 
Alkalinity titrations were completed in the field on $100-\mathrm{mL}$ filtered samples during the December 1990, June 1991, July 1991, and October 1991 sampling events. Each sample was stirred slowly, using a battery-powered magnetic stirrer while a Hach Digital Titrator was used to add 0.16-normal sulfuric acid solution to the sample until a $\mathrm{pH}$ endpoint of 4.5 was reacled. Alkalinity was calculated as the endpoint of the cumulative volume of added acid as a function of $\mathrm{pH}$.

Water samples for analysis of BTEX, total petroleum hydrocarbons (TPH), total organic carbon (TOC), and naphthalene were collected by slowly filling sample-rinsed glass bottles from a bottom-discharge bailer. The bottles were allowed to overflow several seconds, and then samples were either preserved with 3 drops of hydrochloric acid (BTEX samples), preserved vith sulfuric acid (TPH and TOC samples), or not preserved (naphthalene samples). All sample bottles were capped with Teflon-lined bottle caps. If aeration of a BTEX sample was suspected, or if bubbles were observed in a bottle, the sample was discarded, and a new sample was collected. Ground-water samples for analysis of BTEX and naphthalene were collected in 40-mL glass bottles; TPH and TOC samples were collected in 1-L and $200-\mathrm{mL}$ amber glass bottles, respectively. The water samples for analysis of BTEX, TPH, TOC, and naphthalene wrere delivered to a commercial laboratory on the day of collection for analysis by USEPA methods 8020, 418.1, 415.1, and 8020, respectively (U.S. Environmental Protection Agency, 1983; U.S. Environmental Protection Agency, Office of Solid Waste, 1986). The water samples collected on February 28, 1995, for analysis of BTEX were analyzed by USEPA method 8240 (U.S. Environmental Protection Agency, Office of Solid Waste, 1986).

Water samples for analysis of inorganic ions were collected using either a syringe or a peristaltic pump and filtered through a $0.45-\mu \mathrm{m}$ porous-membrane filter into sample-rinsed polyethylene bottles. The inorganic ion samples were packed in ice immediately following collection. Ammonium, calcium, magnesium, potassium, and sodium constituents were quantified in the laboratory by ion-exchange chromatography using chemical suppression and conductivity detection. Chloride, bromide, nitrate, nitrite, phosphate, and sulfate concentrations were determined in the laboratory by ion-exchange chromatography using chemical suppression and conductivity detection as specified in USEPA method 300.0 (U.S. Environmental Protection Agency, 1983).

Water samples for analysis of organic acids were collected in sample-rinsed 40-mL an ber glass bottles similar to the sample-collection method for BTEX. The organic acid samples were not chemically preserved, but were capped with Teflon-lined septa, and placed on ice. Samples were analyzed for acetate, formate, propionate, and isobutyrate by ion-exclusion chromatography using chemical suppression and conductivity detection (Bradley and others, 1993).

Water samples for analysis of lead and arsenic were collected in sample-rinsed 500-mL polyethylene bottles after passing through a $0.45-\mu \mathrm{m}$ porous-membrane filter. Samples were packed on ice and delivered to a commercial laboratory on the day of collection for analysis by USEPA methods 7060 and 7421 (U.S. Environmental Protection Agency, Office of Solid Waste, 1986).

Methane and dissolved inorganic carbon (DIC) samples were collected using a syringe to inject $5 \mathrm{~mL}$ of sample water through a $0.45-\mu \mathrm{m}$ porous-membrane filter into sealed septated vials. The syringe and vials were rinsed with filtered sample water prior to sampling. The samples wrere 
packed in ice to minimize concentration changes due to microbial activity. Concentrations of methane in the head space were quantified by thermal-conductivity detection gas chromatography and converted to concentrations in water using Henry's Law coefficients (Stumm and Morgan, 1981). The DIC samples were acidified in the laboratory with a 42.5 percent phosphoric acid solution. Headspace carbon dioxide concentrations were quantified by thermal-conductivity detection gas chromatography. Dissolved inorganic carbon concentrations were then calculated using Henry's Law coefficients (Stumm and Morgan, 1981)

Laboratory alkalinity values were determined by end-point titration for seven samples collected on July 8,1991 . For water from all wells where a pH value and DIC concentration was determined (including the seven samples for which laboratory titrations were completed), alkalinity values were calculated using the formula (Stumm and Morgan, 1981):

$$
[\text { Alkalinity }]=\mathrm{C}\left(\alpha_{1}+2 \alpha_{2}\right)+\left[\mathrm{OH}^{-}\right]-\left[\mathrm{H}^{+}\right]
$$

where

$\mathrm{C}$ is the concentration of DIC,

$$
\begin{aligned}
& \alpha_{1}=\left(\frac{\left[H^{+}\right]}{K_{1}}+1+\frac{K_{2}}{\left[H^{+}\right]}\right)^{-1} \\
& \alpha_{2}=\left(\frac{\left[H^{+}\right]^{2}}{K_{1} K_{2}}+\frac{\left[H^{+}\right]}{K_{2}}+1\right)^{-1}
\end{aligned}
$$

$\left[\mathrm{H}^{+}\right]$is the activity of hydrogen ion,

$\left[\mathrm{OH}^{-}\right]$is the activity of hydroxyl ion, and

$\mathrm{K}_{1}$ and $\mathrm{K}_{2}$ are equilibrium constants (Garrels and Christ, 1965).

For quality control and assurance, replicate samples for all analyses were collected and analyzed on at least 10 percent of the total number of individual analyses for each sample event. Replicate samples not showing analytical agreement were reanalyzed when possible. The vast majority of the wells were sampled using equipment dedicated to each respective well. In such cases, there was little or no potential for cross contamination. Thus, equipment blanks generally were not collected. Selected equipment blanks were collected in the few instances where sampling equipment was used for multiple wells. 
Ground-water samples were collected for analysis of hydrogen gas using the bubble-strip method of Chapelle and McMahon (1991). A stream of water was pumped from the well through a gas-sampling bulb at an approximate rate of $600 \mathrm{~mL}$ per minute. An injected bubble of nitrogen in the bulb asymptotically collected hydrogen and other soluble gases until equilibrium was achieved. Once equilibrium was achieved (less than 5-percent change in 5 minutes, which typically occurred within 15 minutes of initiating the flow of water through the bulb), gas was extracted from the bulb using a gas-tight syringe. Hydrogen was measured on a gas chromatograph equipped with a reduction gas detector. The detection limit of this method for samples collected at this site varied between 0.1 and $0.5 \mathrm{nM}$. Hydrogen samples typically were not collected if the ground water at the well was aerobic. All hydrogen samples were collected in duplicate. Each duplicate was separately analyzed; however, hydrogen values were reported as average values. Differences between duplicate samples were typically less than 10 percent.

Water samples, including two replicates, were collected in July 1991, July 1992, and May 1993 from 17 wells located on and adjacent to the facility for the analysis of volatile-organic and extractable-organic compounds. This analysis provided a list of tentatively identified compounds in the water samples, an estimated concentration of each tentatively identified compound, and in some cases, a number that represents the probability that the compound was correctly identifed. Volatile-organic samples were collected in 40-mL glass vials and preserved with hydrochloric acid. Extractable samples were collected in 1-L amber glass bottles and not chemically preserved. The volatile and extractable samples were placed on ice and delivered to a commerial laboratory on the day of collection for analysis by USEPA methods 8240 and 8270 , respectively (U.S. Environmental Protection Agency, Office of Solid Waste, 1986).

\section{Water-Level Measurements}

Water levels were measured in 97 surficial aquifer wells and at 4 surface-water sites located on the facility and adjacent properties. The data were used to characterize the shape and slope of the water-table surface in the study area and to record how this surface changed over time. Water levels were measured on a monthly basis in selected wells. The total number of water-level measurements each month varied during the study; however, all monthly measurements were made within an 8-hour period.

Water levels in the surficial aquifer also were measured by continuous water-level recordors (recorded at 15-minute intervals) in three 6-in. ID wells (W-103, W-107, and W-108) located on the facility (pl. 1). Elevations, relative to sea level, were determined for established measuring points at each well and surface-water site by differential leveling to provide a common datım. Depths to water from the measuring point were made using a weighted steel tape. At least two measurements were made in each well to ensure accuracy. Water-level measurements at surfecewater sites were obtained using a stadia rod to determine the vertical distance from the water surface to the permanent measuring points. All measurements were corrected to sea level.

\section{Lake-Bottom-Sediment Collection and Analysis}

Four lake-bottom sediment samples were collected from three locations in Gold Cup Springs Lake on February 8, 1994 (pl. 1). Two samples, identified as Outfall-1 and Input-2, w'ere collected immediately downstream of stream outfalls. A replicate sample (Outfall-1R) was collected from a separate hole, located about 1.5-ft downstream from Outfall-1, because the 
sample volume obtained at Outfall-1 was insufficient for splitting. Another sample, identified as Lake Center, was collected from approximately the middle of the lake and downstream from both

outfalls. Samples were collected using a coring device that consisted of a 2 -in. ID PVC coring tube with an internal water-tight plunger (smaller diameter inner rod with rubber O-ring at bottom). The bottom of the coring device was advanced through the lake-water column with the rubber seal of the internal plunger situated at the lip of the coring tube. The coring device was pushed through the accumulated mud at the bottom of the lake until a solid bottom was encountered. As the coring tube was hammered into the bottom sediments, the internal plunger remained in place at the top of the solid sediments. The coring tube was advanced approximately $1 \mathrm{ft}$ into the bottom sediments. The device allowed sample collection with minimal influence from surface water. Care was taken to minimize lateral movement, which would result in the introduction of surface water into the cored hole.

Samples were extruded by the plunger and immediately placed into clean glass containers with Teflon-lined caps. Sediments were packed tightly into the containers to minimize trapped air space. The coring device was scrubbed with detergent and deionized water prior to collecting each sample. The samples were packed in ice and immediately transported to a commercial laboratory for analysis. Lake-bottom sediments were analyzed for various volatile-organic compounds using USEPA methods 8010 and 8020, extractable-organic compounds using USEPA method 8270, and metals using USEPA methods 6010 and 7471 (U.S. Environmental Protection Agency, Office of Solid Waste, 1986). The constituents analyzed and their detection limits are listed in table 3 (at end of report).

\section{WATER-QUALITY DATA}

Water-quality data collected at the facility between December 1990 and January 1996 were separated into three groups: water-quality constituents and properties, organic water-chemistry data, and inorganic water-chemistry data (tables 4-6, at end of report). Water-quality constituent and property measurements in the field included the determination of dissolved oxygen, hydrogen, hydrogen sulfide, iron, and titrated alkalinity concentrations, $\mathrm{pH}$, specific conductance, and water temperature. The organic data included concentrations of volatile-organic compounds (BTEX), extractable-organic compounds (TPH and naphthalene), TOC, methane, and organic acid compounds (formate, acetate, propionate, and isobutyrate). Inorganic data included concentrations of major ions, calculated alkalinity, DIC, and metals (arsenic and lead).

Continuous specific conductance data were recorded at 15-minute intervals for various periods of record for wells MWGS-33A, MWGS-33B, MWGS-34A, and MWGS-34B (pl. 1). Specific conductance data were recorded at well MWGS-33A between February 5, 1992 and December 5, 1995, and at well MWGS-33B between February 21, 1992 and December 4, 1995 (fig. 1). Specific conductance data were recorded at wells MWGS-34A and MWGS-34B between February 5, 1992 and December 5, 1995 (fig. 1). 
$\mathbf{a}$

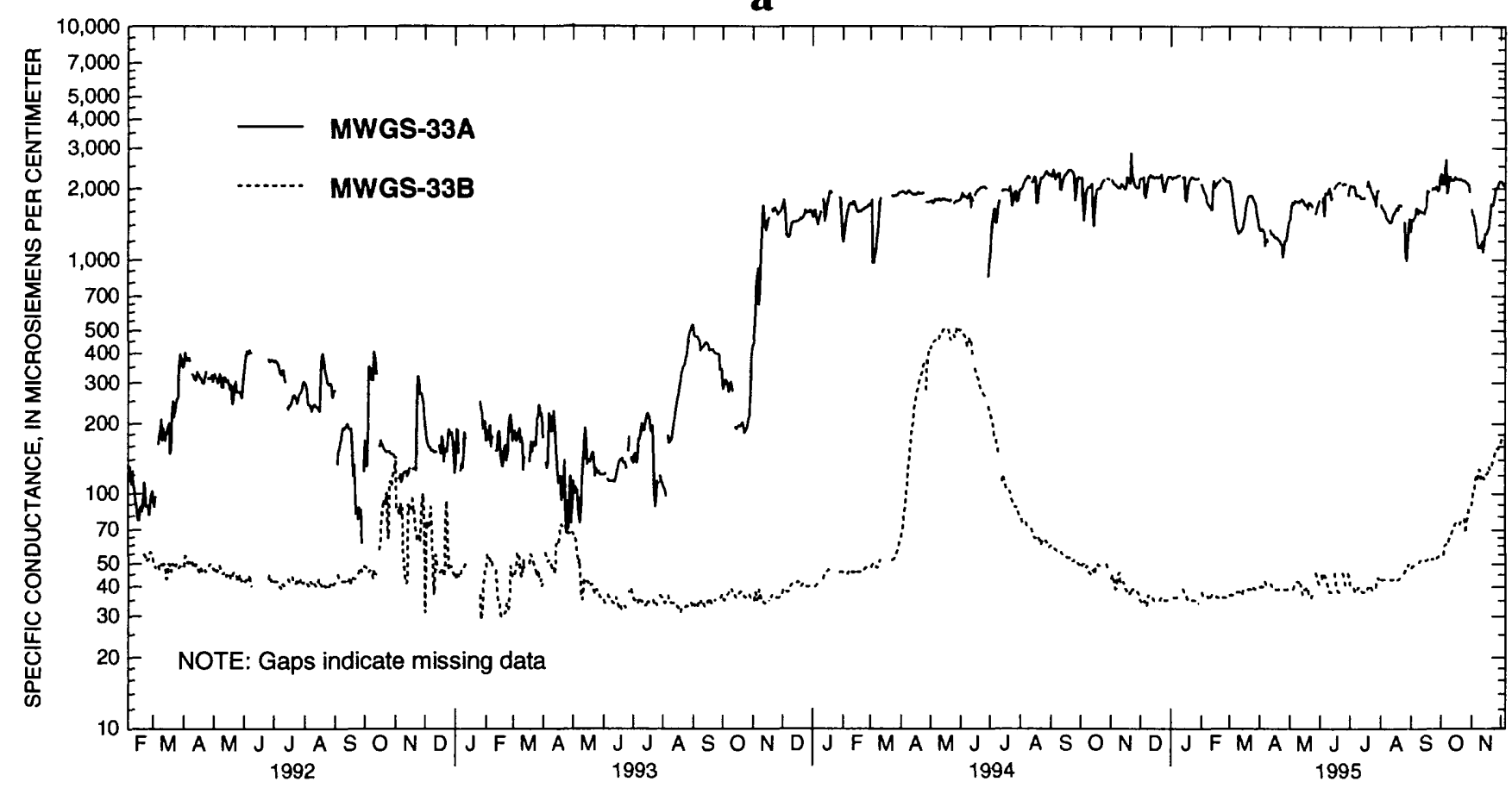

b

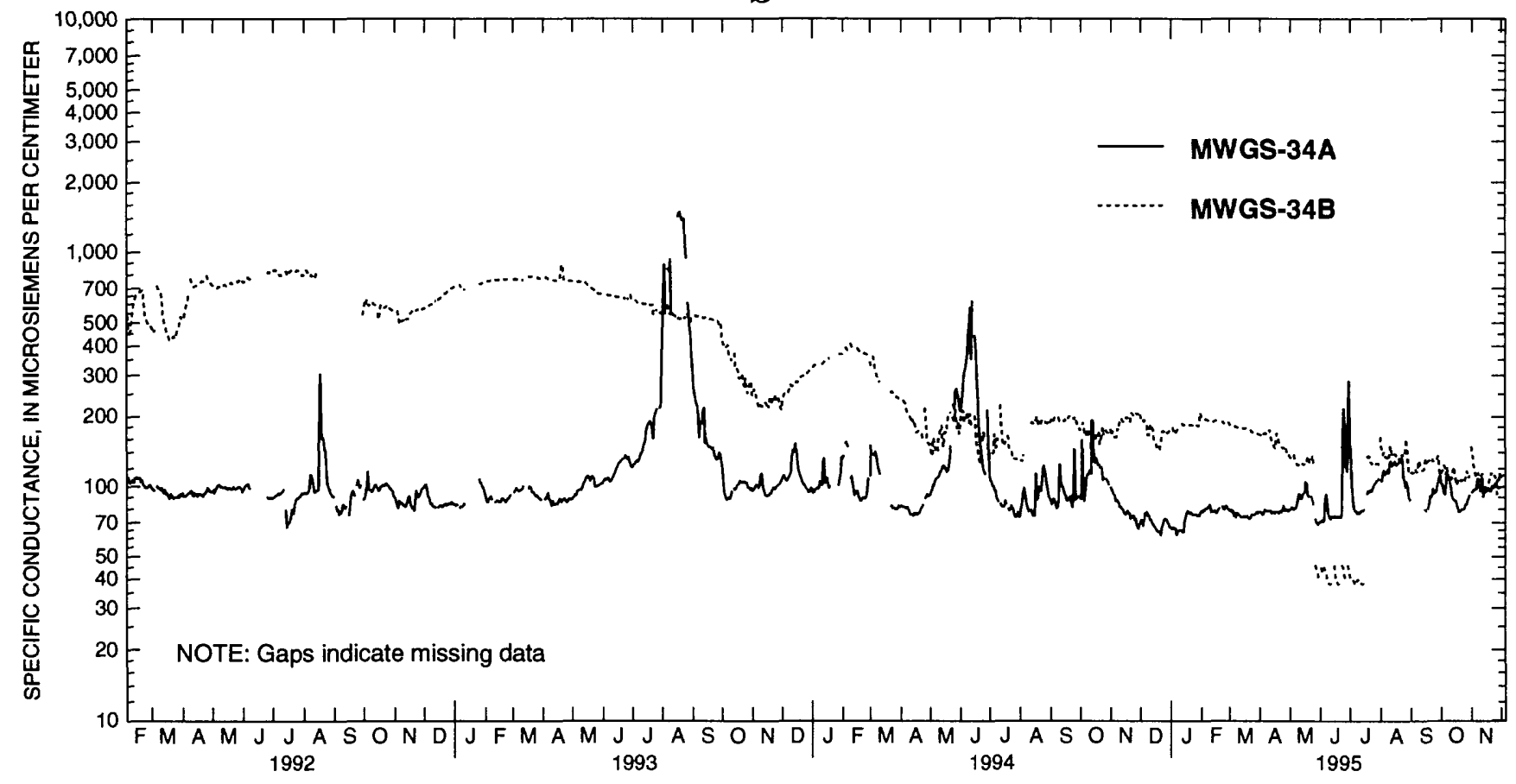

Figure 1. Specific conductance in (a) wells MWGS-33A and MWGS-33B and (b) wells MWGS-34A and MWGS-34B at the Defense Fuel Supply Point, Hanahan, S.C., February 5, 1992 to December 5, 1995. 
Results of the tentative identification of volatile- and extractable-organic compounds using comparison of mass spectral data are listed in table 7 (at end of report). These results list tentatively identified volatile- and extractable-organic compounds with probability numbers for identification that exceed 80 percent for the 19 water samples (includes 2 replicate samples) collected in 17 wells at or near the facility. Sample MWGS-20R was not analyzed for the volatile fraction of organic compounds. Methylene chloride found in analytical blanks associated with the samples from wells EW-05 and EW-07, collected in June 1992, and MWGS-40B and MWGS40BR, collected in July 1992 may represent laboratory-induced contamination (Pete Ballou, General Engineering Laboratories, written commun., 1992).

Equipment blanks were collected for BTEX analyses as necessary. In most instances, individual BTEX compounds were not detected at concentrations exceeding the minimum detection limits of $2 \mu \mathrm{g} / \mathrm{L}$ for benzene, toluene, and ethylbenzene, and $4 \mu \mathrm{g} / \mathrm{L}$ for total xylener (table 5). On January 12, 1994, an equipment blank collected prior to sampling well PW-01A was found to contain $9.1 \mu \mathrm{g} / \mathrm{L}$ benzene, $2.1 \mu \mathrm{g} / \mathrm{L}$ toluene, $2.5 \mu \mathrm{g} / \mathrm{L}$ ethylbenzene, and $7.3 \mu \mathrm{g} / \mathrm{L}$ total xylenes. However, concentrations of these compounds in water from well PW-01A were found to be below the minimum detection limits. On April 26, 1994, an equipment blank collected prior to sampling well PW-02 was found to contain $2.1 \mu \mathrm{g} / \mathrm{L}$ ethylbenzene $(0.1 \mu \mathrm{g} / \mathrm{L}$ greater than the detection limit). The ethylbenzene concentration detected in the water sample from well PW-02 $(240 \mu \mathrm{g} / \mathrm{L})$, however, was significantly higher than that detected in the equipment blank. Wells for which equipment blanks were collected prior to sampling are denoted in table 5. In three instances when free product was encountered or gross contamination was suspected, equipment blanks were collected after decontamination of sampling equipment to ensure that cleaning techniques were adequate. Analytical results for these three equipment blanks indicated no BTEX concentrations above the minimum detection limits.

\section{WATER-LEVEL DATA}

The water-level data from wells and surface-water sites located at or near the facility are presented in table 8 (at end of report). These data show the changing water-table conditions in the study area for 97 wells and 4 surface-water sites for the period of record between April 1991 and September 1995.

Continuous water-level data were recorded at 15 -minute intervals for various periods of record for wells W-103, W-107, and W-108. Water-level data were recorded at well W-10: between February 28, 1991 and January 9, 1994, and at well W-108 between February 27, 199] and November 30, 1993 (fig. 2). Continuous water-level monitoring was discontinued at both of these wells when free-phase petroleum was discovered floating on the water-table surface in the wells. Continuous water-level data were recorded at well W-107 from December 14, 1993 to December 5, 1995 (fig. 2).

\section{LAKE-BOTTOM-SEDIMENT DATA}

All lake-bottom-sediment analyses for volatile organics, extractable organics, and metals resulted in the detection of the same compounds (table 9). Methylene chloride was the only' volatile organic detected in the lake-bottom sediments. Methylene chloride was not found ir 
analytical blanks associated with these four samples (Karen Blakeney, General Engineering Laboratories, oral commun., 1996); and therefore, detection of this compound in these samples may indicate an actual presence in the lake-bottom sediments. Concentrations of this compound varied between 12 and $19 \mu \mathrm{g} / \mathrm{kg}$ at the 3 sample locations. There were no extractable orgarics detected in any of the samples. Samples analyzed for metals produced detectable concentrations of chromium, lead, and zinc at all three sample locations. The concentrations of chromium and zinc were similar at each sample location, with values that varied between 2.5 and $7.8 \mathrm{mg} / \mathrm{kg}$ for chromium and between 5.4 and $14 \mathrm{mg} / \mathrm{kg}$ for zinc. The concentrations of lead determined for the three sample locations were $54,3.5,3.9$, and $6.5 \mathrm{mg} / \mathrm{kg}$ for locations Outfall-1, Outfall- $1 \mathrm{R}$, Infrut2 , and Lake Center, respectively (pl. 1).

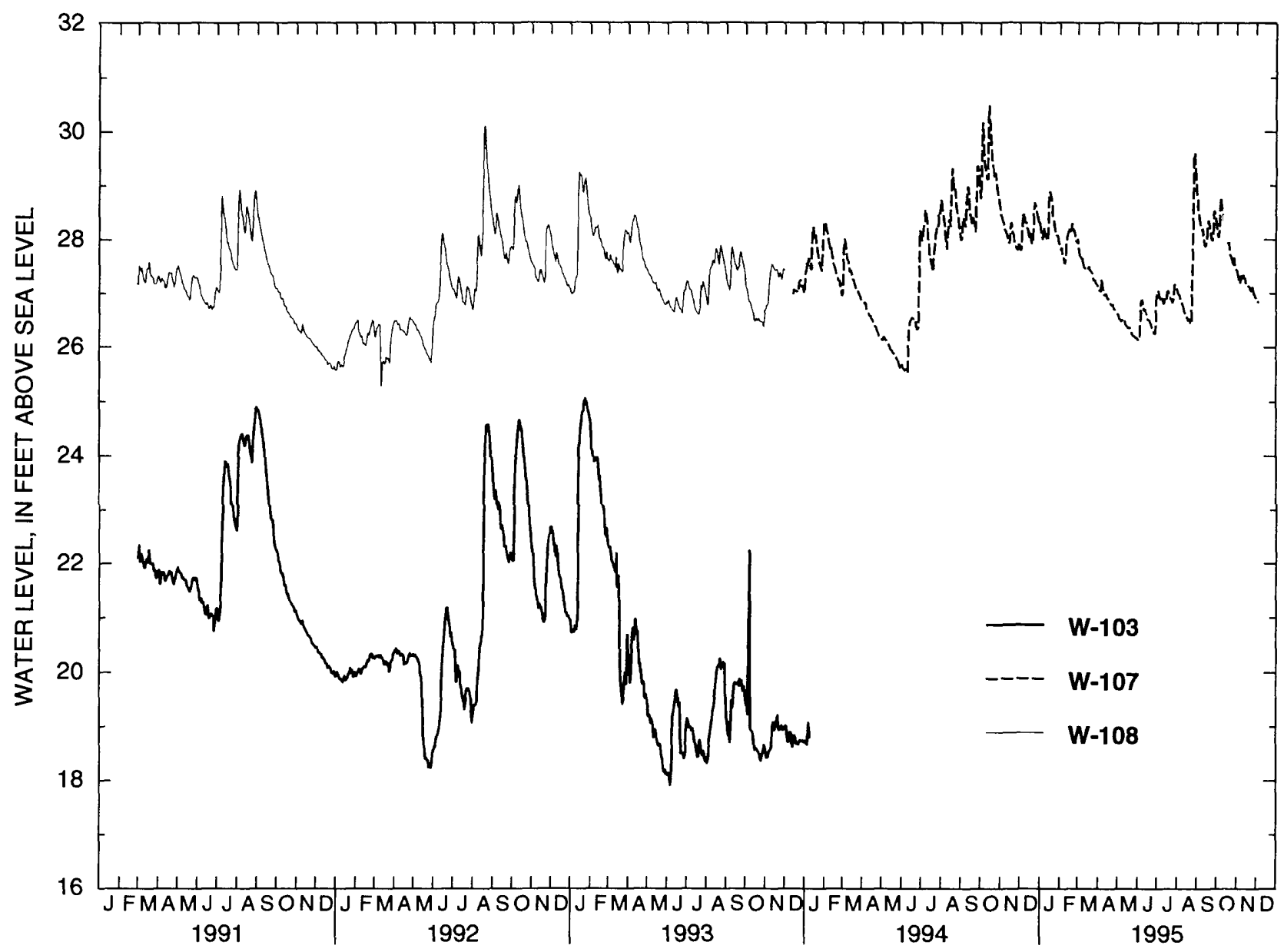

Figure 2. Hydrograph of wells W-103, W-107, and W-108 at the Defense Fuel Supply Point, Hanahan, S.C., February 27, 1991 to December 5, 1995. 


\section{SUMMARY}

This report presents the results of a study to monitor the changes in surface- and groundwater quality and water-table elevations in wells located on the Defense Fuel Supply Point and adjacent properties, Hanahan, S.C. Fieldwork completed between December 1990 and January 1996 included drilling to obtain lithologic descriptions of the sediments of the surficial aquifer and to allow the installation of monitoring wells; collection of surface- and ground-water samples to delineate contaminated areas and to monitor temporal changes in the distribution of the contaminant plumes; measurement of water levels in wells and streams to determine the direction of ground-water flow; and collection of lake-bottom sediments to determine whether fuel-related contaminants were present.

Eighty-five monitoring wells and piezometers were installed at or near the DFSP facility by the USGS or under direction of the USGS. All wells were installed in the surficial aquifer from June 1990 to June 1994. Ninety-one wells and 13 surface-water sites located on and adjacent to the facility were sampled intermittently during this investigation. Water levels were measured monthly in 97 wells and at 4 surface-water sites located at or near the facility using a steel tape (wells) and a stadia rod (surface-water sites). Water levels in the surficial aquifer also were measured by continuous water-level recorders (recorded at 15 -minute intervals) in three 6-in. ID wells located at the facility. Lake-bottom-sediment samples were collected from three locations in Gold Cup Springs Lake on February 8, 1994, and analyzed for volatile- and extractable-organic compounds and metals. 


\section{REFERENCES}

American Society for Testing and Materials, 1994, Annual Book of Standards, Section 4, v. 04.08, Philadelphia, Penn.

Bradley, P.M., Chapelle, F.H., and Vroblesky, D.A., 1993, Does lead affect microbial metabolism in aquifer sediments under different terminal electron accepting conditions?:

Geomicrobiology Journal, v.11, p. 85-94.

Chapelle, F.H., and McMahon, P.B., 1991, Geochemistry of dissolved inorganic carbon in a coastal plain aquifer: 1 . Sulfate from confining beds as an oxidant in microbial $\mathrm{CO}_{2}$ production, Journal of Hydrology, no. 127, p. 85-108.

Garrels, R.M., and Christ, C.L., 1965, Solutions, Minerals, and Equilibria: Harper \& Row, New York, $450 \mathrm{p}$.

Hach Company, 1992, Water Analysis Handbook: Hach Company, Loveland, Colorado, 831 p.

RMT, Inc., 1988, Final Report, aquifer evaluation, Defense Fuel Supply Point, Charleston, Carolina: Consultant's report to Defense Fuel Supply Center, January 1988, 22 p.

Stookey, L.L., 1970, FerroZine, a new spectrophotometric reagent for iron, Analytical Chemistry, p. 779-781.

Stumm, Werner, and Morgan, J.J., 1981, Aquatic Chemistry, 2nd ed.: Wiley, New York, $78 \mathrm{C}$ p.

U.S. Army Environmental Hygiene Agency, 1975, Water quality geohydrologic consultation no. 24-020-76.

U.S. Environmental Protection Agency, 1983, Methods for chemical analysis of water and waste, EPA-600/4-79-020: U.S. Environmental Protection Agency, Washington, D.C.

U.S. Environmental Protection Agency, Office of Solid Waste, 1986, Test Methods for Evaluating Solid Waste, Physical/Chemical Methods, SW-846 (3rd ed.): U.S. Environmental Protection Agency, Washington, D.C., 1919 p.

Vroblesky, D.A., and Chapelle, F.H., 1994, Temporal and spatial changes of terminal electronaccepting processes in a petroleum hydrocarbon-contaminated aquifer and the significance for contaminant biodegradation, Water Resources Research, v. 30, no. 5, p. 1561-1570.

Vroblesky, D.A., Robertson, J.F., Petkewich, M.D., Chapelle, F.H., Bradley, P.M., and Landmeyer, J.E., in press, Remediation of petroleum hydrocarbon-contaminated ground water in the vicinity of a jet-fuel tank farm, Hanahan, South Carolina: U.S. Geological Survey Water-Resources Investigations Report 96-4251.

Wentworth, C.K., 1922, A scale of grade and class terms for clastic sediment: Journal of Geology, v. 30, p. 377-392.

Wood, W.W., 1976, Guidelines for collection and field analysis of ground-water samples for selected unstable constituents: U.S. Geological Survey Techniques of Water-Resources Investigations, book 1, chap. D2, $24 \mathrm{p}$. 
TABLES 1-9 


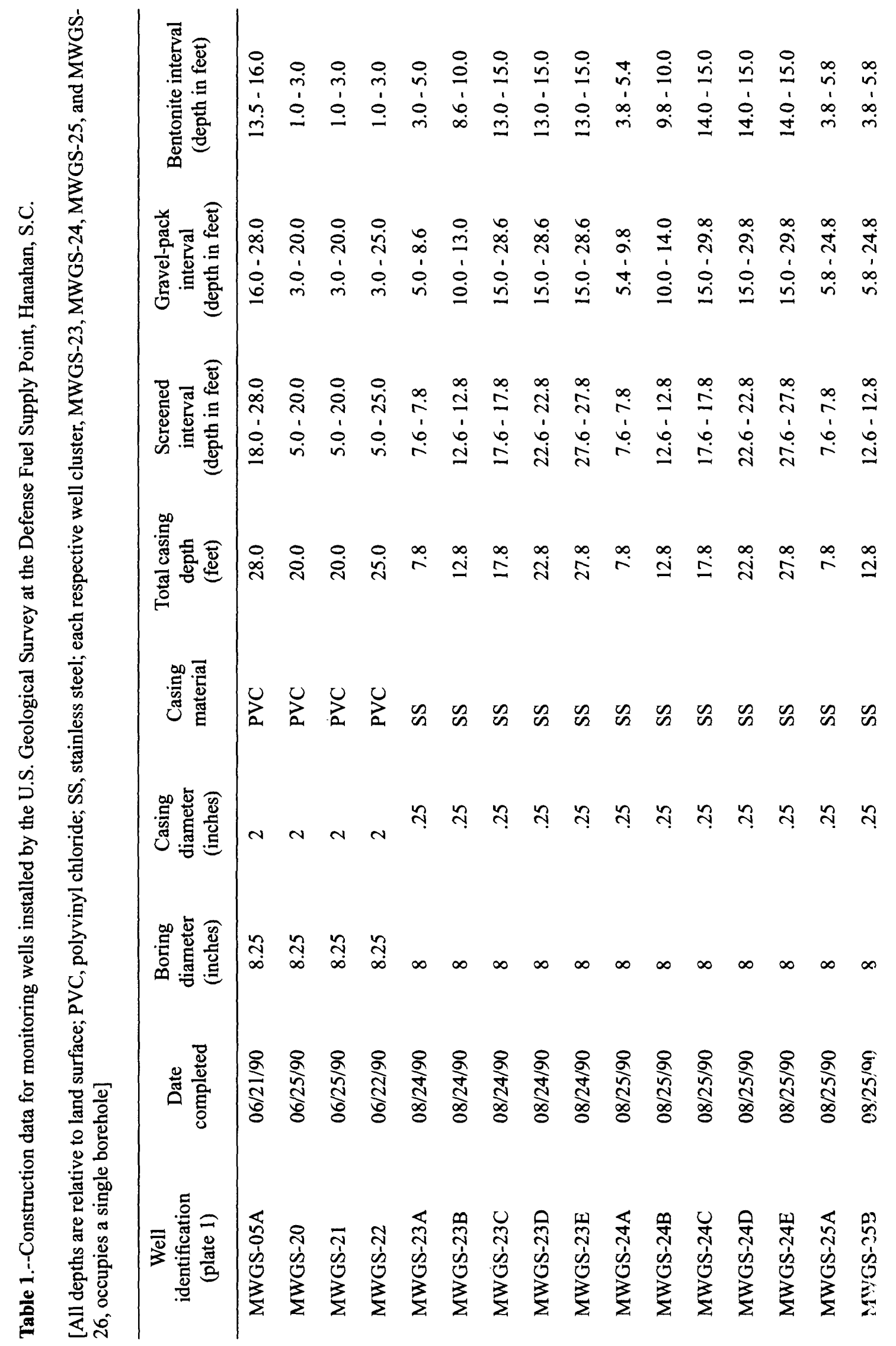




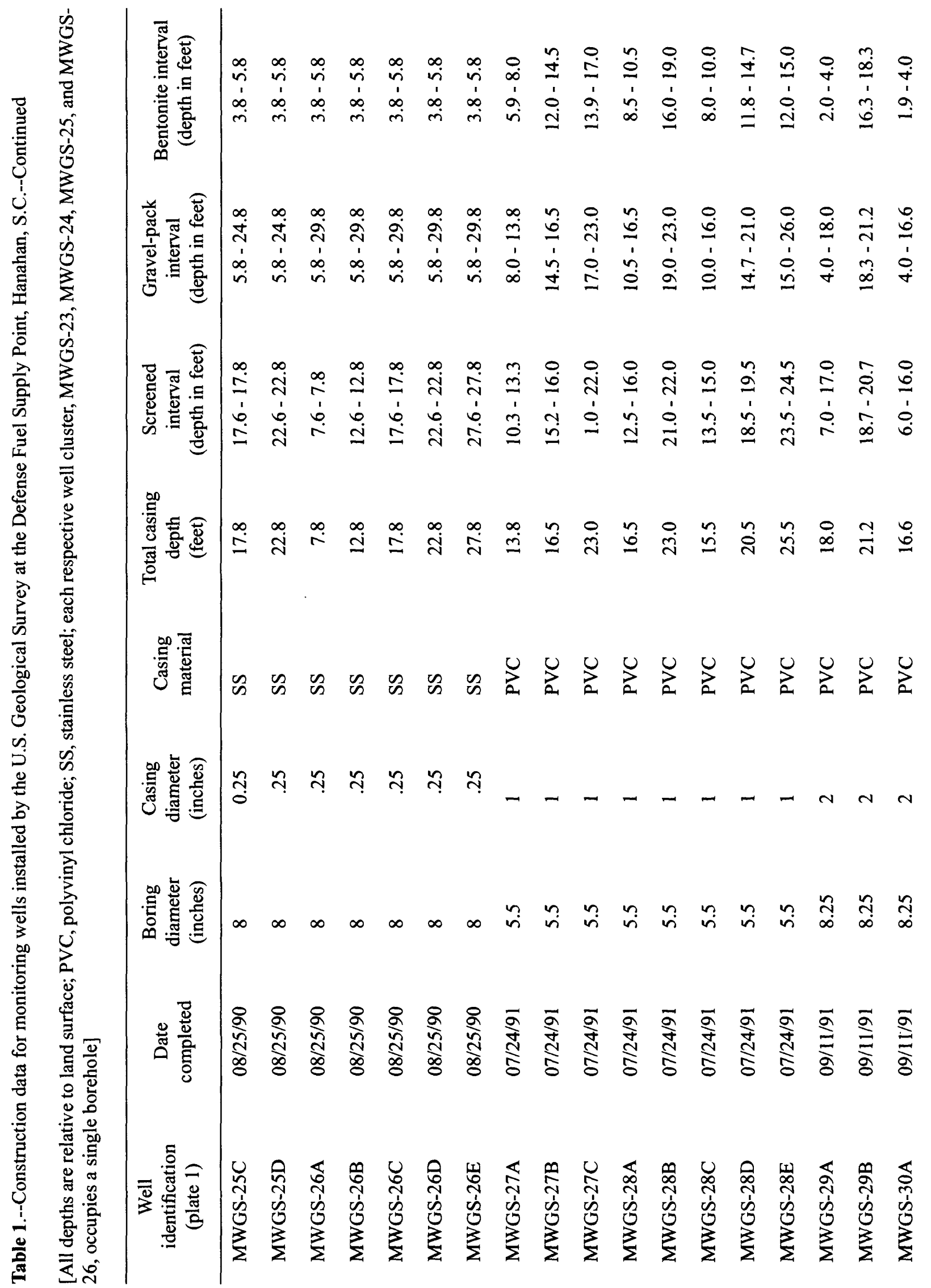




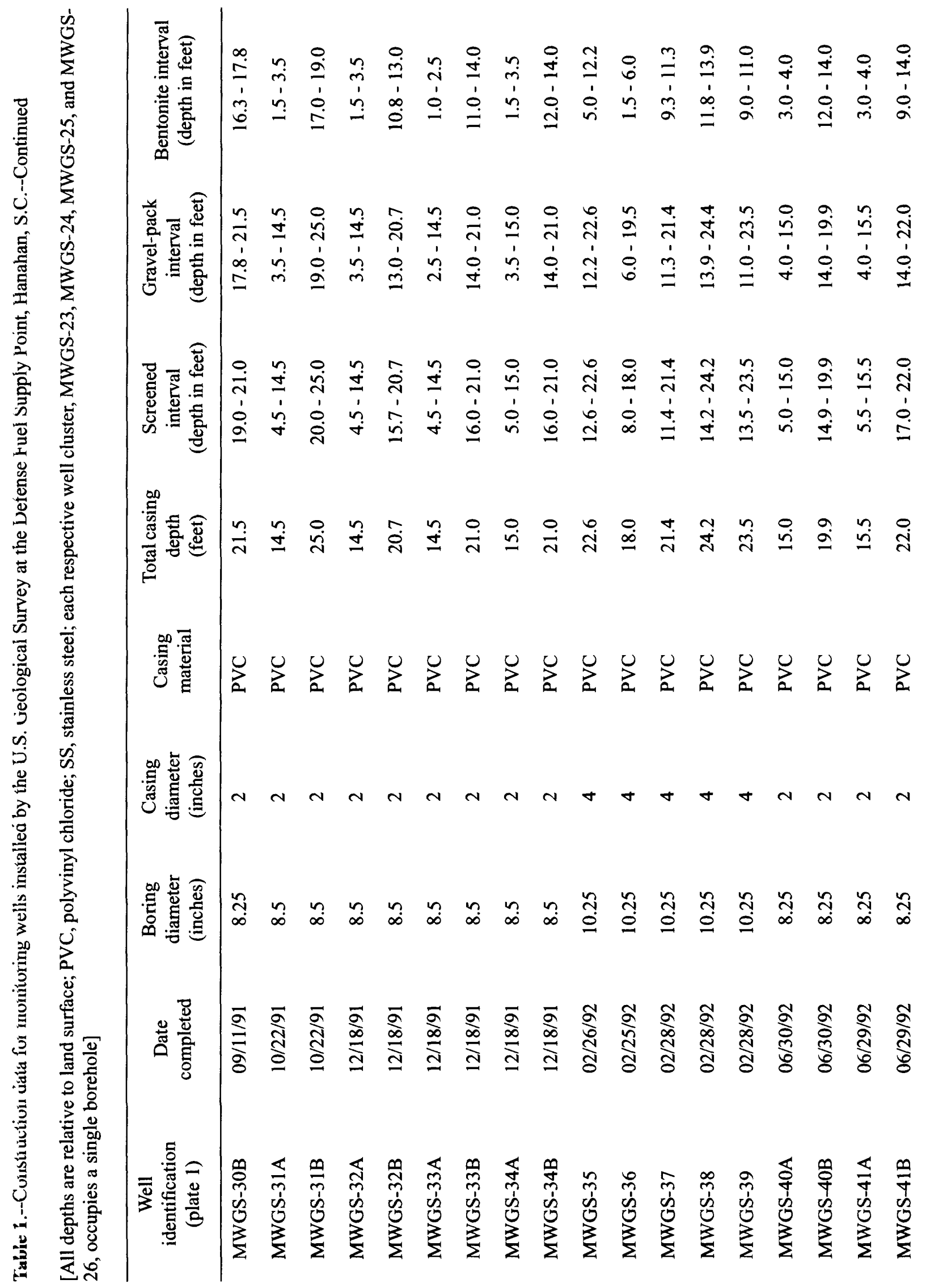




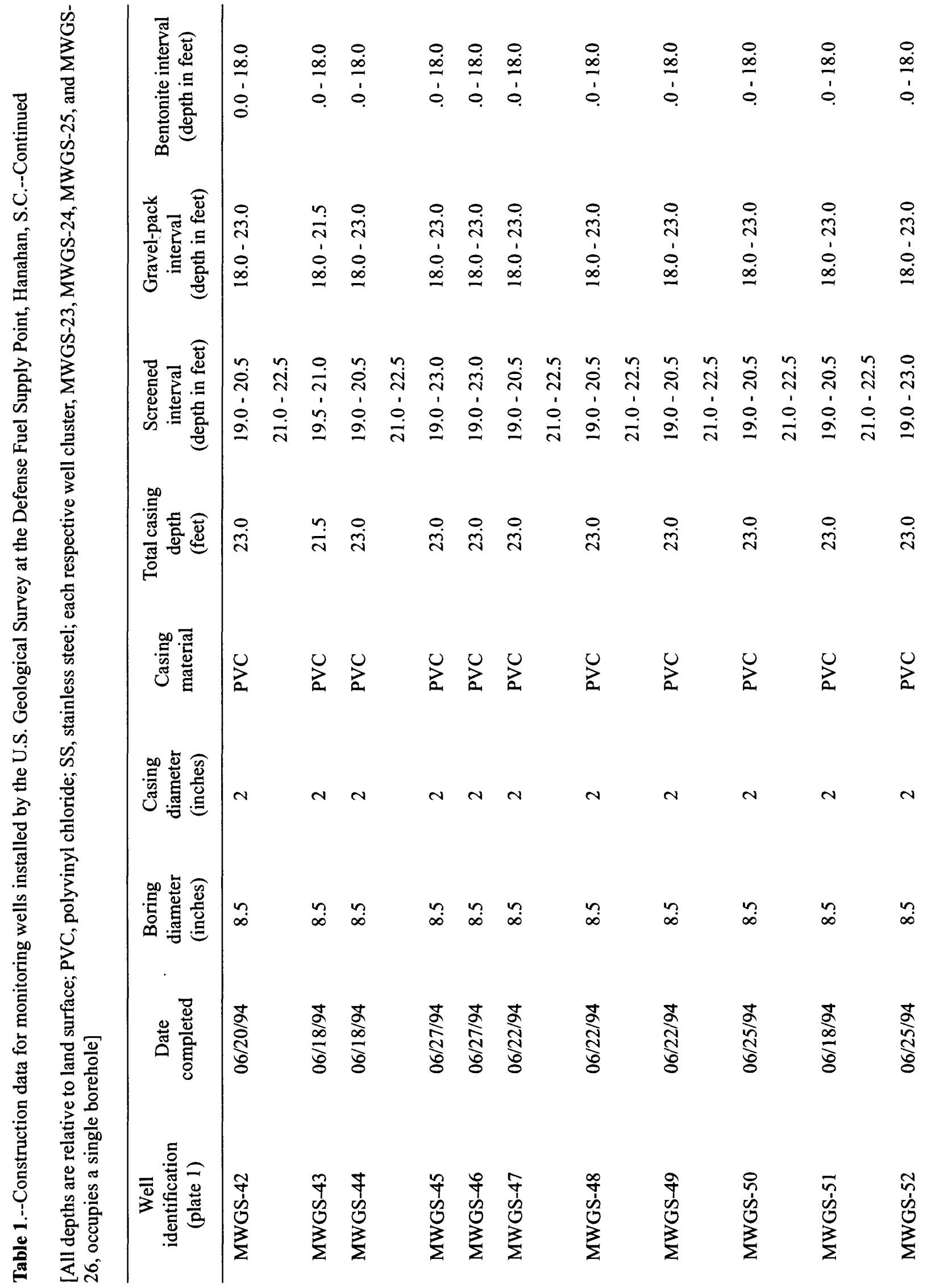




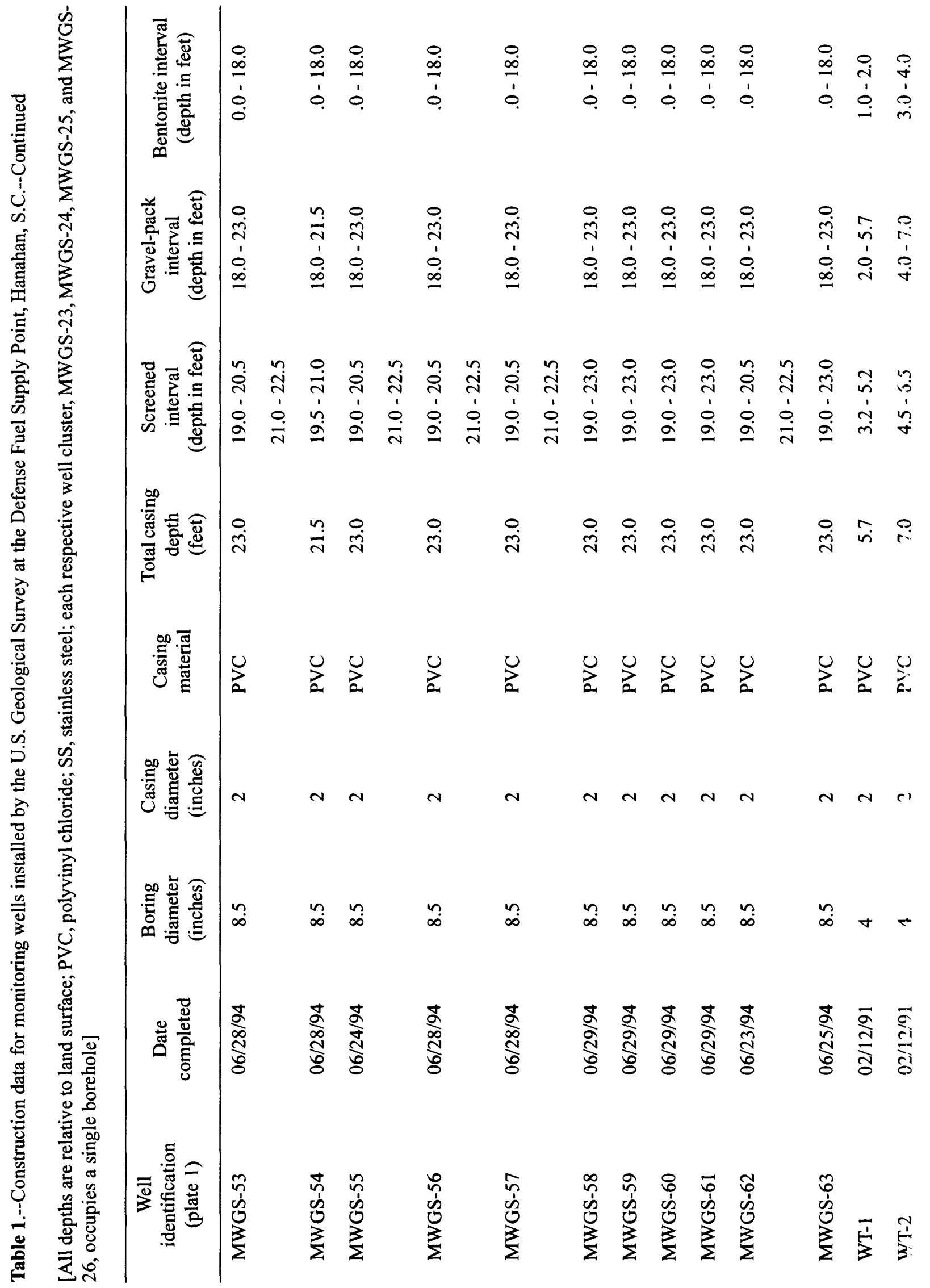




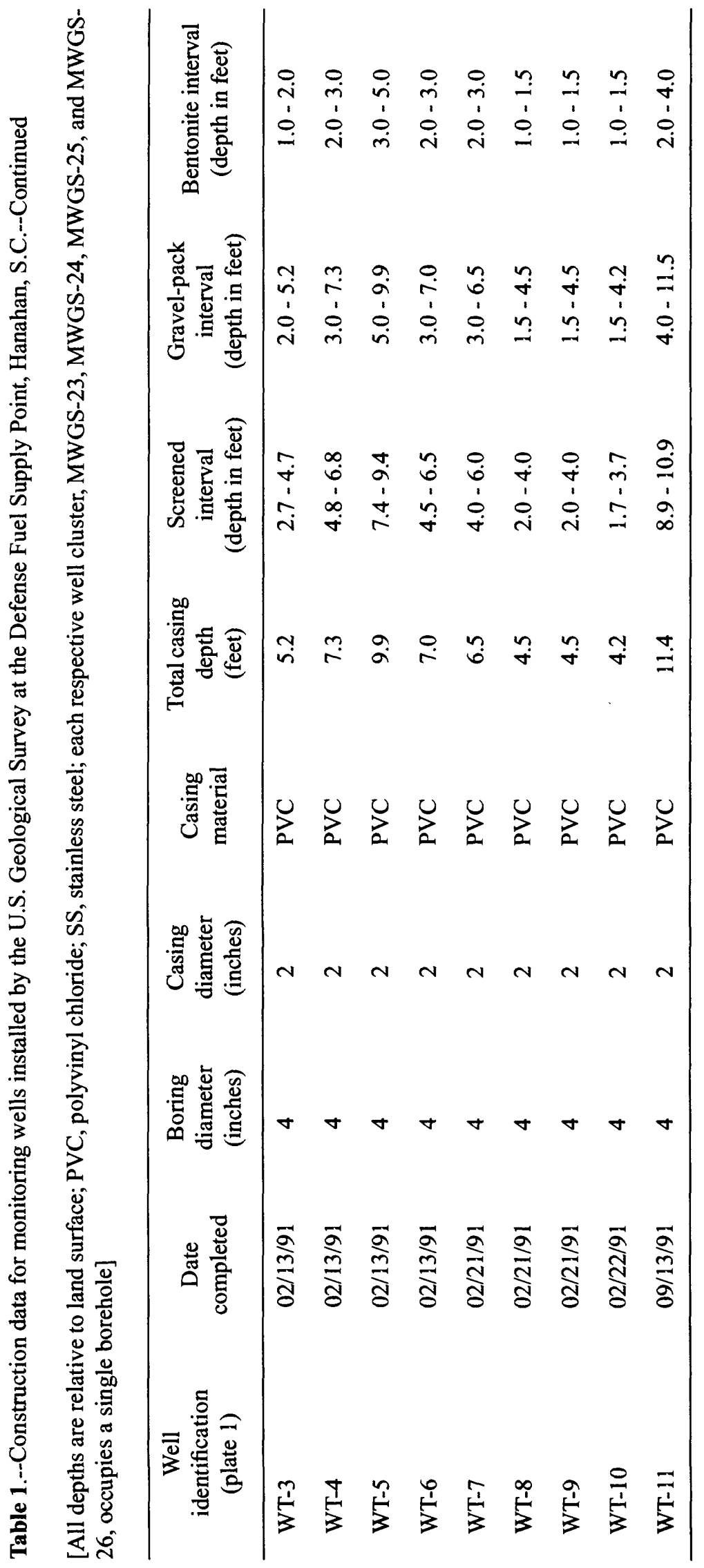




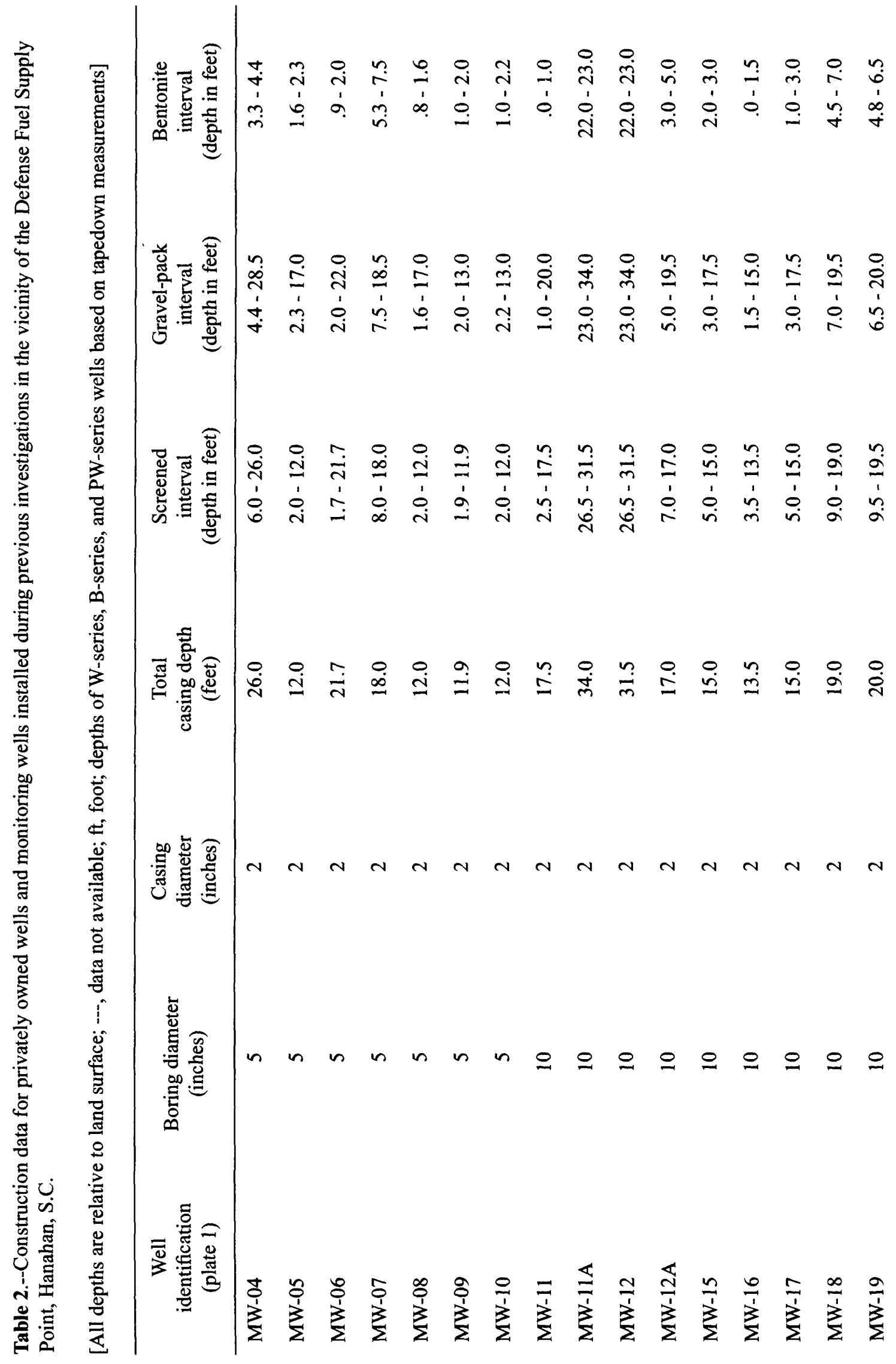




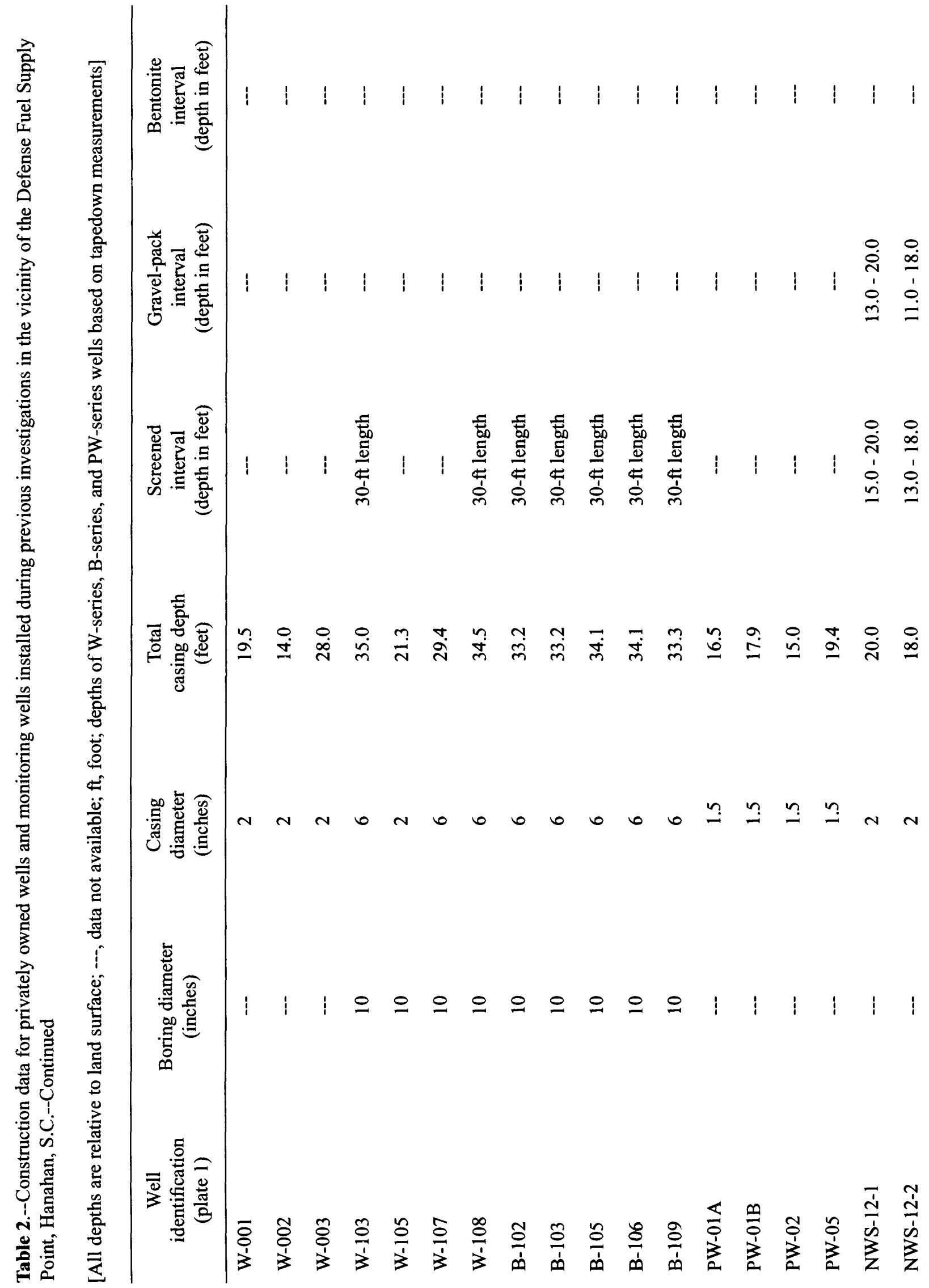




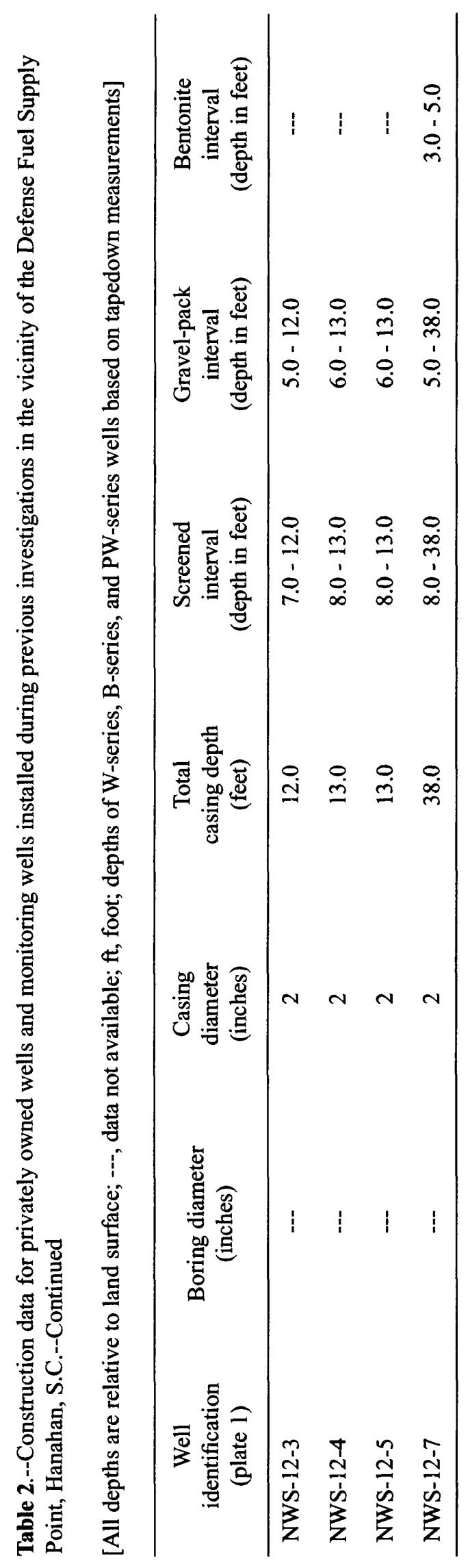


Table 3.--Analytical detection limits for volatile-organic, extractable-organic, and metal analyses determined for lake-bottom-sediment samples collected from Gold Cup Springs Lake, Hanahan, S.C., February 8, 1994

[All organic compound detection-limit concentrations are reported in micrograms per kilogram ( $\mu \mathrm{g} / \mathrm{kg}$ ); all metal detection-limit concentrations are reported in milligrams per kilogram $(\mathrm{mg} / \mathrm{kg})$; PCB, polychlorinated biphenyls; BHC, benzene hexachloride]

\begin{tabular}{|c|c|}
\hline Constituent & Detection limit \\
\hline \multicolumn{2}{|c|}{ Volatile organics $^{1}$} \\
\hline 1,1,1-Trichloroethane & 10.0 \\
\hline 1,1,2,2-Tetrachloroethane & 10.0 \\
\hline 1,1,2-Trichloroethane & 10.0 \\
\hline 1,1-Dichloroethane & 10.0 \\
\hline 1,1-Dichloroethylene & 10.0 \\
\hline 1,2-Dichlorobenzene & 10.0 \\
\hline 1,2-Dichloroethane & 10.0 \\
\hline 1,2-Dichloropropane & 10.0 \\
\hline 1,2-trans-Dichloroethylene & 10.0 \\
\hline 1,3-Dichlorobenzene & 10.0 \\
\hline 1,4-Dichlorobenzene & 10.0 \\
\hline 2-Chloroethyl vinyl ether & 10.0 \\
\hline Benzene & 10.0 \\
\hline Bromoform & 10.0 \\
\hline Carbon tetrachloride & 10.0 \\
\hline Chlorobenzene & 10.0 \\
\hline Chlorodibromomethane & 10.0 \\
\hline Chloroethane & 10.0 \\
\hline Chloroform & 10.0 \\
\hline Dichlorobromomethane & 10.0 \\
\hline Dichlorodifluoromethane & 10.0 \\
\hline Ethylbenzene & 10.0 \\
\hline Methyl bromide & 10.0 \\
\hline Methyl chloride & 10.0 \\
\hline
\end{tabular}


Table 3.--Analytical detection limits for volatile-organic, extractable-organic, and metal analyses determined for lake-bottom-sediment samples collected from Gold Cup Springs Lake, Hanahan, S.C., February 8, 1994 --Continued

[All organic compound detection-limit concentrations are reported in micrograms per kilogram $(\mu \mathrm{g} / \mathrm{kg})$; all metal detection-limit concentrations are reported in milligrams per kilogram $(\mathrm{mg} / \mathrm{kg})$; $\mathrm{PCB}$, polychlorinated biphenyls: BHC, benzene hexachloride]

\begin{tabular}{|c|c|c|}
\hline Constituent & & Detection limit \\
\hline Methylene chloride & & 10.0 \\
\hline Tetrachloroethylene & & 10.0 \\
\hline Toluene & & 10.0 \\
\hline Trichloroethylene & & 10.0 \\
\hline Trichlorofluoromethane & & 10.0 \\
\hline Vinyl chloride & & 10.0 \\
\hline cis-1,3-Dichloropropylene & & 10.0 \\
\hline \multirow[t]{2}{*}{ trans-1,3-Dichloropropylene } & & 10.0 \\
\hline & $\begin{array}{l}\text { Extractable organics }{ }^{2} \\
\text { (Priority pollutant acid) }\end{array}$ & \\
\hline 2,4,6-Trichlorophenol & & 6,540 \\
\hline 2,4-Dichlorophenol & & 6,540 \\
\hline 2,4-Dimethylphenol & & 6,540 \\
\hline 2,4-Dinitrophenol & & 13,100 \\
\hline 2-Chlorophenol & & 6,540 \\
\hline 2-Nitrophenol & & 6,540 \\
\hline 2-Methyl-4,6-dinitrophenol & & 6,540 \\
\hline 4-Nitrophenol & & 124,000 \\
\hline 4-Chloro-3-methylphenol & & 6,540 \\
\hline Pentachlorophenol & & 6,540 \\
\hline Phenol & & 6,540 \\
\hline
\end{tabular}

Extractable organics ${ }^{2}$

(Priority pollutant base/neutral)

1,2,4-Trichlorobenzene

6,540

1,2-Diphenylhydrazine

13,100

2,4-Dinitrotoluene

6,540 
Table 3.--Analytical detection limits for volatile-organic, extractable-organic, and metal analyses determined for lake-bottom-sediment samples collected from Gold Cup Springs Lake, Hanahan, S.C., February 8, 1994 --Continued

[All organic compound detection-limit concentrations are reported in micrograms per kilogram $(\mu \mathrm{g} / \mathrm{kg})$; all metal detection-limit concentrations are reported in milligrams per kilogram $(\mathrm{mg} / \mathrm{kg}) ; \mathrm{PCB}$, polychlorinated biphenyls; BHC, benzene hexachloride]

\begin{tabular}{|c|c|}
\hline Constituent & Detection limit \\
\hline 2,6-Dinitrotoluene & 6,540 \\
\hline 2-Chloronaphthalene & 6,540 \\
\hline 3,3'-Dichlorobenzidine & 13,100 \\
\hline 4,4'-Dichlorodiphenyldichloroethane (DDD) & 13,100 \\
\hline 4,4'-Dichlorodiphenyldichloroethylene (DDE) & 13,100 \\
\hline 4,4'-Dichlorodiphenyltrichloroethane (DDT) & 13,100 \\
\hline 4-Bromophenyl phenyl ether & 13,100 \\
\hline 4-Chlorophenyl phenyl ether & 6,540 \\
\hline Acenaphthene & 6,540 \\
\hline Acenaphthalene & 6,540 \\
\hline Aldrin & 22,000 \\
\hline Anthracene & 6,540 \\
\hline Benzidine & 13,100 \\
\hline Benzo(a)anthracene & 6,540 \\
\hline Benzo(a)pyrene & 6,540 \\
\hline Benzo(b)fluoranthene & 12,200 \\
\hline Benzo(ghi)perylene & 6,540 \\
\hline Benzo(k)fluoranthene & 16,400 \\
\hline Butyl benzyl phthalate & 6,540 \\
\hline Chlordane & 13,100 \\
\hline Chrysene & 13,100 \\
\hline Di-n-butyl phthalate & 13,100 \\
\hline Di-n-octyl phthalate & 13,100 \\
\hline Dibenzo(a,h)anthracene & 6,540 \\
\hline Dieldrin & 23,900 \\
\hline Diethyl phthalate & 6,540 \\
\hline
\end{tabular}


Table 3.--Analytical detection limits for volatile-organic, extractable-organic, and metal analyses determined for lake-bottom-sediment samples collected from Gold Cup Springs Lake, Hanahan, S.C., February 8, 1994 --Continued

[All organic compound detection-limit concentrations are reported in micrograms per kilogram $(\mu \mathrm{g} / \mathrm{kg})$; all me ${ }^{+} \mathrm{l}$ detection-limit concentrations are reported in milligrams per kilogram $(\mathrm{mg} / \mathrm{kg}) ; \mathrm{PCB}$, polychlorinated biphenyls; $\mathrm{BHC}$, benzene hexachloride]

\begin{tabular}{|c|c|}
\hline Constituent & Detection limit \\
\hline Dimethyl phthalate & 6,540 \\
\hline Endosulfan I & 13,100 \\
\hline Endosulfan II & 13,100 \\
\hline Endosulfan sulfate & 13,100 \\
\hline Endrin & 24,600 \\
\hline Endrin aldehyde & 44,700 \\
\hline Fluoranthene & 6,540 \\
\hline Fluorene & 6,540 \\
\hline Heptachlor & 29,200 \\
\hline Heptachlor epoxide & 13,100 \\
\hline Hexachlorobenzene & 6,540 \\
\hline Hexachlorobutadiene & 6,540 \\
\hline Hexachlorocyclopentadiene & 14,300 \\
\hline Hexachloroethane & 6,540 \\
\hline Indeno(1,2,3-c,d)pyrene & 13,100 \\
\hline Isophorone & 6,540 \\
\hline N-Nitrosodimethylamine & 6,540 \\
\hline N-Nitrosodiphenylamine & 13,100 \\
\hline N-Nitrosodipropylamine & 6,540 \\
\hline Naphthalene & 6,540 \\
\hline Nitrobenzene & 6,540 \\
\hline PCB-1016 & 13,100 \\
\hline PCB-1221 & 13,100 \\
\hline PCB-1232 & 13,100 \\
\hline PCB-1242 & 13,100 \\
\hline PCB- 1248 & 13,100 \\
\hline
\end{tabular}


Table 3.--Analytical detection limits for volatile-organic, extractable-organic, and metal analyses determined for lake-bottom-sediment samples collected from Gold Cup Springs Lake, Hanahan, S.C., February 8, 1994 --Continued

[All organic compound detection-limit concentrations are reported in micrograms per kilogram $(\mu \mathrm{g} / \mathrm{kg})$; all metal detection-limit concentrations are reported in milligrams per kilogram $(\mathrm{mg} / \mathrm{kg}) ; \mathrm{PCB}$, polychlorinated biphenyls; BHC, benzene hexachloride]

\begin{tabular}{|c|c|}
\hline Constituent & Detection limit \\
\hline PCB-1254 & 13,100 \\
\hline PCB-1260 & 13,100 \\
\hline Phenanthrene & 6,540 \\
\hline Pyrene & 6,540 \\
\hline Toxaphene & 13,100 \\
\hline alpha-BHC & 13,100 \\
\hline beta-BHC & 13,100 \\
\hline bis(2-Chloroethoxy)methane & 6,540 \\
\hline bis(2-Chloroethyl)ether & 6,540 \\
\hline bis(2-Chloroisopropyl)ether & 6,540 \\
\hline bis(2-Ethylhexyl)phthalate & 13,100 \\
\hline delta-BHC & 13,100 \\
\hline gamma-BHC & 37,700 \\
\hline \multicolumn{2}{|c|}{ Metals $^{3}$} \\
\hline Antimony & 2.50 \\
\hline Arsenic & 2.50 \\
\hline Beryllium & 2.50 \\
\hline Cadmium & 2.50 \\
\hline Chromium & 2.50 \\
\hline Copper & 2.50 \\
\hline Lead & 2.50 \\
\hline Mercury & 0.200 \\
\hline Nickel & 2.50 \\
\hline Selenium & 2.50 \\
\hline
\end{tabular}


Table 3.--Analytical detection limits for volatile-organic, extractable-organic, and metal analyses determined for lake-bottom-sediment samples collected from Gold Cup Springs Lake, Hanahan, S.C., February 8, 1994 --Continued

[All organic compound detection-limit concentrations are reported in micrograms per kilogram $(\mu \mathrm{g} / \mathrm{kg})$; all metal detection-limit concentrations are reported in milligrams per kilogram $(\mathrm{mg} / \mathrm{kg}) ; \mathrm{PCB}$, polychlorinated biphenyls; BHC, benzene hexachloride]

\begin{tabular}{lcc}
\hline & Constituent & Detection limit \\
\hline Silver & 2.50 \\
Thallium & 2.50 \\
Zinc & 2.50 \\
\hline
\end{tabular}

${ }^{1}$ U.S. Environmental Protection Agency Methods 8010 and 8020.

${ }^{2}$ U.S. Environmental Protection Agency Method 8270.

${ }^{3}$ U.S. Environmental Protection Agency Methods 6010 and 7471. 


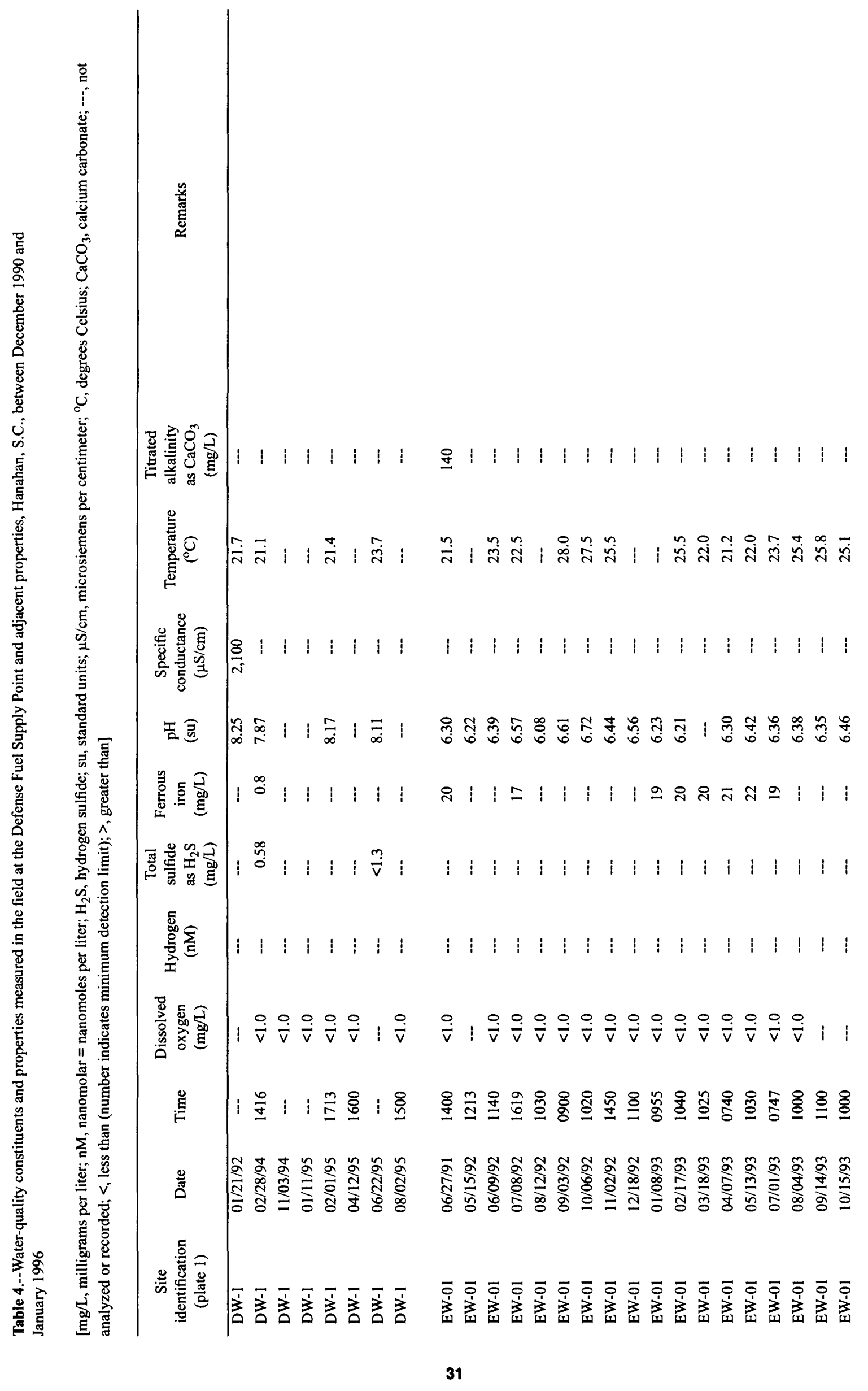




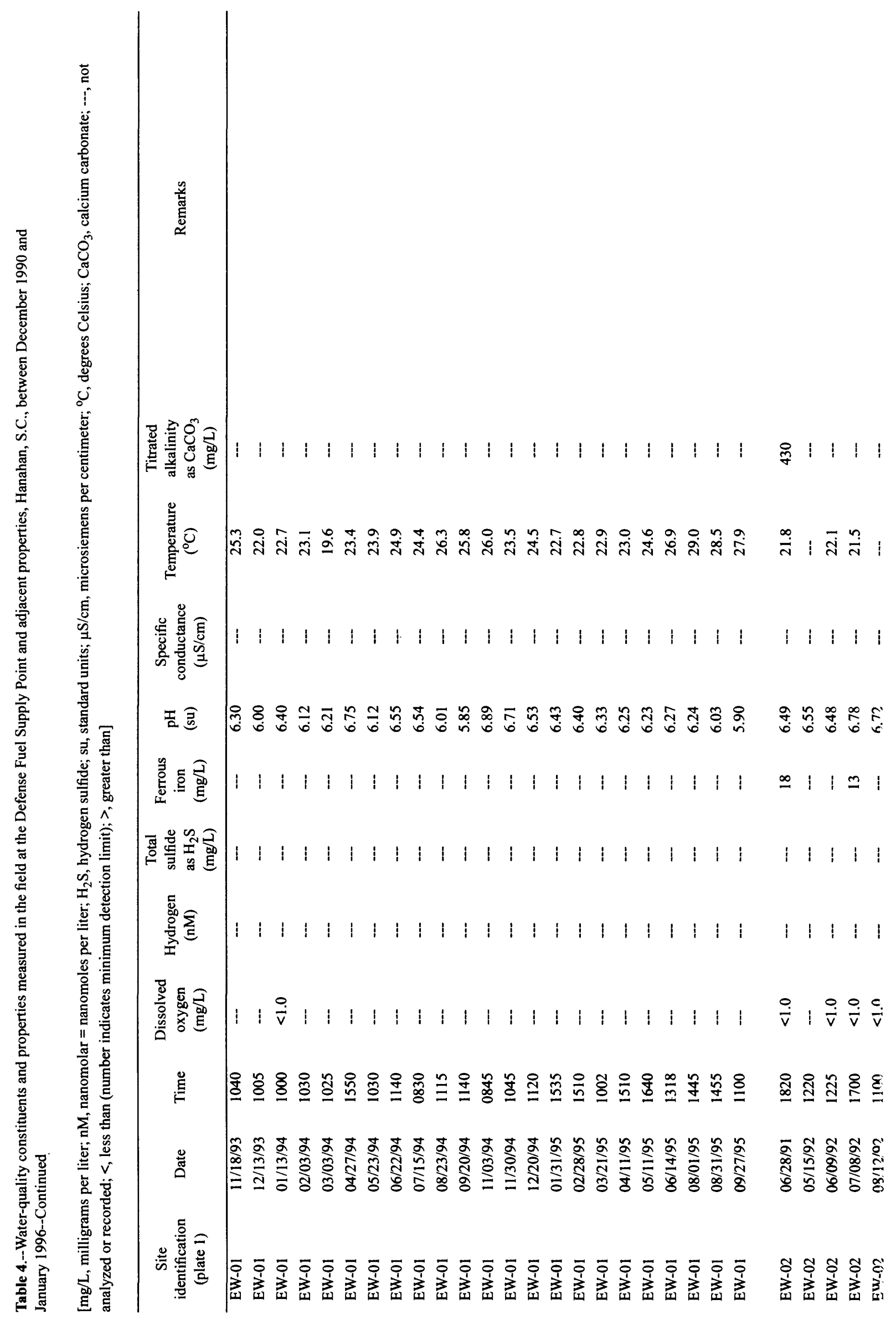




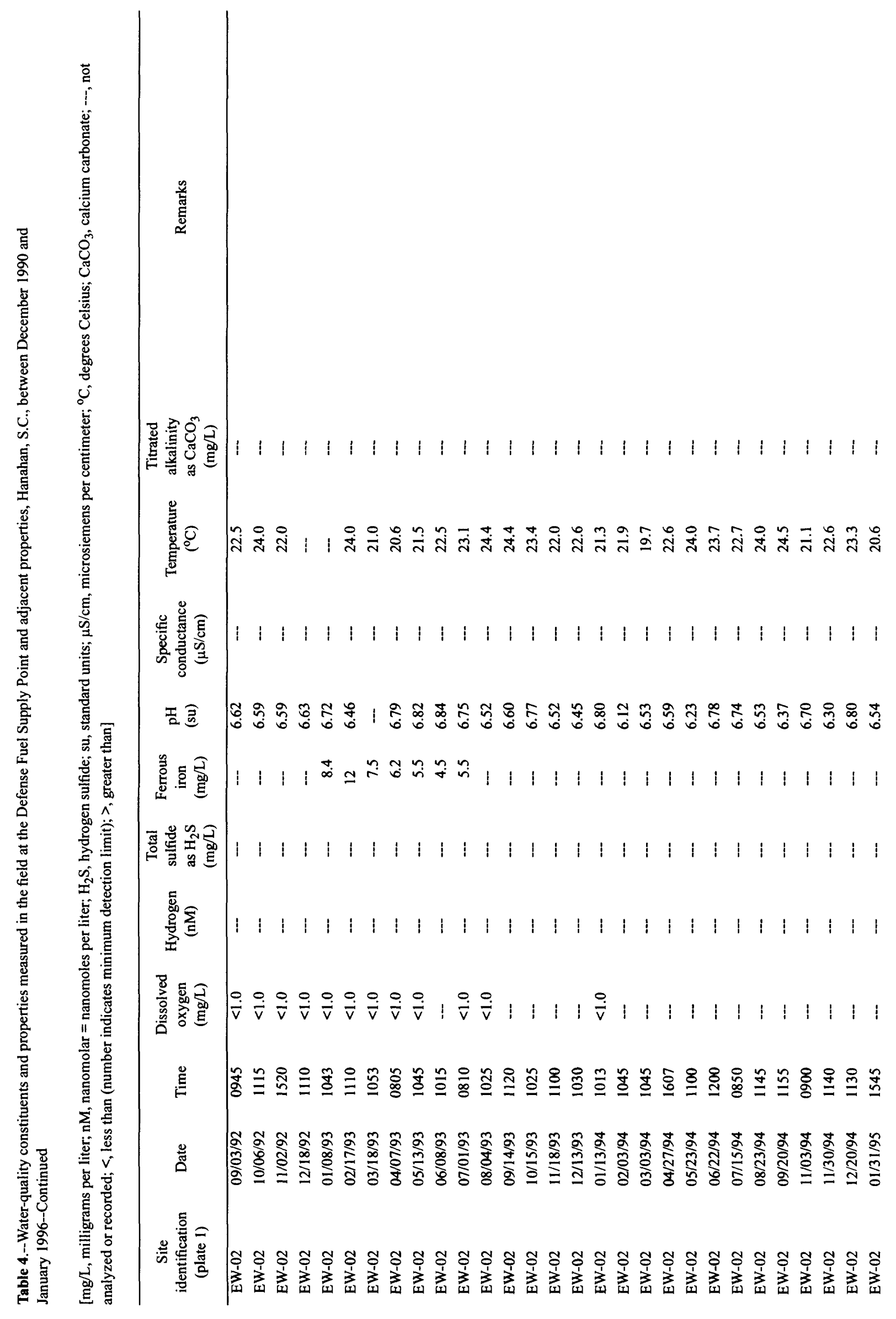




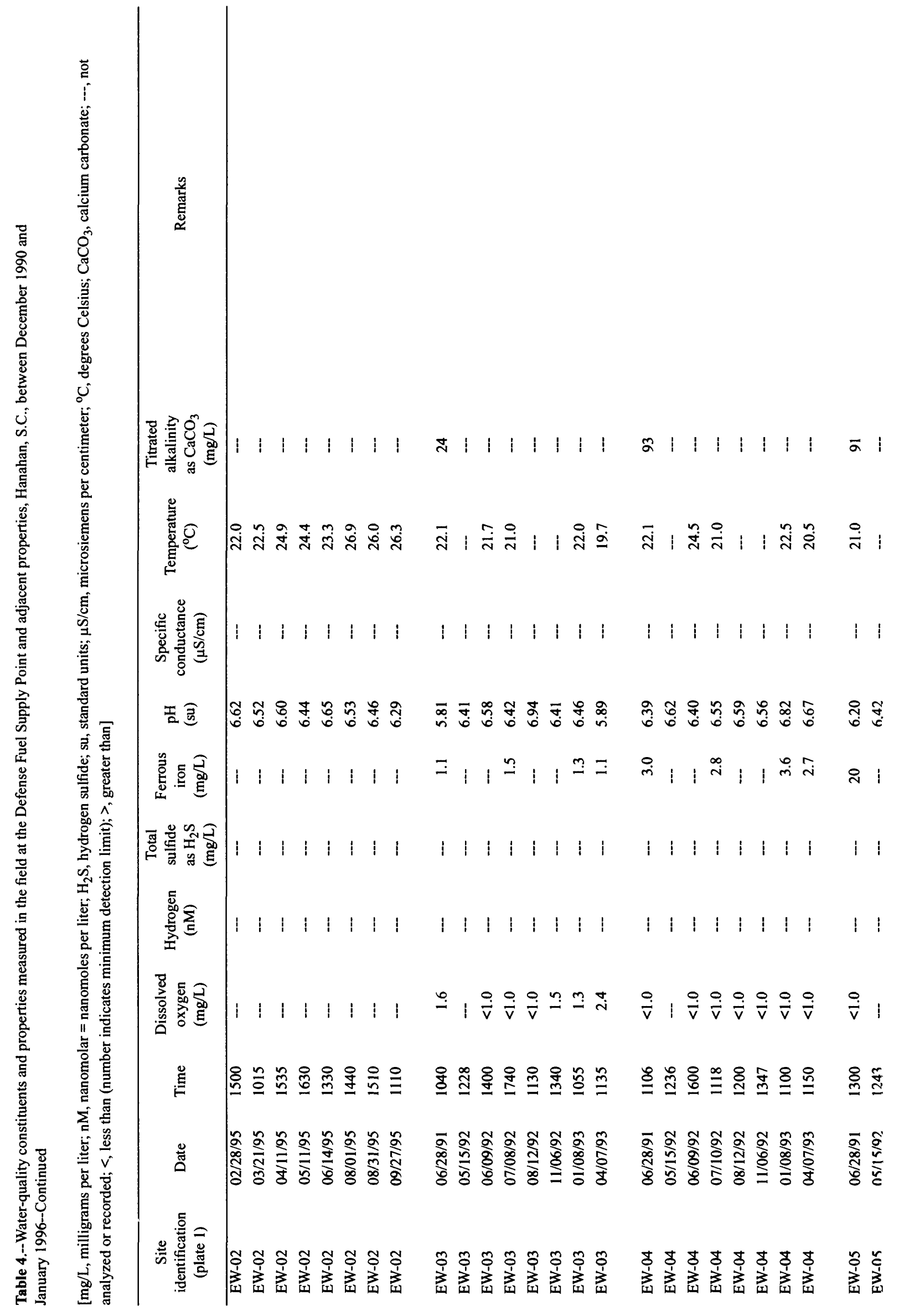




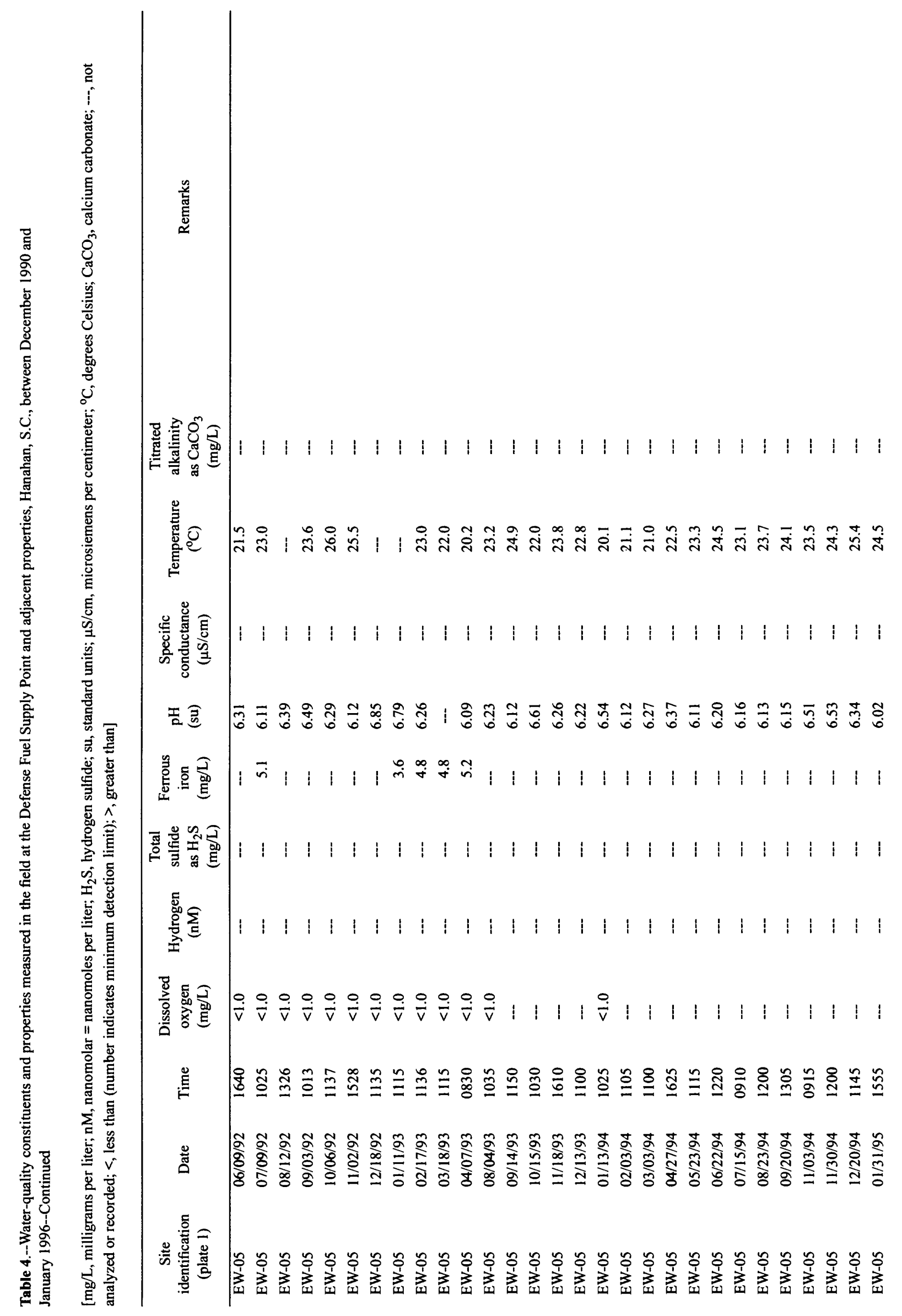




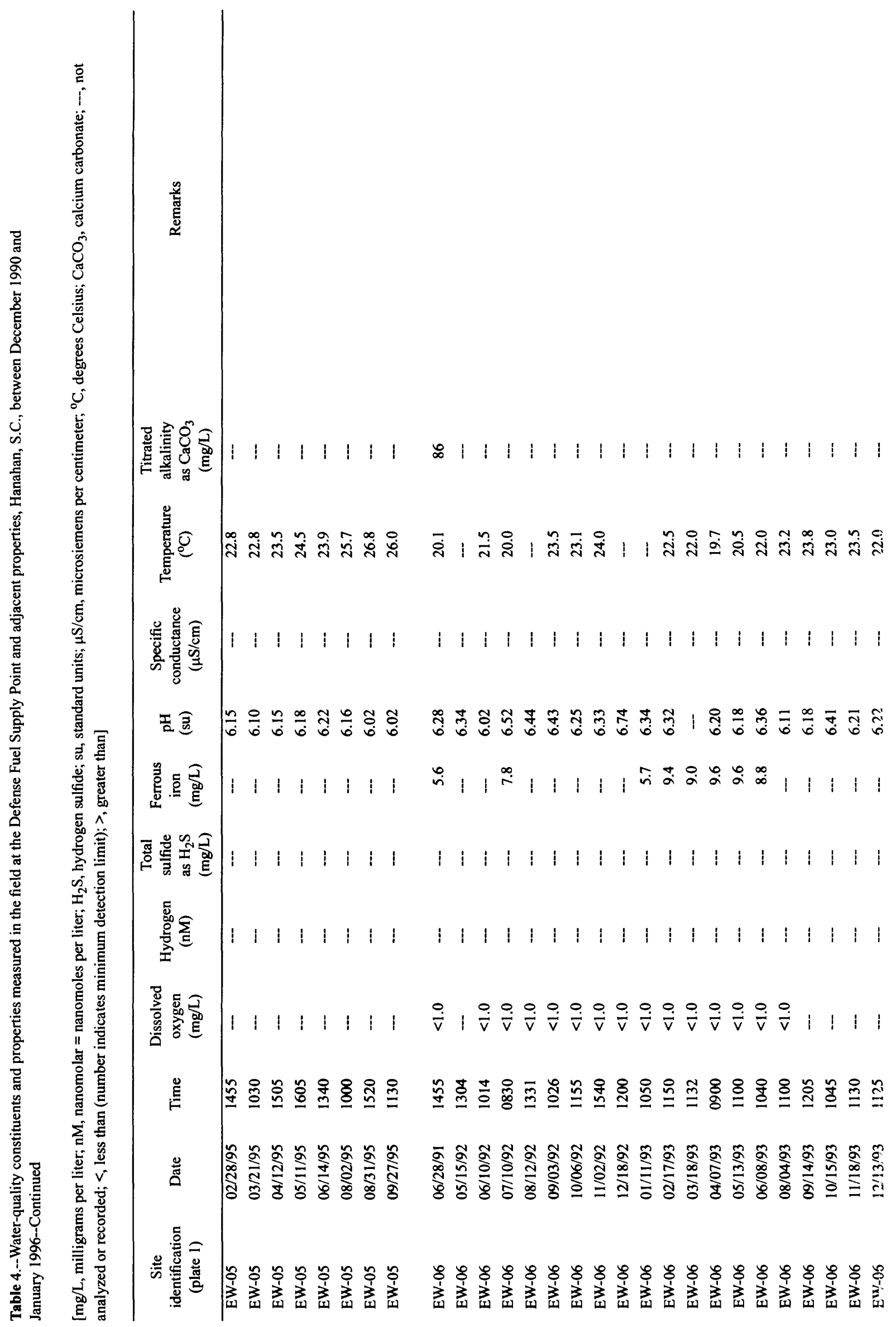




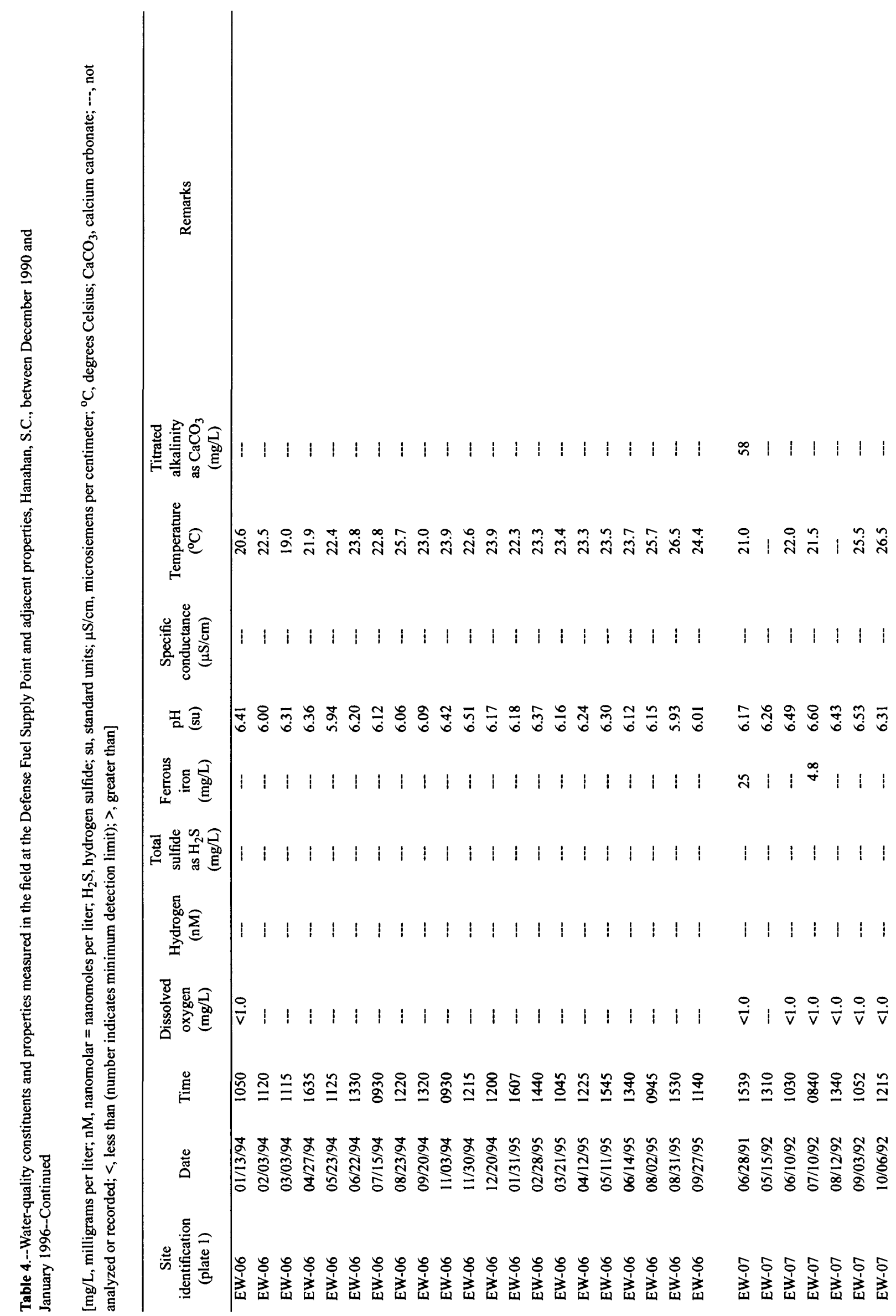




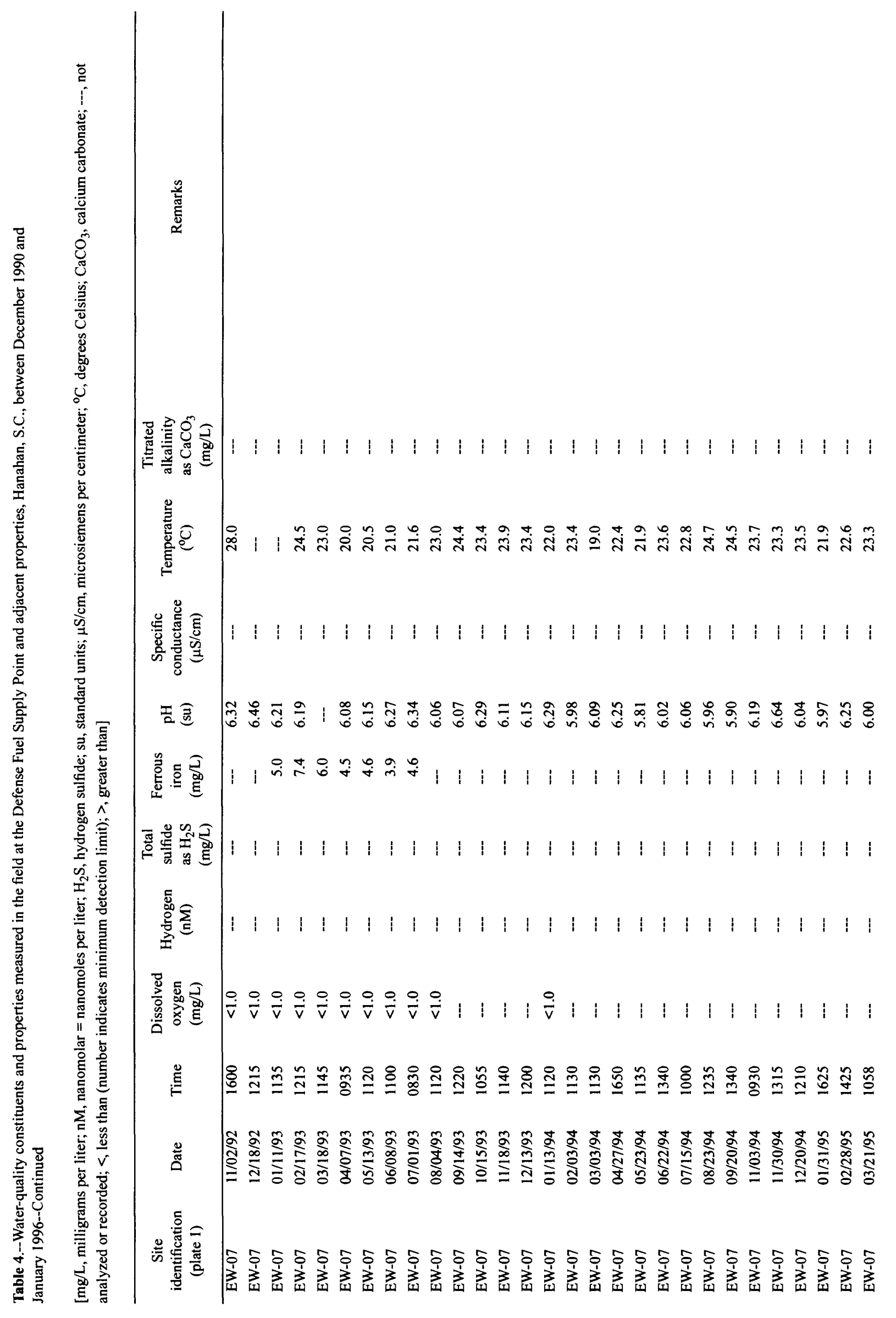




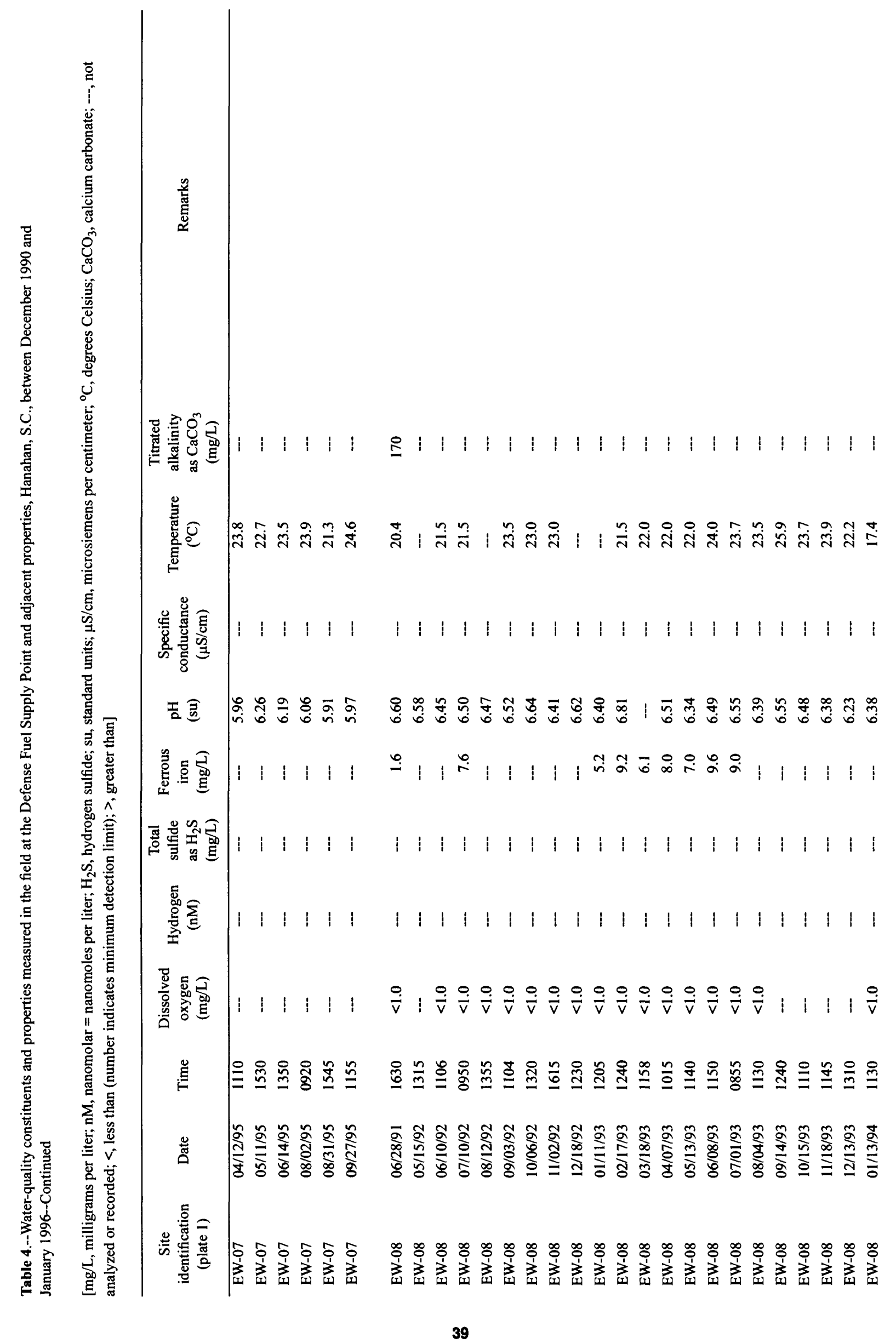




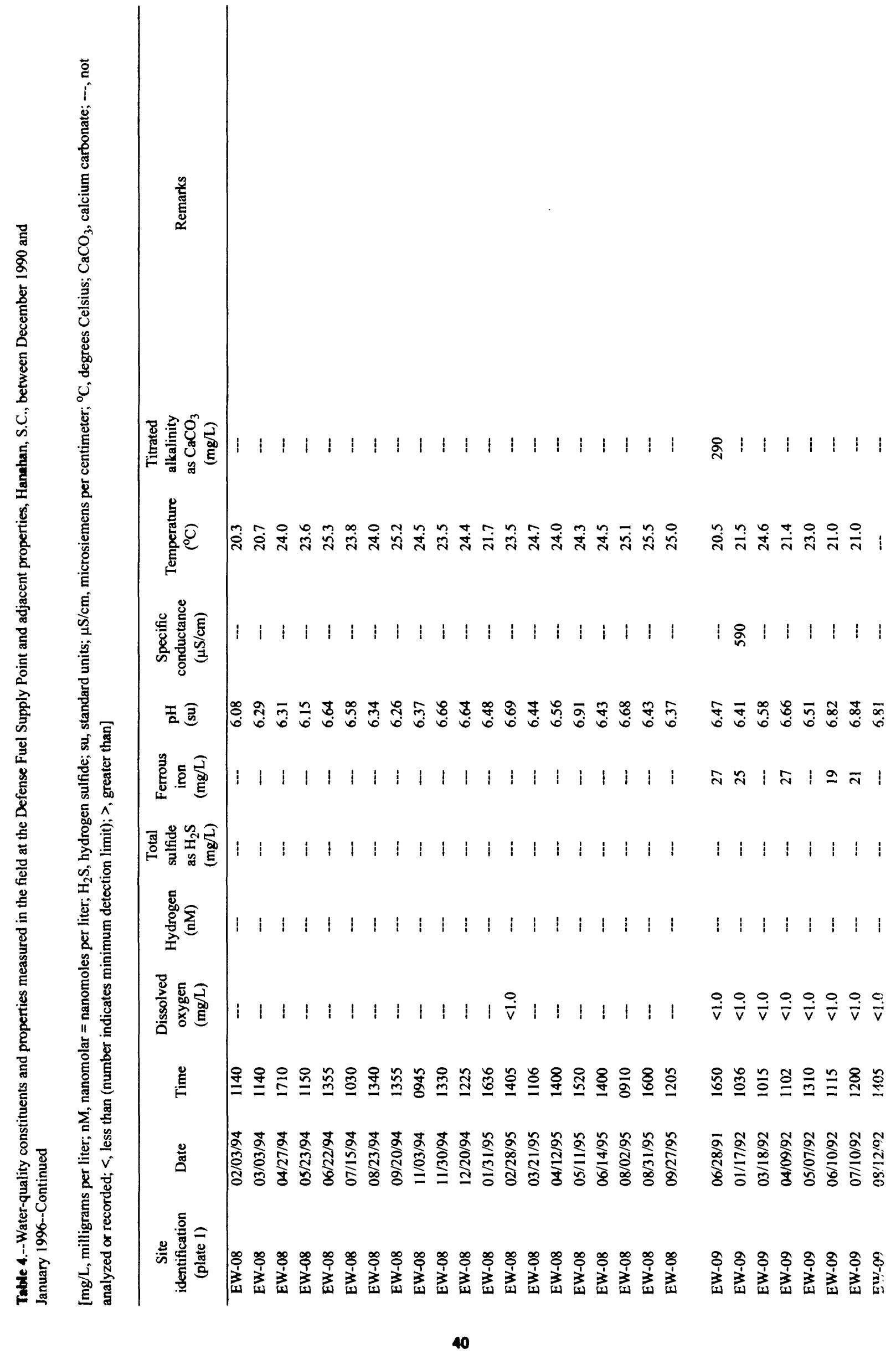




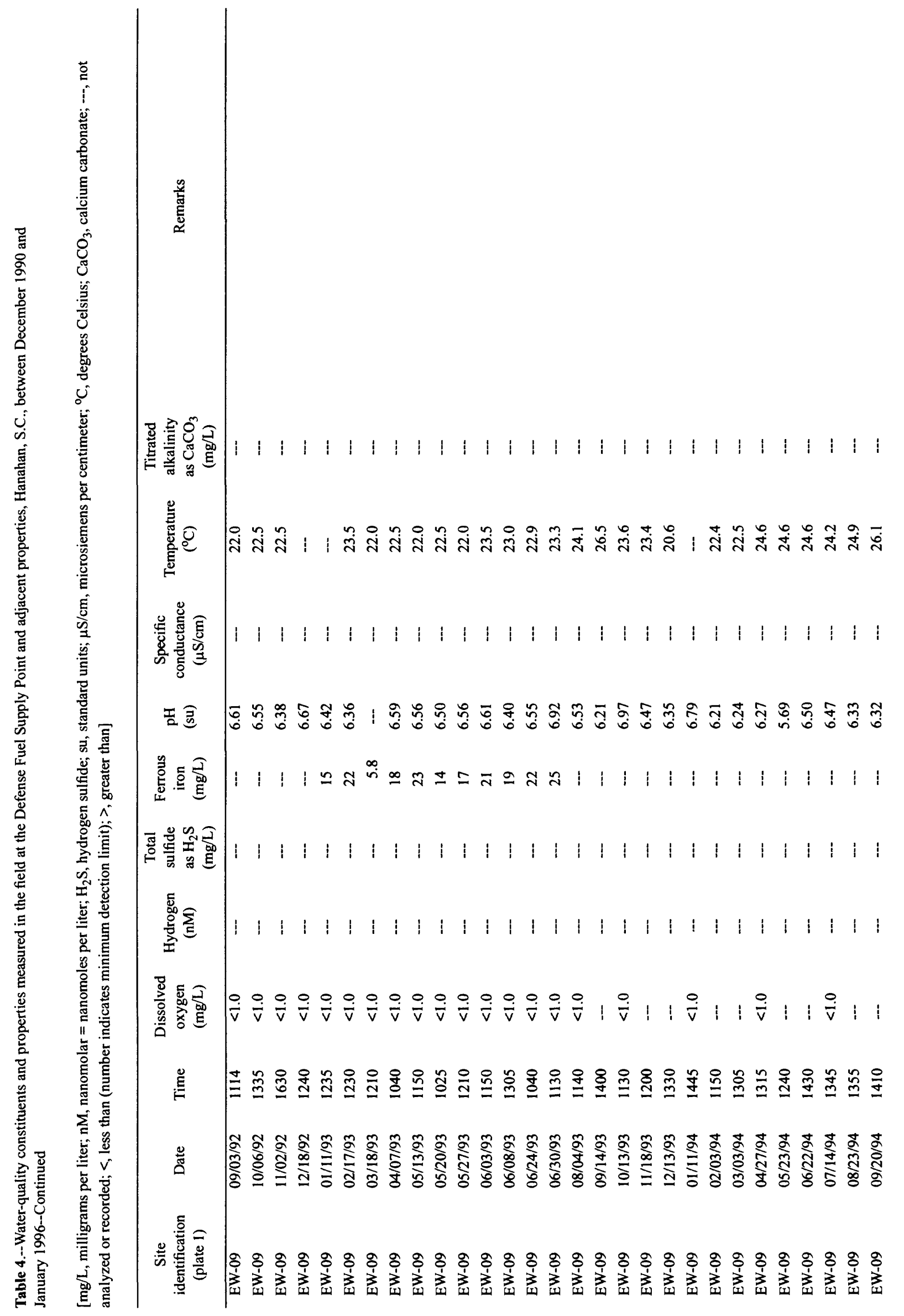




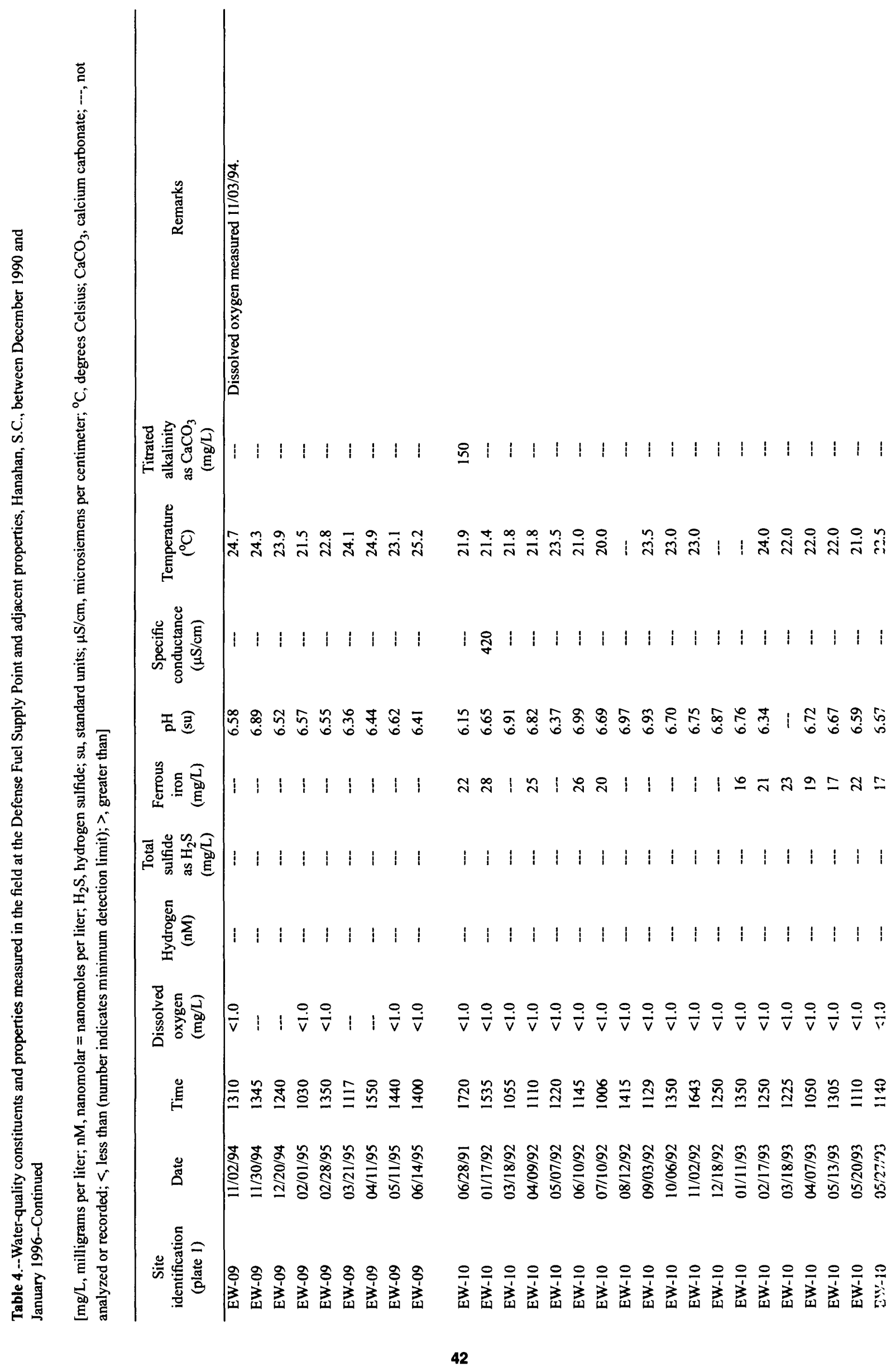




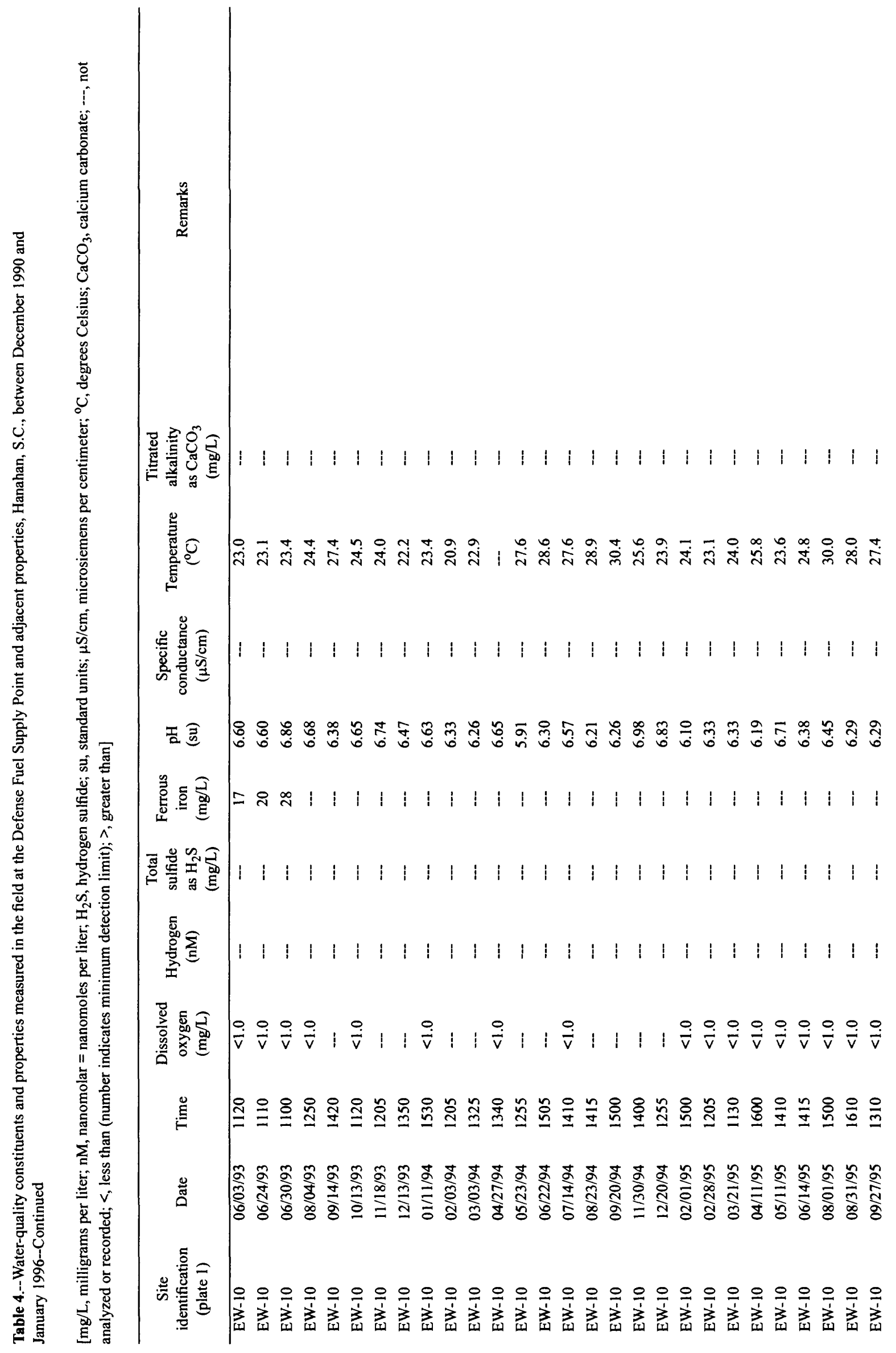




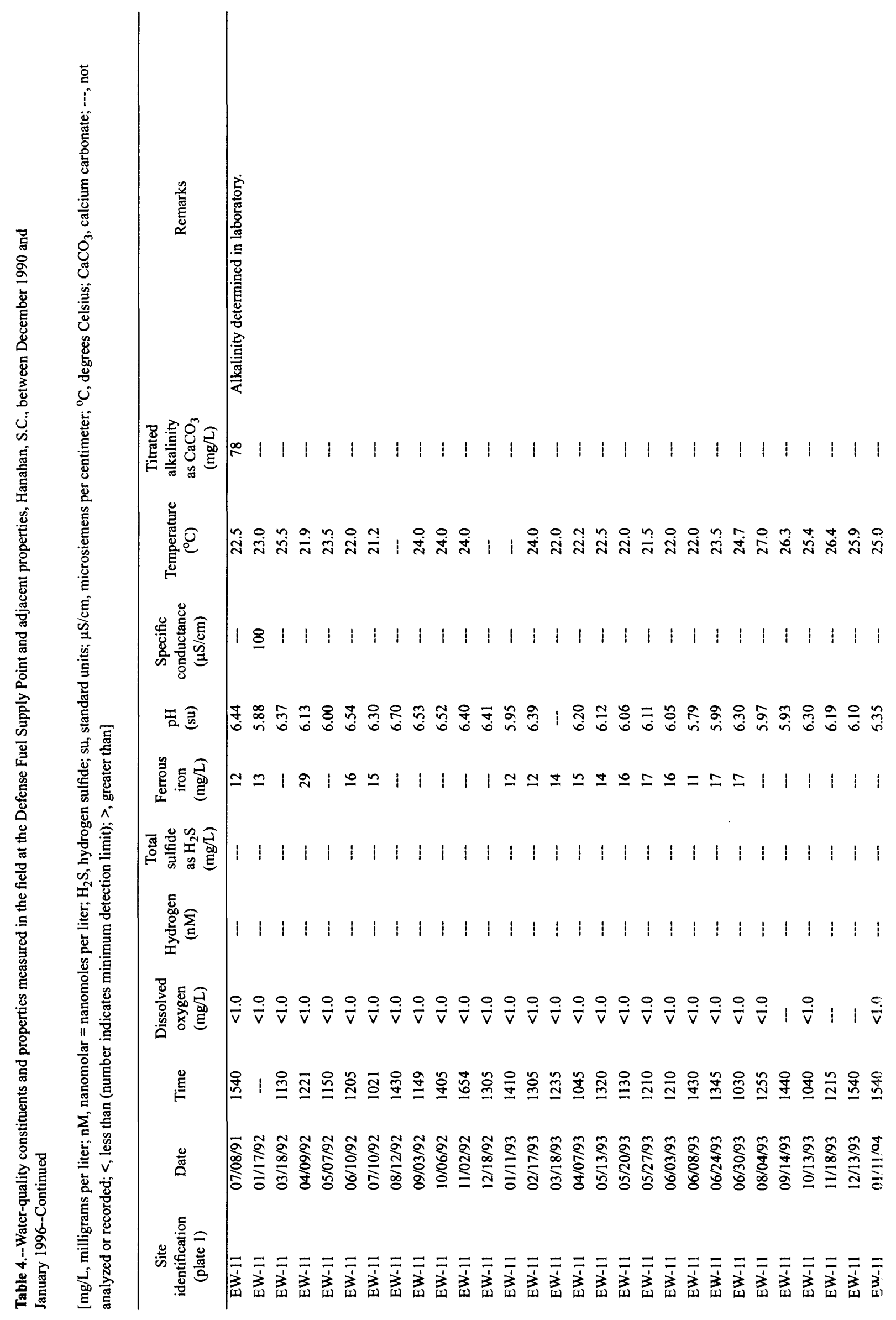




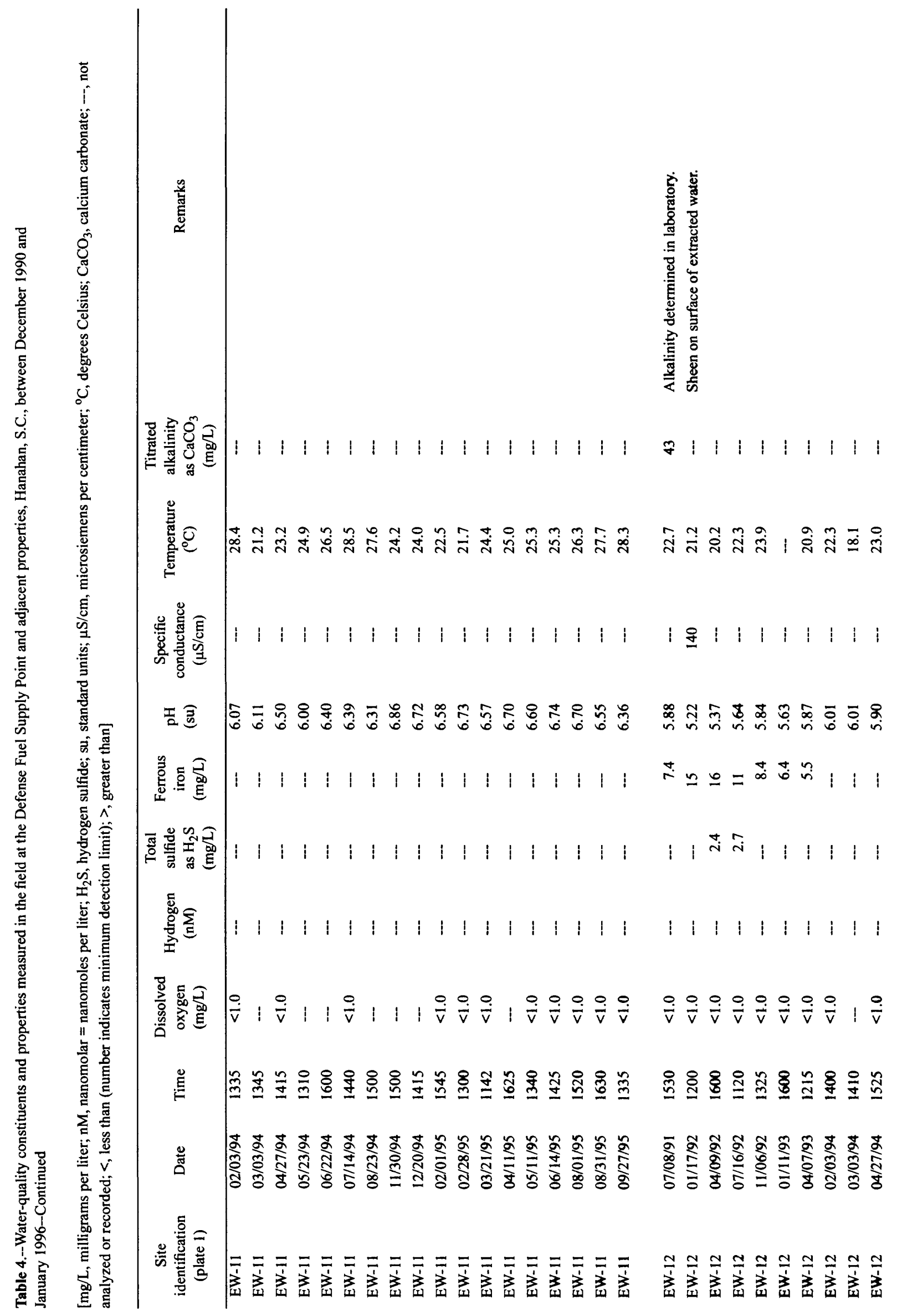




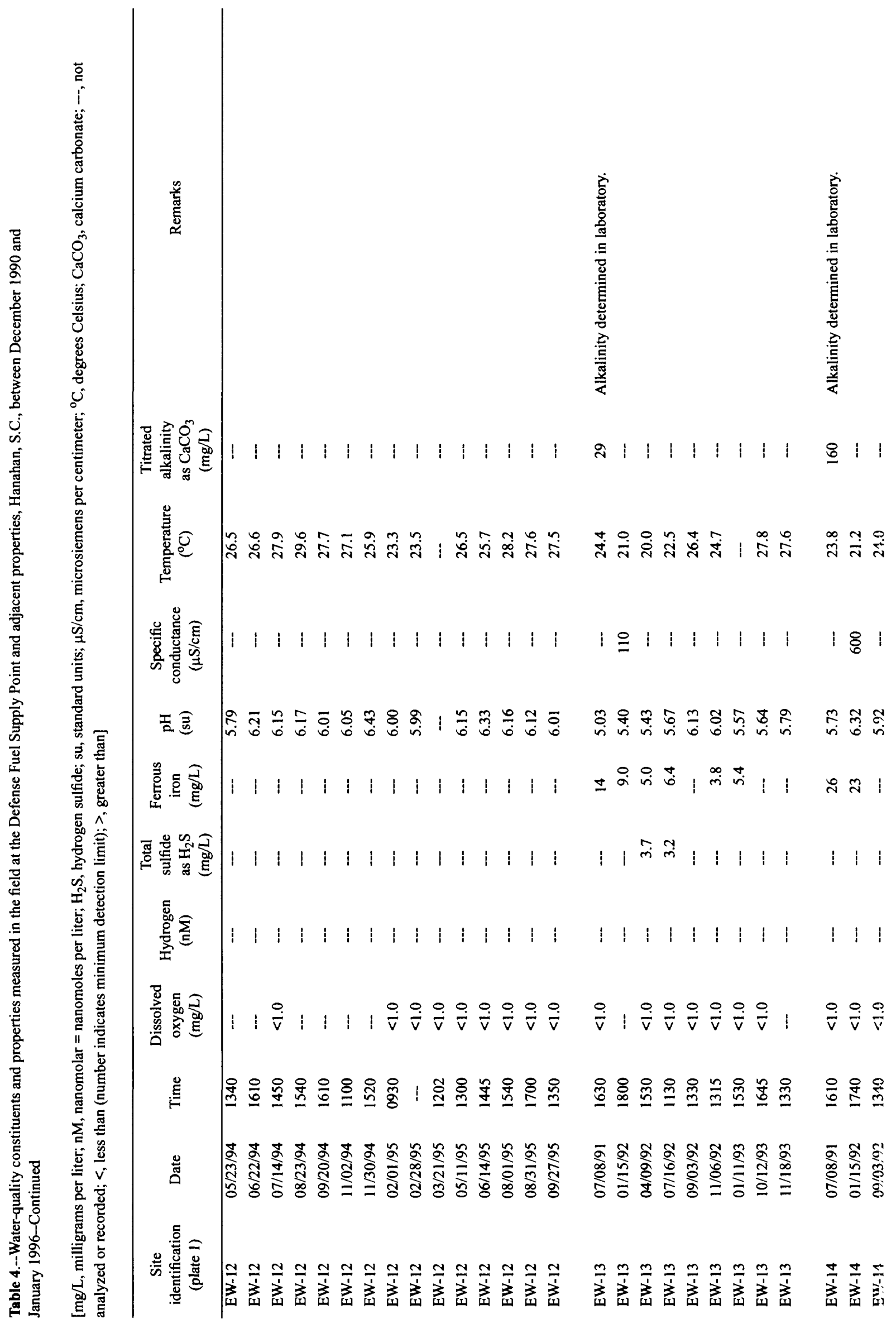




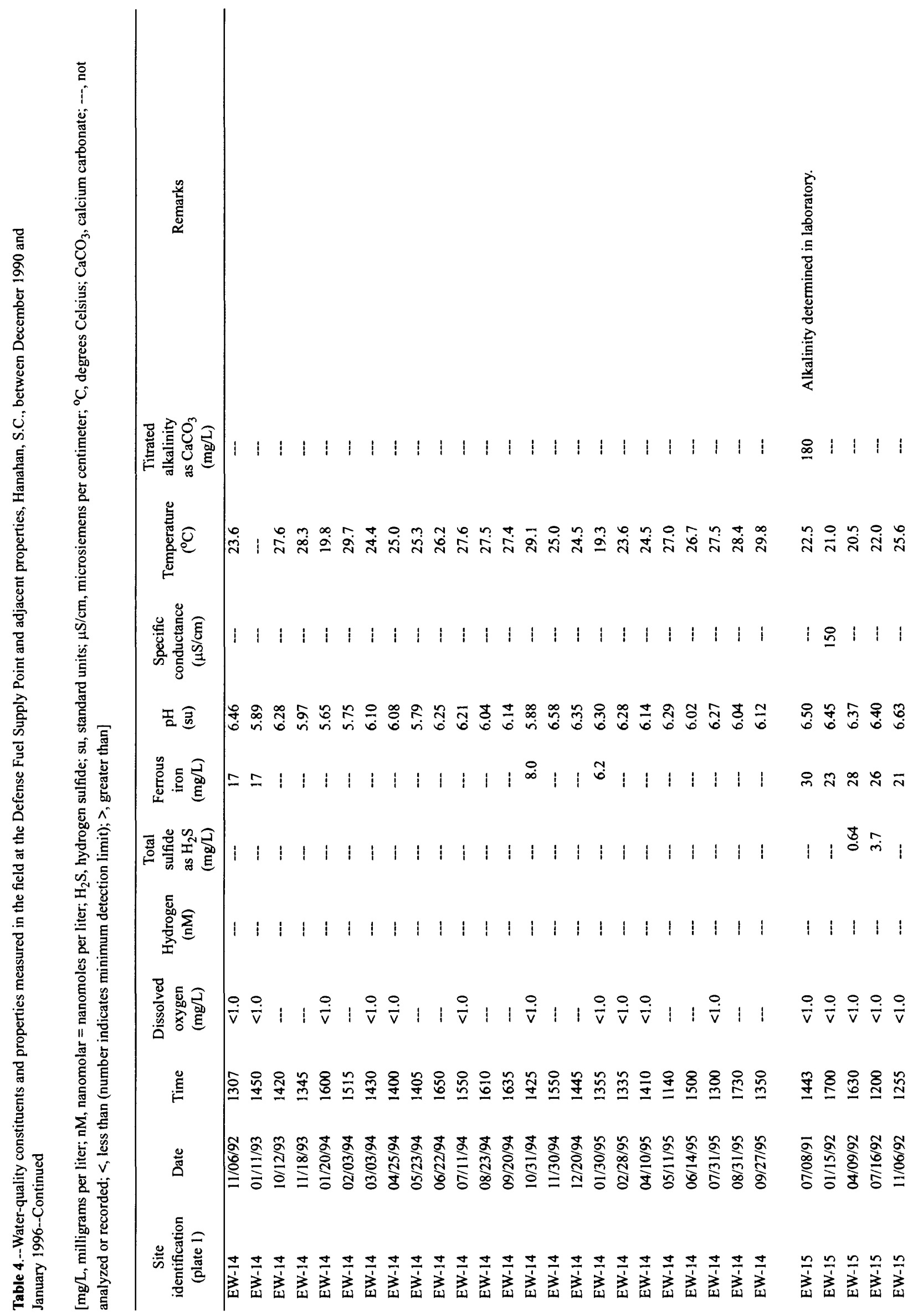




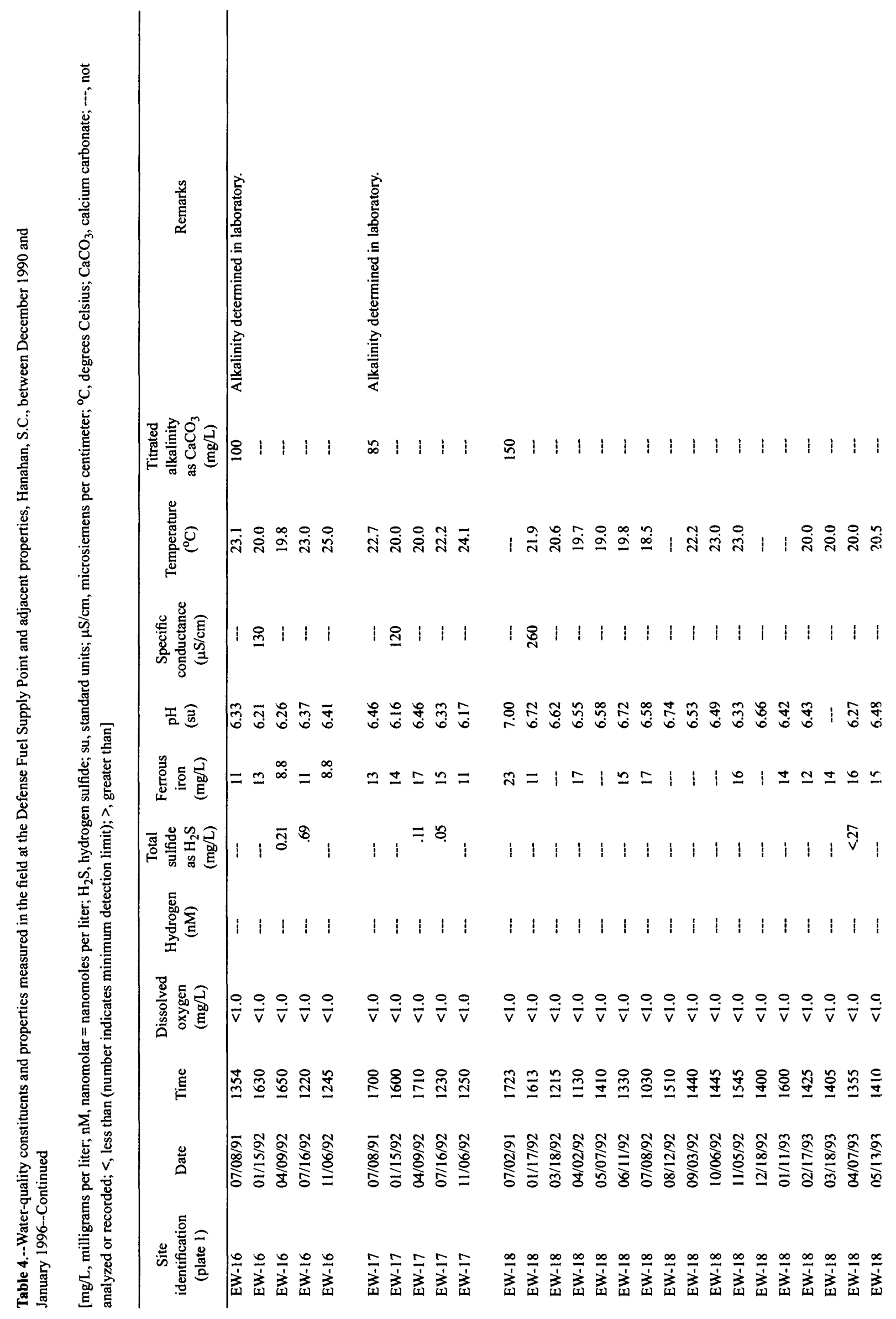




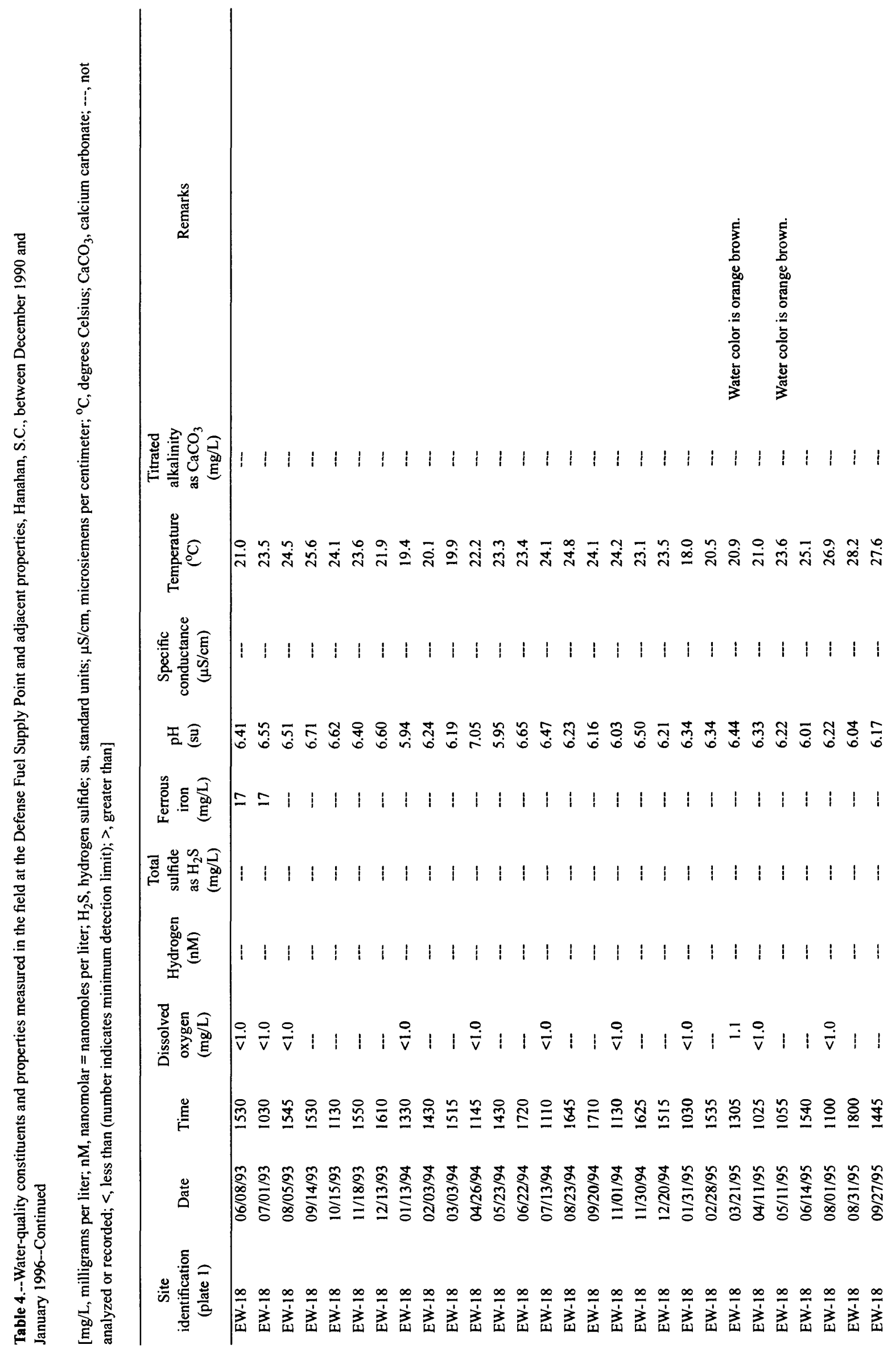




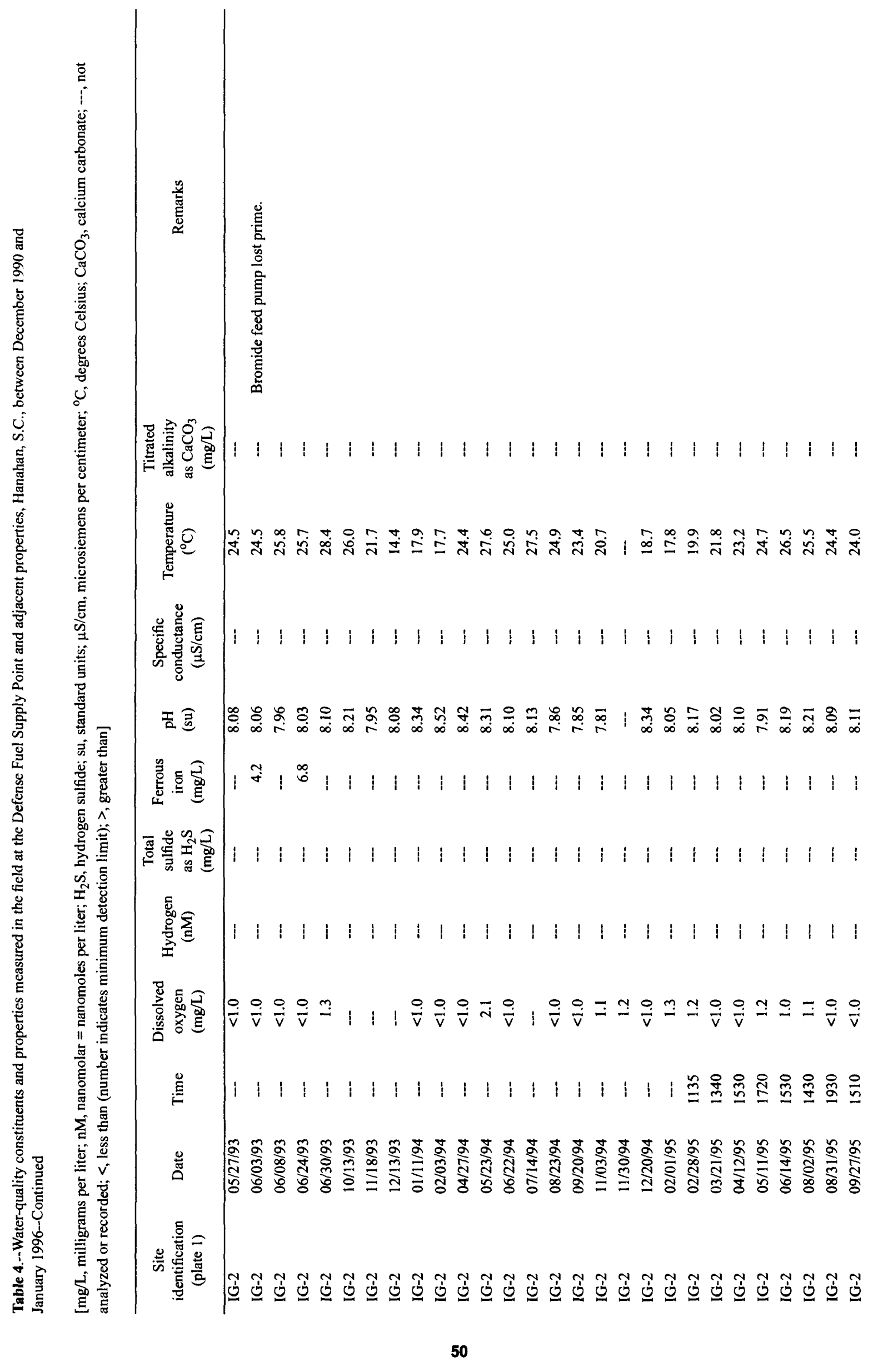




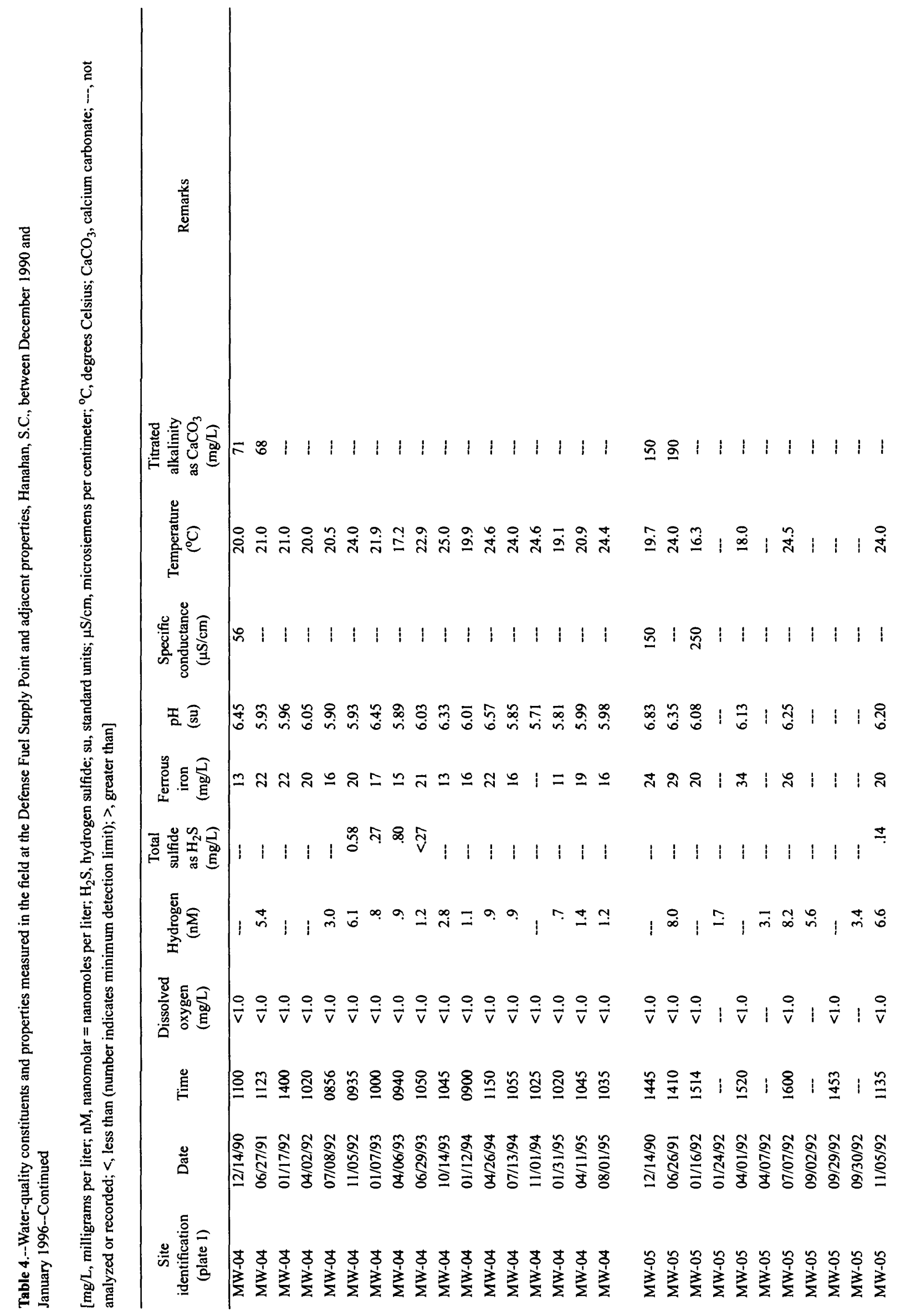




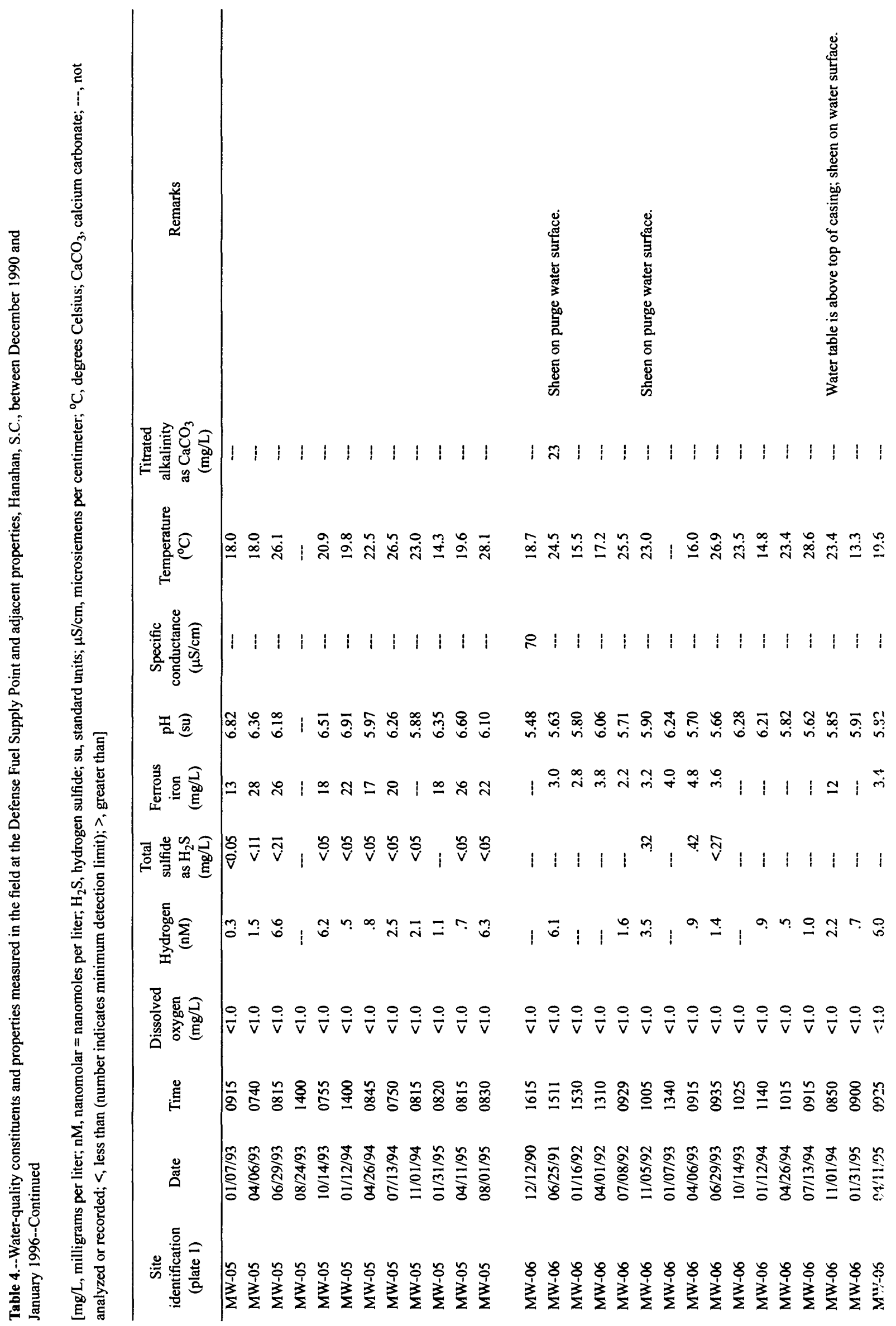




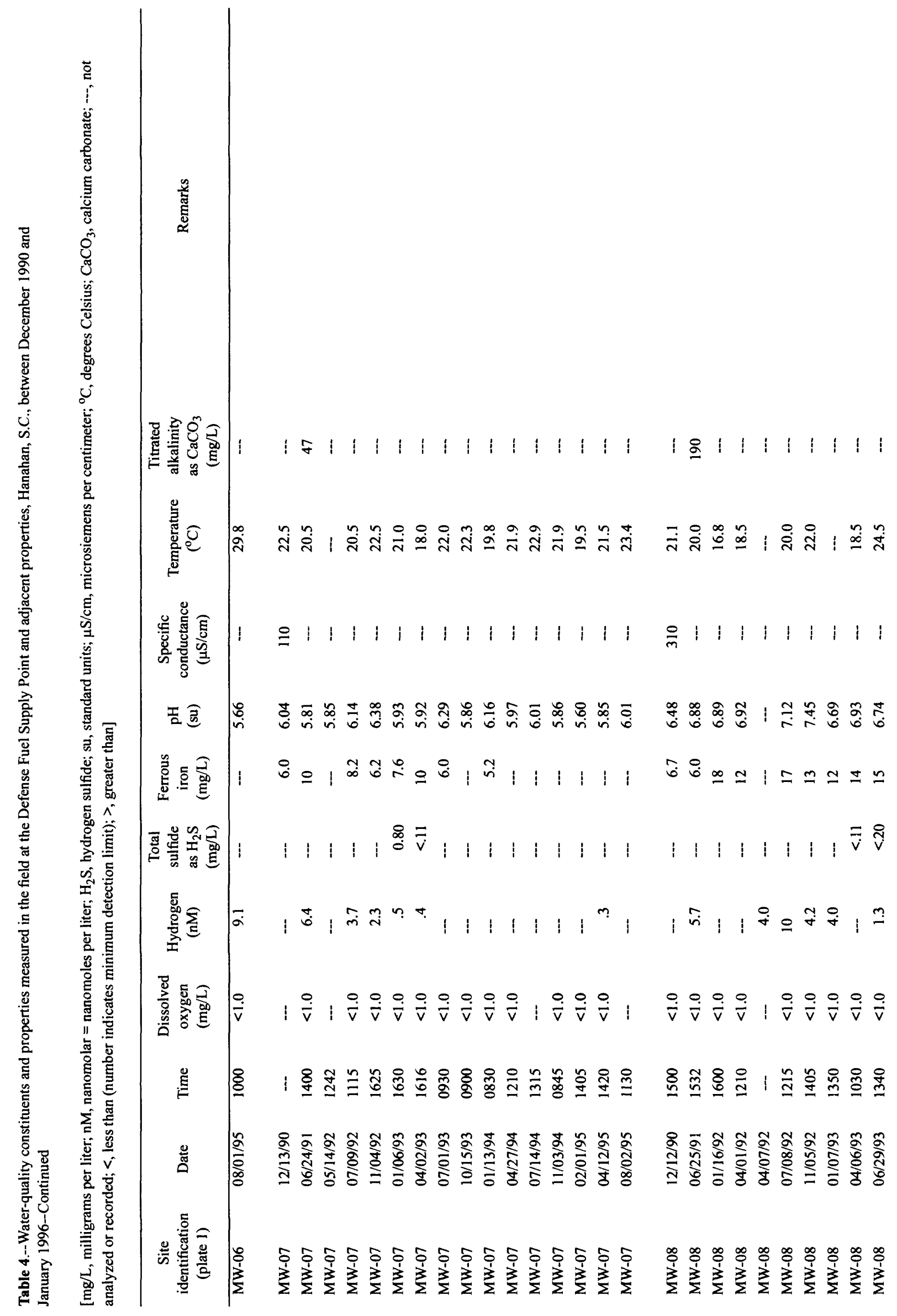




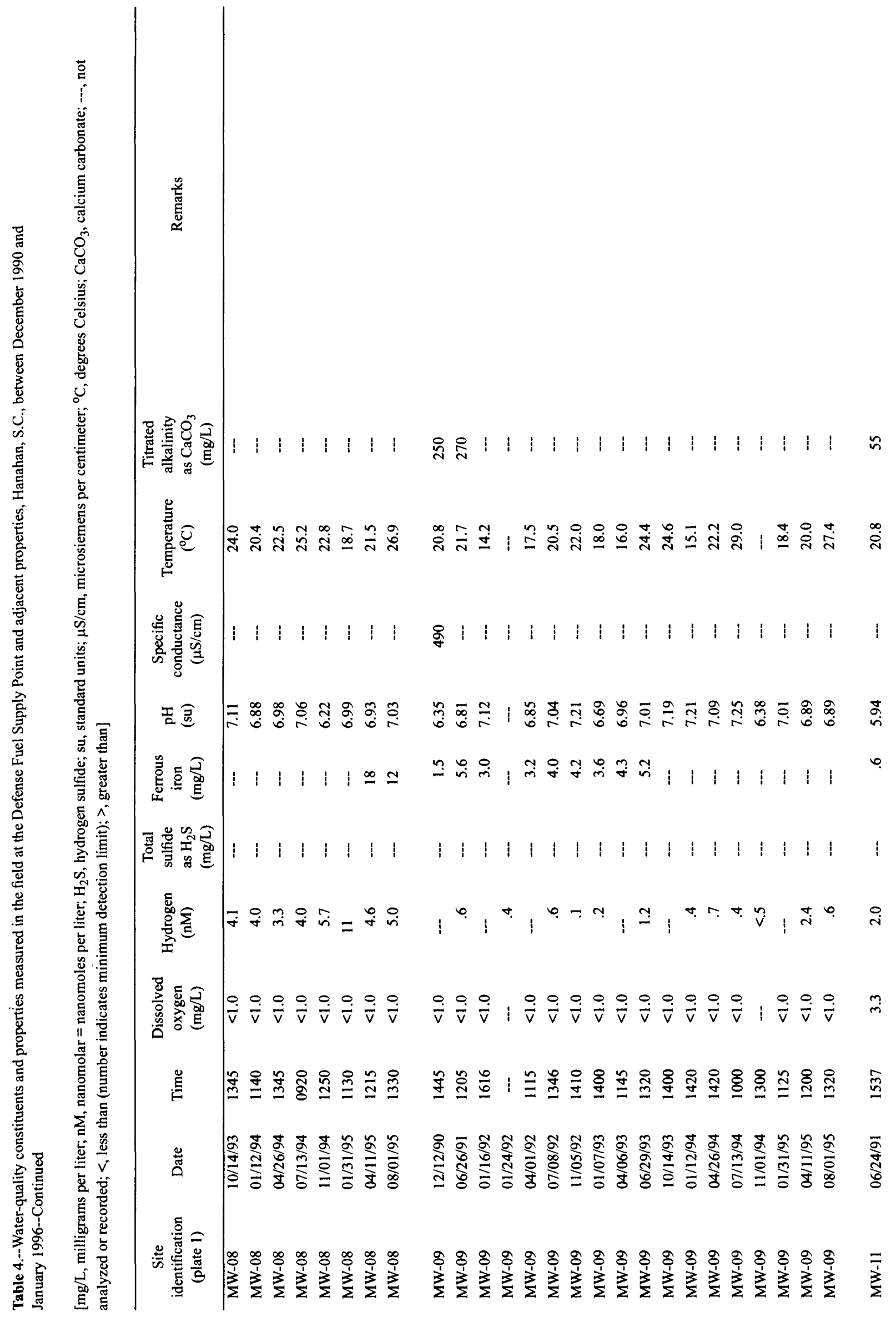




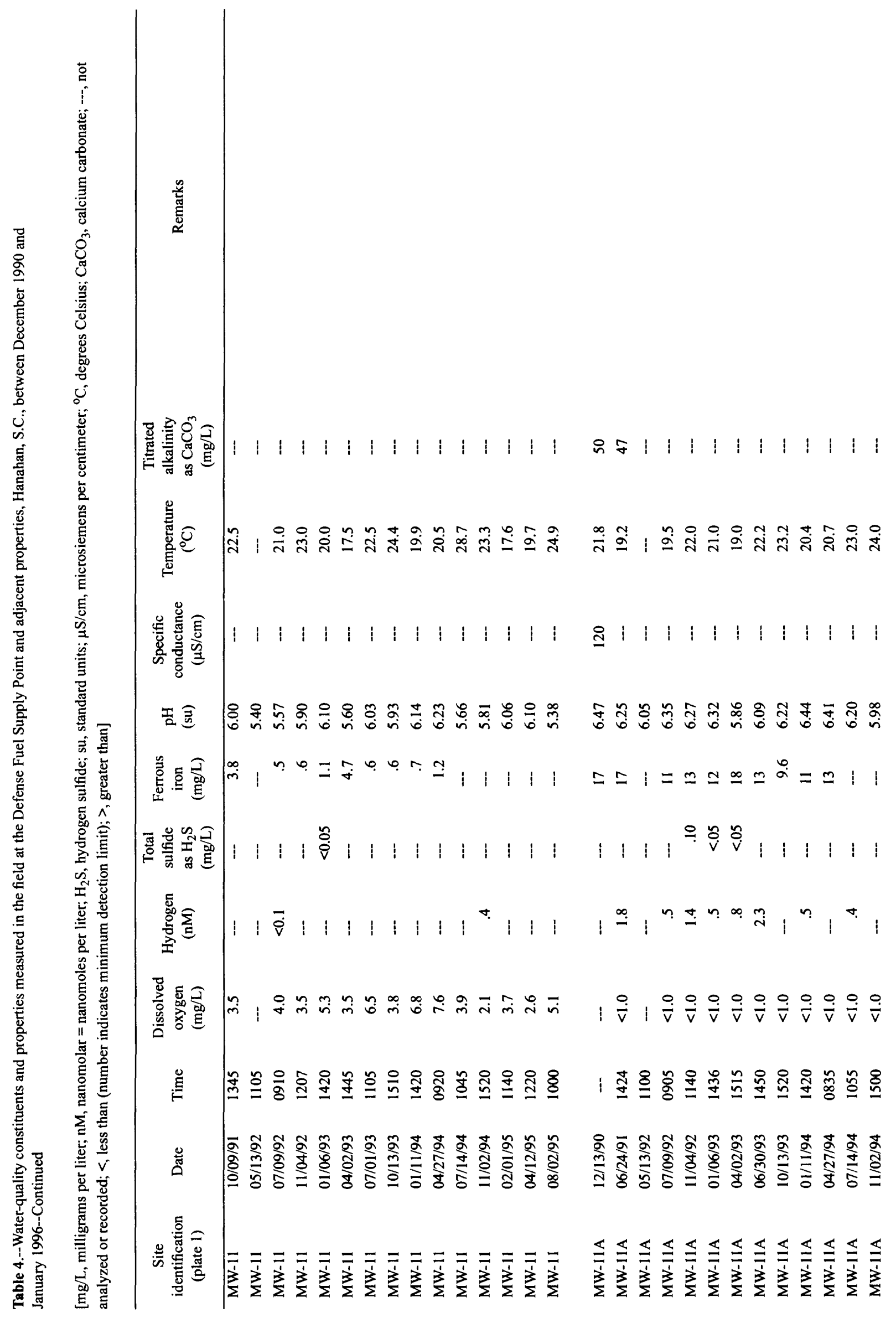




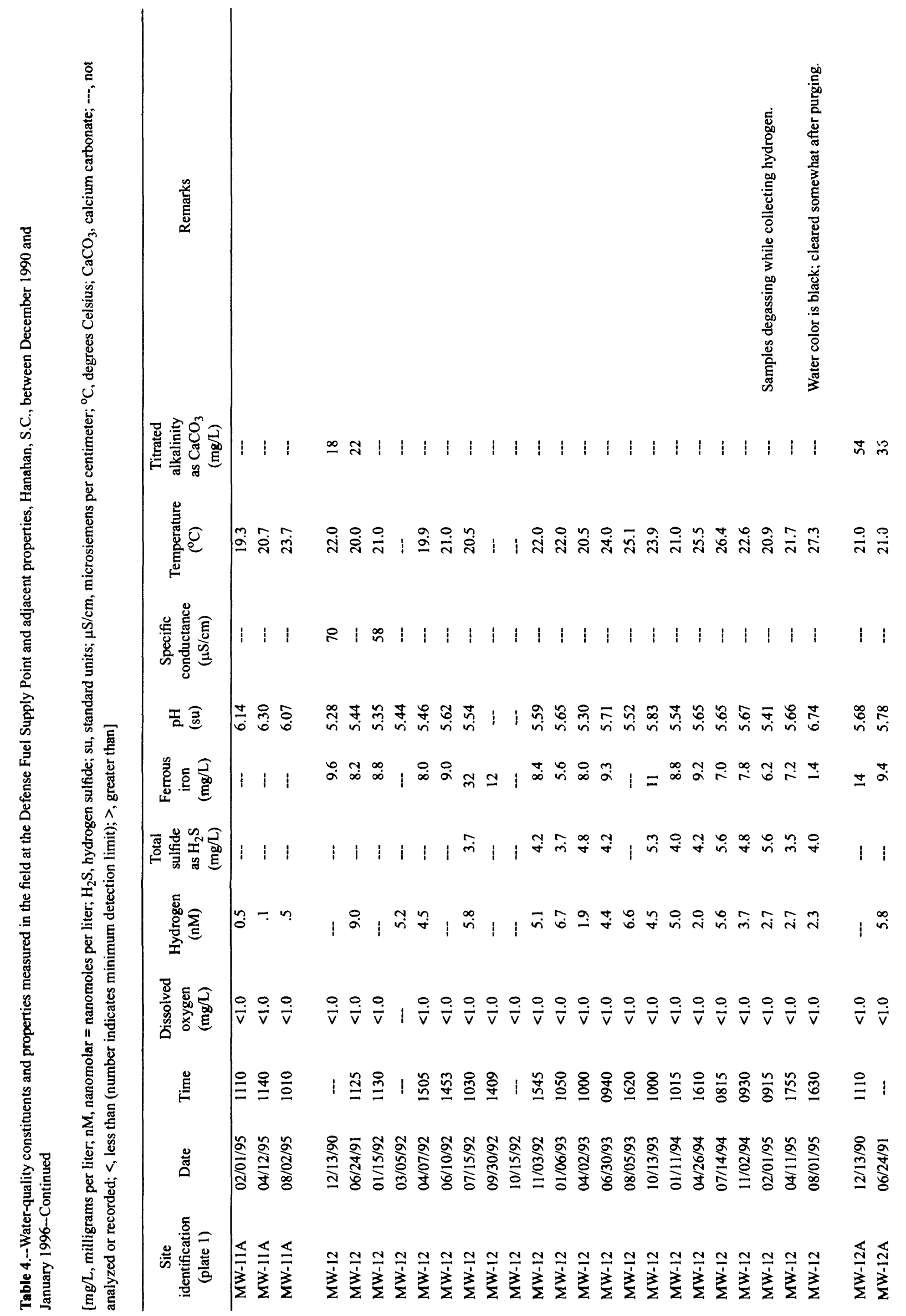




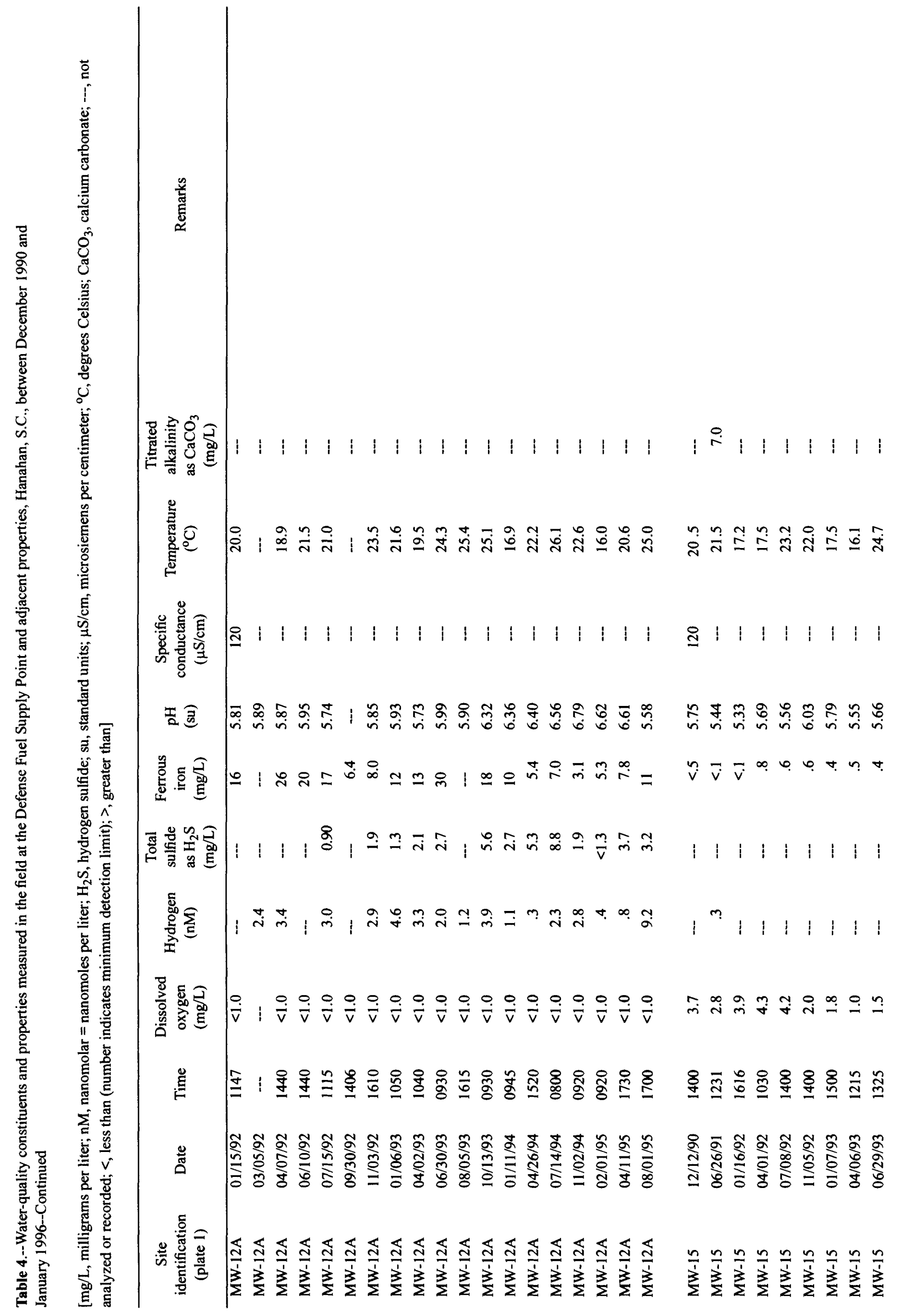




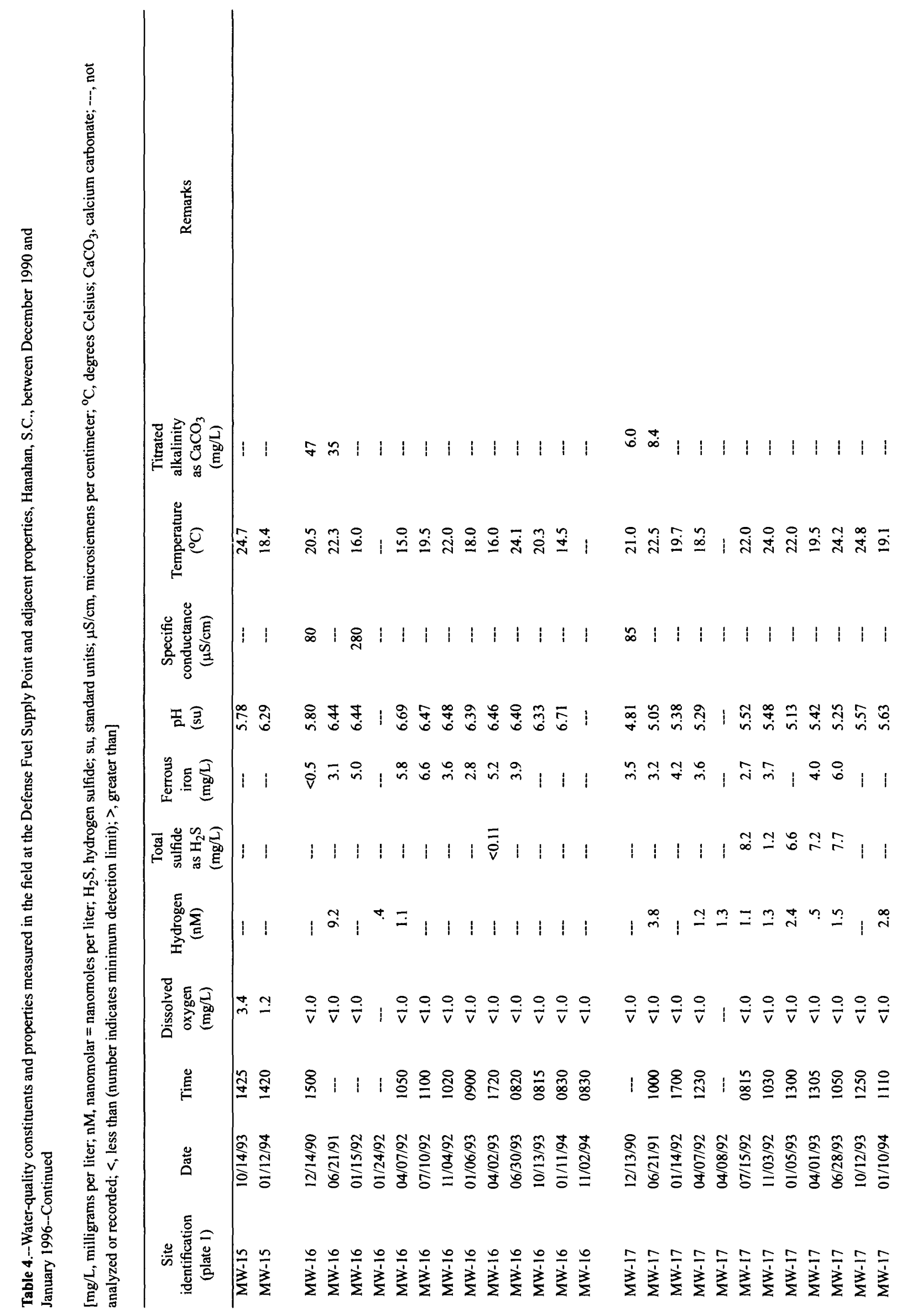




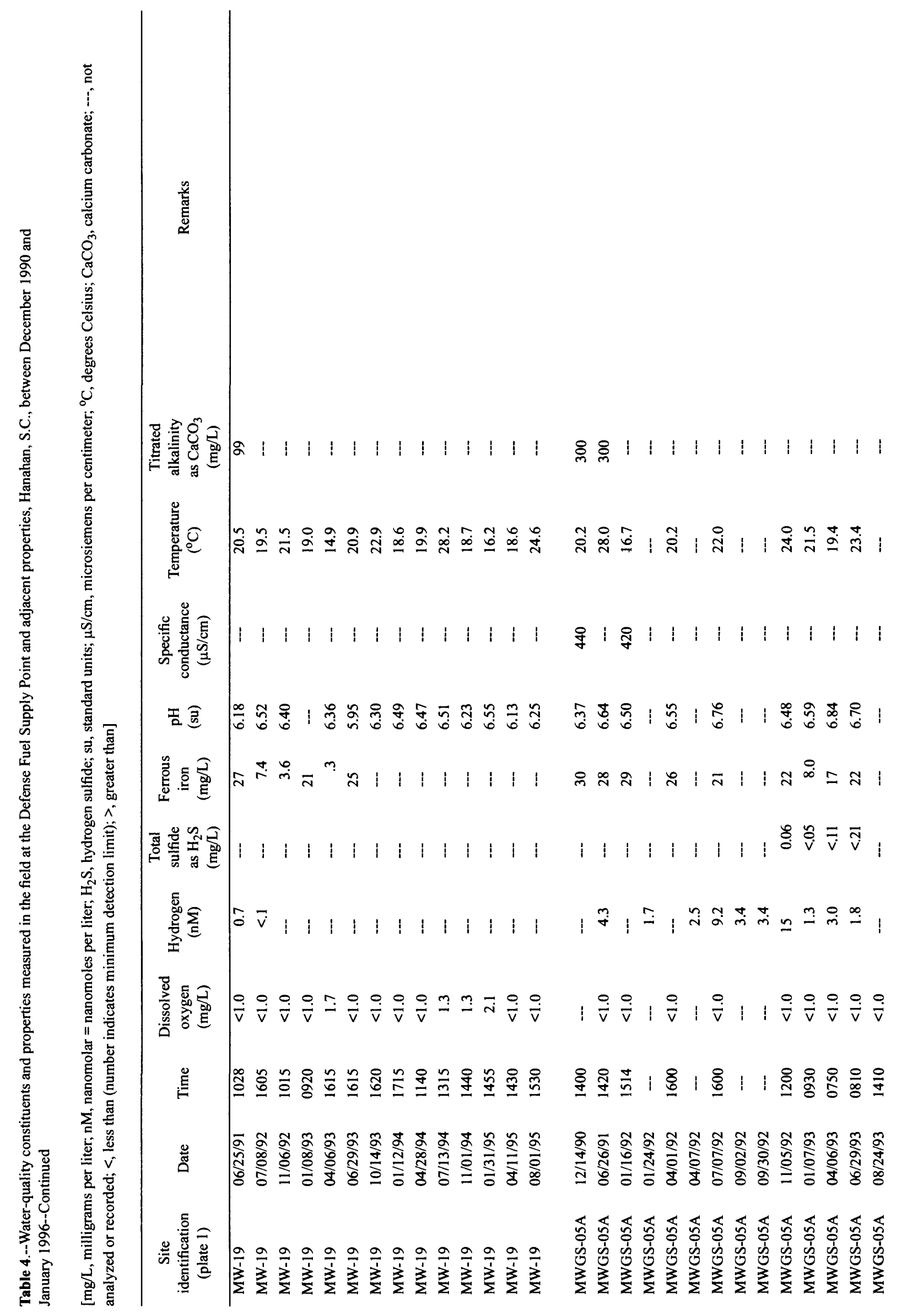




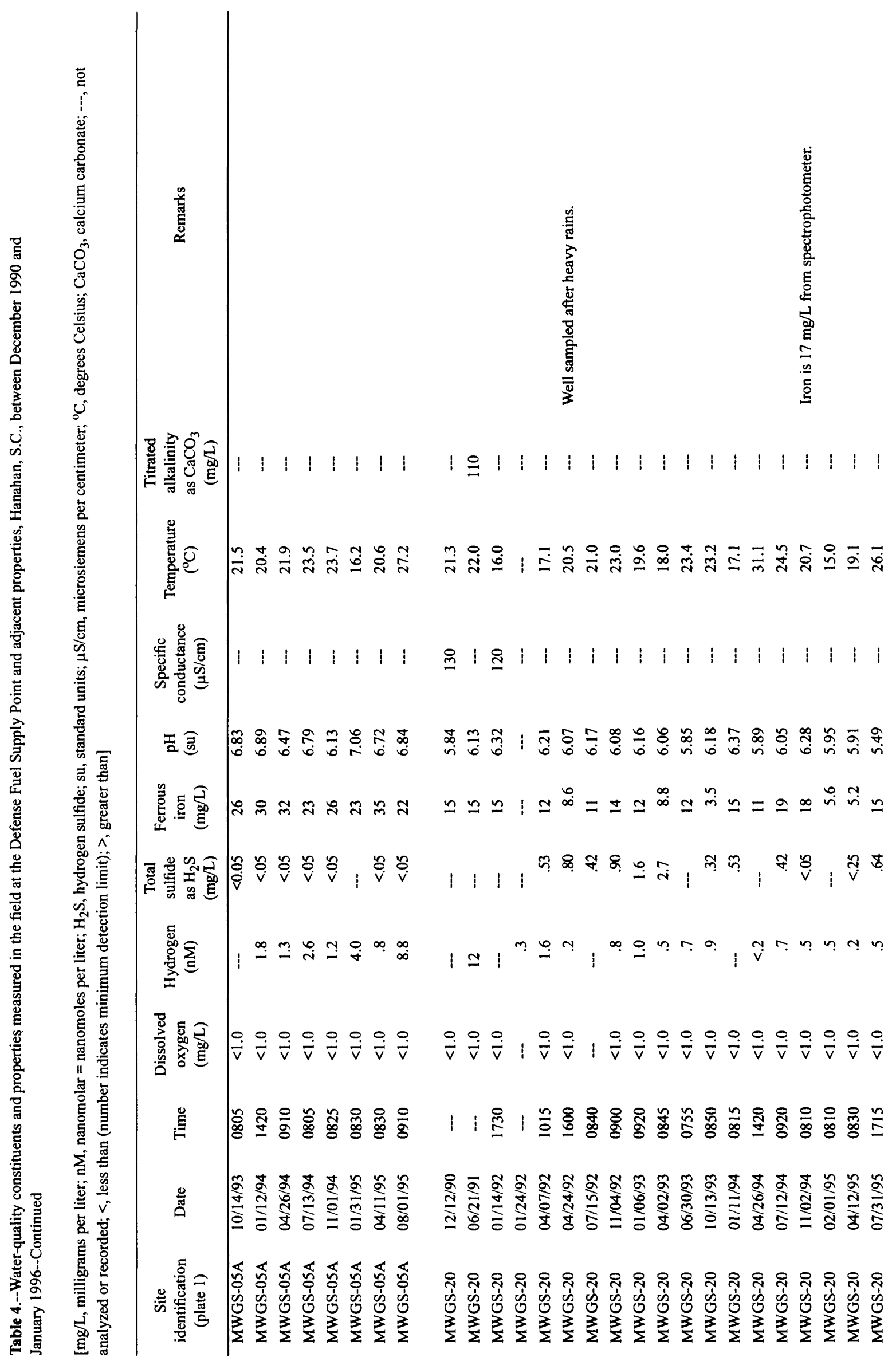




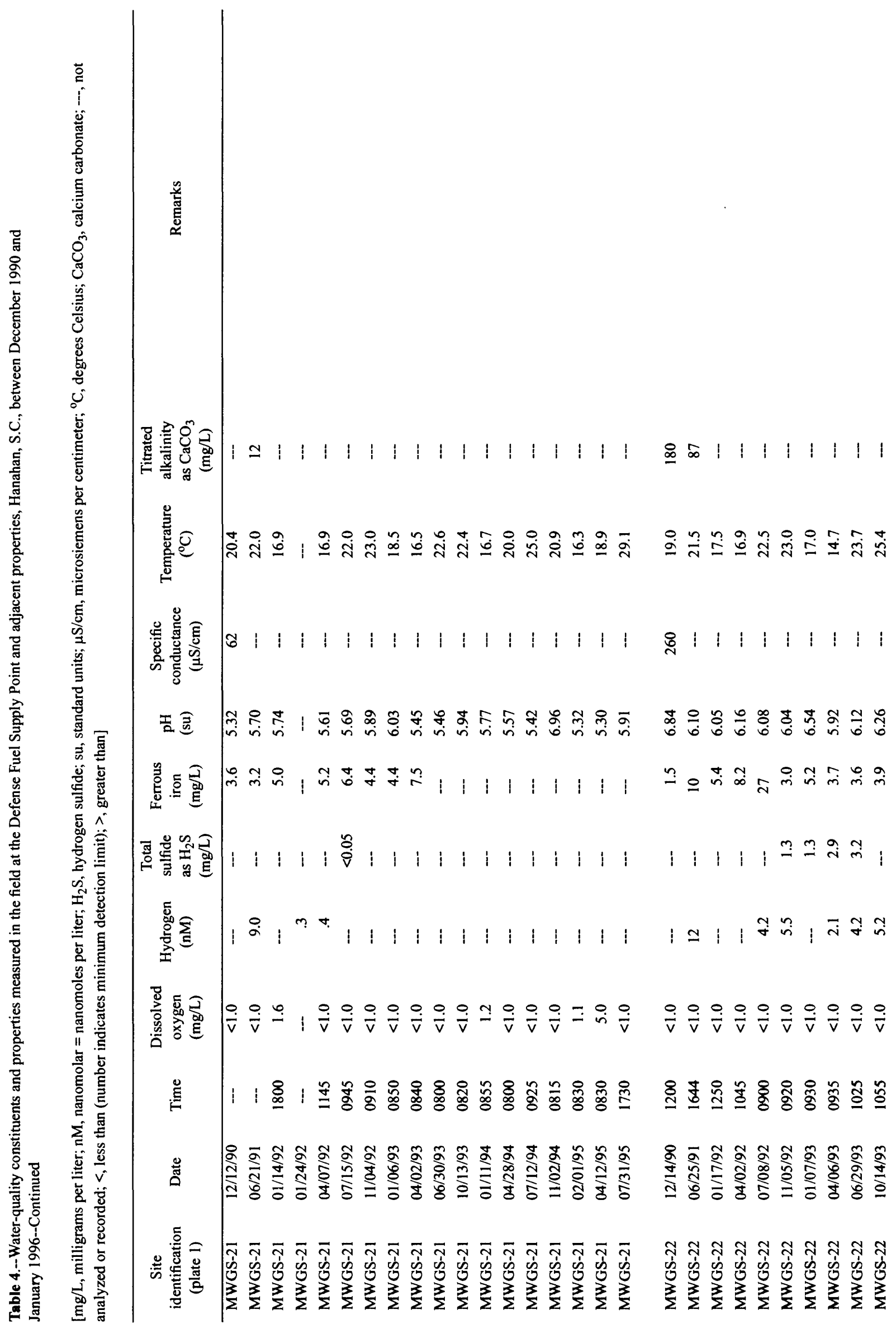




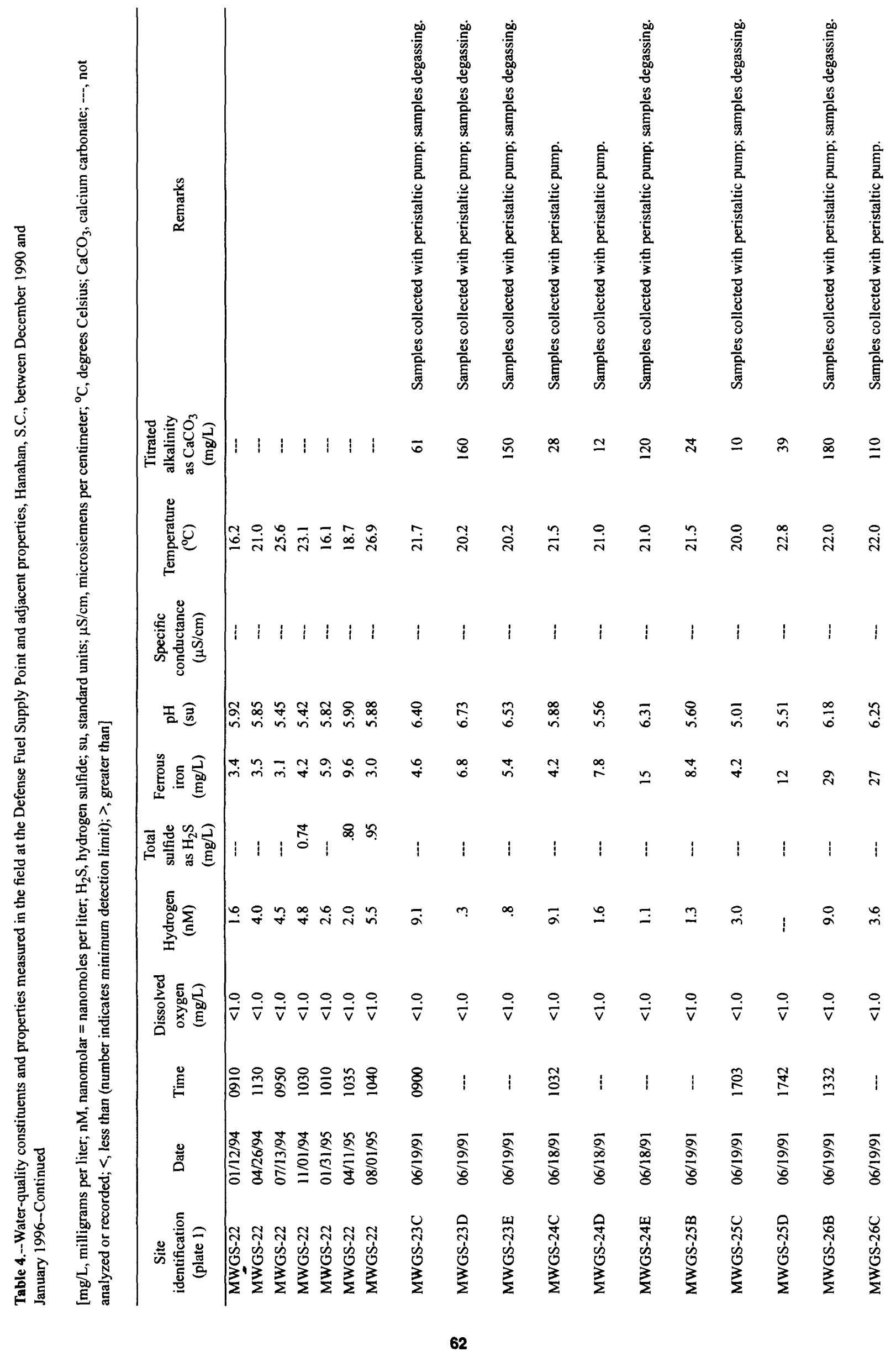




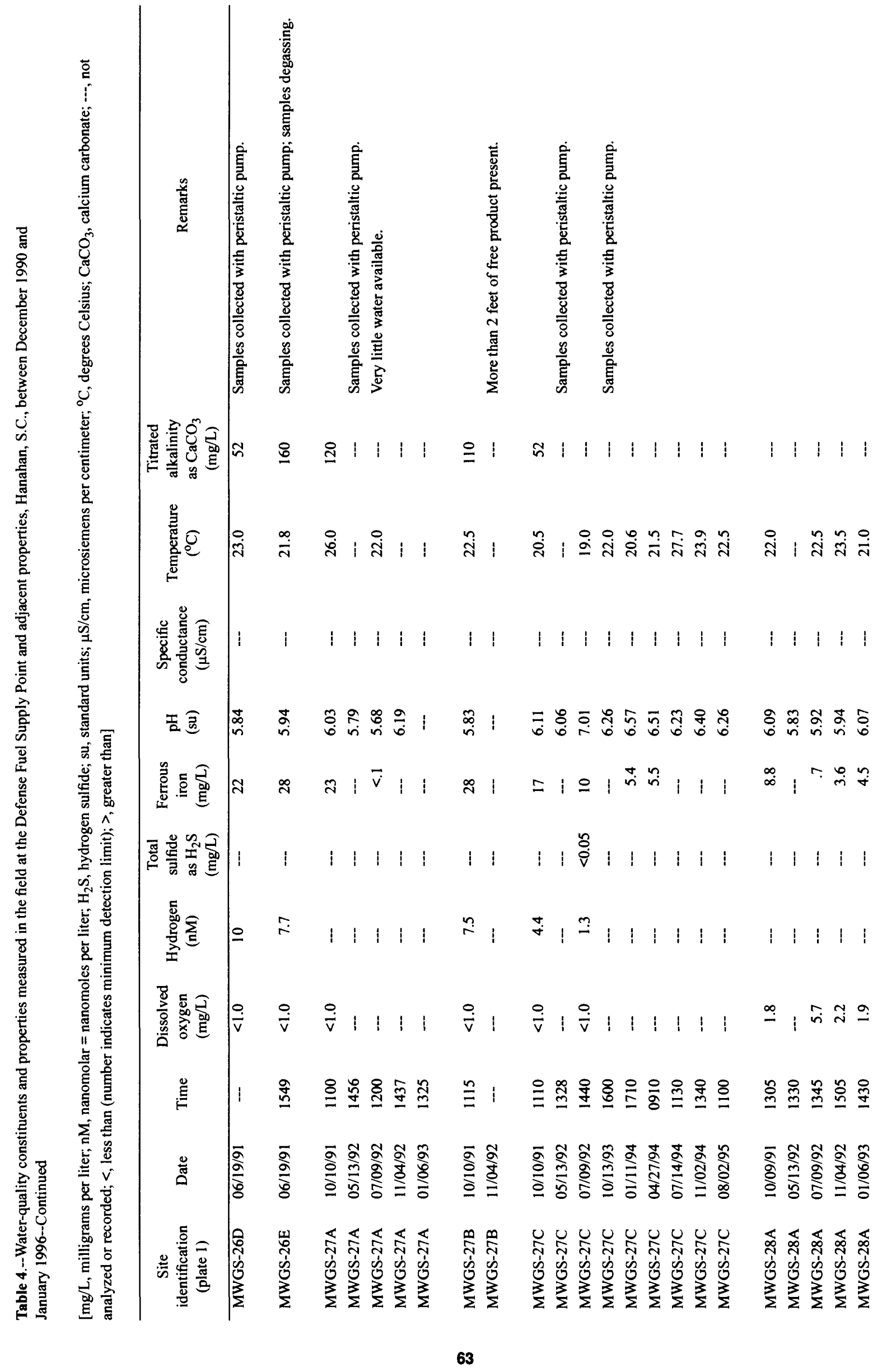




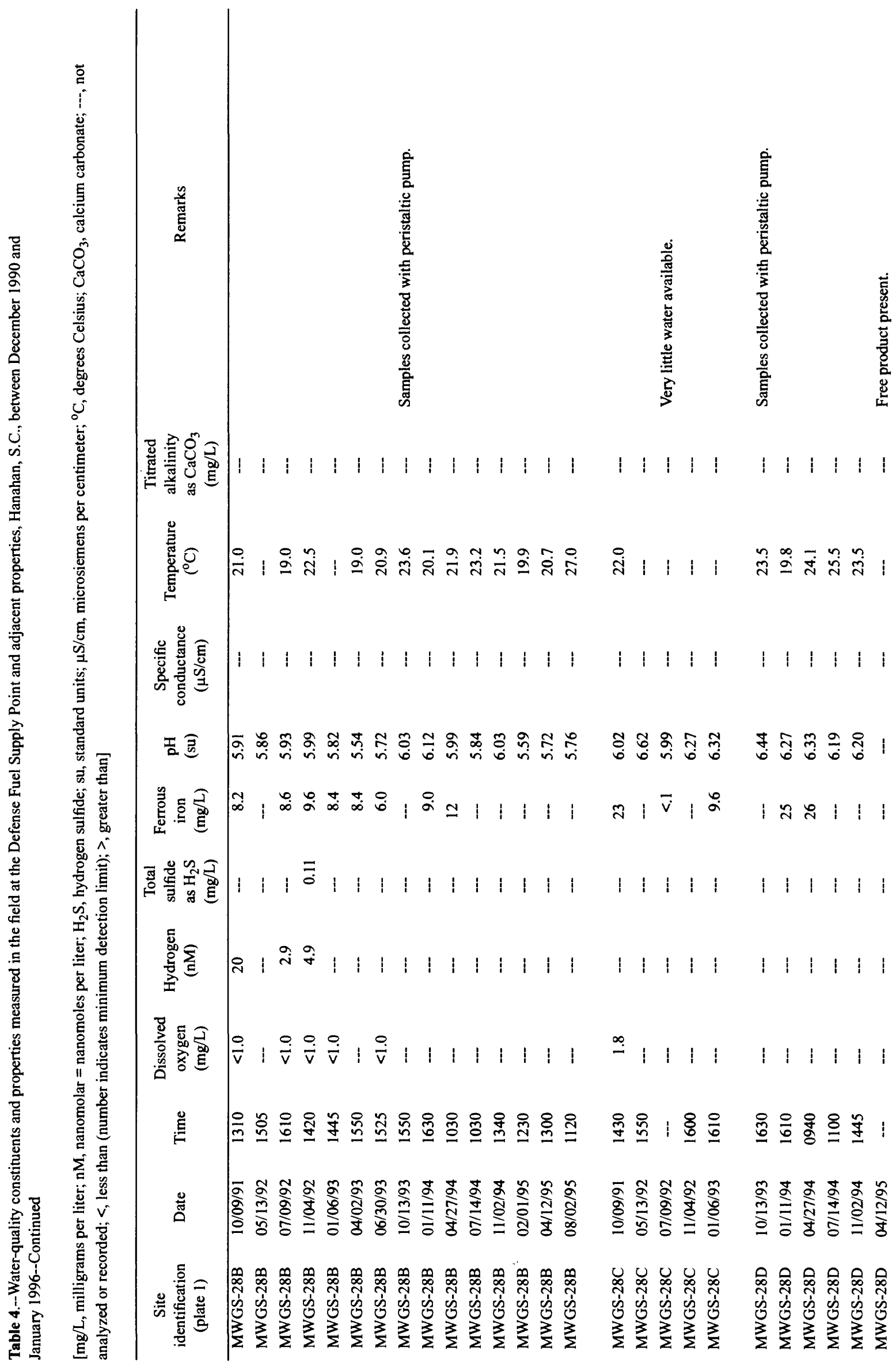




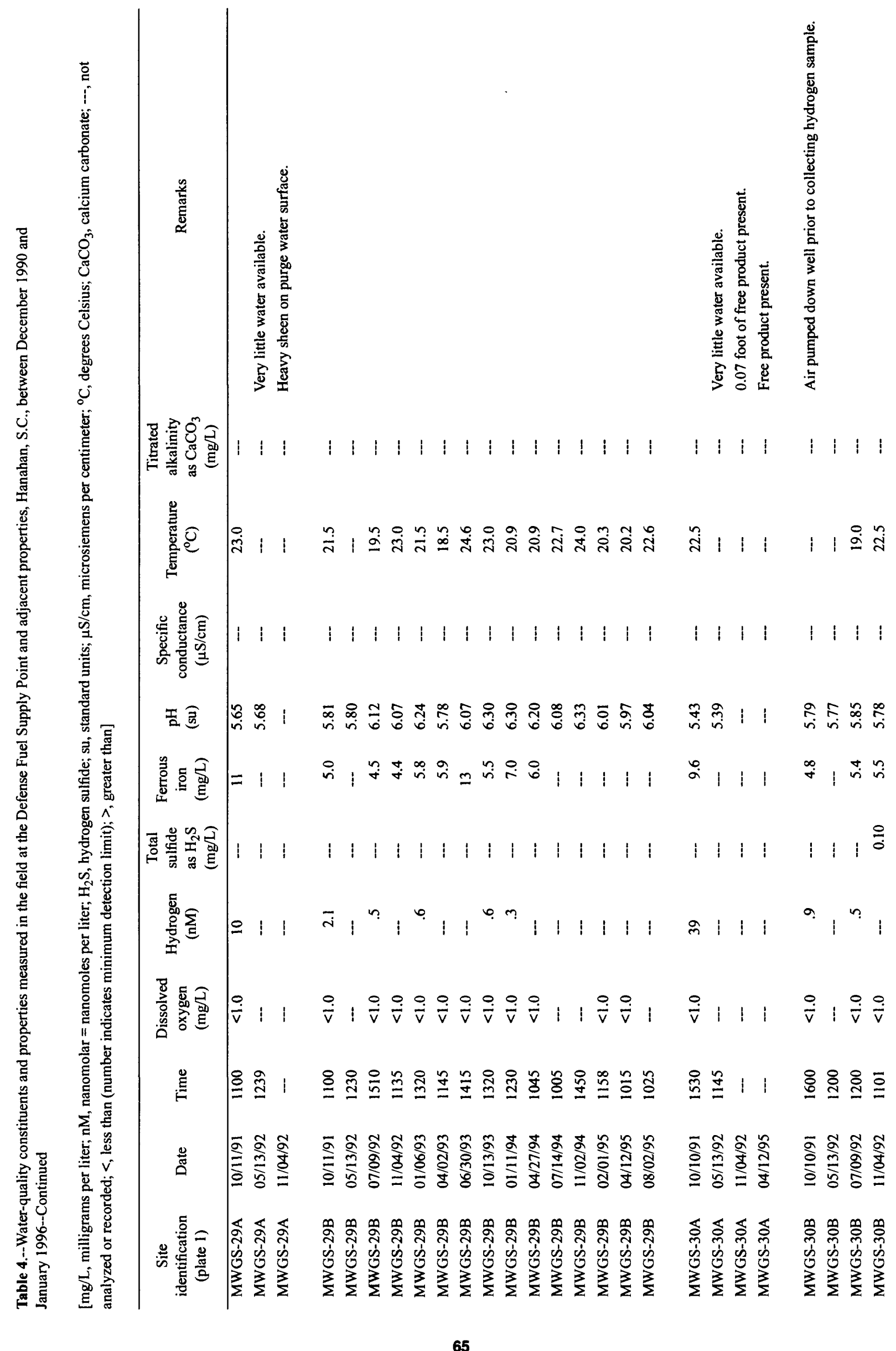




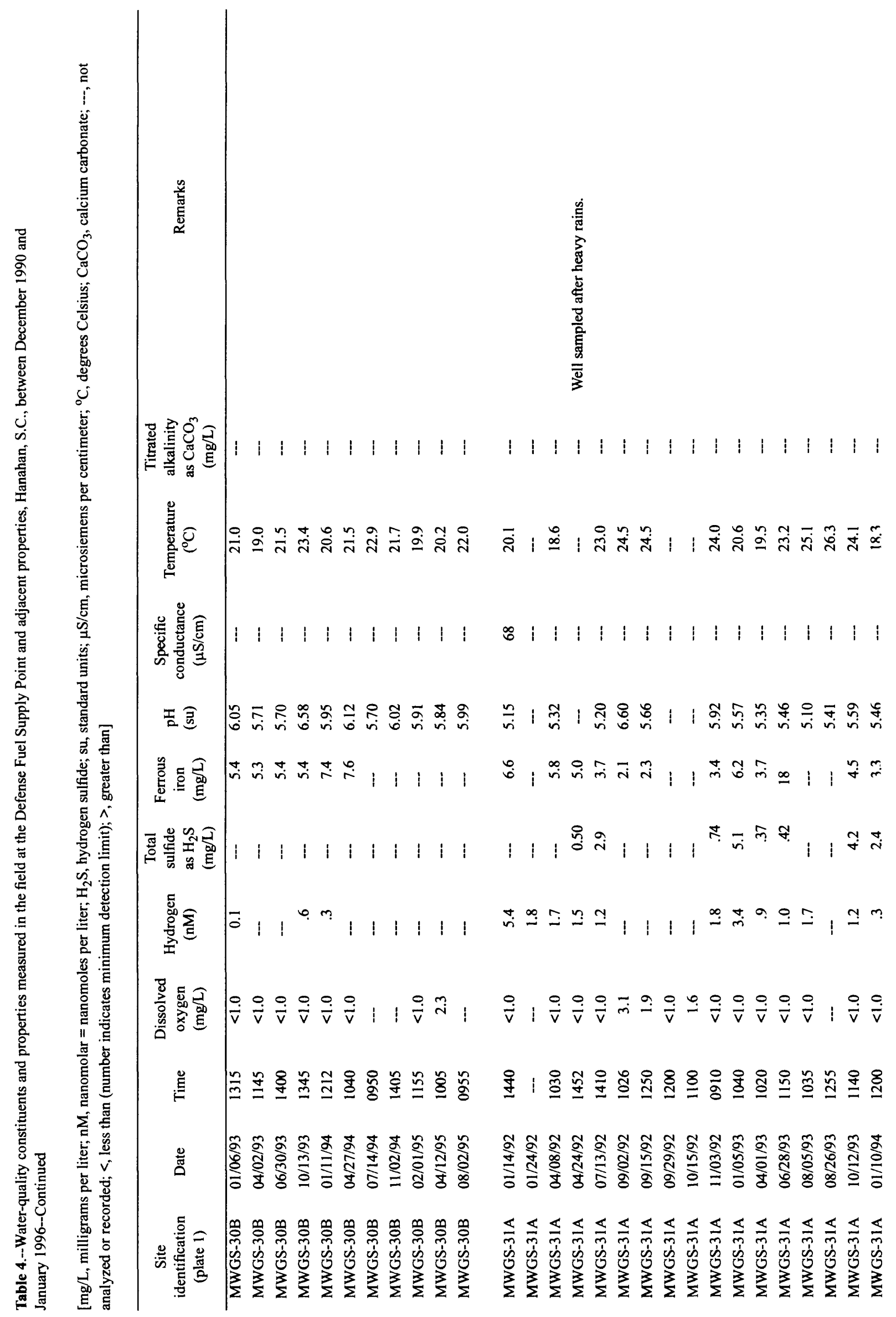




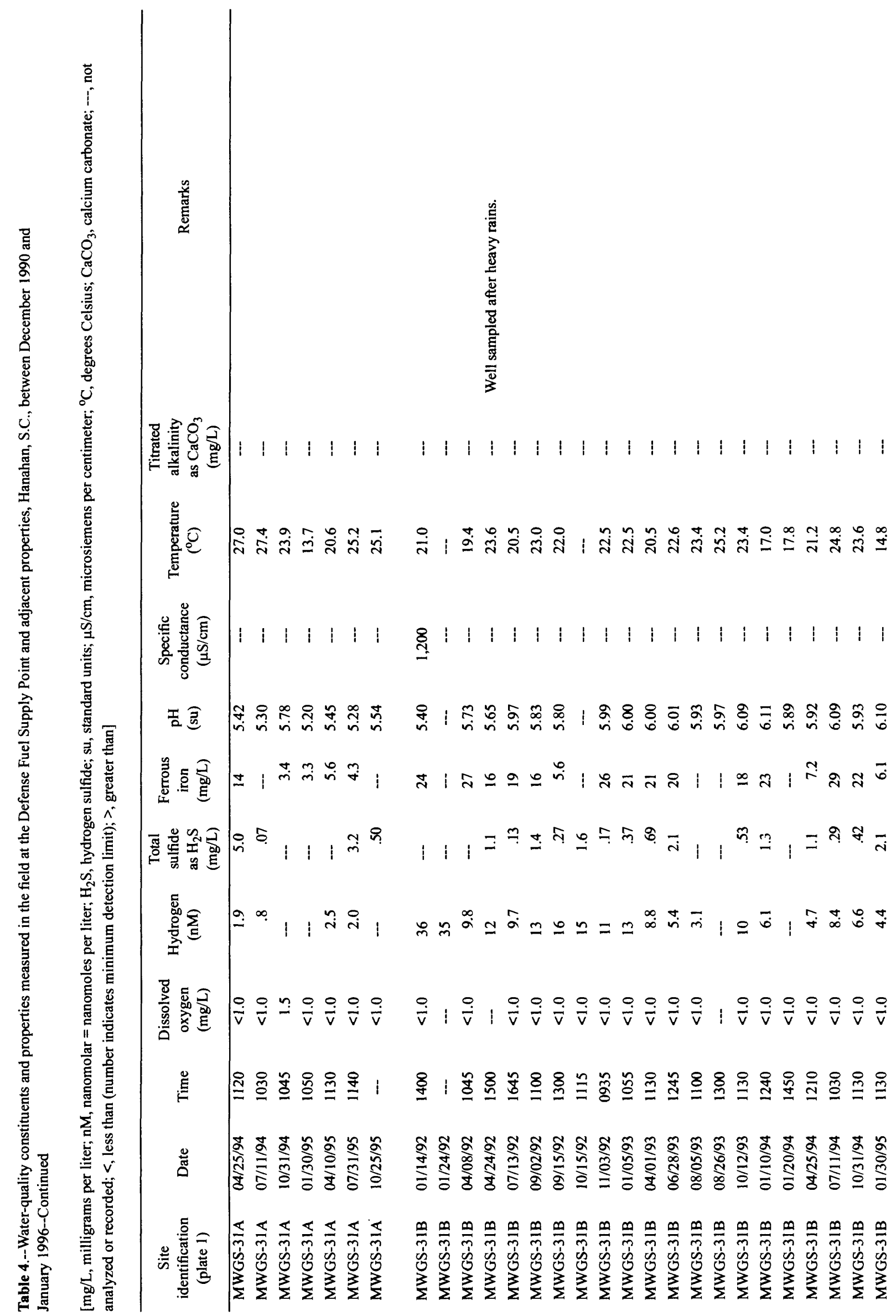




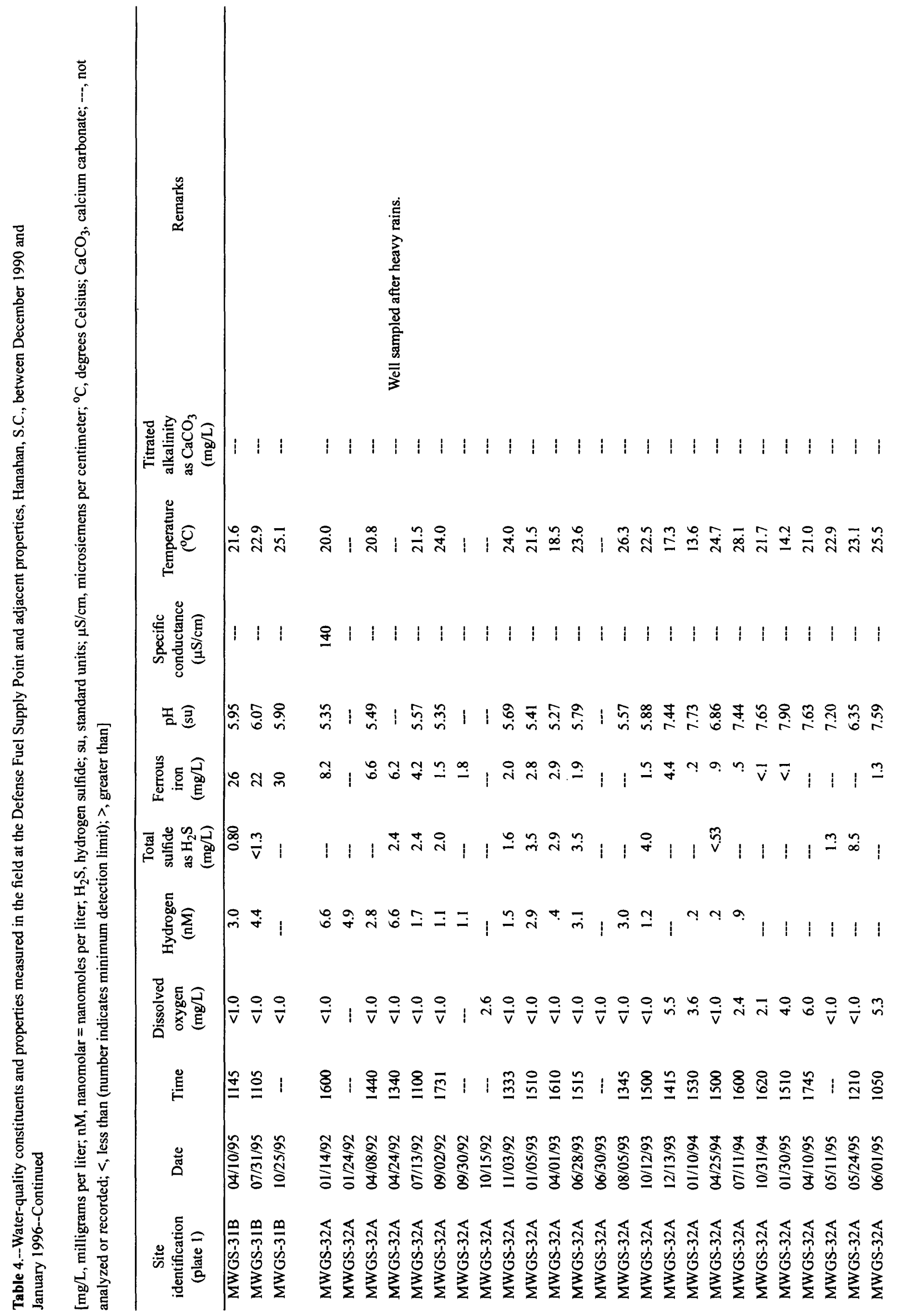




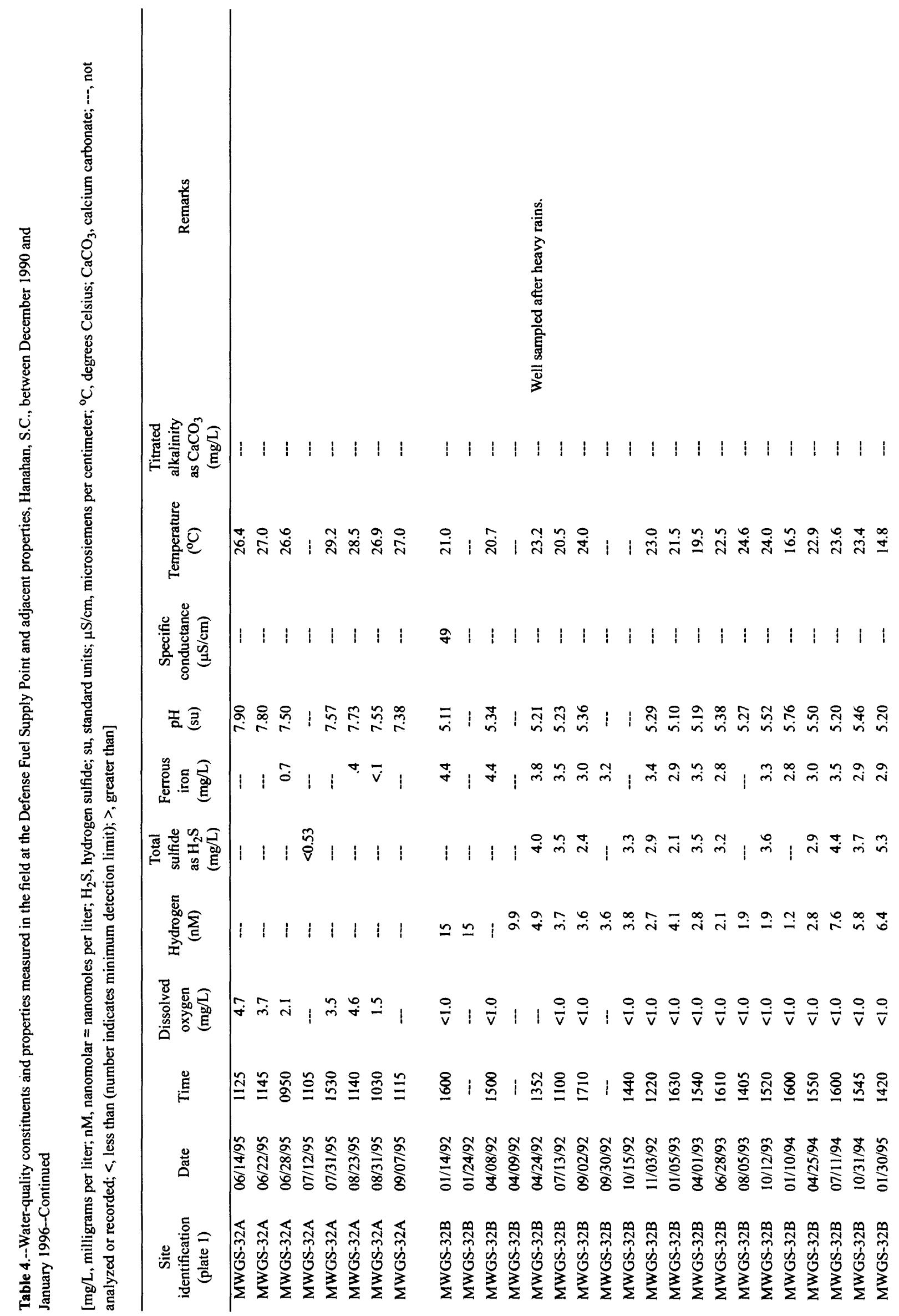




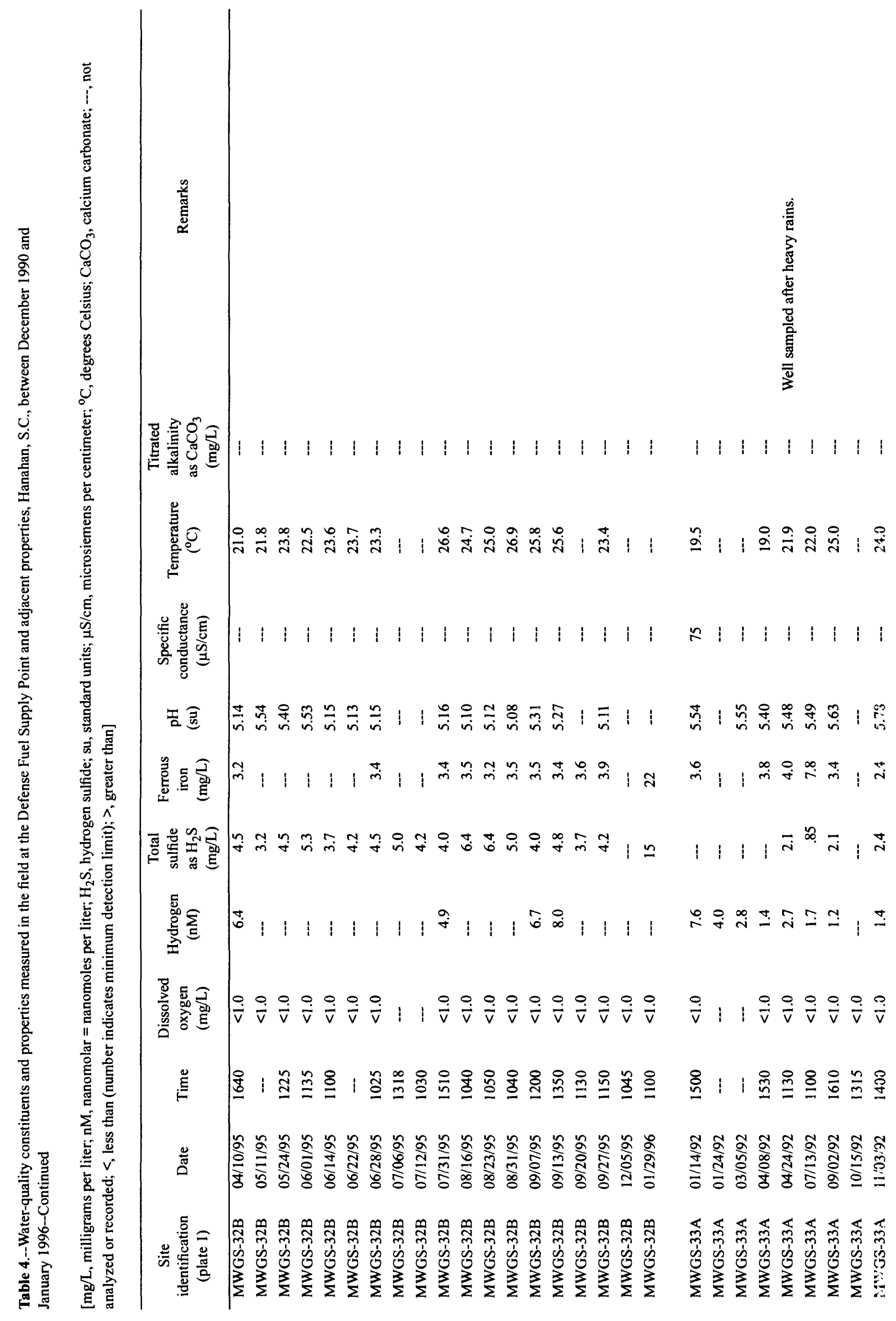




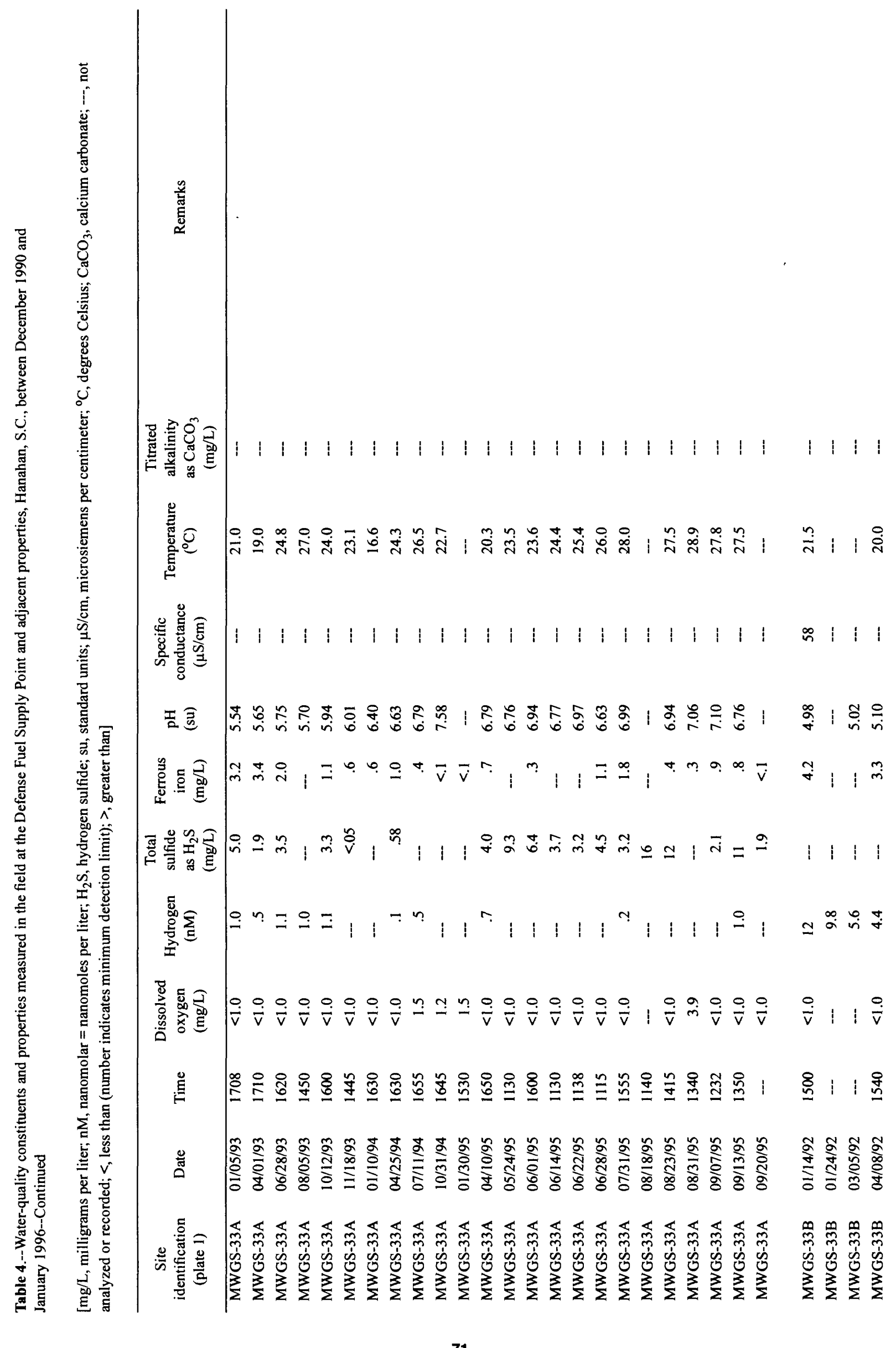




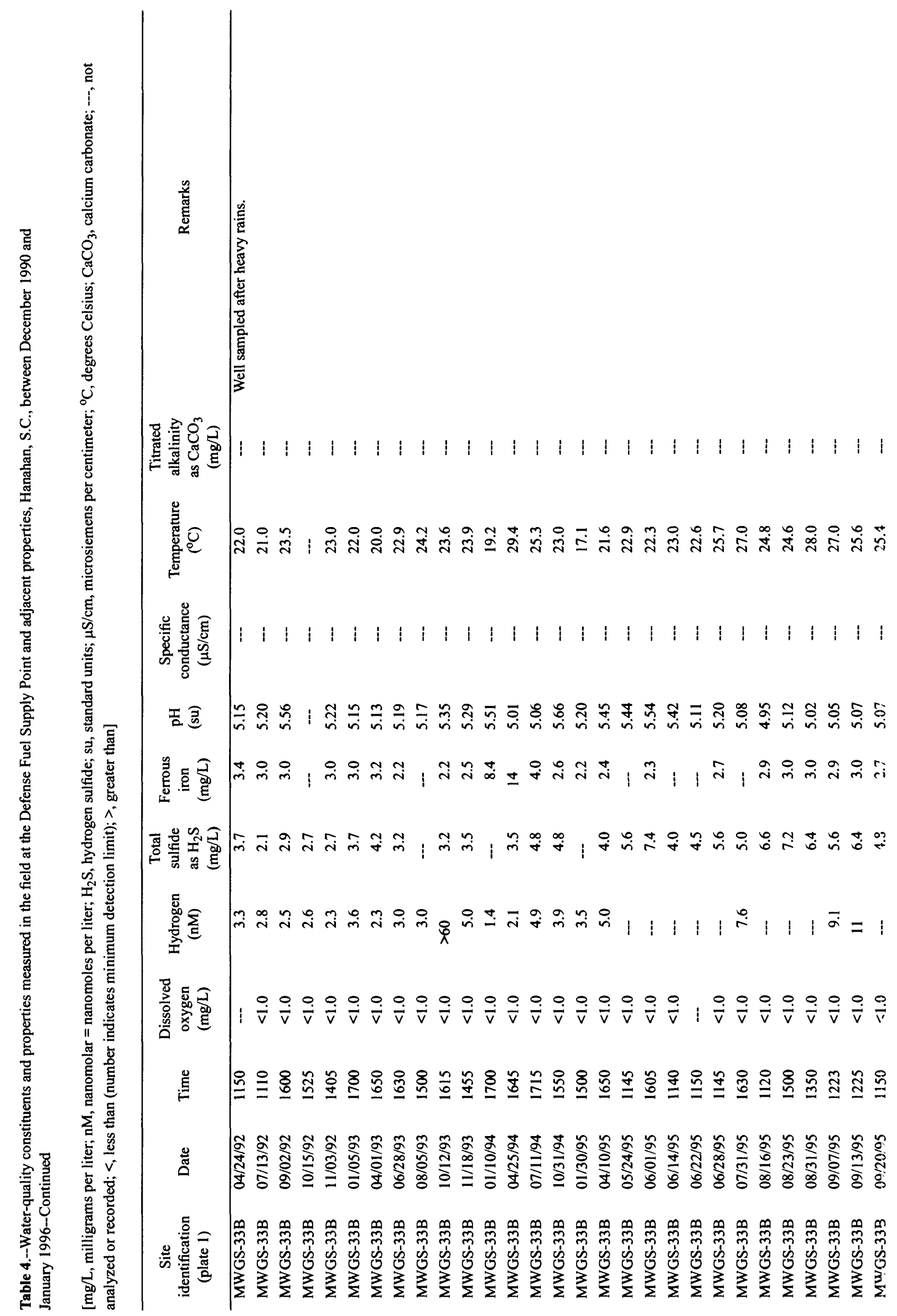




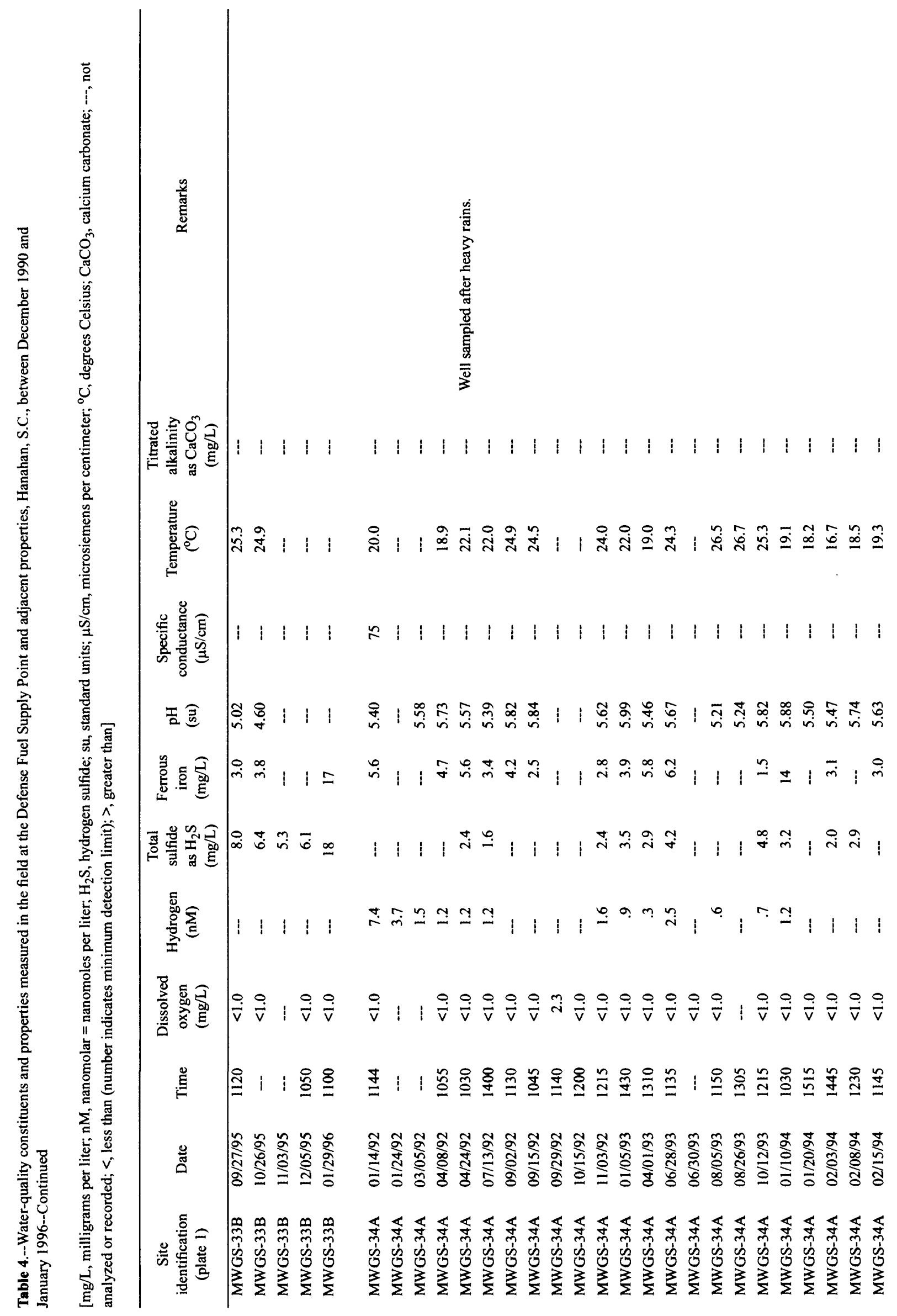




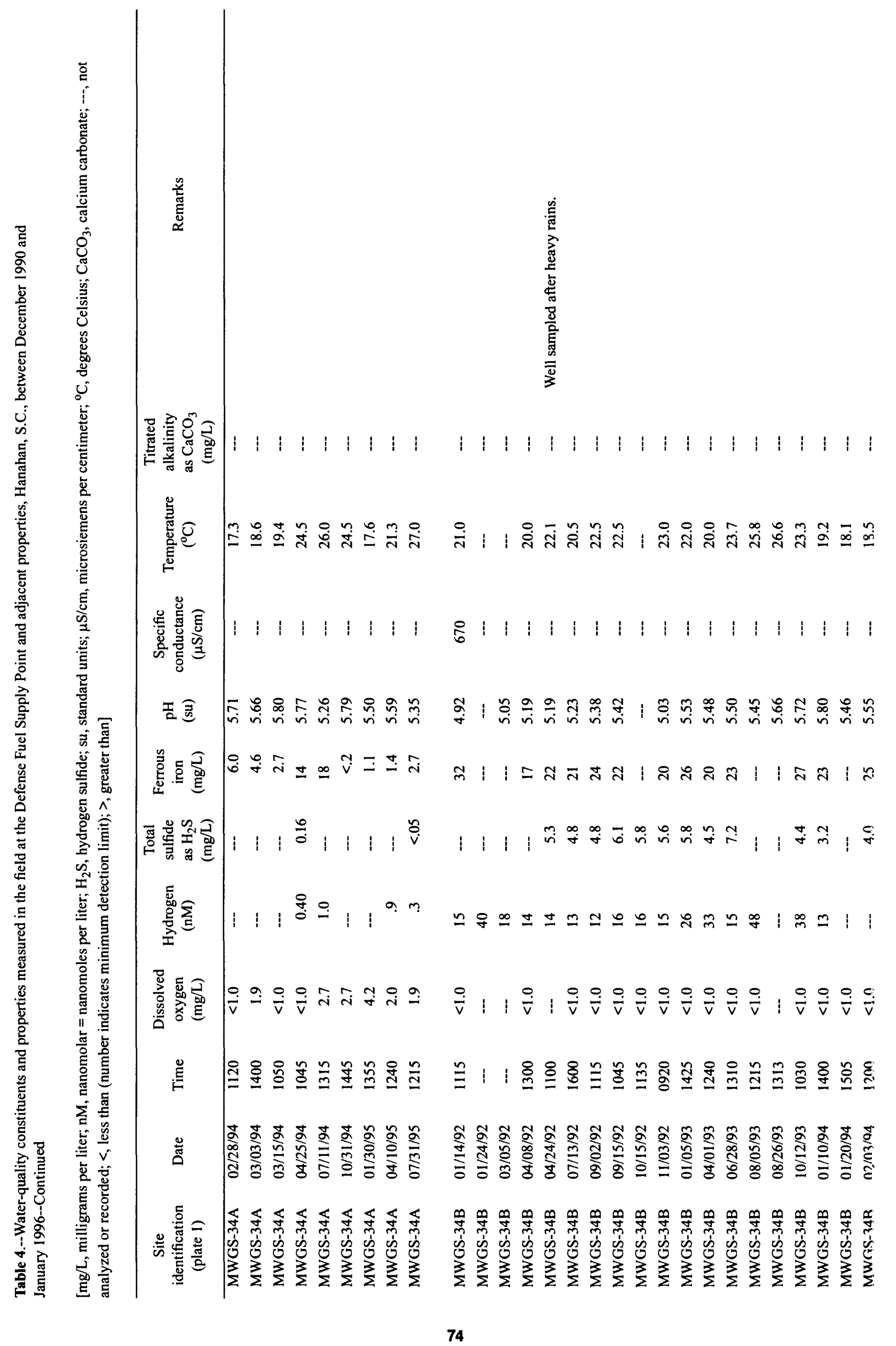




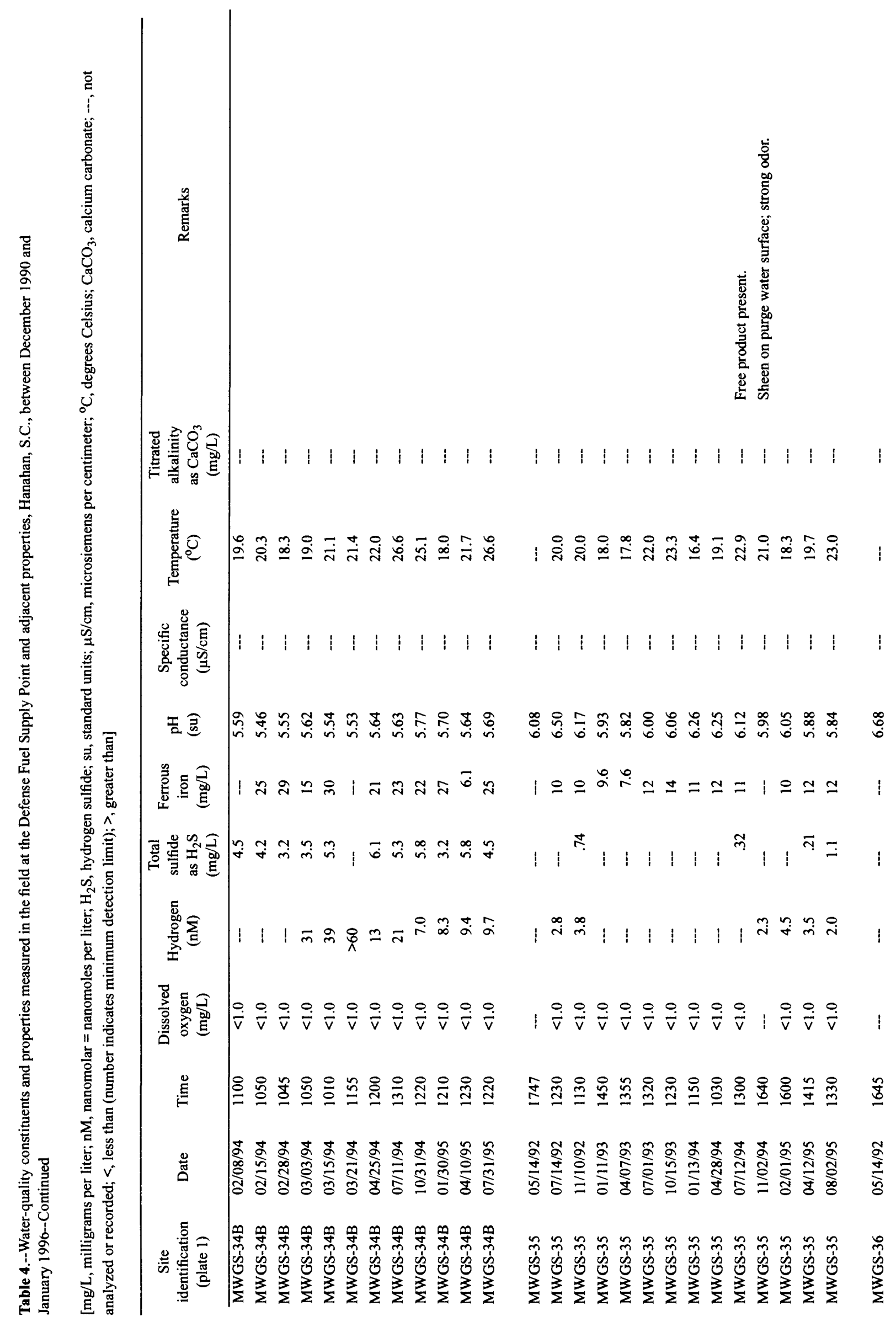




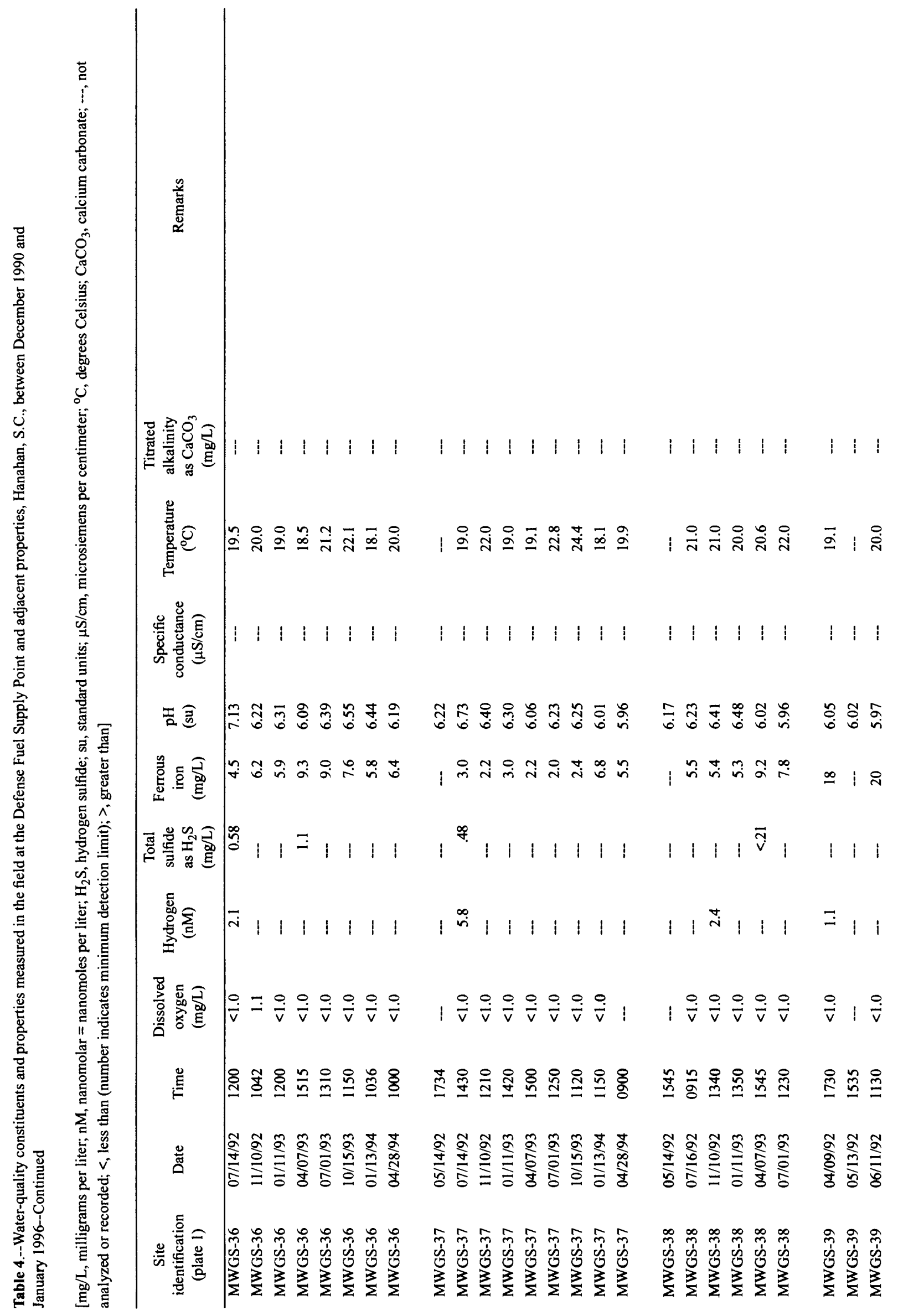




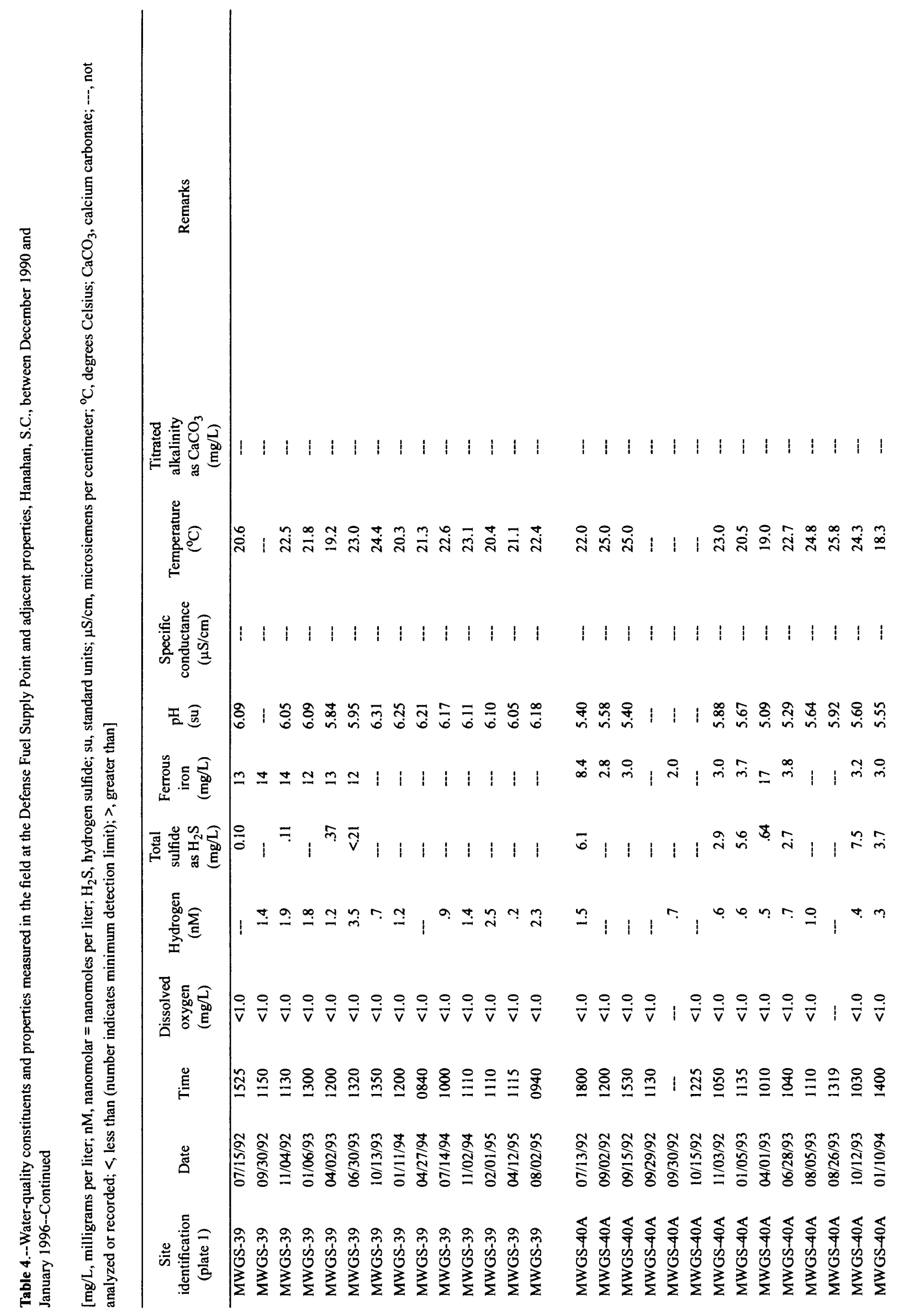




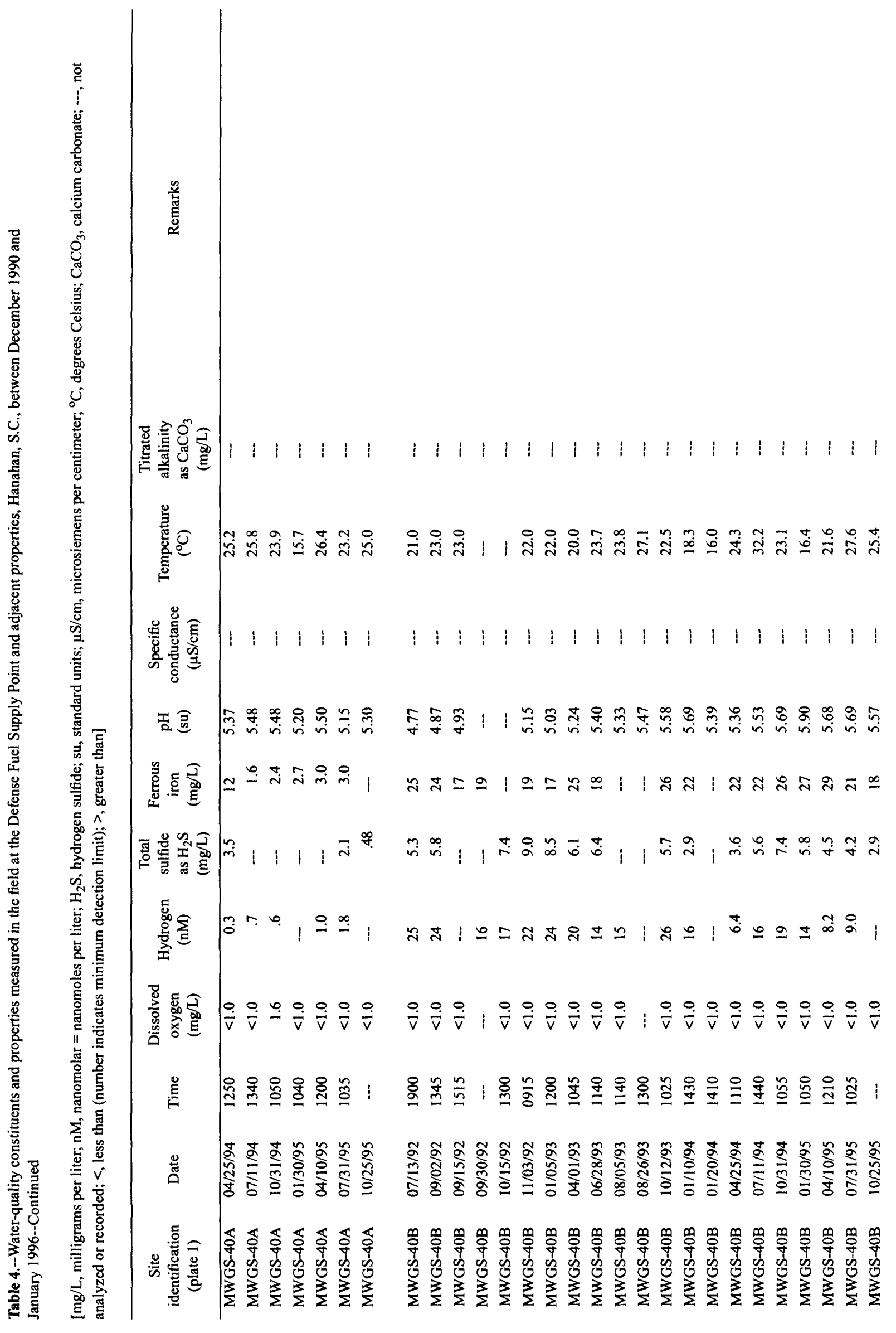




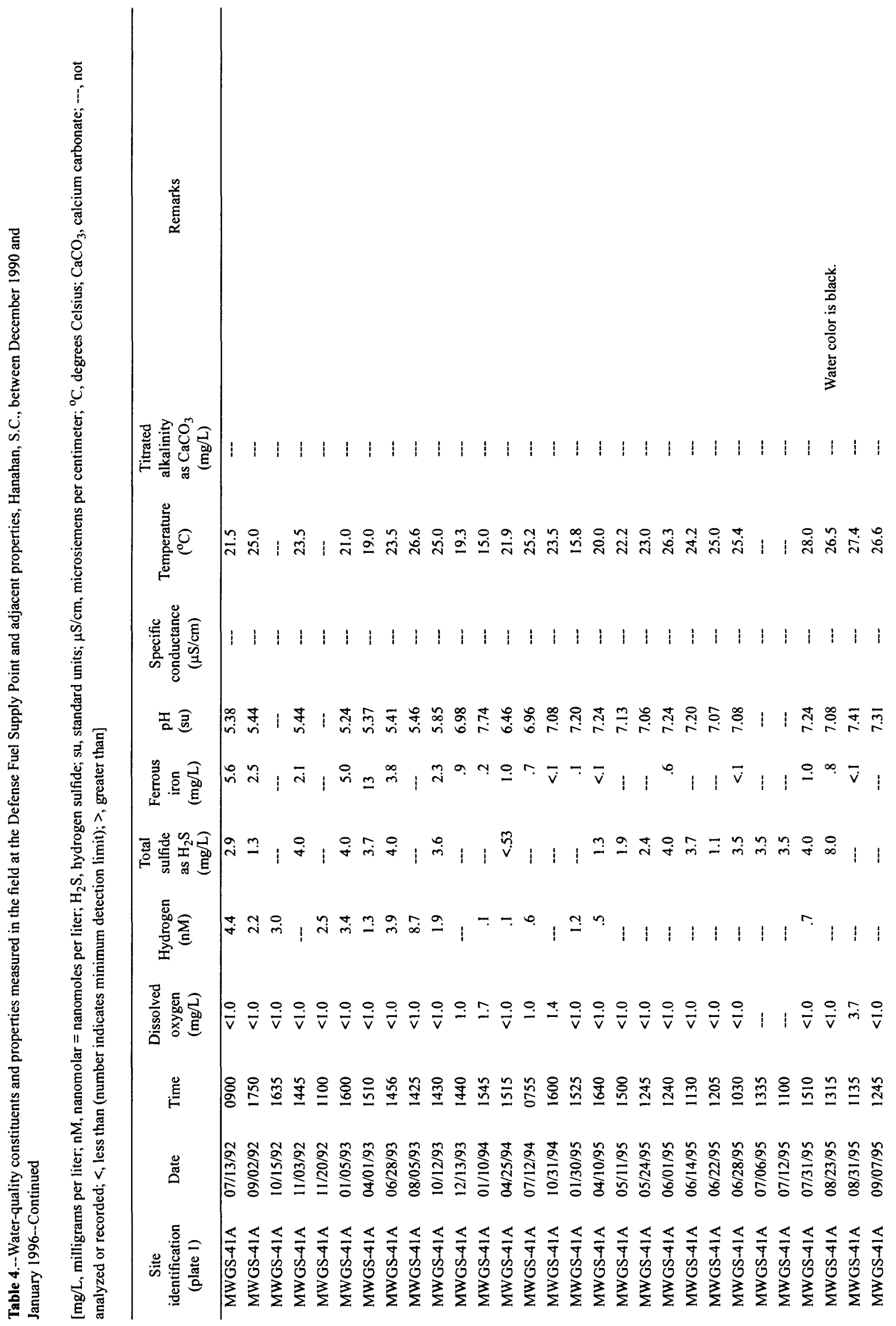




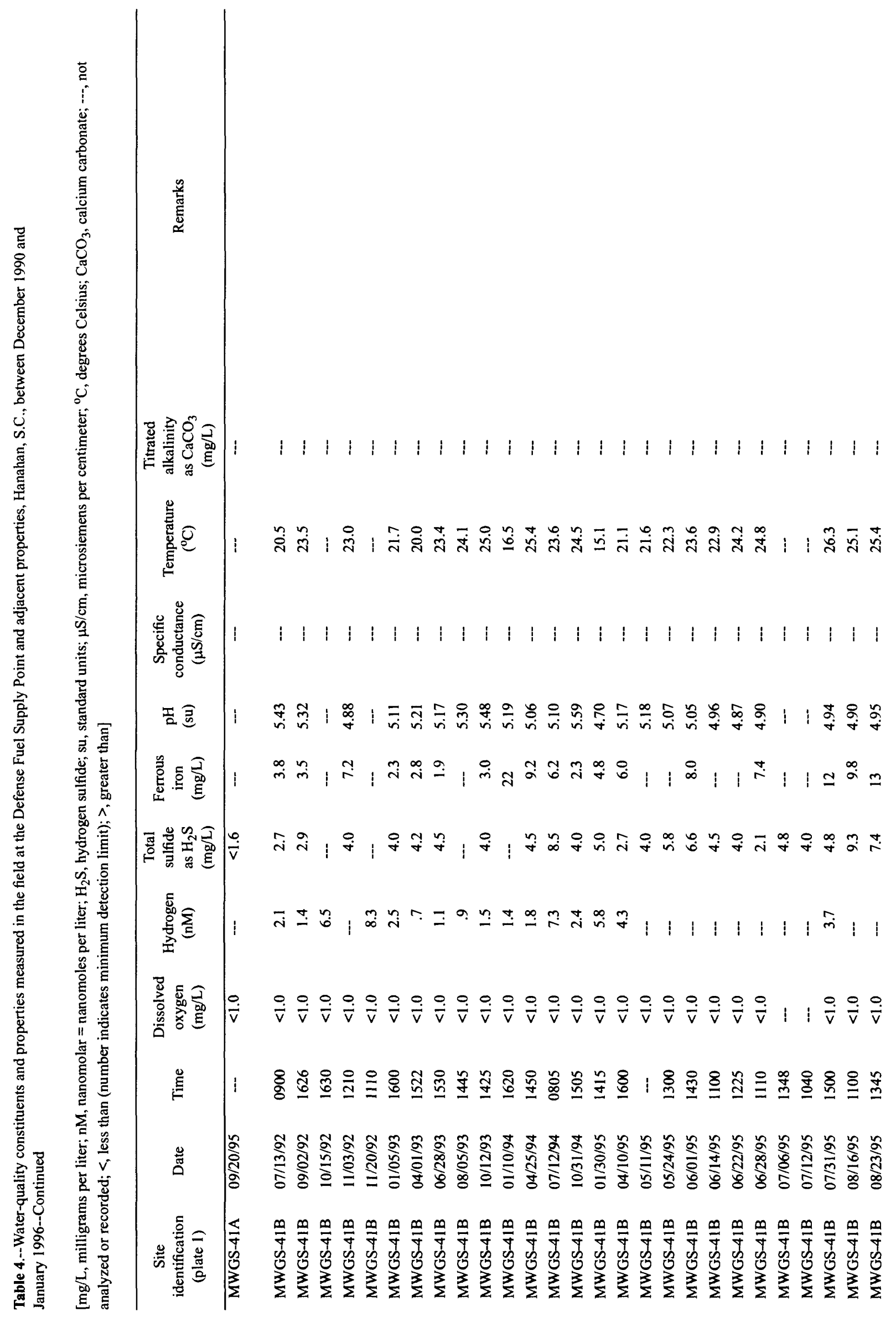




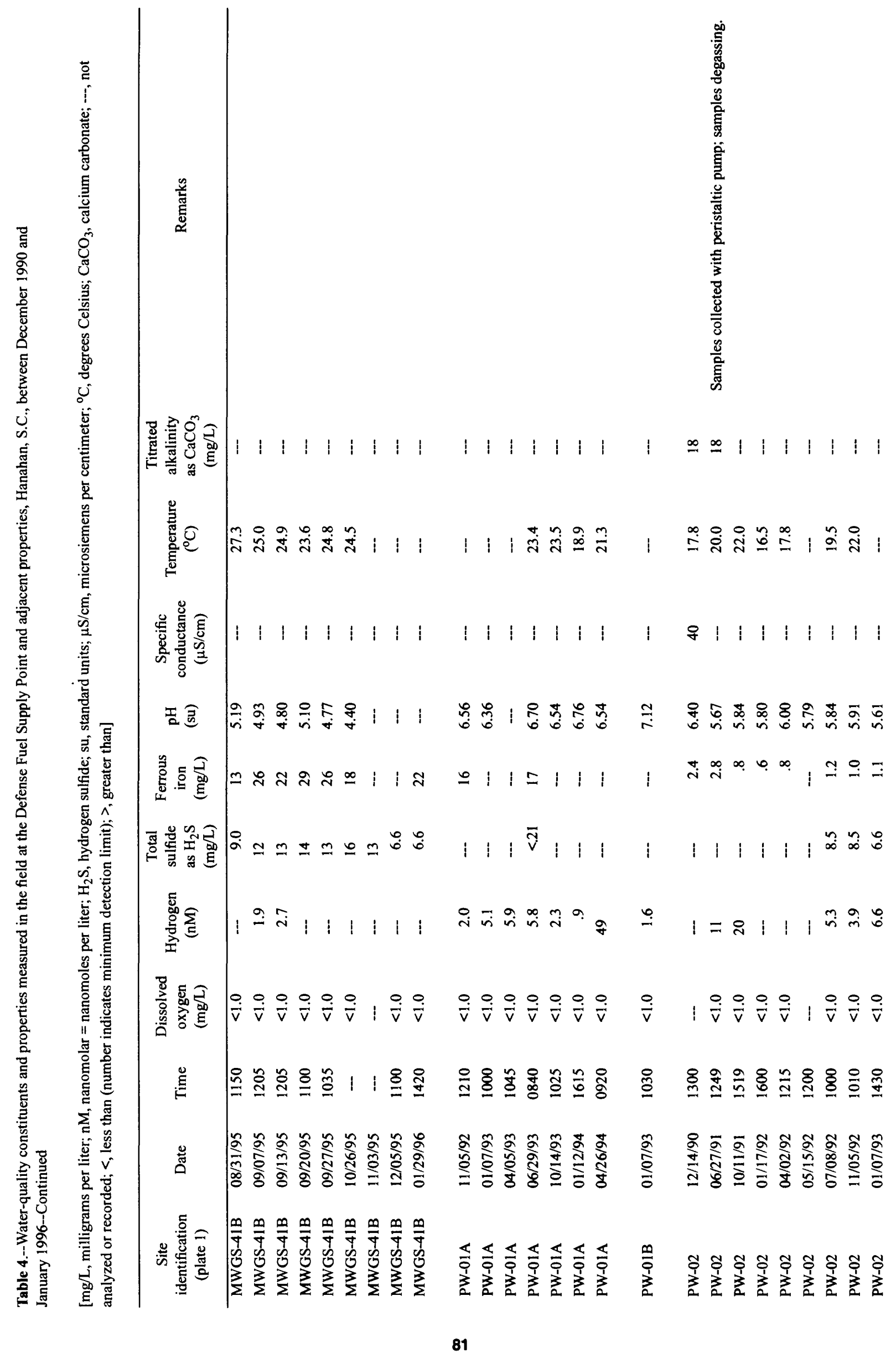




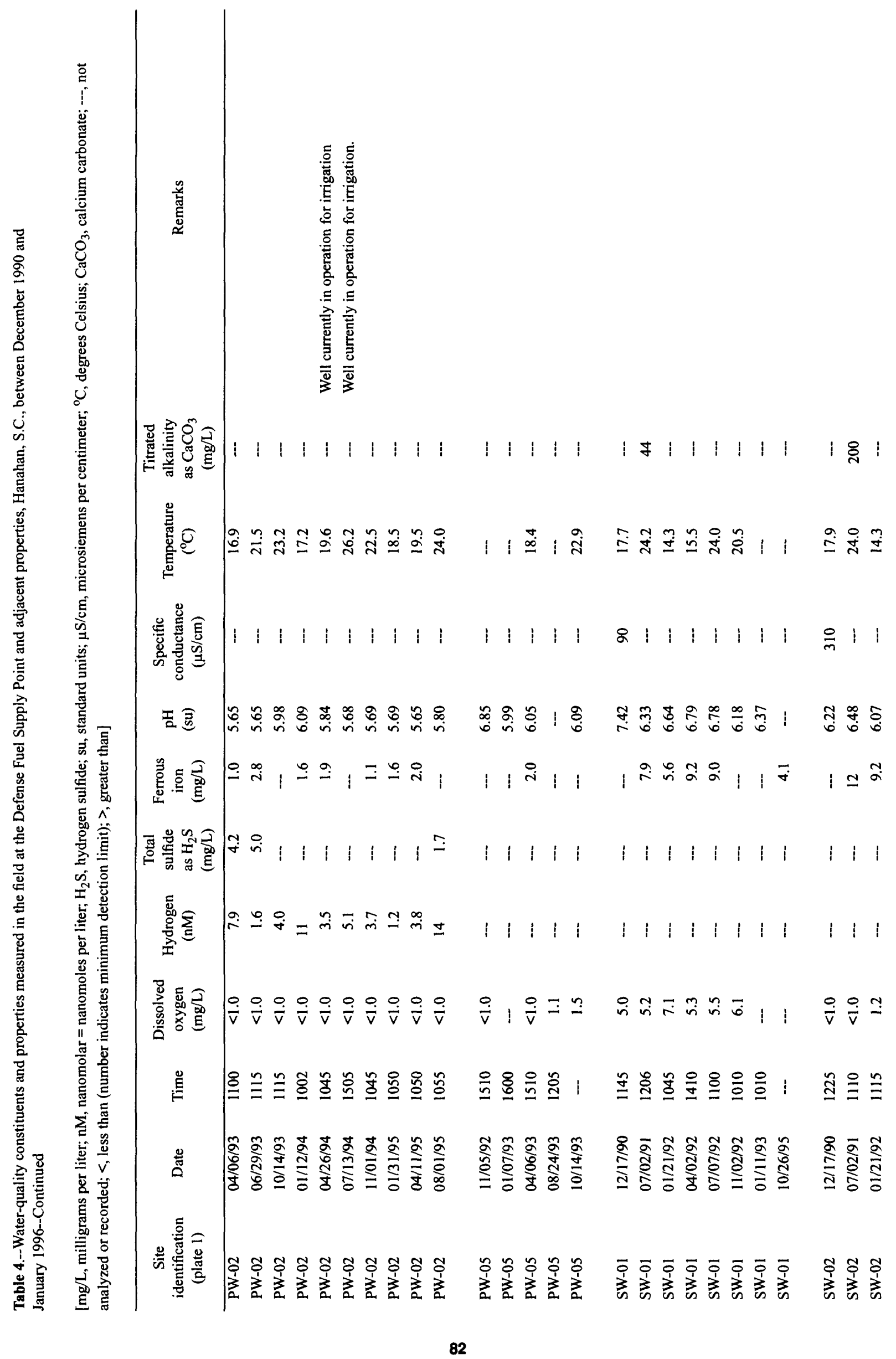




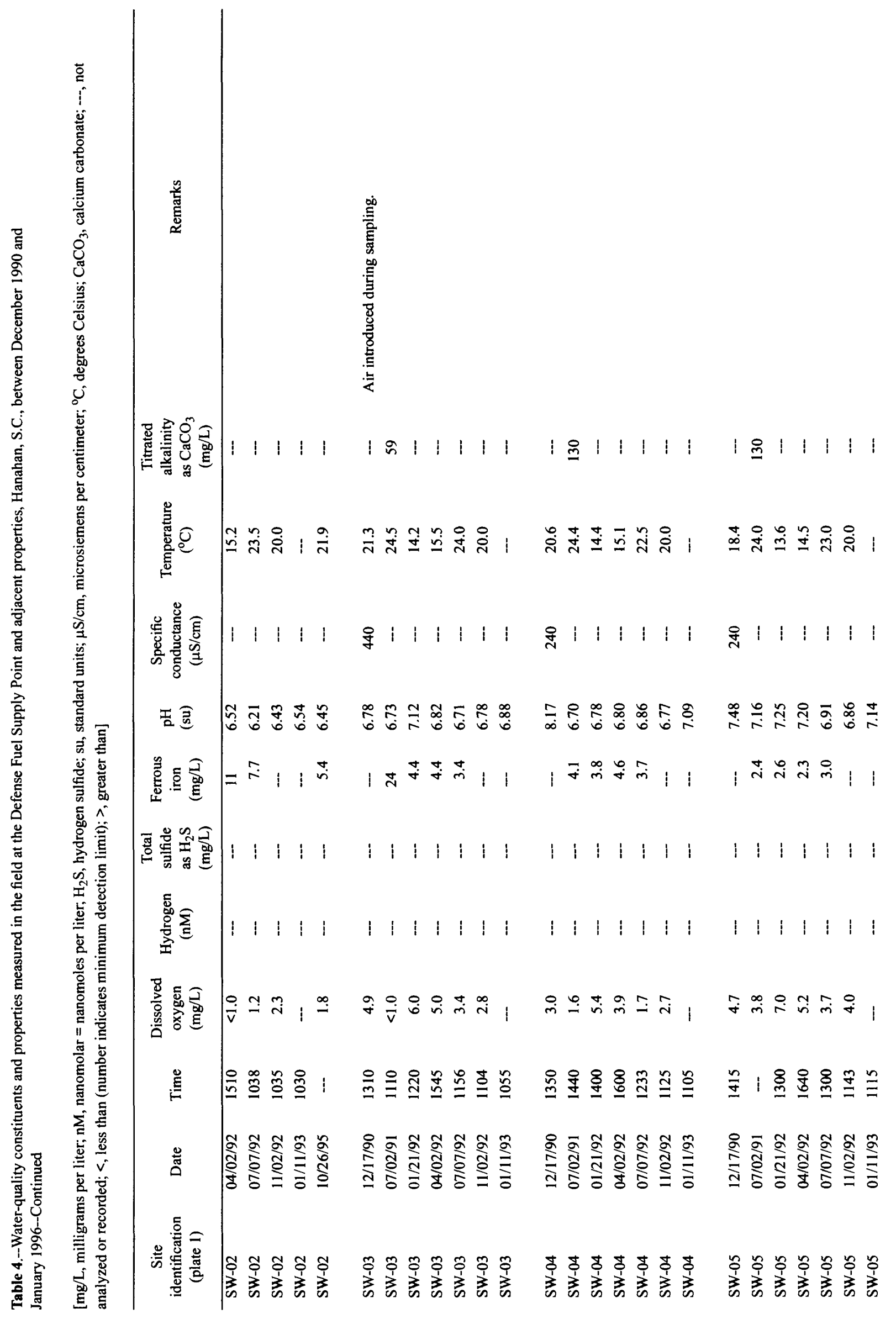




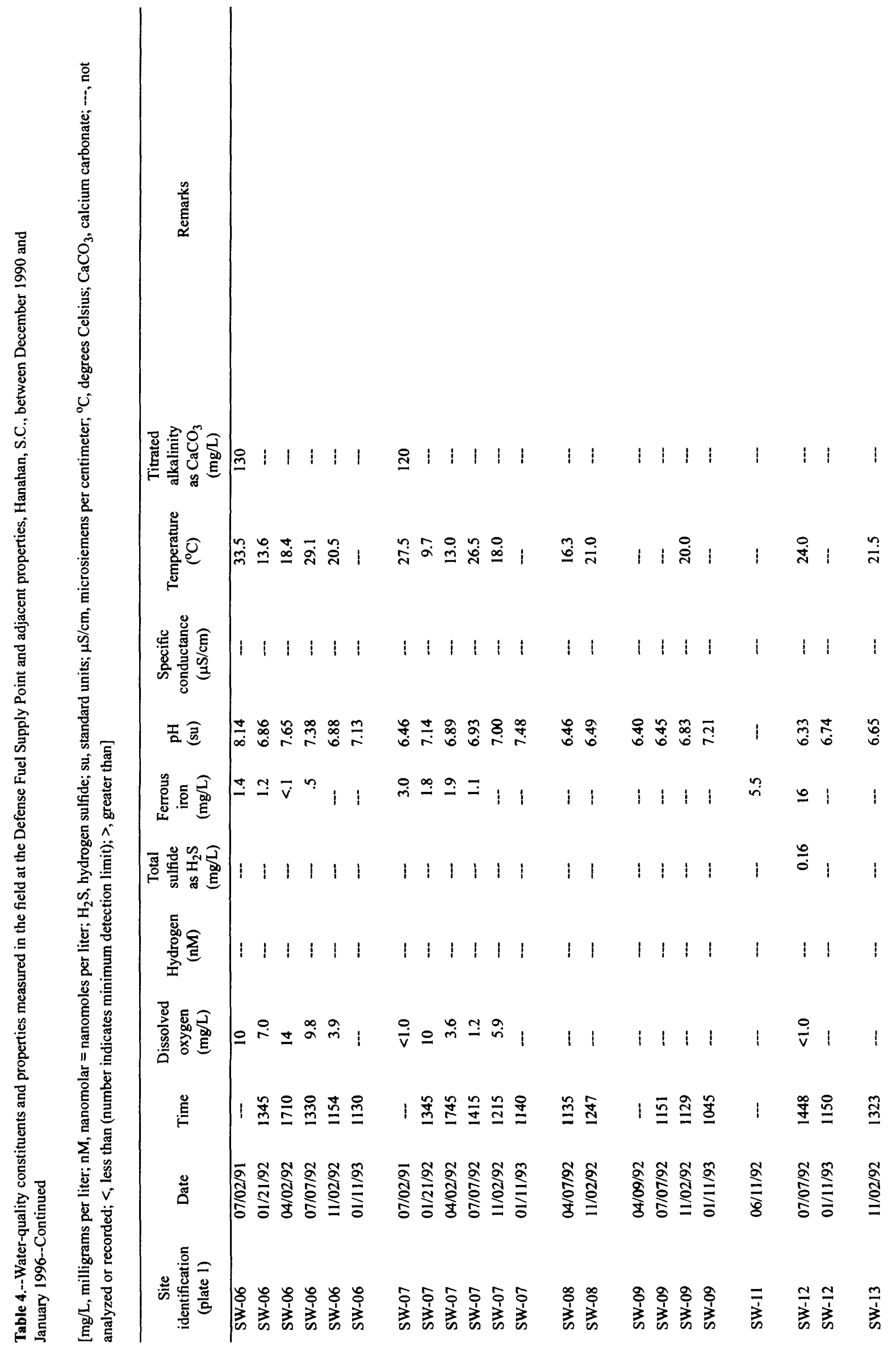




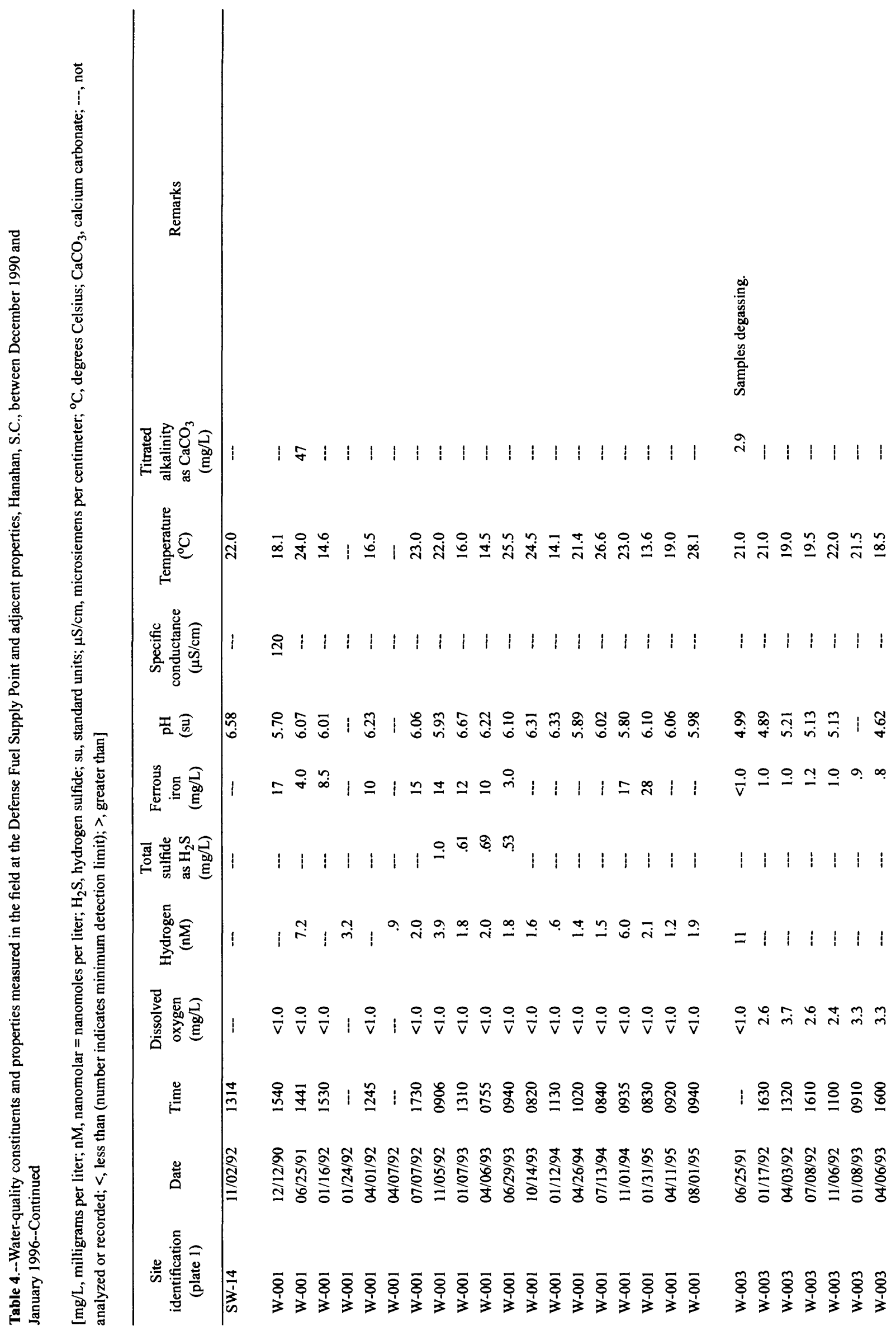




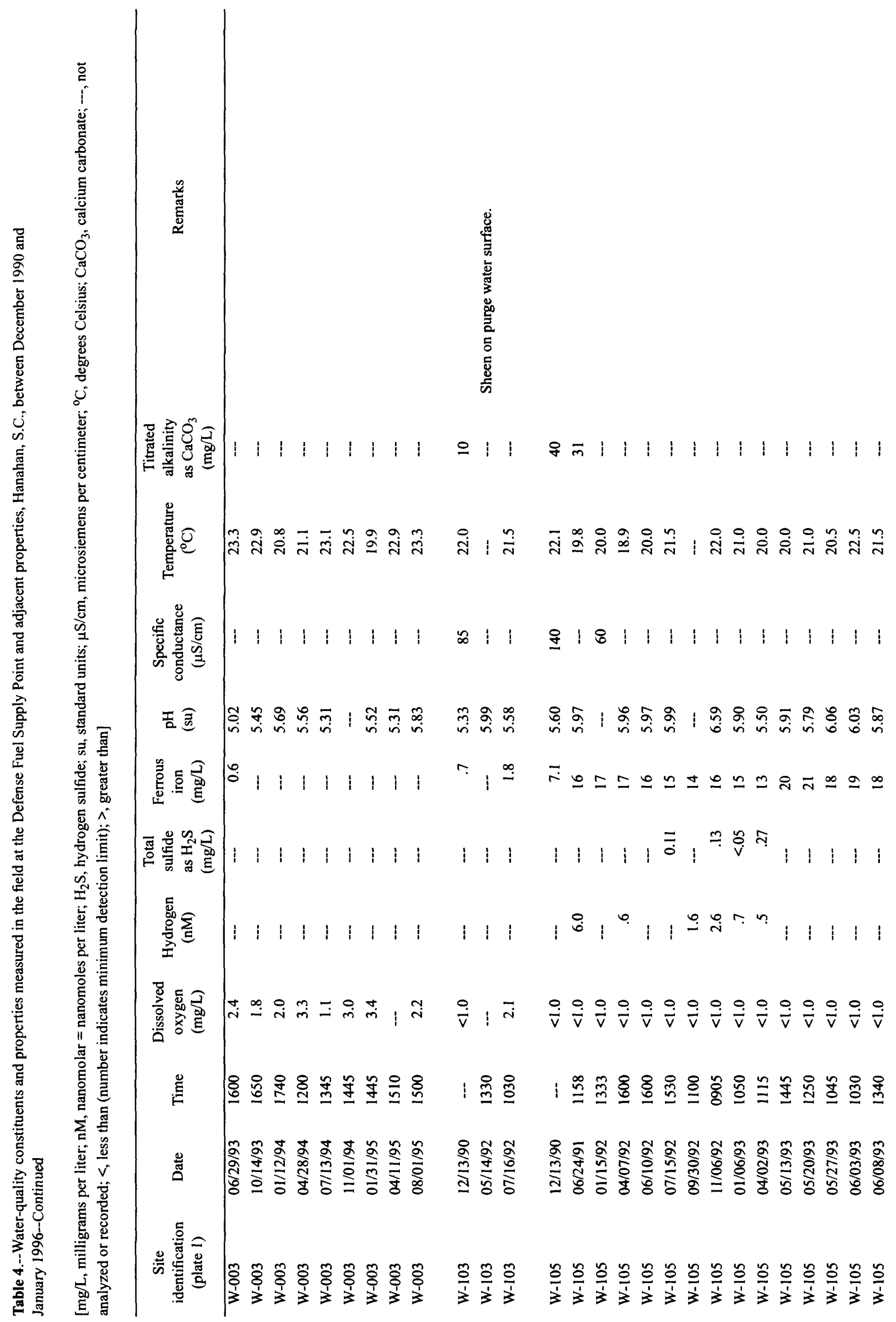




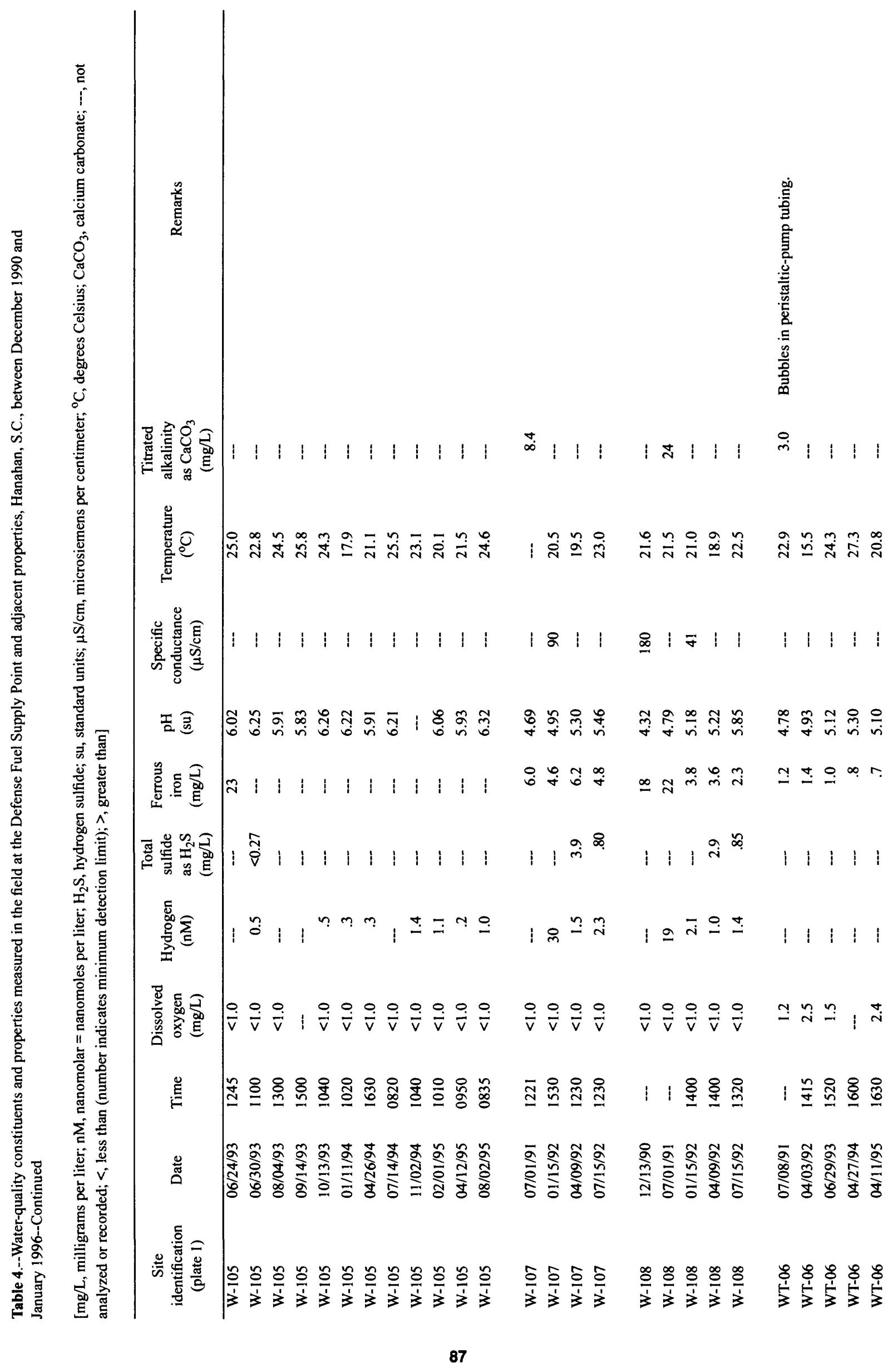




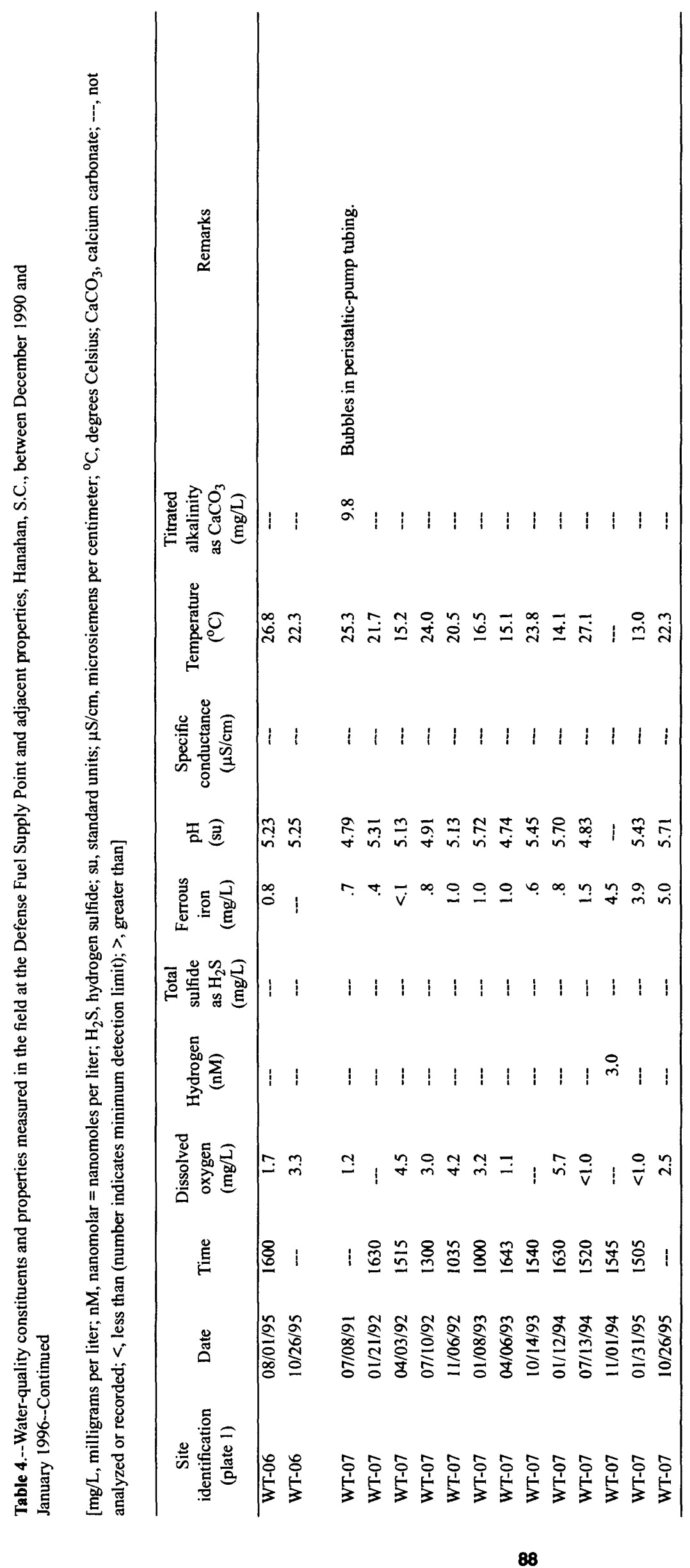




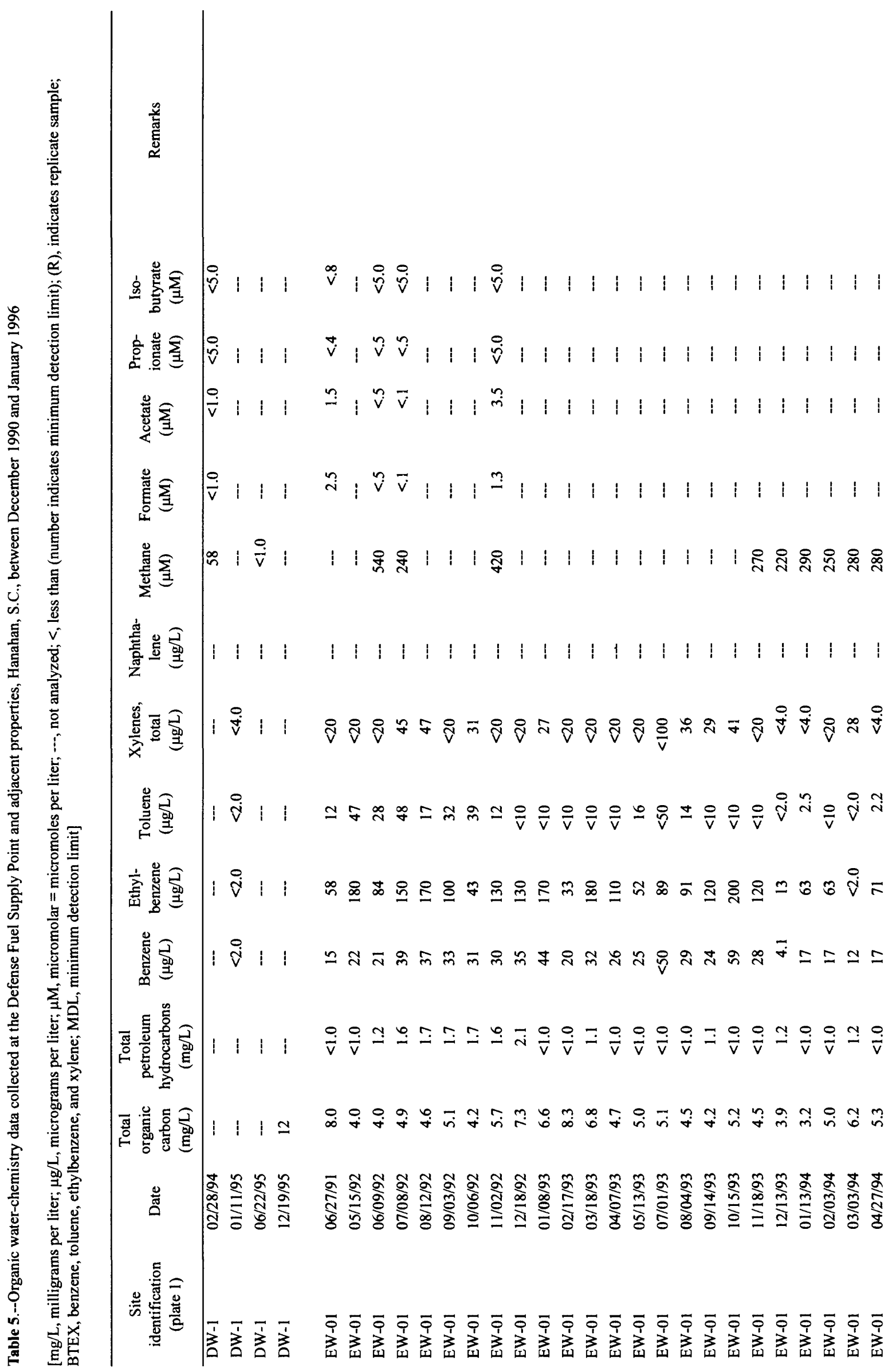




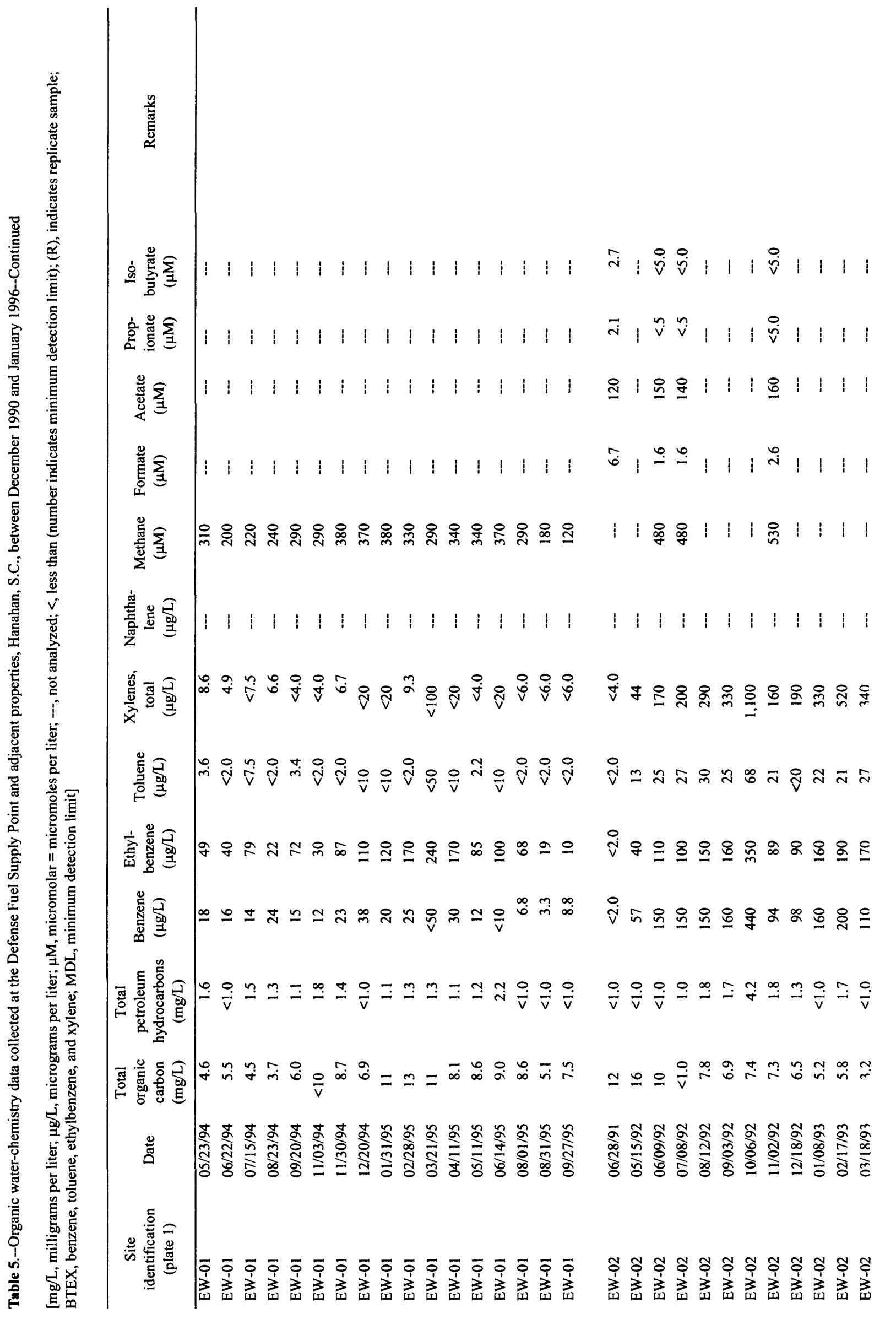




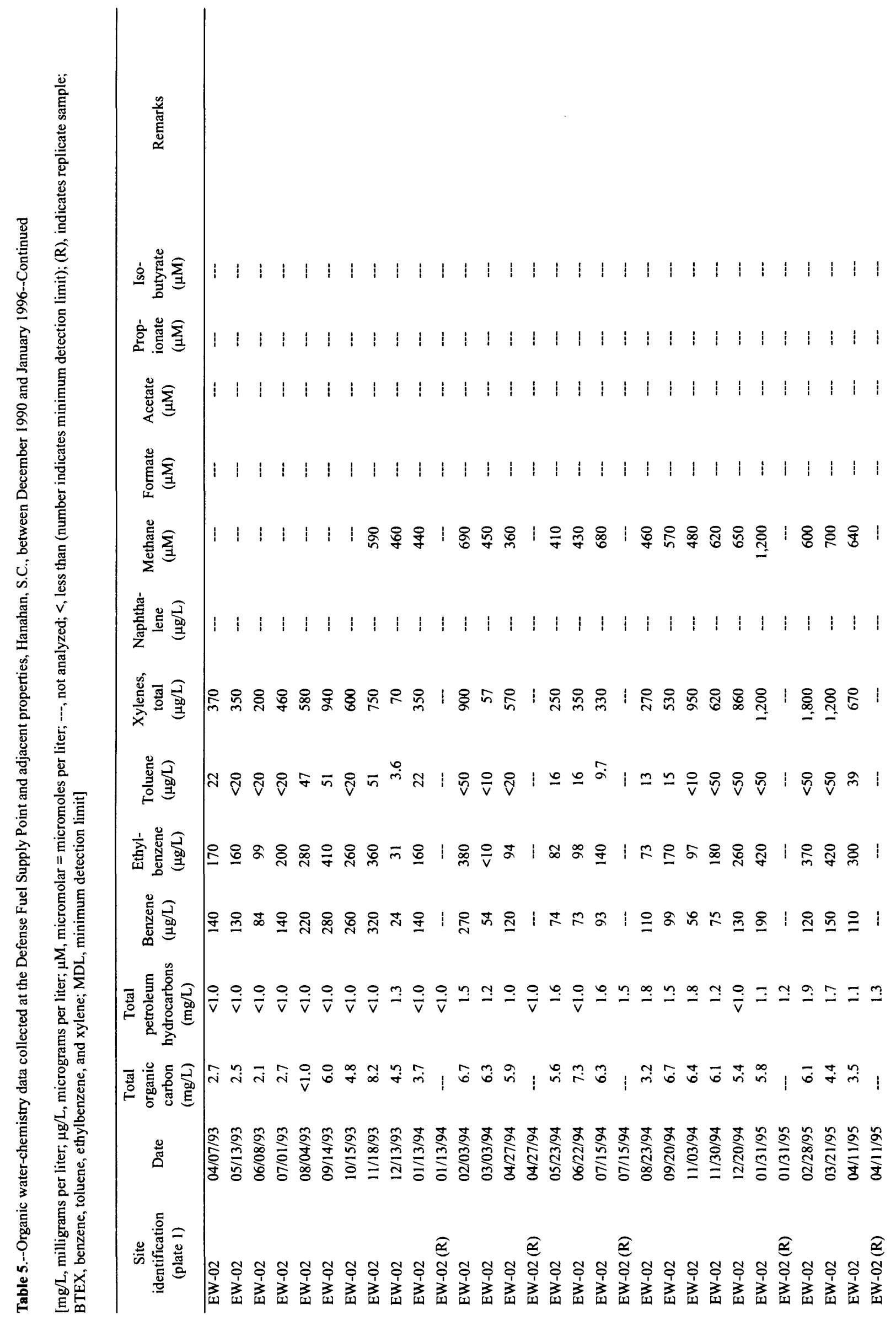




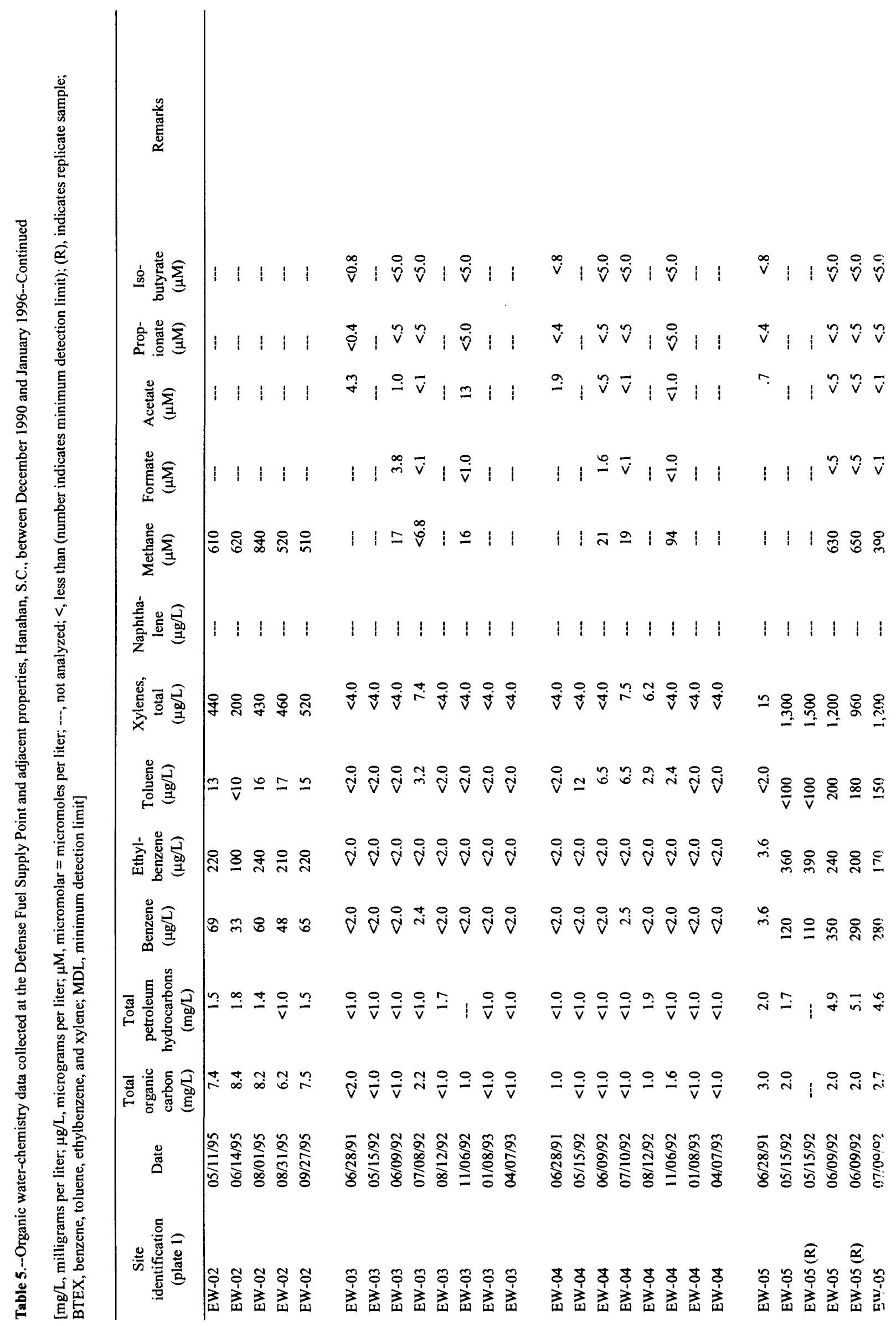




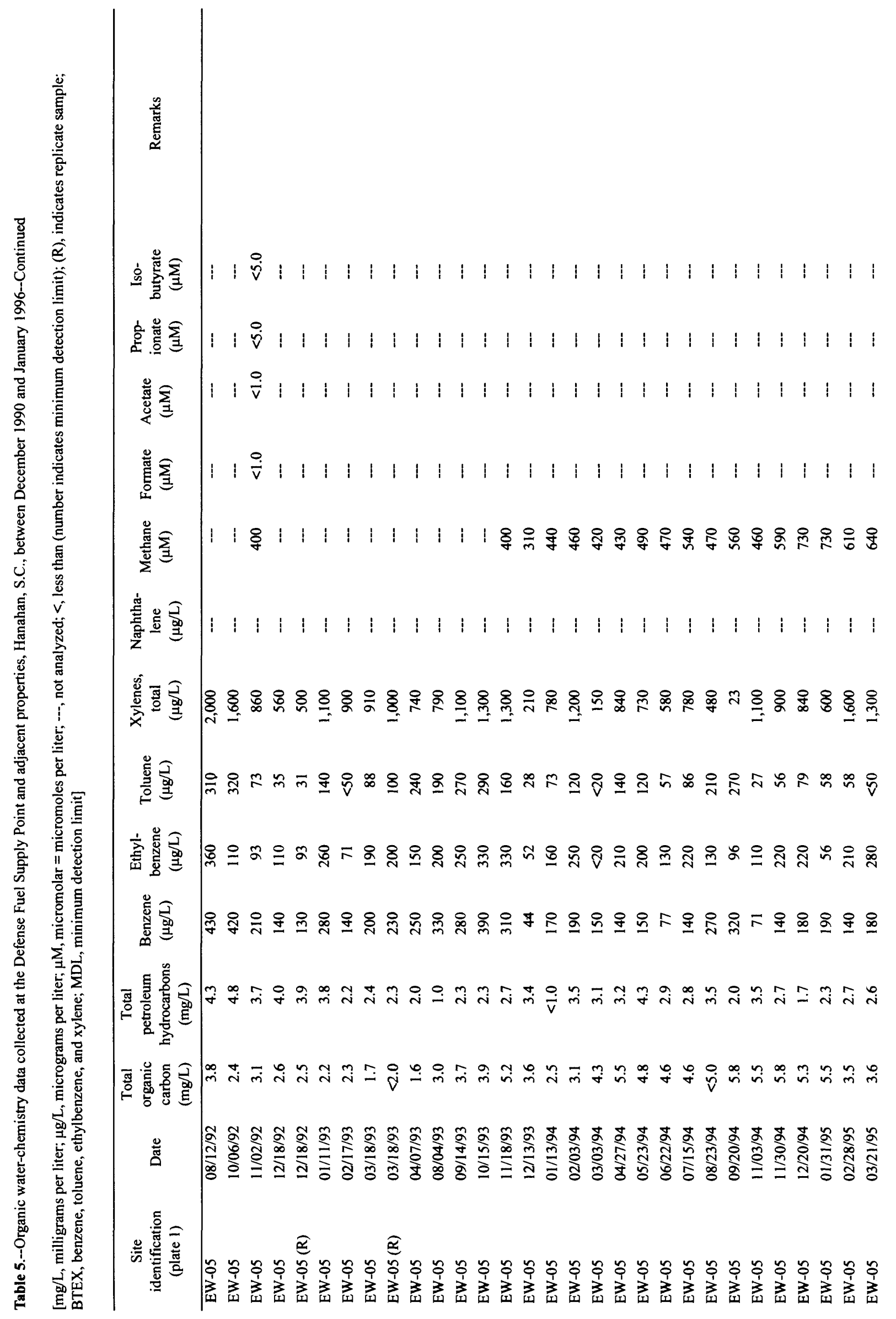




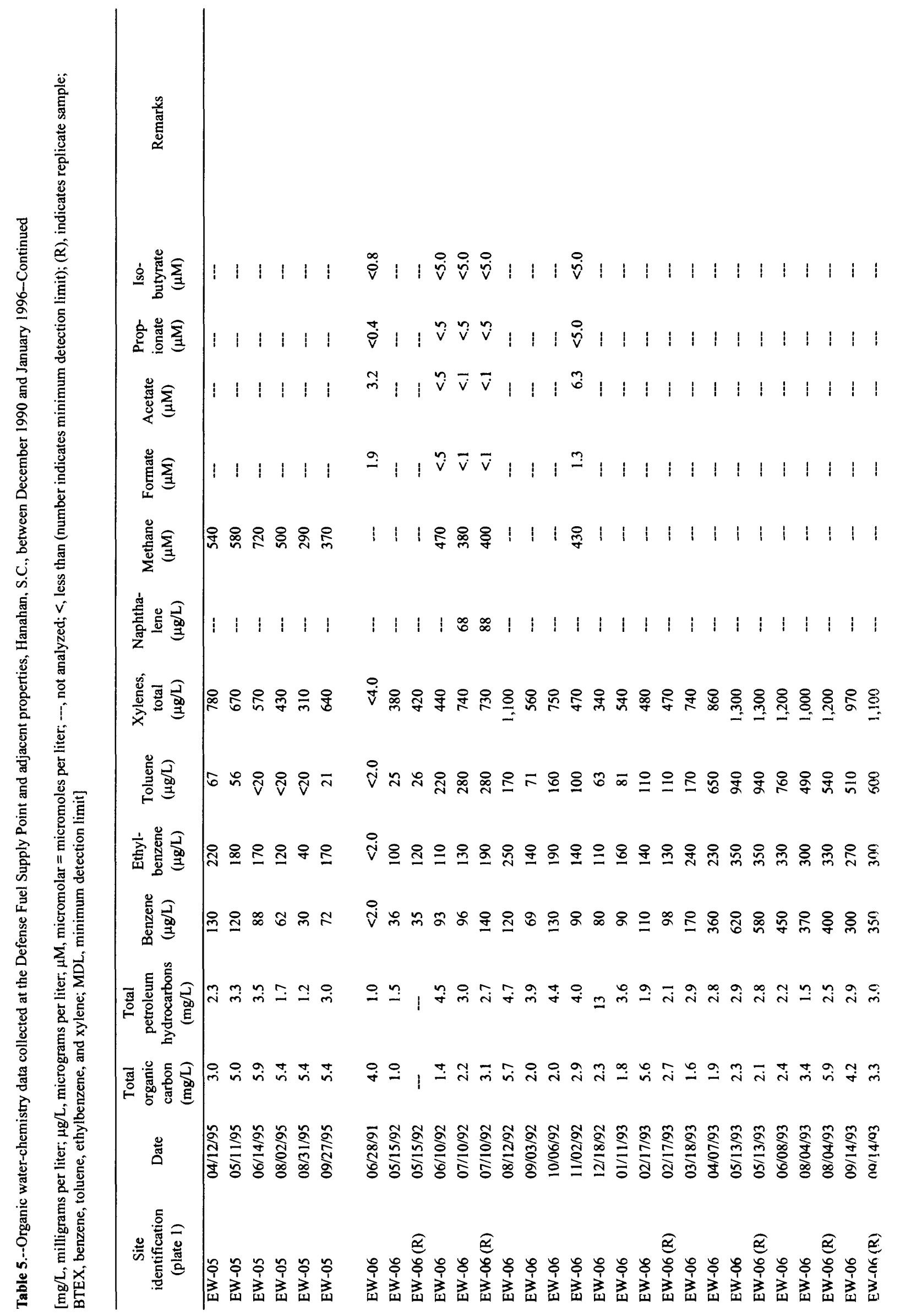




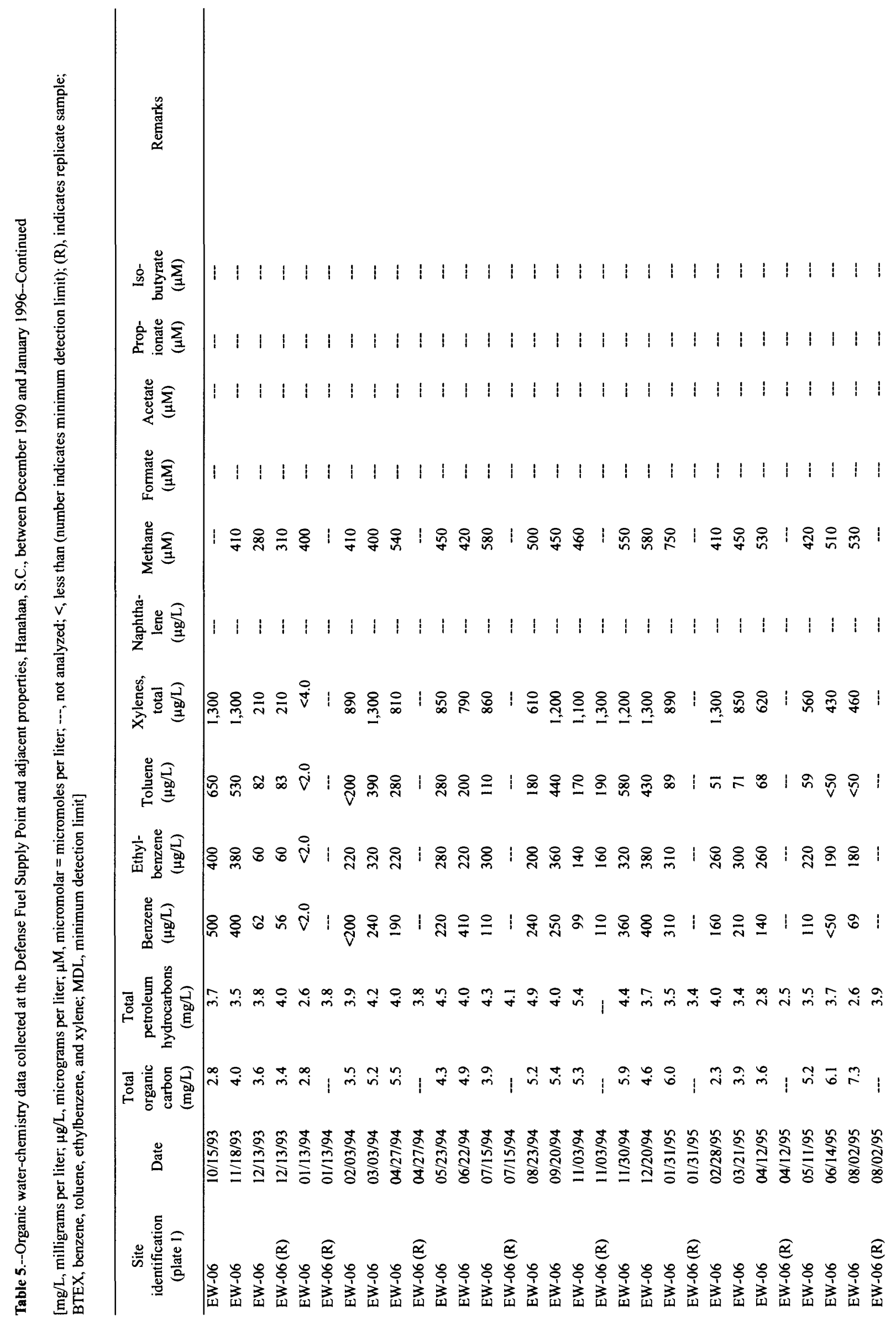




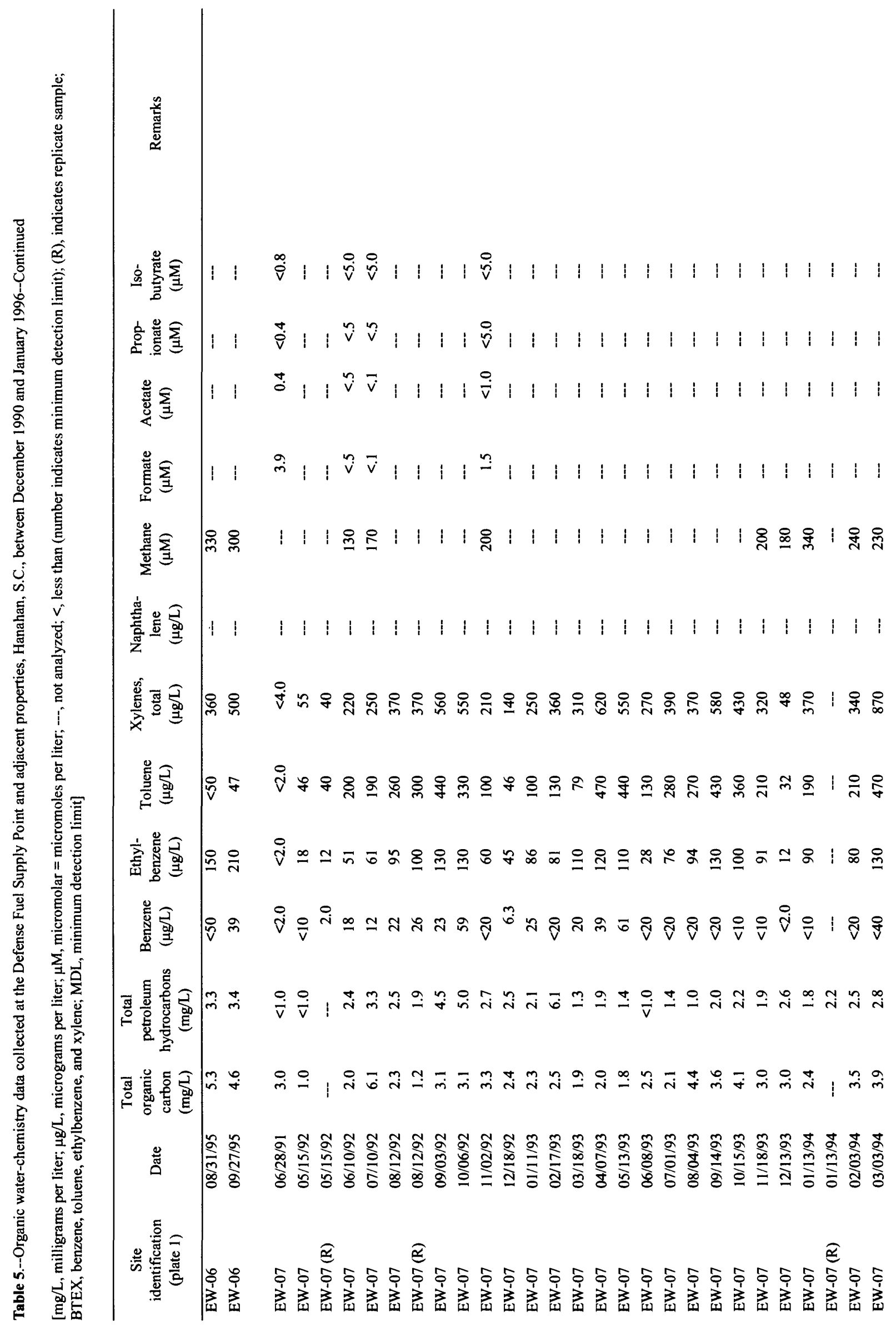




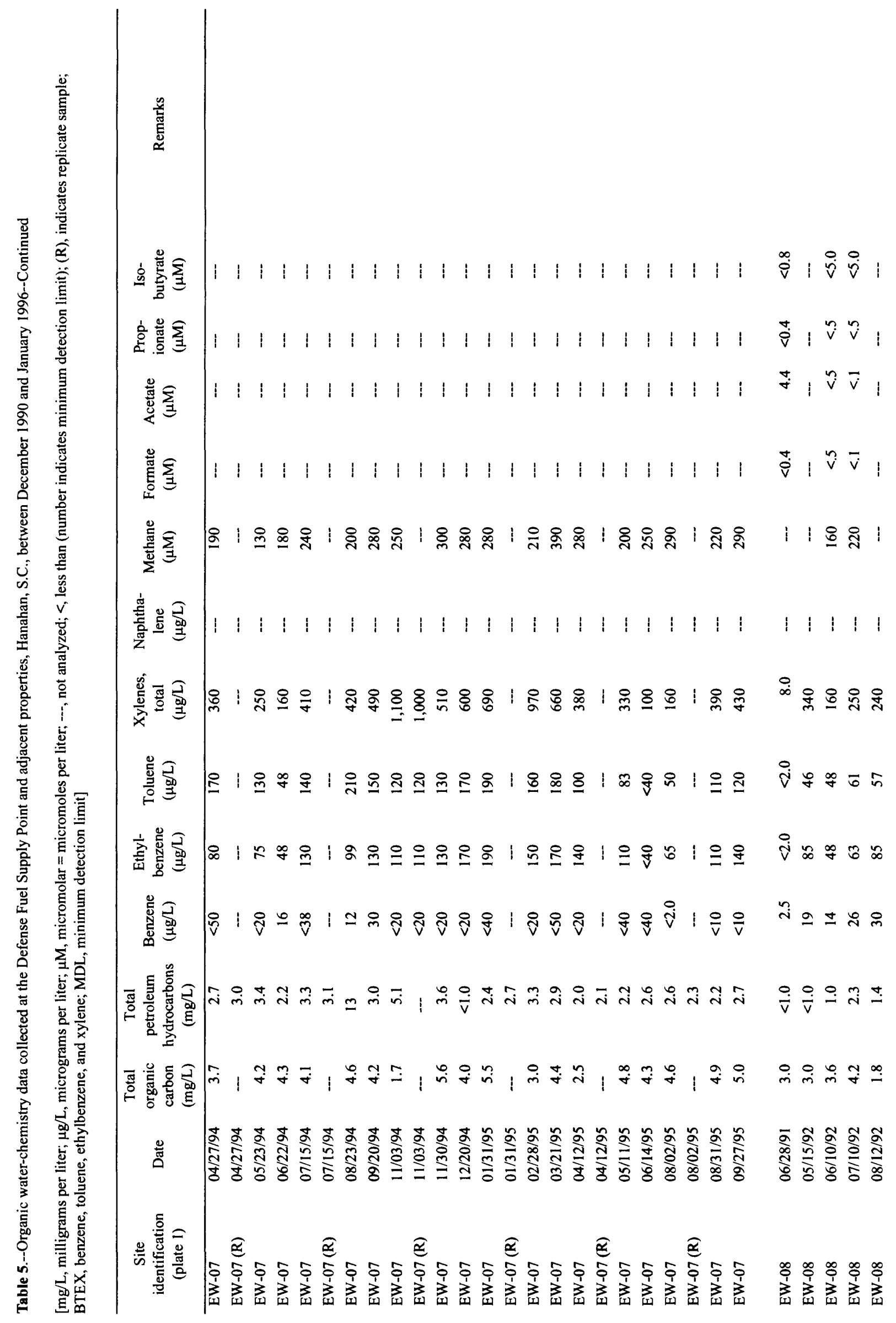




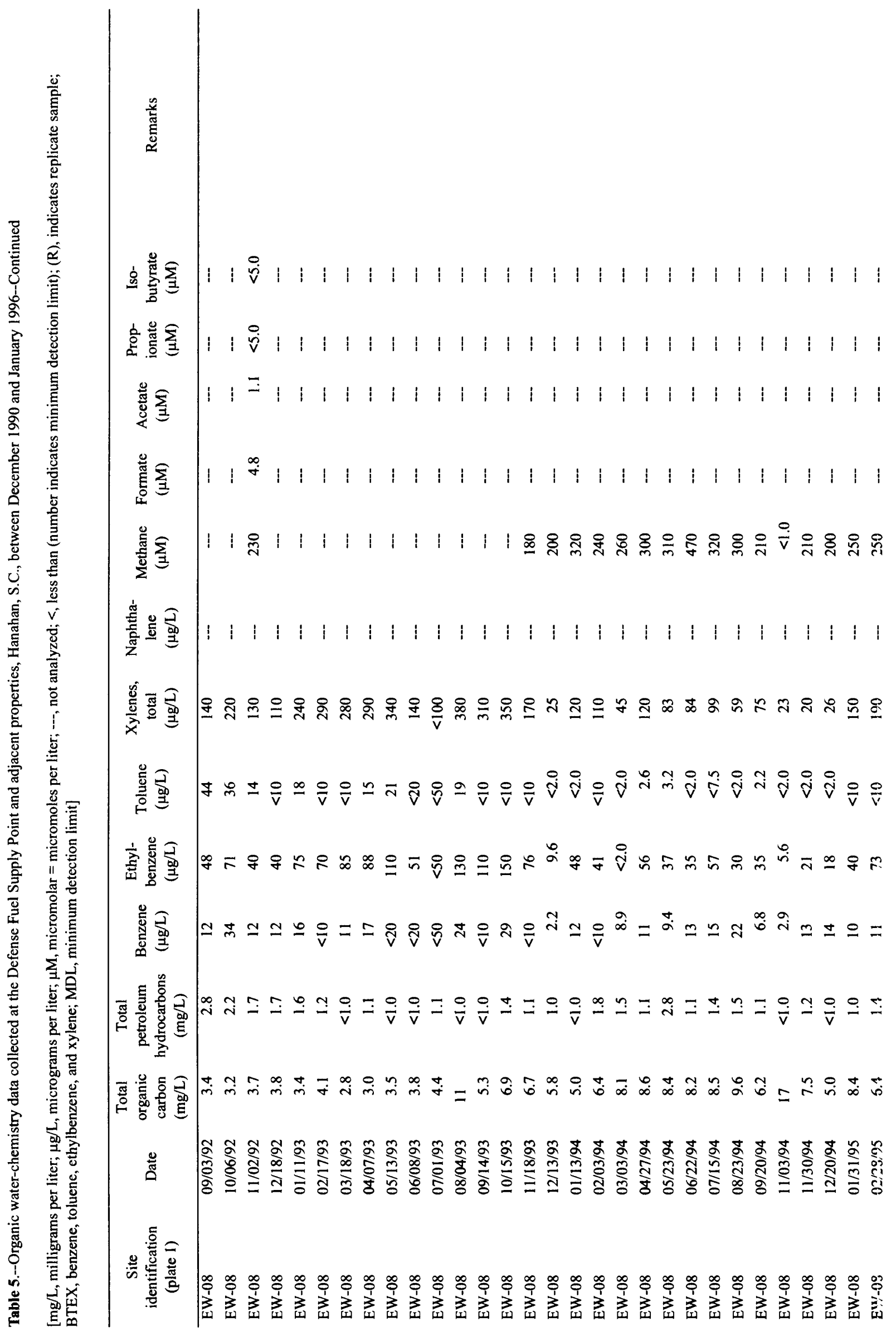




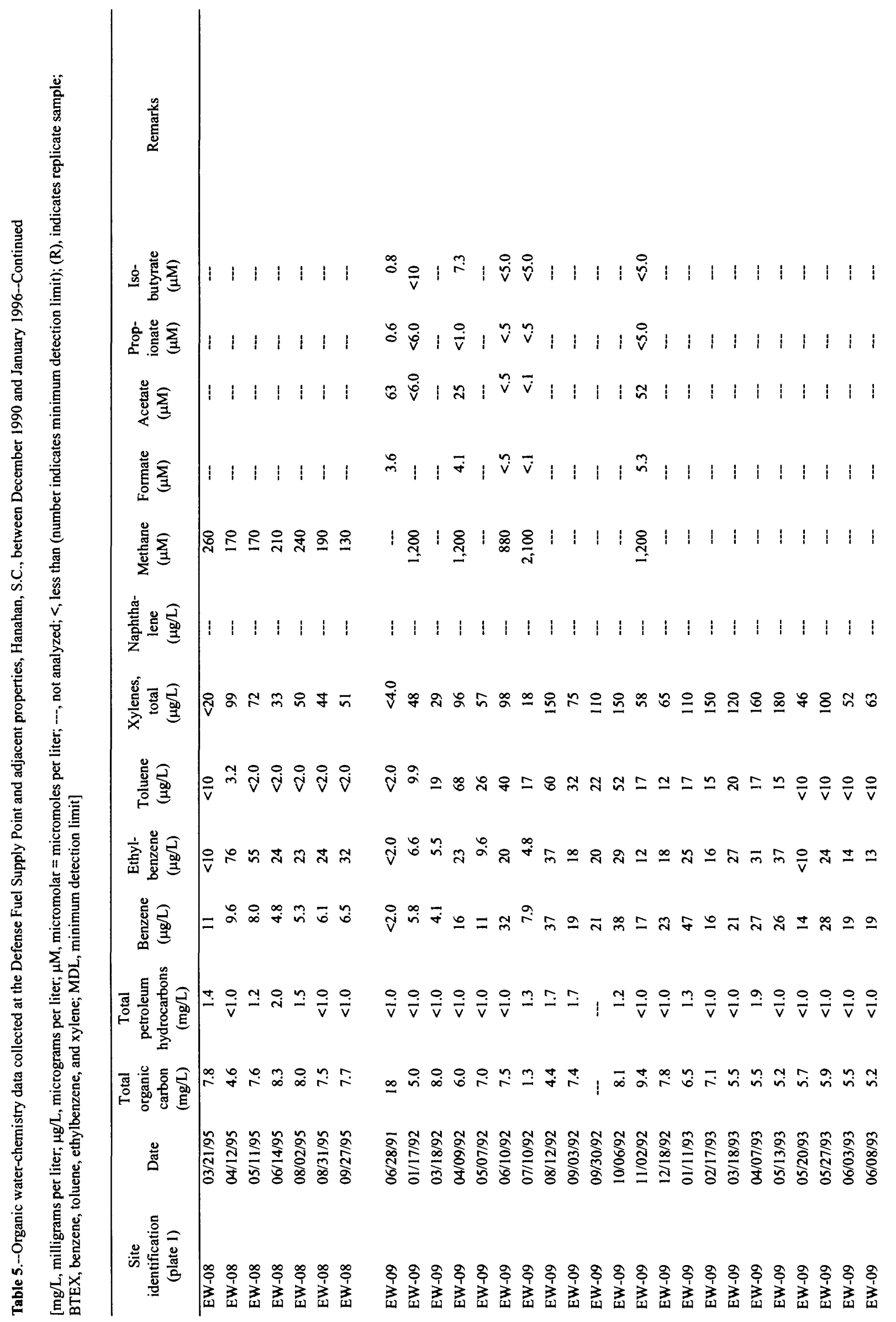




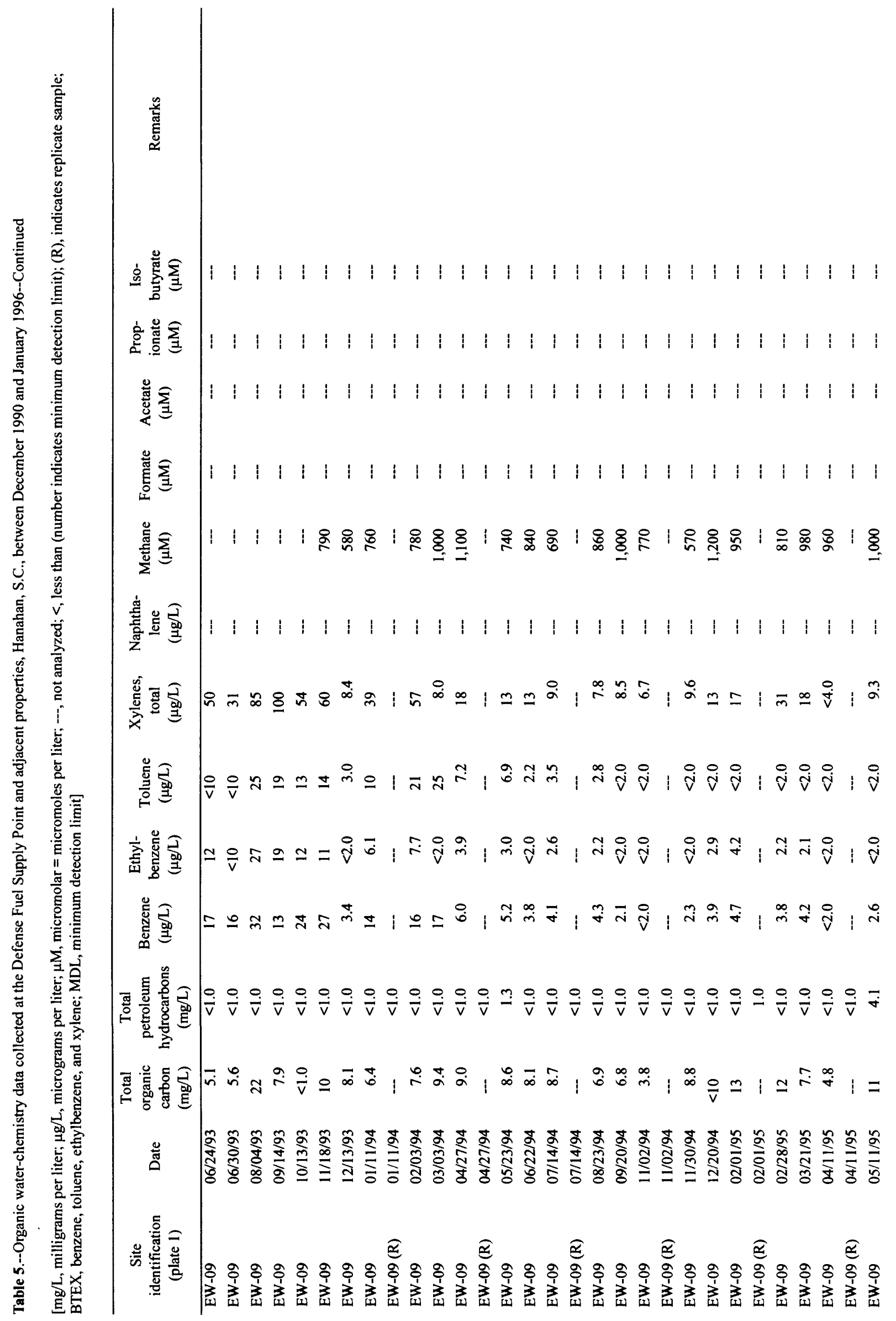




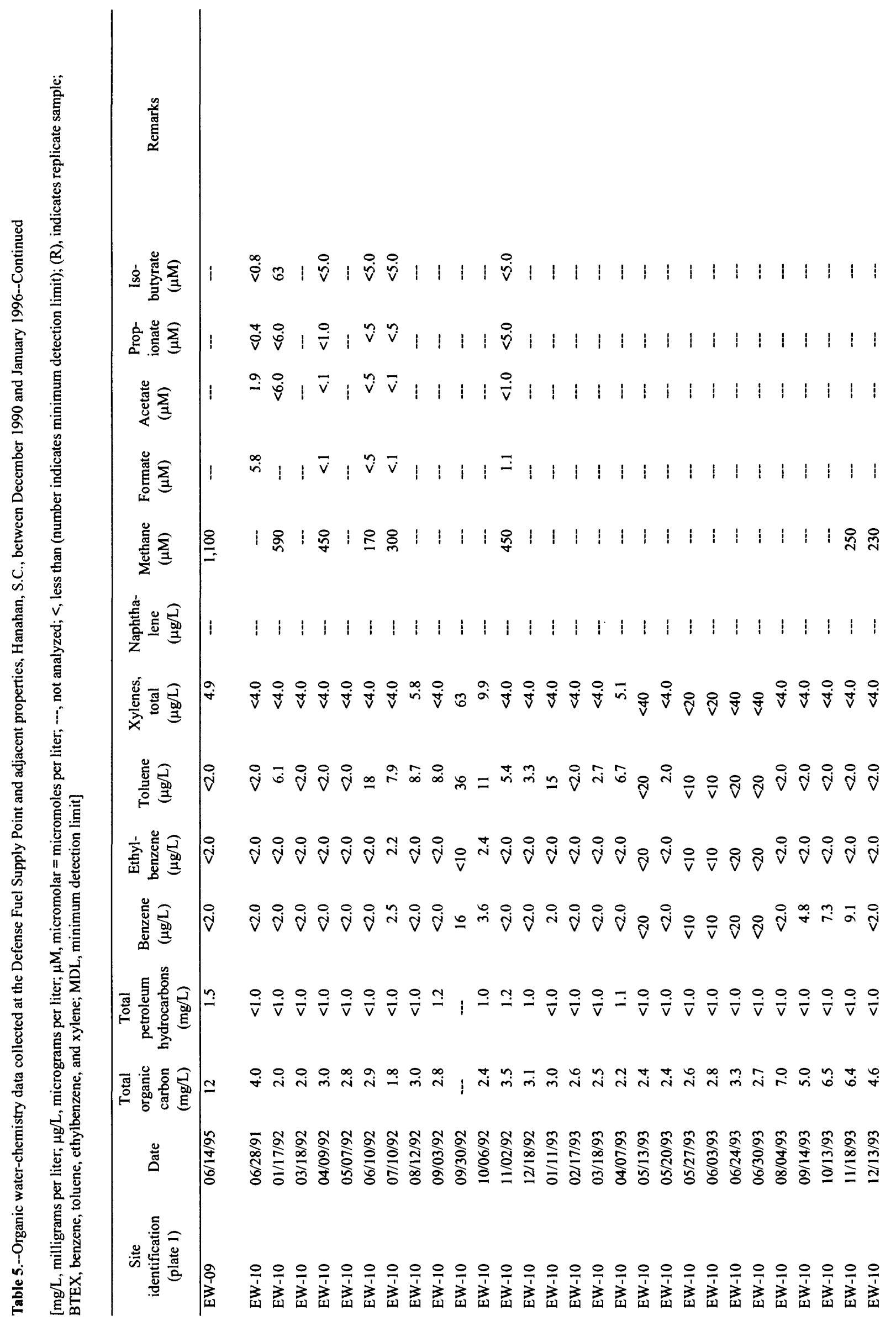




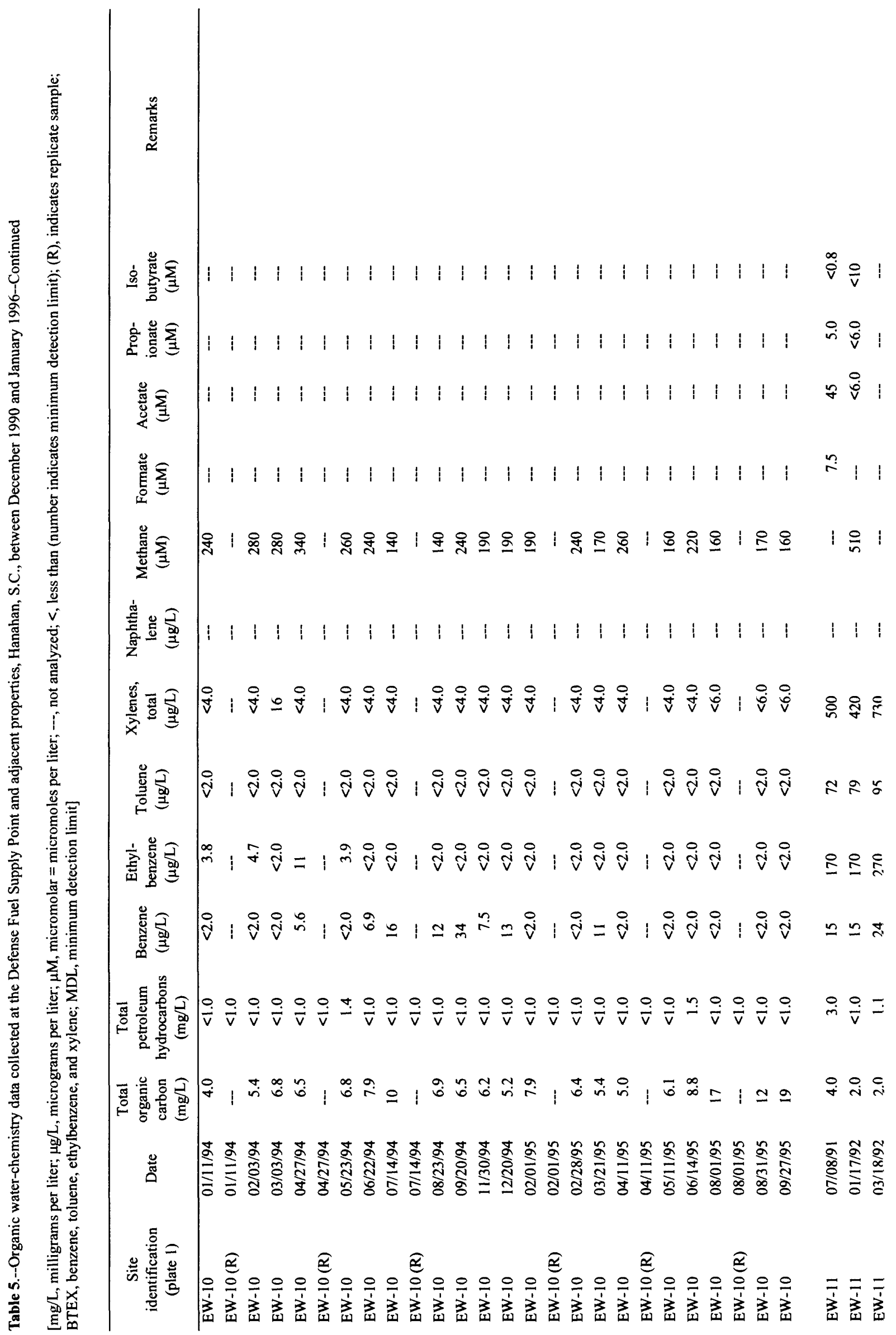




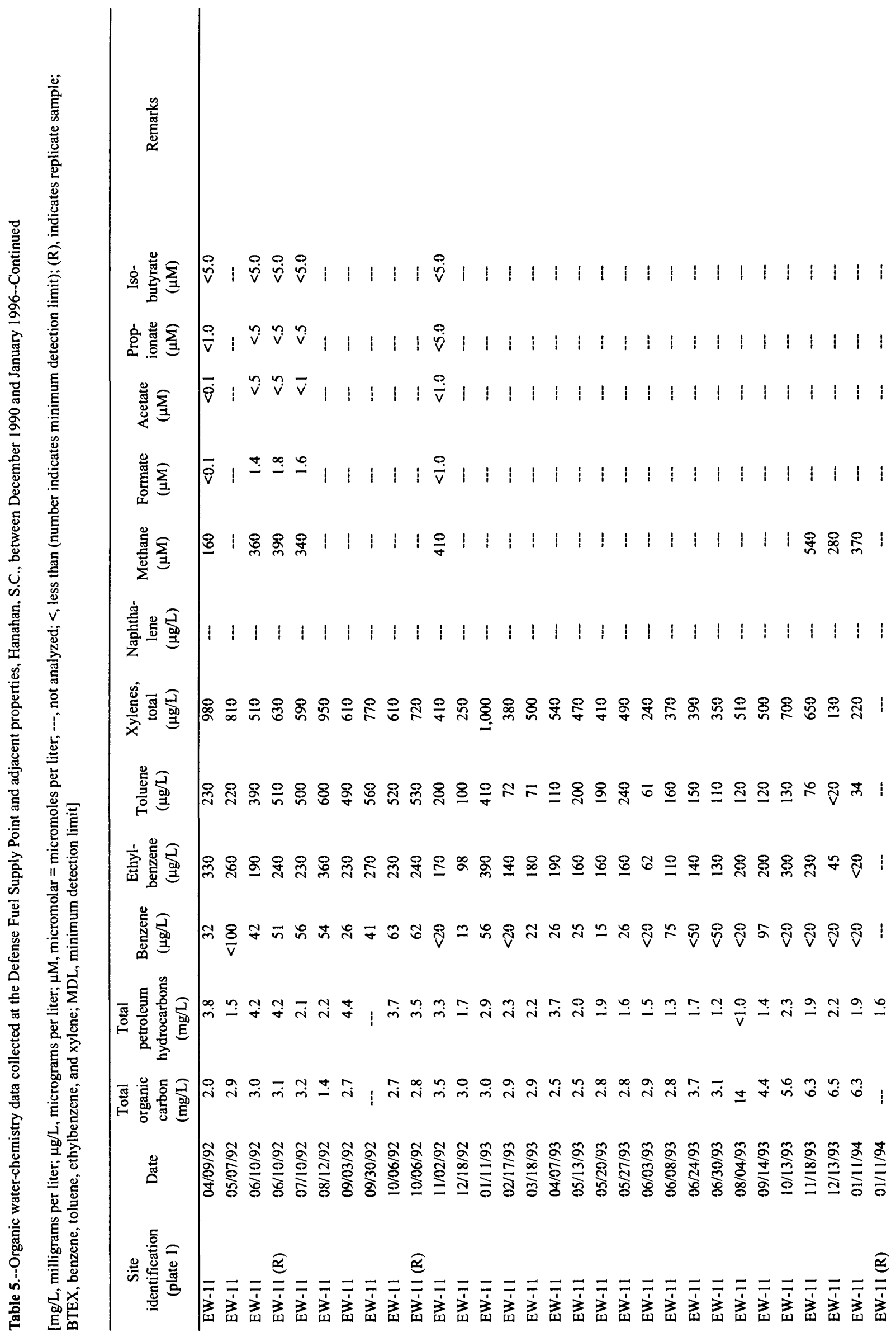




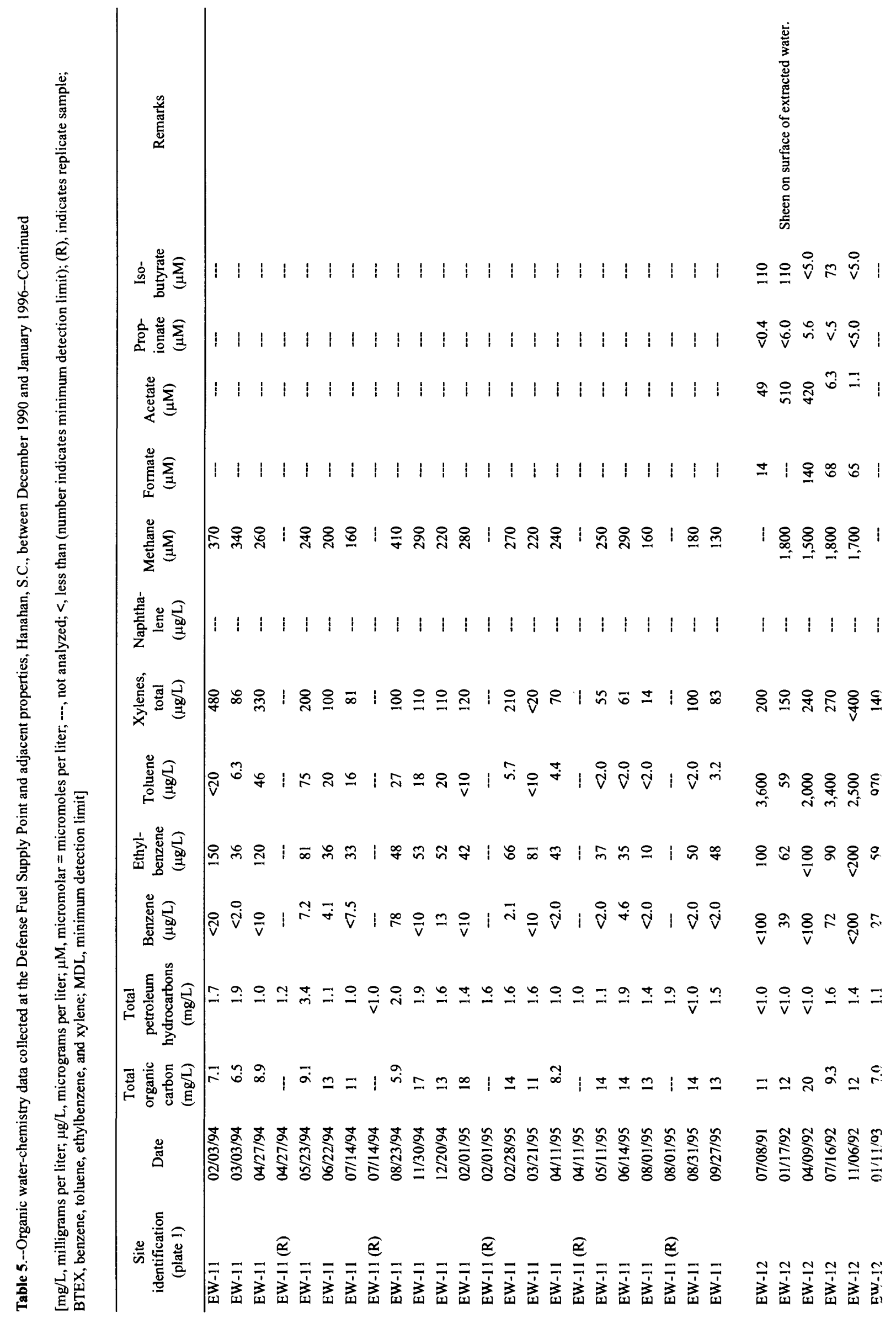




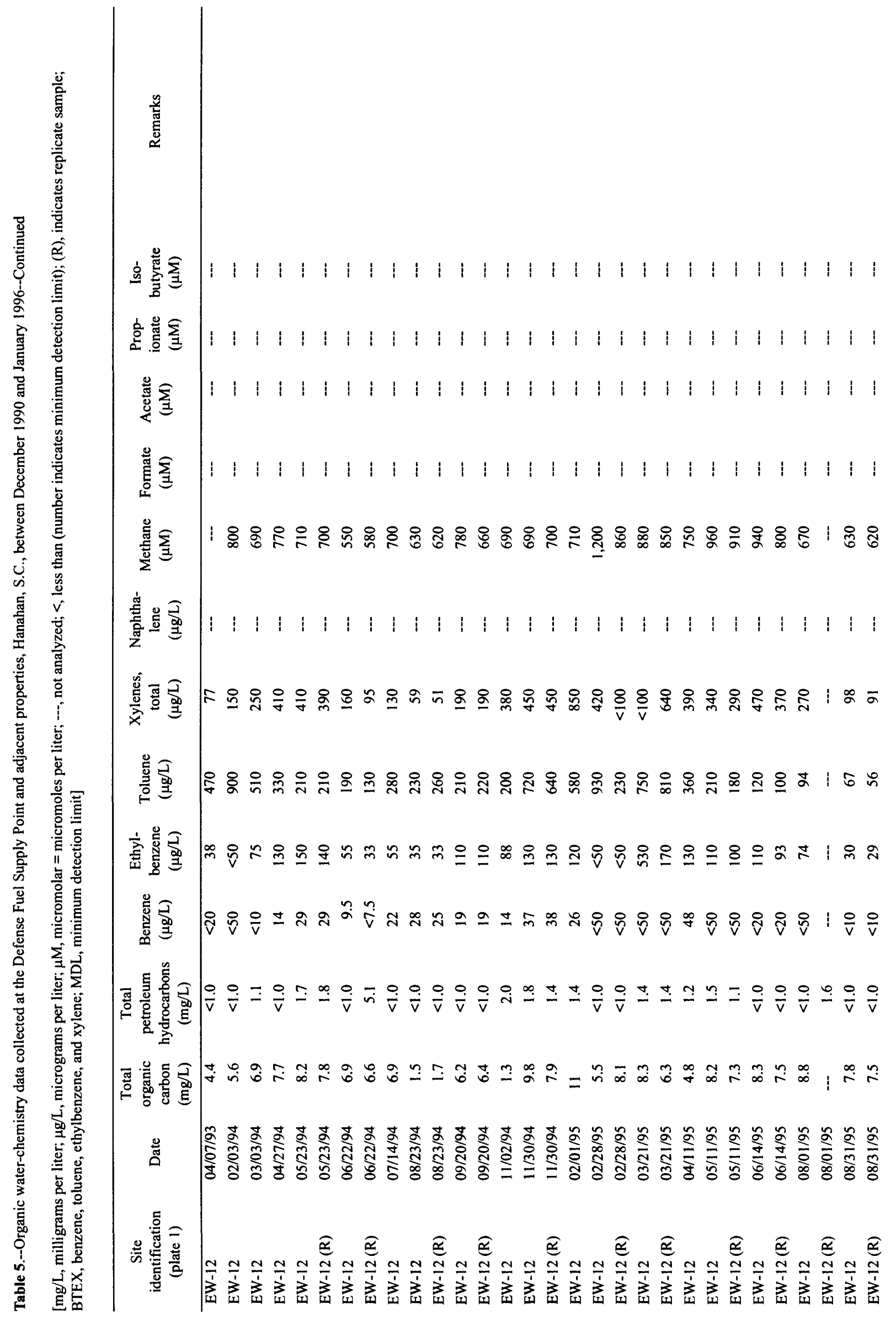




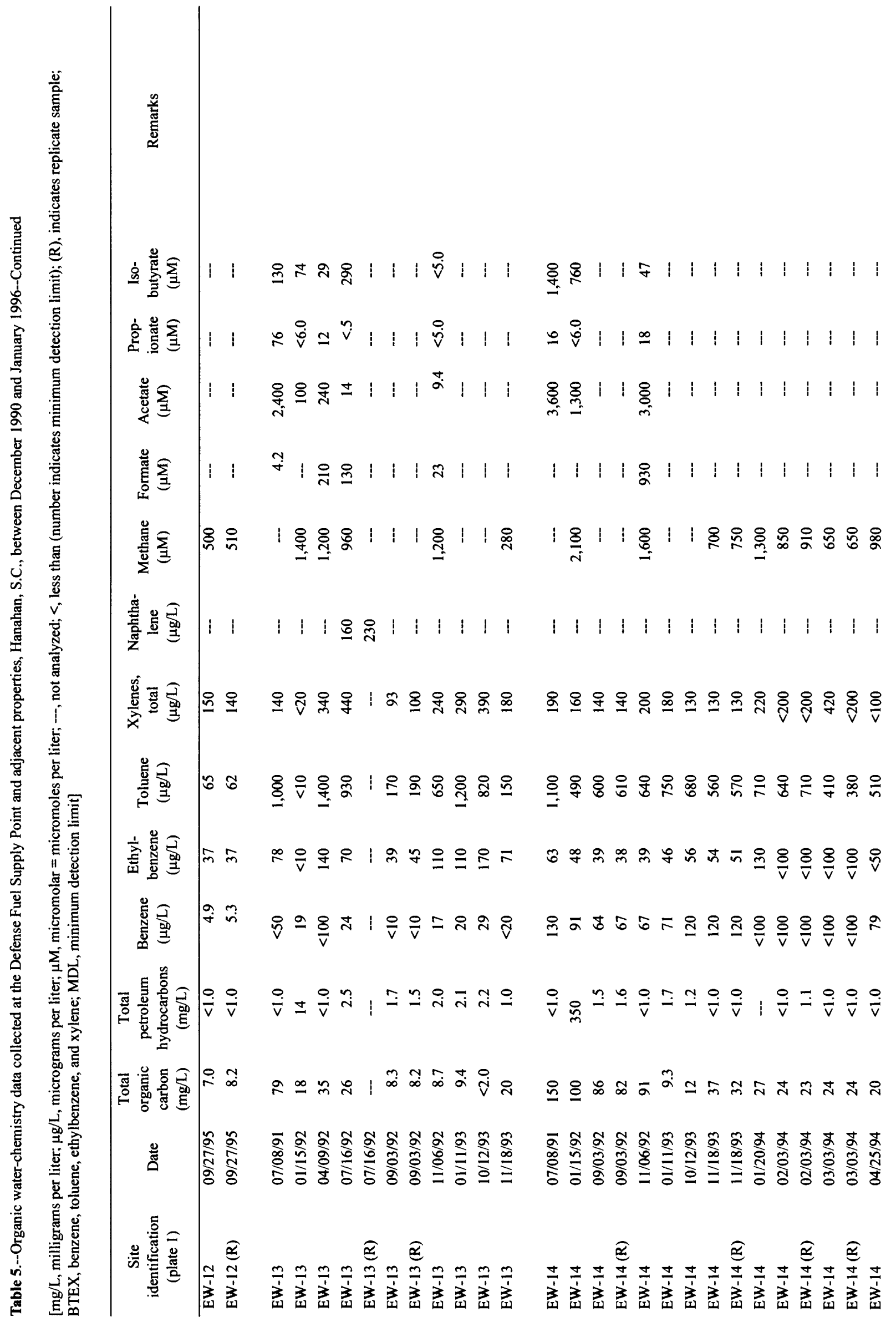




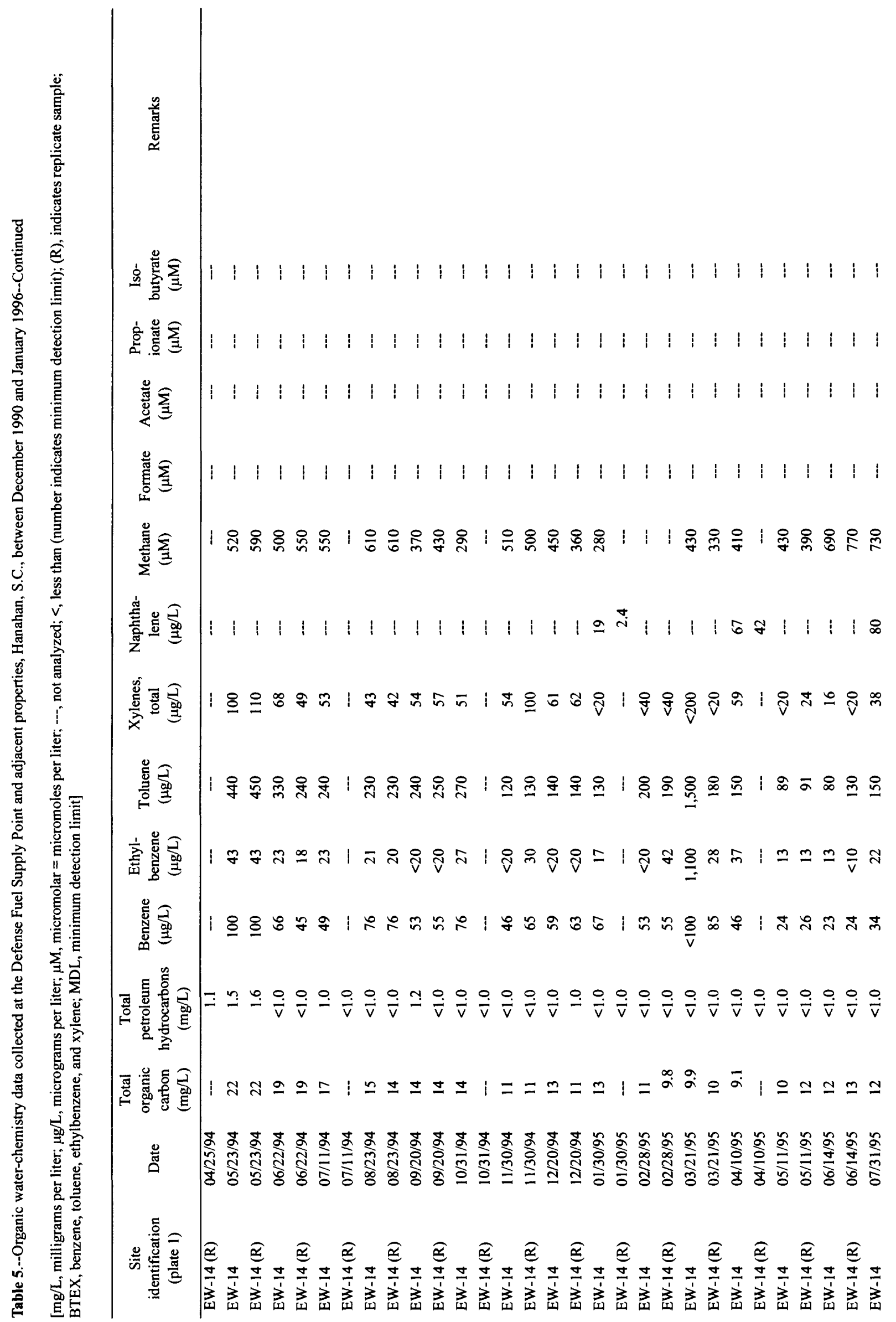




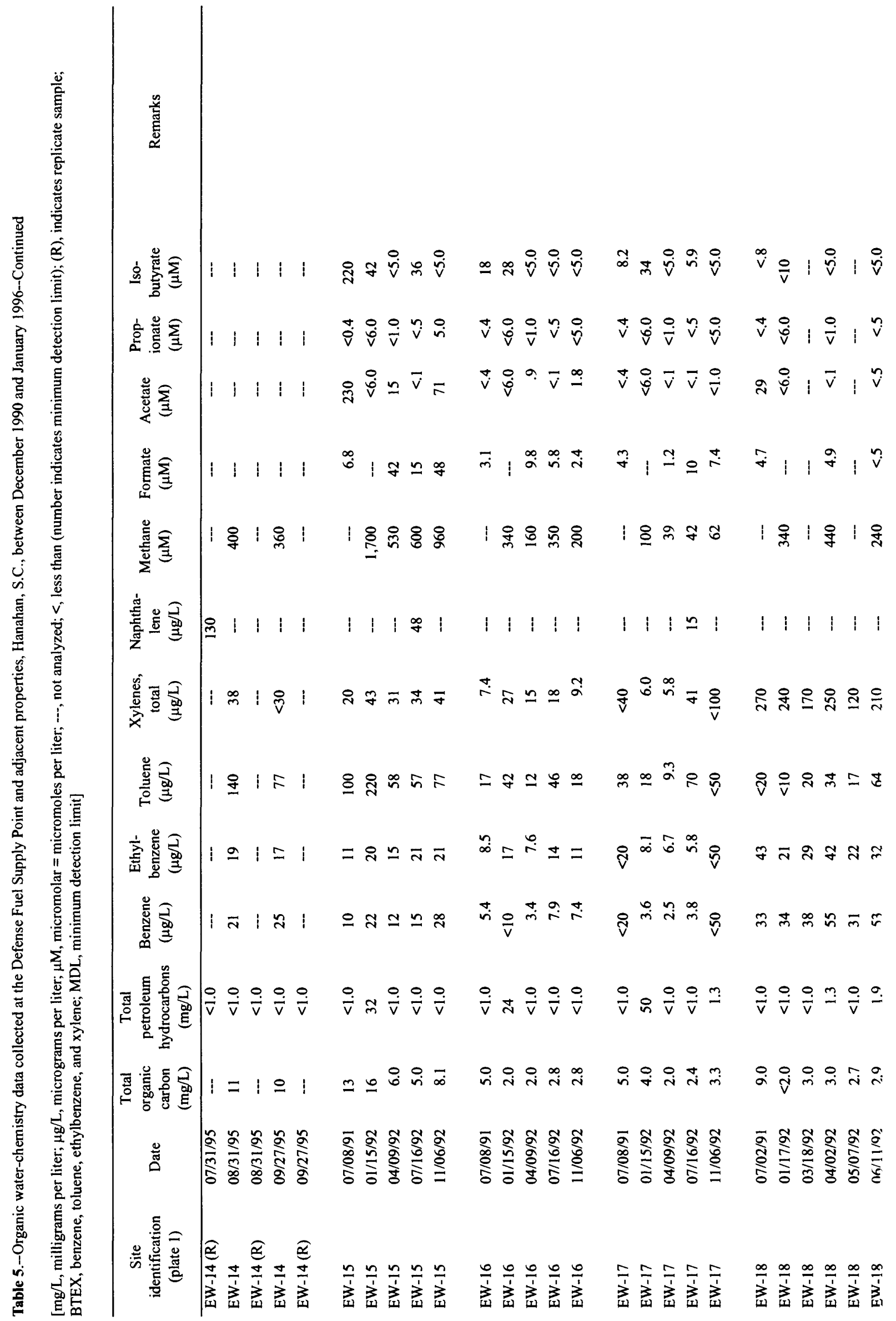




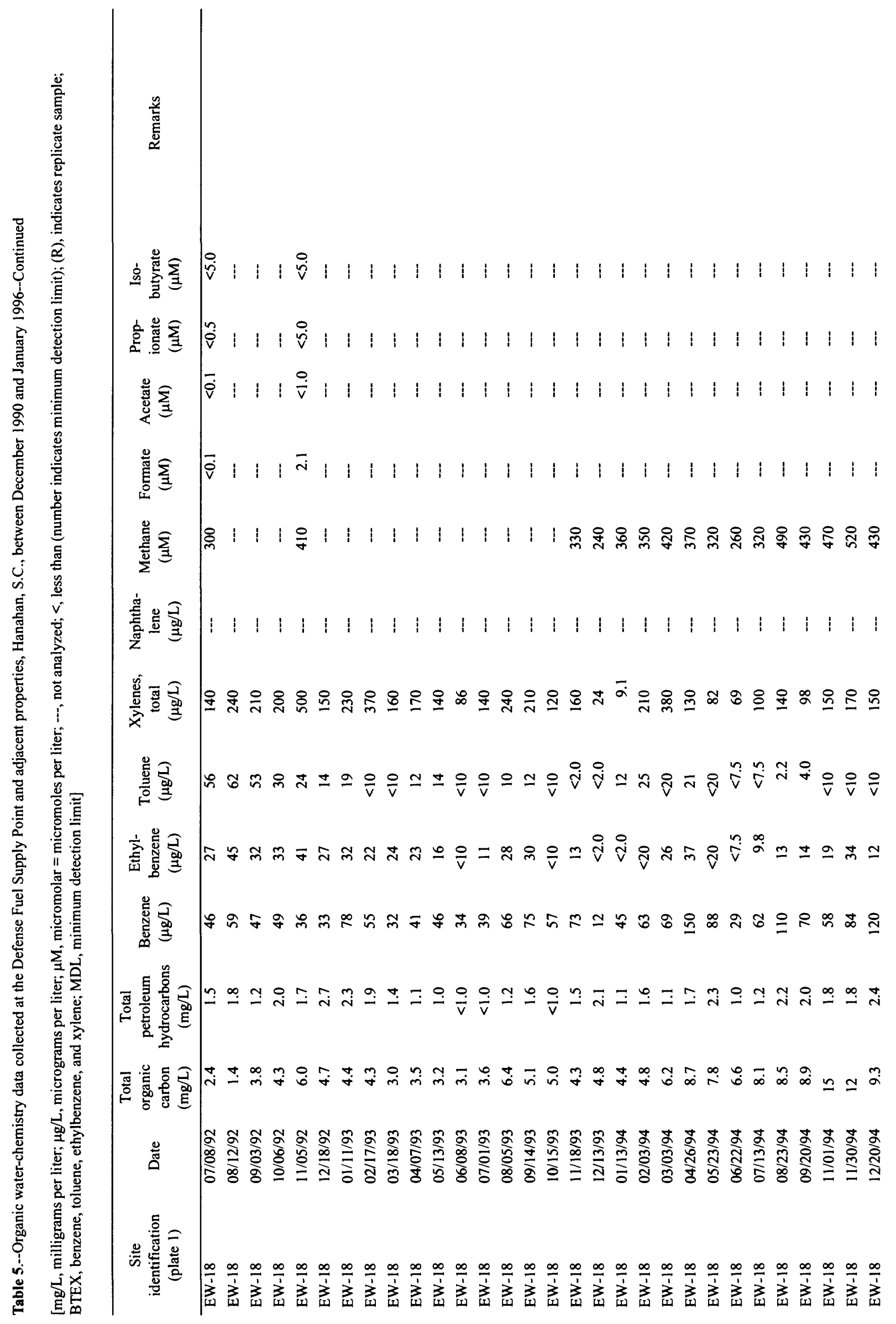




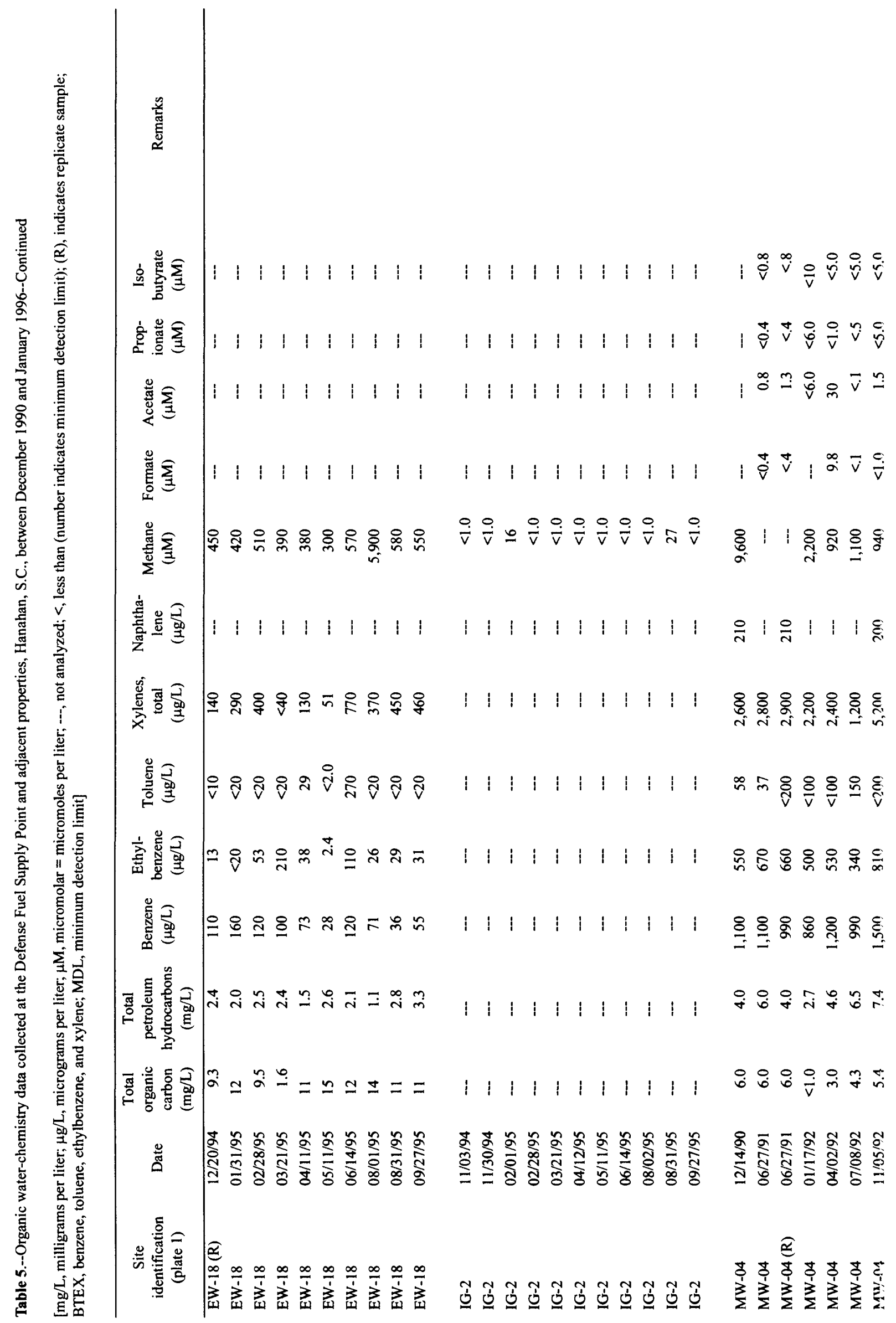




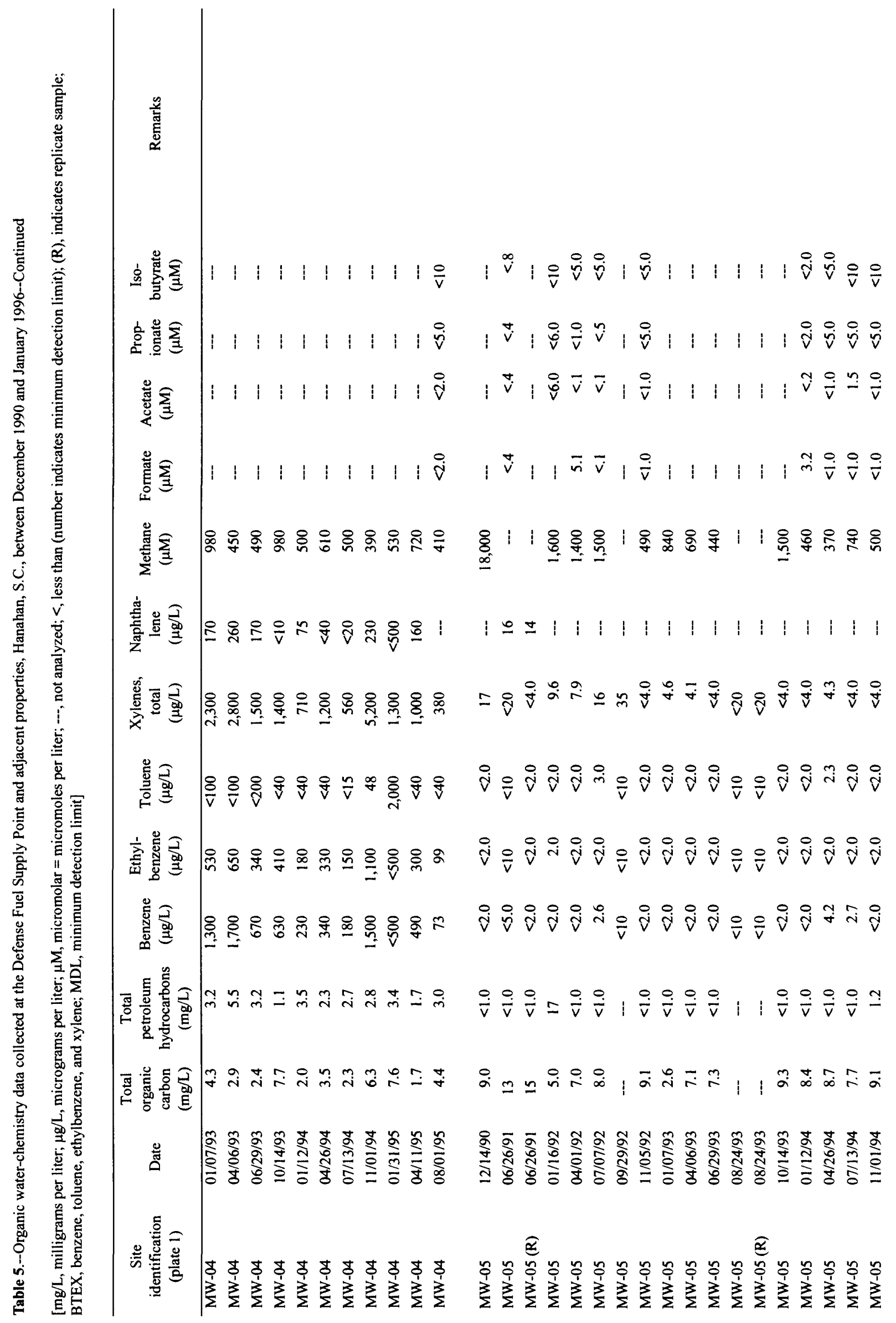




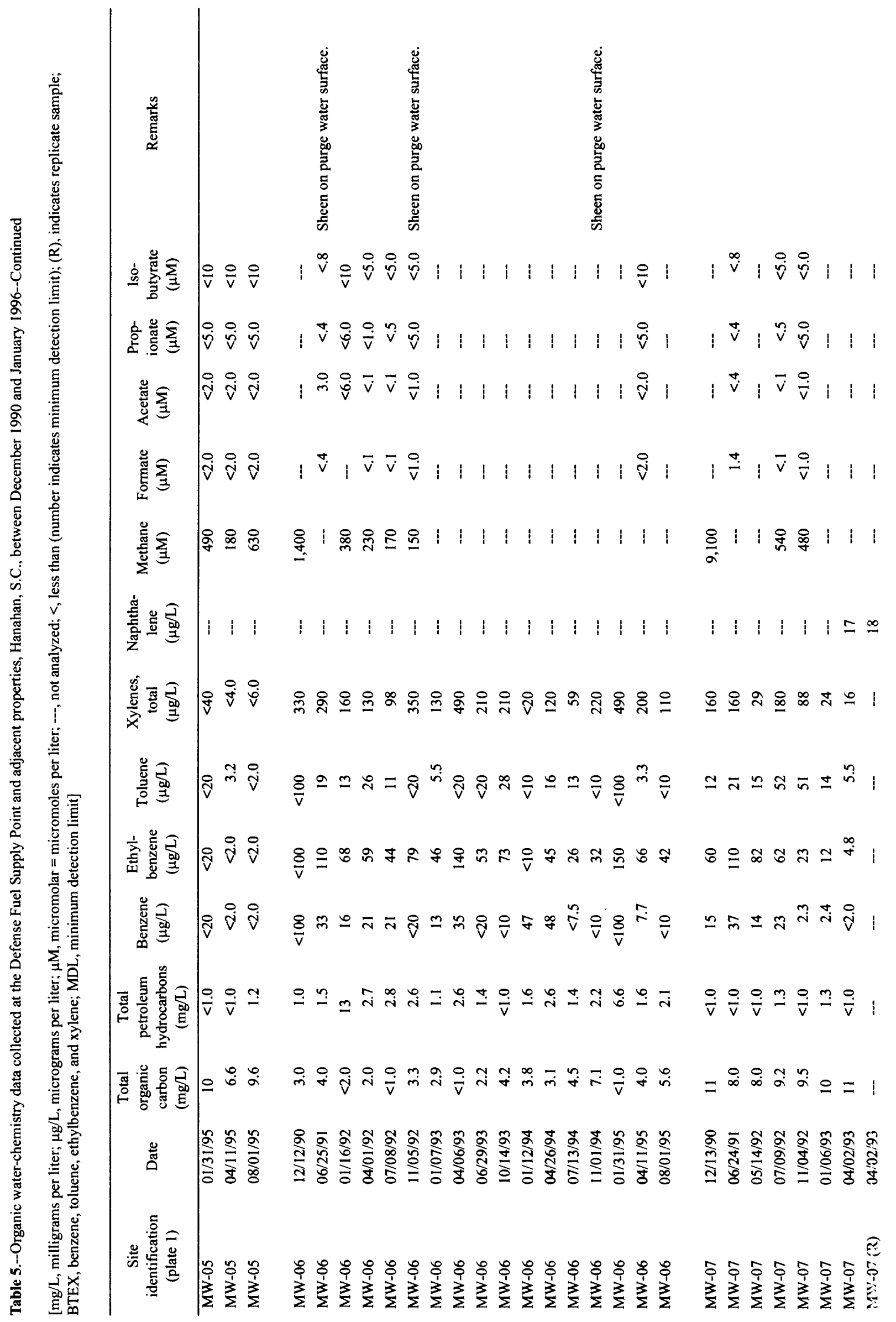




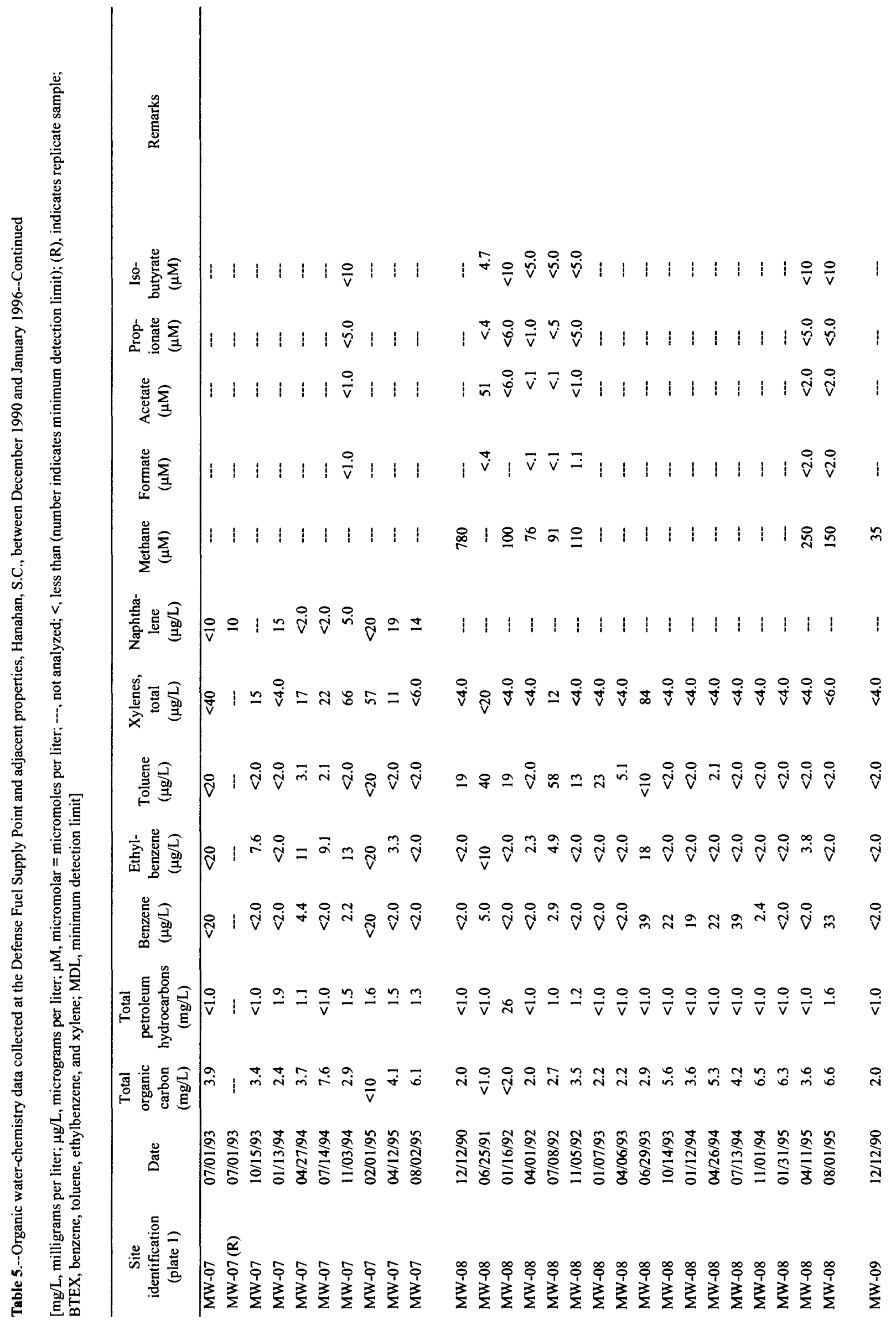




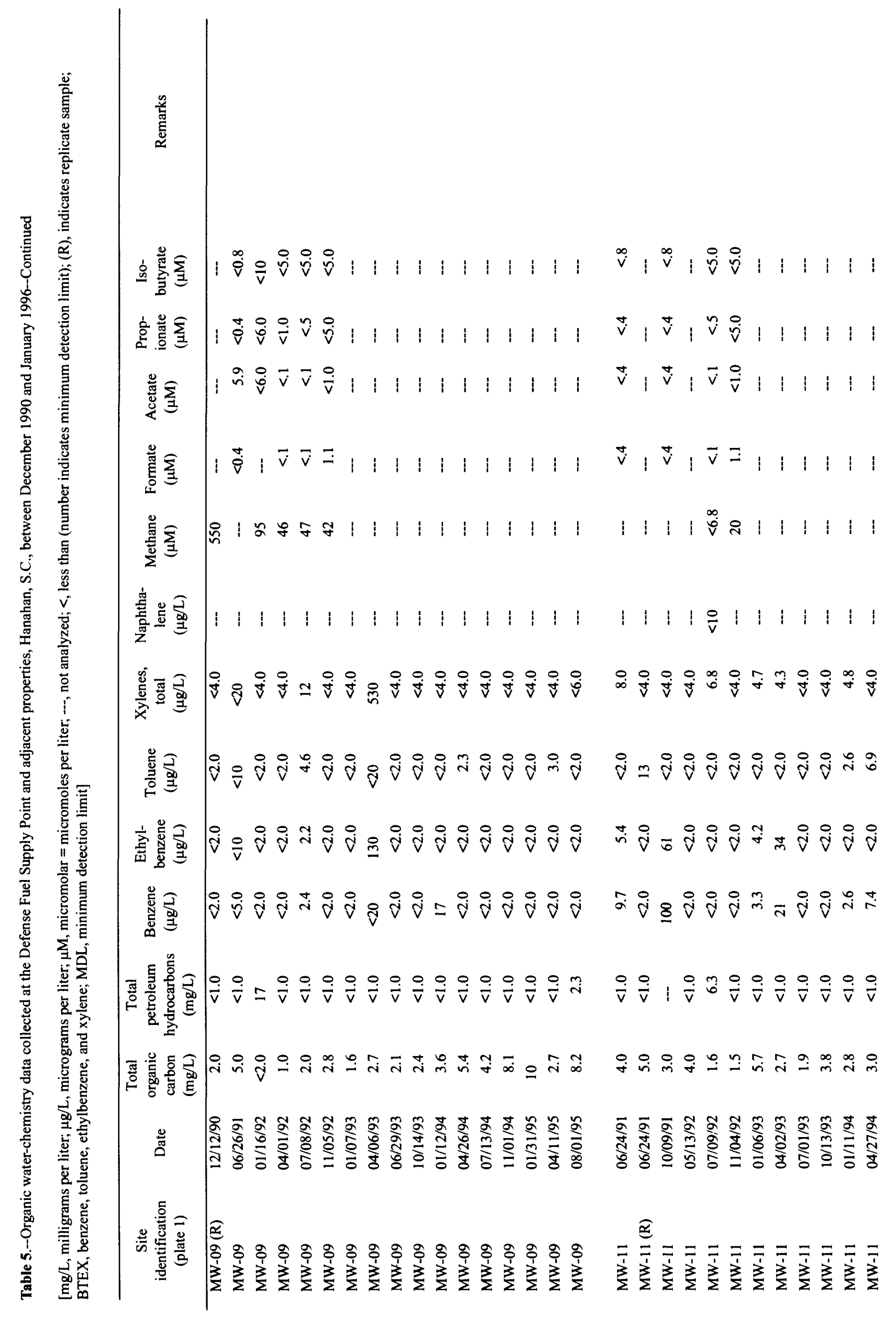




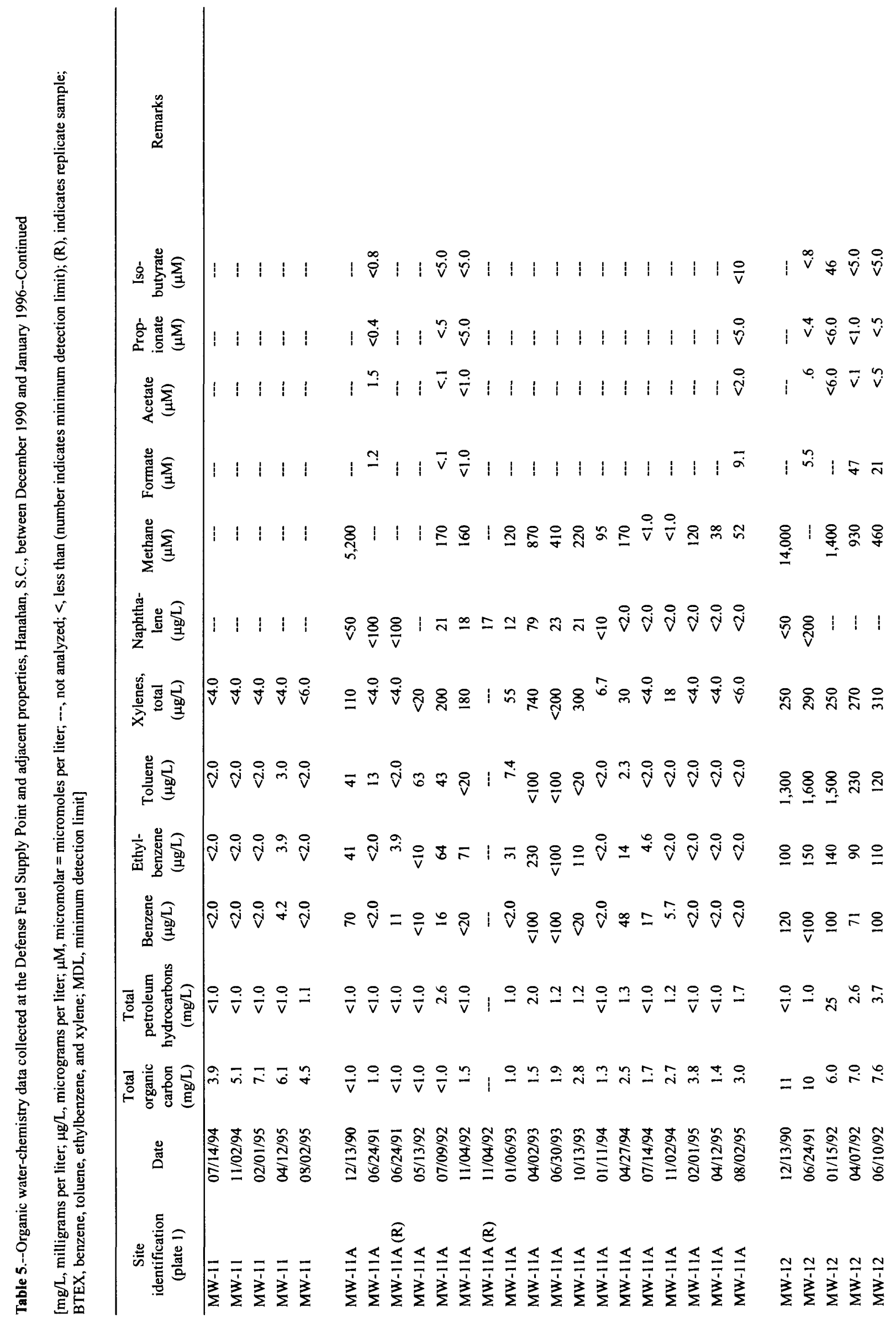




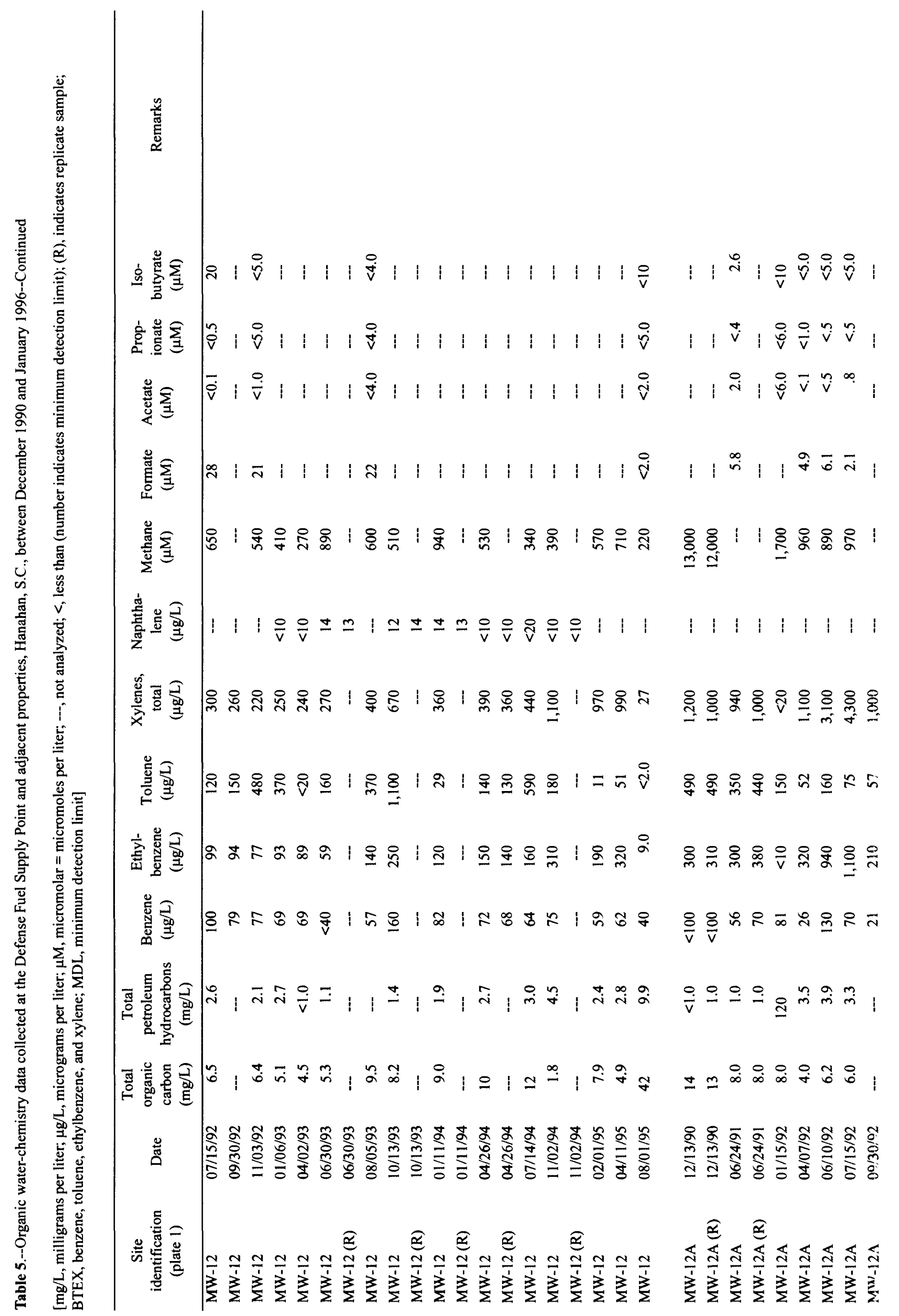




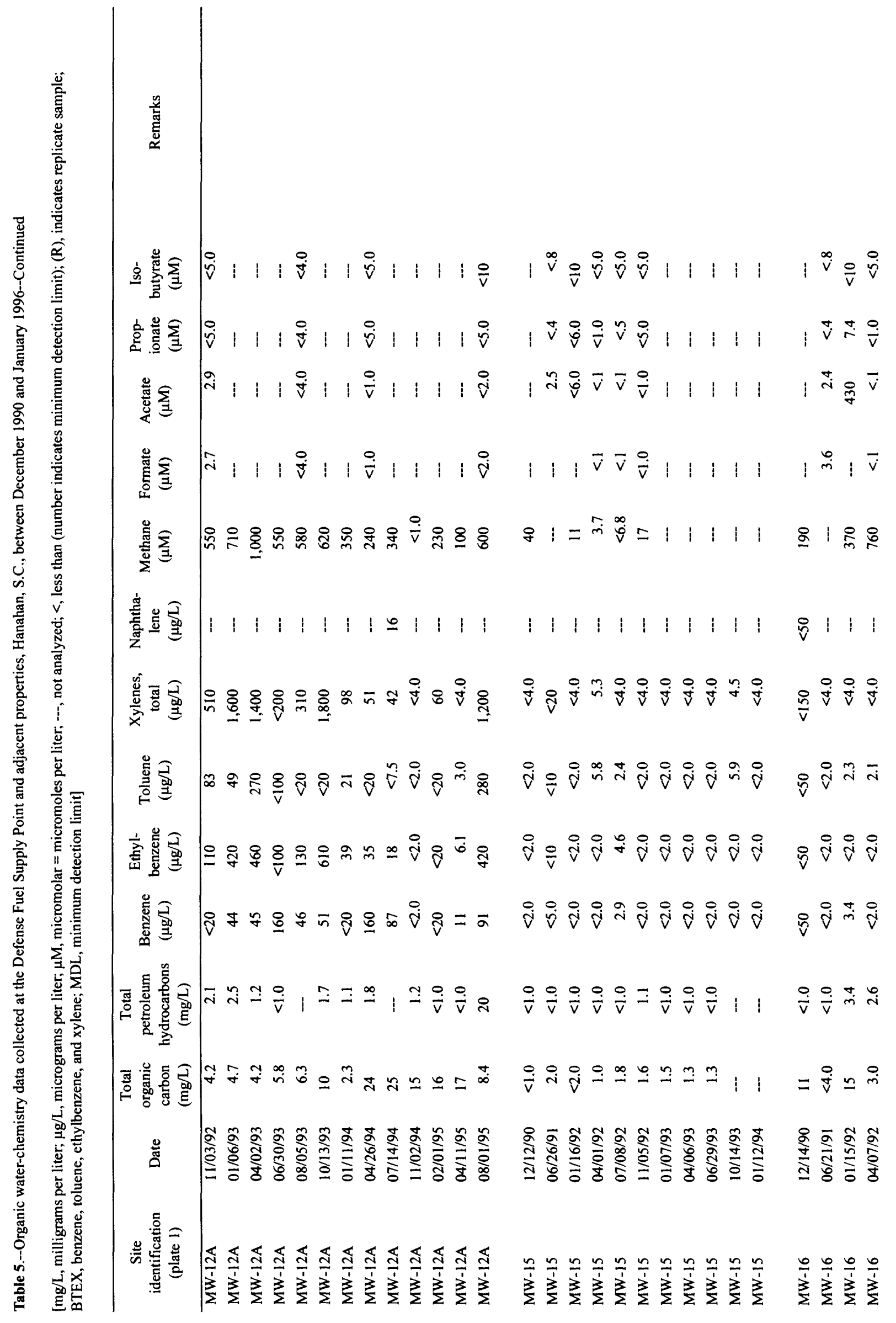




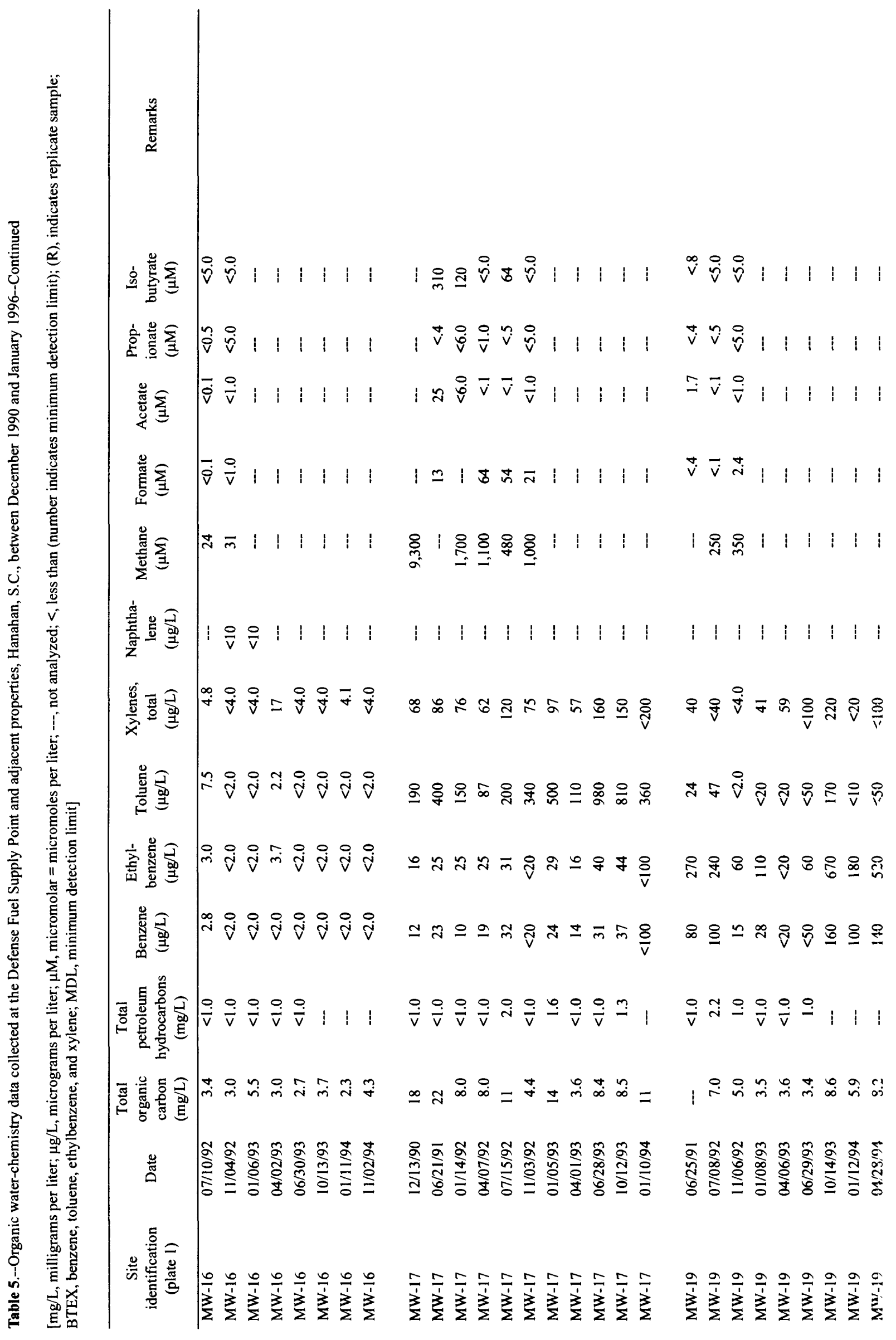




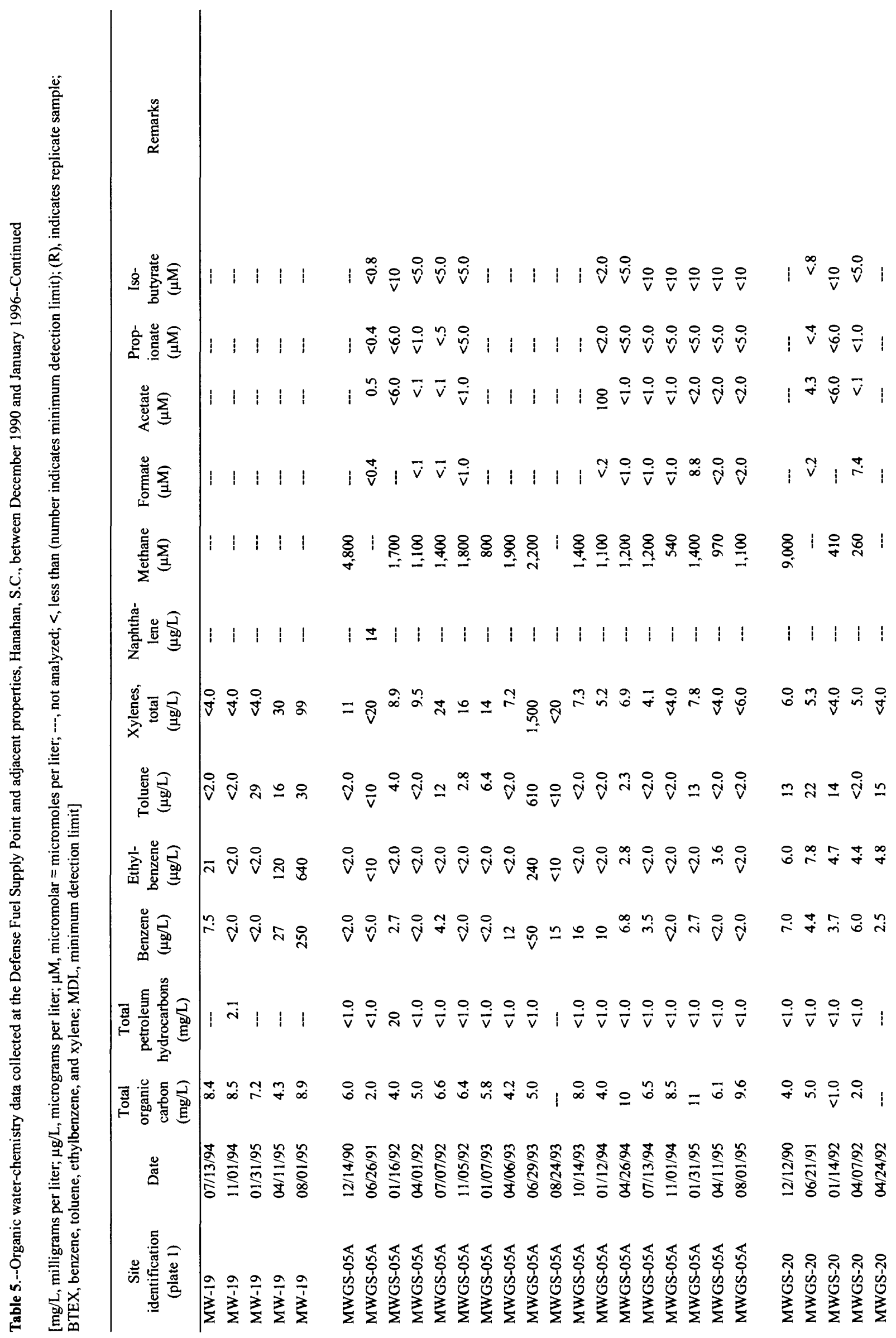




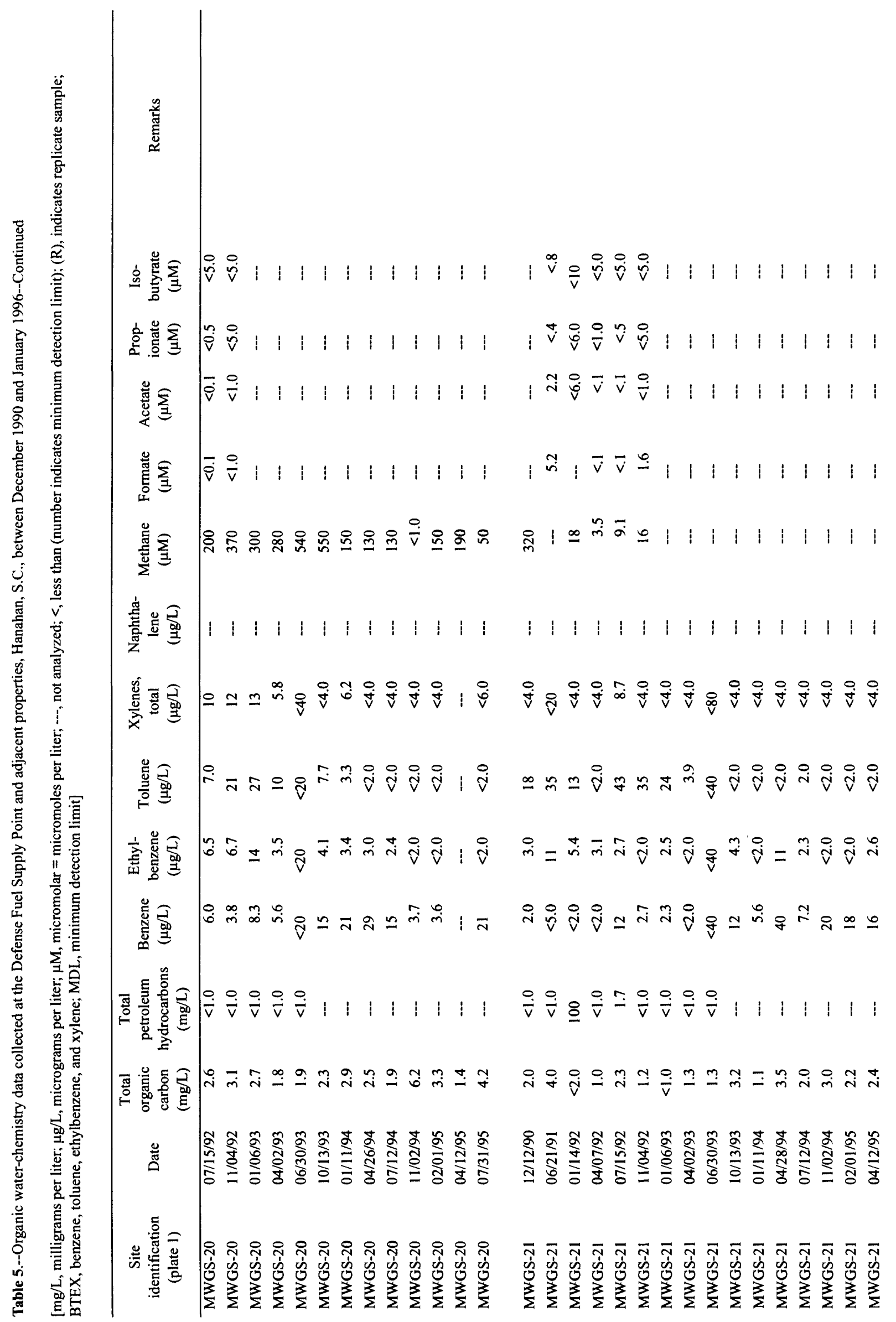




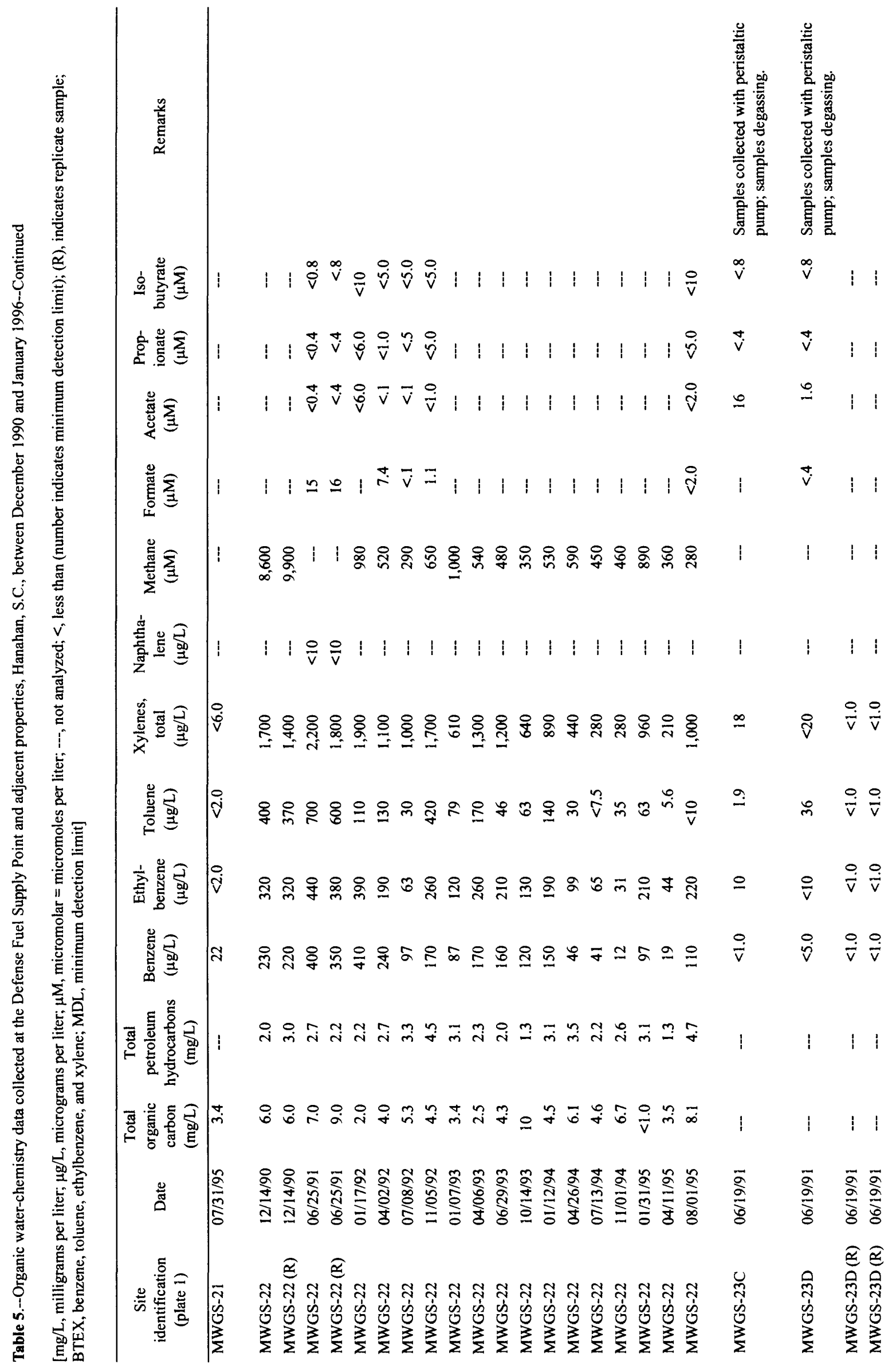




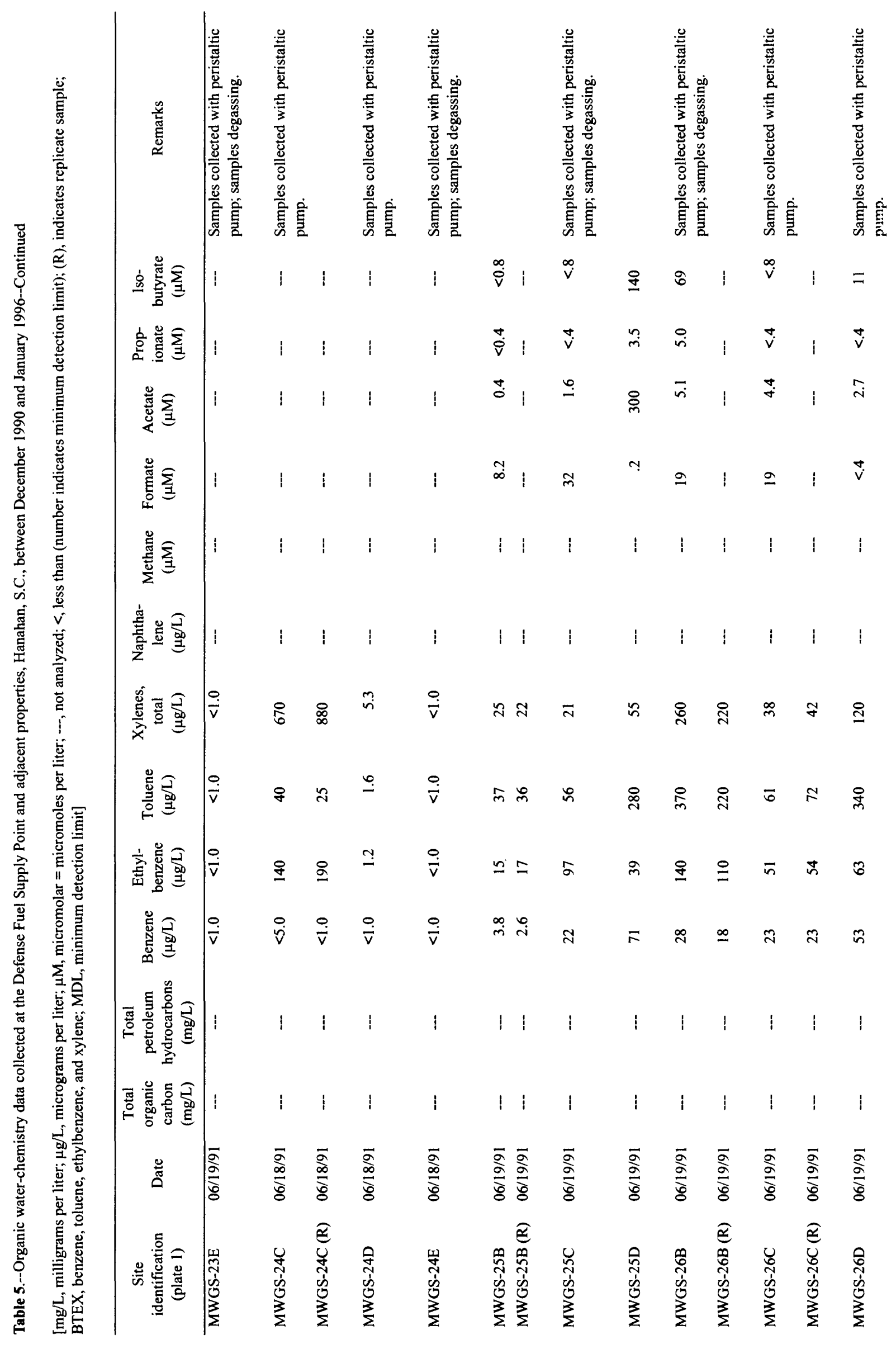




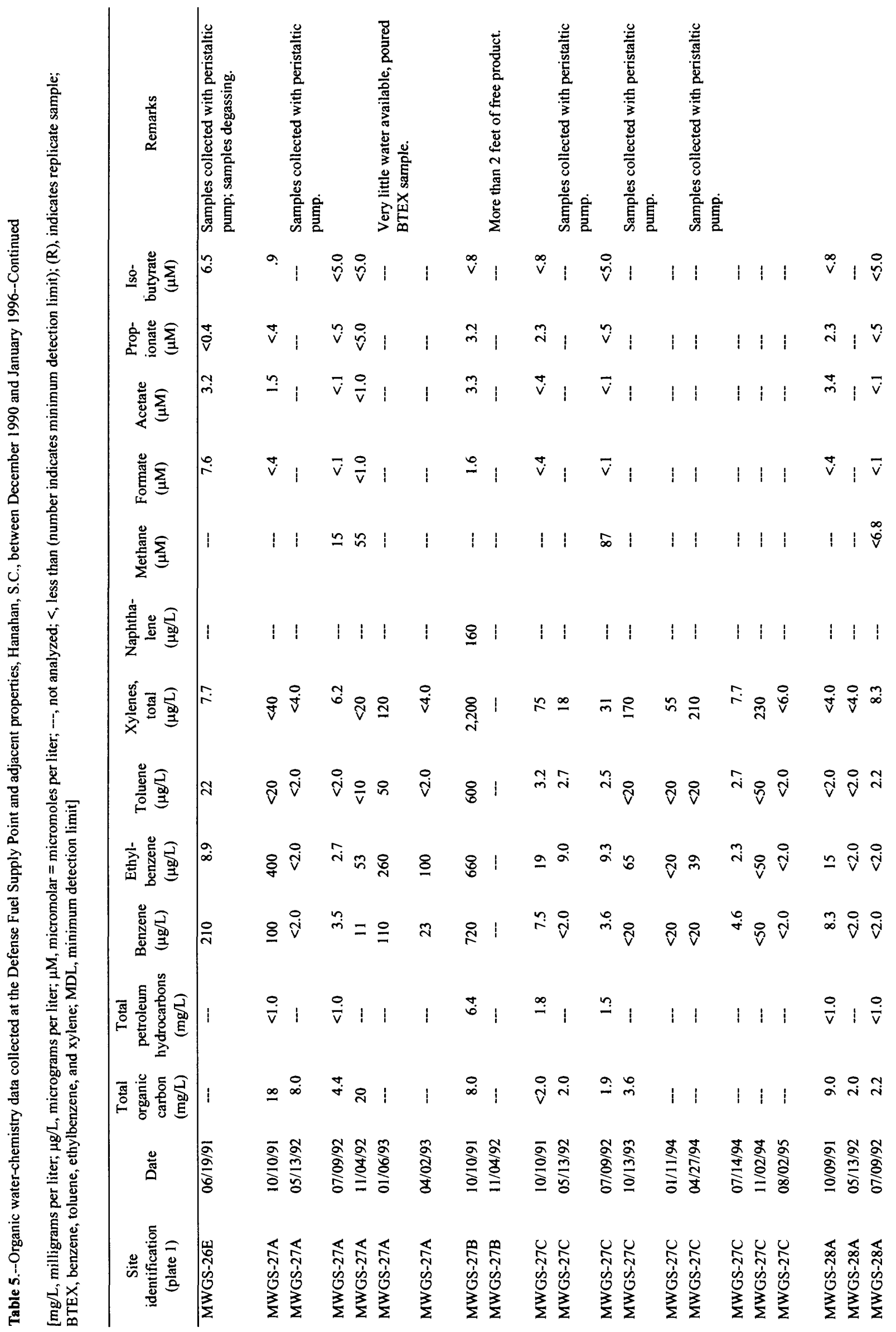




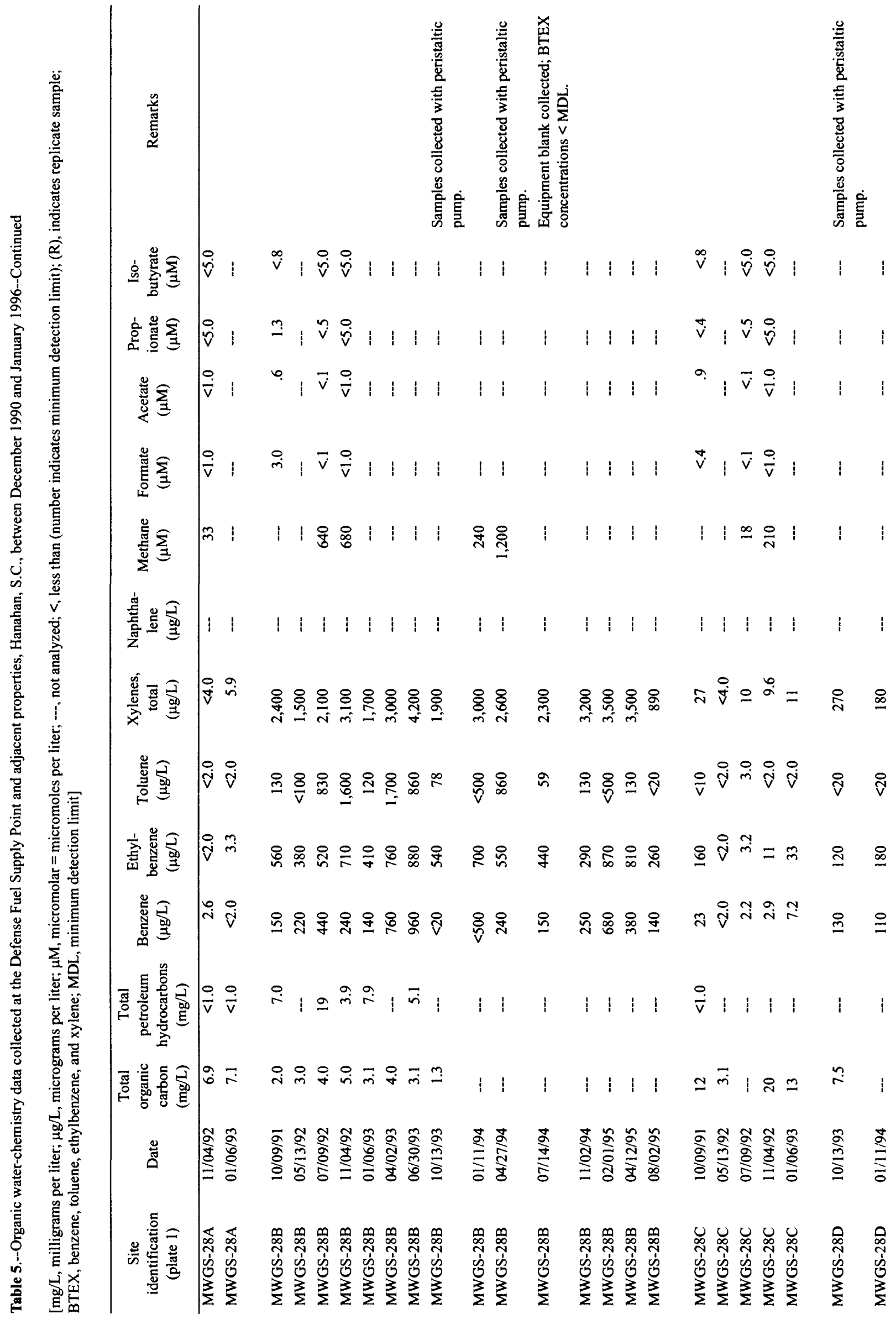




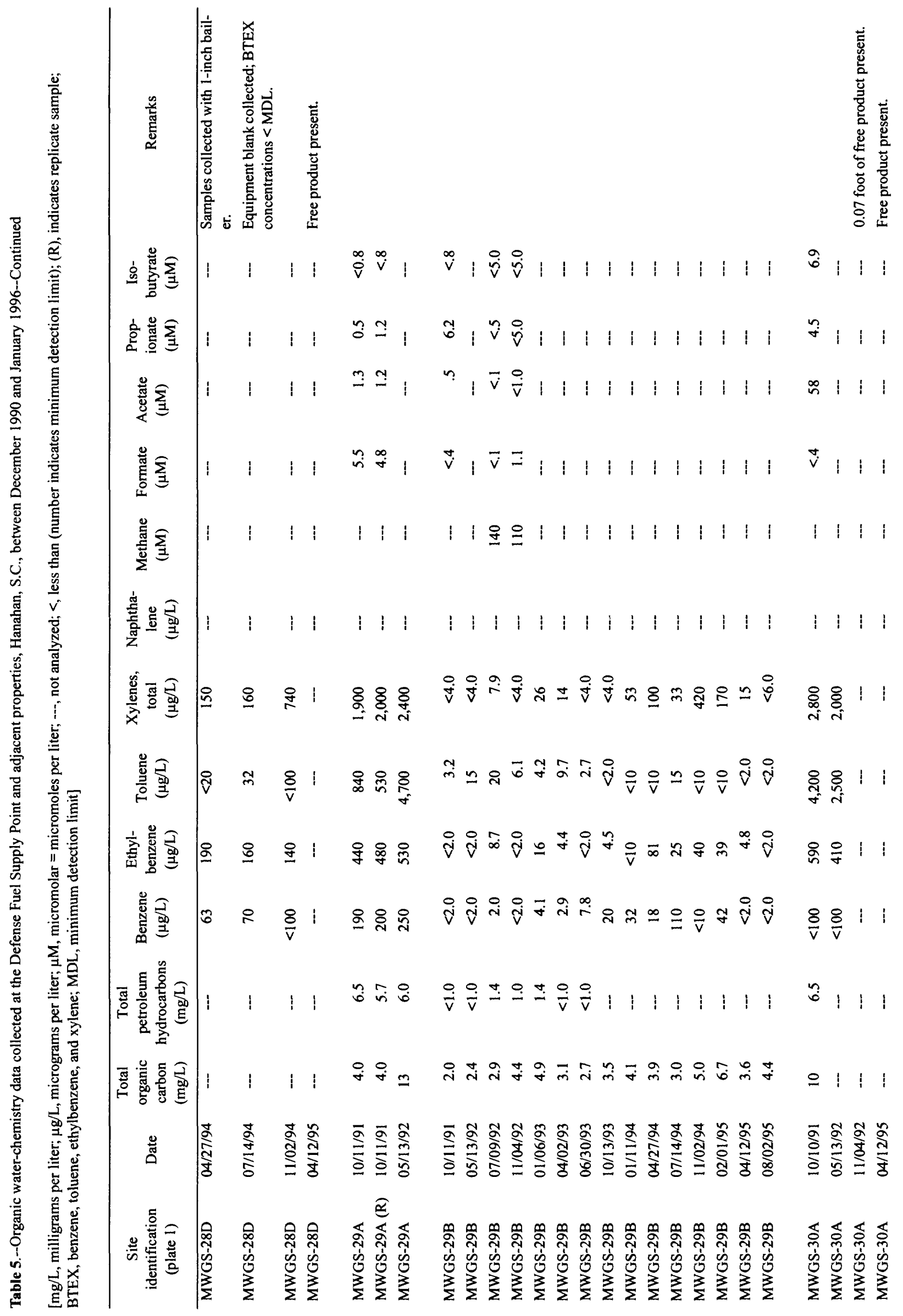




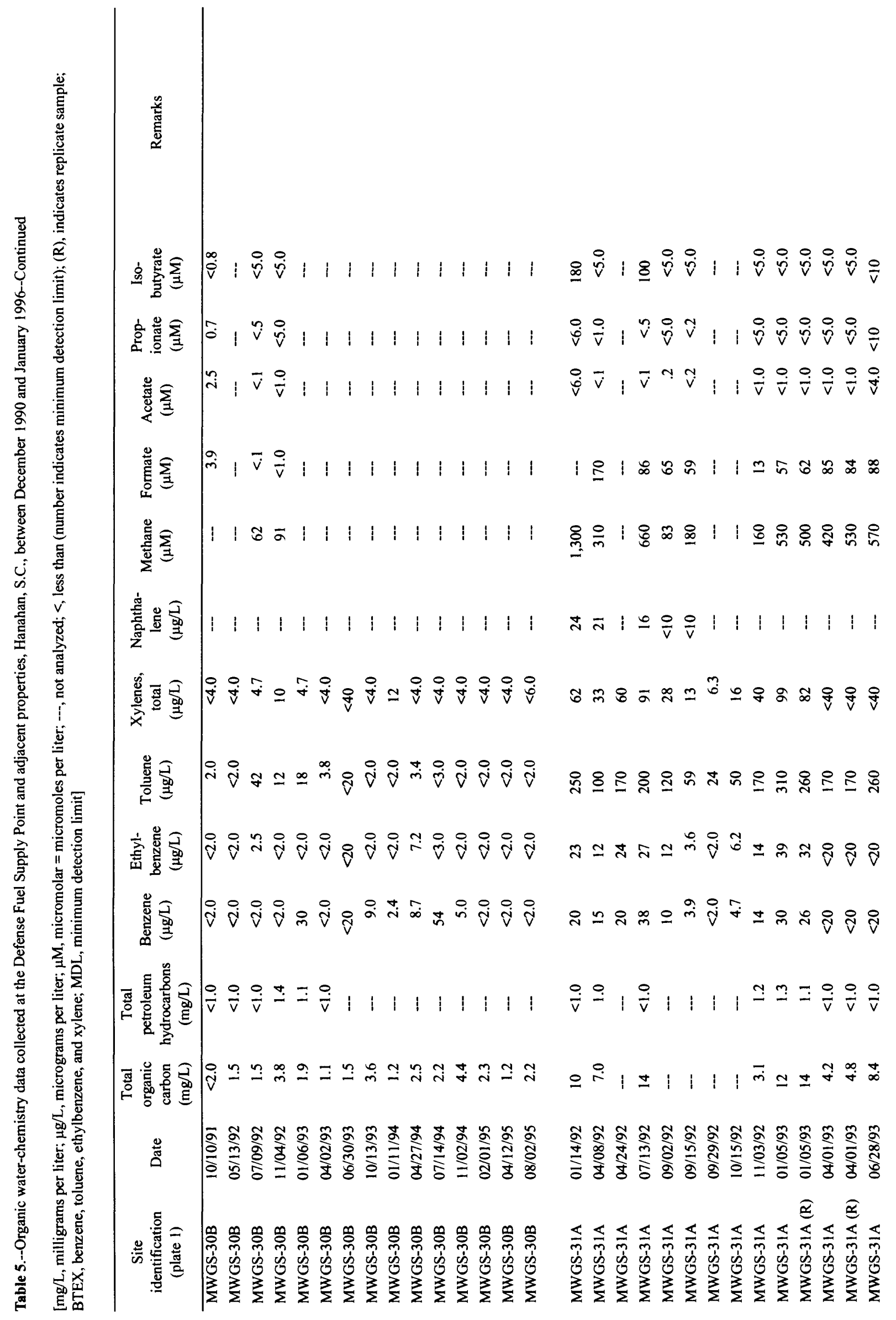




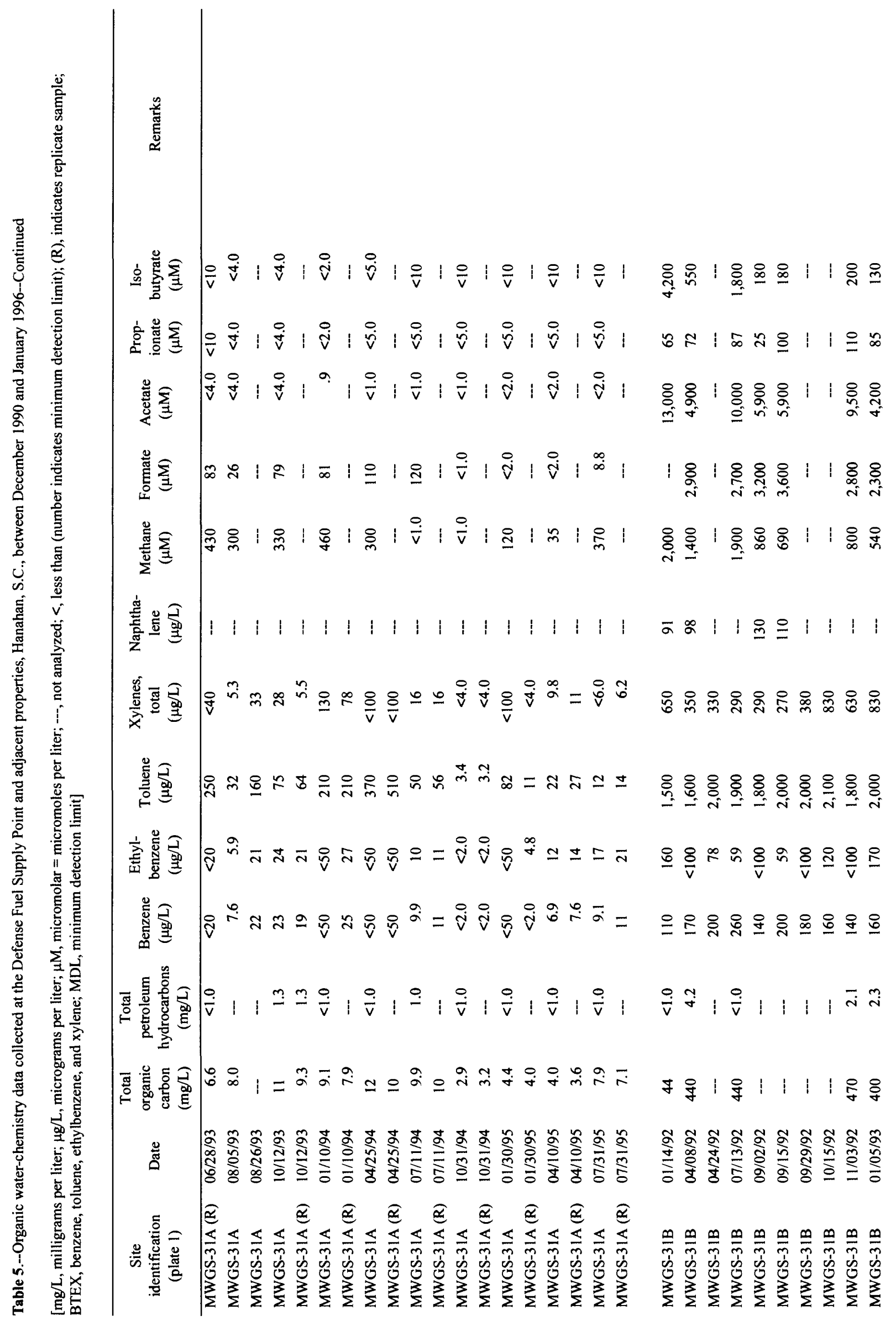




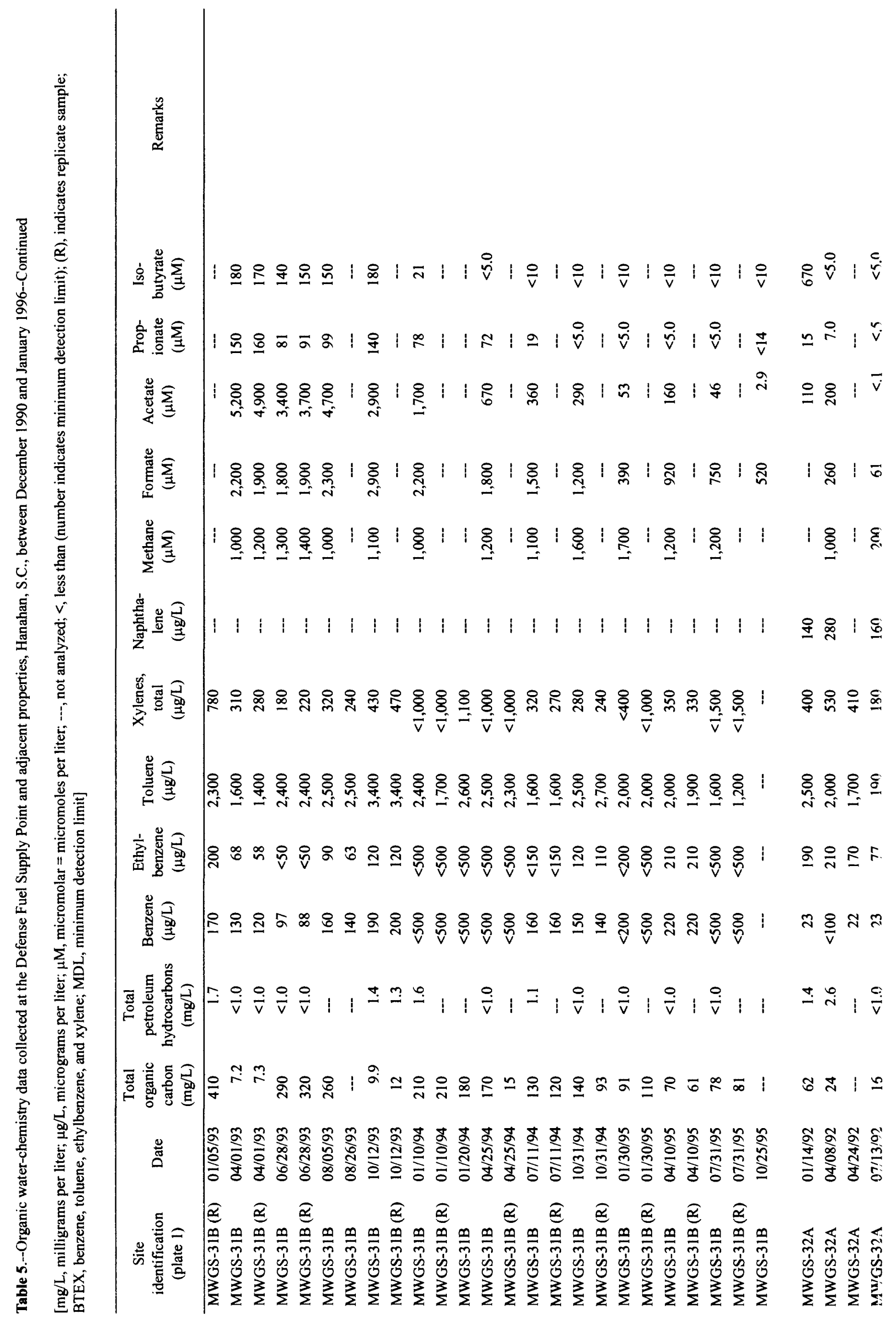




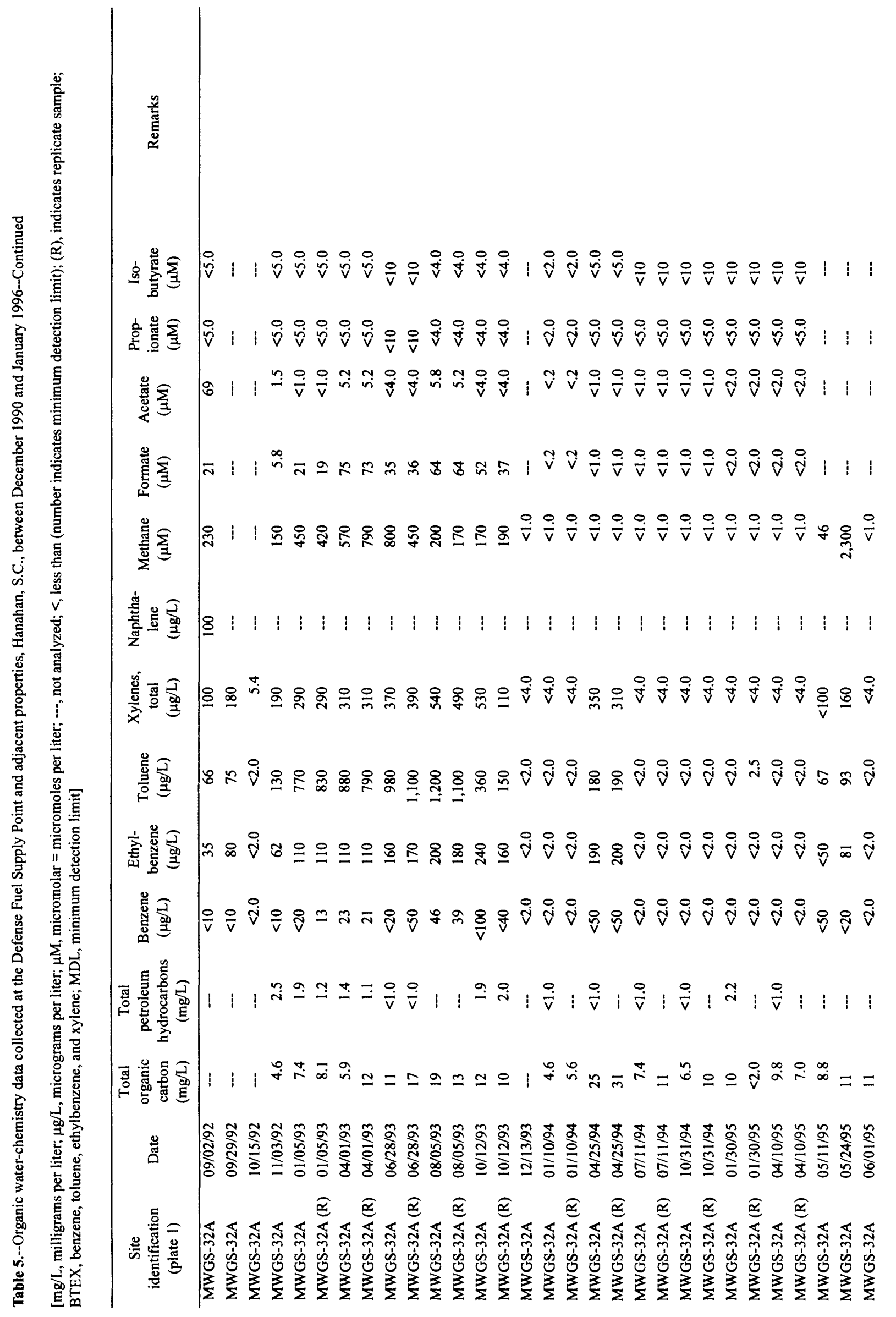




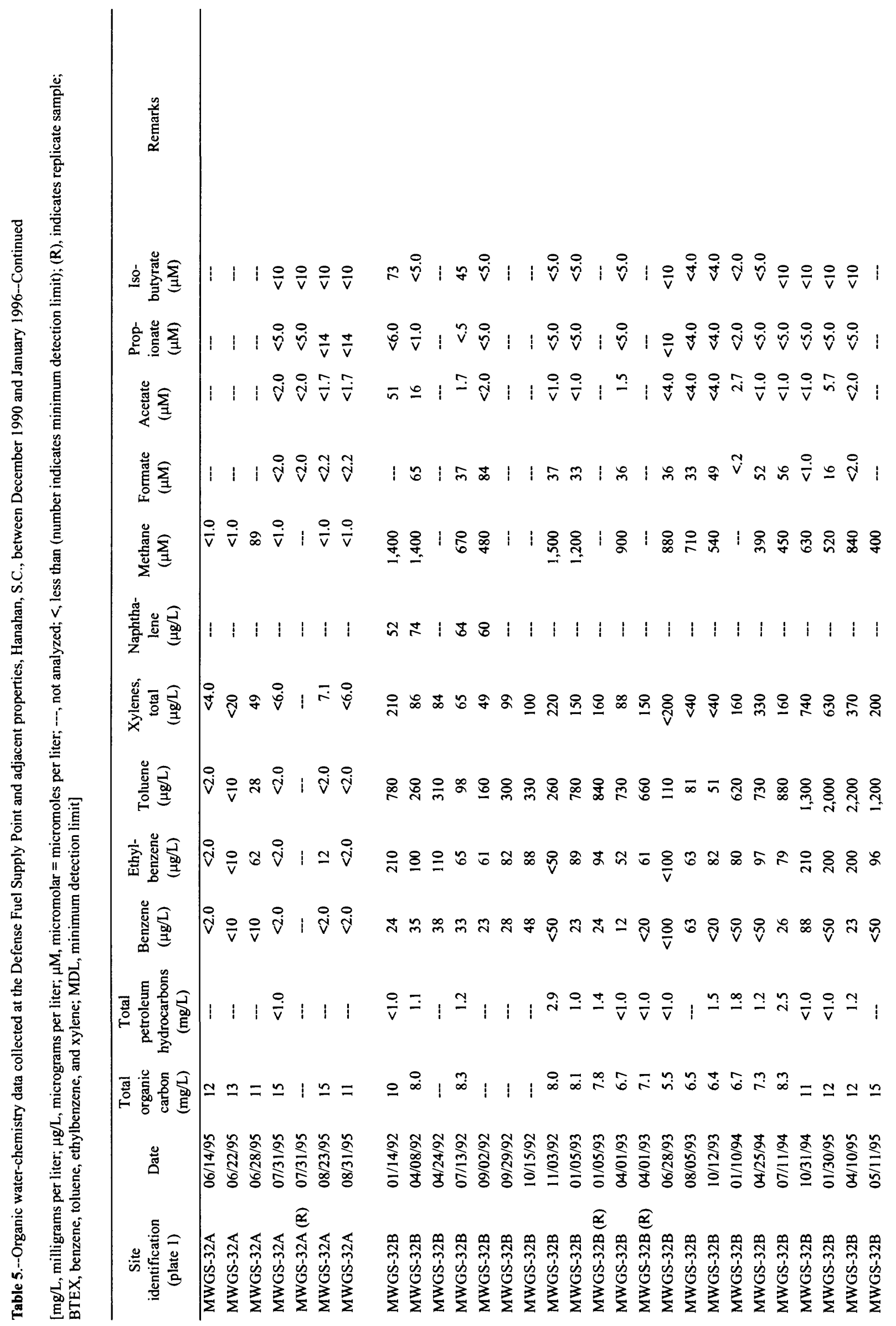




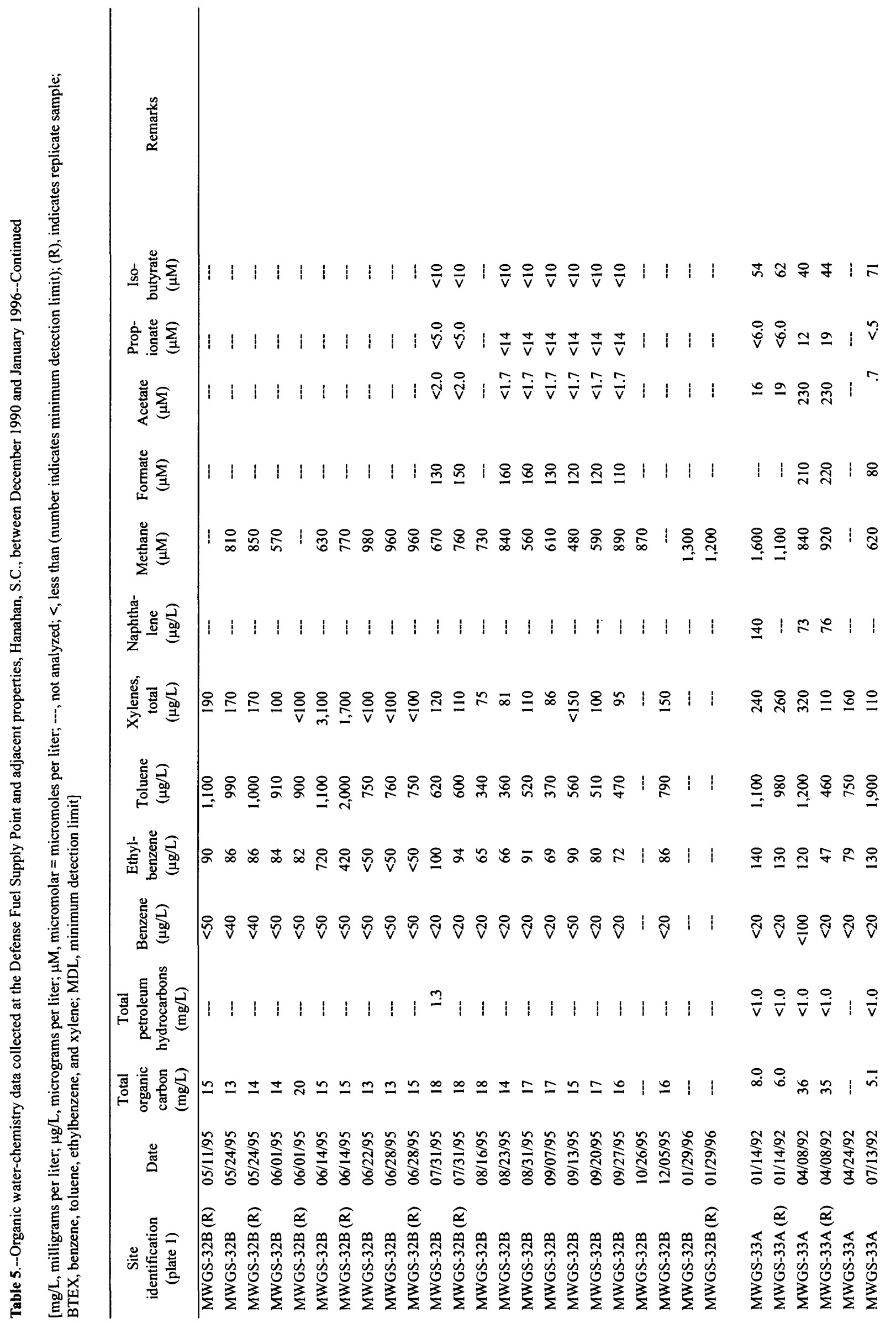




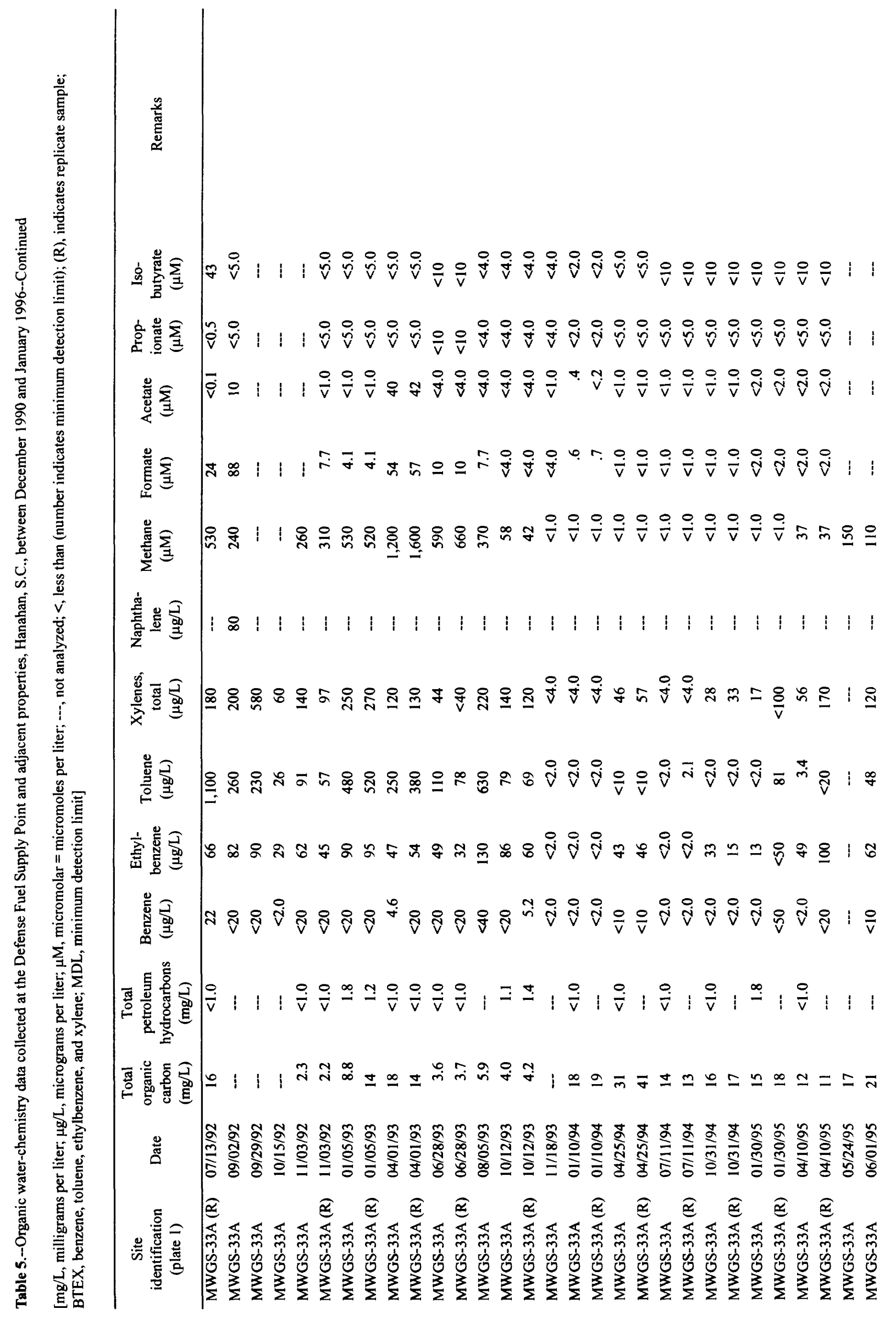




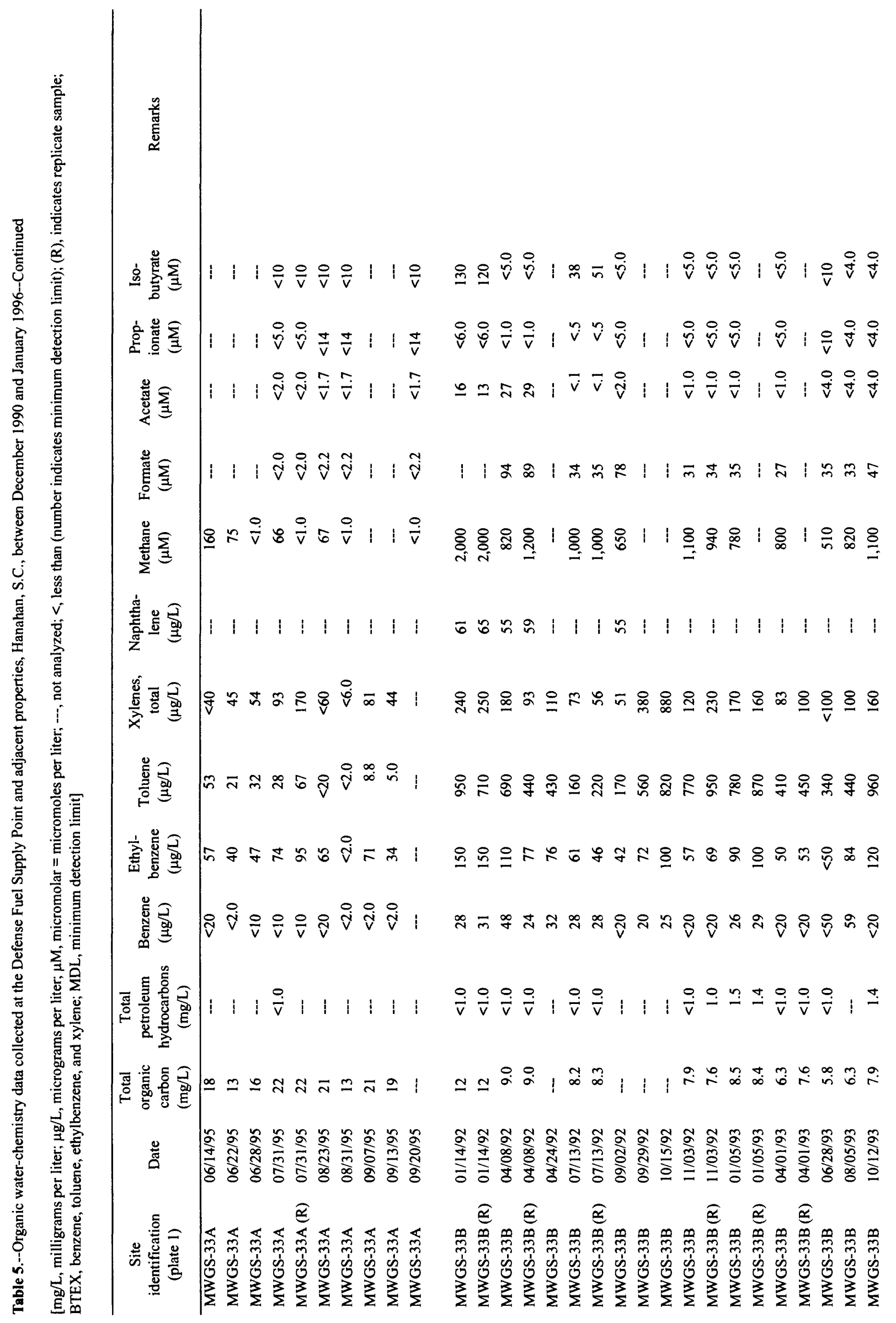




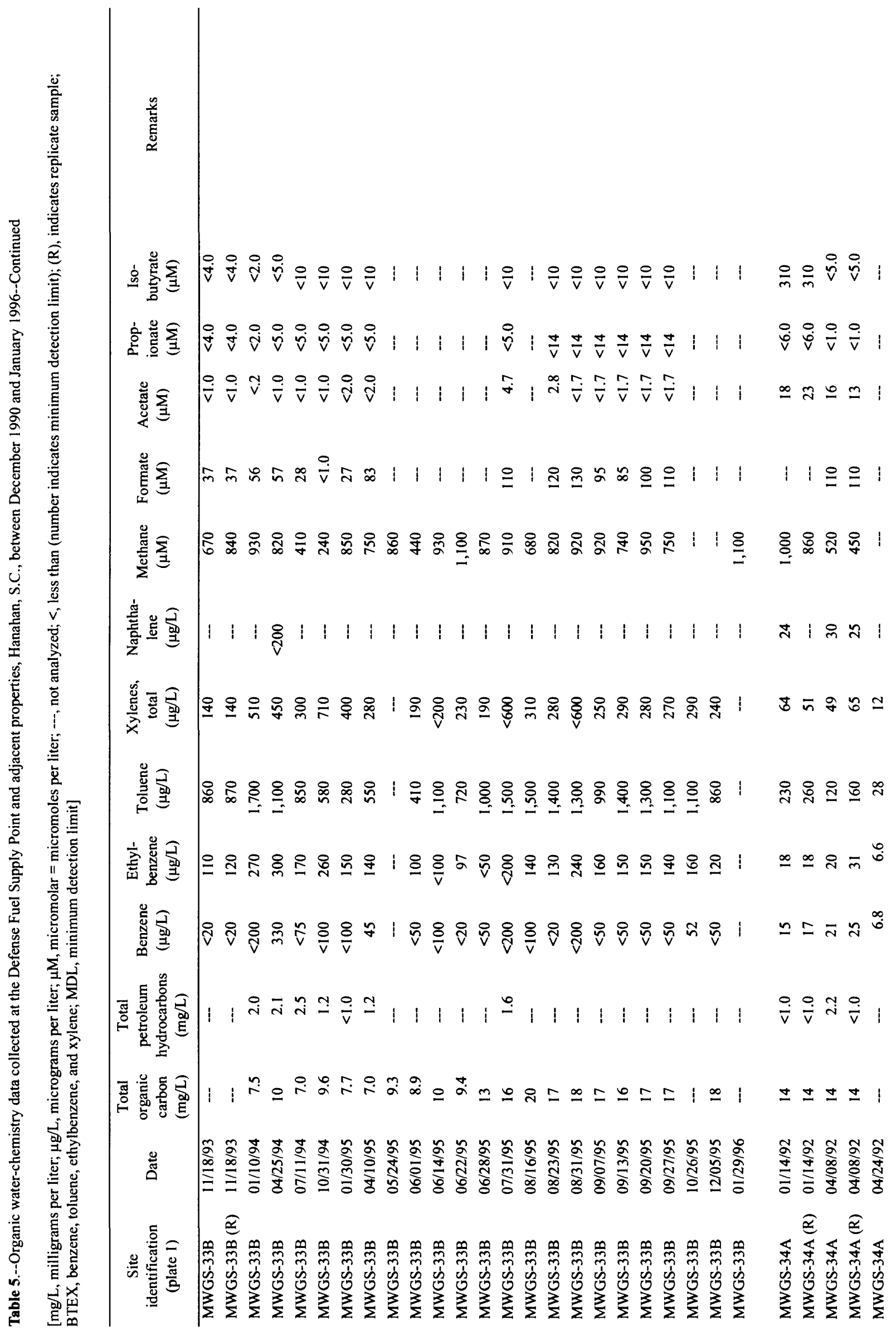




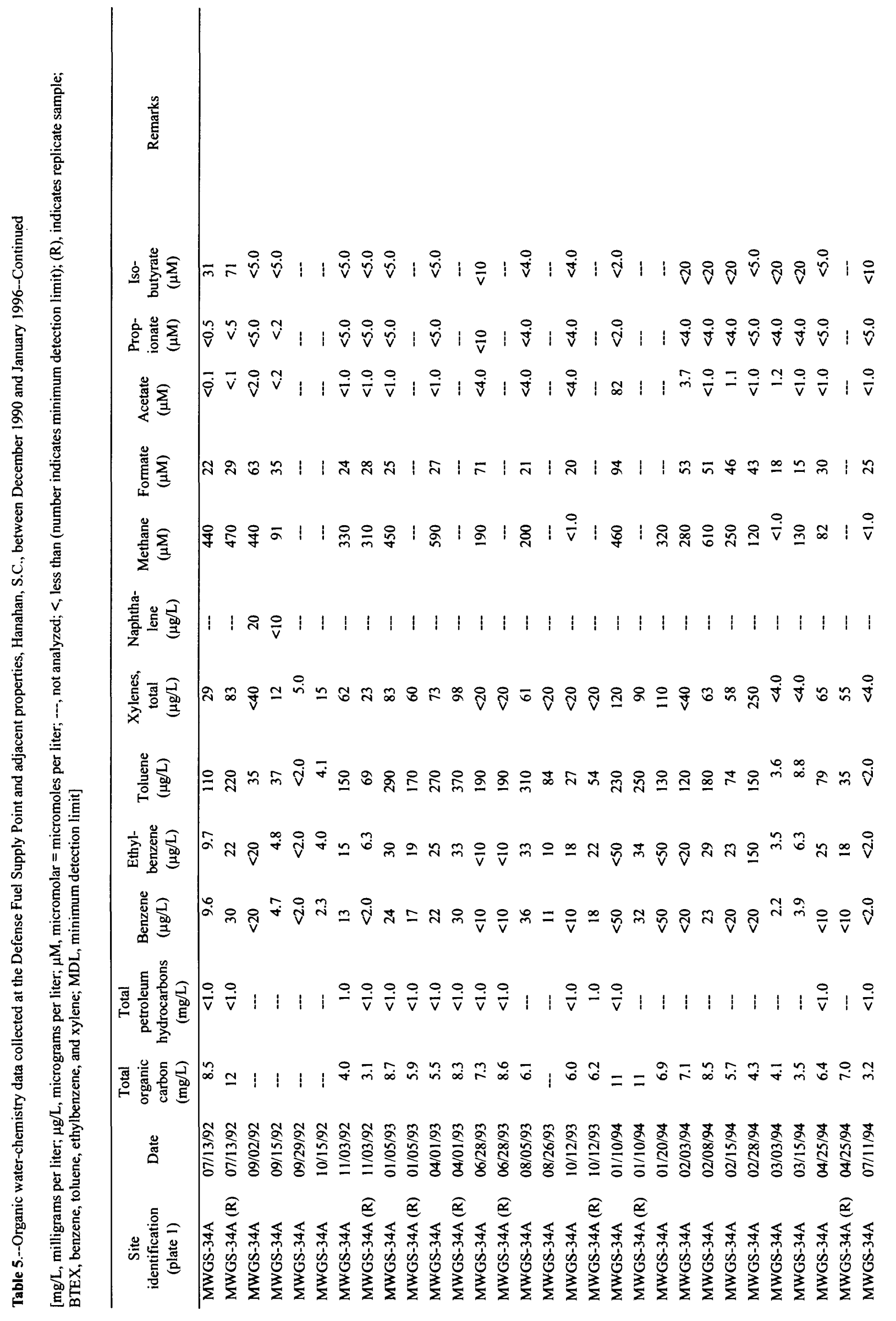




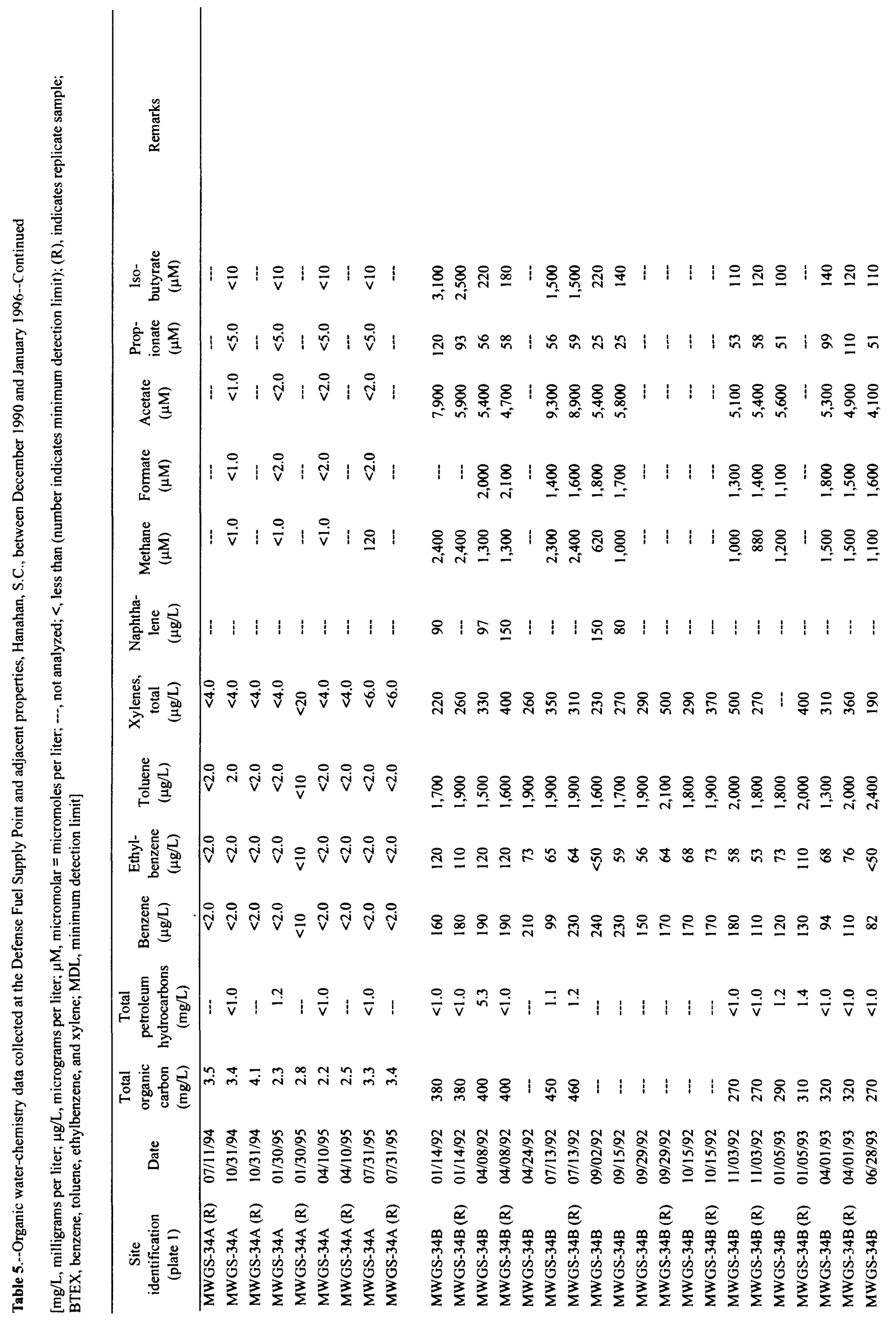




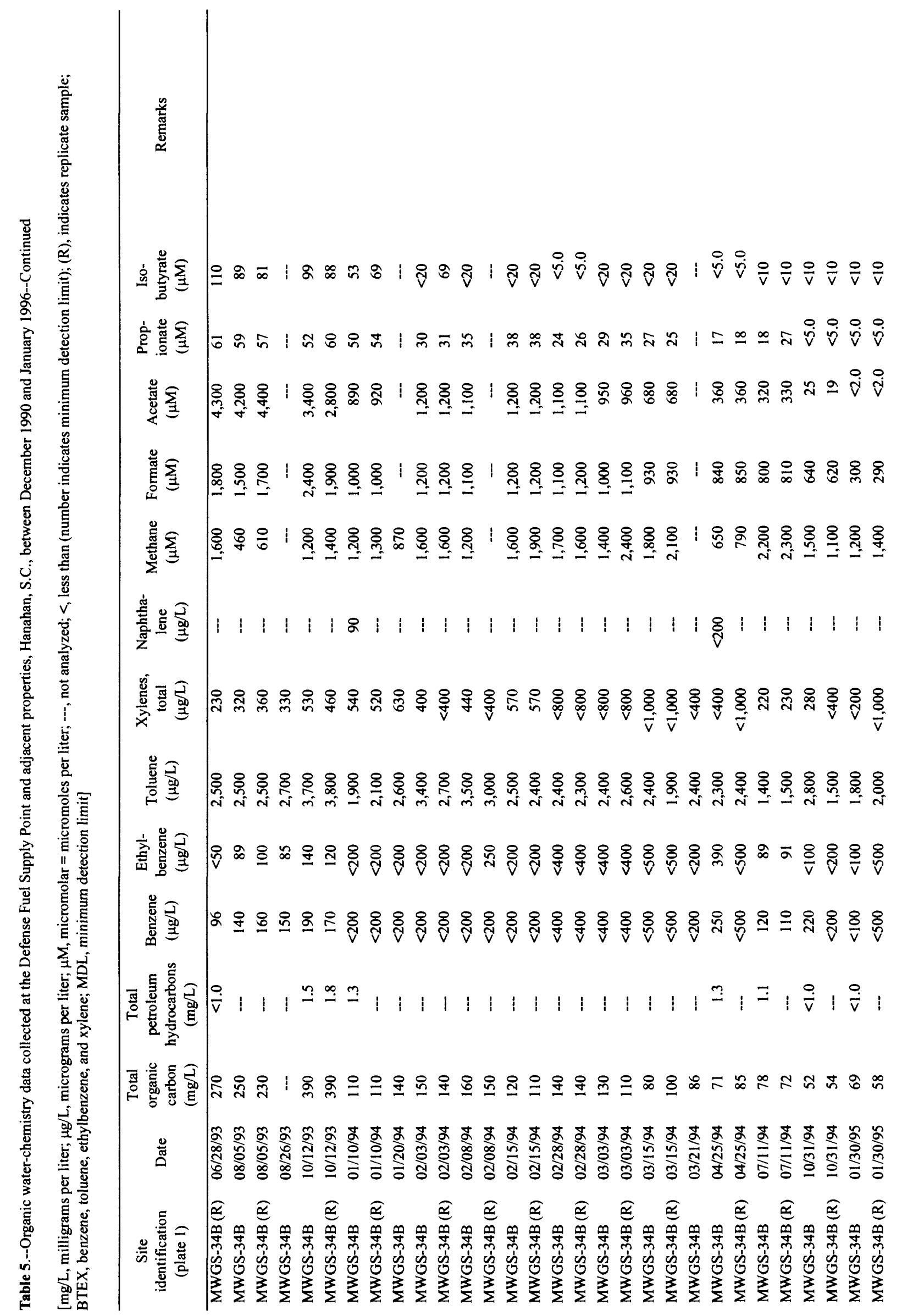




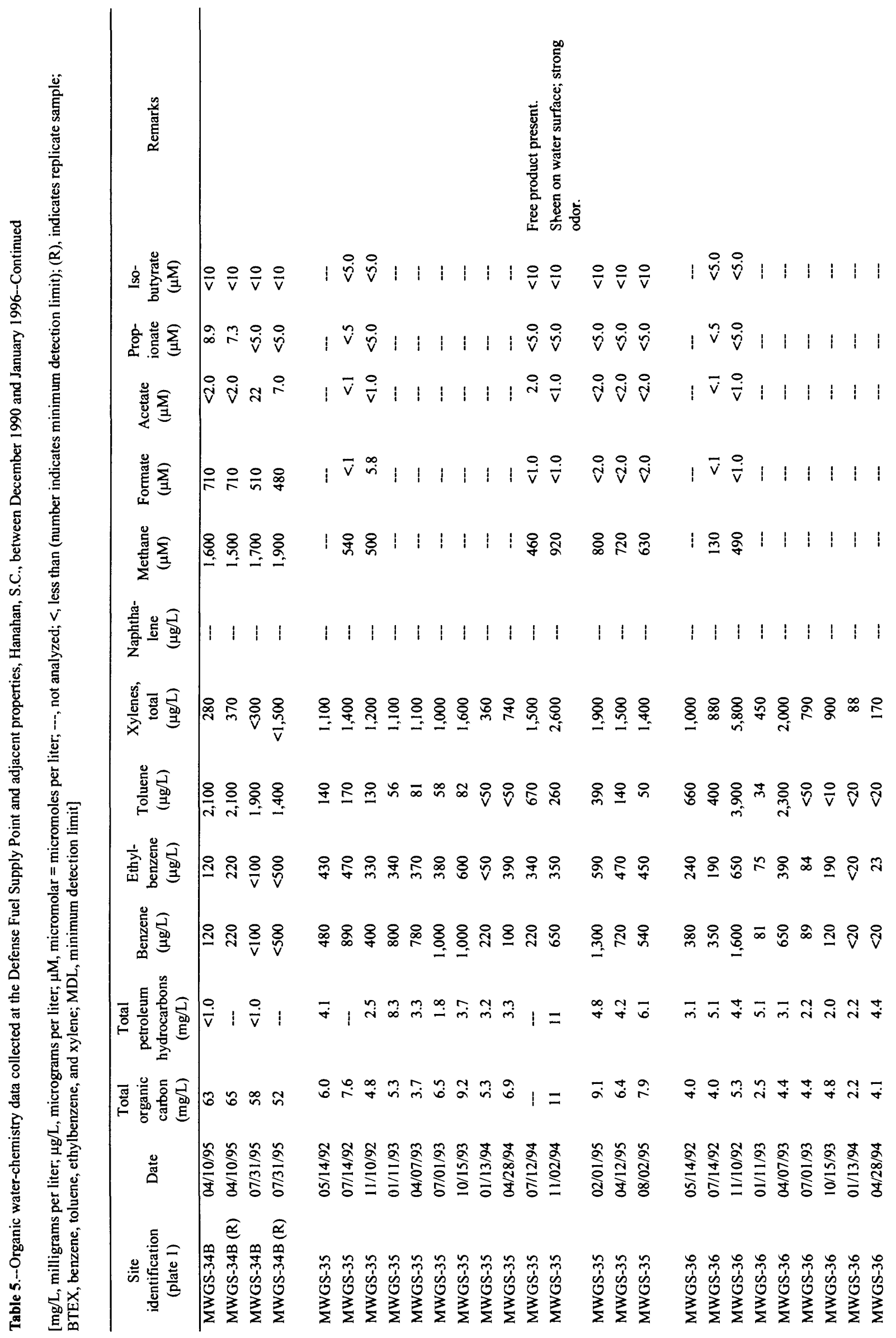




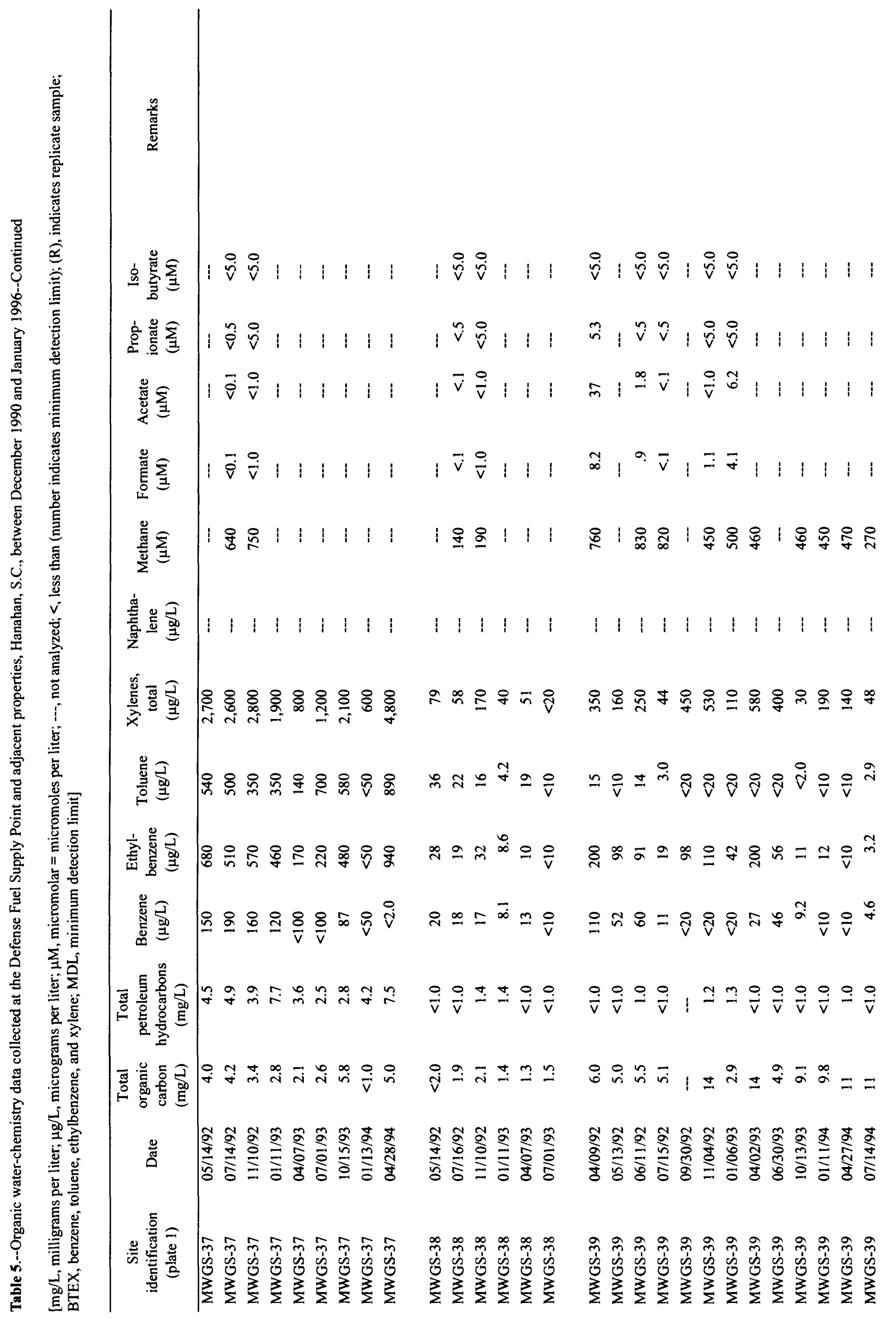




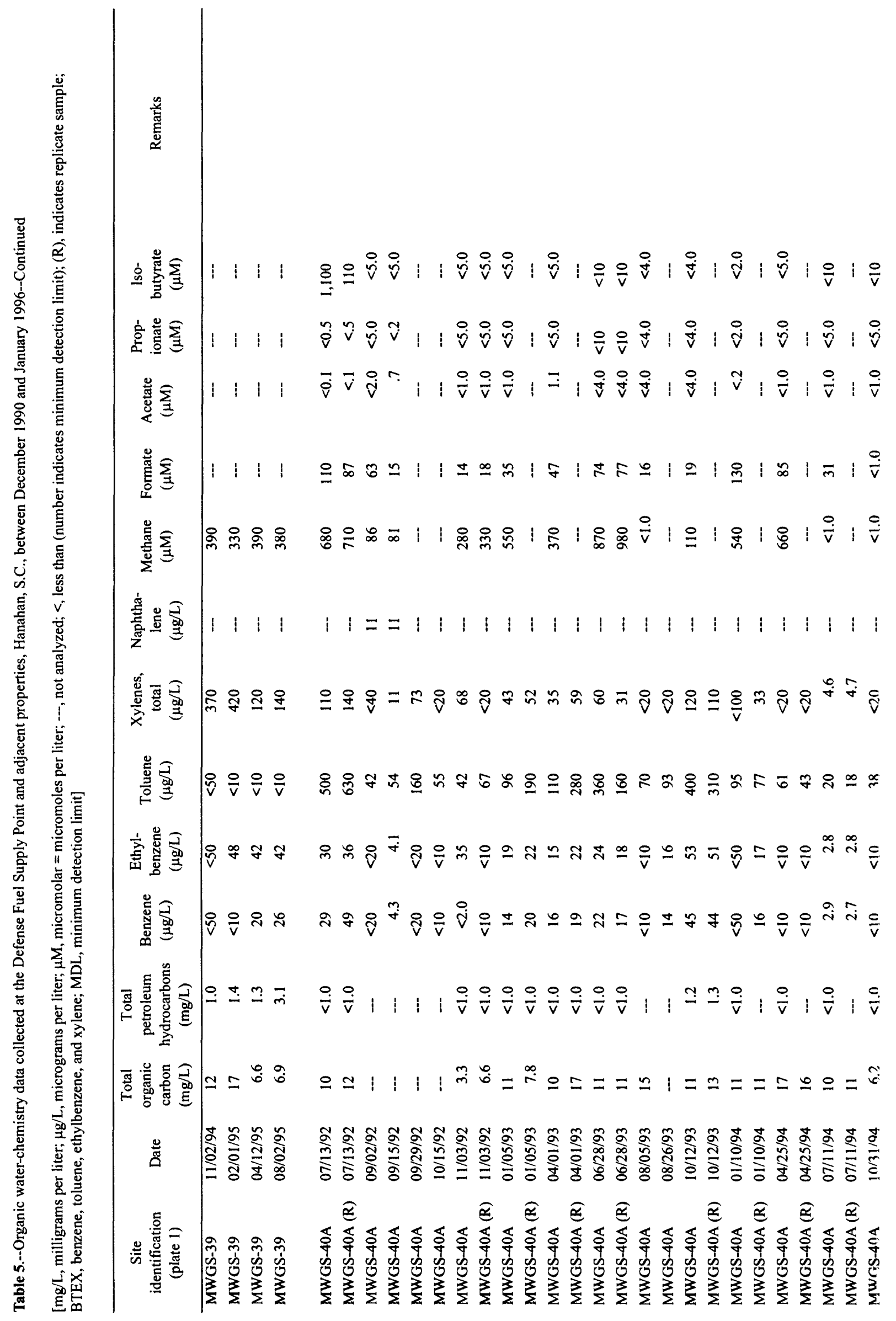




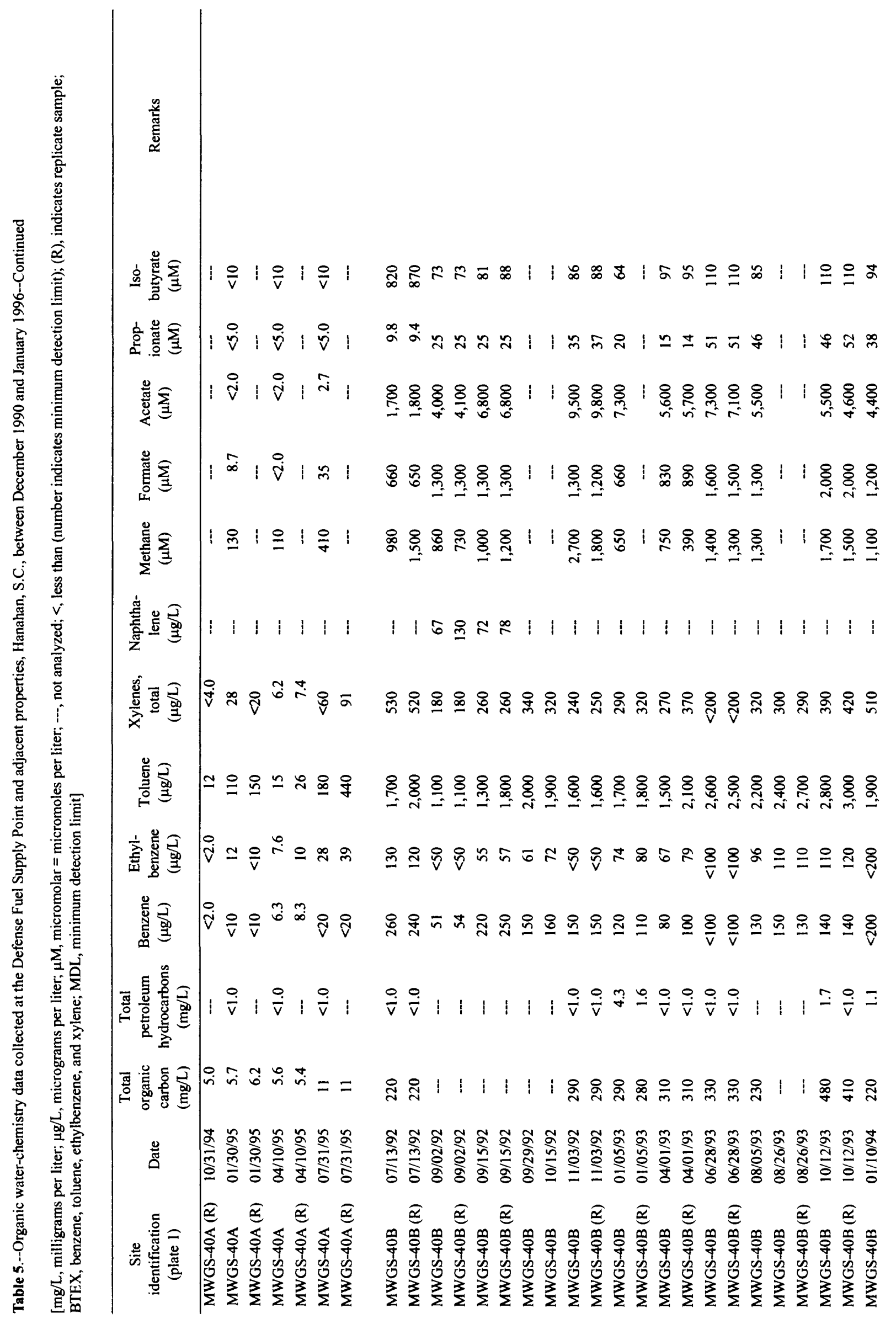




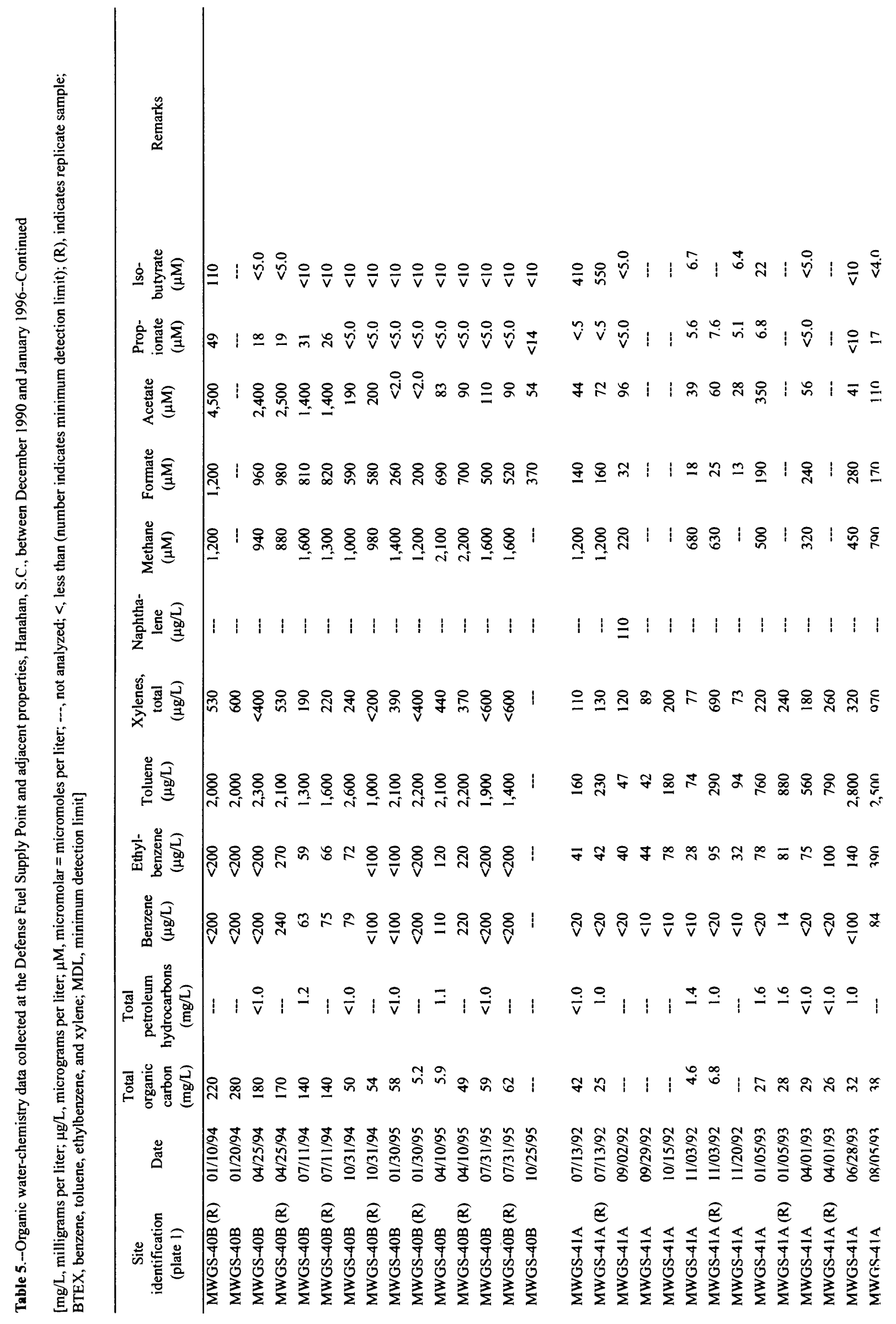




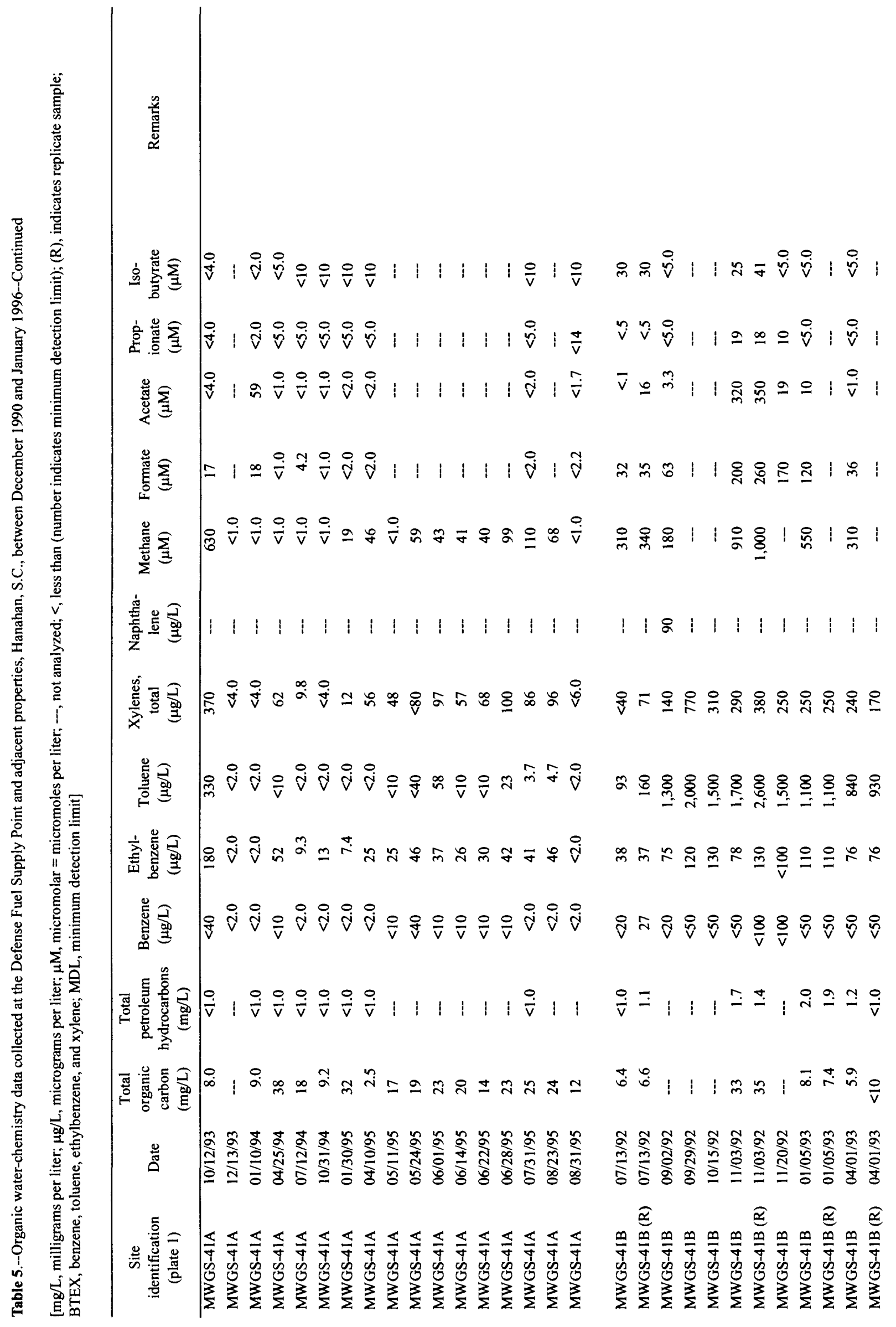




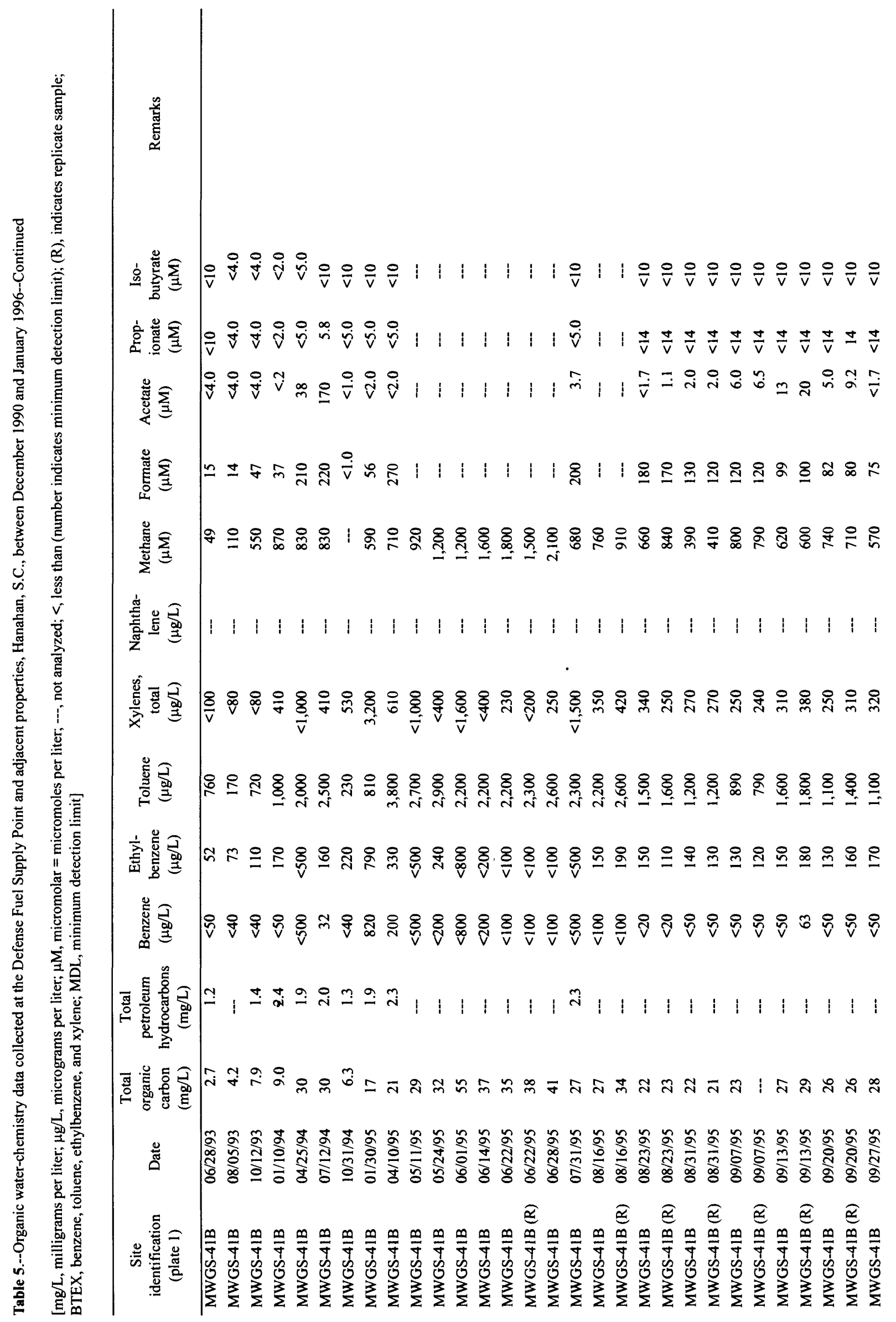




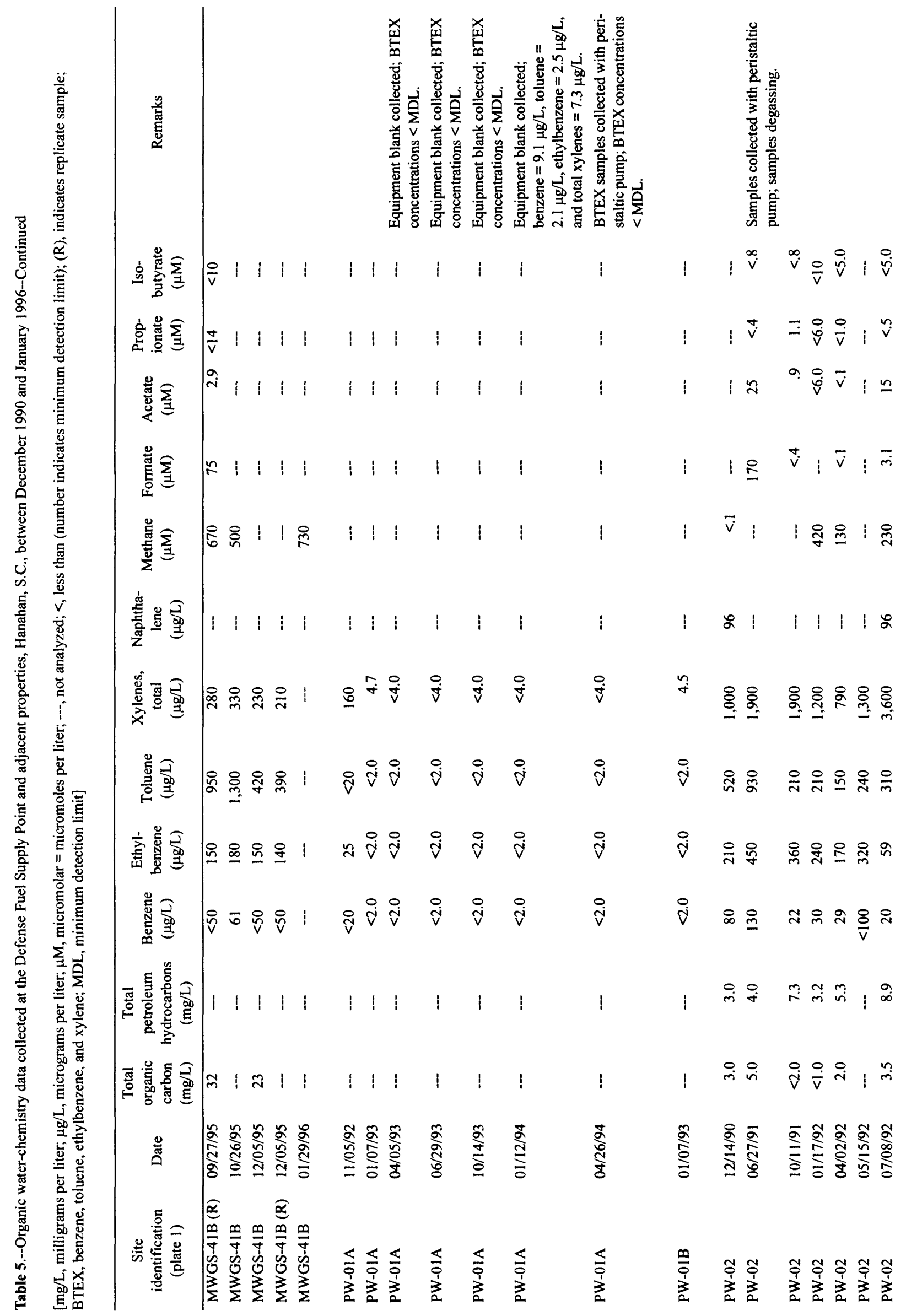




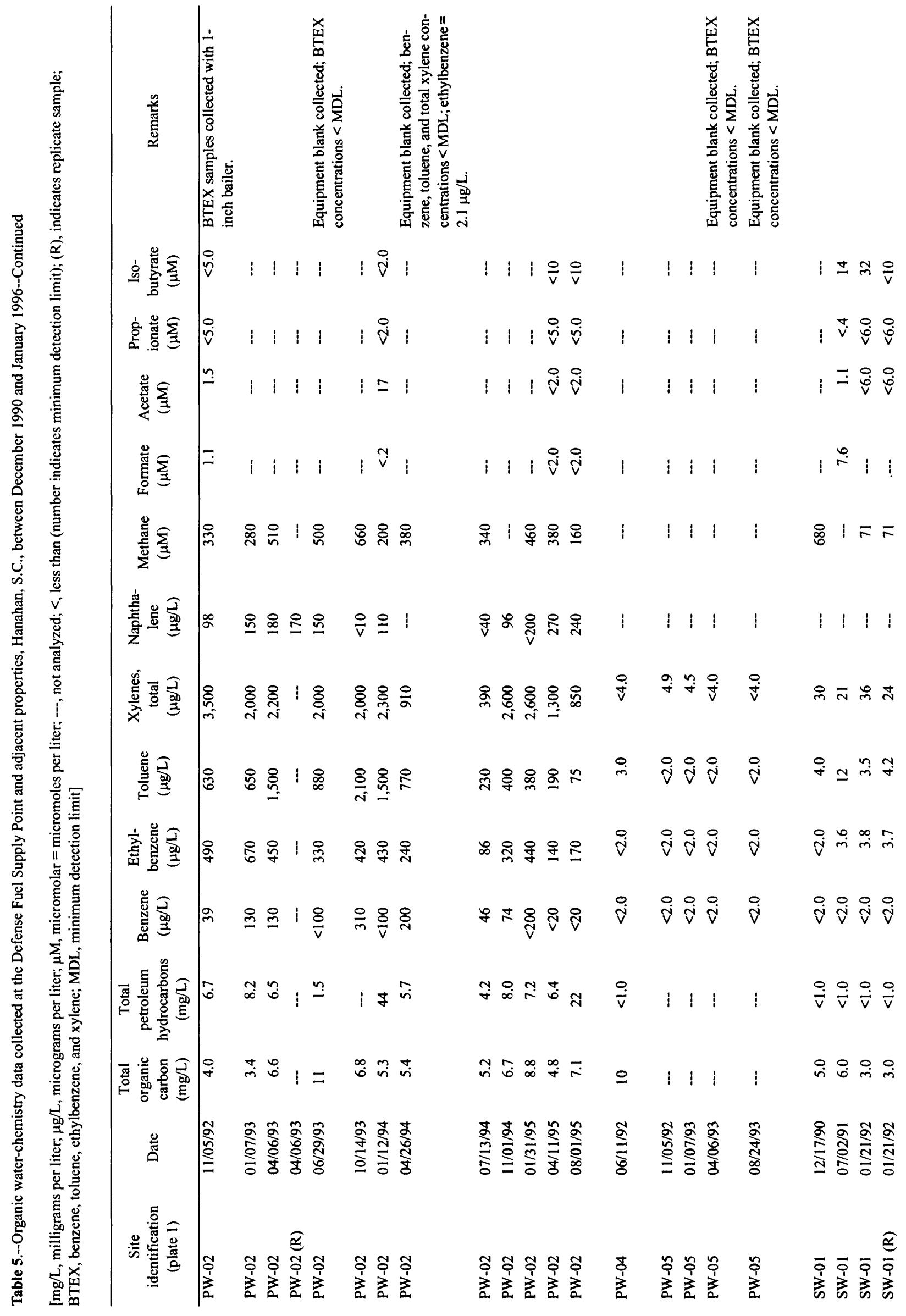




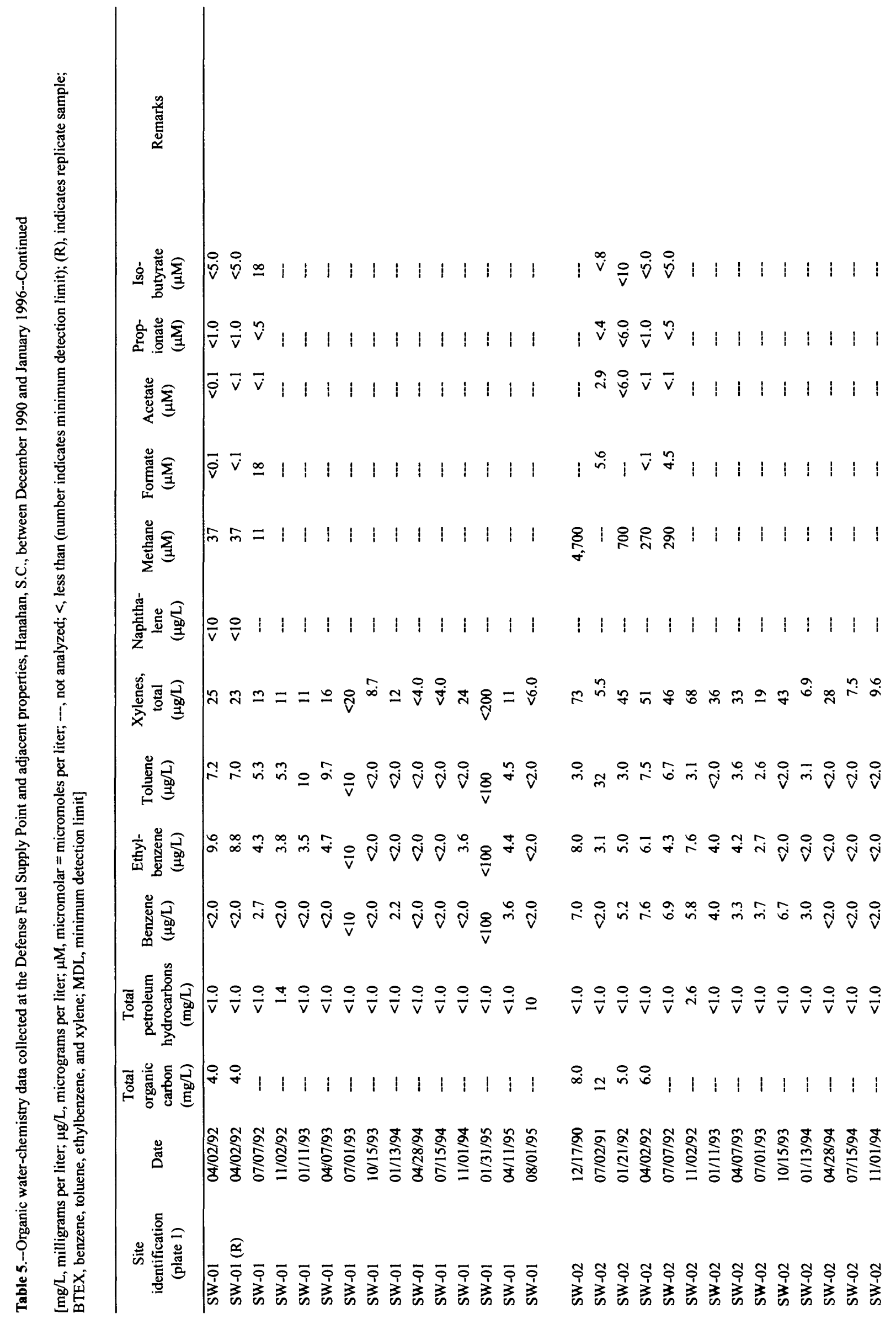




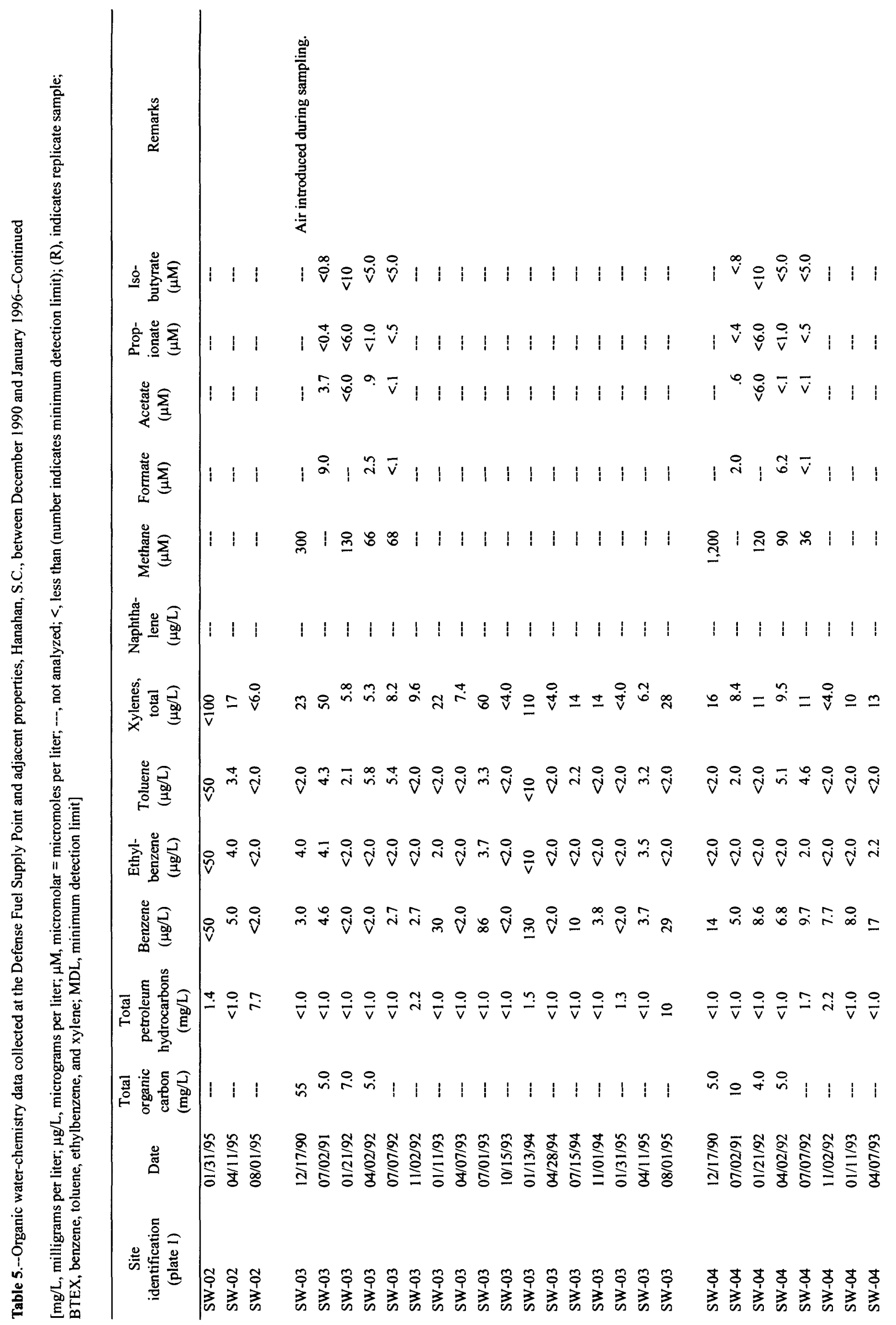




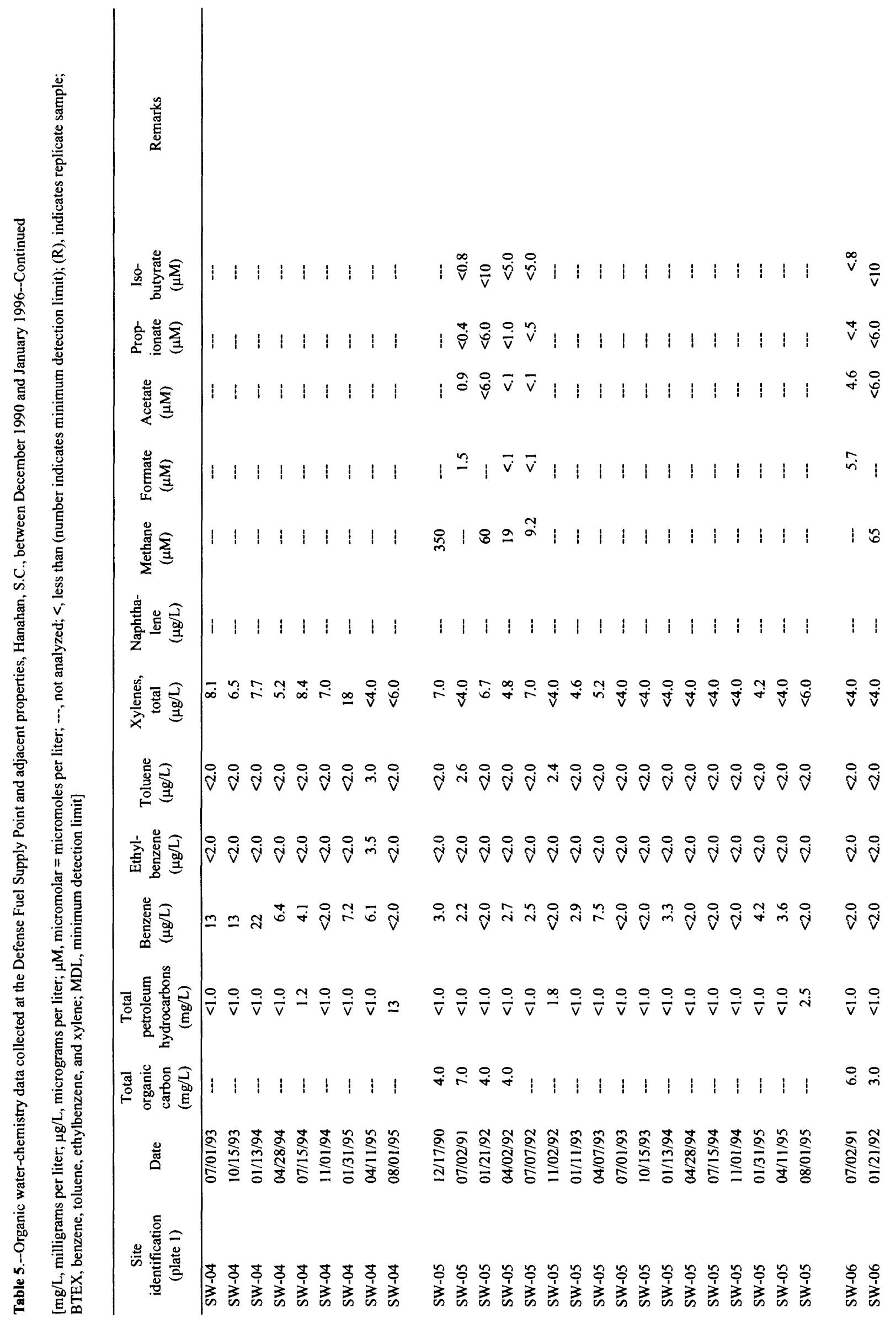




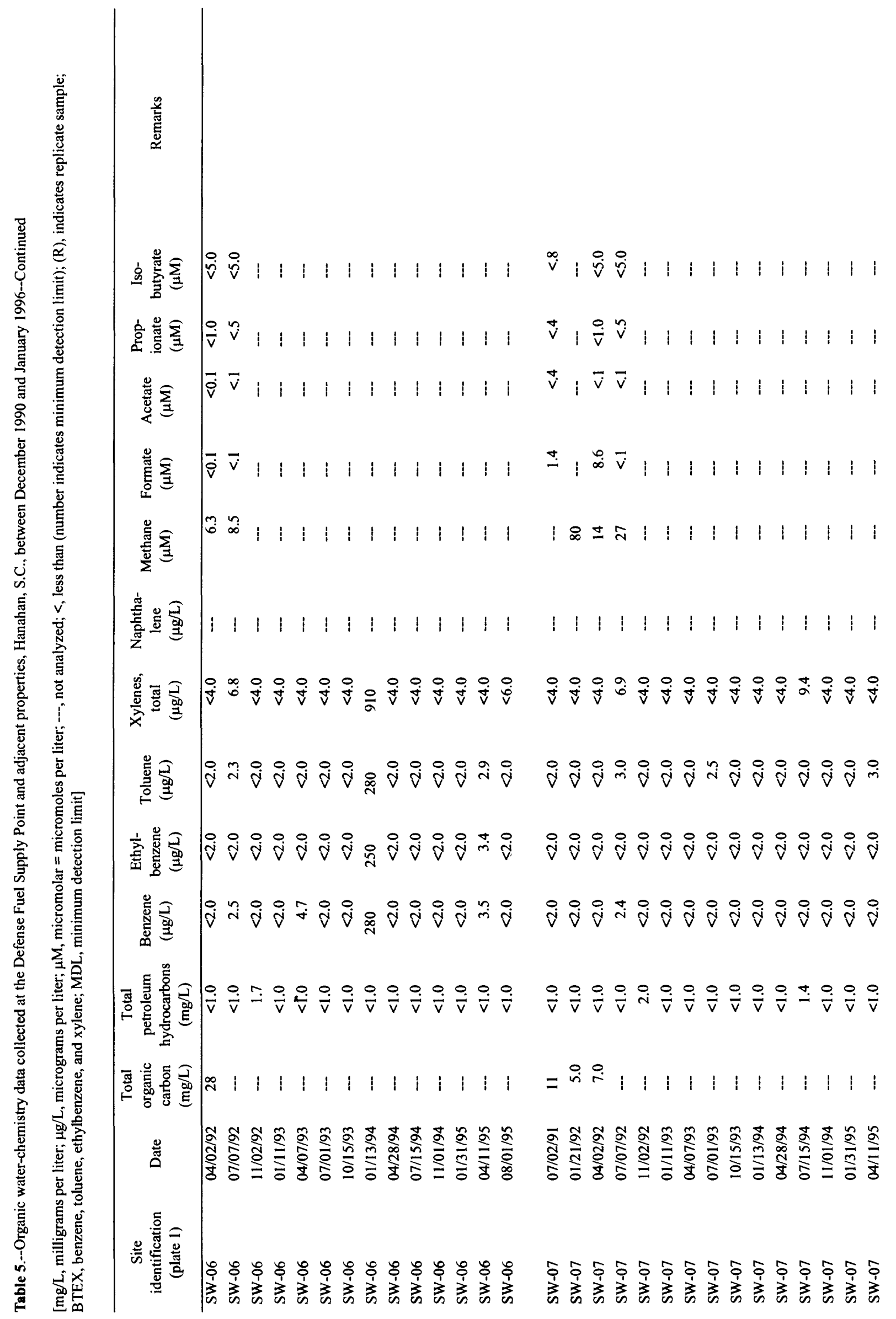




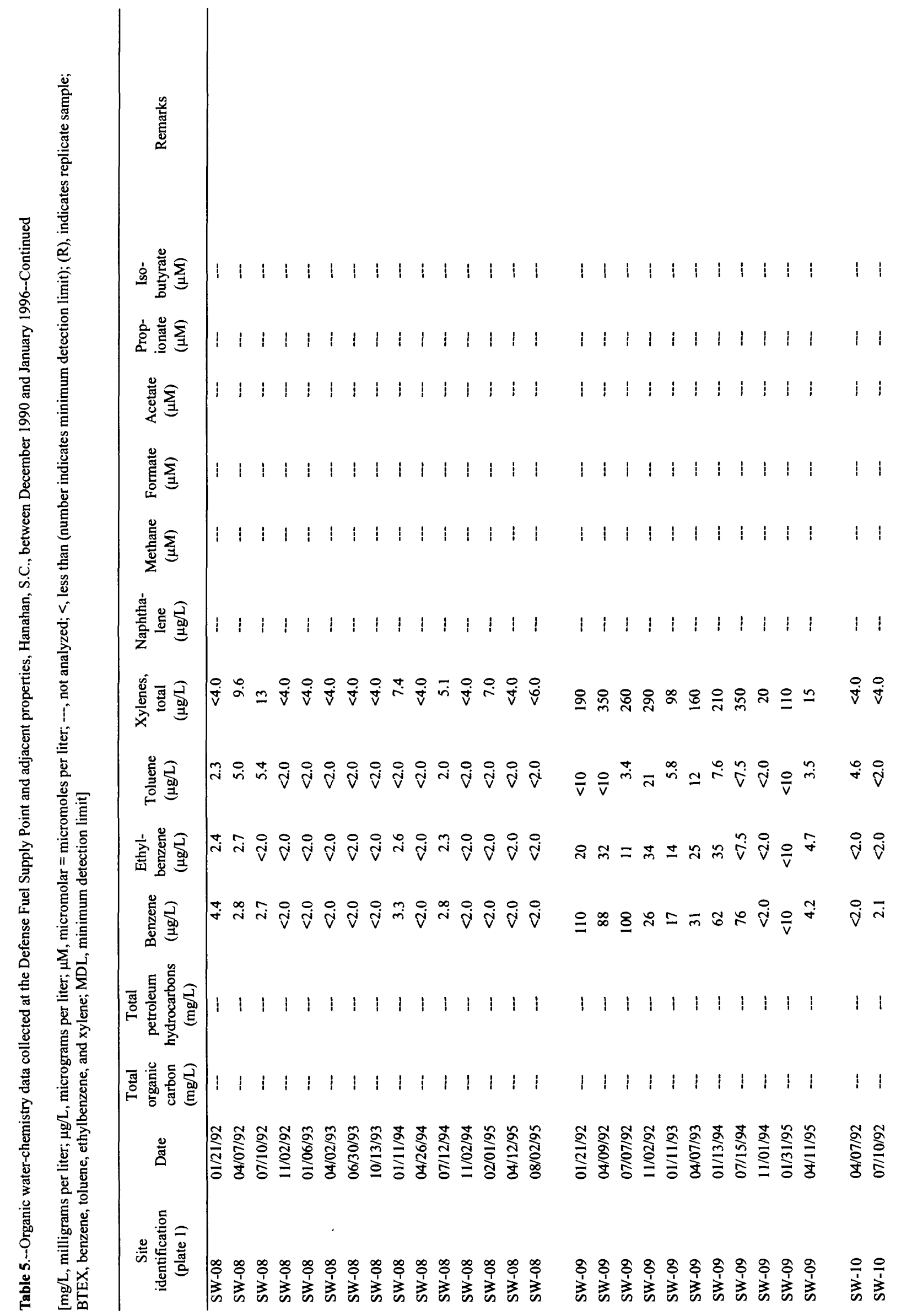




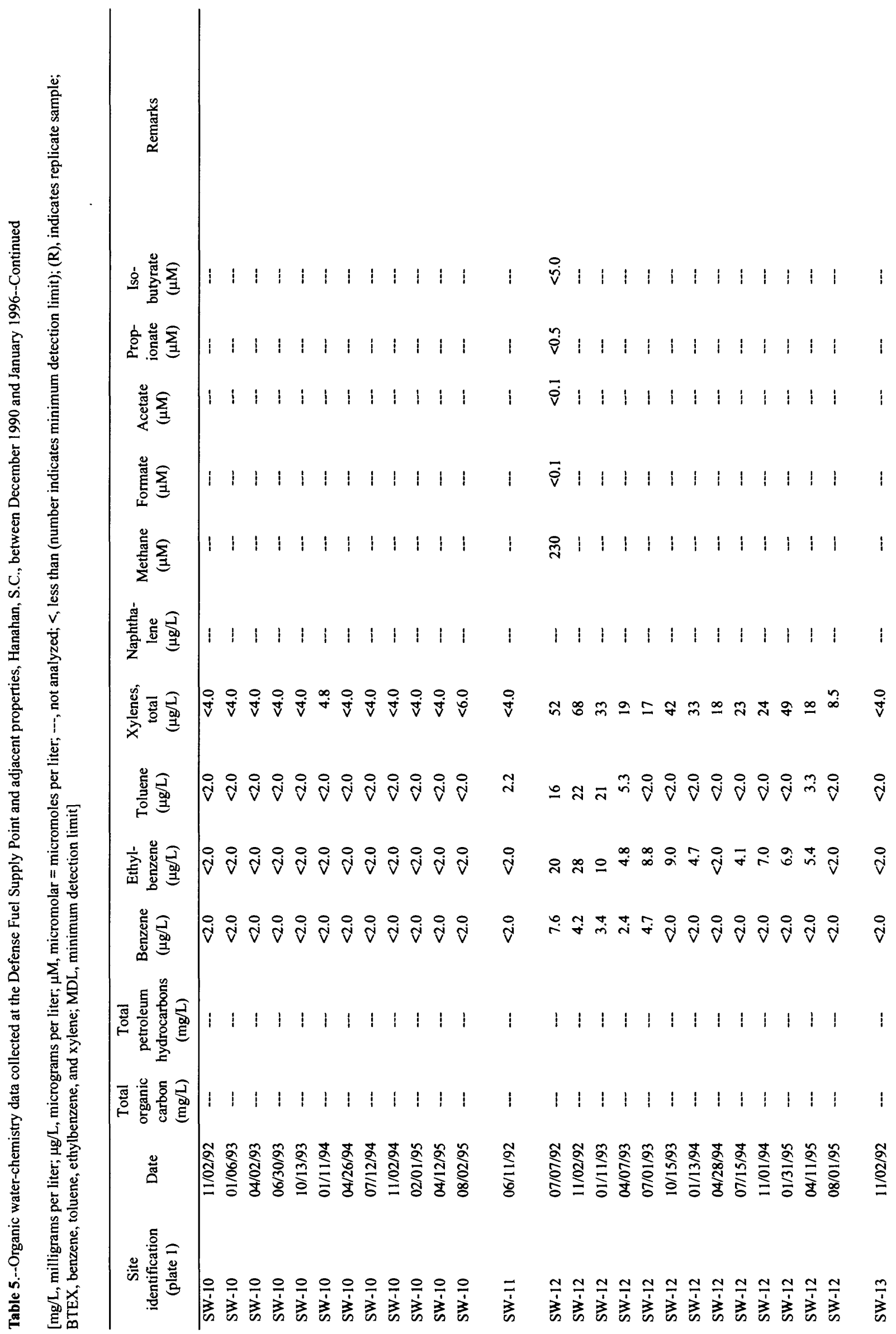




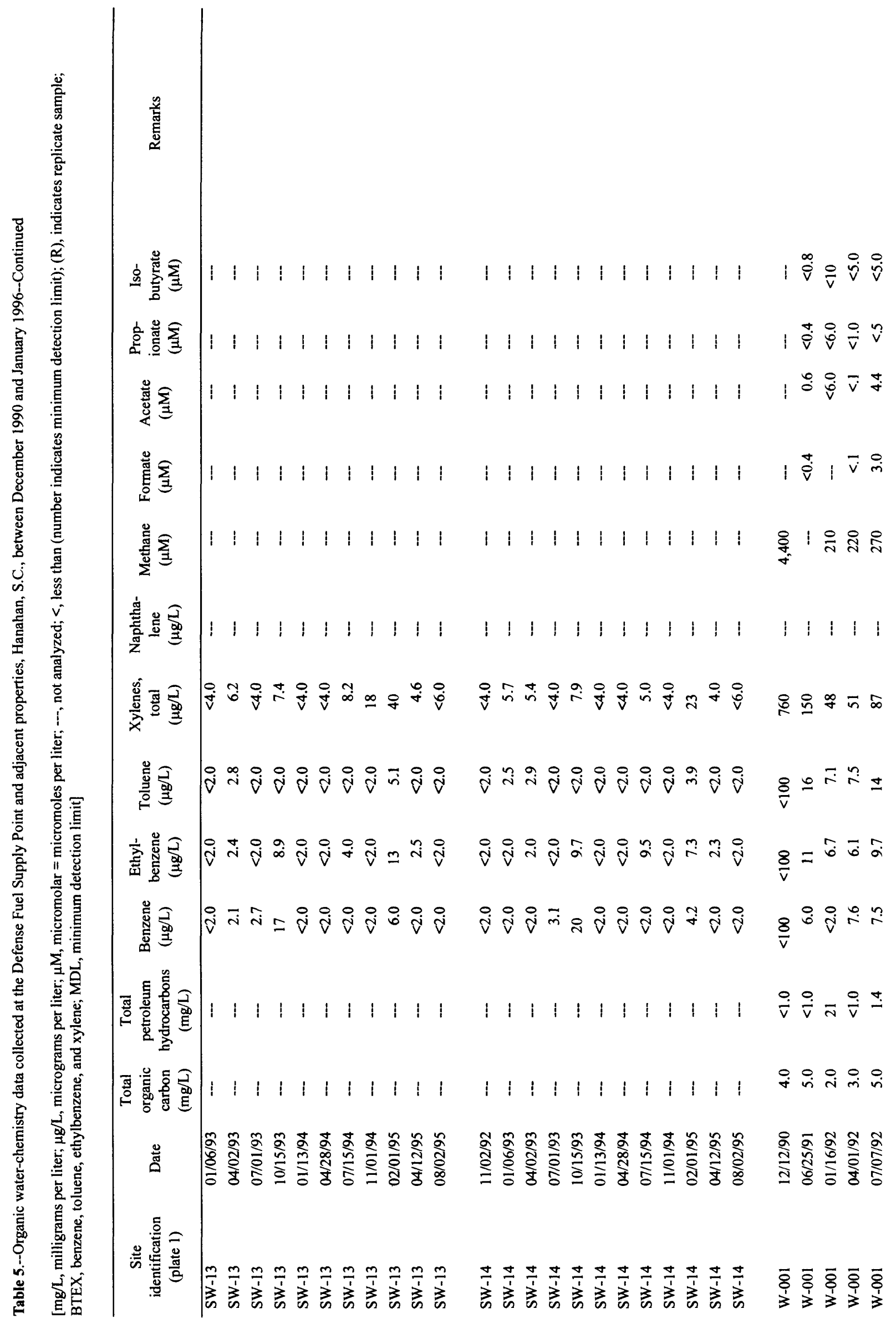




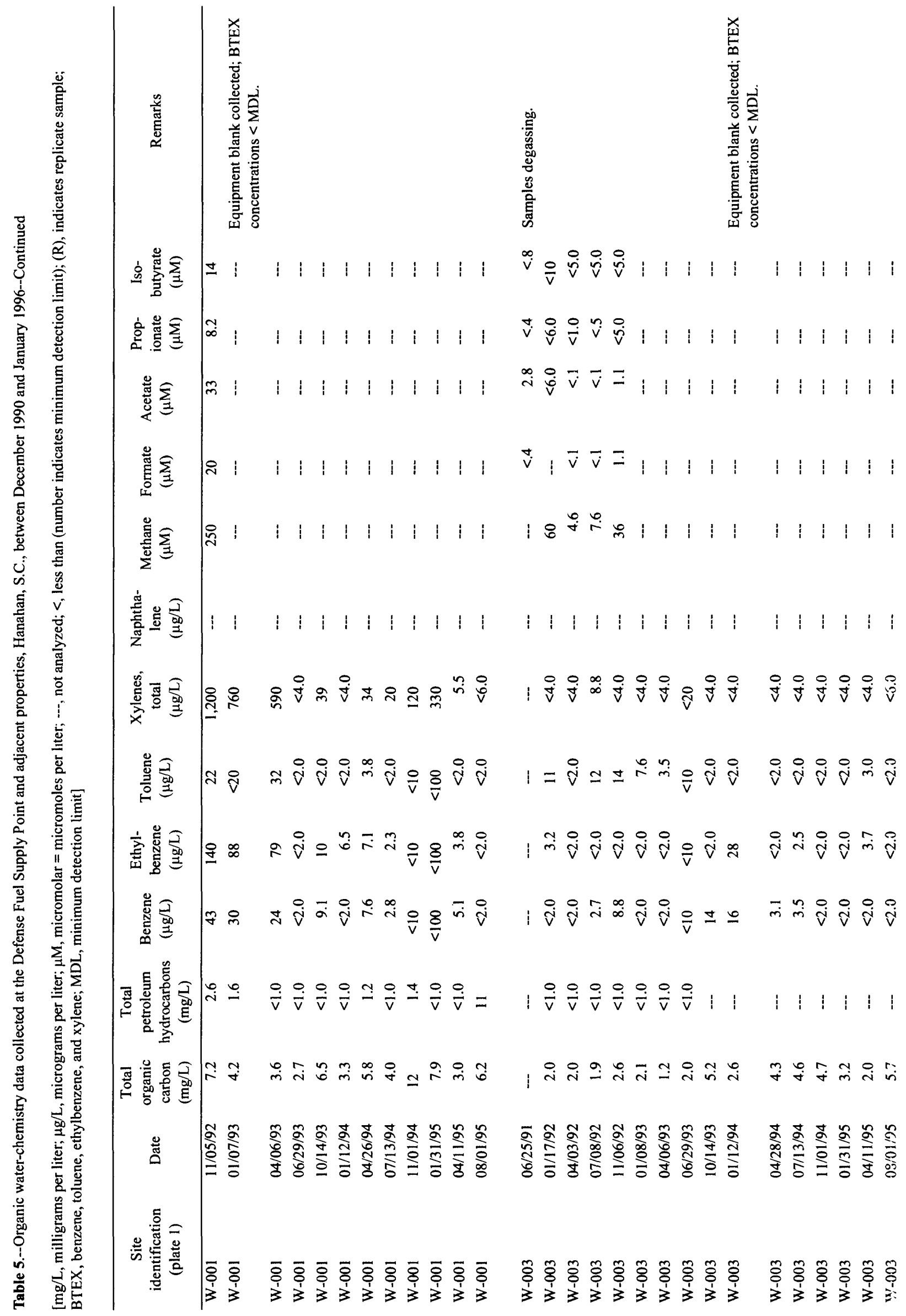




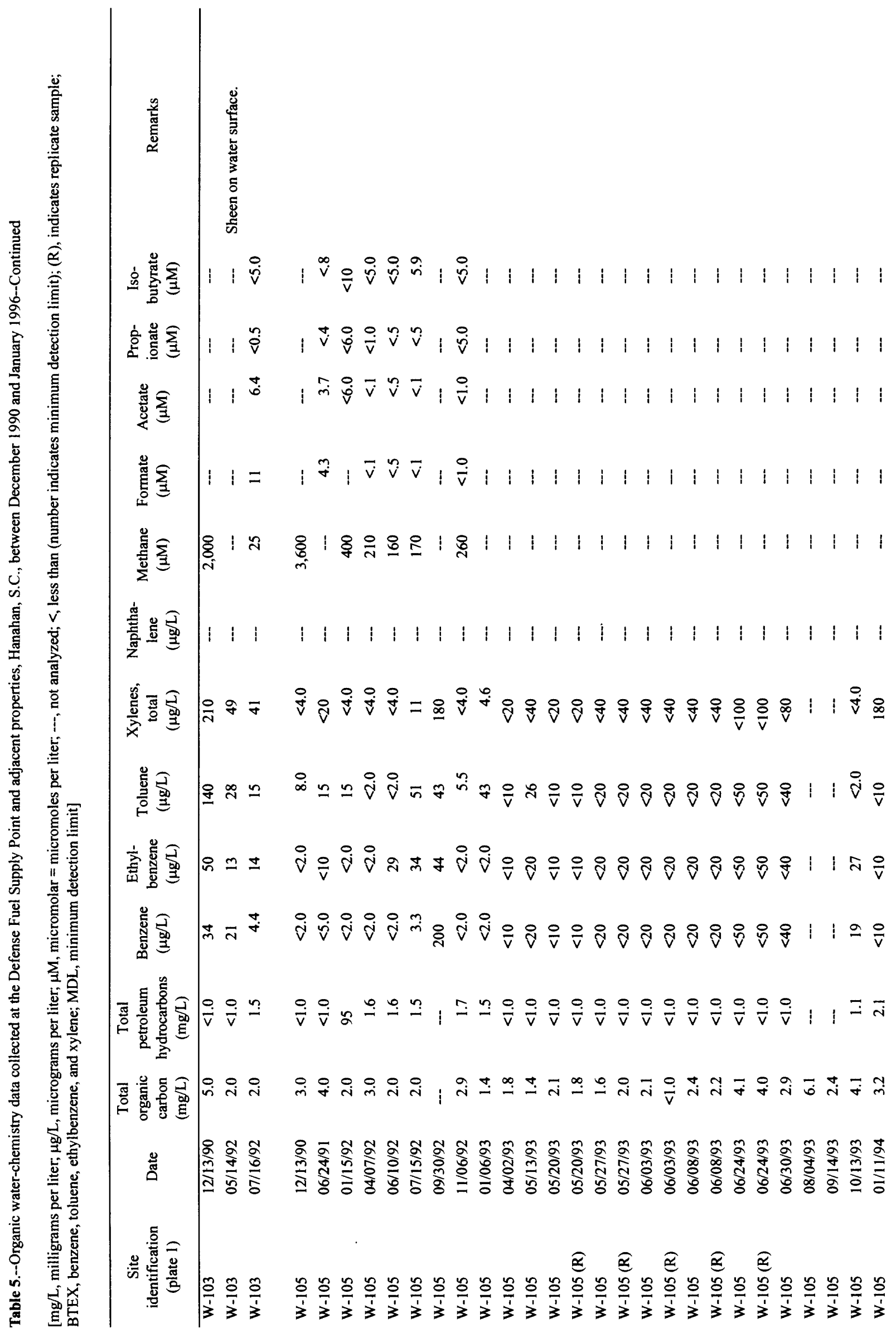




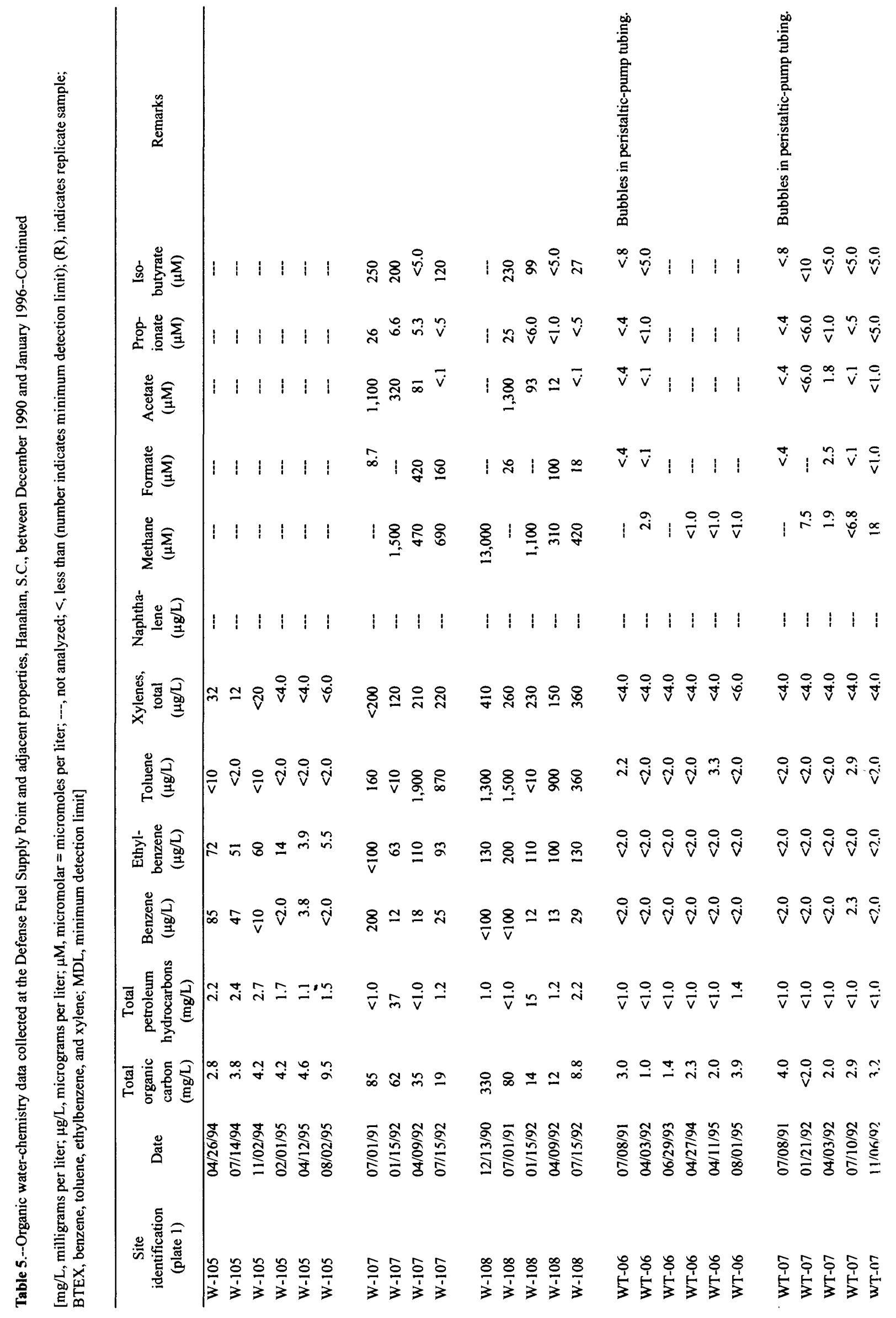




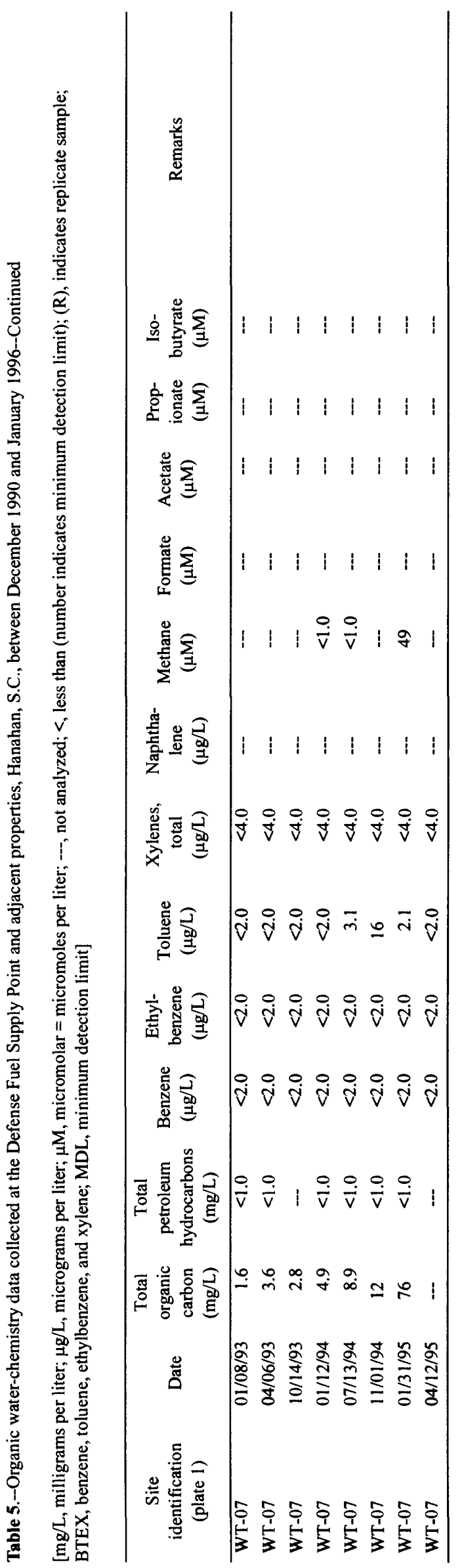




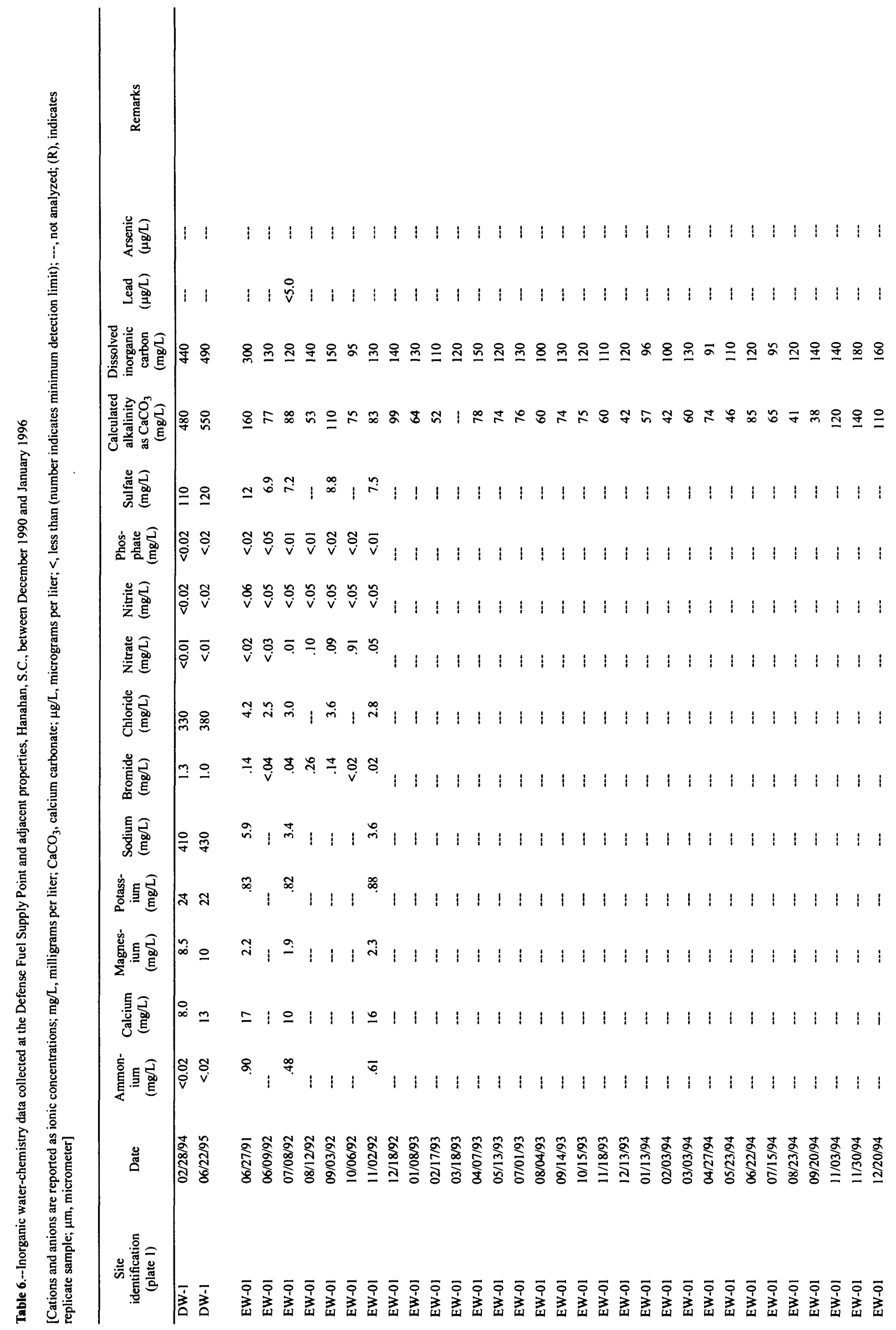




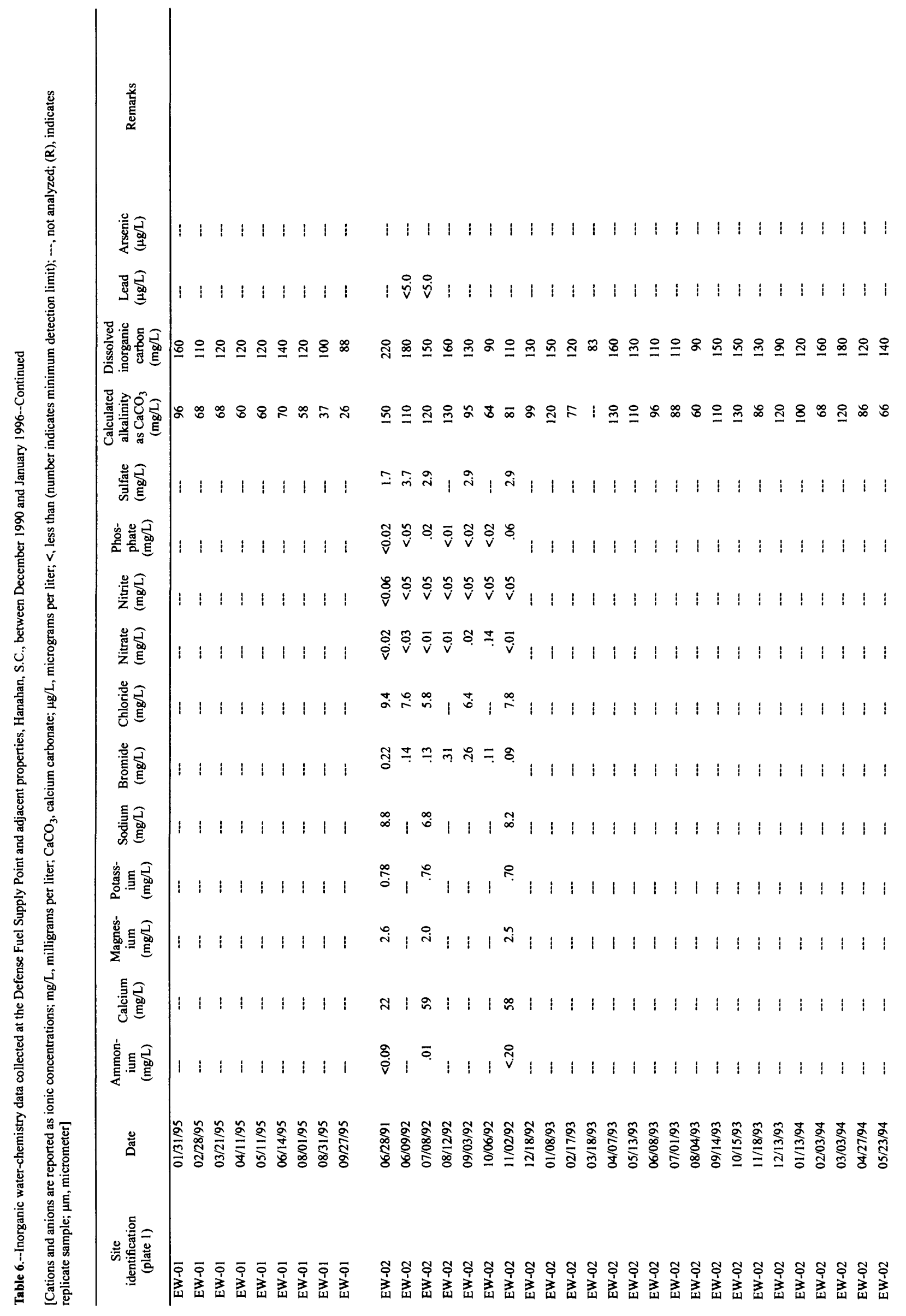




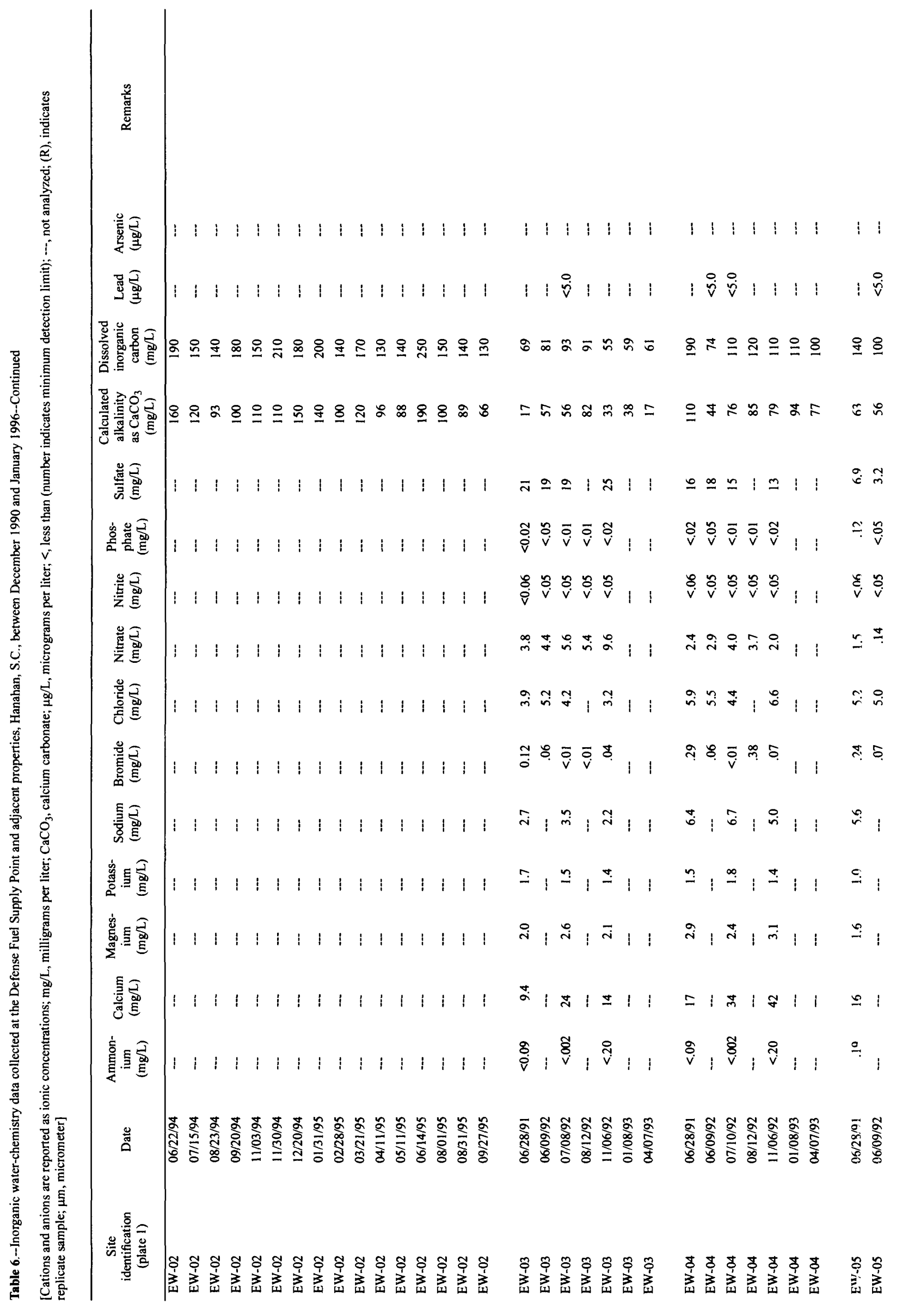




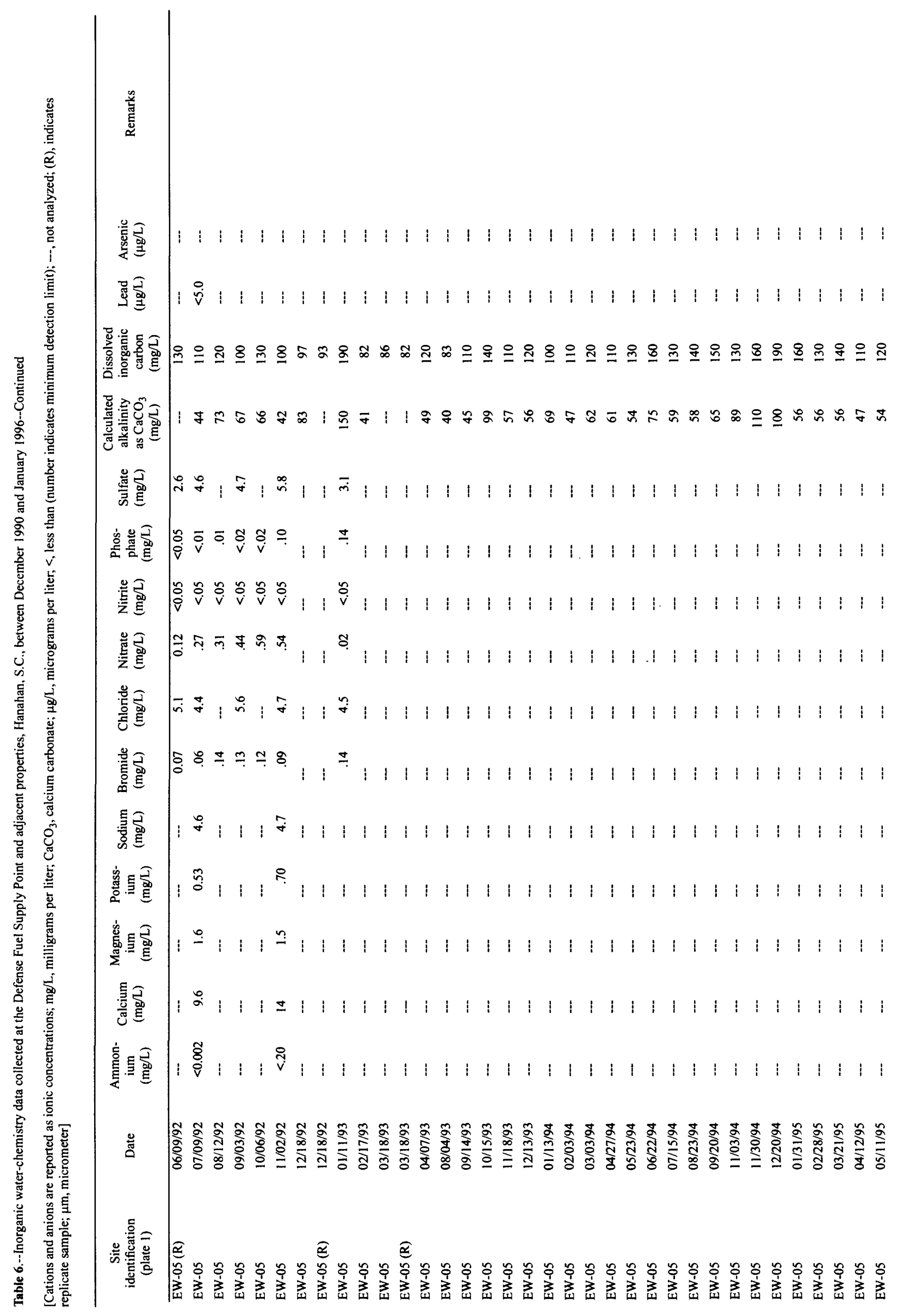




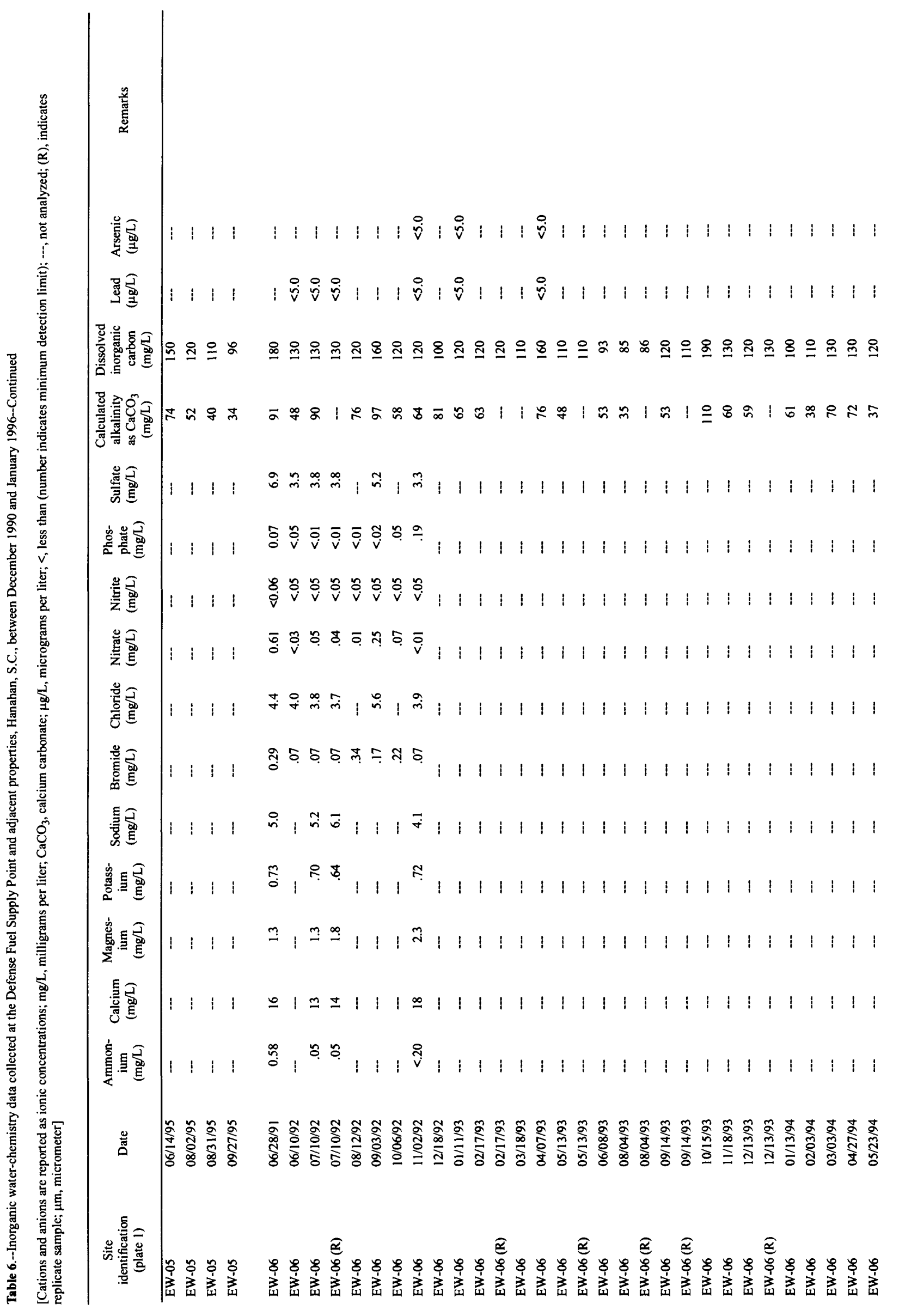




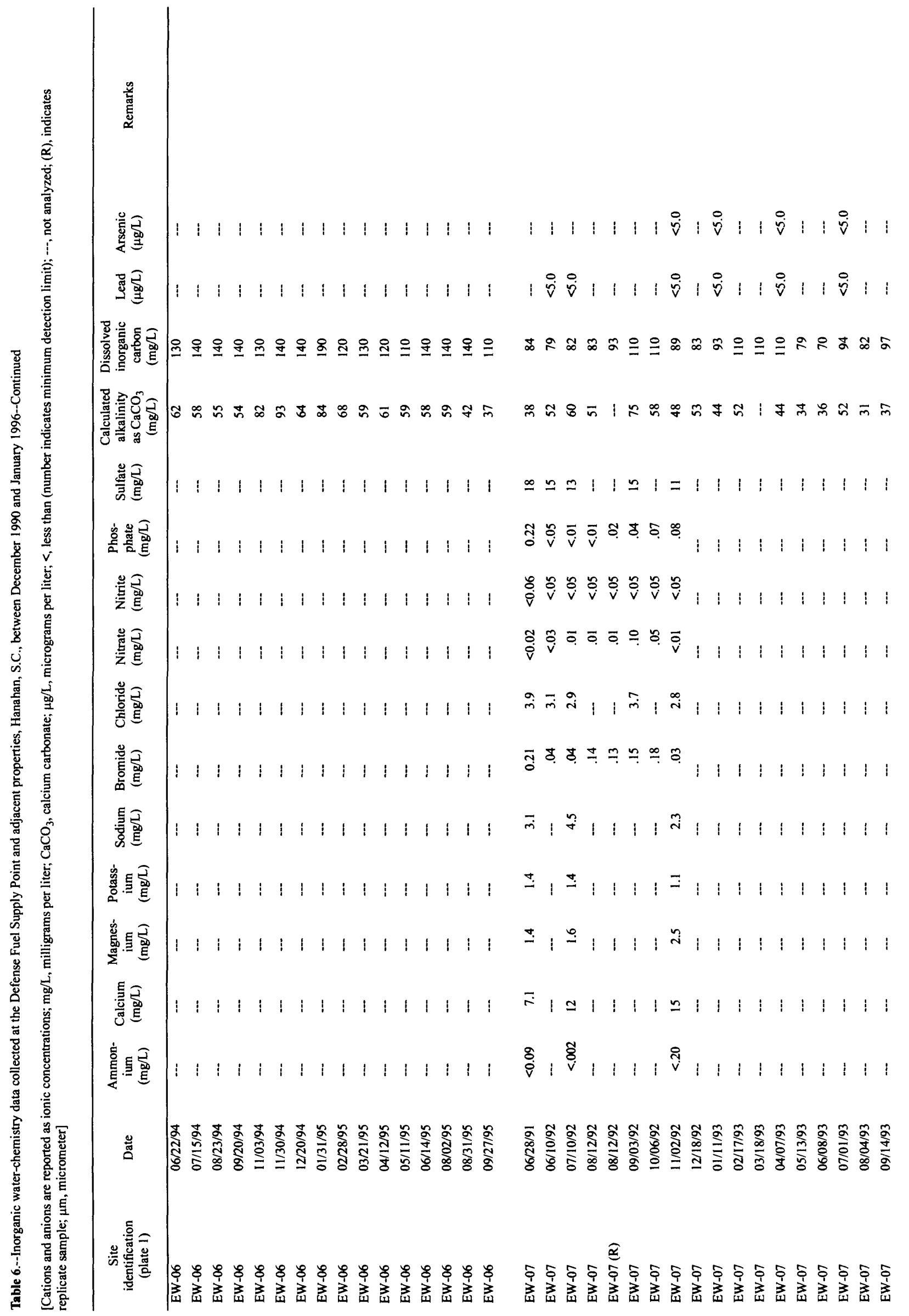




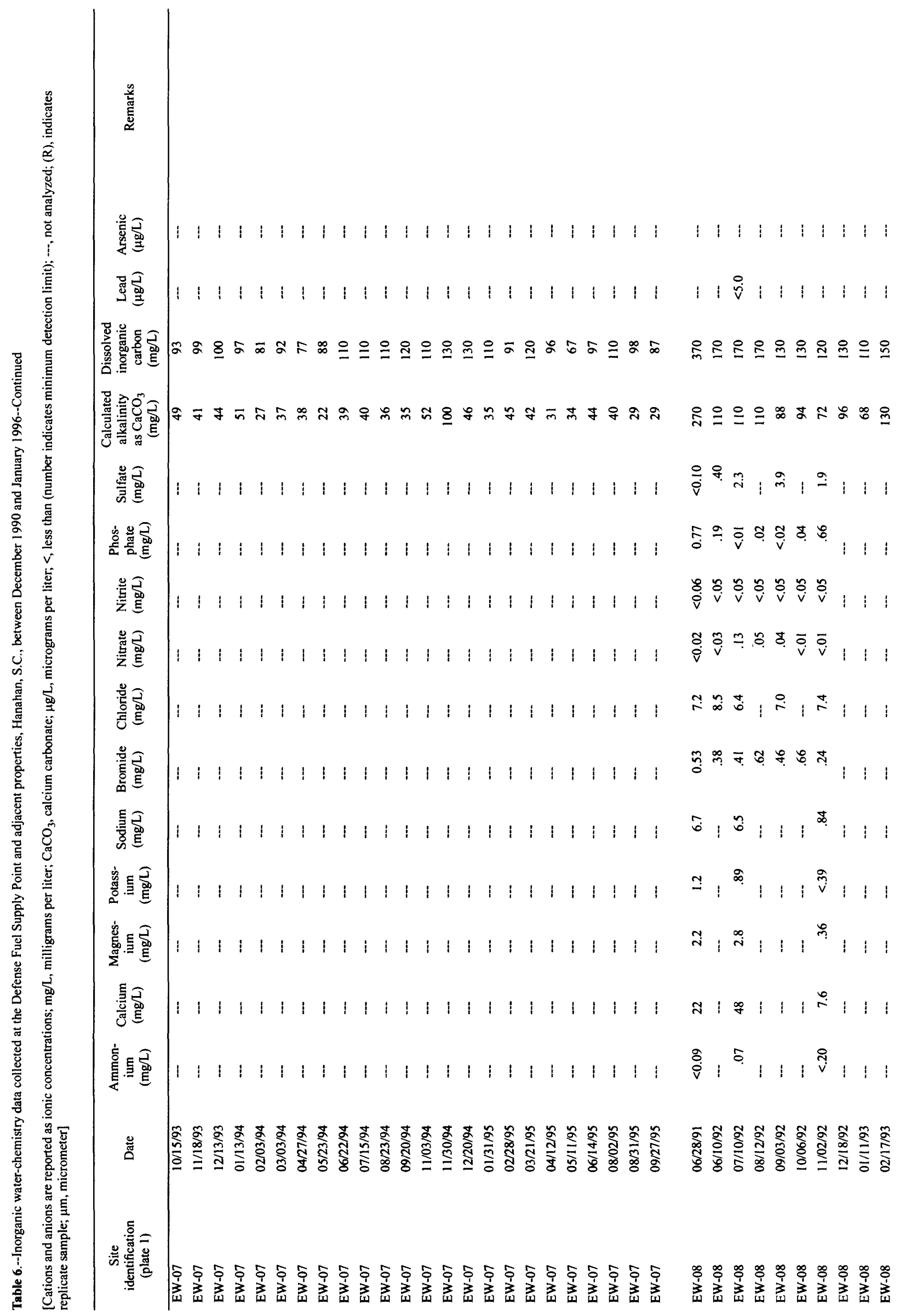




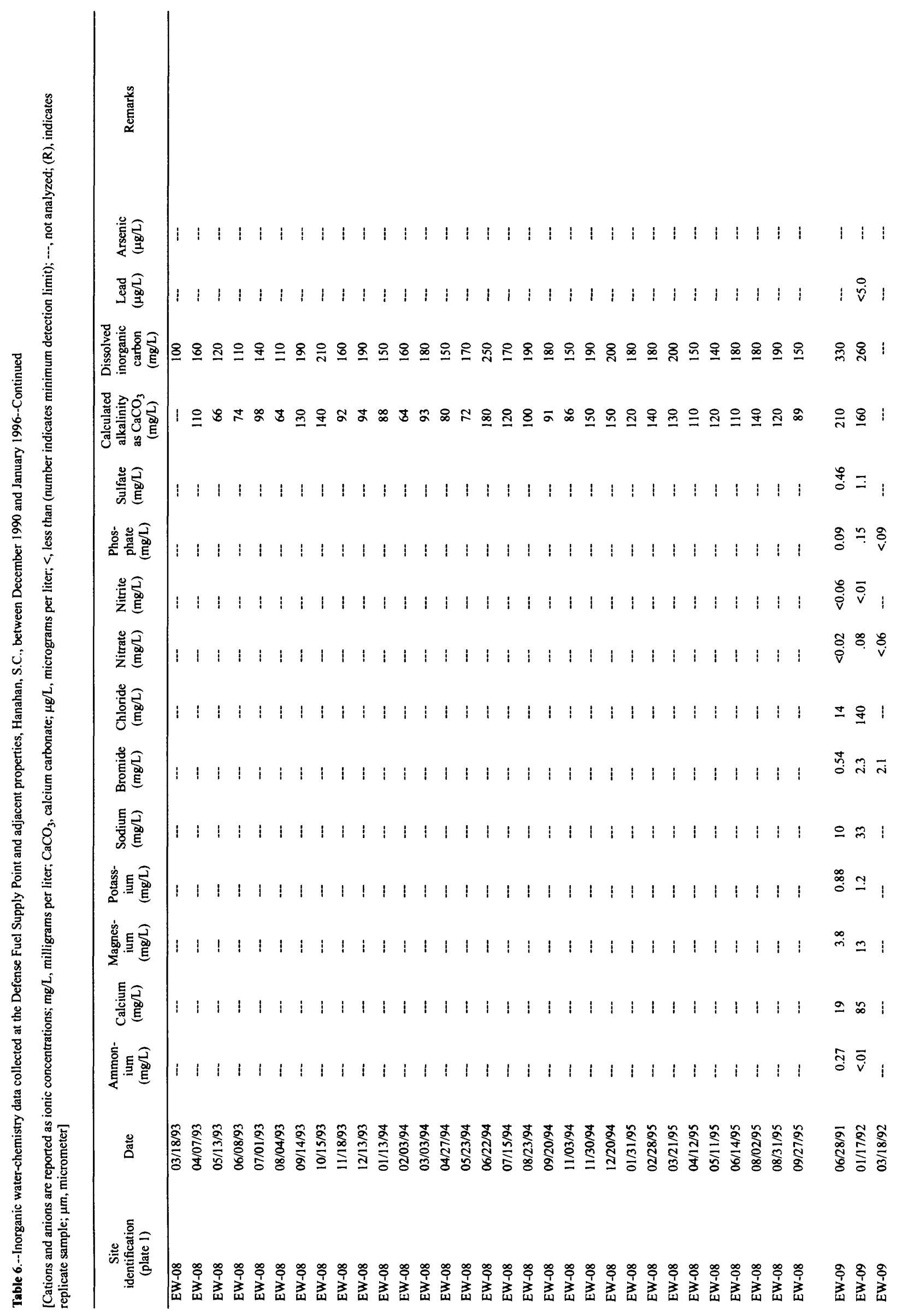




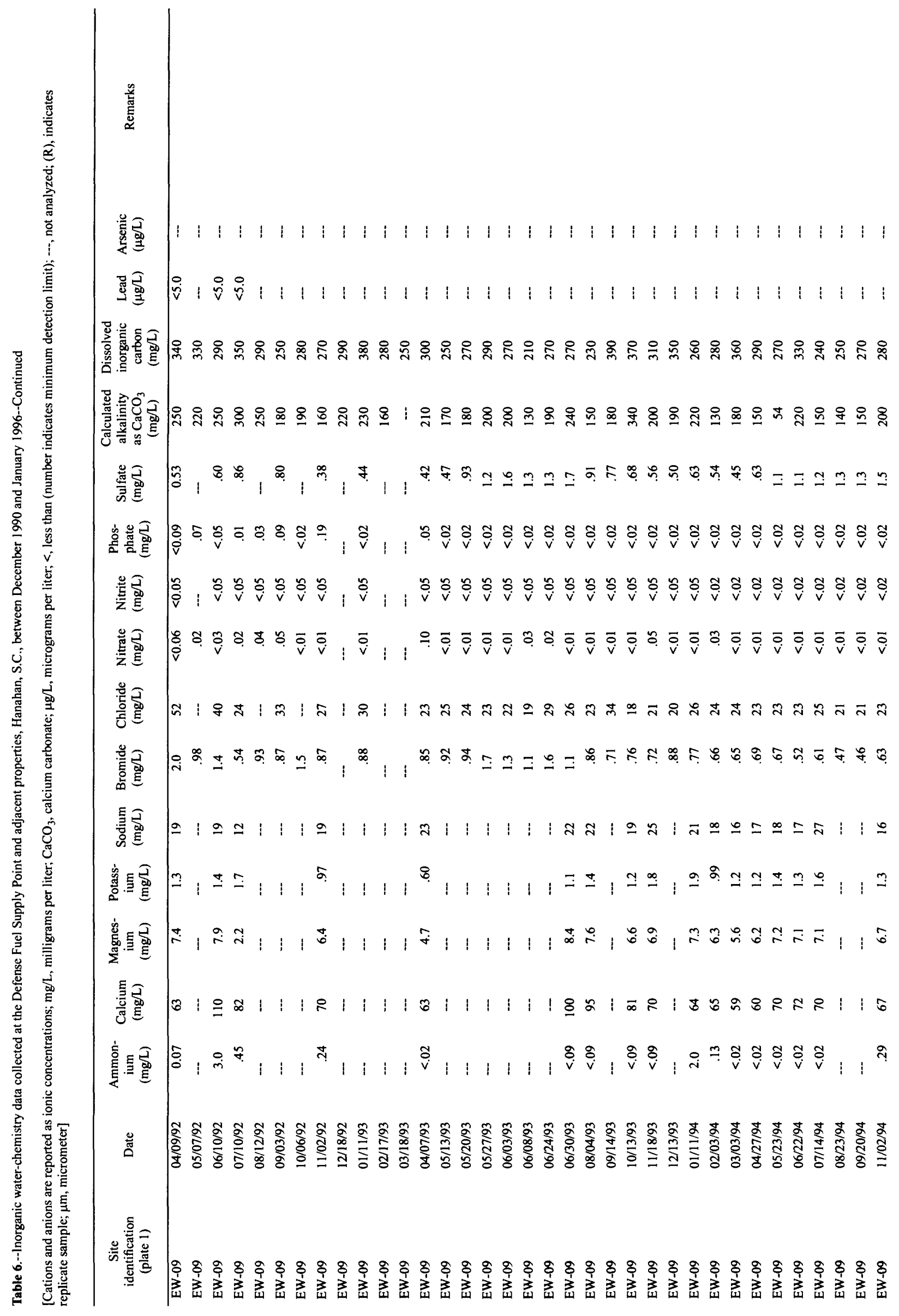




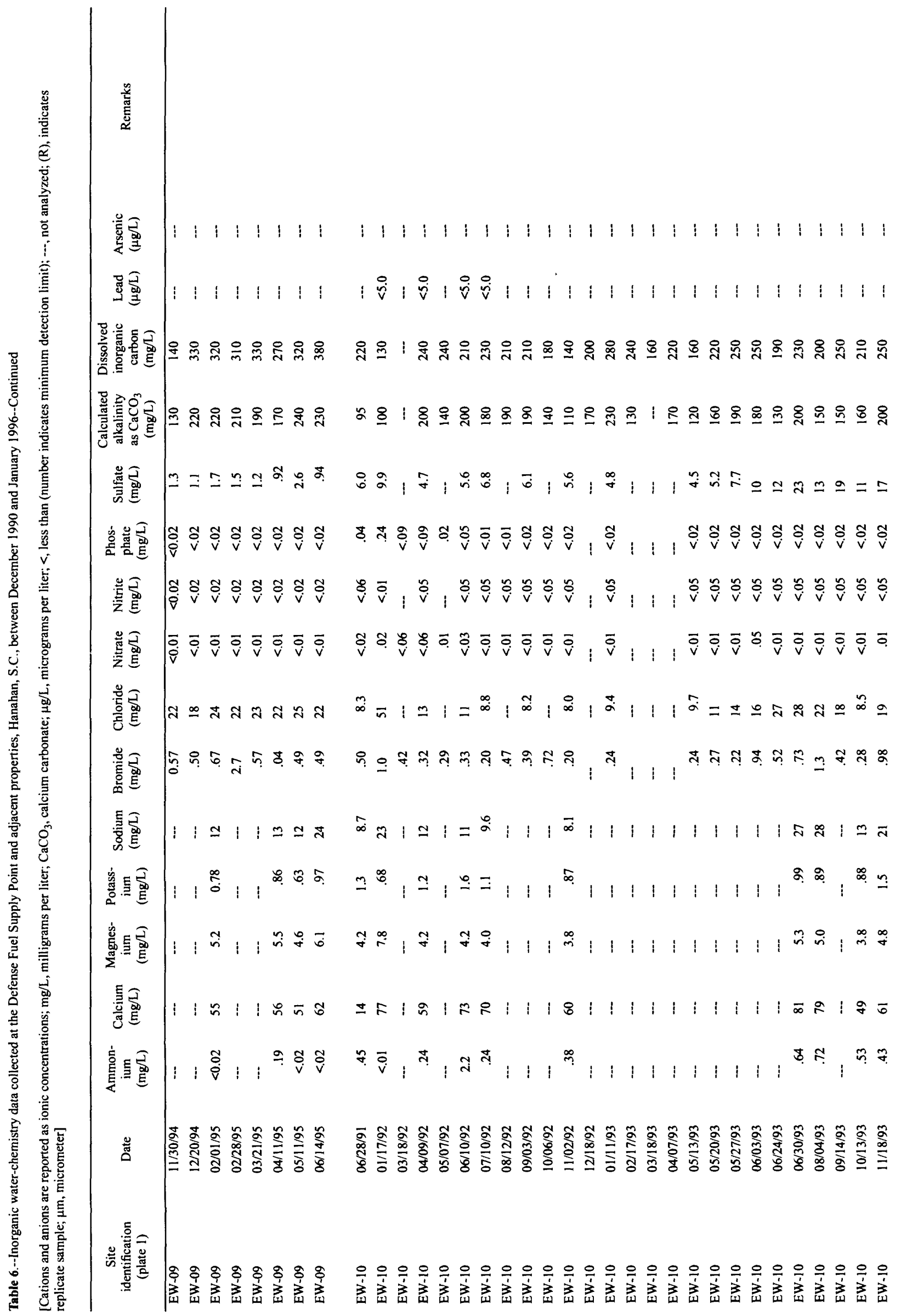




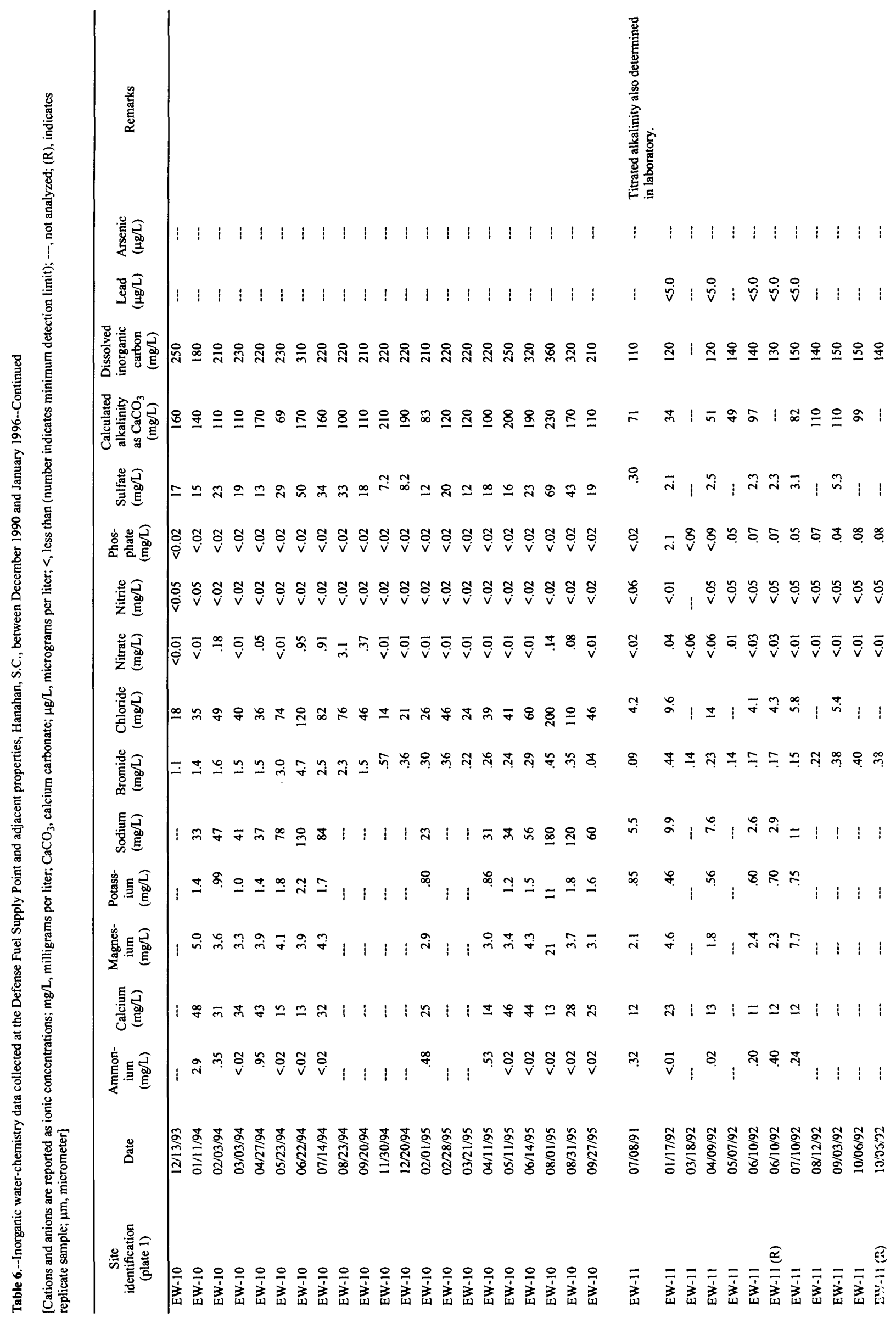




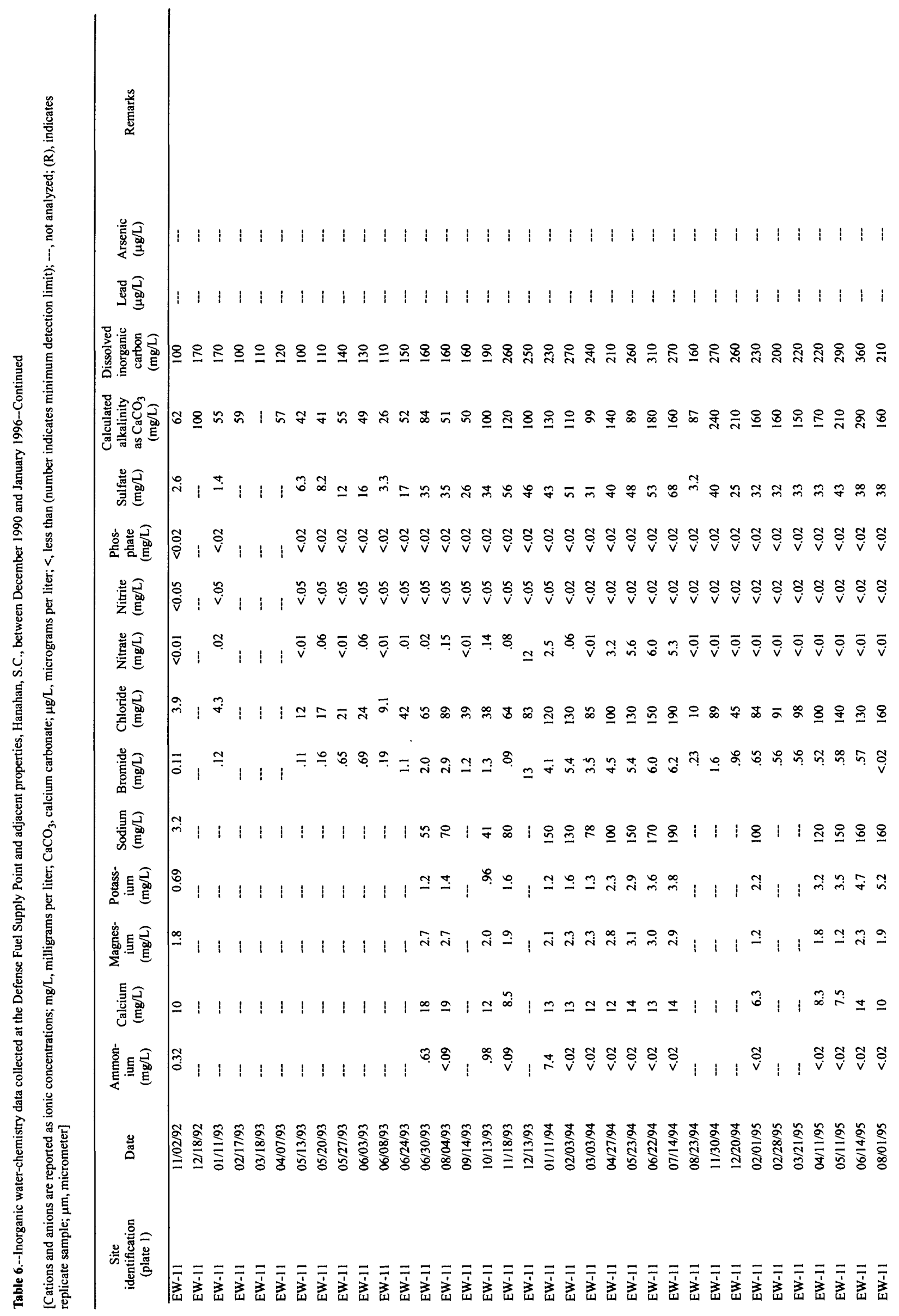




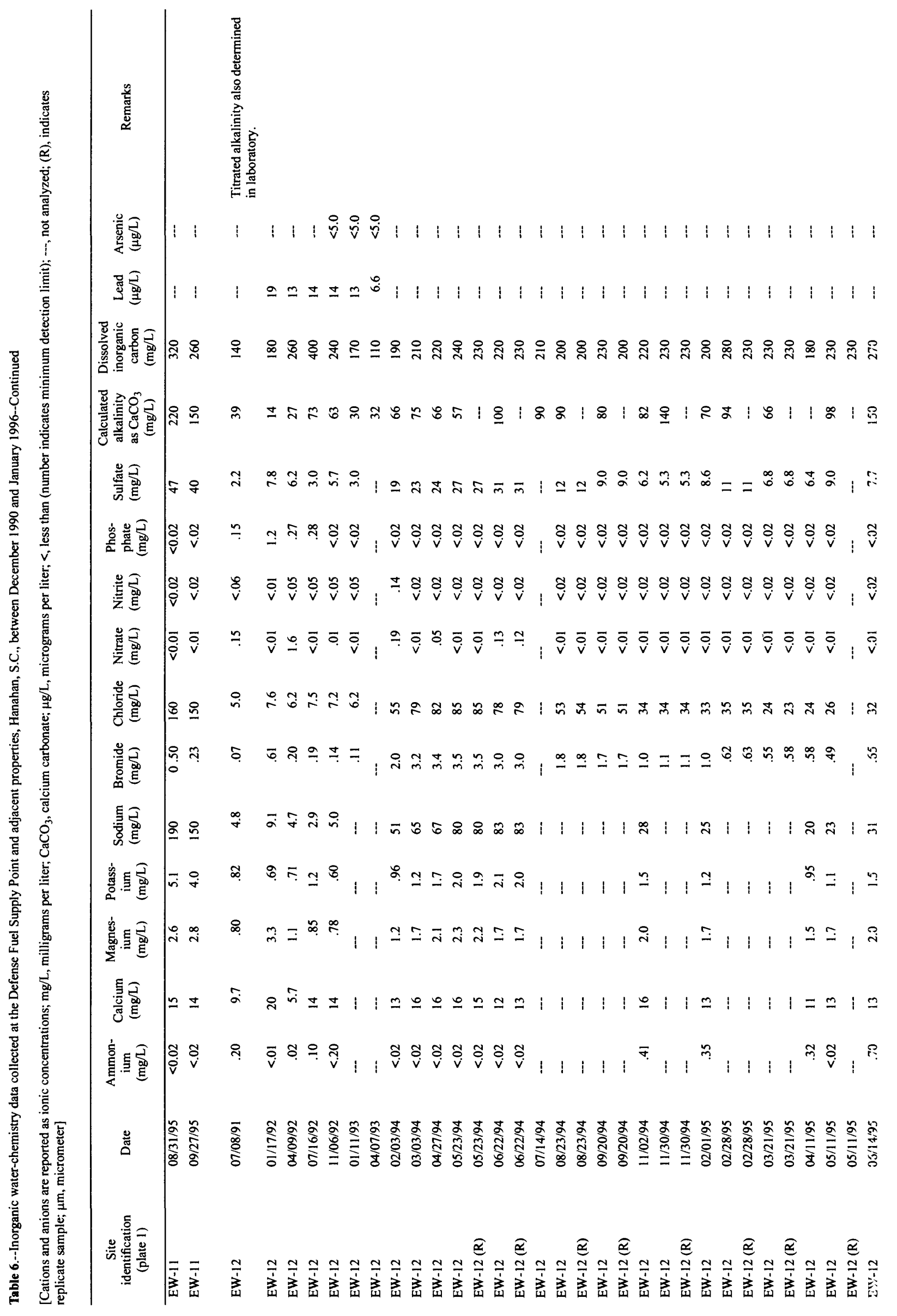




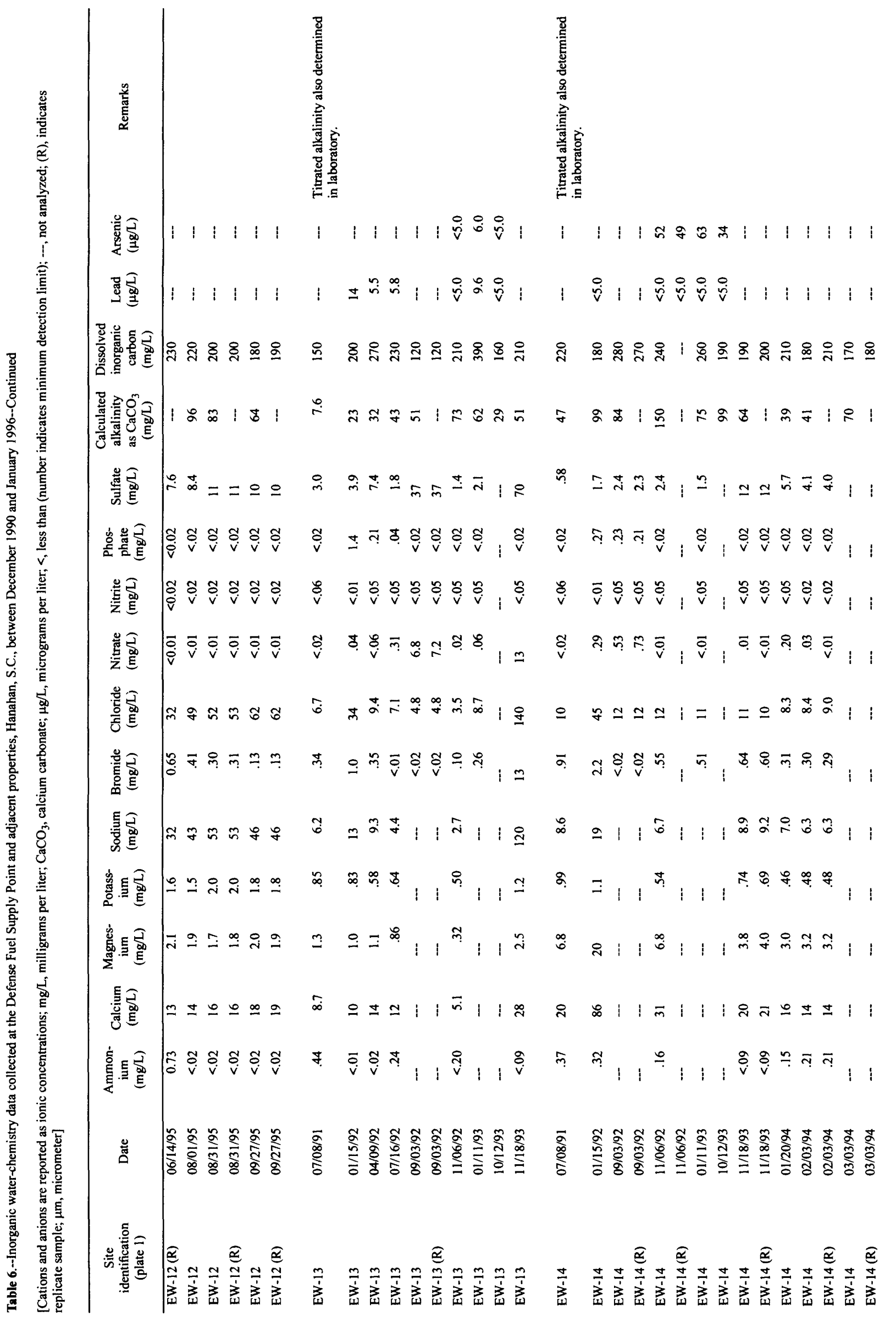




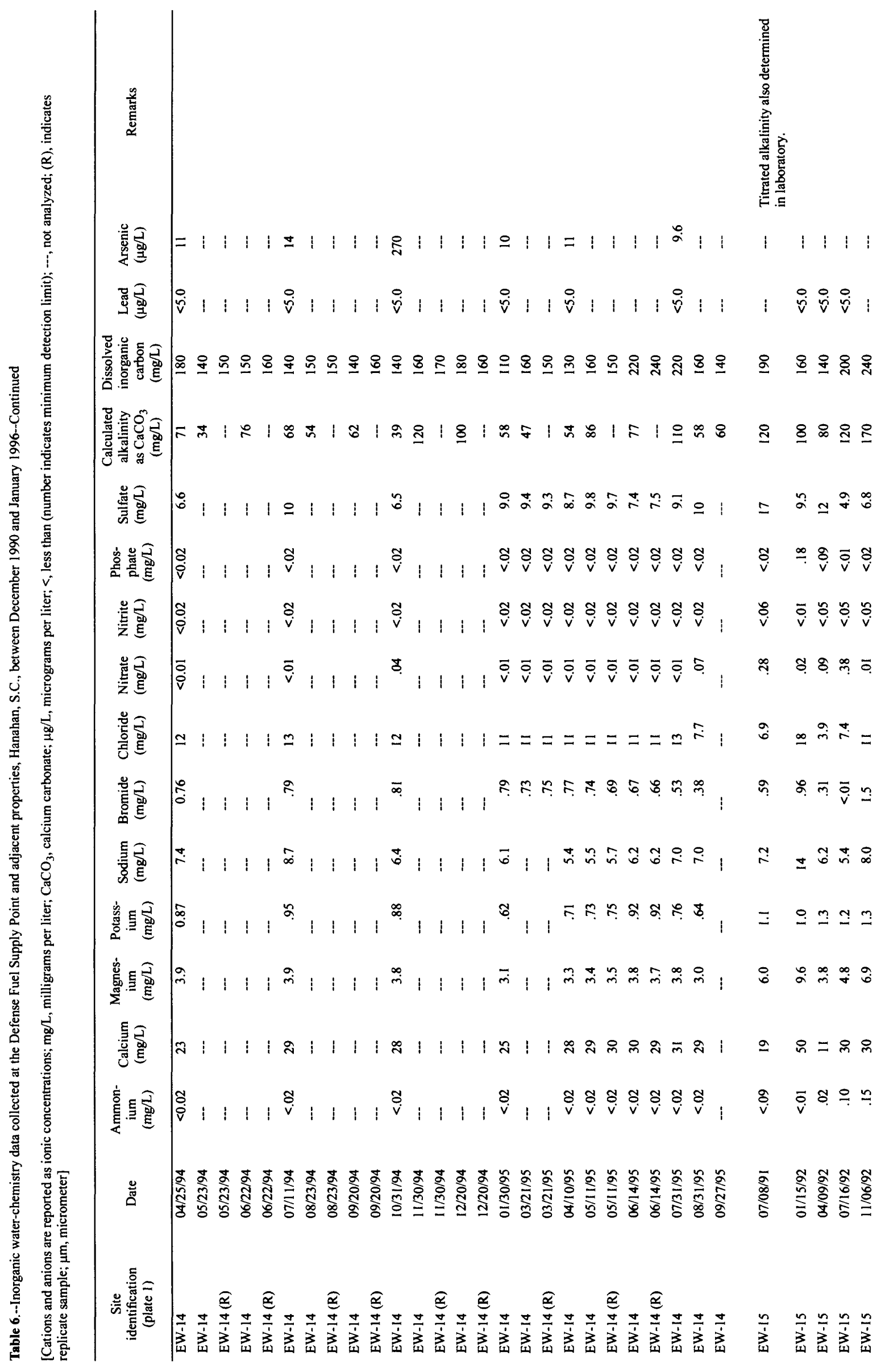




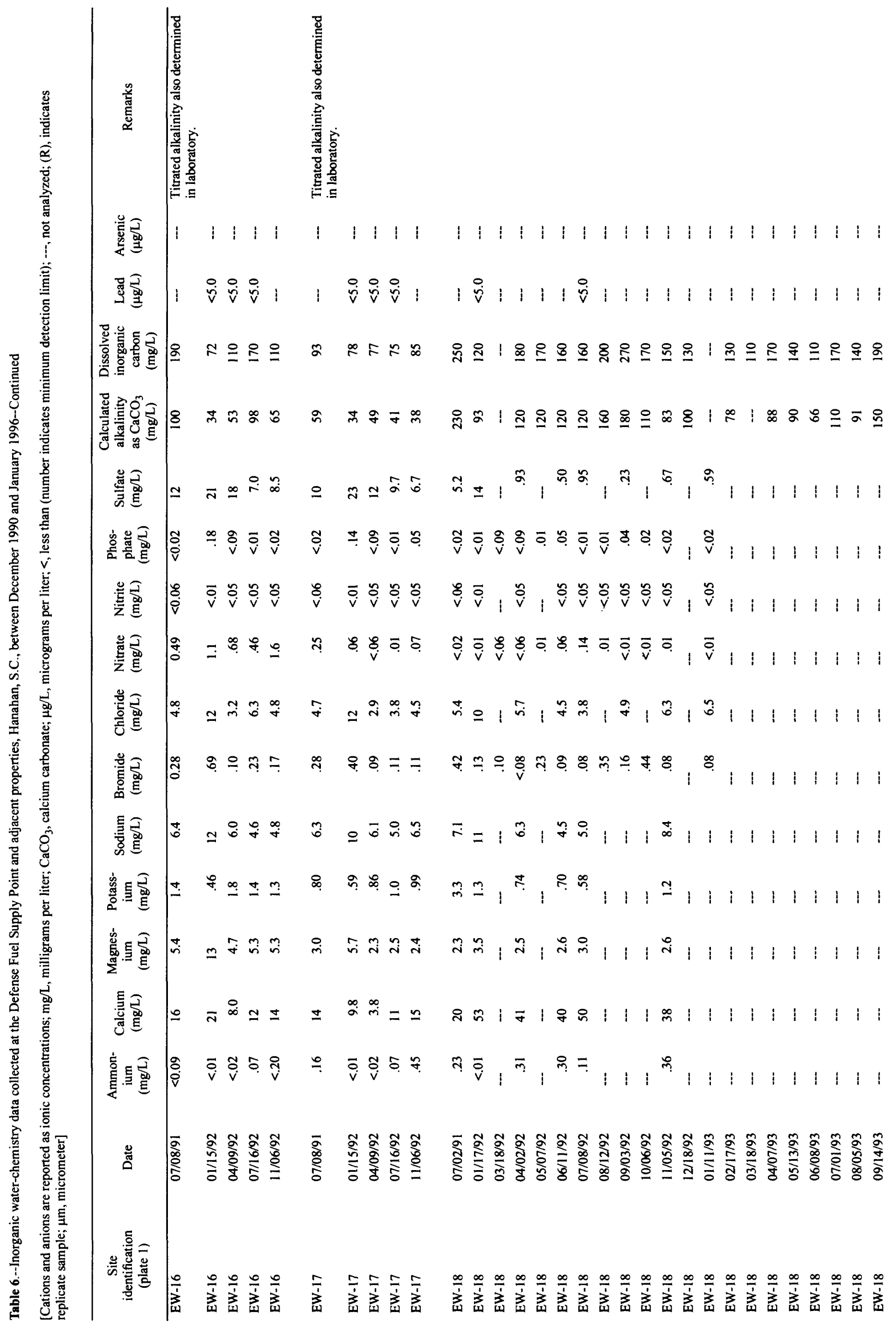




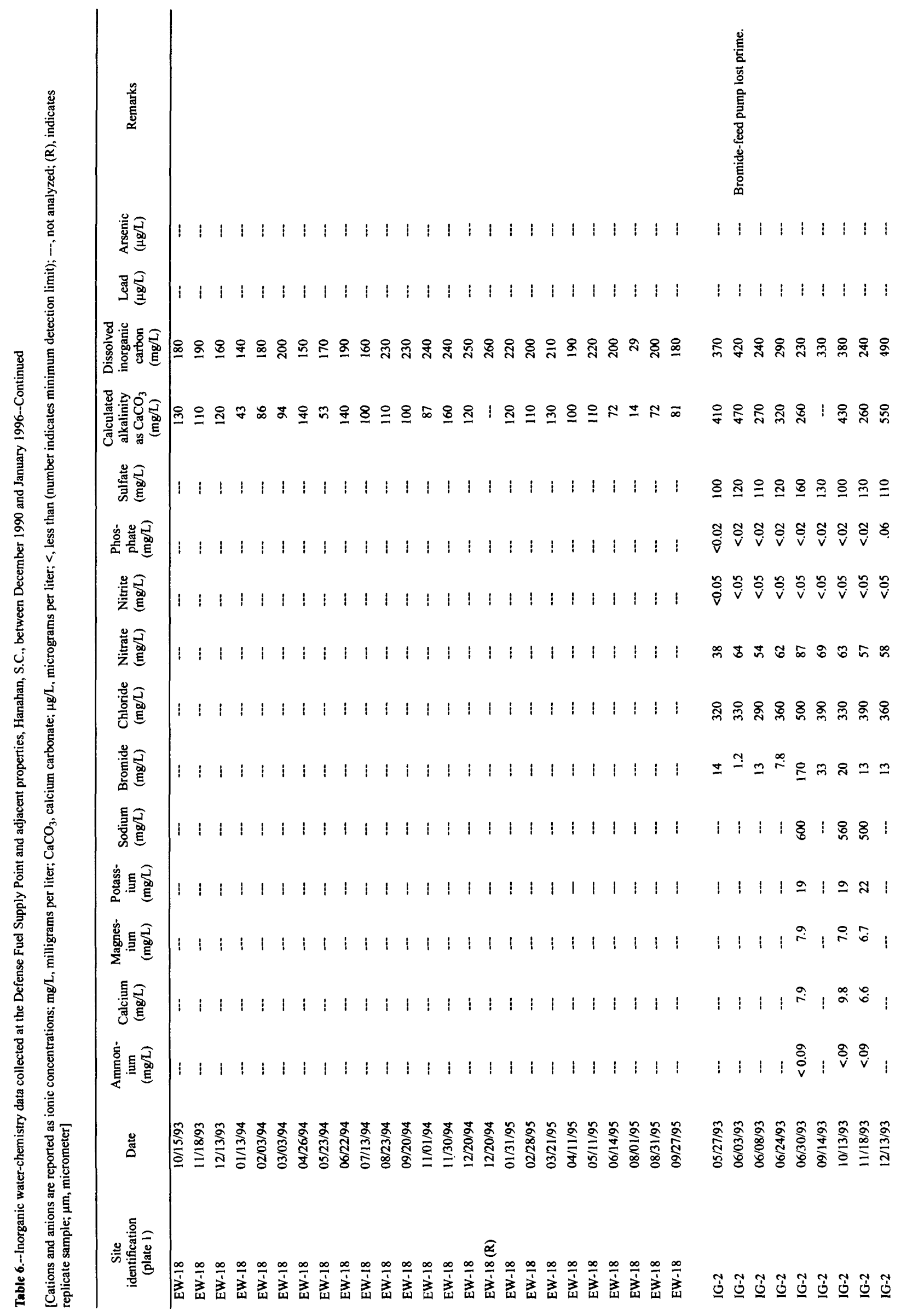




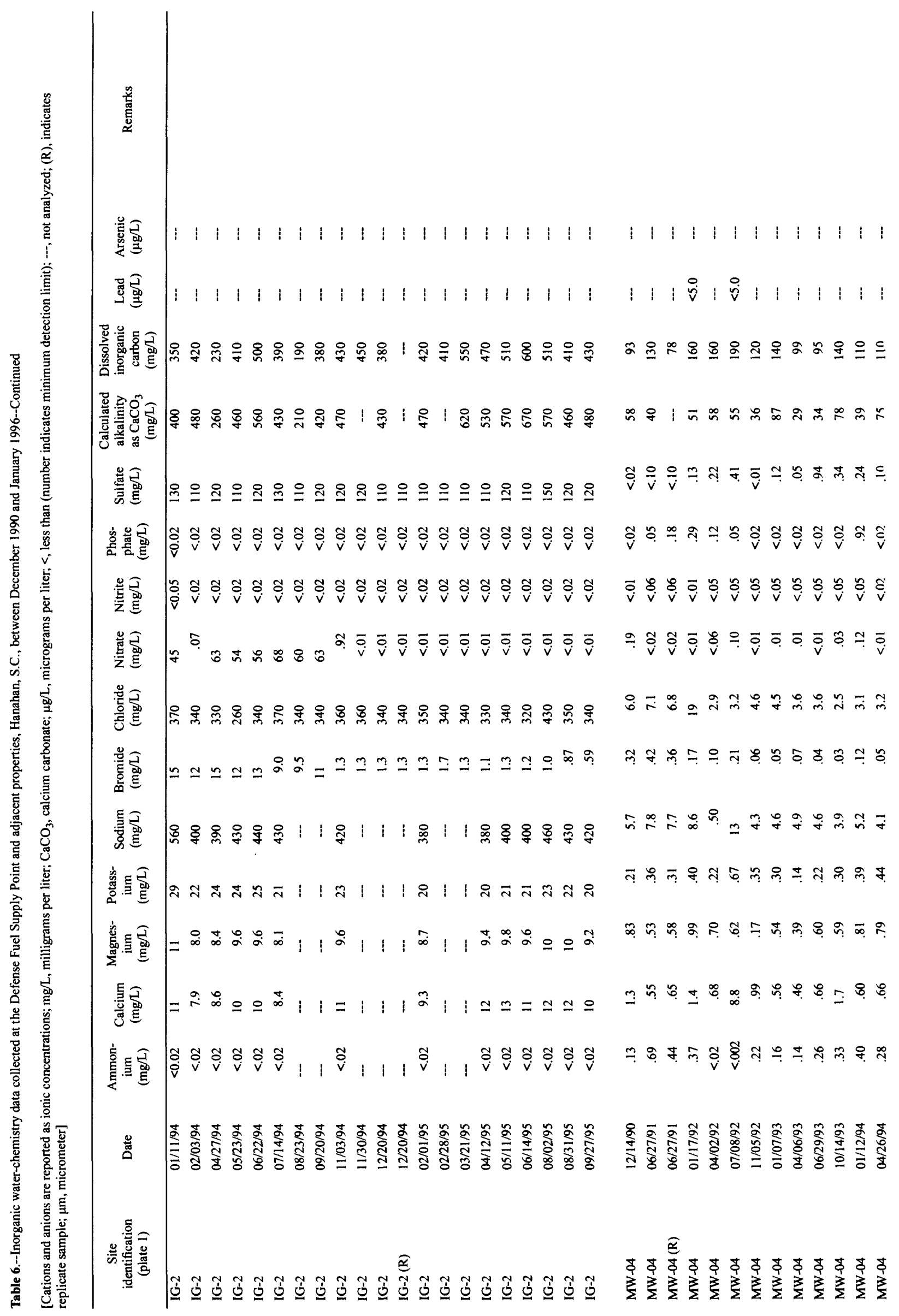




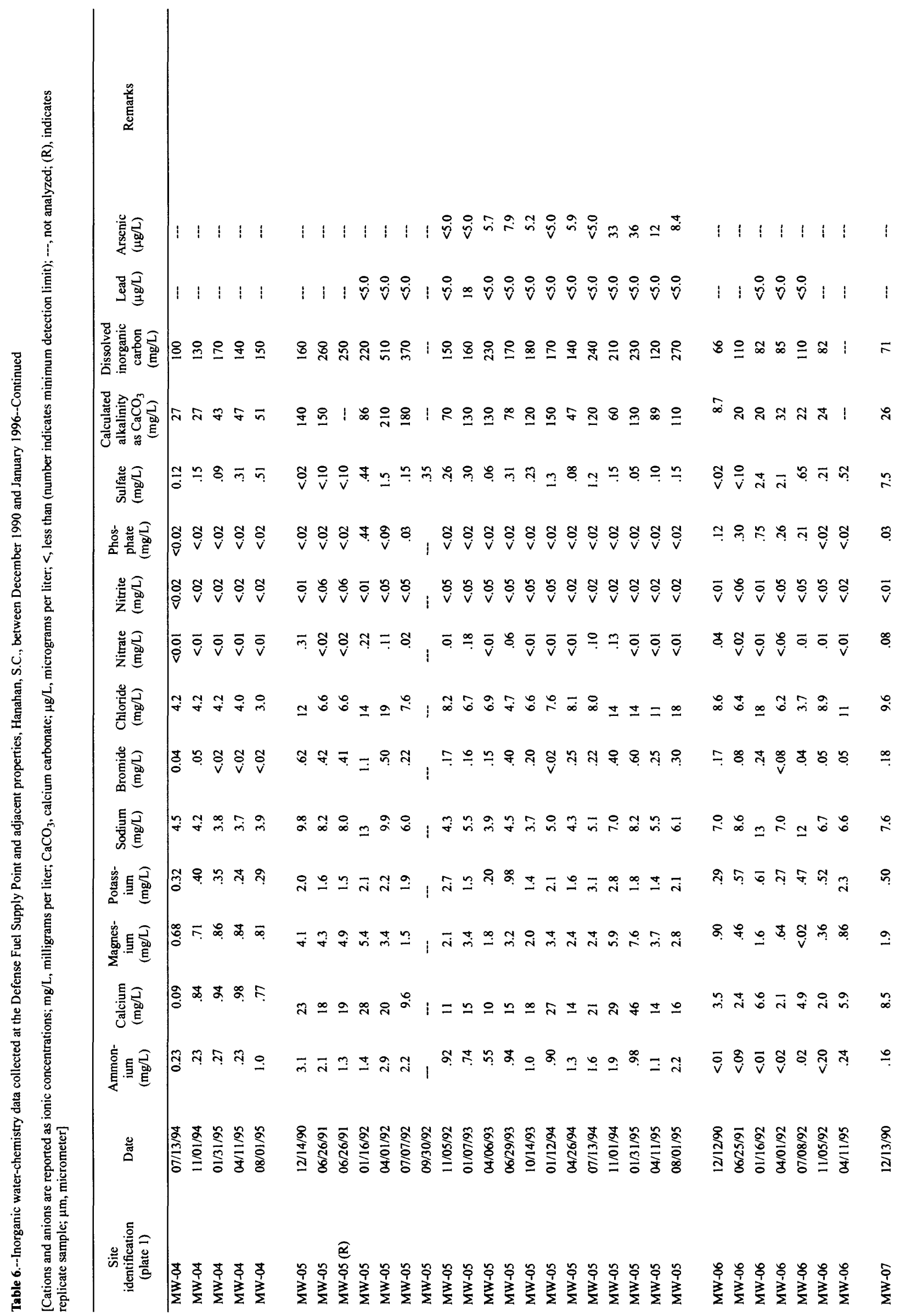




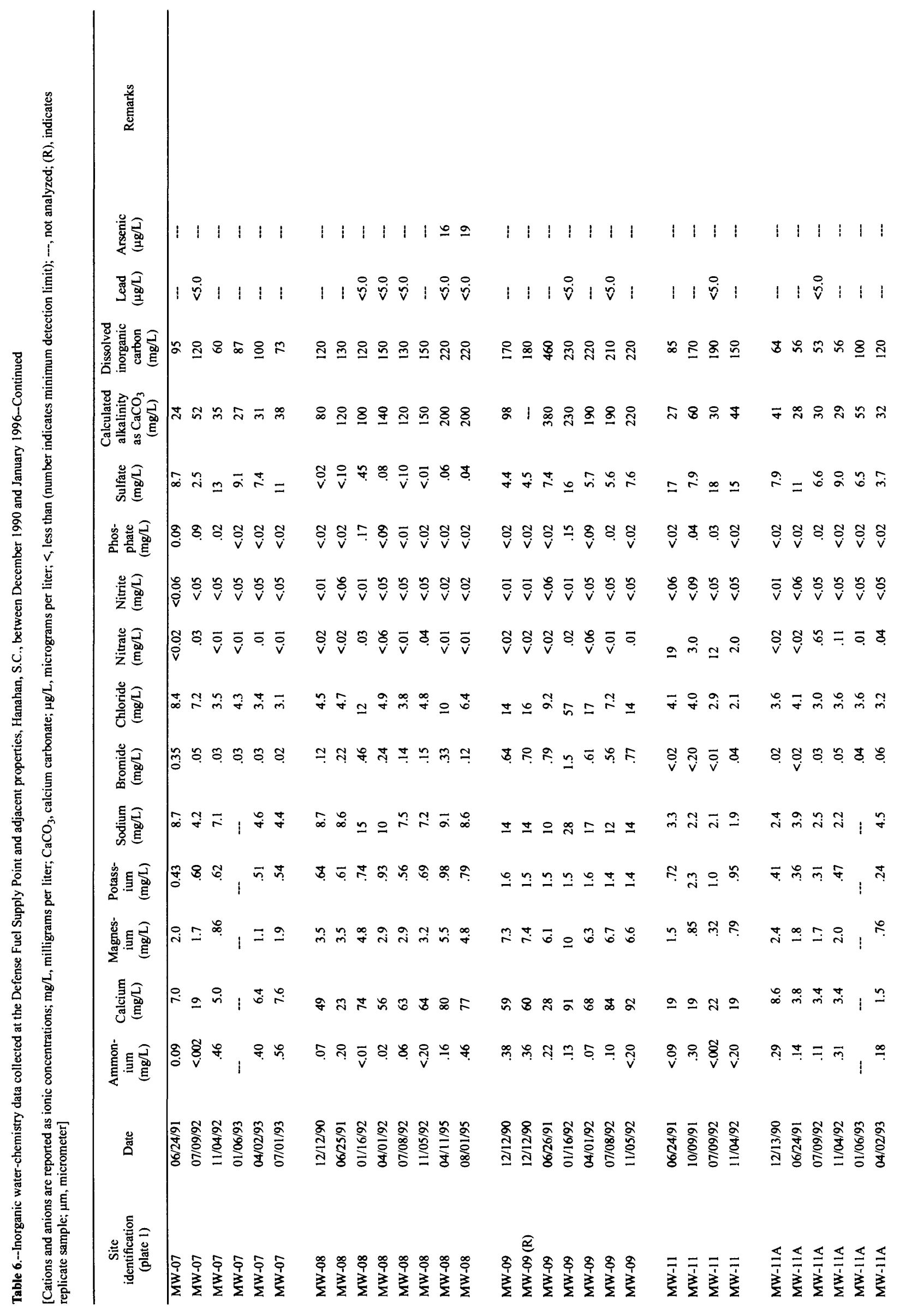




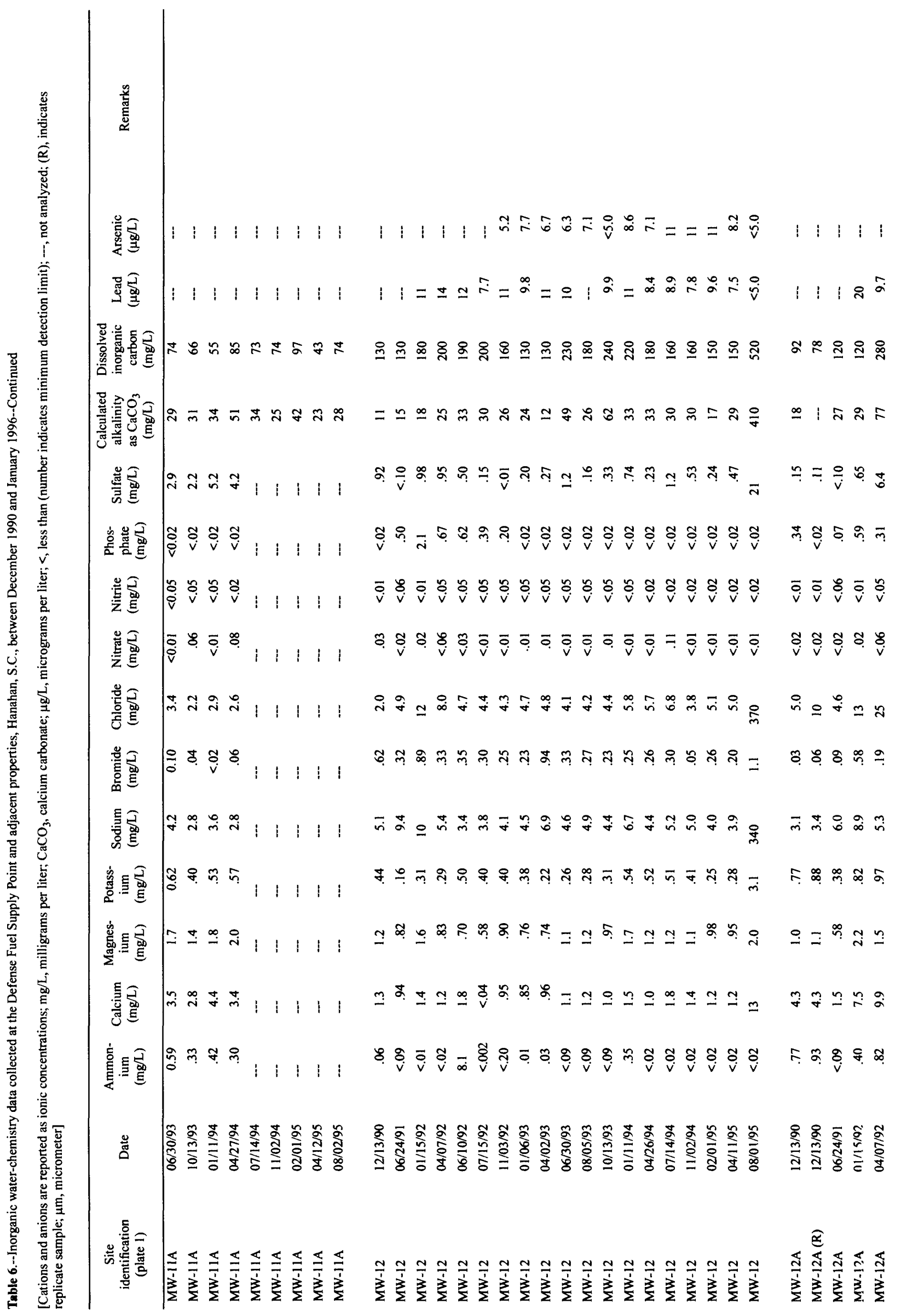




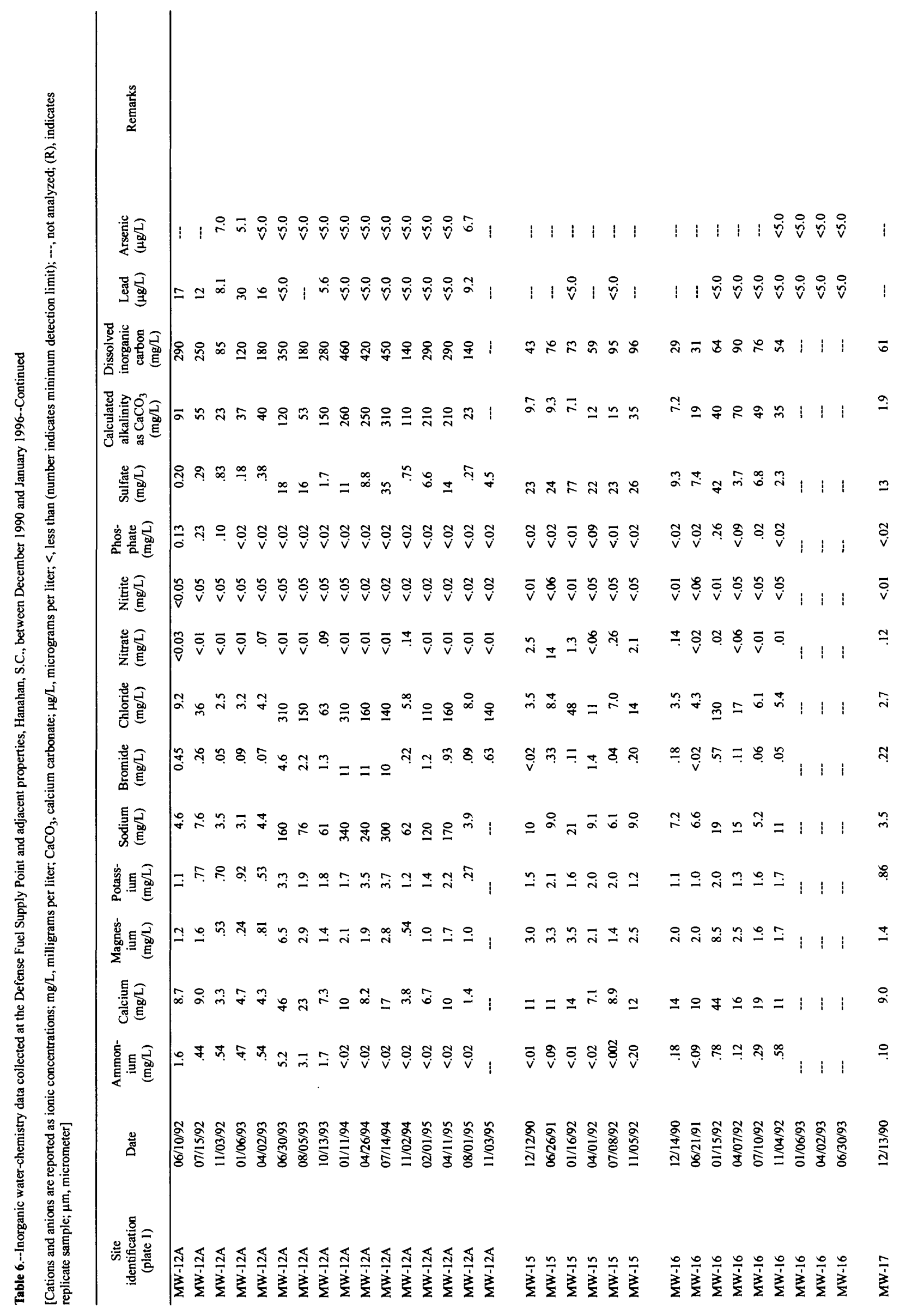




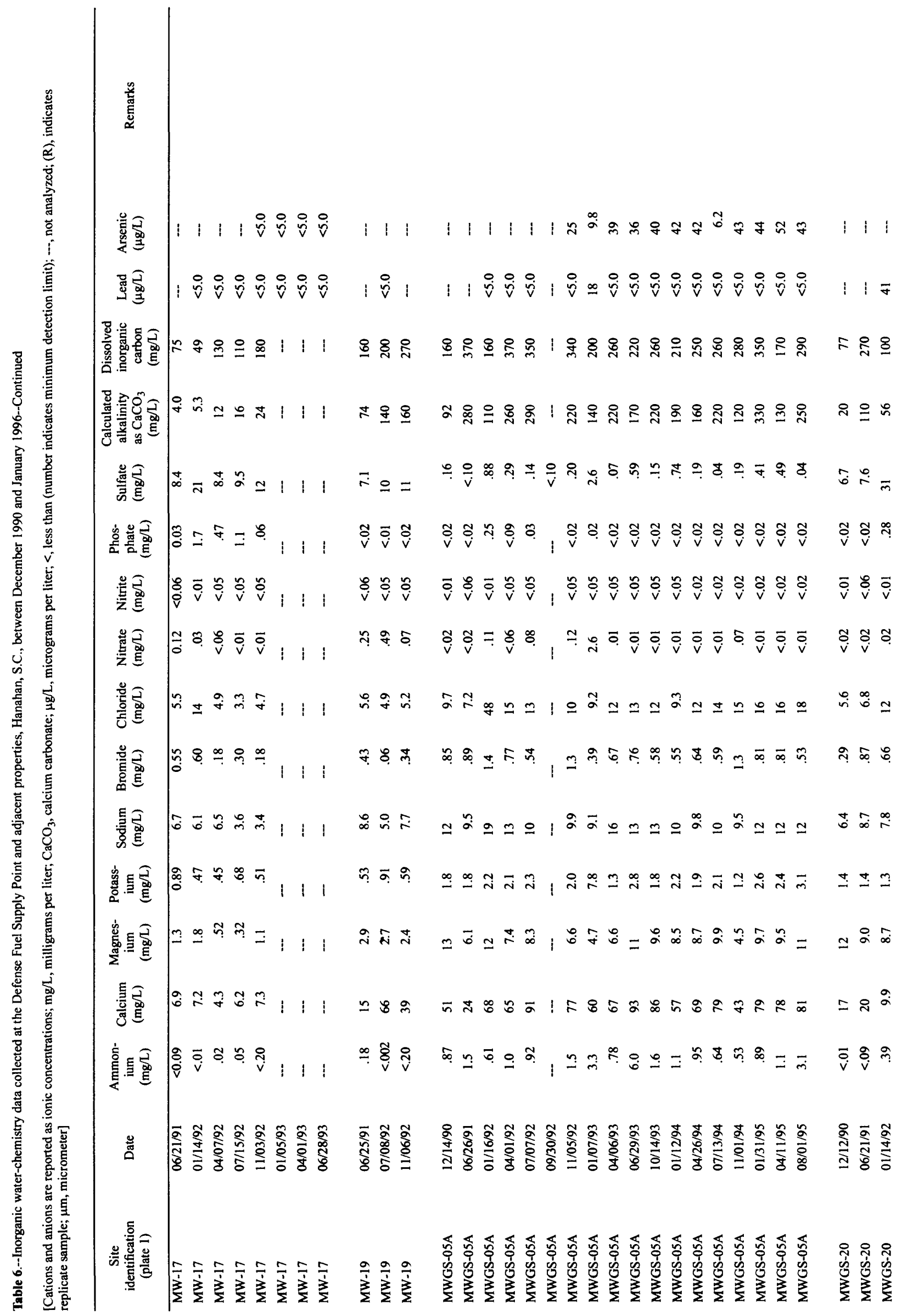




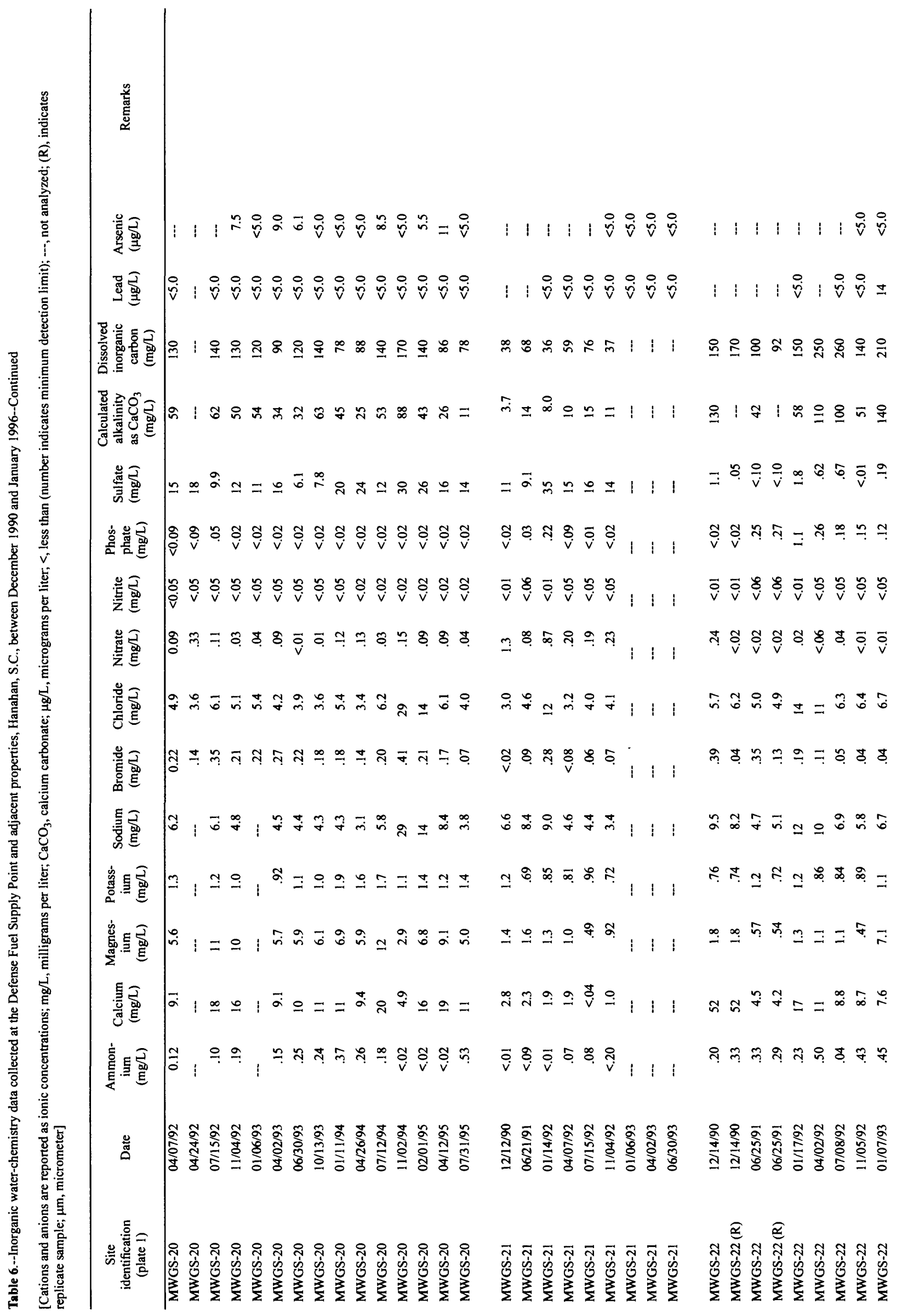




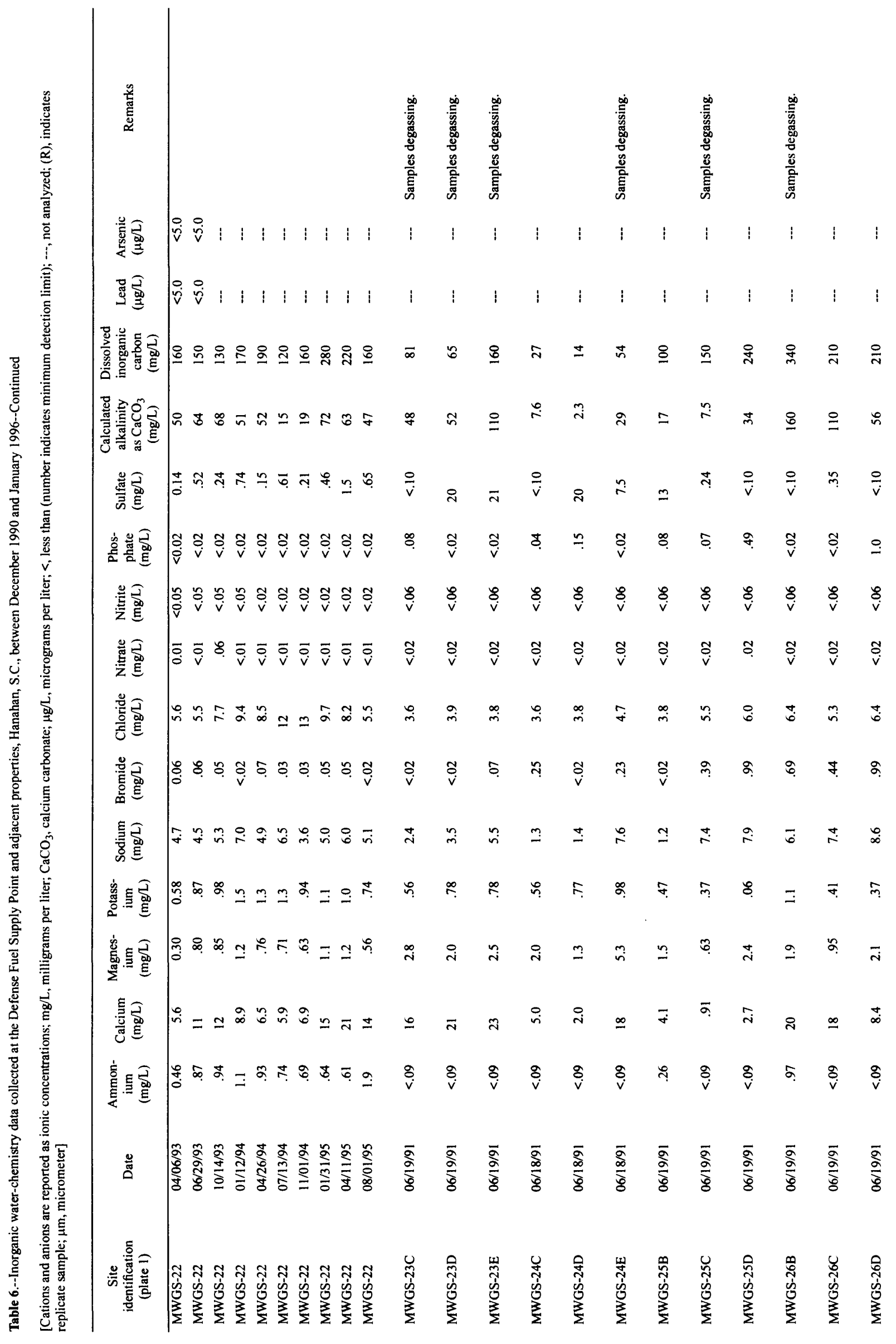




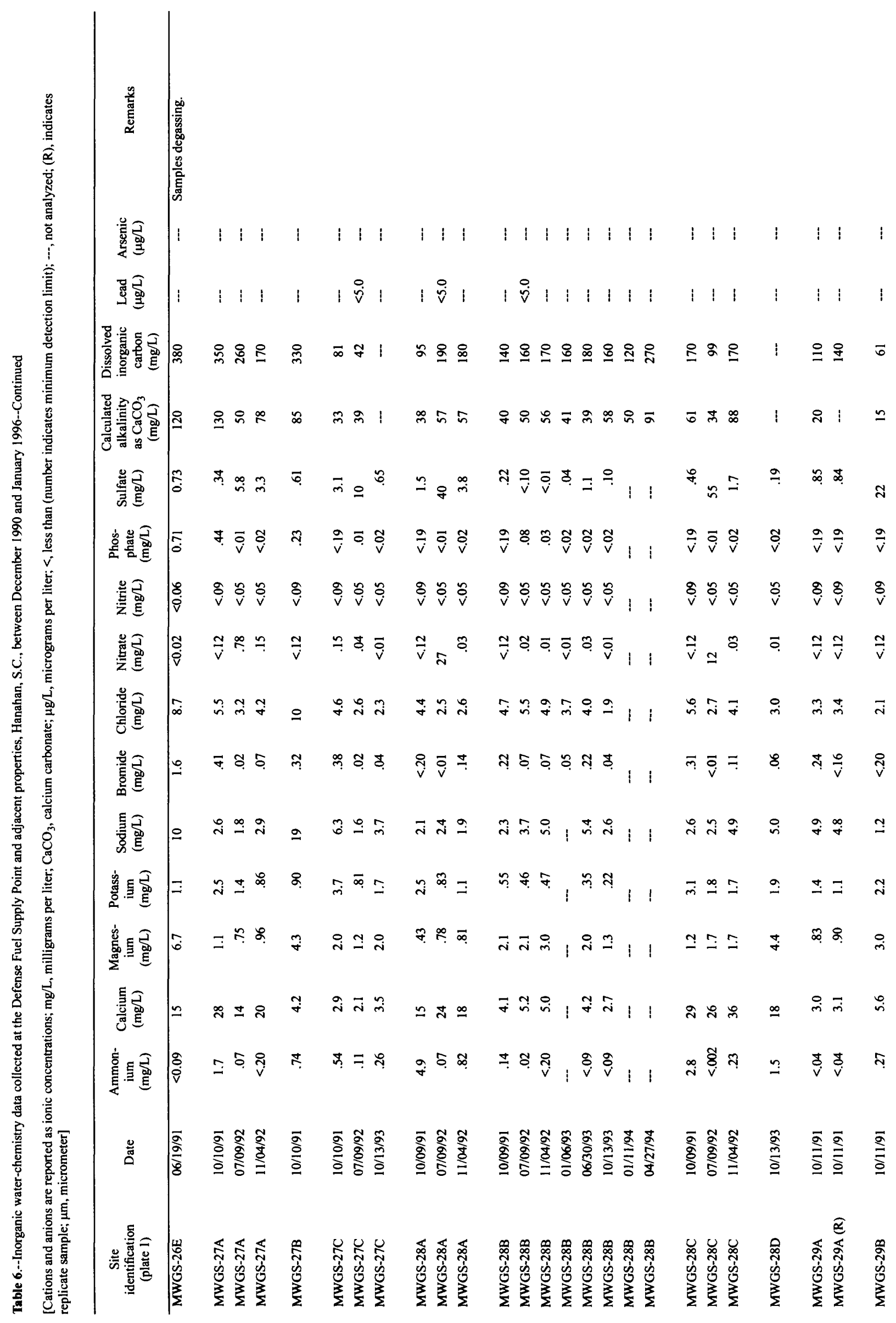




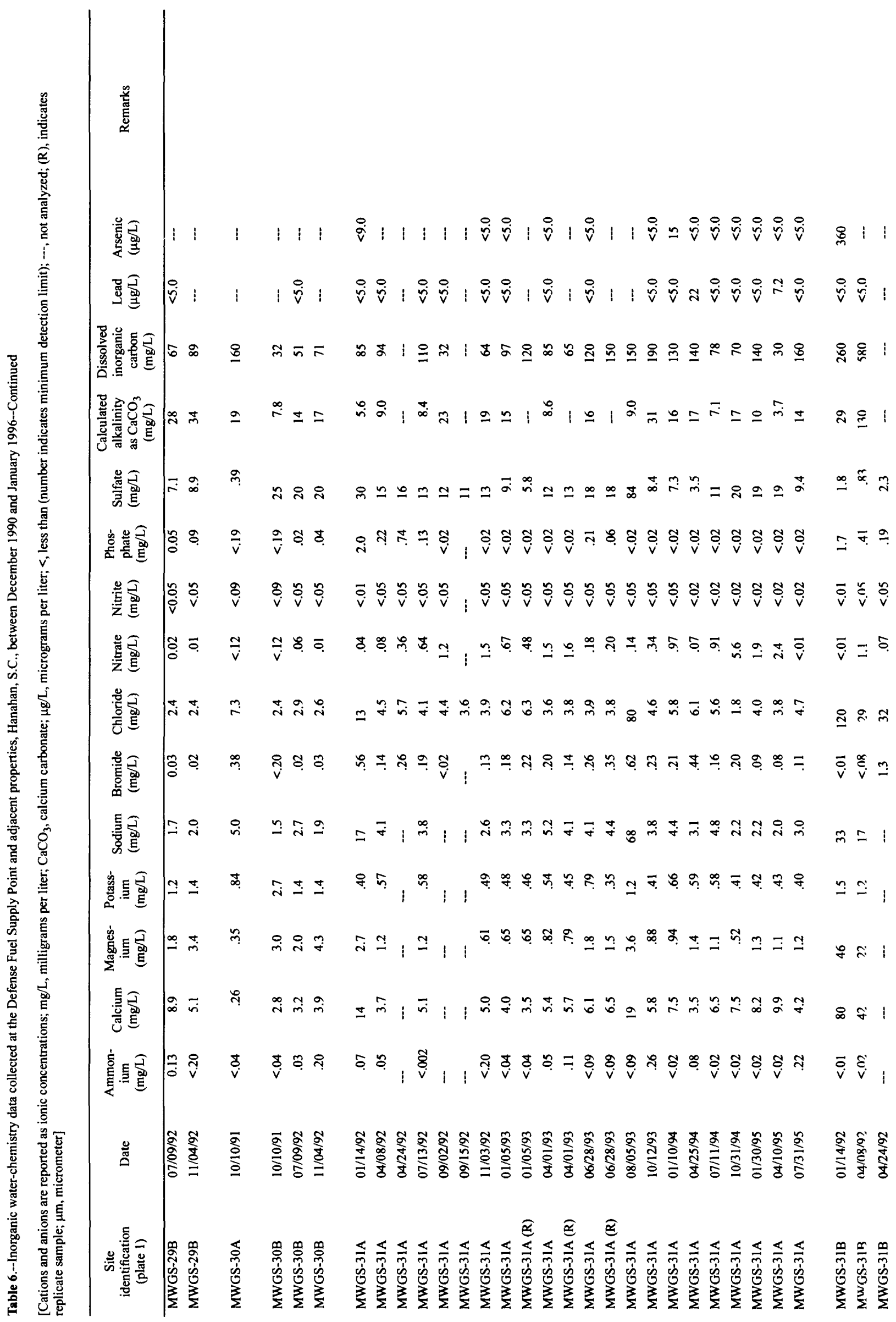




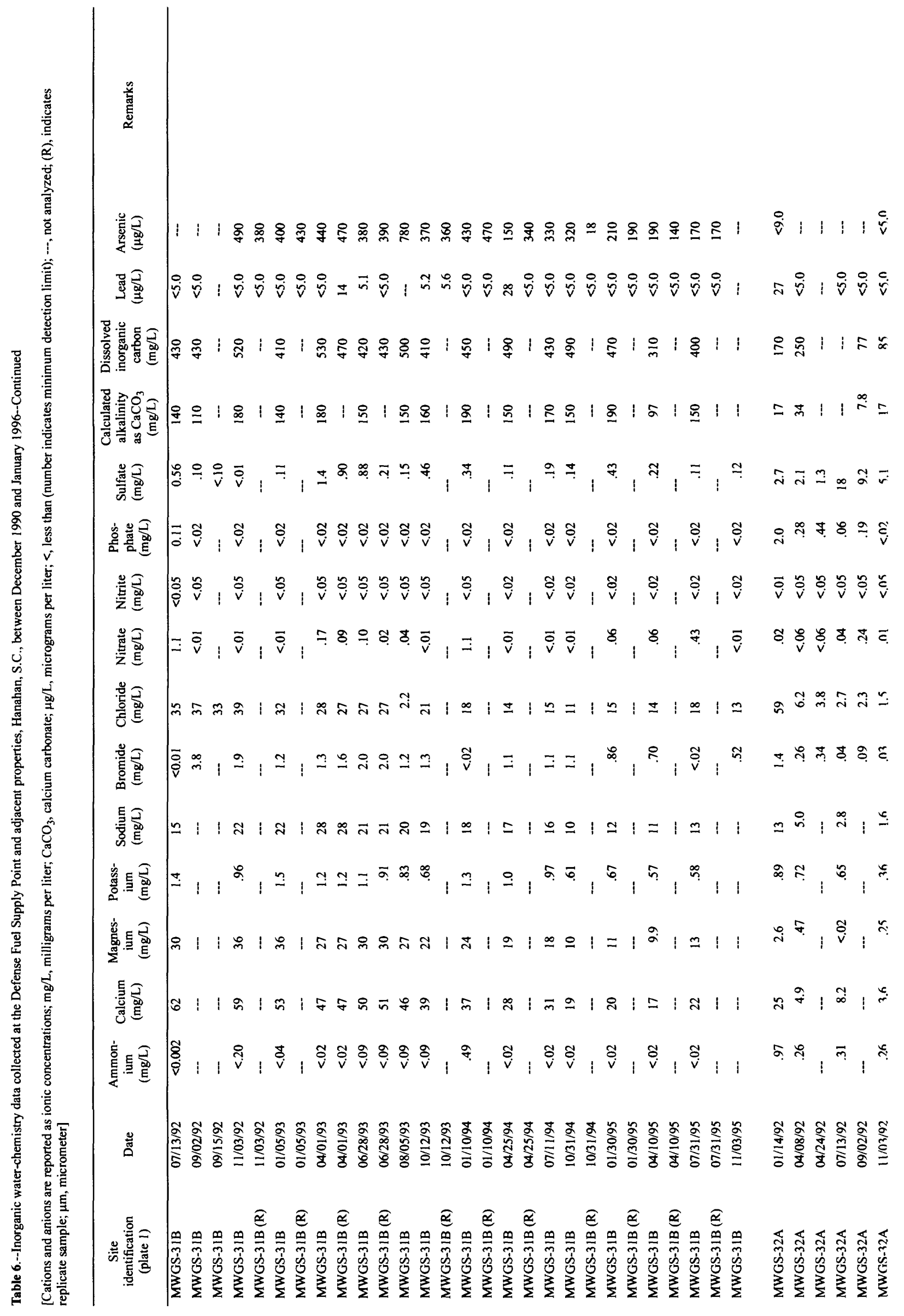




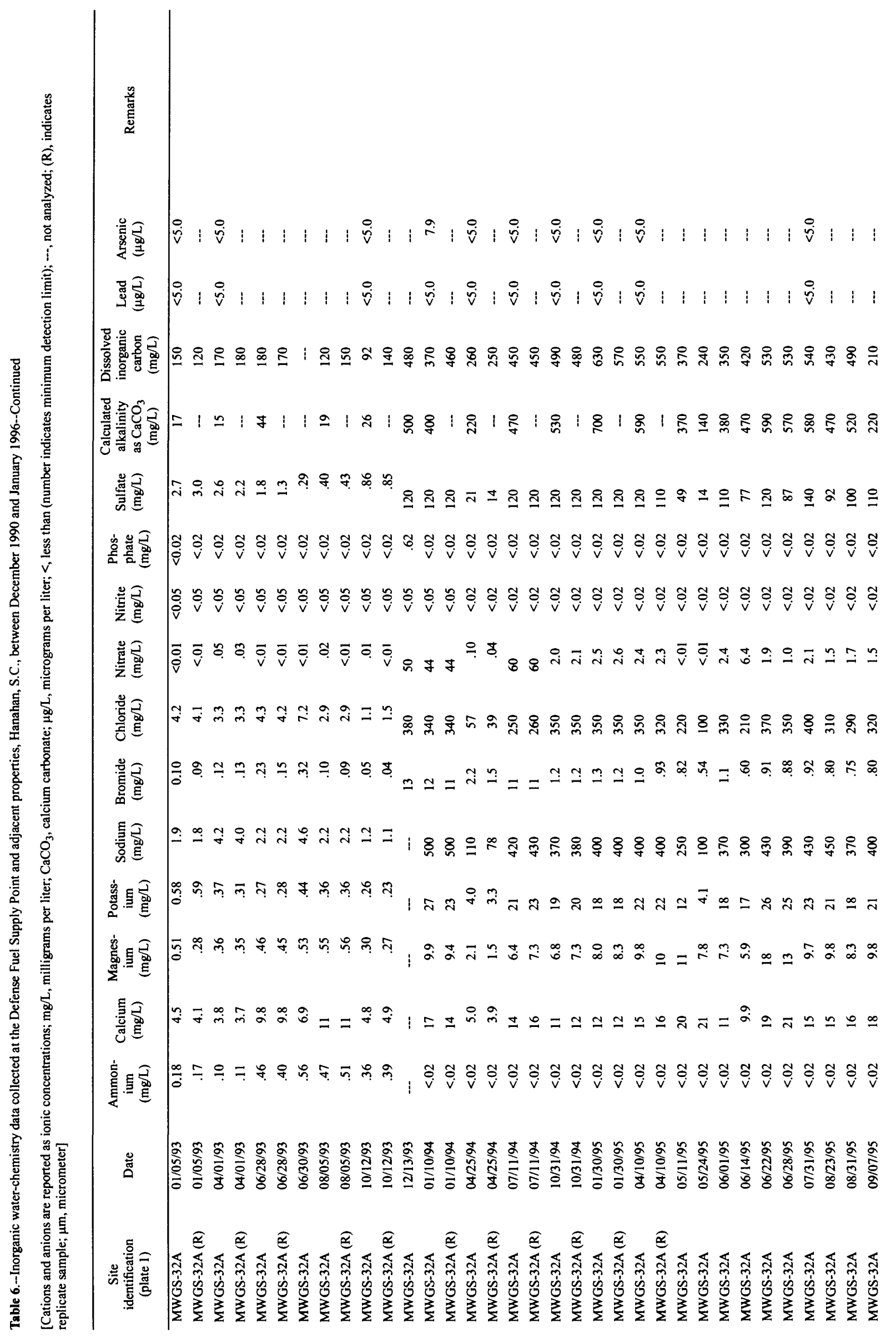




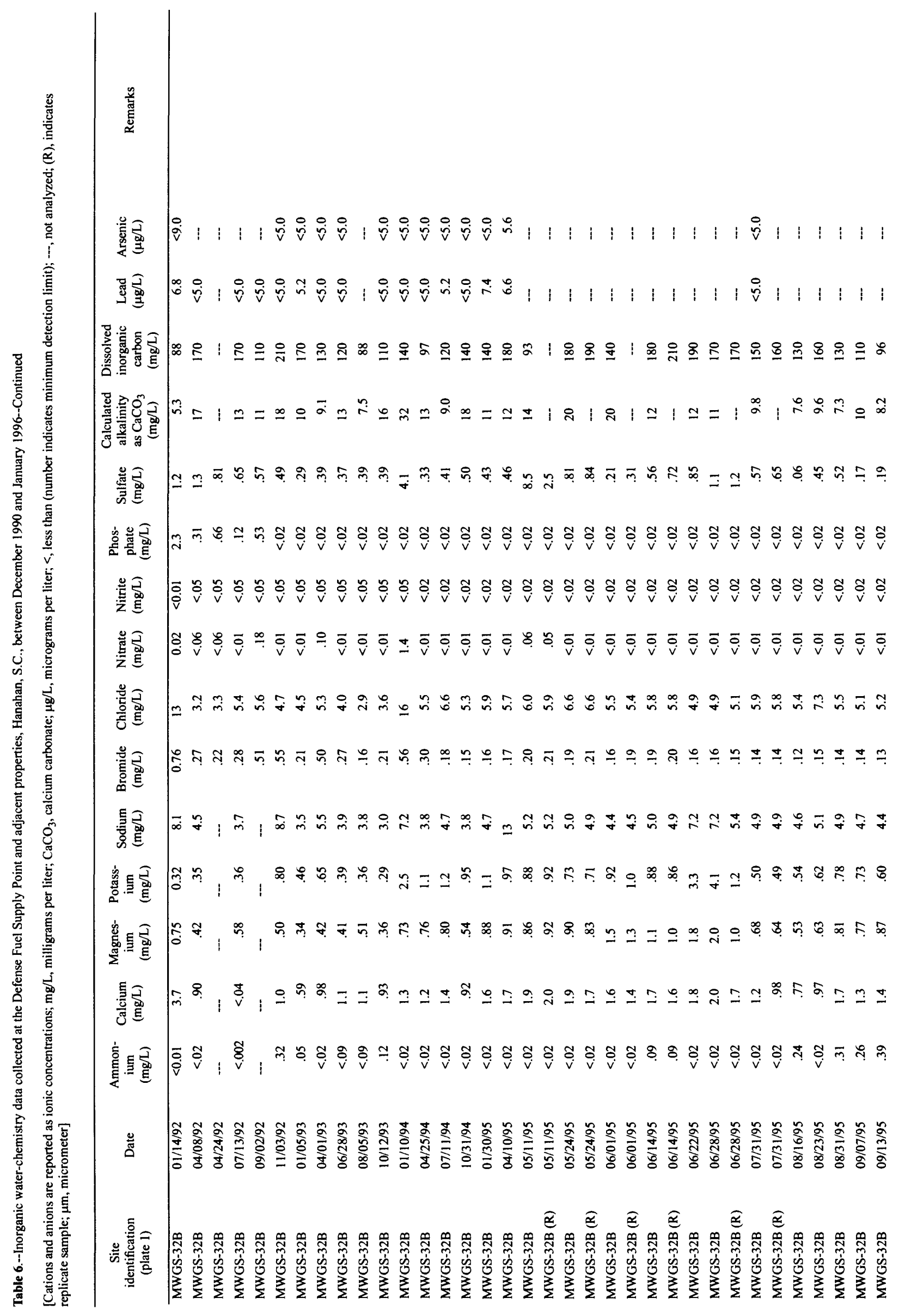




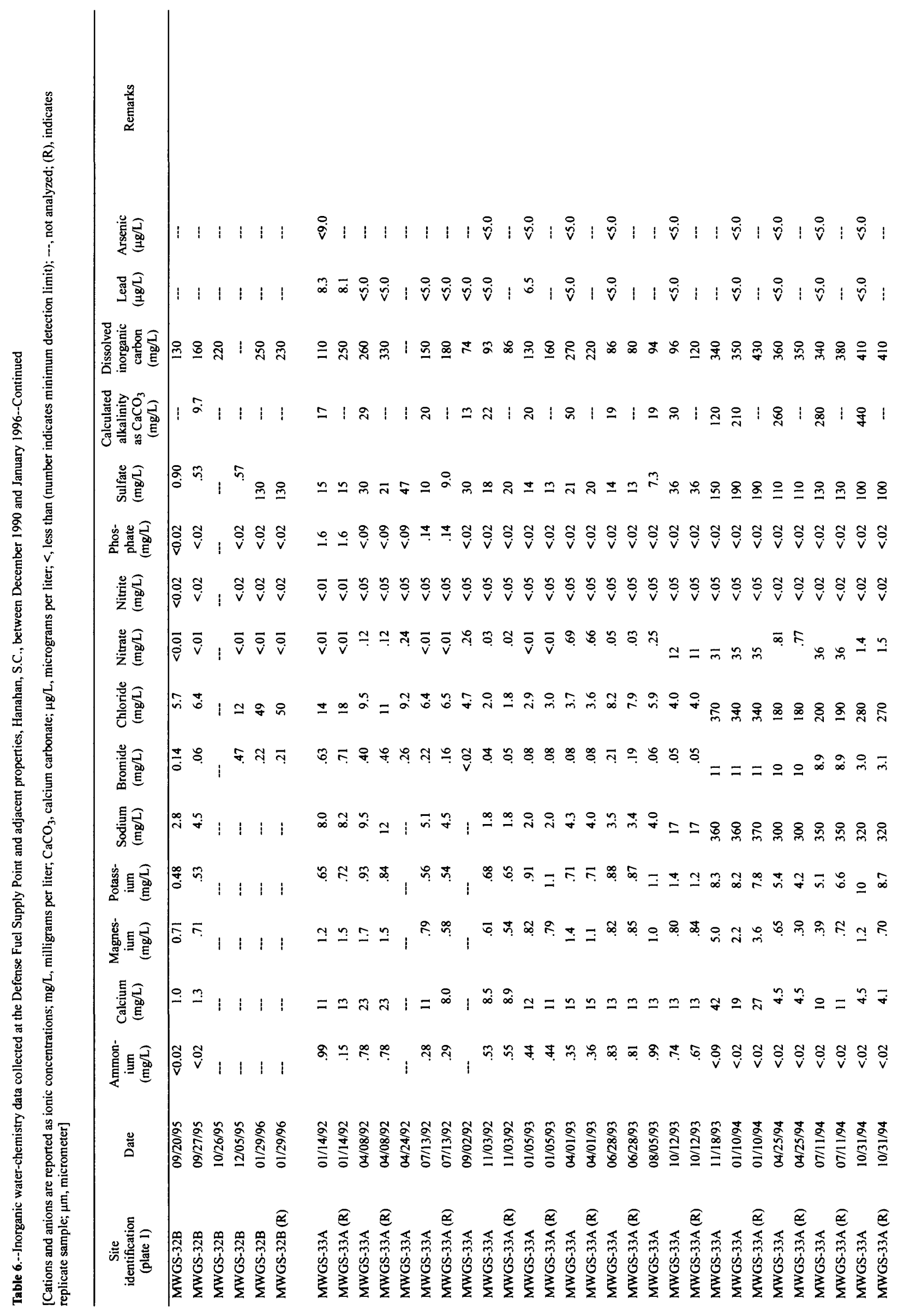




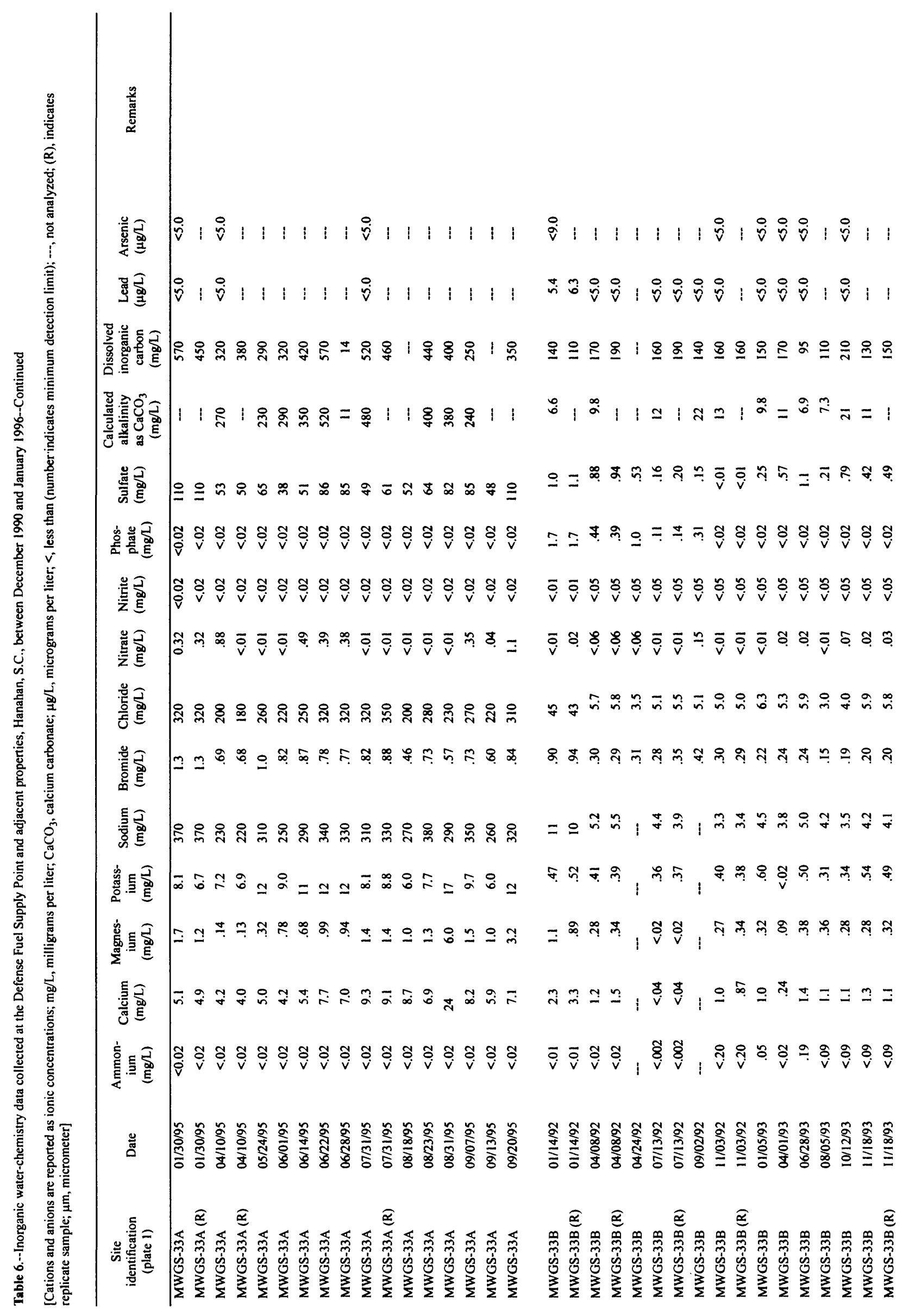




\begin{tabular}{|c|c|c|c|c|c|}
\hline 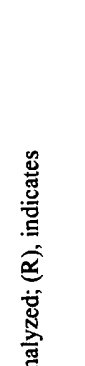 & 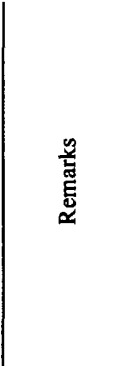 & & 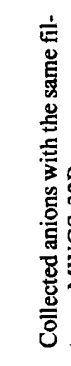 & & \\
\hline i⿱ & 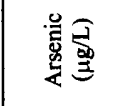 & 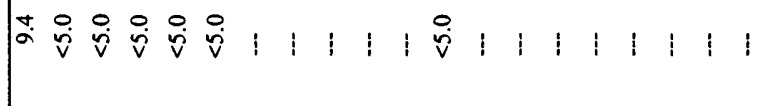 & & 1 & $\simeq 1: 1: 111:$ i \\
\hline 言 & $\stackrel{g}{\Xi}$ & 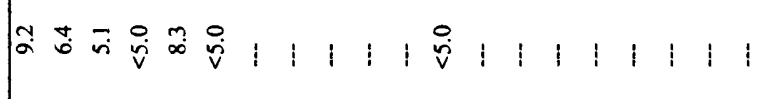 & 11 & 1 & 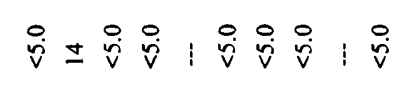 \\
\hline 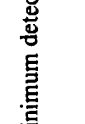 & 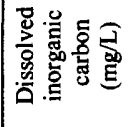 & 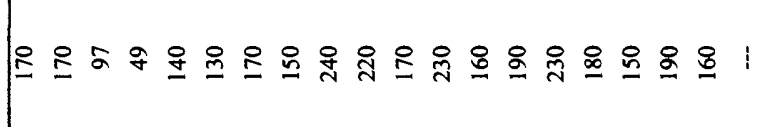 & 1 & 요 & 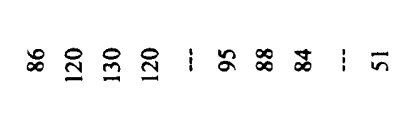 \\
\hline 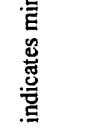 & 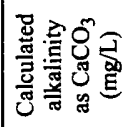 & 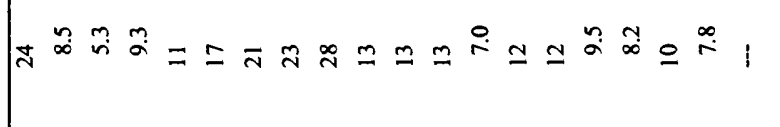 & 1 & $i$ & $\hat{a}:$ iิ: \\
\hline 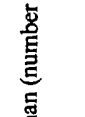 & 总 & i & 0.4 & An & 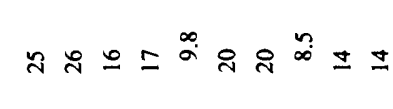 \\
\hline 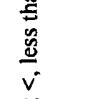 & 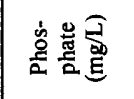 & 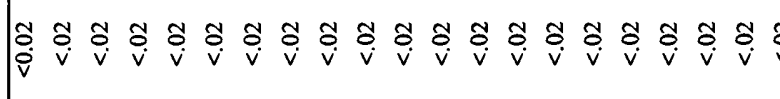 & $\underset{v}{\tilde{\sigma}} \underset{v}{\tilde{v}}$ & $\underset{v}{\tilde{V}}$ & 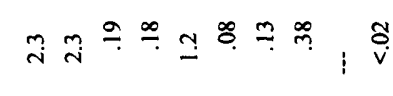 \\
\hline 离 & 曾总 & 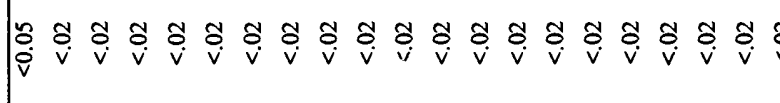 & $\underset{v}{\tilde{v}} \underset{v}{\tilde{v}}$ & $\stackrel{\text { ș }}{\mathrm{v}}$ & 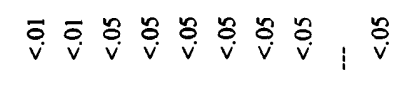 \\
\hline 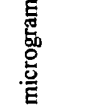 & 兽点 & 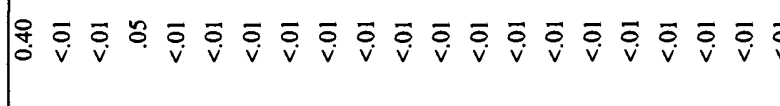 & $\bar{o}_{v} \bar{o}_{v}$ & $\overline{\vec{i}}$ & 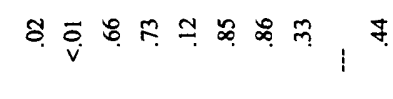 \\
\hline 离 & 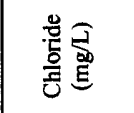 & 웅 & $\stackrel{\sim}{\bar{n}}$ & 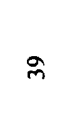 & 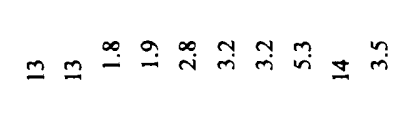 \\
\hline 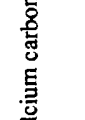 & 曾施 & 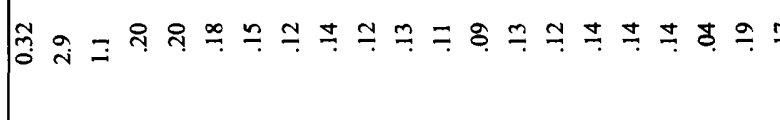 & $=$ 구 & $\stackrel{\circ}{\ddots}$ & 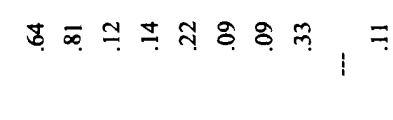 \\
\hline $\begin{array}{l}\bar{\delta} \\
\hat{0} \\
\tilde{\delta}\end{array}$ & 量名 & 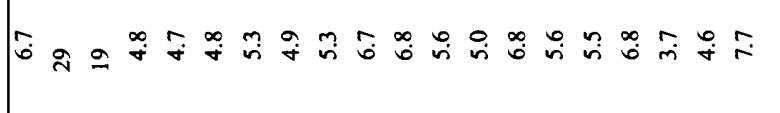 & 11 & 1 & 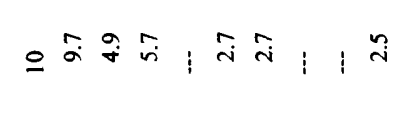 \\
\hline 言 & 产 & 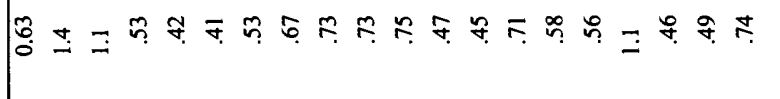 & 11 & 1 & केष \\
\hline 宸 & 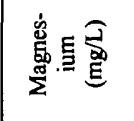 & 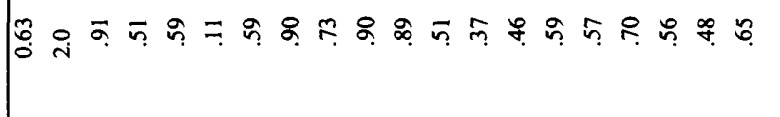 & 11 & $\vdots$ & $\cong \cong$ \\
\hline 要 & 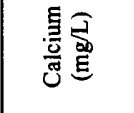 & 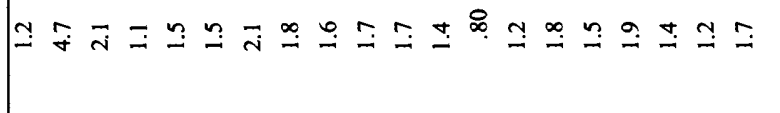 & 1 & $:$ & 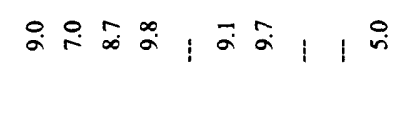 \\
\hline 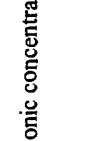 & 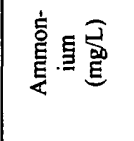 & 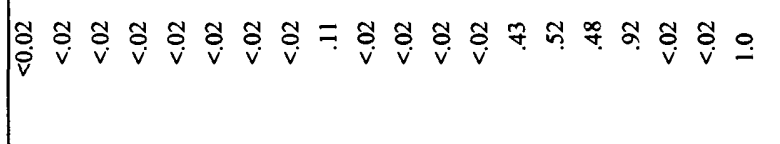 & 1 & 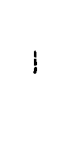 & 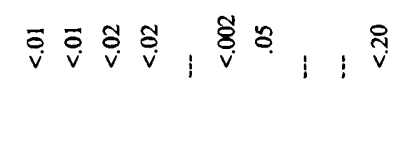 \\
\hline 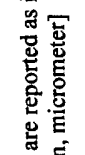 & 产 & 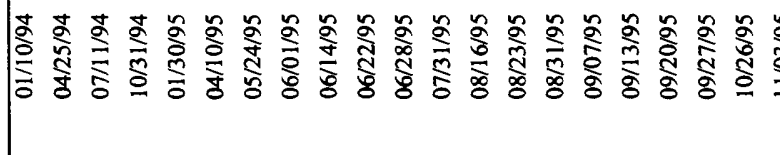 & 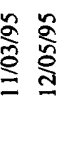 & ڤั & 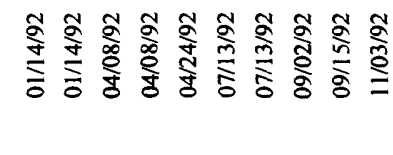 \\
\hline 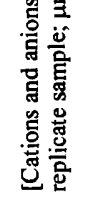 & 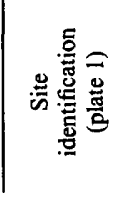 & 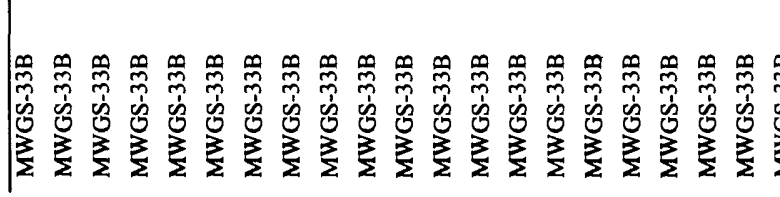 & 站 & 忢 & 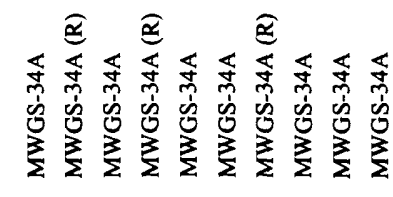 \\
\hline
\end{tabular}




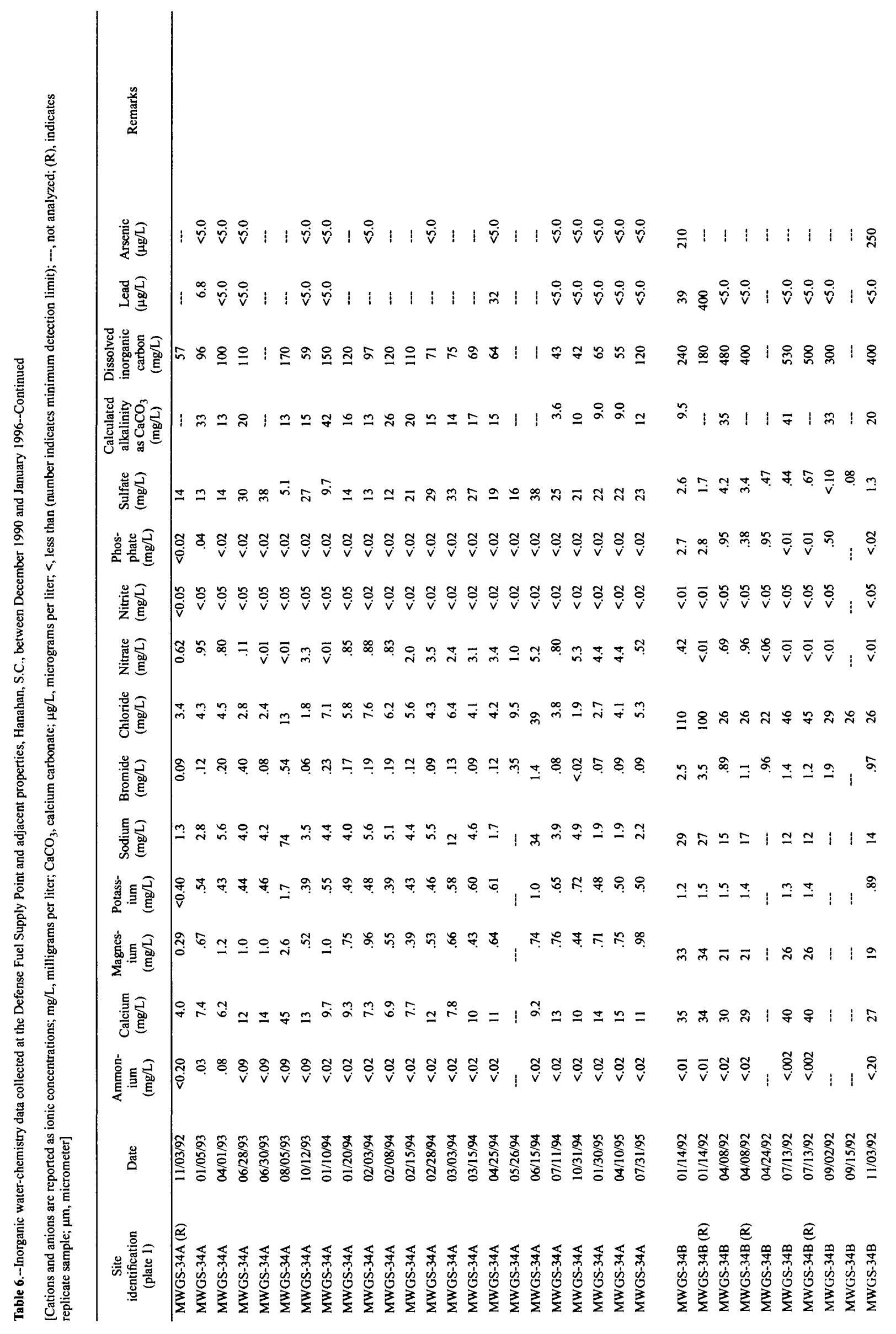




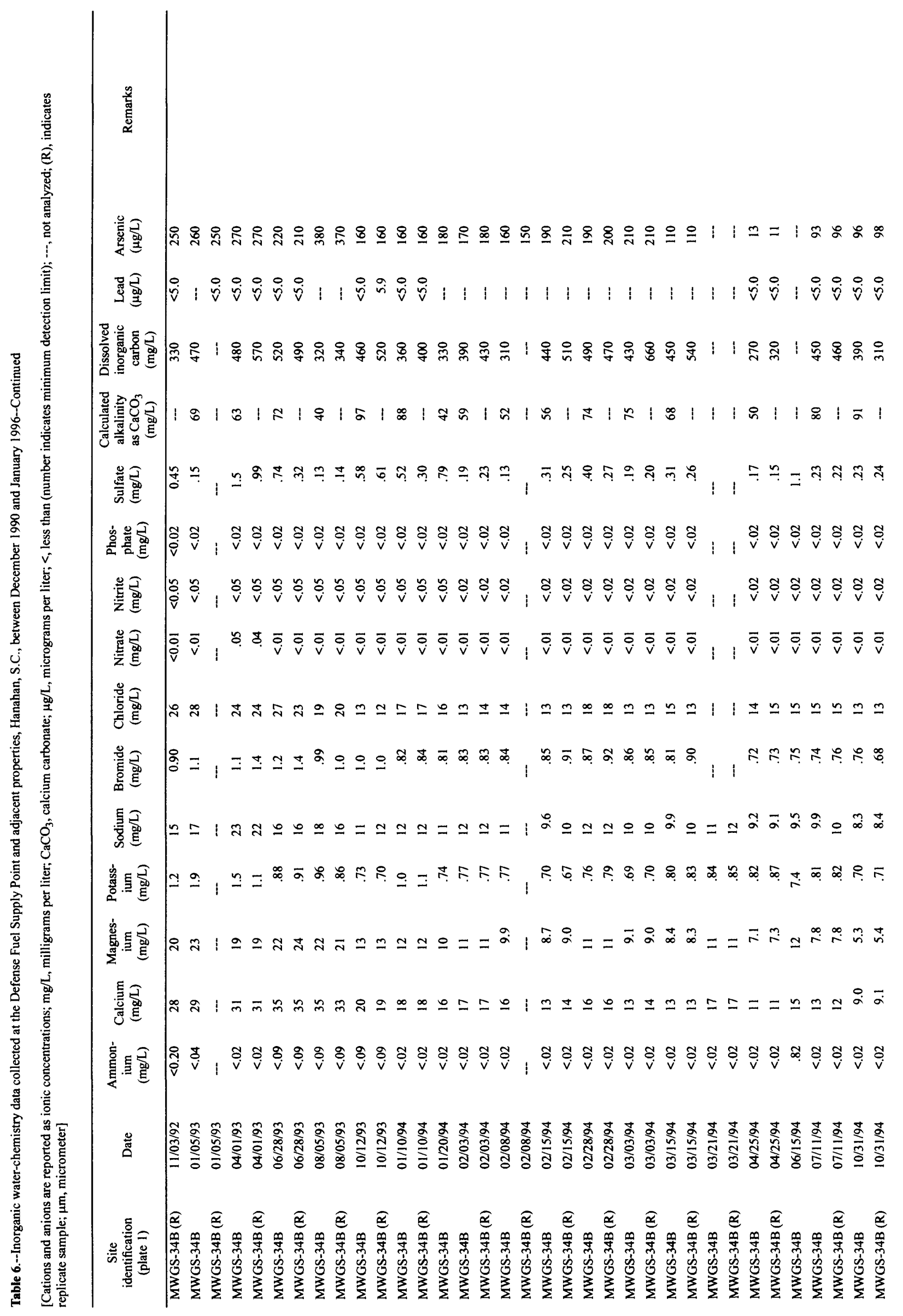




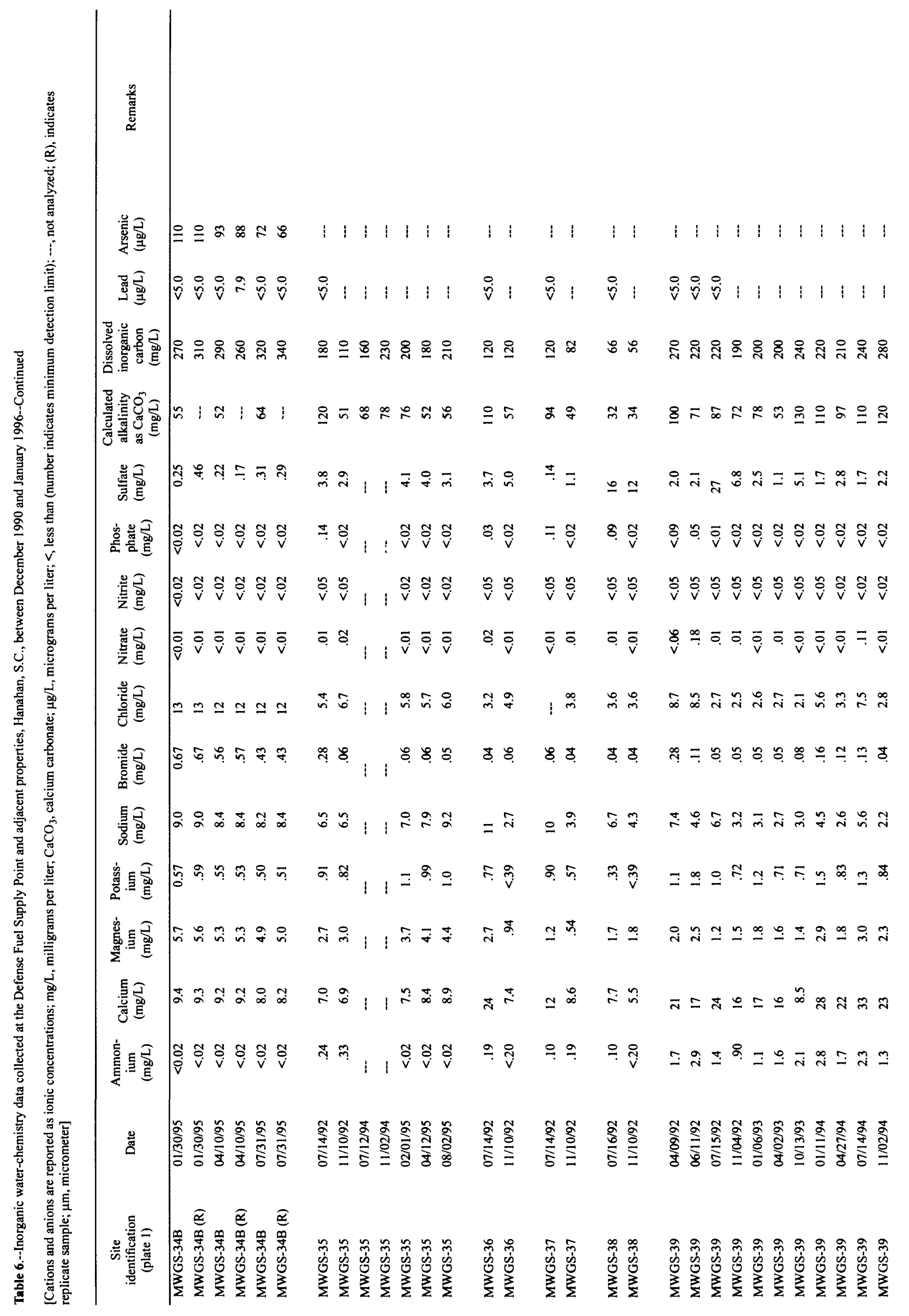




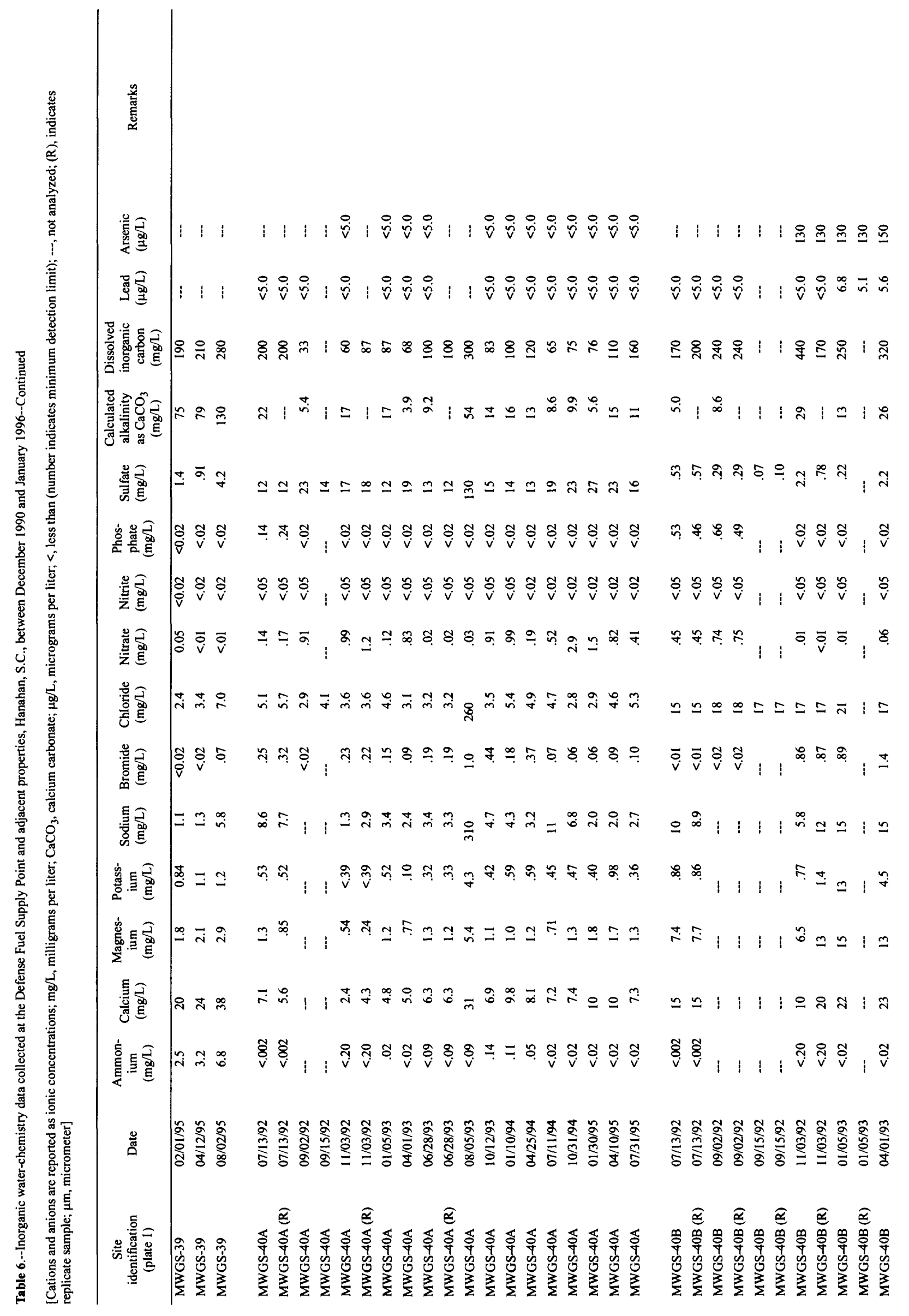




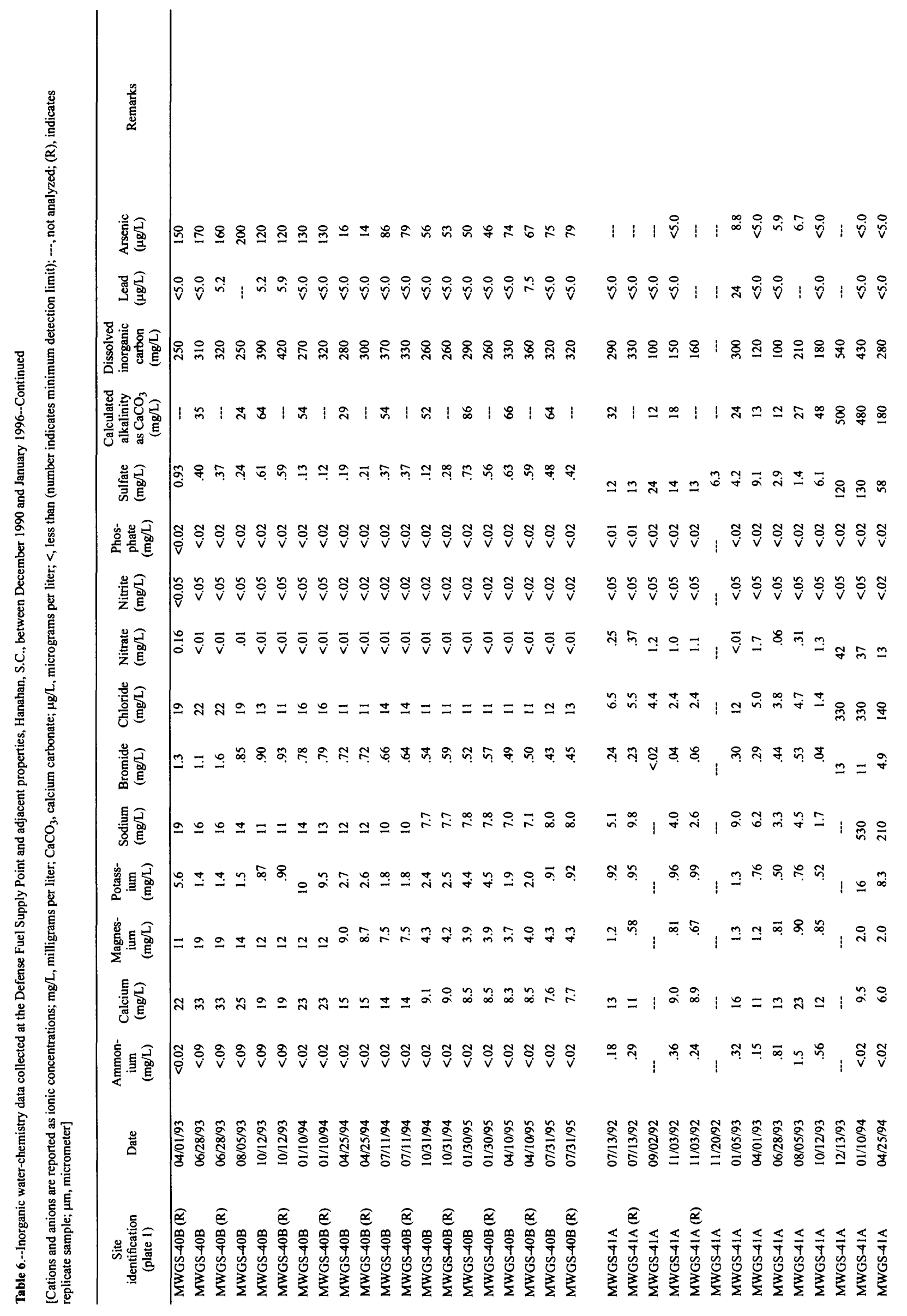




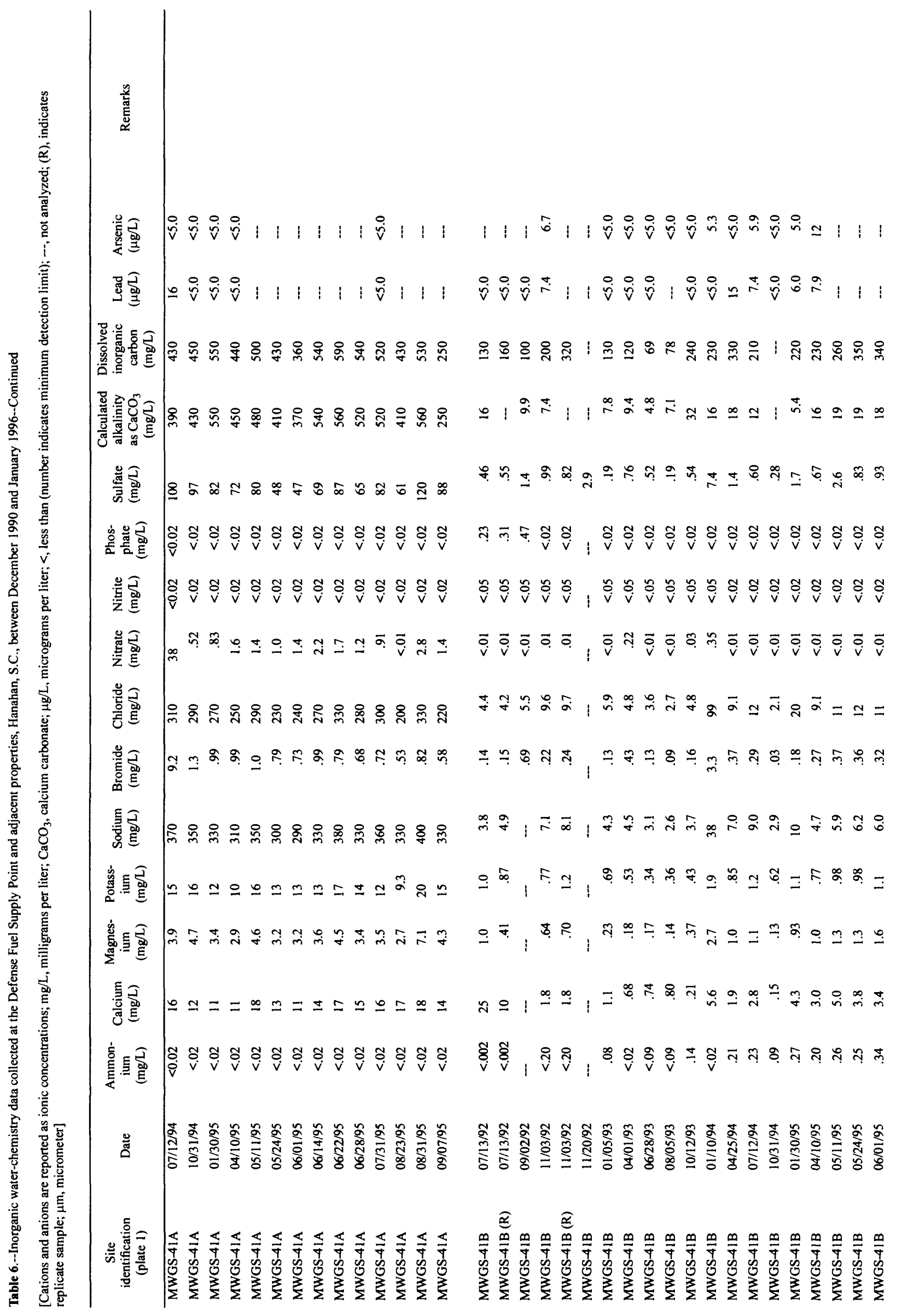




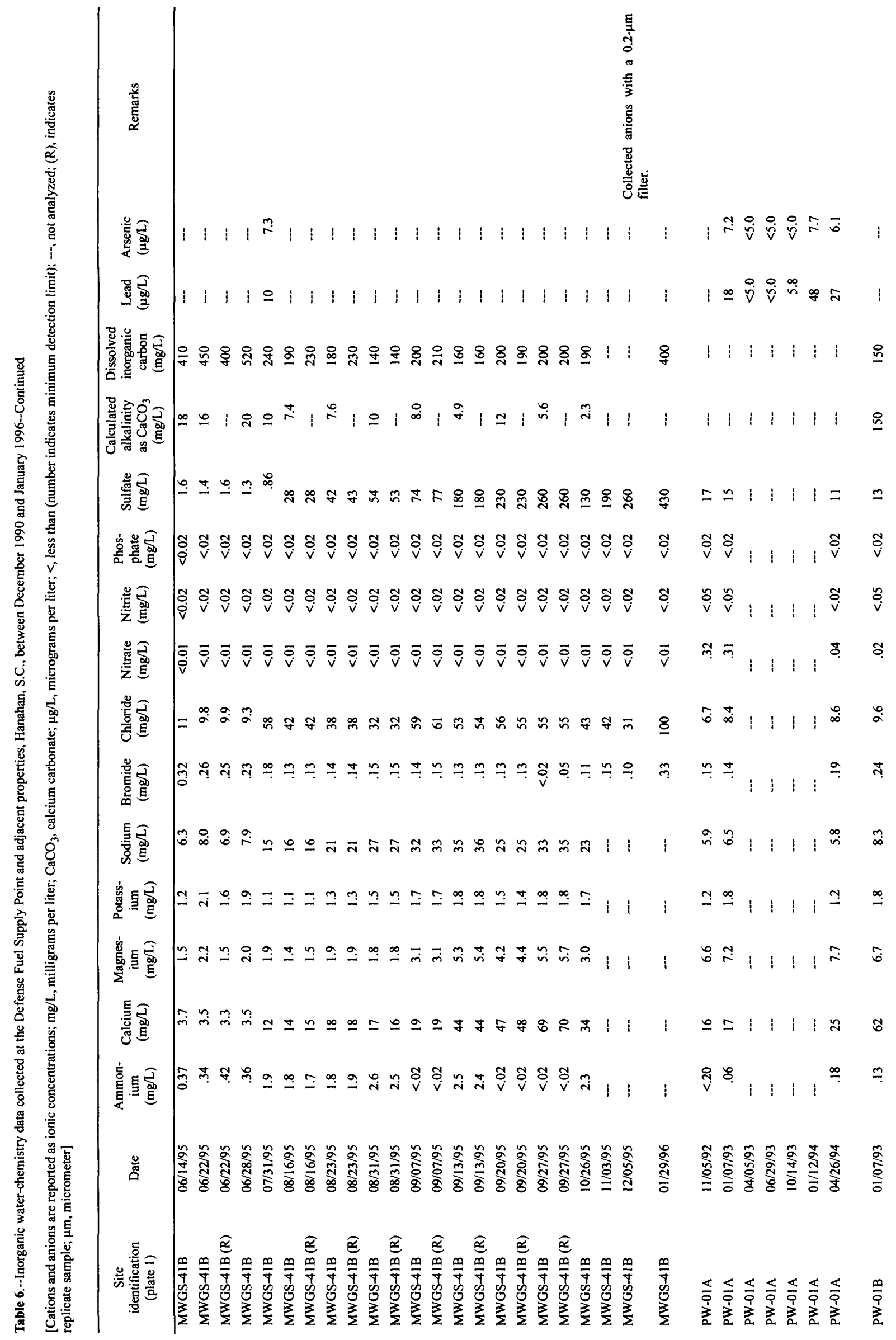




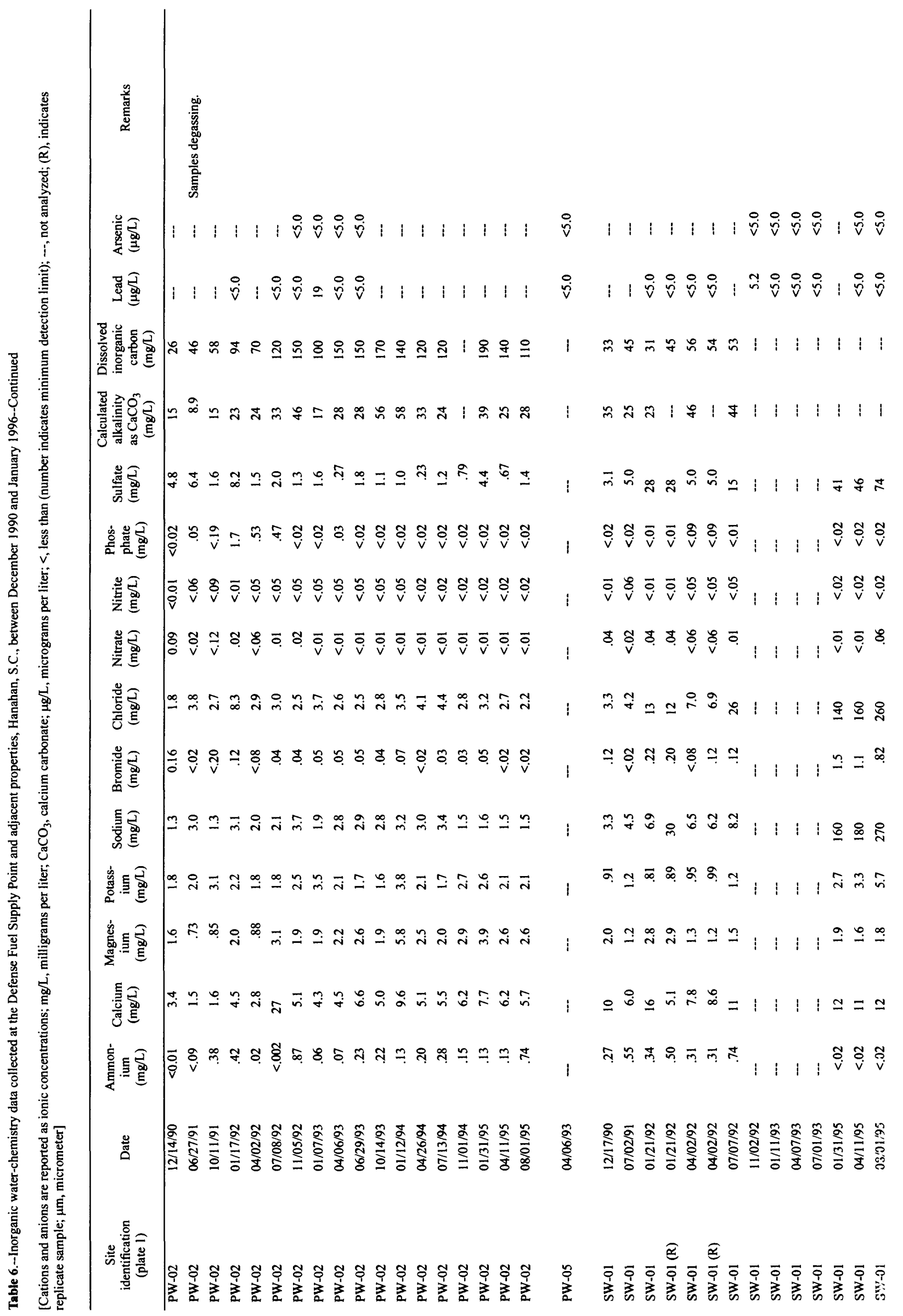




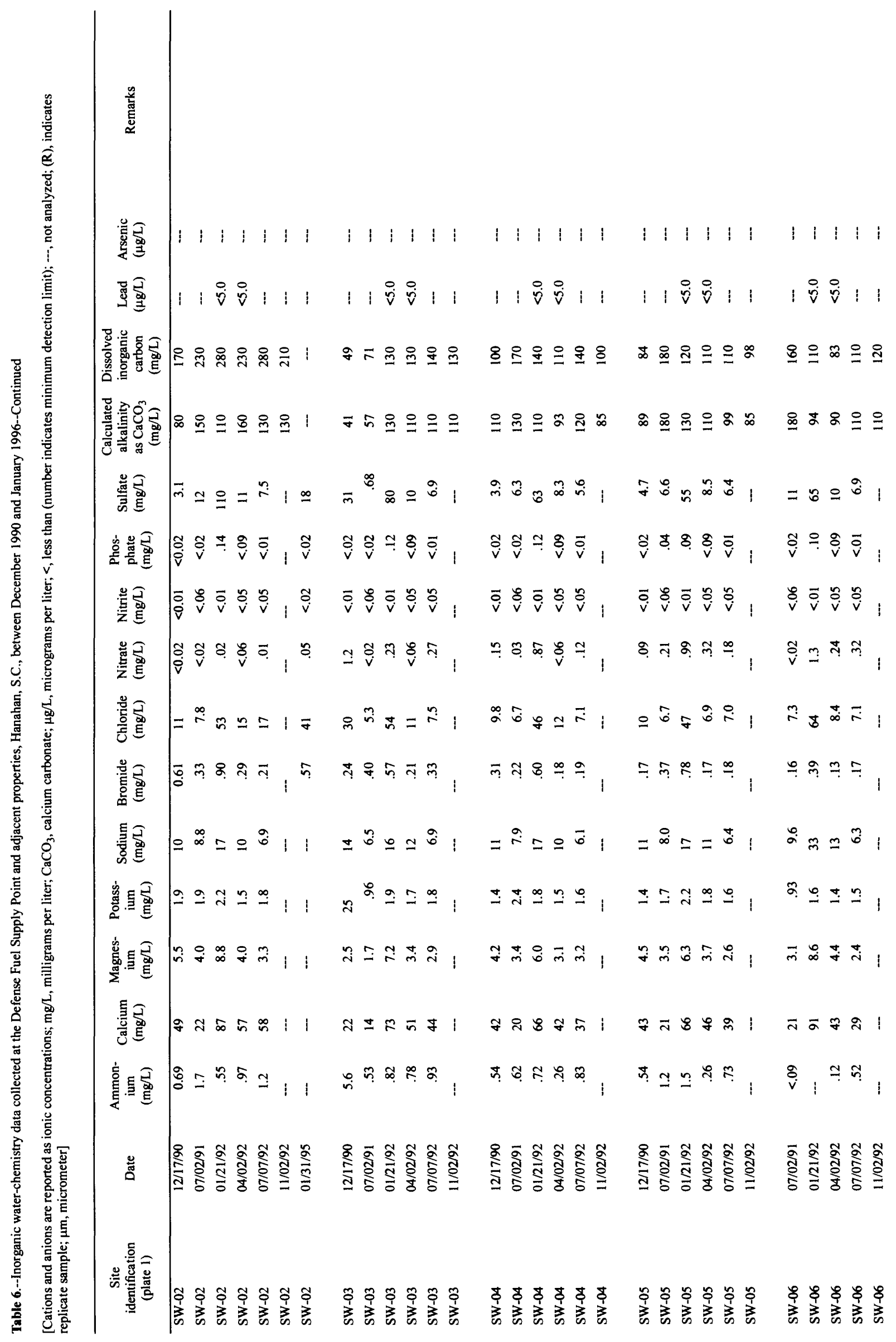




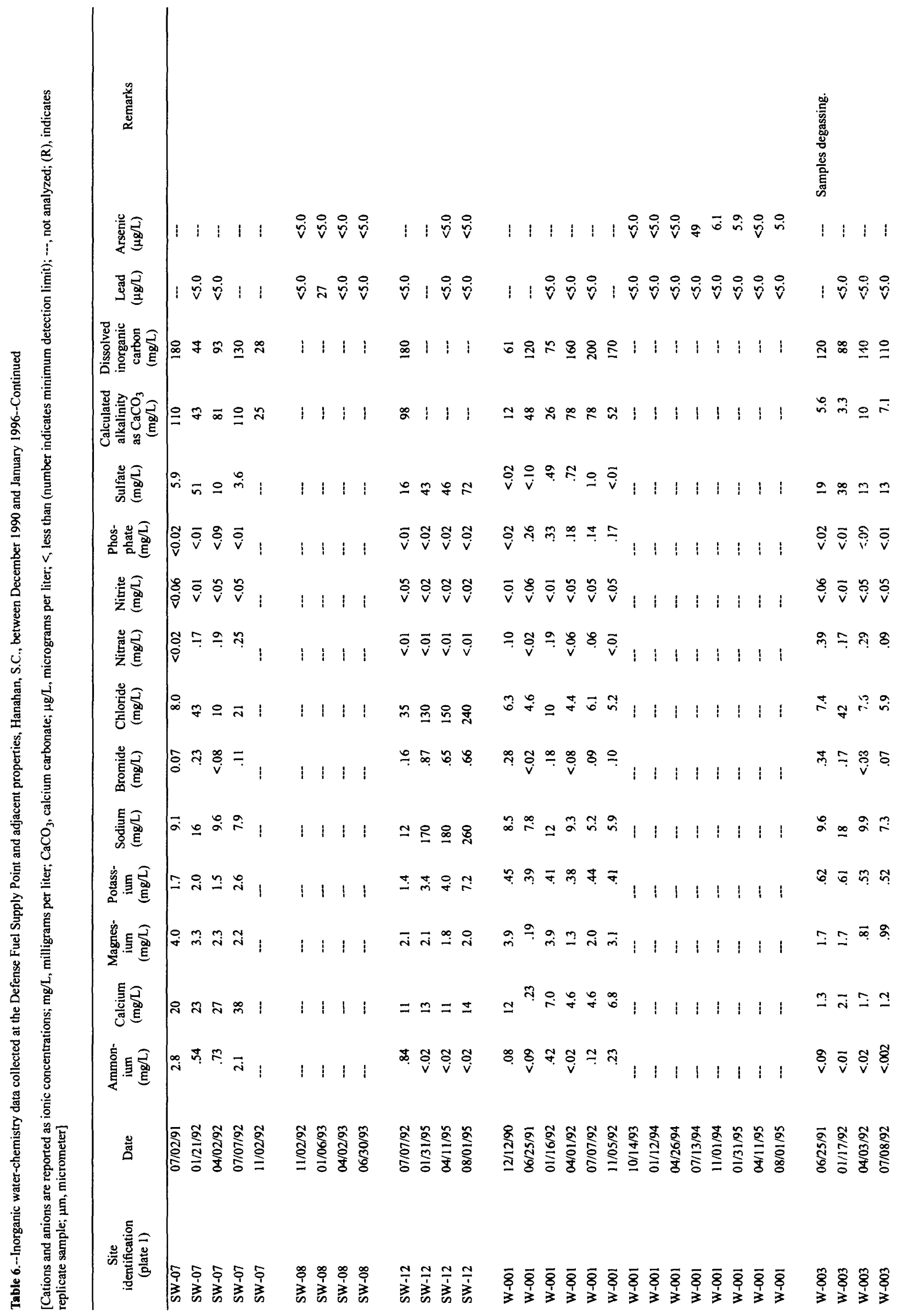




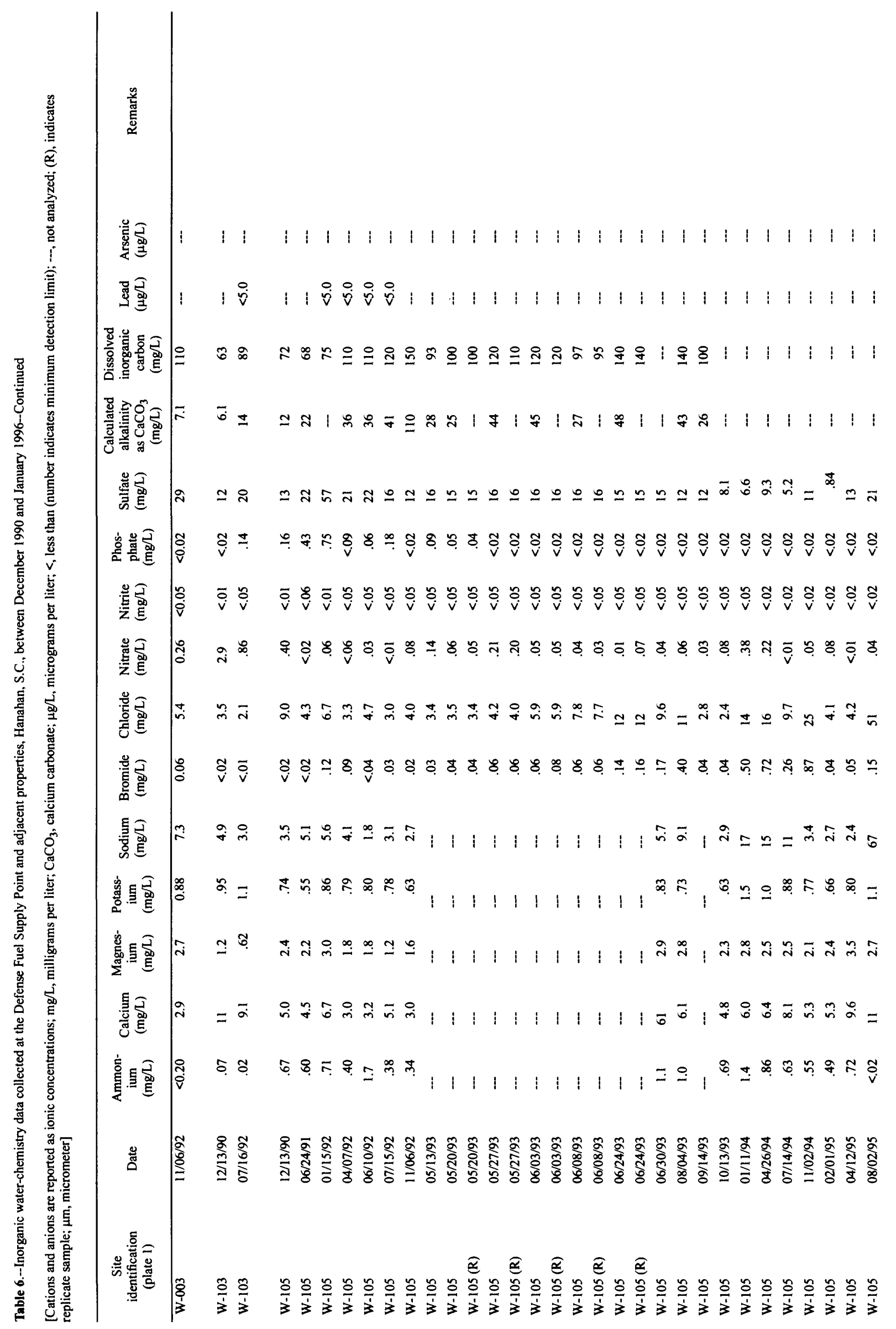




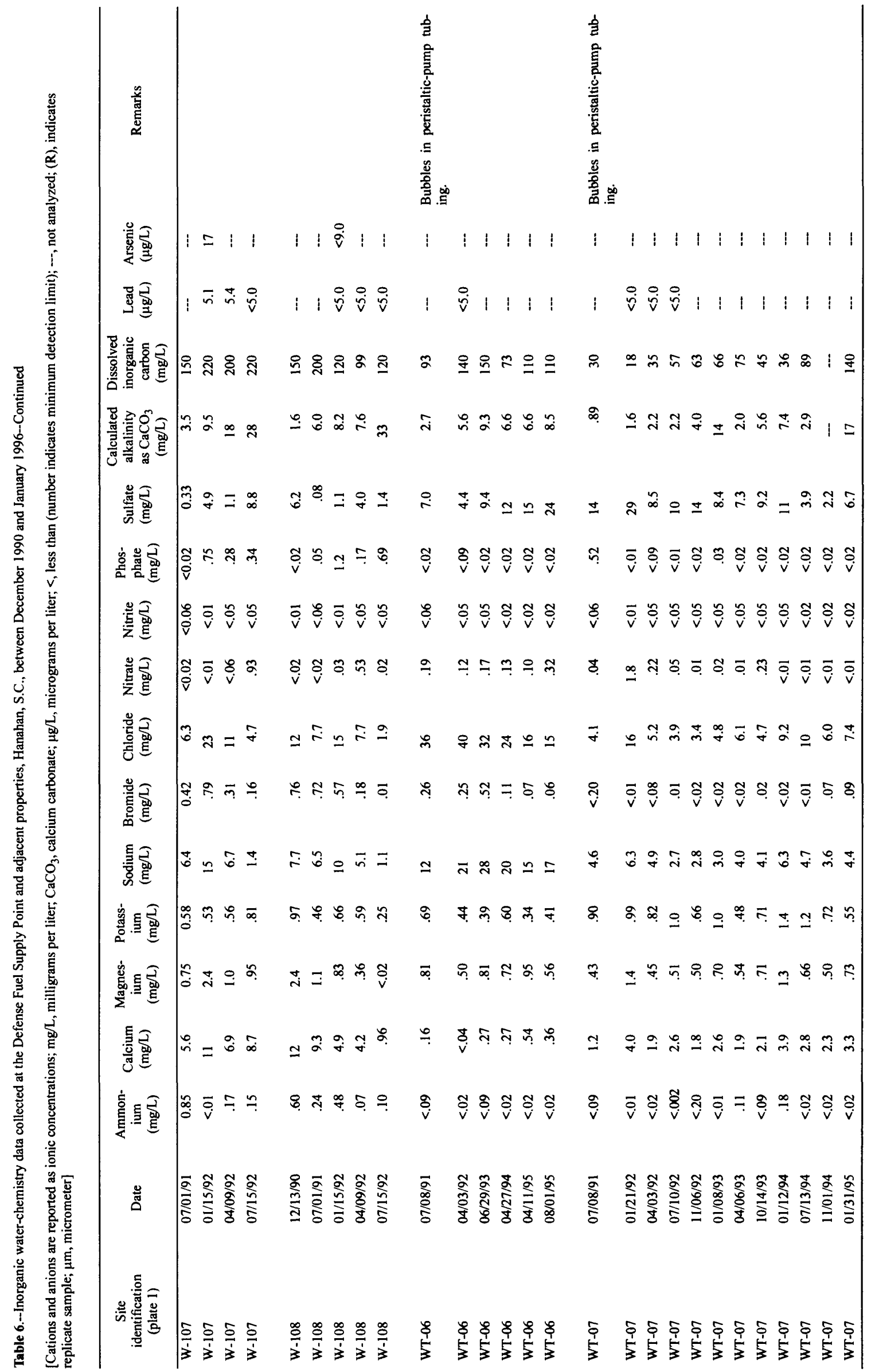


Table 7.--Tentative identification and estimated concentrations of volatile- and extractable-organic compounds using comparison of mass spectral data collected at the Defense Fuel Supply Point and adjacent properties, Hanahan, S.C.

[ $\mu \mathrm{g} / \mathrm{L}$, micrograms per liter; ---, data not available]

\begin{tabular}{|c|c|c|c|}
\hline Well identification & Compound & $\begin{array}{c}\text { Estimated } \\
\text { concentration } \\
(\mu \mathrm{g} / \mathrm{L})\end{array}$ & $\begin{array}{c}\text { Probability } \\
\text { base matching } \\
\text { (percent) }\end{array}$ \\
\hline \multicolumn{4}{|c|}{ July 1991 analysis } \\
\hline MW-05 & None detected & -- & -- \\
\hline \multirow[t]{4}{*}{ MWGS-20 } & Naphthalene & 18 & $\cdots$ \\
\hline & Benzene & 11 & $-\cdots$ \\
\hline & Toluene & 72 & -- \\
\hline & Ethylbenzene & 18 & -- \\
\hline MWGS-20R & Naphthalene & 19 & -- \\
\hline \multirow[t]{5}{*}{ W-107 } & Naphthalene & 71 & -- \\
\hline & Nitrobenzene & 11 & -- \\
\hline & Toluene & 1,600 & --- \\
\hline & Ethylbenzene & 130 & $\cdots$ \\
\hline & Xylene & 280 & -- \\
\hline \multicolumn{4}{|c|}{ July 1992 analysis } \\
\hline \multirow[t]{9}{*}{ MWGS-34B } & C9 Alkylbenzene & 41 & $\cdots$ \\
\hline & C9 Alkylbenzene & 18 & -- \\
\hline & Trimethylbenzene (isomer) & 120 & 96 \\
\hline & Tetramethylbenzene (isomer) & 6 & 94 \\
\hline & Dimethylnaphthalene & 16 & 97 \\
\hline & Dimethyl disulfide & 150 & 97 \\
\hline & Methylundecane & 120 & 83 \\
\hline & Dodecane & 950 & 97 \\
\hline & C9 Alkylbenzene & 150 & 97 \\
\hline
\end{tabular}


Table 7.-Tentative identification and estimated concentrations of volatile- and extractable-organic compounds using comparison of mass spectral data collected at the Defense Fuel Supply Point and adjacent properties, Hanahan, S.C.--Continued

[ $\mu \mathrm{g} / \mathrm{L}$, micrograms per liter; ---, data not available $]$

\begin{tabular}{|c|c|c|c|}
\hline Well identification & Compound & $\begin{array}{c}\text { Estimated } \\
\text { concentration } \\
(\mu \mathrm{g} / \mathrm{L})\end{array}$ & $\begin{array}{c}\text { Probability } \\
\text { base matching } \\
\text { (percent) }\end{array}$ \\
\hline \multicolumn{4}{|c|}{ July 1992 analysis-Continued } \\
\hline \multirow[t]{2}{*}{ MWGS-34B--Continued } & C9 Alkylbenzene & 100 & 96 \\
\hline & Methylnaphthalene & 220 & 87 \\
\hline \multirow[t]{9}{*}{ MWGS-40A } & C9 Alkylbenzene & 12 & -- \\
\hline & C9 Alkylbenzene & 10 & --- \\
\hline & C10 Alkylbenzene & 16 & --- \\
\hline & Tetramethylbenzene (isomer) & 19 & 89 \\
\hline & Methyl-naphthalene & 22 & 93 \\
\hline & C9 Alkylbenzene & 40 & --- \\
\hline & Trimethylbenzene (isomer) & 90 & 97 \\
\hline & C9 Alkylbenzene & 30 & 95 \\
\hline & C10 Alkylbenzene & 30 & 83 \\
\hline \multirow[t]{9}{*}{ MWGS-40B } & Trimethylbenzene (isomer) & 50 & 97 \\
\hline & Unknown & 60 & -- \\
\hline & Unknown & 80 & --- \\
\hline & Unknown & 70 & --- \\
\hline & Octanoic Acid & 110 & 83 \\
\hline & Unknown & 60 & --- \\
\hline & Toluene & 220 & --- \\
\hline & Ethylbenzene & 40 & --- \\
\hline & Xylene (isomer) & 40 & --- \\
\hline \multirow[t]{3}{*}{ MWGS-41A } & C9 Alkylbenzene & 26 & --- \\
\hline & Tetramethylbezene (isomer) & 15 & 96 \\
\hline & C9 Alkylbenzene & 70 & -- \\
\hline
\end{tabular}


Table 7.--Tentative identification and estimated concentrations of volatile- and extractable-organic compounds using comparison of mass spectral data collected at the Defense Fuel Supply Point and adjacent properties, Hanahan, S.C.--Continued

$[\mu \mathrm{g} / \mathrm{L}$, micrograms per liter; ---, data not available]

\begin{tabular}{|c|c|c|c|}
\hline Well identification & Compound & $\begin{array}{c}\text { Estimated } \\
\text { concentration } \\
(\mu \mathrm{g} / \mathrm{L})\end{array}$ & $\begin{array}{c}\text { Probability } \\
\text { base matching } \\
\text { (percent) }\end{array}$ \\
\hline \multicolumn{4}{|c|}{ July 1992 analysis-Continued } \\
\hline \multirow[t]{4}{*}{ MWGS-41A--Continued } & C9 Alkylbenzene & 50 & $-\cdots$ \\
\hline & Trimethylbenzene (isomer) & 160 & 97 \\
\hline & C9 Alkylbenzene & 60 & -- \\
\hline & C10 Alkylbenzene & 30 & $\cdots$ \\
\hline \multirow[t]{4}{*}{ MWGS-31B } & C9 Alkylbenzene & 34 & -- \\
\hline & C9 Alkylbenzene & 16 & -- \\
\hline & Trimethylbenzene (isomer) & 100 & 95 \\
\hline & Dimethylnaphthalene & 10 & 95 \\
\hline \multirow[t]{11}{*}{ MWGS-40BR } & Toluene & 250 & -- \\
\hline & Ethylbenzene & 22 & -- \\
\hline & Xylene & 49 & -- \\
\hline & Dimethylnaphthalene & 10 & -- \\
\hline & Propylbenzene & 17 & -- \\
\hline & 2-Methylnaphthalene & 50 & -- \\
\hline & C9 Alkylbenzene & 34 & -- \\
\hline & Trimethylbenzene (isomer) & 23 & -- \\
\hline & C9 Alkylbenzene & 17 & -- \\
\hline & Trimethylbenzene (isomer) & 82 & --- \\
\hline & Tetramethylbenzene & 25 & -- \\
\hline MWGS-34A & None detected & -- & -- \\
\hline \multirow[t]{3}{*}{ MWGS-41B } & Unknown & 50 & -- \\
\hline & Methylbutane & 60 & 89 \\
\hline & Dimethylpentane (isomer) & 90 & 95 \\
\hline
\end{tabular}


Table 7.-Tentative identification and estimated concentrations of volatile- and extractable-organic compounds using comparison of mass spectral data collected at the Defense Fuel Supply Point and adjacent properties, Hanahan, S.C.--Continued

[ $\mu \mathrm{g} / \mathrm{L}$, micrograms per liter; ---, data not available]

\begin{tabular}{|c|c|c|c|}
\hline Well identification & Compound & $\begin{array}{c}\text { Estimated } \\
\text { concentration } \\
(\mu \mathrm{g} / \mathrm{L})\end{array}$ & $\begin{array}{c}\text { Probability } \\
\text { base matching } \\
\text { (percent) }\end{array}$ \\
\hline \multicolumn{4}{|c|}{ July 1992 analysis-Continued } \\
\hline \multirow[t]{7}{*}{ MWGS-4IB--Continued } & Dimethylpentane (isomer) & 120 & 95 \\
\hline & Dimethylpentane (isomer) & 170 & 86 \\
\hline & Trimethylpentane (isomer) & 80 & 96 \\
\hline & Trimethylpentane (isomer) & 90 & 89 \\
\hline & C9 Alkylbenzene & 30 & --- \\
\hline & Trimethylbenzene (isomer) & 50 & 96 \\
\hline & Methylnaphthalene & 50 & 94 \\
\hline \multirow[t]{8}{*}{ MWGS-33A } & 2-Methylbutane & 30 & 89 \\
\hline & Propylbenzene & 40 & 97 \\
\hline & C9 Alkylbenzene & 110 & -- \\
\hline & Trimethylbenzene (isomer) & 50 & 97 \\
\hline & C9 Alkylbenzene & 70 & --- \\
\hline & Trimethylbenzene & 240 & 97 \\
\hline & C9 Alkylbenzene & 110 & --- \\
\hline & C10 Alkylbenzene & 30 & -- \\
\hline \multirow[t]{8}{*}{ MWGS-33B } & 2-Methylbutane & 30 & 89 \\
\hline & Dimethylpentane & 80 & 95 \\
\hline & Trimethylpentane & 50 & 86 \\
\hline & Trimethylpentane & 70 & 89 \\
\hline & C10 Alkylbenzene & 60 & 89 \\
\hline & Trimethylpentane & 60 & 96 \\
\hline & C10 Alkylbenzene & 40 & 81 \\
\hline & Unknown hydrocarbon & 160 & --. \\
\hline
\end{tabular}


Table 7.-Tentative identification and estimated concentrations of volatile- and extractable-organic compounds using comparison of mass spectral data collected at the Defense Fuel Supply Point and adjacent properties, Hanahan, S.C.--Continued

[ $\mu \mathrm{g} / \mathrm{L}$, micrograms per liter; ---, data not available]

\begin{tabular}{|c|c|c|c|}
\hline Well identification & Compound & $\begin{array}{c}\text { Estimated } \\
\text { concentration } \\
(\mu \mathrm{g} / \mathrm{L})\end{array}$ & $\begin{array}{c}\text { Probability } \\
\text { base matching } \\
\text { (percent) }\end{array}$ \\
\hline \multicolumn{4}{|c|}{ July 1992 analysis-Continued } \\
\hline \multirow[t]{13}{*}{ EW-05 } & Methylcylcopentane & 450 & 93 \\
\hline & Cyclohexane & 500 & 97 \\
\hline & Methylcyclohexane & 300 & 96 \\
\hline & C9 Alkylbenzene & 200 & 97 \\
\hline & Trimethylbenzene (isomers) & 250 & 97 \\
\hline & Xylenes (total) & 1,600 & 100 \\
\hline & Trimethylbenzene (isomer) & 31 & 97 \\
\hline & Trimethylbenzene (isomer) & 62 & 96 \\
\hline & Trimethylbenzene (isomer) & 43 & 97 \\
\hline & C10 Alkylbenzene & 15 & 97 \\
\hline & Tetramethylbenzene (isomer) & 12 & 94 \\
\hline & C11 Alkylnaphthalene & 39 & 95 \\
\hline & C12 Alkylnaphthalene & 12 & 99 \\
\hline \multirow[t]{8}{*}{ EW-07 } & Trimethylpentane (isomer) & 30 & 86 \\
\hline & Cyclohexane & 110 & 95 \\
\hline & Methylcyclohexane & 70 & 96 \\
\hline & C9 Alkylbenzene & 30 & 97 \\
\hline & Trimethylbenzene (isomers) & 40 & 97 \\
\hline & 3-Methylpentane & 40 & 86 \\
\hline & Methylcyclopentane & 140 & 89 \\
\hline & $\begin{array}{l}\text { 1,3,5-Triazine-2,4-diamine, } \\
\text { 6-metoxy N, N'bis }\end{array}$ & 19 & 99 \\
\hline
\end{tabular}


Table 7.--Tentative identification and estimated concentrations of volatile- and extractable-organic compounds using comparison of mass spectral data collected at the Defense Fuel Supply Point and adjacent properties, Hanaha , S.C.--Continued

[ $\mu \mathrm{g} / \mathrm{L}$, micrograms per liter; ---, data not available]

\begin{tabular}{|c|c|c|c|}
\hline Well identification & Compound & $\begin{array}{c}\text { Estimated } \\
\text { concentration } \\
(\mu \mathrm{g} / \mathrm{L})\end{array}$ & $\begin{array}{c}\text { Probability } \\
\text { base matching } \\
\text { (percent) }\end{array}$ \\
\hline \multicolumn{4}{|c|}{ May 1993 analysis } \\
\hline \multirow[t]{5}{*}{ MW-11A } & Pentane & 250 & 87 \\
\hline & C5H10 Unknown hydrocarbons & 300 & --- \\
\hline & C6H12 Unknown hydrocarbons & 350 & --- \\
\hline & Cyclohexane & 700 & 95 \\
\hline & Methylcyclohexane & 250 & 96 \\
\hline \multirow[t]{8}{*}{ MW-12 } & Methylbutane & 380 & 84 \\
\hline & C5H12 Unknown hydrocarbons & 70 & --- \\
\hline & Unknown hydrocarbons & 70 & --- \\
\hline & Unknown hydrocarbons & 140 & --- \\
\hline & Dimethylpentane (isomer) & 270 & 95 \\
\hline & Trimethylpentane (isomer) & 200 & 83 \\
\hline & Trimethylpentane (isomer) & 90 & 86 \\
\hline & Trimethylpentane (isomer) & 130 & 89 \\
\hline \multirow[t]{8}{*}{ MWGS-37 } & Pentane & 150 & 86 \\
\hline & C5H10 Unknown hydrocarbons & 200 & -- \\
\hline & Unknown hydrocarbons & 200 & --- \\
\hline & $\mathrm{C} 6 \mathrm{H} 12$ Unknown hydrocarbons & 800 & --- \\
\hline & Cyclohexane & 900 & 95 \\
\hline & Methylcyclohexane & 300 & 94 \\
\hline & C9H12 Alkylbenzene (isomer) & 200 & 95 \\
\hline & C9H12 Alkylbenzene (isomer) & 200 & 96 \\
\hline
\end{tabular}


Table 8.--Water-level data collected at the Defense Fuel Supply Point and adjacent properties, Hanahan, S.C., between April 1991 and September 1995

[ft, feet; ---, data not available; WL, water-level well; EW, extraction well; MW, monitoring well; SW, surface-water site; -, negative depth to water indicates water-level altitude is above measuring point]

\begin{tabular}{|c|c|c|c|c|c|c|c|}
\hline $\begin{array}{c}\text { Site } \\
\text { (plate 1) }\end{array}$ & $\begin{array}{l}\text { Site } \\
\text { type }\end{array}$ & Date & Time & $\begin{array}{l}\text { Measuring-point } \\
\text { altitude } \\
\text { (ft above } \\
\text { sea level) }\end{array}$ & $\begin{array}{l}\text { Depth to water } \\
\text { (ft below } \\
\text { measuring point) }\end{array}$ & $\begin{array}{l}\text { Water-level } \\
\text { altitude } \\
\text { (ft above } \\
\text { sea level) }\end{array}$ & Remarks \\
\hline B-102 & $\overline{W L}$ & $05 / 28 / 91$ & -- & 40.17 & 8.02 & 32.15 & \\
\hline B-102 & WL & $07 / 01 / 91$ & --- & 40.17 & 8.56 & 31.61 & \\
\hline B-102 & WL & $08 / 06 / 91$ & --- & 40.17 & 6.36 & 33.81 & \\
\hline B-102 & WL & 08/30/91 & $\cdots$ & 40.17 & 5.90 & 34.27 & \\
\hline B-102 & WL & $09 / 27 / 91$ & $\cdots$ & 40.17 & 7.22 & 32.95 & \\
\hline B-102 & WL & $10 / 29 / 91$ & -- & 40.17 & 8.75 & 31.42 & \\
\hline B-102 & WL & $12 / 02 / 91$ & --- & 40.17 & 10.30 & 29.87 & \\
\hline B-102 & WL & $01 / 02 / 92$ & $-\cdot-$ & 40.17 & 11.26 & 28.91 & \\
\hline B-102 & WL & $02 / 04 / 92$ & -- & 40.17 & 8.70 & 31.47 & \\
\hline B-102 & WL & $03 / 06 / 92$ & --- & 40.17 & 8.90 & 31.27 & \\
\hline B-102 & WL & $03 / 31 / 92$ & --- & 40.17 & 8.76 & 31.41 & \\
\hline B-102 & WL & $05 / 01 / 92$ & -- & 40.17 & 8.92 & 31.25 & \\
\hline B-102 & WL & $06 / 11 / 92$ & 1534 & 40.17 & 7.96 & 32.21 & \\
\hline B-102 & WL & $07 / 07 / 92$ & 1430 & 40.17 & 7.88 & 32.29 & \\
\hline B-102 & WL & $08 / 07 / 92$ & 1412 & 40.17 & 8.12 & 32.05 & \\
\hline B-102 & WL & $08 / 31 / 92$ & 1256 & 40.17 & 5.71 & 34.46 & \\
\hline B-102 & WL & $10 / 07 / 92$ & 1344 & 40.17 & 5.25 & 34.92 & \\
\hline B-102 & WL & $10 / 28 / 92$ & 1406 & 40.17 & 6.38 & 33.79 & \\
\hline B-102 & WL & $12 / 03 / 92$ & 1250 & 40.17 & 6.23 & 33.94 & \\
\hline B-102 & WL & $01 / 04 / 93$ & 1220 & 40.17 & 7.49 & 32.68 & \\
\hline B-102 & WL & $02 / 04 / 93$ & 1340 & 40.17 & 5.81 & 34.36 & \\
\hline B-102 & WL & $03 / 05 / 93$ & 1225 & 40.17 & 6.44 & 33.73 & \\
\hline B-102 & WL & $03 / 30 / 93$ & 1150 & 40.17 & 5.78 & 34.39 & \\
\hline B-102 & WL & $05 / 06 / 93$ & 1300 & 40.17 & 6.88 & 33.29 & \\
\hline B-102 & WL & $04 / 30 / 91$ & 1210 & 40.17 & 7.81 & 32.36 & \\
\hline B-103 & WL & $04 / 30 / 91$ & 1130 & 41.01 & 10.68 & 30.33 & \\
\hline B-103 & WL & $05 / 28 / 91$ & -- & 41.01 & 10.91 & 30.10 & \\
\hline B-103 & WL & $07 / 01 / 91$ & -- & 41.01 & 10.10 & 30.91 & \\
\hline B-103 & WL & $08 / 06 / 91$ & -- & 41.01 & 9.98 & 31.03 & \\
\hline B-103 & WL & $08 / 30 / 91$ & -- & 41.01 & 9.78 & 31.23 & \\
\hline B-103 & WL & $09 / 27 / 91$ & -- & 41.01 & 10.96 & 30.05 & \\
\hline B-103 & WL & $10 / 29 / 91$ & -- & 41.01 & 11.53 & 29.48 & \\
\hline B-103 & WL & $12 / 02 / 91$ & --- & 41.01 & 11.85 & 29.16 & \\
\hline B-103 & WL & $01 / 02 / 92$ & -- & 41.01 & 12.13 & 28.88 & \\
\hline B-103 & WL & $02 / 04 / 92$ & -- & 41.01 & 11.23 & 29.78 & \\
\hline B-103 & WL & $03 / 06 / 92$ & $\cdots$ & 41.01 & 11.30 & 29.71 & \\
\hline B-103 & WL & $03 / 31 / 92$ & $-\cdots$ & 41.01 & 11.22 & 29.79 & \\
\hline B-103 & WL & $05 / 01 / 92$ & --- & 41.01 & 11.33 & 29.68 & \\
\hline B-103 & WL & $06 / 11 / 92$ & 1510 & 41.01 & 10.49 & 30.52 & \\
\hline B-103 & WL & $07 / 07 / 92$ & 1500 & 41.01 & 10.96 & 30.05 & \\
\hline B-103 & WL & $08 / 07 / 92$ & 1353 & 41.01 & 10.82 & 30.19 & \\
\hline B-103 & WL & $08 / 31 / 92$ & 1229 & 41.01 & 9.61 & 31.40 & \\
\hline
\end{tabular}


Table 8.--Water-level data collected at the Defense Fuel Supply Point and adjacent properties, Hanahan, S.C., between April 1991 and September 1995--Continued

[ft, feet; ---, data not available; WL, water-level well; EW, extraction well; MW, monitoring well; SW, surface-water site; -, negative depth to water indicates water-level altitude is above measuring point]

\begin{tabular}{|c|c|c|c|c|c|c|c|}
\hline $\begin{array}{c}\text { Site } \\
\text { (plate 1) }\end{array}$ & $\begin{array}{l}\text { Site } \\
\text { type }\end{array}$ & Date & Time & $\begin{array}{l}\text { Measuring-point } \\
\text { altitude } \\
\text { (ft above } \\
\text { sea level) }\end{array}$ & $\begin{array}{l}\text { Depth to water } \\
\text { (ft below } \\
\text { measuring point) }\end{array}$ & $\begin{array}{l}\text { Water-level } \\
\text { altitude } \\
\text { (ft above } \\
\text { sea level) }\end{array}$ & Remarks \\
\hline B-103 & WL & $10 / 07 / 92$ & 1226 & 41.01 & 9.56 & 31.45 & \\
\hline B-103 & WL & $10 / 28 / 92$ & 1342 & 41.01 & 10.36 & 30.65 & \\
\hline B-103 & WL & $12 / 03 / 92$ & 1226 & 41.01 & 10.19 & 30.82 & \\
\hline B-103 & WL & $01 / 04 / 93$ & 1210 & 41.01 & 10.90 & 30.11 & \\
\hline B-103 & WL & $02 / 04 / 93$ & 1337 & 41.01 & 9.89 & 31.12 & \\
\hline B-103 & WL & $03 / 05 / 93$ & 1212 & 41.01 & 10.23 & 30.78 & \\
\hline B-103 & WL & $03 / 30 / 93$ & 1132 & 41.01 & 9.99 & 31.02 & \\
\hline B-103 & WL & $05 / 06 / 93$ & 1150 & 41.01 & 10.69 & 30.32 & \\
\hline B-103 & WL & $05 / 28 / 93$ & 1145 & 41.01 & 11.16 & 29.85 & \\
\hline B-103 & WL & $07 / 07 / 93$ & 1135 & 41.01 & 11.10 & 29.91 & \\
\hline B-103 & WL & $08 / 06 / 93$ & 1114 & 41.01 & 11.00 & 30.01 & \\
\hline B-103 & WL & $09 / 03 / 93$ & 1131 & 41.01 & 10.90 & 30.11 & \\
\hline B-103 & WL & $09 / 30 / 93$ & 1149 & 41.01 & 10.71 & 30.30 & \\
\hline B-103 & WL & $10 / 28 / 93$ & 1128 & 41.01 & 11.37 & 29.64 & \\
\hline B-103 & WL & $12 / 01 / 93$ & 1437 & 41.01 & 11.05 & 29.96 & \\
\hline B-103 & WL & $01 / 06 / 94$ & 1152 & 41.01 & 10.84 & 30.17 & \\
\hline B-103 & WL & $02 / 01 / 94$ & 1115 & 41.01 & 9.98 & 31.03 & \\
\hline B-103 & WL & $03 / 04 / 94$ & 1130 & 41.01 & 9.99 & 31.02 & \\
\hline B-105 & WL & $04 / 30 / 91$ & 1150 & 34.85 & 1.88 & 32.97 & \\
\hline B-105 & WL & $05 / 28 / 91$ & --- & 34.85 & 2.30 & 32.55 & \\
\hline B-105 & WL & $07 / 01 / 91$ & -- & 34.85 & 2.59 & 32.26 & \\
\hline B-105 & WL & $08 / 06 / 91$ & -- & 34.85 & 1.83 & 33.02 & \\
\hline B-105 & WL & $08 / 30 / 91$ & -- & 34.85 & 1.63 & 33.22 & \\
\hline B-105 & WL & $09 / 27 / 91$ & -- & 34.85 & 3.11 & 31.74 & \\
\hline B-105 & WL & $10 / 29 / 91$ & --- & 34.85 & 3.69 & 31.16 & \\
\hline B-105 & WL & $12 / 02 / 91$ & --- & 34.85 & 3.85 & 31.00 & \\
\hline B-105 & WL & $01 / 02 / 92$ & --- & 34.85 & 3.61 & 31.24 & \\
\hline B-105 & WL & $02 / 04 / 92$ & $\cdots$ & 34.85 & 2.13 & 32.72 & \\
\hline B-105 & WL & $03 / 06 / 92$ & -- & 34.85 & 2.67 & 32.18 & \\
\hline B-105 & WL & $03 / 31 / 92$ & --- & 34.85 & 2.05 & 32.80 & \\
\hline B-105 & WL & $05 / 01 / 92$ & --- & 34.85 & 2.83 & 32.02 & \\
\hline B-105 & WL & $06 / 11 / 92$ & 1517 & 34.85 & 1.28 & 33.57 & \\
\hline B-105 & WL & $07 / 07 / 92$ & 1448 & 34.85 & 2.93 & 31.92 & \\
\hline B-105 & WL & 08/07/92 & 1401 & 34.85 & 2.52 & 32.33 & \\
\hline B-105 & WL & $08 / 31 / 92$ & 1237 & 34.85 & 2.10 & 32.75 & \\
\hline B-105 & WL & $10 / 07 / 92$ & 1237 & 34.85 & 1.59 & 33.26 & \\
\hline B-105 & WL & $10 / 28 / 92$ & 1354 & 34.85 & 2.62 & 32.23 & \\
\hline B-105 & WL & $12 / 03 / 92$ & 1237 & 34.85 & 1.98 & 32.87 & \\
\hline B-105 & WL & $01 / 04 / 93$ & 1215 & 34.85 & 2.54 & 32.31 & \\
\hline B-105 & WL & $02 / 04 / 93$ & 1347 & 34.85 & 2.10 & 32.75 & \\
\hline B-105 & WL & $03 / 05 / 93$ & 1221 & 34.85 & 1.70 & 33.15 & \\
\hline B-105 & WL & $03 / 30 / 93$ & 1147 & 34.85 & 1.74 & 33.11 & \\
\hline B-105 & WL & $05 / 06 / 93$ & 1249 & 34.85 & 2.80 & 32.05 & \\
\hline B-105 & WL & $05 / 28 / 93$ & 1154 & 34.85 & 3.38 & 31.47 & \\
\hline
\end{tabular}


Table 8.--Water-level data collected at the Defense Fuel Supply Point and adjacent properties, Hanahan, S.C., between April 1991 and September 1995--Continued

[ft, feet; ---, data not available; WL, water-level well; EW, extraction well; MW, monitoring well; SW, surface-water site; -, negative depth to water indicates water-level altitude is above measuring point]

\begin{tabular}{|c|c|c|c|c|c|c|c|}
\hline $\begin{array}{c}\text { Site } \\
\text { (plate 1) }\end{array}$ & $\begin{array}{l}\text { Site } \\
\text { type }\end{array}$ & Date & Time & $\begin{array}{l}\text { Measuring-point } \\
\text { altitude } \\
\text { (ft above } \\
\text { sea level) }\end{array}$ & $\begin{array}{l}\text { Depth to water } \\
\text { (ft below } \\
\text { measuring point) }\end{array}$ & $\begin{array}{l}\text { Water-level } \\
\text { altitude } \\
\text { (ft above } \\
\text { sea level) }\end{array}$ & Remarks \\
\hline B-105 & WL & $07 / 07 / 93$ & 1145 & 34.85 & 3.10 & 31.75 & \\
\hline B-105 & WL & 09/03/93 & 1140 & 34.85 & 3.24 & 31.61 & \\
\hline B-105 & WL & $09 / 30 / 93$ & 1150 & 34.85 & 2.67 & 32.18 & \\
\hline B-105 & WL & $10 / 28 / 93$ & 1140 & 34.85 & 3.38 & 31.47 & \\
\hline B-105 & WL & $12 / 01 / 93$ & 1451 & 34.85 & 2.51 & 32.34 & \\
\hline B-105 & WL & $01 / 06 / 94$ & 1158 & 34.85 & 1.80 & 33.05 & \\
\hline B-105 & WL & $02 / 01 / 94$ & 1144 & 34.85 & 1.41 & 33.44 & \\
\hline B-105 & WL & $03 / 04 / 94$ & 1030 & 34.85 & 1.49 & 33.36 & Measuring point appeared disturbed. \\
\hline B-106 & WL & $04 / 30 / 91$ & 1155 & 39.08 & 6.11 & 32.97 & \\
\hline B-106 & WL & $05 / 28 / 91$ &.-- & 39.08 & 6.51 & 32.57 & \\
\hline B-106 & WL & $07 / 01 / 91$ & --- & 39.08 & 6.95 & 32.13 & \\
\hline B-106 & WL & $08 / 06 / 91$ & -- & 39.08 & 5.37 & 33.71 & \\
\hline B-106 & WL & $08 / 30 / 91$ & -- & 39.08 & 5.03 & 34.05 & \\
\hline B-106 & WL & $09 / 27 / 91$ & --- & 39.08 & 6.76 & 32.32 & \\
\hline B-106 & WL & $10 / 29 / 91$ & -- & 39.08 & 7.67 & 31.41 & \\
\hline B-106 & WL & $12 / 02 / 91$ & -- & 39.08 & 8.62 & 30.46 & \\
\hline B-106 & WL & $01 / 02 / 92$ & -- & 39.08 & 8.72 & 30.36 & \\
\hline B-106 & WL & $02 / 04 / 92$ & - & 39.08 & 6.32 & 32.76 & \\
\hline B-106 & WL & $03 / 06 / 92$ & -- & 39.08 & 6.87 & 32.21 & \\
\hline B-106 & WL & $03 / 31 / 92$ & -- & 39.08 & 6.43 & 32.65 & \\
\hline B-106 & WL & $05 / 01 / 92$ & -- & 39.08 & 7.01 & 32.07 & \\
\hline B-106 & WL & $06 / 11 / 92$ & 1519 & 39.08 & 5.83 & 33.25 & \\
\hline B-106 & WL & $07 / 07 / 92$ & 1441 & 39.08 & 6.64 & 32.44 & \\
\hline B-106 & WL & $08 / 07 / 92$ & 1404 & 39.08 & 6.84 & 32.24 & \\
\hline B-106 & WL & $08 / 31 / 92$ & 1241 & 39.08 & 5.29 & 33.79 & \\
\hline B-106 & WL & $10 / 07 / 92$ & 1241 & 39.08 & 4.85 & 34.23 & \\
\hline B-106 & WL & $10 / 28 / 92$ & 1355 & 39.08 & 6.05 & 33.03 & \\
\hline B-106 & WL & $12 / 03 / 92$ & 1244 & 39.08 & 5.60 & 33.48 & \\
\hline B-106 & WL & $01 / 04 / 93$ & 1220 & 39.08 & 6.53 & 32.55 & \\
\hline B-106 & WL & $02 / 04 / 93$ & 1343 & 39.08 & 5.52 & 33.56 & \\
\hline B-106 & WL & $03 / 05 / 93$ & 1216 & 39.08 & 5.67 & 33.41 & \\
\hline B-106 & WL & $03 / 30 / 93$ & 1140 & 39.08 & 5.20 & 33.88 & \\
\hline B-106 & WL & $05 / 06 / 93$ & 1252 & 39.08 & 6.48 & 32.60 & \\
\hline B-106 & WL & $05 / 28 / 93$ & 1151 & 39.08 & 7.20 & 31.88 & \\
\hline B-106 & WL & $07 / 07 / 93$ & 1141 & 39.08 & 7.40 & 31.68 & \\
\hline B-106 & WL & $08 / 06 / 93$ & 1119 & 39.08 & 6.96 & 32.12 & \\
\hline B-106 & WL & 09/03/93 & 1134 & 39.08 & 7.24 & 31.84 & \\
\hline B-106 & WL & $09 / 30 / 93$ & 1155 & 39.08 & 6.56 & 32.52 & \\
\hline B-106 & WL & $10 / 28 / 93$ & 1134 & 39.08 & 7.66 & 31.42 & \\
\hline B-106 & WL & $12 / 01 / 93$ & 1441 & 39.08 & 6.92 & 32.16 & \\
\hline B-106 & WL & $01 / 06 / 94$ & 1202 & 39.08 & 6.19 & 32.89 & \\
\hline B-106 & WL & $02 / 01 / 94$ & 1110 & 39.08 & 5.18 & 33.90 & \\
\hline B-106 & WL & $03 / 04 / 94$ & 1125 & 39.08 & 6.15 & 32.93 & \\
\hline
\end{tabular}


Table 8.--Water-level data collected at the Defense Fuel Supply Point and adjacent properties, Hanahan, S.C., between April 1991 and September 1995--Continued

[ft, feet; ---, data not available; WL, water-level well; EW, extraction well; MW, monitoring well; SW, surface-water site; -, negative depth to water indicates water-level altitude is above measuring point]

\begin{tabular}{|c|c|c|c|c|c|c|c|}
\hline $\begin{array}{c}\text { Site } \\
\text { (plate 1) }\end{array}$ & $\begin{array}{l}\text { Site } \\
\text { type }\end{array}$ & Date & Time & $\begin{array}{l}\text { Measuring-point } \\
\text { altitude } \\
\text { (ft above } \\
\text { sea level) }\end{array}$ & $\begin{array}{l}\text { Depth to water } \\
\text { (ft below } \\
\text { measuring point) }\end{array}$ & $\begin{array}{l}\text { Water-level } \\
\text { altitude } \\
\text { (ft above } \\
\text { sea level) }\end{array}$ & Remarks \\
\hline B-109 & WL & $04 / 30 / 91$ & 1220 & 38.70 & 6.90 & 31.80 & \\
\hline B-109 & WL & $05 / 28 / 91$ & --- & 38.70 & 7.29 & 31.41 & \\
\hline B-109 & WL & $07 / 01 / 91$ & --- & 38.70 & 7.99 & 30.71 & \\
\hline B-109 & WL & $08 / 06 / 91$ & -- & 38.70 & 5.77 & 32.93 & \\
\hline B-109 & WL & $08 / 30 / 91$ & -- & 38.70 & 5.10 & 33.60 & \\
\hline B-109 & WL & $09 / 27 / 91$ & -- & 38.70 & 7.11 & 31.59 & \\
\hline B-109 & WL & $10 / 29 / 91$ & -- & 38.70 & 8.65 & 30.05 & \\
\hline B-109 & WL & $12 / 02 / 91$ & --- & 38.70 & 9.82 & 28.88 & \\
\hline B-109 & WL & $01 / 02 / 92$ & --- & 38.70 & 10.67 & 28.03 & \\
\hline B-109 & WL & $02 / 04 / 92$ & -- & 38.70 & 7.78 & 30.92 & \\
\hline B-109 & WL & $03 / 06 / 92$ & --- & 38.70 & 8.24 & 30.46 & \\
\hline B-109 & WL & $03 / 31 / 92$ & --- & 38.70 & 7.85 & 30.85 & \\
\hline B-109 & WL & $05 / 01 / 92$ & -- & 38.70 & 8.23 & 30.47 & \\
\hline B-109 & WL & $06 / 11 / 92$ & 1539 & 38.70 & 6.74 & 31.96 & \\
\hline B-109 & WL & $07 / 07 / 92$ & 1425 & 38.70 & 7.57 & 31.13 & \\
\hline B-109 & WL & $08 / 07 / 92$ & 1423 & 38.70 & 7.88 & 30.82 & \\
\hline B- 109 & WL & $08 / 31 / 92$ & 1302 & 38.70 & 5.45 & 33.25 & \\
\hline B-109 & WL & $10 / 07 / 92$ & 1339 & 38.70 & 4.93 & 33.77 & \\
\hline B-109 & WL & $10 / 28 / 92$ & 1410 & 38.70 & 6.22 & 32.48 & \\
\hline B-109 & WL & $12 / 03 / 92$ & 1245 & 38.70 & 5.73 & 32.97 & \\
\hline B-109 & WL & $01 / 04 / 93$ & 1215 & 38.70 & 7.09 & 31.61 & \\
\hline B-109 & WL & $02 / 04 / 93$ & 1330 & 38.70 & 5.33 & 33.37 & \\
\hline B-109 & WL & $03 / 05 / 93$ & 1220 & 38.70 & 5.60 & 33.10 & \\
\hline B-109 & WL & $03 / 30 / 93$ & 1145 & 38.70 & 5.18 & 33.52 & \\
\hline B-109 & WL & $05 / 06 / 93$ & 1255 & 38.70 & 6.63 & 32.07 & \\
\hline B-109 & WL & $05 / 28 / 93$ & 1205 & 38.70 & 7.76 & 30.94 & \\
\hline B-109 & WL & $07 / 07 / 93$ & 1155 & 38.70 & 8.45 & 30.25 & \\
\hline B-109 & WL & $08 / 06 / 93$ & 1130 & 38.70 & 8.27 & 30.43 & \\
\hline B-109 & WL & $09 / 03 / 93$ & 1145 & 38.70 & 7.91 & 30.79 & \\
\hline B-109 & WL & $09 / 30 / 93$ & 1140 & 38.70 & 7.17 & 31.53 & \\
\hline B-109 & WL & $10 / 28 / 93$ & 1140 & 38.70 & 8.72 & 29.98 & \\
\hline B-109 & WL & $12 / 01 / 93$ & 1450 & 38.70 & 8.08 & 30.62 & \\
\hline B-109 & WL & $01 / 06 / 94$ & 1210 & 38.70 & 7.59 & 31.11 & \\
\hline B-109 & WL & $02 / 01 / 94$ & 1100 & 38.70 & 5.80 & 32.90 & \\
\hline B-109 & WL & $03 / 04 / 94$ & 1115 & 38.70 & 5.84 & 32.86 & \\
\hline EW-01 & EW & $04 / 30 / 91$ & 1511 & 36.21 & 9.77 & 26.44 & \\
\hline EW-0l & EW & $05 / 28 / 91$ & --- & 36.21 & 9.95 & 26.26 & \\
\hline EW-01 & EW & $05 / 14 / 92$ & 1005 & 36.27 & 11.10 & 25.17 & New measuring point established. \\
\hline EW-02 & EW & $04 / 30 / 91$ & 1340 & 35.76 & 14.56 & 21.20 & \\
\hline EW-02 & EW & $05 / 28 / 91$ & -- & 35.76 & 10.68 & 25.08 & \\
\hline EW-02 & EW & $05 / 14 / 92$ & 1019 & 35.83 & 11.89 & 23.94 & New measuring point established. \\
\hline EW-03 & EW & $04 / 30 / 91$ & 1505 & 32.53 & 11.03 & 21.50 & \\
\hline
\end{tabular}


Table 8.--Water-level data collected at the Defense Fuel Supply Point and adjacent properties, Hanahan, S.C., between April 1991 and September 1995--Continued

[ft, feet; ---, data not available; WL, water-level well; EW, extraction well; MW, monitoring well; SW, surface-water site; -, negative depth to water indicates water-level altitude is above measuring point]

\begin{tabular}{|c|c|c|c|c|c|c|c|}
\hline $\begin{array}{c}\text { Site } \\
\text { (plate 1) }\end{array}$ & $\begin{array}{l}\text { Site } \\
\text { type }\end{array}$ & Date & Time & $\begin{array}{l}\text { Measuring-point } \\
\text { altitude } \\
\text { (ft above } \\
\text { sea level) }\end{array}$ & $\begin{array}{l}\text { Depth to water } \\
\text { (ft below } \\
\text { measuring point) }\end{array}$ & $\begin{array}{l}\text { Water-level } \\
\text { altitude } \\
\text { (ft above } \\
\text { sea level) }\end{array}$ & Remarks \\
\hline$\overline{\text { EW-03 }}$ & EW & $05 / 28 / 91$ & 1505 & 32.53 & 11.23 & 21.30 & \\
\hline EW-03 & EW & $05 / 14 / 92$ & 1036 & 32.59 & 12.49 & 20.10 & New measuring point established. \\
\hline EW-08 & EW & $04 / 30 / 91$ & 1459 & 37.50 & 16.01 & 21.49 & \\
\hline EW-08 & EW & $05 / 28 / 91$ & --- & 37.50 & 16.10 & 21.40 & \\
\hline EW-08 & EW & $05 / 14 / 92$ & 1042 & 37.56 & 17.74 & 19.82 & New measuring point established. \\
\hline EW-09 & EW & $04 / 30 / 91$ & 1356 & 37.86 & 16.44 & 21.42 & \\
\hline EW-09 & EW & $05 / 28 / 91$ & -- & 37.86 & 16.52 & 21.34 & \\
\hline EW-09 & EW & $02 / 04 / 92$ & 1208 & 37.94 & 25.23 & 12.71 & New measuring point established. \\
\hline EW-09 & EW & $05 / 01 / 92$ & 1536 & 37.94 & 17.13 & 20.81 & \\
\hline EW-09 & EW & $05 / 01 / 92$ & 1052 & 37.94 & 20.89 & 17.05 & \\
\hline EW-10 & EW & $04 / 30 / 91$ & 1356 & 37.77 & 16.90 & 20.87 & \\
\hline EW-10 & EW & $05 / 28 / 91$ & -- & 37.77 & 16.92 & 20.85 & \\
\hline EW-10 & EW & $02 / 04 / 92$ & 1115 & 37.84 & 17.72 & 20.12 & New measuring point established. \\
\hline EW-10 & EW & $05 / 01 / 92$ & 1539 & 37.84 & 17.44 & 20.40 & \\
\hline EW-10 & EW & $05 / 01 / 92$ & 1047 & 37.84 & 18.99 & 18.85 & \\
\hline EW-11 & EW & $04 / 30 / 91$ & 1407 & 37.15 & 13.03 & 24.12 & \\
\hline EW-11 & EW & $05 / 28 / 91$ & 1444 & 37.15 & 13.09 & 24.06 & \\
\hline EW-11 & EW & $02 / 04 / 92$ & 1228 & 37.18 & 23.30 & 13.88 & New measuring point established. \\
\hline EW-11 & EW & $05 / 01 / 92$ & 1042 & 37.18 & 16.94 & 20.24 & \\
\hline EW-11 & EW & $05 / 01 / 92$ & 1542 & 37.18 & 13.48 & 23.70 & \\
\hline EW-12 & EW & $04 / 30 / 91$ & 1413 & 36.56 & 10.64 & 25.92 & \\
\hline EW-12 & EW & $05 / 28 / 91$ & 1440 & 36.56 & 10.82 & 25.74 & \\
\hline EW-13 & EW & $04 / 30 / 91$ & 1418 & 36.30 & 9.60 & 26.70 & \\
\hline EW-13 & EW & $05 / 28 / 91$ & 1431 & 36.30 & 9.83 & 26.47 & \\
\hline EW-13 & EW & $02 / 04 / 92$ & - & 36.36 & 10.25 & 26.11 & $\begin{array}{l}\text { Pre-start-up water levels; new measuring } \\
\text { point established. }\end{array}$ \\
\hline EW-13 & EW & $02 / 12 / 92$ & - & 36.36 & 12.91 & 23.45 & \\
\hline EW-13 & EW & $02 / 20 / 92$ & 1531 & 36.36 & 11.40 & 24.96 & \\
\hline EW-13 & EW & $02 / 25 / 92$ & 0931 & 36.36 & 10.41 & 25.95 & \\
\hline EW-13 & EW & $02 / 25 / 92$ & 1520 & 36.36 & 13.20 & 23.16 & \\
\hline EW-13 & EW & $03 / 09 / 92$ & 1106 & 36.36 & 10.45 & 25.91 & \\
\hline EW-14 & EW & $04 / 30 / 91$ & 1449 & 36.06 & 9.24 & 26.82 & \\
\hline EW-14 & EW & $05 / 28 / 91$ & 1427 & 36.06 & 9.47 & 26.59 & \\
\hline EW-14 & EW & $02 / 04 / 92$ & --- & 36.10 & 9.59 & 26.51 & $\begin{array}{l}\text { Pre-start-up water levels; new measuring } \\
\text { point established. }\end{array}$ \\
\hline EW-14 & EW & $02 / 12 / 92$ & --- & 36.10 & 10.10 & 26.00 & \\
\hline EW-14 & EW & $02 / 20 / 92$ & 1526 & 36.10 & 12.07 & 24.03 & \\
\hline EW-14 & EW & $02 / 25 / 92$ & 0916 & 36.10 & 9.83 & 26.27 & \\
\hline
\end{tabular}


Table 8.--Water-level data collected at the Defense Fuel Supply Point and adjacent properties, Hanahan, S.C., between April 1991 and September 1995--Continued

[ft, feet; ---, data not available; WL, water-level well; EW, extraction well; MW, monitoring well; SW, surface-water site; -, negative depth to wateindicates water-level altitude is above measuring point]

\begin{tabular}{|c|c|c|c|c|c|c|c|}
\hline $\begin{array}{c}\text { Site } \\
\text { (plate 1) }\end{array}$ & $\begin{array}{l}\text { Site } \\
\text { type }\end{array}$ & Date & Time & $\begin{array}{l}\text { Measuring-point } \\
\text { altitude } \\
\text { (ft above } \\
\text { sea level) }\end{array}$ & $\begin{array}{l}\text { Depth to water } \\
\text { (ft below } \\
\text { measuring point) }\end{array}$ & $\begin{array}{l}\text { Water-level } \\
\text { altitude } \\
\text { (ft above } \\
\text { sea level) }\end{array}$ & Remarks \\
\hline EW-14 & EW & $02 / 25 / 92$ & 1515 & 36.10 & 10.00 & 26.10 & \\
\hline EW-14 & EW & $03 / 09 / 92$ & 1112 & 36.10 & 9.80 & 26.30 & \\
\hline EW-15 & EW & $05 / 28 / 91$ & --- & 34.82 & 7.71 & 27.11 & \\
\hline EW-15 & EW & $03 / 09 / 92$ & --- & 34.88 & 7.96 & 26.92 & New measuring point established. \\
\hline EW-16 & EW & $04 / 30 / 91$ & 1425 & 35.65 & 7.93 & 27.72 & \\
\hline EW-16 & EW & $05 / 28 / 91$ & 1420 & 35.65 & 8.10 & 27.55 & \\
\hline EW-17 & EW & $04 / 30 / 91$ & 1430 & 35.75 & 7.75 & 28.00 & \\
\hline EW-17 & EW & $05 / 28 / 91$ & 1410 & 35.75 & 7.94 & 27.81 & \\
\hline EW-18 & EW & $04 / 30 / 91$ & 1054 & 21.76 & 6.43 & 15.33 & \\
\hline MW-04 & MW & $04 / 30 / 91$ & 1048 & 27.82 & 10.67 & 17.15 & \\
\hline MW-04 & MW & $05 / 28 / 91$ & 1200 & 27.82 & 10.84 & 16.98 & \\
\hline MW-04 & MW & $07 / 01 / 91$ & --- & 27.82 & 11.21 & 16.61 & \\
\hline MW-04 & MW & $08 / 06 / 91$ & --- & 27.82 & 10.04 & 17.78 & \\
\hline MW-04 & MW & $08 / 30 / 91$ & --- & 27.82 & 9.70 & 18.12 & \\
\hline MW-04 & MW & $09 / 27 / 91$ & --- & 27.82 & 10.50 & 17.32 & \\
\hline MW-04 & MW & $10 / 29 / 91$ & --- & 27.82 & 11.24 & 16.58 & \\
\hline MW-04 & MW & $12 / 02 / 91$ & --- & 27.82 & 11.70 & 16.12 & \\
\hline MW-04 & MW & $01 / 02 / 92$ & -- & 27.82 & 12.04 & 15.78 & \\
\hline MW-04 & MW & $02 / 04 / 92$ & --- & 27.82 & 12.18 & 15.64 & \\
\hline MW-04 & MW & $03 / 06 / 92$ & -.- & 27.82 & 12.23 & 15.59 & \\
\hline MW-04 & MW & $03 / 31 / 92$ & --- & 27.82 & 11.95 & 15.87 & \\
\hline MW-04 & MW & $05 / 01 / 92$ & --- & 27.82 & 12.21 & 15.61 & \\
\hline MW-04 & MW & $06 / 11 / 92$ & 1650 & 27.82 & 12.46 & 15.36 & \\
\hline MW-04 & MW & $07 / 07 / 92$ & 1026 & 27.82 & 12.20 & 15.62 & \\
\hline MW-04 & MW & $08 / 31 / 92$ & 1340 & 27.82 & 10.74 & 17.08 & \\
\hline MW-04 & MW & $10 / 07 / 92$ & 1440 & 27.82 & 10.42 & 17.40 & \\
\hline MW-04 & MW & $10 / 28 / 92$ & 1321 & 27.82 & 10.49 & 17.33 & \\
\hline MW-04 & MW & $12 / 03 / 92$ & 1402 & 27.82 & 10.49 & 17.33 & \\
\hline MW-04 & MW & $01 / 04 / 93$ & 1335 & 27.82 & 11.14 & 16.68 & \\
\hline MW-04 & MW & $02 / 04 / 93$ & 1420 & 27.82 & 9.58 & 18.24 & \\
\hline MW-04 & MW & $03 / 05 / 93$ & 1347 & 27.82 & 10.16 & 17.66 & \\
\hline MW-04 & MW & $03 / 30 / 93$ & 1251 & 27.82 & 10.58 & 17.24 & \\
\hline MW-04 & MW & $05 / 06 / 93$ & 1305 & 27.82 & 11.18 & 16.64 & \\
\hline MW-04 & MW & $05 / 28 / 93$ & 1254 & 27.82 & 11.76 & 16.06 & \\
\hline MW-04 & MW & $07 / 07 / 93$ & 1310 & 27.82 & 12.24 & 15.58 & \\
\hline MW-04 & MW & $08 / 06 / 93$ & 1316 & 27.82 & 12.53 & 15.29 & \\
\hline MW-04 & MW & $09 / 03 / 93$ & 1215 & 27.82 & 12.37 & 15.45 & \\
\hline MW-04 & MW & $09 / 30 / 93$ & 1309 & 27.82 & 12.15 & 15.67 & \\
\hline MW-04 & MW & $10 / 28 / 93$ & 1245 & 27.82 & 12.73 & 15.09 & \\
\hline MW-04 & MW & $12 / 01 / 93$ & 1535 & 27.82 & 12.70 & 15.12 & \\
\hline
\end{tabular}


Table 8.--Water-level data collected at the Defense Fuel Supply Point and adjacent properties, Hanahan, S.C., between April 1991 and September 1995--Continued

[ft, feet; ---, data not available; WL, water-level well; EW, extraction well; MW, monitoring well; SW, surface-water site; -, negative depth to water indicates water-level altitude is above measuring point]

\begin{tabular}{|c|c|c|c|c|c|c|c|}
\hline $\begin{array}{c}\text { Site } \\
\text { (plate 1) }\end{array}$ & $\begin{array}{l}\text { Site } \\
\text { type }\end{array}$ & Date & Time & $\begin{array}{l}\text { Measuring-point } \\
\text { altitude } \\
\text { (ft above } \\
\text { sea level) }\end{array}$ & $\begin{array}{l}\text { Depth to water } \\
\text { (ft below } \\
\text { measuring point) }\end{array}$ & $\begin{array}{l}\text { Water-level } \\
\text { altitude } \\
\text { (ft above } \\
\text { sea level) }\end{array}$ & Remarks \\
\hline MW-04 & MW & $01 / 06 / 94$ & 1322 & 27.82 & 12.56 & 15.26 & \\
\hline MW-04 & MW & $02 / 01 / 94$ & 1300 & 27.82 & 11.70 & 16.12 & \\
\hline MW-04 & MW & $03 / 04 / 94$ & 1230 & 27.82 & 11.32 & 16.50 & \\
\hline MW-04 & MW & $04 / 04 / 94$ & 1159 & 27.82 & 11.74 & 16.08 & \\
\hline MW-04 & MW & $05 / 06 / 94$ & 1302 & 27.82 & 12.54 & 15.28 & \\
\hline MW-04 & MW & $06 / 03 / 94$ & 1230 & 27.82 & 13.11 & 14.71 & \\
\hline MW-04 & MW & $07 / 08 / 94$ & 1215 & 27.82 & 12.10 & 15.72 & \\
\hline MW-04 & MW & $08 / 05 / 94$ & 1452 & 27.82 & 11.30 & 16.52 & \\
\hline MW-04 & MW & $09 / 07 / 94$ & 1330 & 27.82 & 10.79 & 17.03 & \\
\hline MW-04 & MW & $10 / 06 / 94$ & 1303 & 27.82 & 9.59 & 18.23 & \\
\hline MW-04 & MW & $11 / 09 / 94$ & 1338 & 27.82 & 9.64 & 18.18 & \\
\hline MW-04 & MW & $12 / 06 / 94$ & 1205 & 27.82 & 10.24 & 17.58 & \\
\hline MW-04 & MW & $01 / 10 / 95$ & 1641 & 27.82 & 10.25 & 17.57 & \\
\hline MW-04 & MW & $02 / 02 / 95$ & 1416 & 27.82 & 10.10 & 17.72 & \\
\hline MW-04 & MW & $03 / 02 / 95$ & 1550 & 27.82 & 10.26 & 17.56 & \\
\hline MW-04 & MW & $04 / 05 / 95$ & 1206 & 27.82 & 10.98 & 16.84 & \\
\hline MW-04 & MW & $05 / 02 / 95$ & 1416 & 27.82 & 11.50 & 16.32 & \\
\hline MW-04 & MW & $06 / 09 / 95$ & 1250 & 27.82 & 12.16 & 15.66 & \\
\hline MW-04 & MW & $07 / 06 / 95$ & 1217 & 27.82 & 12.24 & 15.58 & \\
\hline MW-04 & MW & $08 / 10 / 95$ & 1305 & 27.82 & 12.19 & 15.63 & \\
\hline MW-04 & MW & $09 / 13 / 95$ & 1436 & 27.82 & 10.53 & 17.29 & \\
\hline MW-05 & MW & $04 / 30 / 91$ & 1120 & 20.00 & 2.03 & 17.97 & \\
\hline MW-05 & MW & $05 / 28 / 91$ & 1215 & 20.00 & 2.11 & 17.89 & \\
\hline MW-05 & MW & $07 / 01 / 91$ & - & 20.00 & 2.26 & 17.74 & \\
\hline MW-05 & MW & $08 / 06 / 91$ & -- & 20.00 & 1.64 & 18.36 & \\
\hline MW-05 & MW & $08 / 30 / 91$ & -- & 20.00 & 1.51 & 18.49 & \\
\hline MW-05 & MW & $09 / 27 / 91$ & --- & 20.00 & 2.05 & 17.95 & \\
\hline MW-05 & MW & $10 / 29 / 91$ & -- & 20.00 & 2.60 & 17.40 & \\
\hline MW-05 & MW & $12 / 02 / 91$ & -- & 20.00 & 3.27 & 16.73 & \\
\hline MW-05 & MW & $01 / 02 / 92$ & -- & 20.00 & 3.92 & 16.08 & \\
\hline MW-05 & MW & $02 / 04 / 92$ & -- & 20.00 & 3.55 & 16.45 & \\
\hline MW-05 & MW & $03 / 06 / 92$ & -- & 20.00 & 3.45 & 16.55 & \\
\hline MW-05 & MW & $03 / 31 / 92$ & -- & 20.00 & 3.33 & 16.67 & \\
\hline MW-05 & MW & $05 / 01 / 92$ & -- & 20.00 & 3.41 & 16.59 & \\
\hline MW-05 & MW & $06 / 11 / 92$ & 1732 & 20.00 & 3.29 & 16.71 & \\
\hline MW-05 & MW & $07 / 07 / 92$ & 1100 & 20.00 & 3.05 & 16.95 & \\
\hline MW-05 & MW & $08 / 31 / 92$ & 1340 & 20.00 & 1.85 & 18.15 & \\
\hline MW-05 & MW & $10 / 07 / 92$ & 1443 & 20.00 & 1.57 & 18.43 & \\
\hline MW-05 & MW & $10 / 28 / 92$ & 1348 & 20.00 & 1.94 & 18.06 & \\
\hline MW-05 & MW & $12 / 03 / 92$ & 1414 & 20.00 & 1.76 & 18.24 & \\
\hline MW-05 & MW & $01 / 04 / 93$ & 1355 & 20.00 & 2.11 & 17.89 & \\
\hline MW-05 & MW & $02 / 04 / 93$ & 1432 & 20.00 & 1.54 & 18.46 & \\
\hline MW-05 & MW & $03 / 05 / 93$ & 1355 & 20.00 & 1.60 & 18.40 & \\
\hline MW-05 & MW & $03 / 30 / 93$ & 1305 & 20.00 & 1.45 & 18.55 & \\
\hline
\end{tabular}


Table 8.--Water-level data collected at the Defense Fuel Supply Point and adjacent properties, Hanahan, S.C., between April 1991 and September 1995--Continued

[ft, feet; ---, data not available; WL, water-level well; EW, extraction well; MW, monitoring well; SW, surface-water site; -, negative depth to water indicates water-level altitude is above measuring point]

\begin{tabular}{|c|c|c|c|c|c|c|c|}
\hline $\begin{array}{c}\text { Site } \\
\text { (plate 1) }\end{array}$ & $\begin{array}{l}\text { Site } \\
\text { type }\end{array}$ & Date & Time & $\begin{array}{l}\text { Measuring-point } \\
\text { altitude } \\
\text { (ft above } \\
\text { sea level) }\end{array}$ & $\begin{array}{l}\text { Depth to water } \\
\text { (ft below } \\
\text { measuring point) }\end{array}$ & $\begin{array}{l}\text { Water-level } \\
\text { altitude } \\
\text { (ft above } \\
\text { sea level) }\end{array}$ & Remarks \\
\hline MW-05 & MW & $05 / 06 / 93$ & 1335 & 20.00 & 1.79 & 18.21 & \\
\hline MW-05 & MW & $05 / 28 / 93$ & 1307 & 20.00 & 1.57 & 18.43 & \\
\hline MW-05 & MW & $07 / 07 / 93$ & 1334 & 20.00 & 2.35 & 17.65 & \\
\hline MW-05 & MW & $08 / 06 / 93$ & 1311 & 20.00 & 2.49 & 17.51 & \\
\hline MW-05 & MW & 09/03/93 & 1149 & 20.00 & 2.64 & 17.36 & \\
\hline MW-05 & MW & 09/30/93 & 1246 & 20.00 & 2.48 & 17.52 & \\
\hline MW-05 & MW & $10 / 28 / 93$ & 1325 & 20.00 & 2.80 & 17.20 & \\
\hline MW-05 & MW & $12 / 01 / 93$ & 1515 & 20.00 & 2.73 & 17.27 & \\
\hline MW-05 & MW & $01 / 06 / 94$ & 1304 & 20.00 & 2.27 & 17.73 & \\
\hline MW-05 & MW & $02 / 01 / 94$ & 1234 & 20.00 & 1.93 & 18.07 & \\
\hline MW-05 & MW & $03 / 04 / 94$ & 1212 & 20.00 & 1.73 & 18.27 & \\
\hline MW-05 & MW & $04 / 04 / 94$ & 1131 & 20.00 & 2.24 & 17.76 & \\
\hline MW-05 & MW & $05 / 06 / 94$ & 1227 & 20.00 & 2.80 & 17.20 & \\
\hline MW-05 & MW & $06 / 03 / 94$ & 1200 & 20.00 & 3.20 & 16.80 & \\
\hline MW-05 & MW & $07 / 08 / 94$ & 1238 & 20.00 & 2.12 & 17.88 & \\
\hline MW-05 & MW & $08 / 05 / 94$ & 1506 & 20.00 & 2.15 & 17.85 & \\
\hline MW-05 & MW & $09 / 07 / 94$ & 1345 & 20.00 & 1.80 & 18.20 & \\
\hline MW-05 & MW & $10 / 06 / 94$ & 1331 & 20.00 & 1.09 & 18.91 & \\
\hline MW-05 & MW & $11 / 09 / 94$ & 1401 & 20.00 & 1.57 & 18.43 & \\
\hline MW-05 & MW & $12 / 06 / 94$ & 1230 & 20.00 & 1.35 & 18.65 & \\
\hline MW-05 & MW & $01 / 10 / 95$ & 1700 & 20.00 & 1.47 & 18.53 & \\
\hline MW-05 & MW & $02 / 02 / 95$ & 1428 & 20.00 & 1.62 & 18.38 & \\
\hline MW-05 & MW & $03 / 02 / 95$ & 1602 & 20.00 & 1.46 & 18.54 & \\
\hline MW-05 & MW & $04 / 05 / 95$ & 1226 & 20.00 & 1.94 & 18.06 & \\
\hline MW-05 & MW & $05 / 02 / 95$ & 1347 & 20.00 & 2.24 & 17.76 & \\
\hline MW-05 & MW & $06 / 09 / 95$ & 1305 & 20.00 & 2.63 & 17.37 & \\
\hline MW-05 & MW & $07 / 06 / 95$ & 1227 & 20.00 & 2.74 & 17.26 & \\
\hline MW-05 & MW & $08 / 10 / 95$ & 1313 & 20.00 & 2.76 & 17.24 & \\
\hline MW-05 & MW & $09 / 13 / 95$ & 1501 & 20.00 & 1.76 & 18.24 & \\
\hline MW-06 & MW & $04 / 30 / 91$ & 1102 & 16.34 & .02 & 16.32 & \\
\hline MW-06 & MW & $05 / 28 / 91$ & 1213 & 16.34 & .08 & 16.26 & \\
\hline MW-06 & MW & $07 / 01 / 91$ & -- & 16.34 & .22 & 16.12 & \\
\hline MW-06 & MW & $08 / 06 / 91$ & --- & 16.34 & -.22 & 16.56 & \\
\hline MW-06 & MW & $08 / 30 / 91$ &.-- & 16.34 & -.28 & 16.62 & \\
\hline MW-06 & MW & $09 / 27 / 91$ & --- & 16.34 & .05 & 16.29 & \\
\hline MW-06 & MW & $10 / 29 / 91$ & -- & 16.34 & .35 & 15.99 & \\
\hline MW-06 & MW & $12 / 02 / 91$ & --- & 16.34 & .52 & 15.82 & \\
\hline MW-06 & MW & $01 / 02 / 92$ & --- & 16.34 & .69 & 15.65 & \\
\hline MW-06 & MW & $02 / 04 / 92$ & --. & 16.34 & .80 & 15.54 & \\
\hline MW-06 & MW & $03 / 06 / 92$ & $\cdots$ & 16.34 & .98 & 15.36 & \\
\hline MW-06 & MW & $03 / 31 / 92$ & -- & 16.34 & .71 & 15.63 & \\
\hline MW-06 & MW & $05 / 01 / 92$ & 1412 & 16.34 & 1.08 & 15.26 & \\
\hline MW-06 & MW & $06 / 11 / 92$ & 1707 & 16.34 & .59 & 15.75 & \\
\hline MW-06 & MW & $07 / 07 / 92$ & 1035 & 16.34 & 1.05 & 15.29 & \\
\hline
\end{tabular}


Table 8.--Water-level data collected at the Defense Fuel Supply Point and adjacent properties, Hanahan, S.C., between April 1991 and September 1995--Continued

[ft, feet; ---, data not available; WL, water-level well; EW, extraction well; MW, monitoring well; SW, surface-water site; -, negative depth to water indicates water-level altitude is above measuring point]

\begin{tabular}{|c|c|c|c|c|c|c|c|}
\hline $\begin{array}{c}\text { Site } \\
\text { (plate 1) }\end{array}$ & $\begin{array}{l}\text { Site } \\
\text { type }\end{array}$ & Date & Time & $\begin{array}{l}\text { Measuring-point } \\
\text { altitude } \\
\text { (ft above } \\
\text { sea level) }\end{array}$ & $\begin{array}{l}\text { Depth to water } \\
\text { ( } \mathrm{ft} \text { below } \\
\text { measuring point) }\end{array}$ & $\begin{array}{l}\text { Water-level } \\
\text { altitude } \\
\text { (ft above } \\
\text { sea level) }\end{array}$ & Remarks \\
\hline MW-06 & MW & $08 / 31 / 92$ & 1322 & 16.34 & 0.03 & 16.31 & \\
\hline MW-06 & MW & $10 / 07 / 92$ & 1438 & 16.34 & -.24 & 16.58 & \\
\hline MW-06 & MW & $10 / 28 / 92$ & 1340 & 16.34 & .05 & 16.29 & \\
\hline MW-06 & MW & $12 / 03 / 92$ & 1407 & 16.34 & .07 & 16.27 & \\
\hline MW-06 & MW & $01 / 04 / 93$ & 1325 & 16.34 & .40 & 15.94 & \\
\hline MW-06 & MW & $02 / 04 / 93$ & 1414 & 16.34 & -.08 & 16.42 & \\
\hline MW-06 & MW & $03 / 05 / 93$ & 1347 & 16.34 & -.01 & 16.35 & \\
\hline MW-06 & MW & $03 / 30 / 93$ & 1300 & 16.34 & .30 & 16.04 & \\
\hline MW-06 & MW & $05 / 06 / 93$ & 1313 & 16.34 & .77 & 15.57 & \\
\hline MW-06 & MW & $05 / 28 / 93$ & 1300 & 16.34 & 1.16 & 15.18 & \\
\hline MW-06 & MW & $07 / 07 / 93$ & 1323 & 16.34 & 1.10 & 15.24 & \\
\hline MW-06 & MW & $08 / 06 / 93$ & 1324 & 16.34 & 1.05 & 15.29 & \\
\hline MW-06 & MW & $09 / 03 / 93$ & 1207 & 16.34 & 1.35 & 14.99 & \\
\hline MW-06 & MW & $09 / 30 / 93$ & 1257 & 16.34 & 1.02 & 15.32 & \\
\hline MW-06 & MW & $10 / 28 / 93$ & 1253 & 16.34 & 1.40 & 14.94 & \\
\hline MW-06 & MW & $12 / 01 / 93$ & 1532 & 16.34 & 1.32 & 15.02 & \\
\hline MW-06 & MW & $01 / 06 / 94$ & 1320 & 16.34 & 1.04 & 15.30 & \\
\hline MW-06 & MW & $02 / 01 / 94$ & 1245 & 16.34 & .30 & 16.04 & \\
\hline MW-06 & MW & $03 / 04 / 94$ & 1225 & 16.34 & .23 & 16.11 & \\
\hline MW-06 & MW & $04 / 04 / 94$ & 1145 & 16.34 & .90 & 15.44 & \\
\hline MW-06 & MW & $05 / 06 / 94$ & 1241 & 16.34 & 1.48 & 14.86 & \\
\hline MW-06 & MW & $06 / 03 / 94$ & 1217 & 16.34 & 1.96 & 14.38 & \\
\hline MW-06 & MW & $07 / 08 / 94$ & 1222 & 16.34 & .46 & 15.88 & \\
\hline MW-06 & MW & $08 / 05 / 94$ & 1457 & 16.34 & .27 & 16.07 & \\
\hline MW-06 & MW & $09 / 07 / 94$ & 1322 & 16.34 & .13 & 16.21 & \\
\hline MW-06 & MW & $10 / 06 / 94$ & 1326 & 16.34 & -.33 & 16.67 & \\
\hline MW-06 & MW & $11 / 09 / 94$ & 1348 & 16.34 & -.21 & 16.55 & \\
\hline MW-06 & MW & $12 / 06 / 94$ & 1218 & 16.34 & -.23 & 16.57 & \\
\hline MW-06 & MW & $01 / 10 / 95$ & 1649 & 16.34 & .16 & 16.18 & \\
\hline MW-06 & MW & $02 / 02 / 95$ & 1423 & 16.34 & .03 & 16.31 & \\
\hline MW-06 & MW & $03 / 02 / 95$ & 1606 & 16.34 & .17 & 16.17 & \\
\hline MW-06 & MW & $04 / 05 / 95$ & 1219 & 16.34 & .60 & 15.74 & \\
\hline MW-06 & MW & $05 / 02 / 95$ & 1354 & 16.34 & .82 & 15.52 & \\
\hline MW-06 & MW & $06 / 09 / 95$ & 1315 & 16.34 & 1.05 & 15.29 & \\
\hline MW-06 & MW & $07 / 06 / 95$ & 1234 & 16.34 & 1.04 & 15.30 & \\
\hline MW-06 & MW & $08 / 10 / 95$ & 1320 & 16.34 & 1.03 & 15.31 & \\
\hline MW-06 & MW & $09 / 13 / 95$ & 1442 & 16.34 & .12 & 16.22 & \\
\hline MW-07 & MW & $04 / 30 / 91$ & 0930 & 38.44 & 12.63 & 25.81 & \\
\hline MW-07 & MW & $05 / 28 / 91$ & --- & 38.44 & 12.68 & 25.76 & \\
\hline MW-07 & MW & $07 / 01 / 91$ & --- & 38.44 & 13.26 & 25.18 & \\
\hline MW-07 & MW & $08 / 06 / 91$ & -- & 38.44 & 11.27 & 27.17 & \\
\hline MW-07 & MW & $08 / 30 / 91$ & --- & 38.44 & 10.52 & 27.92 & \\
\hline MW-07 & MW & $09 / 27 / 91$ & --- & 38.44 & 12.12 & 26.32 & \\
\hline MW-07 & MW & $10 / 29 / 91$ & -- & 38.44 & 13.31 & 25.13 & \\
\hline
\end{tabular}


Table 8.--Water-level data collected at the Defense Fuel Supply Point and adjacent properties, Hanahan, S.C., between April 1991 and September 1995--Continued

[ft, feet; ---, data not available; WL, water-level well; EW, extraction well; MW, monitoring well; SW, surface-water site; -, negative depth to water indicates water-level altitude is above measuring point]

\begin{tabular}{|c|c|c|c|c|c|c|c|}
\hline $\begin{array}{c}\text { Site } \\
\text { (plate 1) }\end{array}$ & $\begin{array}{l}\text { Site } \\
\text { type }\end{array}$ & Date & Time & $\begin{array}{c}\text { Measuring-point } \\
\text { altitude } \\
\text { (ft above } \\
\text { sea level) }\end{array}$ & $\begin{array}{l}\text { Depth to water } \\
\text { (ft below } \\
\text { measuring point) }\end{array}$ & $\begin{array}{l}\text { Water-level } \\
\text { altitude } \\
\text { (ft above } \\
\text { sea level) }\end{array}$ & Remarks \\
\hline MW-07 & MW & $12 / 02 / 91$ & -- & 38.44 & 14.23 & 24.21 & \\
\hline MW-07 & MW & $01 / 02 / 92$ & --- & 38.44 & 14.95 & 23.49 & \\
\hline MW-07 & MW & $02 / 04 / 92$ & --- & 38.44 & 14.70 & 23.74 & \\
\hline MW-07 & MW & $03 / 06 / 92$ & --- & 38.44 & 14.26 & 24.18 & \\
\hline MW-07 & MW & $03 / 31 / 92$ & -- & 38.44 & 14.27 & 24.17 & \\
\hline MW-07 & MW & $05 / 01 / 92$ & -- & 38.44 & 14.17 & 24.27 & \\
\hline MW-07 & MW & $05 / 14 / 92$ & 1020 & 38.44 & 14.27 & 24.17 & \\
\hline MW-07 & MW & $06 / 11 / 92$ & 1100 & 38.44 & 14.64 & 23.80 & \\
\hline MW-07 & MW & $07 / 07 / 92$ & 1804 & 38.44 & 13.67 & 24.77 & \\
\hline MW-07 & MW & $08 / 07 / 92$ & 0943 & 38.44 & 14.02 & 24.42 & \\
\hline MW-07 & MW & $08 / 31 / 92$ & 1024 & 38.44 & 11.54 & 26.90 & \\
\hline MW-07 & MW & $10 / 07 / 92$ & 1029 & 38.44 & 11.09 & 27.35 & \\
\hline MW-07 & MW & $10 / 28 / 92$ & 1100 & 38.44 & 11.80 & 26.64 & \\
\hline MW-07 & MW & $12 / 03 / 92$ & 0948 & 38.44 & 11.82 & 26.62 & \\
\hline MW-07 & MW & $01 / 04 / 93$ & 0959 & 38.44 & 12.82 & 25.62 & \\
\hline MW-07 & MW & $02 / 04 / 93$ & 1024 & 38.44 & 10.77 & 27.67 & \\
\hline MW-07 & MW & $03 / 05 / 93$ & 1012 & 38.44 & 11.52 & 26.92 & \\
\hline MW-07 & MW & $03 / 30 / 93$ & 0927 & 38.44 & 11.69 & 26.75 & \\
\hline MW-07 & MW & $05 / 06 / 93$ & 0957 & 38.44 & 12.68 & 25.76 & \\
\hline MW-07 & MW & $05 / 28 / 93$ & 1000 & 38.44 & 13.60 & 24.84 & \\
\hline MW-07 & MW & $07 / 07 / 93$ & 0948 & 38.44 & 14.24 & 24.20 & \\
\hline MW-07 & MW & $08 / 06 / 93$ & 0938 & 38.44 & 14.45 & 23.99 & \\
\hline MW-07 & MW & $09 / 03 / 93$ & 1000 & 38.44 & 13.78 & 24.66 & \\
\hline MW-07 & MW & $09 / 30 / 93$ & 1137 & 38.44 & 13.53 & 24.91 & \\
\hline MW-07 & MW & $10 / 28 / 93$ & 0948 & 38.44 & 14.50 & 23.94 & \\
\hline MW-07 & MW & $12 / 01 / 93$ & 0952 & 38.44 & 14.59 & 23.85 & \\
\hline MW-07 & MW & $01 / 06 / 94$ & 1002 & 38.44 & 15.06 & 23.38 & \\
\hline MW-07 & MW & $02 / 01 / 94$ & 0948 & 38.44 & 13.97 & 24.47 & \\
\hline MW-07 & MW & $03 / 04 / 94$ & 0945 & 38.44 & 13.47 & 24.97 & \\
\hline MW-07 & MW & $04 / 04 / 94$ & 0939 & 38.44 & 13.67 & 24.77 & \\
\hline MW-07 & MW & $05 / 06 / 94$ & 1029 & 38.44 & 14.65 & 23.79 & \\
\hline MW-07 & MW & $06 / 03 / 94$ & 0958 & 38.44 & 15.41 & 23.03 & \\
\hline MW-07 & MW & $07 / 08 / 94$ & 1000 & 38.44 & 14.52 & 23.92 & \\
\hline MW-07 & MW & $08 / 05 / 94$ & 1255 & 38.44 & 12.53 & 25.91 & \\
\hline MW-07 & MW & $09 / 07 / 94$ & 1005 & 38.44 & 11.89 & 26.55 & \\
\hline MW-07 & MW & $10 / 06 / 94$ & 1025 & 38.44 & 9.82 & 28.62 & \\
\hline MW-07 & MW & $11 / 09 / 94$ & 1205 & 38.44 & 10.66 & 27.78 & \\
\hline MW-07 & MW & $12 / 06 / 94$ & 1005 & 38.44 & 11.58 & 26.86 & \\
\hline MW-07 & MW & $01 / 10 / 95$ & 1205 & 38.44 & 11.78 & 26.66 & \\
\hline MW-07 & MW & $02 / 02 / 95$ & 1250 & 38.44 & 11.63 & 26.81 & \\
\hline MW-07 & MW & $03 / 02 / 95$ & 1406 & 38.44 & 11.83 & 26.61 & \\
\hline MW-07 & MW & $04 / 05 / 95$ & 1004 & 38.44 & 12.92 & 25.52 & \\
\hline MW-07 & MW & $05 / 02 / 95$ & 1051 & 38.44 & 13.60 & 24.84 & \\
\hline MW-07 & MW & $06 / 09 / 95$ & 1005 & 38.44 & 14.53 & 23.91 & \\
\hline MW-07 & MW & $07 / 06 / 95$ & 0957 & 38.44 & 14.51 & 23.93 & \\
\hline
\end{tabular}


Table 8.--Water-level data collected at the Defense Fuel Supply Point and adjacent properties, Hanahan, S.C., between April 1991 and September 1995--Continued

[ft, feet; ---, data not available; WL, water-level well; EW, extraction well; MW, monitoring well; SW, surface-water site; -, negative depth to water indicates water-level altitude is above measuring point]

\begin{tabular}{|c|c|c|c|c|c|c|c|}
\hline $\begin{array}{c}\text { Site } \\
\text { (plate 1) }\end{array}$ & $\begin{array}{l}\text { Site } \\
\text { type }\end{array}$ & Date & Time & $\begin{array}{l}\text { Measuring-point } \\
\text { altitude } \\
\text { (ft above } \\
\text { sea level) }\end{array}$ & $\begin{array}{l}\text { Depth to water } \\
\text { (ft below } \\
\text { measuring point) }\end{array}$ & $\begin{array}{l}\text { Water-level } \\
\text { altitude } \\
\text { (ft above } \\
\text { sea level) }\end{array}$ & Remarks \\
\hline MW-07 & MW & $08 / 10 / 95$ & 1035 & 38.44 & 13.99 & 24.45 & \\
\hline MW-07 & MW & $09 / 13 / 95$ & 1115 & 38.44 & 11.70 & 26.74 & \\
\hline MW-08 & MW & $04 / 30 / 91$ & 1140 & 12.08 & .07 & 12.01 & \\
\hline MW-08 & MW & $05 / 28 / 91$ & 1220 & 12.08 & .22 & 11.86 & \\
\hline MW-08 & MW & $08 / 06 / 91$ & --- & 12.08 & -.34 & 12.42 & \\
\hline MW-08 & MW & $08 / 30 / 91$ & -- & 12.08 & -.29 & 12.37 & \\
\hline MW-08 & MW & $09 / 27 / 91$ & --- & 12.08 & .23 & 11.85 & \\
\hline MW-08 & MW & $10 / 29 / 91$ & --- & 12.08 & .55 & 11.53 & \\
\hline MW-08 & MW & $12 / 02 / 91$ & $\cdots$ & 12.08 & .64 & 11.44 & \\
\hline MW-08 & MW & $01 / 02 / 92$ & $\ldots$ & 12.08 & .85 & 11.23 & \\
\hline MW-08 & MW & $02 / 04 / 92$ & -- & 12.08 & .68 & 11.40 & \\
\hline MW-08 & MW & $03 / 06 / 92$ & -- & 12.08 & 1.27 & 10.81 & \\
\hline MW-08 & MW & $03 / 31 / 92$ & -- & 12.08 & 1.05 & 11.03 & \\
\hline MW-08 & MW & $05 / 01 / 92$ & 1416 & 12.08 & 1.03 & 11.05 & \\
\hline MW-08 & MW & $06 / 11 / 92$ & 1710 & 12.08 & .64 & 11.44 & \\
\hline MW-08 & MW & $07 / 07 / 92$ & 1040 & 12.08 & .97 & 11.11 & \\
\hline MW-08 & MW & $08 / 31 / 92$ & 1315 & 12.08 & .12 & 11.96 & \\
\hline MW-08 & MW & $10 / 07 / 92$ & 1435 & 12.08 & -.15 & 12.23 & \\
\hline MW-08 & MW & $10 / 28 / 92$ & 1356 & 12.08 & .12 & 11.96 & \\
\hline MW-08 & MW & $12 / 03 / 92$ & 1405 & 12.08 & -.10 & 12.18 & \\
\hline MW-08 & MW & $01 / 04 / 93$ & 1335 & 12.08 & .38 & 11.70 & \\
\hline MW-08 & MW & $02 / 04 / 93$ & 1442 & 12.08 & -.27 & 12.35 & \\
\hline MW-08 & MW & $03 / 05 / 93$ & 1342 & 12.08 & -.21 & 12.29 & \\
\hline MW-08 & MW & $03 / 30 / 93$ & 1306 & 12.08 & -.07 & 12.15 & \\
\hline MW-08 & MW & $05 / 06 / 93$ & 1322 & 12.08 & -.23 & 12.31 & \\
\hline MW-08 & MW & $05 / 28 / 93$ & 1308 & 12.08 & .32 & 11.76 & \\
\hline MW-08 & MW & $07 / 07 / 93$ & 1330 & 12.08 & .10 & 11.98 & \\
\hline MW-08 & MW & $08 / 06 / 93$ & 1300 & 12.08 & .36 & 11.72 & \\
\hline MW-08 & MW & $09 / 03 / 93$ & 1150 & 12.08 & .83 & 11.25 & \\
\hline MW-08 & MW & $09 / 30 / 93$ & 1245 & 12.08 & .85 & 11.23 & \\
\hline MW-08 & MW & $10 / 28 / 93$ & 1258 & 12.08 & .36 & 11.72 & \\
\hline MW-08 & MW & $12 / 01 / 93$ & 1515 & 12.08 & .60 & 11.48 & \\
\hline MW-08 & MW & $01 / 06 / 94$ & 1310 & 12.08 & .50 & 11.58 & \\
\hline MW-08 & MW & $02 / 01 / 94$ & 1240 & 12.08 & -.26 & 12.34 & \\
\hline MW-08 & MW & $03 / 04 / 94$ & 1220 & 12.08 & .09 & 11.99 & \\
\hline MW-08 & MW & $04 / 04 / 94$ & 1146 & 12.08 & .75 & 11.33 & \\
\hline MW-08 & MW & $05 / 06 / 94$ & 1309 & 12.08 & .34 & 11.74 & \\
\hline MW-08 & MW & $06 / 03 / 94$ & 1220 & 12.08 & .90 & 11.18 & \\
\hline MW-08 & MW & $07 / 08 / 94$ & 1234 & 12.08 & .14 & 11.94 & \\
\hline MW-08 & MW & $08 / 05 / 94$ & 1459 & 12.08 & -.24 & 12.32 & \\
\hline MW-08 & MW & $09 / 07 / 94$ & 1325 & 12.08 & .01 & 12.07 & \\
\hline MW-08 & MW & $10 / 06 / 94$ & 1325 & 12.08 & -.36 & 12.44 & \\
\hline MW-08 & MW & $11 / 09 / 94$ & 1350 & 12.08 & -.44 & 12.52 & \\
\hline MW-08 & MW & $12 / 06 / 94$ & 1220 & 12.08 & -.31 & 12.39 & \\
\hline
\end{tabular}


Table 8.--Water-level data collected at the Defense Fuel Supply Point and adjacent properties, Hanahan, S.C., between April 1991 and September 1995--Continued

[ft, feet; ---, data not available; WL, water-level well; EW, extraction well; MW, monitoring well; SW, surface-water site; -, negative depth to water indicates water-level altitude is above measuring point]

\begin{tabular}{|c|c|c|c|c|c|c|c|}
\hline $\begin{array}{c}\text { Site } \\
\text { (plate 1) }\end{array}$ & $\begin{array}{l}\text { Site } \\
\text { type }\end{array}$ & Date & Time & $\begin{array}{l}\text { Measuring-point } \\
\text { altitude } \\
\text { (ft above } \\
\text { sea level) }\end{array}$ & $\begin{array}{l}\text { Depth to water } \\
\text { (ft below } \\
\text { measuring point) }\end{array}$ & $\begin{array}{l}\text { Water-level } \\
\text { altitude } \\
\text { (ft above } \\
\text { sea level) }\end{array}$ & Remarks \\
\hline MW-08 & MW & $01 / 10 / 95$ & 1801 & 12.08 & -0.12 & 12.20 & \\
\hline MW-08 & MW & $02 / 02 / 95$ & 1444 & 12.08 & -.43 & 12.51 & \\
\hline MW-08 & MW & $03 / 02 / 95$ & 1614 & 12.08 & -.34 & 12.42 & \\
\hline MW-08 & MW & $04 / 05 / 95$ & 1231 & 12.08 & .24 & 11.84 & \\
\hline MW-08 & MW & $05 / 02 / 95$ & 1356 & 12.08 & .16 & 11.92 & \\
\hline MW-08 & MW & $06 / 09 / 95$ & 1320 & 12.08 & .55 & 11.53 & \\
\hline MW-08 & MW & $07 / 06 / 95$ & 1236 & 12.08 & .71 & 11.37 & \\
\hline MW-08 & MW & $08 / 10 / 95$ & 1322 & 12.08 & .33 & 11.75 & \\
\hline MW-08 & MW & $09 / 13 / 95$ & 1444 & 12.08 & .14 & 11.94 & \\
\hline MW-09 & MW & $04 / 30 / 91$ & 1145 & 9.06 & .51 & 8.55 & \\
\hline MW-09 & MW & $05 / 28 / 91$ & 1227 & 9.06 & .69 & 8.37 & \\
\hline MW-09 & MW & $07 / 01 / 91$ & --- & 9.06 & 1.06 & 8.00 & \\
\hline MW-09 & MW & $08 / 06 / 91$ & -- & 9.06 & .44 & 8.62 & \\
\hline MW-09 & MW & $08 / 30 / 91$ & -- & 9.06 & .25 & 8.81 & \\
\hline MW-09 & MW & $09 / 27 / 91$ & -- & 9.06 & .78 & 8.28 & \\
\hline MW-09 & MW & $10 / 29 / 91$ & - & 9.06 & 1.05 & 8.01 & \\
\hline MW-09 & MW & $12 / 02 / 91$ & --- & 9.06 & 1.12 & 7.94 & \\
\hline MW-09 & MW & $01 / 02 / 92$ & --- & 9.06 & 1.25 & 7.81 & \\
\hline MW-09 & MW & $02 / 04 / 92$ & --- & 9.06 & 1.19 & 7.87 & \\
\hline MW-09 & MW & $03 / 06 / 92$ & --- & 9.06 & 1.31 & 7.75 & \\
\hline MW-09 & MW & $03 / 31 / 92$ & -- & 9.06 & 1.14 & 7.92 & \\
\hline MW-09 & MW & $05 / 01 / 92$ & 1419 & 9.06 & 1.45 & 7.61 & \\
\hline MW-09 & MW & $06 / 11 / 92$ & 1700 & 9.06 & 1.01 & 8.05 & \\
\hline MW-09 & MW & $07 / 07 / 92$ & 1042 & 9.06 & 1.21 & 7.85 & \\
\hline MW-09 & MW & $08 / 31 / 92$ & 1310 & 9.06 & .58 & 8.48 & \\
\hline MW-09 & MW & $10 / 07 / 92$ & 1420 & 9.06 & .28 & 8.78 & \\
\hline MW-09 & MW & $10 / 28 / 92$ & --- & 9.06 & .53 & 8.53 & \\
\hline MW-09 & MW & $12 / 03 / 92$ & 1400 & 9.06 & .48 & 8.58 & \\
\hline MW-09 & MW & $01 / 04 / 93$ & 1330 & 9.06 & .65 & 8.41 & \\
\hline MW-09 & MW & $02 / 04 / 93$ & 1400 & 9.06 & .16 & 8.90 & \\
\hline MW-09 & MW & $03 / 05 / 93$ & 1328 & 9.06 & .17 & 8.89 & \\
\hline MW-09 & MW & $03 / 30 / 93$ & 1302 & 9.06 & .10 & 8.96 & \\
\hline MW-09 & MW & $05 / 06 / 93$ & 1320 & 9.06 & .44 & 8.62 & \\
\hline MW-09 & MW & $05 / 28 / 93$ & 1306 & 9.06 & .76 & 8.30 & \\
\hline MW-09 & MW & $07 / 07 / 93$ & 1323 & 9.06 & .87 & 8.19 & \\
\hline MW-10 & MW & $04 / 30 / 91$ & 1150 & 6.16 & .82 & 5.34 & \\
\hline MW-10 & MW & $05 / 28 / 91$ & 1224 & 6.16 & .92 & 5.24 & \\
\hline MW-10 & MW & $09 / 27 / 91$ & -.- & 6.16 & 1.00 & 5.16 & \\
\hline MW-10 & MW & $10 / 29 / 91$ & --- & 6.16 & 1.21 & 4.95 & \\
\hline MW-10 & MW & $12 / 02 / 91$ & -- & 6.16 & 1.36 & 4.80 & \\
\hline MW-10 & MW & $01 / 02 / 92$ & -- & 6.16 & 1.39 & 4.77 & \\
\hline MW-10 & MW & $02 / 04 / 92$ & --- & 6.16 & 1.11 & 5.05 & \\
\hline MW-10 & MW & $03 / 06 / 92$ & - & 6.16 & 1.19 & 4.97 & \\
\hline
\end{tabular}


Table 8.--Water-level data collected at the Defense Fuel Supply Point and adjacent properties, Hanahan, S.C., between April 1991 and September 1995--Continued

[ft, feet; ---, data not available; WL, water-level well; EW, extraction well; MW, monitoring well; SW, surface-water site; -, negative depth to water indicates water-level altitude is above measuring point]

\begin{tabular}{|c|c|c|c|c|c|c|c|}
\hline $\begin{array}{c}\text { Site } \\
\text { (plate 1) }\end{array}$ & $\begin{array}{l}\text { Site } \\
\text { type }\end{array}$ & Date & Time & $\begin{array}{c}\text { Measuring-point } \\
\text { altitude } \\
\text { (ft above } \\
\text { sea level) }\end{array}$ & $\begin{array}{l}\text { Depth to water } \\
\text { ( } \mathrm{ft} \text { below } \\
\text { measuring point) }\end{array}$ & $\begin{array}{l}\text { Water-level } \\
\text { altitude } \\
\text { (ft above } \\
\text { sea level) }\end{array}$ & Remarks \\
\hline MW-10 & MW & $03 / 31 / 92$ & --- & 6.16 & 1.05 & 5.11 & \\
\hline MW-10 & MW & $05 / 01 / 92$ & 1425 & 6.16 & 1.29 & 4.87 & \\
\hline MW-10 & MW & $06 / 11 / 92$ & 1655 & 6.16 & .92 & 5.24 & \\
\hline MW-10 & MW & $07 / 07 / 92$ & 1055 & 6.16 & 1.24 & 4.92 & \\
\hline MW-10 & MW & $08 / 31 / 92$ & 1300 & 6.16 & .77 & 5.39 & \\
\hline MW-10 & MW & $10 / 07 / 92$ & 1410 & 6.16 & -.11 & 6.27 & \\
\hline MW-10 & MW & $10 / 28 / 92$ & 1451 & 6.16 & .84 & 5.32 & \\
\hline MW-10 & MW & $12 / 03 / 92$ & 1350 & 6.16 & .68 & 5.48 & \\
\hline MW-10 & MW & $01 / 04 / 93$ & 1320 & 6.16 & .96 & 5.20 & \\
\hline$M W-10$ & MW & $02 / 04 / 93$ & 1355 & 6.16 & .49 & 5.67 & \\
\hline MW-10 & MW & $03 / 05 / 93$ & 1330 & 6.16 & .71 & 5.45 & \\
\hline MW-10 & MW & $03 / 30 / 93$ & 1254 & 6.16 & .71 & 5.45 & \\
\hline MW-10 & MW & $05 / 06 / 93$ & 1315 & 6.16 & .95 & 5.21 & \\
\hline MW-10 & MW & $05 / 28 / 93$ & 1300 & 6.16 & .77 & 5.39 & \\
\hline MW-10 & MW & $07 / 07 / 93$ & 1316 & 6.16 & .99 & 5.17 & \\
\hline MW-11 & MW & $04 / 30 / 91$ & 0950 & 37.42 & 14.07 & 23.35 & \\
\hline MW-11 & MW & $05 / 28 / 91$ & -.- & 37.42 & 14.17 & 23.25 & \\
\hline MW-11 & MW & $07 / 01 / 91$ & --- & 37.42 & 15.00 & 22.42 & \\
\hline MW-11 & MW & $08 / 06 / 91$ & -- & 37.42 & 12.92 & 24.50 & \\
\hline MW-11 & MW & $08 / 30 / 91$ & -- & 37.42 & 12.65 & 24.77 & \\
\hline MW-11 & MW & $09 / 27 / 91$ & -- & 37.42 & 13.63 & 23.79 & \\
\hline MW-11 & MW & $10 / 29 / 91$ & --- & 37.42 & 14.67 & 22.75 & \\
\hline MW-11 & MW & $11 / 21 / 91$ & 1313 & 37.42 & 15.60 & 21.82 & \\
\hline MW-11 & MW & $12 / 02 / 91$ & -.. & 37.42 & 16.21 & 21.21 & \\
\hline MW-11 & MW & $12 / 16 / 91$ & --- & 37.42 & 17.29 & 20.13 & \\
\hline MW-11 & MW & $01 / 02 / 92$ & --. & 37.42 & 17.43 & 19.99 & \\
\hline MW-11 & MW & $02 / 04 / 92$ & --- & 37.42 & 17.04 & 20.38 & \\
\hline MW-11 & MW & $03 / 06 / 92$ & -- & 37.42 & 15.80 & 21.62 & \\
\hline MW-11 & MW & $03 / 31 / 92$ & -- & 37.42 & 15.73 & 21.69 & \\
\hline MW-11 & MW & $05 / 01 / 92$ & $\ldots$ & 37.42 & 15.43 & 21.99 & \\
\hline MW-11 & MW & $05 / 14 / 92$ & 1045 & 37.42 & 17.00 & 20.42 & \\
\hline MW-11 & MW & $06 / 11 / 92$ & 1130 & 37.42 & 17.98 & 19.44 & \\
\hline MW-11 & MW & $07 / 07 / 92$ & 1747 & 37.42 & 14.23 & 23.19 & \\
\hline MW-11 & MW & $08 / 07 / 92$ & 1126 & 37.42 & 16.98 & 20.44 & \\
\hline MW-11 & MW & $08 / 31 / 92$ & 1040 & 37.42 & 12.72 & 24.70 & \\
\hline MW-11 & MW & $10 / 07 / 92$ & 1055 & 37.42 & 12.99 & 24.43 & \\
\hline MW-11 & MW & $10 / 28 / 92$ & 1124 & 37.42 & 13.26 & 24.16 & \\
\hline MW-11 & MW & $12 / 03 / 92$ & 1027 & 37.42 & 13.35 & 24.07 & \\
\hline MW-11 & MW & $01 / 04 / 93$ & 1021 & 37.42 & 14.25 & 23.17 & \\
\hline$M W-11$ & MW & $02 / 04 / 93$ & 1101 & 37.42 & 12.80 & 24.62 & \\
\hline MW-11 & MW & $03 / 05 / 93$ & 1038 & 37.42 & 13.57 & 23.85 & \\
\hline MW-11 & MW & $03 / 30 / 93$ & 0948 & 37.42 & 13.81 & 23.61 & \\
\hline$M W-11$ & MW & $05 / 06 / 93$ & 1019 & 37.42 & 14.30 & 23.12 & \\
\hline MW-11 & MW & $05 / 28 / 93$ & 1020 & 37.42 & 15.67 & 21.75 & \\
\hline
\end{tabular}


Table 8.--Water-level data collected at the Defense Fuel Supply Point and adjacent properties, Hanahan, S.C., between April 1991 and September 1995--Continued

[ft, feet; ---, data not available; WL, water-level well; EW, extraction well; MW, monitoring well; SW, surface-water site; -, negative depth to water indicates water-level altitude is above measuring point]

\begin{tabular}{|c|c|c|c|c|c|c|c|}
\hline $\begin{array}{c}\text { Site } \\
\text { (plate 1) }\end{array}$ & $\begin{array}{l}\text { Site } \\
\text { type }\end{array}$ & Date & Time & $\begin{array}{l}\text { Measuring-point } \\
\text { altitude } \\
\text { (ft above } \\
\text { sea level) }\end{array}$ & $\begin{array}{l}\text { Depth to water } \\
\text { (ft below } \\
\text { measuring point) }\end{array}$ & $\begin{array}{l}\text { Water-level } \\
\text { altitude } \\
\text { (ft above } \\
\text { sea level) }\end{array}$ & Remarks \\
\hline MW-11 & $\overline{M W}$ & $07 / 07 / 93$ & 1009 & 37.42 & 16.97 & 20.45 & \\
\hline MW-11 & MW & $08 / 06 / 93$ & 1002 & 37.42 & 17.48 & 19.94 & \\
\hline MW-11 & MW & $09 / 03 / 93$ & 1016 & 37.42 & 14.21 & 23.21 & \\
\hline MW-11 & MW & $09 / 30 / 93$ & 1119 & 37.42 & 14.09 & 23.33 & \\
\hline MW-11 & MW & $10 / 28 / 93$ & 1006 & 37.42 & 15.81 & 21.61 & \\
\hline MW-11 & MW & $12 / 01 / 93$ & 1008 & 37.42 & 16.22 & 21.20 & \\
\hline MW-11 & MW & $01 / 06 / 94$ & 1026 & 37.42 & 17.99 & 19.43 & \\
\hline MW-11 & MW & $02 / 01 / 94$ & 1020 & 37.42 & 14.93 & 22.49 & \\
\hline MW-11 & MW & $03 / 04 / 94$ & 1004 & 37.42 & 14.30 & 23.12 & \\
\hline MW-11 & MW & $04 / 04 / 94$ & 0955 & 37.42 & 14.70 & 22.72 & \\
\hline MW-11 & MW & $05 / 06 / 94$ & 1047 & 37.42 & 18.38 & 19.04 & \\
\hline MW-11 & MW & $06 / 03 / 94$ & 1018 & 37.42 & 19.58 & 17.84 & \\
\hline MW-11 & MW & $07 / 08 / 94$ & 1020 & 37.42 & 13.78 & 23.64 & \\
\hline MW-11 & MW & $08 / 05 / 94$ & 1315 & 37.42 & 13.05 & 24.37 & \\
\hline MW-11 & MW & $09 / 07 / 94$ & 1035 & 37.42 & 13.26 & 24.16 & \\
\hline MW-11 & MW & $10 / 06 / 94$ & 1050 & 37.42 & 11.41 & 26.01 & \\
\hline MW-11 & MW & $11 / 09 / 94$ & 1230 & 37.42 & 12.70 & 24.72 & \\
\hline MW-11 & MW & $12 / 06 / 94$ & 1038 & 37.42 & 13.60 & 23.82 & \\
\hline MW-11 & MW & $01 / 10 / 95$ & 1223 & 37.42 & 13.43 & 23.99 & \\
\hline MW-11 & MW & $02 / 02 / 95$ & 1312 & 37.42 & 13.40 & 24.02 & \\
\hline MW-11 & MW & $03 / 02 / 95$ & 1428 & 37.42 & 13.65 & 23.77 & \\
\hline MW-11 & MW & $04 / 05 / 95$ & 1024 & 37.42 & 14.24 & 23.18 & \\
\hline MW-11 & MW & $05 / 02 / 95$ & 1026 & 37.42 & 14.86 & 22.56 & \\
\hline MW-11 & MW & $06 / 09 / 95$ & 1027 & 37.42 & 16.67 & 20.75 & \\
\hline MW-11 & MW & $07 / 06 / 95$ & 1018 & 37.42 & 16.45 & 20.97 & \\
\hline MW-11 & MW & $08 / 10 / 95$ & 1100 & 37.42 & 14.27 & 23.15 & \\
\hline MW-11 & MW & $09 / 13 / 95$ & 1131 & 37.42 & 12.79 & 24.63 & \\
\hline MW-11A & MW & $04 / 30 / 91$ & 1000 & 37.27 & 15.50 & 21.77 & \\
\hline$M W-11 A$ & MW & $05 / 28 / 91$ & $\cdots$ & 37.27 & 15.76 & 21.51 & \\
\hline$M W-11 A$ & MW & $07 / 01 / 91$ & - & 37.27 & 16.24 & 21.03 & \\
\hline $\mathrm{MW}-11 \mathrm{~A}$ & MW & $08 / 06 / 91$ & --- & 37.27 & 14.34 & 22.93 & \\
\hline MW-11A & MW & $08 / 30 / 91$ & -- & 37.27 & 13.91 & 23.36 & \\
\hline MW-11A & MW & $09 / 27 / 91$ & -- & 37.27 & 15.32 & 21.95 & \\
\hline MW-11A & MW & $10 / 29 / 91$ & --- & 37.27 & 16.33 & 20.94 & \\
\hline MW-11A & MW & $11 / 21 / 91$ & 1313 & 37.27 & 16.75 & 20.52 & \\
\hline$M W-11 A$ & MW & $12 / 02 / 91$ & -- & 37.27 & 17.02 & 20.25 & \\
\hline $\mathrm{MW}-11 \mathrm{~A}$ & MW & $01 / 02 / 92$ & -- & 37.27 & 17.55 & 19.72 & \\
\hline$M W-11 A$ & MW & $02 / 04 / 92$ & --- & 37.27 & 17.39 & 19.88 & \\
\hline MW-11A & MW & $03 / 06 / 92$ & --- & 37.27 & 17.22 & 20.05 & \\
\hline MW-11A & MW & $03 / 31 / 92$ & -- & 37.27 & 17.15 & 20.12 & \\
\hline MW-11A & MW & $05 / 01 / 92$ & 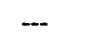 & 37.27 & 17.19 & 20.08 & \\
\hline$M W-11 A$ & MW & $05 / 14 / 92$ & 1040 & 37.27 & 17.45 & 19.82 & \\
\hline$M W-11 A$ & MW & $06 / 11 / 92$ & 1125 & 37.27 & 18.78 & 18.49 & \\
\hline MW-11A & MW & $07 / 07 / 92$ & 1750 & 37.27 & 17.77 & 19.50 & \\
\hline
\end{tabular}


Table 8.--Water-level data collected at the Defense Fuel Supply Point and adjacent properties, Hanahan, S.C., between April 1991 and September 1995--Continued

[ft, feet; ---, data not available; WL, water-level well; EW, extraction well; MW, monitoring well; SW, surface-water site; -, negative depth to water indicates water-level altitude is above measuring point]

\begin{tabular}{|c|c|c|c|c|c|c|c|}
\hline $\begin{array}{c}\text { Site } \\
\text { (plate 1) }\end{array}$ & $\begin{array}{l}\text { Site } \\
\text { type }\end{array}$ & Date & Time & $\begin{array}{l}\text { Measuring-point } \\
\text { altitude } \\
\text { (ft above } \\
\text { sea level) }\end{array}$ & $\begin{array}{l}\text { Depth to water } \\
\text { (ft below } \\
\text { measuring point) }\end{array}$ & $\begin{array}{l}\text { Water-level } \\
\text { altitude } \\
\text { (ft above } \\
\text { sea level) }\end{array}$ & Remarks \\
\hline $\mathrm{MW}-11 \mathrm{~A}$ & MW & $08 / 07 / 92$ & 1124 & 37.27 & 18.21 & 19.06 & \\
\hline MW-11A & MW & $08 / 31 / 92$ & 1042 & 37.27 & 15.32 & 21.95 & \\
\hline MW-11A & MW & $10 / 07 / 92$ & 1051 & 37.27 & 15.12 & 22.15 & \\
\hline MW-11A & MW & $10 / 28 / 92$ & 1127 & 37.27 & 15.71 & 21.56 & \\
\hline MW-11A & MW & $12 / 03 / 92$ & 1023 & 37.27 & 15.80 & 21.47 & \\
\hline MW-11A & MW & $01 / 04 / 93$ & 1019 & 37.27 & 16.70 & 20.57 & \\
\hline $\mathrm{MW}-11 \mathrm{~A}$ & MW & $02 / 04 / 93$ & 1055 & 37.27 & 14.90 & 22.37 & \\
\hline$M W-11 A$ & MW & $03 / 05 / 93$ & 1035 & 37.27 & 15.61 & 21.66 & \\
\hline MW-11A & MW & $03 / 30 / 93$ & 0951 & 37.27 & 18.14 & 19.13 & \\
\hline$M W-11 A$ & MW & $05 / 06 / 93$ & 1015 & 37.27 & 18.38 & 18.89 & \\
\hline $\mathrm{MW}-11 \mathrm{~A}$ & MW & $05 / 28 / 93$ & 1018 & 37.27 & 18.95 & 18.32 & \\
\hline MW-11A & MW & $07 / 07 / 93$ & 1005 & 37.27 & 17.70 & 19.57 & \\
\hline MW-11A & MW & $08 / 06 / 93$ & 1000 & 37.27 & 18.67 & 18.60 & \\
\hline$M W-11 A$ & MW & $09 / 03 / 93$ & 1019 & 37.27 & 18.74 & 18.53 & \\
\hline MW-11A & MW & $09 / 30 / 93$ & 1123 & 37.27 & 18.14 & 19.13 & \\
\hline MW-11A & MW & $10 / 28 / 93$ & 1004 & 37.27 & 19.00 & 18.27 & \\
\hline $\mathrm{MW}-11 \mathrm{~A}$ & MW & $12 / 01 / 93$ & 1010 & 37.27 & 19.10 & 18.17 & \\
\hline MW-11A & MW & $01 / 06 / 94$ & 1023 & 37.27 & 19.08 & 18.19 & \\
\hline $\mathrm{MW}-11 \mathrm{~A}$ & MW & $02 / 01 / 94$ & 1018 & 37.27 & 17.86 & 19.11 & \\
\hline$M W-11 \mathrm{~A}$ & MW & $03 / 04 / 94$ & 1006 & 37.27 & 17.50 & 19.77 & \\
\hline MW-11A & MW & $04 / 04 / 94$ & 0958 & 37.27 & 17.99 & 19.28 & \\
\hline MW-11A & MW & $05 / 06 / 94$ & 1045 & 37.27 & 18.95 & 18.32 & \\
\hline MW-11A & MW & $06 / 03 / 94$ & 1014 & 37.27 & 19.78 & 17.49 & \\
\hline $\mathrm{MW}-11 \mathrm{~A}$ & MW & $07 / 08 / 94$ & 1025 & 37.27 & 18.17 & 19.10 & \\
\hline$M W-11 \mathrm{~A}$ & MW & $08 / 05 / 94$ & 1312 & 37.27 & 16.43 & 20.84 & \\
\hline MW-11A & MW & $09 / 07 / 94$ & 1030 & 37.27 & 16.24 & 21.03 & \\
\hline $\mathrm{MW}-11 \mathrm{~A}$ & MW & $10 / 06 / 94$ & 1047 & 37.27 & 13.70 & 23.57 & \\
\hline MW-11A & MW & $11 / 09 / 94$ & 1227 & 37.27 & 15.28 & 21.99 & \\
\hline MW-11A & MW & $12 / 06 / 94$ & 1032 & 37.27 & 16.10 & 21.17 & \\
\hline $\mathrm{MW}-11 \mathrm{~A}$ & MW & $01 / 10 / 95$ & 1225 & 37.27 & 15.92 & 21.35 & \\
\hline MW-11A & MW & $02 / 02 / 95$ & 1310 & 37.27 & 15.65 & 21.62 & \\
\hline MW-11A & MW & $03 / 02 / 95$ & 1425 & 37.27 & 15.68 & 21.59 & \\
\hline MW-11A & MW & $04 / 05 / 95$ & 1025 & 37.27 & 16.60 & 20.67 & \\
\hline MW-11A & MW & $05 / 02 / 95$ & 1123 & 37.27 & 17.22 & 20.05 & \\
\hline MW-11A & MW & $06 / 09 / 95$ & 1025 & 37.27 & 18.14 & 19.13 & \\
\hline MW-11A & MW & $07 / 06 / 95$ & 1016 & 37.27 & 17.99 & 19.28 & \\
\hline $\mathrm{MW}-11 \mathrm{~A}$ & MW & $08 / 10 / 95$ & 1055 & 37.27 & 17.54 & 19.73 & \\
\hline MW-11A & MW & $09 / 13 / 95$ & 1129 & 37.27 & 15.27 & 22.00 & \\
\hline MW-12 & MW & $04 / 30 / 91$ & 1030 & 37.28 & 11.22 & 26.06 & \\
\hline MW-12 & MW & $05 / 28 / 91$ & -- & 37.28 & 11.38 & 25.90 & \\
\hline MW-12 & MW & $07 / 01 / 91$ & -- & 37.28 & 11.68 & 25.60 & \\
\hline MW-12 & MW & $08 / 06 / 91$ & -- & 37.28 & 10.38 & 26.90 & \\
\hline MW-12 & MW & $09 / 27 / 91$ & -- & 37.28 & 11.40 & 25.88 & \\
\hline MW-12 & MW & $10 / 29 / 91$ & -- & 37.28 & 12.08 & 25.20 & \\
\hline
\end{tabular}


Table 8.--Water-level data collected at the Defense Fuel Supply Point and adjacent properties, Hanahan, S.C., between April 1991 and September 1995--Continued

[ft, feet; ---, data not available; WL, water-level well; EW, extraction well; MW, monitoring well; SW, surface-water site; -, negative depth to wateindicates water-level altitude is above measuring point]

\begin{tabular}{|c|c|c|c|c|c|c|c|}
\hline $\begin{array}{c}\text { Site } \\
\text { (plate 1) }\end{array}$ & $\begin{array}{l}\text { Site } \\
\text { type }\end{array}$ & Date & Time & $\begin{array}{l}\text { Measuring-point } \\
\text { altitude } \\
\text { (ft above } \\
\text { sea level) }\end{array}$ & $\begin{array}{l}\text { Depth to water } \\
\text { (ft below } \\
\text { measuring point) }\end{array}$ & $\begin{array}{l}\text { Water-level } \\
\text { altitude } \\
\text { (ft above } \\
\text { sea level) }\end{array}$ & Remarks \\
\hline MW-12 & MW & $12 / 02 / 91$ & $\cdots$ & 37.28 & 12.46 & 24.82 & \\
\hline MW-12 & MW & $01 / 02 / 92$ & $\cdots$ & 37.28 & 12.86 & 24.42 & \\
\hline MW-12 & MW & $02 / 04 / 92$ & $\ldots$ & 37.28 & 12.22 & 25.06 & \\
\hline MW-12 & MW & $03 / 06 / 92$ & $\cdots$ & 37.28 & 12.42 & 24.86 & \\
\hline MW-12 & MW & $03 / 31 / 92$ & -- & 37.28 & 12.46 & 24.82 & \\
\hline MW-12 & MW & $05 / 01 / 92$ & $\cdots$ & 37.28 & 12.49 & 24.79 & \\
\hline MW-12 & MW & $06 / 11 / 92$ & 1409 & 37.28 & 12.09 & 25.19 & \\
\hline MW-12 & MW & $07 / 07 / 92$ & 1710 & 37.28 & 12.27 & 25.01 & \\
\hline MW-12 & MW & $08 / 07 / 92$ & 1253 & 37.28 & 12.14 & 25.14 & \\
\hline MW-12 & MW & $08 / 31 / 92$ & 1132 & 37.28 & 10.88 & 26.40 & \\
\hline MW-12 & MW & $10 / 07 / 92$ & 1144 & 37.28 & 10.68 & 26.60 & \\
\hline MW-12 & MW & $10 / 28 / 92$ & 1300 & 37.28 & 11.29 & 25.99 & \\
\hline MW-12 & MW & $12 / 03 / 92$ & 1127 & 37.28 & 11.29 & 25.99 & \\
\hline MW-12 & MW & $01 / 04 / 93$ & 1115 & 37.28 & 12.02 & 25.26 & \\
\hline MW-12 & MW & $02 / 04 / 93$ & 1204 & 37.28 & 11.26 & 26.02 & \\
\hline MW-12 & MW & $03 / 05 / 93$ & 1128 & 37.28 & 11.45 & 25.83 & \\
\hline MW-12 & MW & $03 / 30 / 93$ & 1040 & 37.28 & 11.36 & 25.92 & \\
\hline MW-12 & MW & $05 / 06 / 93$ & 1109 & 37.28 & 11.78 & 25.50 & \\
\hline MW-12 & MW & $05 / 28 / 93$ & 1103 & 37.28 & 12.18 & 25.10 & \\
\hline MW-12 & MW & $07 / 07 / 93$ & 1050 & 37.28 & 11.82 & 25.46 & \\
\hline MW-12 & MW & $08 / 06 / 93$ & 1029 & 37.28 & 11.88 & 25.40 & \\
\hline MW-12 & MW & $09 / 03 / 93$ & 1050 & 37.28 & 11.96 & 25.32 & \\
\hline MW-12 & MW & $09 / 30 / 93$ & 1034 & 37.28 & 11.69 & 25.59 & \\
\hline MW-12 & MW & $10 / 28 / 93$ & 1047 & 37.28 & 12.37 & 24.91 & \\
\hline MW-12 & MW & $12 / 01 / 93$ & 1344 & 37.28 & 11.93 & 25.35 & \\
\hline MW-12 & MW & $01 / 06 / 94$ & 1057 & 37.28 & 11.73 & 25.55 & \\
\hline MW-12 & MW & $02 / 01 / 94$ & 1056 & 37.28 & 11.27 & 26.01 & \\
\hline MW-12 & MW & $03 / 04 / 94$ & 1034 & 37.28 & 11.49 & 25.79 & \\
\hline MW-12 & MW & $04 / 04 / 94$ & 1031 & 37.28 & 12.18 & 25.10 & \\
\hline MW-12 & MW & $05 / 06 / 94$ & 1118 & 37.28 & 12.87 & 24.41 & \\
\hline MW-12 & MW & $06 / 03 / 94$ & 1050 & 37.28 & 13.44 & 23.84 & \\
\hline MW-12 & MW & $07 / 08 / 94$ & 1108 & 37.28 & 10.88 & 26.40 & \\
\hline MW-12 & MW & $08 / 05 / 94$ & 1410 & 37.28 & 10.68 & 26.60 & \\
\hline MW-12 & MW & $09 / 07 / 94$ & 1127 & 37.28 & 10.66 & 26.62 & \\
\hline MW-12 & MW & $10 / 06 / 94$ & 1137 & 37.28 & 9.64 & 27.64 & \\
\hline MW-12 & MW & $11 / 09 / 94$ & 1300 & 37.28 & 10.63 & 26.65 & \\
\hline MW-12 & MW & $12 / 06 / 94$ & 1119 & 37.28 & 10.71 & 26.57 & \\
\hline MW-12 & MW & $01 / 10 / 95$ & 1325 & 37.28 & 10.73 & 26.55 & \\
\hline MW-12 & MW & $02 / 02 / 95$ & 1337 & 37.28 & 11.03 & 26.25 & \\
\hline MW-12 & MW & $03 / 02 / 95$ & 1507 & 37.28 & 11.07 & 26.21 & \\
\hline MW-12 & MW & $04 / 05 / 95$ & 1103 & 37.28 & 11.70 & 25.58 & \\
\hline MW-12 & MW & $05 / 02 / 95$ & 1252 & 37.28 & 12.09 & 25.19 & \\
\hline MW-12 & MW & $06 / 09 / 95$ & 1052 & 37.28 & 11.64 & 24.98 & \\
\hline MW-12 & MW & $08 / 10 / 95$ & 1121 & 37.28 & 12.01 & 25.27 & \\
\hline MW-12 & MW & $09 / 13 / 95$ & 1200 & 37.28 & 11.09 & 26.19 & \\
\hline
\end{tabular}


Table 8.--Water-level data collected at the Defense Fuel Supply Point and adjacent properties, Hanahan, S.C., between April 1991 and September 1995--Continued

[ft, feet; ---, data not available; WL, water-level well; EW, extraction well; MW, monitoring well; SW, surface-water site; -, negative depth to water indicates water-level altitude is above measuring point]

\begin{tabular}{|c|c|c|c|c|c|c|c|}
\hline $\begin{array}{c}\text { Site } \\
\text { (plate 1) }\end{array}$ & $\begin{array}{l}\text { Site } \\
\text { type }\end{array}$ & Date & Time & $\begin{array}{l}\text { Measuring-point } \\
\text { altitude } \\
\text { (ft above } \\
\text { sea level) }\end{array}$ & $\begin{array}{l}\text { Depth to water } \\
\text { (ft below } \\
\text { measuring point) }\end{array}$ & $\begin{array}{l}\text { Water-level } \\
\text { altitude } \\
\text { (ft above } \\
\text { sea level) }\end{array}$ & Remarks \\
\hline$M W-12 A$ & $\overline{M W}$ & $04 / 30 / 91$ & 1035 & 37.73 & 11.32 & 26.41 & \\
\hline$M W-12 A$ & MW & $05 / 28 / 91$ & --- & 37.73 & 11.49 & 26.24 & \\
\hline$M W-12 A$ & MW & $07 / 01 / 91$ & -- & 37.73 & 11.60 & 26.13 & \\
\hline$M W-12 A$ & MW & $08 / 06 / 91$ & -- & 37.73 & 10.39 & 27.34 & \\
\hline$M W-12 A$ & MW & $08 / 30 / 91$ & --- & 37.73 & 10.13 & 27.60 & \\
\hline$M W-12 A$ & MW & $09 / 27 / 91$ & -- & 37.73 & 11.54 & 26.19 & \\
\hline$M W-12 A$ & MW & $10 / 29 / 91$ & -- & 37.73 & 12.17 & 25.56 & \\
\hline $\mathrm{MW}-12 \mathrm{~A}$ & MW & $12 / 02 / 91$ & $\ldots$ & 37.73 & 12.54 & 25.19 & \\
\hline $\mathrm{MW}-12 \mathrm{~A}$ & MW & $12 / 16 / 91$ & --- & 37.73 & 12.72 & 25.01 & \\
\hline$M W-12 A$ & MW & $01 / 02 / 92$ & --- & 37.73 & 12.93 & 24.80 & \\
\hline$M W-12 A$ & MW & $02 / 04 / 92$ & --- & 37.73 & 11.92 & 25.81 & \\
\hline MW-12A & MW & $03 / 06 / 92$ & -- & 37.73 & 12.30 & 25.43 & \\
\hline MW-12A & MW & $03 / 31 / 92$ & --- & 37.73 & 12.33 & 25.40 & \\
\hline $\mathrm{MW}-12 \mathrm{~A}$ & MW & $05 / 01 / 92$ & -- & 37.73 & 12.45 & 25.28 & \\
\hline $\mathrm{MW}-12 \mathrm{~A}$ & MW & $06 / 11 / 92$ & 1413 & 37.73 & 11.73 & 26.00 & \\
\hline $\mathrm{MW}-12 \mathrm{~A}$ & MW & $07 / 07 / 92$ & 1708 & 37.73 & 12.10 & 25.63 & \\
\hline MW-12A & MW & $08 / 07 / 92$ & 1252 & 37.73 & 11.82 & 25.91 & \\
\hline $\mathrm{MW}-12 \mathrm{~A}$ & MW & $08 / 31 / 92$ & 1135 & 37.73 & 10.82 & 26.91 & \\
\hline MW-12A & MW & $10 / 07 / 92$ & 1142 & 37.73 & 10.54 & 27.19 & \\
\hline$M W-12 A$ & MW & $10 / 28 / 92$ & 1258 & 37.73 & 11.23 & 26.50 & \\
\hline $\mathrm{MW}-12 \mathrm{~A}$ & MW & $12 / 03 / 92$ & 1121 & 37.73 & 11.22 & 26.51 & \\
\hline$M W-12 A$ & MW & $01 / 04 / 93$ & 1110 & 37.73 & 11.96 & 25.77 & \\
\hline $\mathrm{MW}-12 \mathrm{~A}$ & MW & $02 / 04 / 93$ & 1202 & 37.73 & 11.19 & 26.54 & \\
\hline $\mathrm{MW}-12 \mathrm{~A}$ & MW & $03 / 05 / 93$ & 1125 & 37.73 & 11.45 & 26.28 & \\
\hline$M W-12 A$ & MW & $03 / 30 / 93$ & 1042 & 37.73 & 11.28 & 26.45 & \\
\hline $\mathrm{MW}-12 \mathrm{~A}$ & MW & $05 / 06 / 93$ & 1107 & 37.73 & 11.57 & 26.16 & \\
\hline $\mathrm{MW}-12 \mathrm{~A}$ & MW & $05 / 28 / 93$ & 1101 & 37.73 & 11.87 & 25.86 & \\
\hline $\mathrm{MW}-12 \mathrm{~A}$ & MW & $07 / 07 / 93$ & 1054 & 37.73 & 11.47 & 26.26 & \\
\hline$M W-12 A$ & MW & $08 / 06 / 93$ & 1032 & 37.73 & 11.56 & 26.17 & \\
\hline$M W-12 A$ & MW & $09 / 03 / 93$ & 1051 & 37.73 & 11.74 & 25.99 & \\
\hline $\mathrm{MW}-12 \mathrm{~A}$ & MW & $09 / 30 / 93$ & 1030 & 37.73 & 11.50 & 26.23 & \\
\hline$M W-12 A$ & MW & $10 / 28 / 93$ & 1049 & 37.73 & 12.11 & 25.62 & \\
\hline$M W-12 A$ & MW & $12 / 01 / 93$ & 1342 & 37.73 & 11.60 & 26.13 & \\
\hline$M W-12 A$ & MW & $01 / 06 / 94$ & 1054 & 37.73 & 11.35 & 26.38 & \\
\hline$M W-12 A$ & MW & $02 / 01 / 94$ & 1059 & 37.73 & 10.87 & 26.86 & \\
\hline$M W-12 A$ & MW & $03 / 04 / 94$ & 1036 & 37.73 & 11.04 & 26.69 & \\
\hline$M W-12 A$ & MW & $04 / 04 / 94$ & 1029 & 37.73 & 11.83 & 25.90 & \\
\hline$M W-12 A$ & MW & $05 / 06 / 94$ & 1116 & 37.73 & 12.43 & 25.30 & \\
\hline$M W-12 A$ & MW & $06 / 03 / 94$ & 1052 & 37.73 & 12.84 & 24.89 & \\
\hline$M W-12 A$ & MW & $08 / 05 / 94$ & 1409 & 37.52 & 10.19 & 27.33 & New measuring point established. \\
\hline$M W-12 A$ & MW & $09 / 07 / 94$ & 1125 & 37.52 & 10.28 & 27.24 & \\
\hline MW-12A & MW & $10 / 06 / 94$ & 1135 & 37.52 & 9.37 & 28.15 & \\
\hline $\mathrm{MW}-12 \mathrm{~A}$ & MW & $11 / 09 / 94$ & 1259 & 37.52 & 10.30 & 27.22 & \\
\hline$M W-12 A$ & MW & $12 / 06 / 94$ & 1118 & 37.52 & 10.22 & 27.30 & \\
\hline MW-12A & MW & $01 / 10 / 95$ & 1323 & 37.52 & 10.34 & 27.18 & \\
\hline
\end{tabular}


Table 8.--Water-level data collected at the Defense Fuel Supply Point and adjacent properties, Hanahan, S.C., between April 1991 and September 1995--Continued

[ft, feet; ---, data not available; WL, water-level well; EW, extraction well; MW, monitoring well; SW, surface-water site; -, negative depth to water indicates water-level altitude is above measuring point]

\begin{tabular}{|c|c|c|c|c|c|c|c|}
\hline $\begin{array}{c}\text { Site } \\
\text { (plate 1) }\end{array}$ & $\begin{array}{l}\text { Site } \\
\text { type }\end{array}$ & Date & Time & $\begin{array}{l}\text { Measuring-point } \\
\text { altitude } \\
\text { (ft above } \\
\text { sea level) }\end{array}$ & $\begin{array}{l}\text { Depth to water } \\
\text { ( } \mathrm{ft} \text { below } \\
\text { measuring point) }\end{array}$ & $\begin{array}{l}\text { Water-level } \\
\text { altitude } \\
\text { (ft above } \\
\text { sea level) }\end{array}$ & Remarks \\
\hline $\mathrm{MW}-12 \mathrm{~A}$ & MW & $02 / 02 / 95$ & 1335 & 37.52 & 10.62 & 26.90 & \\
\hline MW-12A & MW & $03 / 02 / 95$ & 1505 & 37.52 & 10.66 & 26.86 & \\
\hline$M W-12 A$ & MW & $04 / 05 / 95$ & 1105 & 37.52 & 11.32 & 26.20 & \\
\hline$M W-12 A$ & MW & $05 / 02 / 95$ & 1250 & 37.52 & 11.60 & 25.92 & \\
\hline$M W-12 A$ & MW & $06 / 09 / 95$ & 1055 & 37.52 & 12.30 & 25.88 & \\
\hline $\mathrm{MW}-12 \mathrm{~A}$ & MW & $08 / 10 / 95$ & 1120 & 37.52 & 11.51 & 26.01 & \\
\hline$M W-12 A$ & MW & $09 / 13 / 95$ & 1156 & 37.52 & 10.64 & 26.88 & \\
\hline MW-15 & MW & $04 / 30 / 91$ & 1158 & 13.12 & 2.42 & 10.70 & \\
\hline MW-15 & MW & $05 / 28 / 91$ & 1250 & 13.12 & 2.72 & 10.40 & \\
\hline MW-15 & MW & $07 / 01 / 91$ & --- & 13.12 & 2.97 & 10.15 & \\
\hline MW-15 & MW & $08 / 06 / 91$ & -- & 13.12 & 2.27 & 10.85 & \\
\hline MW-15 & MW & $08 / 30 / 91$ & --- & 13.12 & 2.05 & 11.07 & \\
\hline MW-15 & MW & $09 / 27 / 91$ & -- & 13.12 & 2.80 & 10.32 & \\
\hline MW-15 & MW & $10 / 29 / 91$ & -- & 13.12 & 3.30 & 9.82 & \\
\hline MW-15 & MW & $12 / 02 / 91$ & -- & 13.12 & 3.58 & 9.54 & \\
\hline MW-15 & MW & $01 / 02 / 92$ & $\cdots$ & 13.12 & 3.80 & 9.32 & \\
\hline MW-15 & MW & $02 / 04 / 92$ & -- & 13.12 & 3.70 & 9.42 & \\
\hline MW-15 & MW & $03 / 06 / 92$ & -- & 13.12 & 3.96 & 9.16 & \\
\hline MW-15 & MW & $03 / 31 / 92$ & -- & 13.12 & 3.69 & 9.43 & \\
\hline MW-15 & MW & 05/01/92 & -- & 13.12 & 4.01 & 9.11 & \\
\hline MW-15 & MW & $06 / 11 / 92$ & 1705 & 13.12 & 3.21 & 9.91 & \\
\hline MW-15 & MW & $07 / 07 / 92$ & 1050 & 13.12 & 3.70 & 9.42 & \\
\hline MW-15 & MW & $08 / 31 / 92$ & 1305 & 13.12 & 2.76 & 10.36 & \\
\hline MW-15 & MW & $10 / 07 / 92$ & 1415 & 13.12 & 2.18 & 10.94 & \\
\hline MW-15 & MW & $10 / 28 / 92$ & -- & 13.12 & 2.48 & 10.64 & \\
\hline MW-15 & MW & $12 / 03 / 92$ & 1355 & 13.12 & 2.28 & 10.84 & \\
\hline MW-15 & MW & $01 / 04 / 93$ & 1325 & 13.12 & 2.53 & 10.59 & \\
\hline MW-15 & MW & $02 / 04 / 93$ & 1354 & 13.12 & 1.55 & 11.57 & \\
\hline MW-15 & MW & $03 / 05 / 93$ & 1326 & 13.12 & 1.48 & 11.64 & \\
\hline MW-15 & MW & $03 / 30 / 93$ & 1258 & 13.12 & 1.42 & 11.70 & \\
\hline MW-15 & MW & $05 / 06 / 93$ & 1318 & 13.12 & 1.85 & 11.27 & \\
\hline MW-15 & MW & $05 / 28 / 93$ & 1303 & 13.12 & 2.36 & 10.76 & \\
\hline MW-15 & MW & $07 / 07 / 93$ & 1321 & 13.12 & 2.71 & 10.41 & \\
\hline MW-16 & MW & $04 / 30 / 91$ & 1110 & 28.12 & 2.60 & 25.52 & \\
\hline MW-16 & $\mathrm{MW}$ & $05 / 28 / 91$ & -- & 28.12 & 2.75 & 25.37 & \\
\hline MW-16 & MW & $07 / 01 / 91$ & $\ldots$ & 28.12 & 2.83 & 25.29 & \\
\hline MW-16 & MW & $08 / 06 / 91$ & -- & 28.12 & 2.46 & 25.66 & \\
\hline MW-16 & MW & $08 / 30 / 91$ & $-\cdots$ & 28.12 & 2.32 & 25.80 & \\
\hline MW-16 & MW & $09 / 27 / 91$ & -- & 28.12 & 2.89 & 25.23 & \\
\hline MW-16 & MW & $10 / 29 / 91$ & -- & 28.12 & 3.24 & 24.88 & \\
\hline MW-16 & MW & $12 / 02 / 91$ & $\cdots$ & 28.12 & 3.94 & 24.18 & \\
\hline MW-16 & MW & $01 / 02 / 92$ & --- & 28.12 & 4.02 & 24.10 & \\
\hline MW-16 & MW & $02 / 04 / 92$ & -- & 28.12 & 2.95 & 25.17 & \\
\hline
\end{tabular}


Table 8.--Water-level data collected at the Defense Fuel Supply Point and adjacent properties, Hanahan, S.C., between April 1991 and September 1995--Continued

[ft, feet; ---, data not available; WL, water-level well; EW, extraction well; MW, monitoring well; SW, surface-water site; -, negative depth to water indicates water-level altitude is above measuring point]

\begin{tabular}{|c|c|c|c|c|c|c|c|}
\hline $\begin{array}{c}\text { Site } \\
\text { (plate 1) }\end{array}$ & $\begin{array}{l}\text { Site } \\
\text { type }\end{array}$ & Date & Time & $\begin{array}{l}\text { Measuring-point } \\
\text { altitude } \\
\text { (ft above } \\
\text { sea level) }\end{array}$ & $\begin{array}{l}\text { Depth to water } \\
\text { (ft below } \\
\text { measuring point) }\end{array}$ & $\begin{array}{l}\text { Water-level } \\
\text { altitude } \\
\text { (ft above } \\
\text { sea level) }\end{array}$ & Remarks \\
\hline MW-16 & MW & $03 / 06 / 92$ & $\cdots$ & 28.12 & 2.96 & 25.16 & \\
\hline MW-16 & MW & $03 / 31 / 92$ & -- & 28.12 & 2.91 & 25.21 & \\
\hline MW-16 & MW & $05 / 01 / 92$ & -.- & 28.12 & 3.01 & 25.11 & \\
\hline MW-16 & MW & $06 / 11 / 92$ & 1452 & 28.12 & 2.55 & 25.57 & \\
\hline MW-16 & MW & $07 / 07 / 92$ & 1515 & 28.12 & 3.02 & 25.10 & \\
\hline MW-16 & MW & $08 / 07 / 92$ & 1337 & 28.12 & 2.71 & 25.41 & \\
\hline MW-16 & MW & $08 / 31 / 92$ & 1100 & 28.12 & 2.51 & 25.61 & \\
\hline MW-16 & MW & $10 / 07 / 92$ & 1055 & 28.12 & 2.26 & 25.86 & \\
\hline MW-16 & MW & $10 / 28 / 92$ & 1602 & 28.12 & 2.70 & 25.42 & \\
\hline MW-16 & MW & $12 / 03 / 92$ & 1045 & 28.12 & 2.55 & 25.57 & \\
\hline MW-16 & MW & $01 / 04 / 93$ & 1025 & 28.12 & 2.77 & 25.35 & \\
\hline MW-16 & MW & $02 / 04 / 93$ & 1100 & 28.12 & 2.48 & 25.64 & \\
\hline MW-16 & MW & $03 / 05 / 93$ & 1050 & 28.12 & 2.46 & 25.66 & \\
\hline MW-16 & MW & $03 / 30 / 93$ & 1010 & 28.12 & 2.34 & 25.78 & \\
\hline MW-16 & MW & $05 / 06 / 93$ & 1030 & 28.12 & 2.75 & 25.37 & \\
\hline MW-16 & MW & $05 / 28 / 93$ & 1030 & 28.12 & 2.77 & 25.35 & \\
\hline MW-16 & MW & $07 / 07 / 93$ & 1041 & 28.12 & 2.90 & 25.22 & \\
\hline MW-16 & MW & $08 / 06 / 93$ & 1030 & 28.12 & 2.72 & 25.40 & \\
\hline MW-16 & MW & $09 / 03 / 93$ & 1035 & 28.12 & 2.96 & 25.16 & \\
\hline MW-16 & MW & $09 / 30 / 93$ & 1030 & 28.12 & 2.79 & 25.33 & \\
\hline MW-16 & MW & $10 / 28 / 93$ & 1025 & 28.12 & 3.18 & 24.94 & \\
\hline MW-16 & MW & $12 / 01 / 93$ & 1337 & 28.12 & 2.89 & 25.23 & \\
\hline MW-16 & MW & $01 / 06 / 94$ & 1100 & 28.12 & 2.70 & 25.42 & \\
\hline MW-16 & MW & $02 / 01 / 94$ & 1000 & 28.12 & 2.14 & 25.98 & \\
\hline MW-16 & MW & 03/04/94 & 1000 & 28.12 & 2.08 & 26.04 & \\
\hline MW-17 & MW & $04 / 30 / 91$ & 1050 & 36.59 & 8.90 & 27.69 & \\
\hline MW-17 & MW & $05 / 28 / 91$ & -- & 36.59 & 9.19 & 27.40 & \\
\hline MW-17 & MW & $07 / 01 / 91$ & -- & 36.59 & 9.40 & 27.19 & \\
\hline MW-17 & MW & $08 / 06 / 91$ & -- & 36.59 & 8.18 & 28.41 & \\
\hline MW-17 & MW & $08 / 30 / 91$ & -- & 36.59 & 8.04 & 28.55 & \\
\hline MW-17 & MW & $09 / 27 / 91$ & - & 36.59 & 9.30 & 27.29 & \\
\hline MW-17 & MW & $10 / 29 / 91$ & - & 36.59 & 9.93 & 26.66 & \\
\hline MW-17 & MW & $12 / 02 / 91$ & -- & 36.59 & 10.23 & 26.36 & \\
\hline MW-17 & MW & $12 / 16 / 91$ & -- & 36.59 & 10.40 & 26.19 & \\
\hline MW-17 & MW & $01 / 02 / 92$ & -- & 36.59 & 10.54 & 26.05 & \\
\hline MW-17 & MW & $02 / 04 / 92$ & --- & 36.59 & 9.71 & 26.88 & Pre-start-up water levels. \\
\hline MW-17 & MW & $03 / 06 / 92$ & -- & 36.59 & 9.87 & 26.72 & \\
\hline MW-17 & MW & $03 / 31 / 92$ & -- & 36.59 & 9.88 & 26.71 & \\
\hline MW-17 & MW & $05 / 01 / 92$ & --- & 36.59 & 9.84 & 26.75 & \\
\hline MW-17 & MW & $06 / 11 / 92$ & 1530 & 36.59 & 9.36 & 27.23 & \\
\hline MW-17 & MW & 07/07/92 & 1546 & 36.59 & 9.57 & 27.02 & \\
\hline MW-17 & MW & $08 / 07 / 92$ & 1330 & 36.59 & 9.43 & 27.16 & \\
\hline MW-17 & MW & $08 / 31 / 92$ & 1211 & 36.59 & 8.24 & 28.35 & \\
\hline MW-17 & MW & $10 / 07 / 92$ & 1217 & 36.59 & 8.08 & 28.51 & \\
\hline
\end{tabular}


Table 8.--Water-level data collected at the Defense Fuel Supply Point and adjacent properties, Hanahan, S.C., between April 1991 and September 1995--Continued

[ft, feet; ---, data not available; WL, water-level well; EW, extraction well; MW, monitoring well; SW, surface-water site; -, negative depth to water indicates water-level altitude is above measuring point]

\begin{tabular}{|c|c|c|c|c|c|c|c|}
\hline $\begin{array}{c}\text { Site } \\
\text { (plate 1) }\end{array}$ & $\begin{array}{l}\text { Site } \\
\text { type }\end{array}$ & Date & Time & $\begin{array}{l}\text { Measuring-point } \\
\text { altitude } \\
\text { (ft above } \\
\text { sea level) }\end{array}$ & $\begin{array}{l}\text { Depth to water } \\
\text { (ft below } \\
\text { measuring point) }\end{array}$ & $\begin{array}{l}\text { Water-level } \\
\text { altitude } \\
\text { (ft above } \\
\text { sea level) }\end{array}$ & Remarks \\
\hline MW-17 & MW & $10 / 28 / 92$ & 1330 & 36.59 & 8.93 & 27.66 & \\
\hline MW-17 & MW & $12 / 03 / 92$ & 1214 & 36.59 & 8.68 & 27.91 & \\
\hline MW-17 & MW & $01 / 04 / 93$ & 1150 & 36.59 & 9.41 & 27.18 & \\
\hline MW-17 & MW & $02 / 04 / 93$ & 1244 & 36.59 & 8.45 & 28.14 & \\
\hline MW-17 & MW & $03 / 05 / 93$ & 1203 & 36.59 & 8.79 & 27.80 & \\
\hline MW-17 & MW & $03 / 30 / 93$ & 1121 & 36.59 & 8.40 & 28.19 & \\
\hline MW-17 & MW & $05 / 06 / 93$ & 1138 & 36.59 & 9.25 & 27.34 & \\
\hline MW-17 & MW & $05 / 28 / 93$ & 1134 & 36.59 & 9.65 & 26.94 & \\
\hline MW-17 & MW & $07 / 07 / 93$ & 1121 & 36.59 & 9.57 & 27.02 & \\
\hline MW-17 & MW & $08 / 06 / 93$ & 1104 & 36.59 & 9.24 & 27.35 & \\
\hline MW-17 & MW & $09 / 03 / 93$ & 1121 & 36.59 & 9.43 & 27.16 & \\
\hline MW-17 & MW & $09 / 30 / 93$ & 0945 & 36.59 & 9.24 & 27.35 & \\
\hline MW-17 & MW & $10 / 28 / 93$ & 1120 & 36.59 & 10.03 & 26.56 & \\
\hline MW-17 & MW & $12 / 01 / 93$ & 1423 & 36.59 & 9.62 & 26.97 & \\
\hline MW-17 & MW & $01 / 06 / 94$ & 1141 & 36.59 & 9.08 & 27.51 & \\
\hline MW-17 & MW & $02 / 01 / 94$ & 1130 & 36.59 & 8.33 & 28.26 & \\
\hline MW-17 & MW & $03 / 04 / 94$ & 1102 & 36.59 & 8.50 & 28.09 & \\
\hline MW-17 & MW & $04 / 04 / 94$ & 1100 & 36.59 & 9.60 & 26.99 & \\
\hline MW-17 & MW & $05 / 06 / 94$ & 1156 & 36.59 & 10.20 & 26.39 & \\
\hline MW-17 & MW & $06 / 03 / 94$ & 1140 & 36.59 & 10.65 & 25.94 & \\
\hline MW-17 & MW & $07 / 08 / 94$ & 1155 & 36.59 & 8.37 & 28.22 & \\
\hline MW-17 & MW & $08 / 05 / 94$ & 1434 & 36.59 & 8.38 & 28.21 & \\
\hline MW-17 & MW & $09 / 07 / 94$ & 1130 & 36.59 & 8.55 & 28.04 & \\
\hline MW-17 & MW & $10 / 06 / 94$ & 1158 & 36.59 & 6.92 & 29.67 & \\
\hline MW-17 & MW & $11 / 09 / 94$ & 1327 & 36.59 & 8.42 & 28.17 & \\
\hline MW-17 & MW & $12 / 06 / 94$ & 1146 & 36.59 & 8.25 & 28.34 & \\
\hline MW-17 & MW & $01 / 10 / 95$ & 1624 & 36.59 & 8.57 & 28.02 & \\
\hline MW-17 & MW & $02 / 02 / 95$ & 1402 & 36.59 & 8.68 & 27.91 & \\
\hline MW-17 & MW & $03 / 02 / 95$ & 1536 & 36.59 & 8.67 & 27.92 & \\
\hline MW-17 & MW & $04 / 05 / 95$ & 1146 & 36.59 & 9.56 & 27.03 & \\
\hline MW-17 & MW & $05 / 02 / 95$ & 1330 & 36.59 & 9.78 & 26.81 & \\
\hline MW-17 & MW & $06 / 09 / 95$ & 1131 & 36.59 & 9.66 & 26.93 & \\
\hline$M W-17$ & MW & $07 / 06 / 95$ & 1116 & 36.59 & 9.56 & 27.03 & \\
\hline$M W-17$ & MW & $08 / 10 / 95$ & 1252 & 36.59 & 9.66 & 26.93 & \\
\hline MW-17 & MW & $09 / 13 / 95$ & 1212 & 36.59 & 8.60 & 27.99 & \\
\hline MW-18 & MW & $04 / 30 / 91$ & 1007 & 33.94 & 6.44 & 27.50 & \\
\hline MW-18 & MW & $05 / 28 / 91$ & 1129 & 33.94 & 6.77 & 27.17 & \\
\hline MW-18 & MW & $07 / 01 / 91$ & --- & 33.94 & 7.26 & 26.68 & \\
\hline MW-18 & MW & $08 / 06 / 91$ & -- & 33.94 & 5.58 & 28.36 & \\
\hline MW-18 & MW & $08 / 30 / 91$ & --- & 33.94 & 4.84 & 29.10 & \\
\hline$M W-18$ & MW & $09 / 27 / 91$ & --- & 33.94 & 6.73 & 27.21 & \\
\hline MW-18 & MW & $10 / 29 / 91$ & -- & 33.94 & 7.77 & 26.17 & \\
\hline MW-18 & MW & $12 / 02 / 91$ & --- & 33.94 & 8.49 & 25.45 & \\
\hline MW-18 & MW & $01 / 02 / 92$ & $-\cdots$ & 33.94 & 9.04 & 24.90 & \\
\hline
\end{tabular}


Table 8.--Water-level data collected at the Defense Fuel Supply Point and adjacent properties, Hanahan, S.C., between April 1991 and September 1995--Continued

[ft, feet; --., data not available; WL, water-level well; EW, extraction well; MW, monitoring well; SW, surface-water site; -, negative depth to water indicates water-level altitude is above measuring point]

\begin{tabular}{|c|c|c|c|c|c|c|c|}
\hline $\begin{array}{c}\text { Site } \\
\text { (plate 1) }\end{array}$ & $\begin{array}{l}\text { Site } \\
\text { type }\end{array}$ & Date & Time & $\begin{array}{l}\text { Measuring-point } \\
\text { altitude } \\
\text { (ft above } \\
\text { sea level) }\end{array}$ & $\begin{array}{l}\text { Depth to water } \\
\text { (ft below } \\
\text { measuring point) }\end{array}$ & $\begin{array}{l}\text { Water-level } \\
\text { altitude } \\
\text { (ft above } \\
\text { sea level) }\end{array}$ & Remarks \\
\hline MW-18 & MW & $02 / 04 / 92$ & --- & 33.94 & 7.75 & 26.19 & \\
\hline MW-18 & MW & $03 / 06 / 92$ & --- & 33.94 & 7.78 & 26.16 & \\
\hline MW-18 & MW & $03 / 31 / 92$ & --- & 33.94 & 7.46 & 26.48 & \\
\hline MW-18 & MW & $05 / 01 / 92$ & 1338 & 33.94 & 7.78 & 26.16 & \\
\hline MW-18 & MW & $06 / 11 / 92$ & 1630 & 33.94 & 6.89 & 27.05 & \\
\hline MW-18 & MW & $07 / 07 / 92$ & 1305 & 33.94 & 7.51 & 26.43 & \\
\hline MW-18 & MW & $08 / 07 / 92$ & 1452 & 33.94 & 7.37 & 26.57 & \\
\hline MW-18 & MW & $08 / 31 / 92$ & 1142 & 33.94 & 5.76 & 28.18 & \\
\hline MW-18 & MW & $10 / 07 / 92$ & 1200 & 33.94 & 4.80 & 29.14 & \\
\hline MW-18 & MW & $10 / 28 / 92$ & 1519 & 33.94 & 6.22 & 27.72 & \\
\hline MW-18 & MW & $12 / 03 / 92$ & 1150 & 33.94 & 5.73 & 28.21 & \\
\hline MW-18 & MW & $01 / 04 / 93$ & 1125 & 33.94 & 6.77 & 27.17 & \\
\hline MW-18 & MW & $02 / 04 / 93$ & 1205 & 33.94 & 5.04 & 28.90 & \\
\hline MW-18 & MW & $03 / 05 / 93$ & 1145 & 33.94 & 5.10 & 28.84 & \\
\hline MW-18 & MW & $03 / 30 / 93$ & 1103 & 33.94 & 5.00 & 28.94 & \\
\hline MW-18 & MW & $05 / 06 / 93$ & 1130 & 33.94 & 6.58 & 27.36 & \\
\hline MW-18 & MW & $05 / 28 / 93$ & 1121 & 33.94 & 7.55 & 26.39 & \\
\hline MW-18 & MW & $07 / 07 / 93$ & 1140 & 33.94 & 7.93 & 26.01 & \\
\hline MW-18 & MW & $08 / 06 / 93$ & 1128 & 33.94 & 7.57 & 26.37 & \\
\hline MW-18 & MW & $09 / 03 / 93$ & 1110 & 33.94 & 7.70 & 26.24 & \\
\hline MW-18 & MW & $09 / 30 / 93$ & 1110 & 33.94 & 7.13 & 26.81 & \\
\hline MW-18 & MW & $10 / 28 / 93$ & 1100 & 33.94 & 8.29 & 25.65 & \\
\hline MW-18 & MW & $12 / 01 / 93$ & 1410 & 33.94 & 7.92 & 26.02 & \\
\hline MW-18 & MW & $01 / 06 / 94$ & 1140 & 33.94 & 7.53 & 26.41 & \\
\hline MW-18 & MW & $02 / 01 / 94$ & 1030 & 33.94 & 5.81 & 28.13 & \\
\hline MW-18 & MW & $03 / 04 / 94$ & 1045 & 33.94 & 5.68 & 28.26 & \\
\hline MW-19 & MW & $04 / 30 / 91$ & 1019 & 32.07 & 9.31 & 22.76 & \\
\hline MW-19 & MW & $05 / 28 / 91$ & 1150 & 32.07 & 9.43 & 22.64 & \\
\hline MW-19 & MW & $07 / 01 / 91$ & -- & 32.07 & 11.71 & 20.36 & \\
\hline MW-19 & MW & $08 / 06 / 91$ & -- & 32.07 & 8.85 & 23.22 & \\
\hline MW-19 & MW & $08 / 30 / 91$ & -- & 32.07 & 8.25 & 23.82 & \\
\hline MW-19 & MW & $09 / 27 / 91$ & -- & 32.07 & 9.00 & 23.07 & \\
\hline MW-19 & MW & $10 / 29 / 91$ & -- & 32.07 & 9.93 & 22.14 & \\
\hline MW-19 & MW & $12 / 02 / 91$ & -- & 32.07 & 10.62 & 21.45 & \\
\hline MW-19 & MW & $01 / 02 / 92$ & -- & 32.07 & 11.16 & 20.91 & \\
\hline MW-19 & MW & $02 / 04 / 92$ & -- & 32.07 & 10.97 & 21.10 & \\
\hline MW-19 & MW & $03 / 06 / 92$ & -- & 32.07 & 10.73 & 21.34 & \\
\hline MW-19 & MW & $03 / 31 / 92$ & --- & 32.07 & 10.73 & 21.34 & \\
\hline MW-19 & MW & 05/01/92 & --- & 32.07 & 10.70 & 21.37 & \\
\hline MW-19 & MW & $06 / 11 / 92$ & 1642 & 32.07 & 11.03 & 21.04 & \\
\hline MW-19 & MW & $07 / 07 / 92$ & 1145 & 32.07 & 10.42 & 21.65 & \\
\hline MW-19 & MW & $08 / 31 / 92$ & 1220 & 32.07 & 9.15 & 22.92 & \\
\hline MW-19 & MW & $10 / 07 / 92$ & 1240 & 32.07 & 8.85 & 23.22 & \\
\hline MW-19 & MW & $10 / 28 / 92$ & 1541 & 32.07 & 8.87 & 23.20 & \\
\hline
\end{tabular}


Table 8.--Water-level data collected at the Defense Fuel Supply Point and adjacent properties, Hanahan, S.C., between April 1991 and September 1995--Continued

[ft, feet; ---, data not available; WL, water-level well; EW, extraction well; MW, monitoring well; SW, surface-water site; -, negative depth to wat ?r indicates water-level altitude is above measuring point]

\begin{tabular}{|c|c|c|c|c|c|c|c|}
\hline $\begin{array}{c}\text { Site } \\
\text { (plate 1) }\end{array}$ & $\begin{array}{l}\text { Site } \\
\text { type }\end{array}$ & Date & Time & $\begin{array}{l}\text { Measuring-point } \\
\text { altitude } \\
\text { (ft above } \\
\text { sea level) }\end{array}$ & $\begin{array}{l}\text { Depth to water } \\
\text { (ft below } \\
\text { measuring point) }\end{array}$ & $\begin{array}{l}\text { Water-level } \\
\text { altitude } \\
\text { (ft above } \\
\text { sea level) }\end{array}$ & Remarks \\
\hline MW-19 & MW & $12 / 03 / 92$ & 1235 & 32.07 & 8.80 & 23.27 & \\
\hline MW-19 & MW & $01 / 04 / 93$ & 1155 & 32.07 & 9.50 & 22.57 & \\
\hline MW-19 & MW & $02 / 04 / 93$ & 1235 & 32.07 & .7 .95 & 24.12 & \\
\hline MW-19 & MW & $03 / 05 / 93$ & 1210 & 32.07 & 8.41 & 23.66 & \\
\hline MW-19 & MW & $03 / 30 / 93$ & 1132 & 32.07 & 8.37 & 23.70 & \\
\hline MW-19 & MW & $05 / 06 / 93$ & 1201 & 32.07 & 9.17 & 22.90 & \\
\hline MW-19 & MW & $05 / 28 / 93$ & 1153 & 32.07 & 9.86 & 22.21 & \\
\hline MW-19 & MW & $07 / 07 / 93$ & 1219 & 32.07 & 10.62 & 21.45 & \\
\hline MW-19 & MW & $08 / 06 / 93$ & 1200 & 32.07 & 10.94 & 21.13 & \\
\hline MW-19 & MW & $09 / 03 / 93$ & 1130 & 32.07 & 10.64 & 21.43 & \\
\hline MW-19 & MW & $09 / 30 / 93$ & 1130 & 32.07 & 10.39 & 21.68 & \\
\hline MW-19 & MW & $10 / 28 / 93$ & 1125 & 32.07 & 11.05 & 21.02 & \\
\hline MW-19 & MW & $12 / 01 / 93$ & 1440 & 32.07 & 11.15 & 20.92 & \\
\hline MW-19 & MW & $01 / 06 / 94$ & 1205 & 32.07 & 11.30 & 20.77 & \\
\hline MW-19 & MW & $02 / 01 / 94$ & 1055 & 32.07 & 10.50 & 21.57 & \\
\hline MW-19 & MW & $03 / 04 / 94$ & 1105 & 32.07 & 10.02 & 22.05 & \\
\hline MWGS-05A & MW & $04 / 30 / 91$ & 1133 & 20.14 & 2.53 & 17.61 & \\
\hline MWGS-05A & MW & $07 / 01 / 91$ & --- & 20.14 & 2.81 & 17.33 & \\
\hline MWGS-05A & MW & $08 / 06 / 91$ & --- & 20.14 & 1.72 & 18.42 & \\
\hline MWGS-05A & MW & $08 / 30 / 91$ & - & 20.14 & 1.70 & 18.44 & \\
\hline MWGS-05A & MW & $09 / 27 / 91$ & --- & 20.14 & 2.44 & 17.70 & \\
\hline MWGS-05A & MW & $12 / 02 / 91$ & -- & 20.14 & 3.82 & 16.32 & \\
\hline MWGS-05A & MW & $06 / 11 / 92$ & 1733 & 20.14 & 4.01 & 16.13 & \\
\hline MWGS-05A & MW & $07 / 07 / 92$ & 1105 & 20.14 & 3.63 & 16.51 & \\
\hline MWGS-05A & MW & $08 / 31 / 92$ & 1342 & 20.14 & 2.20 & 17.94 & \\
\hline MWGS-05A & MW & $10 / 07 / 92$ & 1456 & 20.14 & 1.85 & 18.29 & \\
\hline MWGS-05A & MW & $10 / 28 / 92$ & 1346 & 20.14 & 2.25 & 17.89 & \\
\hline MWGS-05A & MW & $12 / 03 / 92$ & 1415 & 20.14 & 2.08 & 18.06 & \\
\hline MWGS-05A & MW & $01 / 04 / 93$ & 1357 & 20.14 & 2.44 & 17.70 & \\
\hline MWGS-05A & MW & $02 / 04 / 93$ & 1434 & 20.14 & 1.54 & 18.60 & \\
\hline MWGS-05A & MW & $03 / 05 / 93$ & 1357 & 20.14 & 1.62 & 18.52 & \\
\hline MWGS-05A & MW & $03 / 30 / 93$ & 1308 & 20.14 & 1.46 & 18.68 & \\
\hline MWGS-05A & MW & $05 / 06 / 93$ & 1334 & 20.14 & 1.89 & 18.25 & \\
\hline MWGS-05A & MW & $05 / 28 / 93$ & 1309 & 20.14 & 2.25 & 17.89 & \\
\hline MWGS-05A & MW & $07 / 07 / 93$ & 1335 & 20.14 & 2.56 & 17.58 & \\
\hline MWGS-05A & MW & $08 / 06 / 93$ & 1313 & 20.14 & 2.75 & 17.39 & \\
\hline MWGS-05A & MW & $09 / 03 / 93$ & 1151 & 20.14 & 2.87 & 17.27 & \\
\hline MWGS-05A & MW & $09 / 30 / 93$ & 1248 & 20.14 & 2.65 & 17.49 & \\
\hline MWGS-05A & MW & $10 / 28 / 93$ & 1327 & 20.14 & 3.12 & 17.02 & \\
\hline MWGS-05A & MW & $12 / 01 / 93$ & 1517 & 20.14 & 3.03 & 17.11 & \\
\hline MWGS-05A & MW & $01 / 06 / 94$ & 1307 & 20.14 & 2.98 & 17.16 & \\
\hline MWGS-05A & MW & $02 / 01 / 94$ & 1239 & 20.14 & 2.29 & 17.85 & \\
\hline MWGS-05A & MW & $03 / 04 / 94$ & 1215 & 20.14 & 1.94 & 18.20 & \\
\hline MWGS-05A & MW & $04 / 04 / 94$ & 1134 & 20.14 & 2.47 & 17.67 & \\
\hline
\end{tabular}


Table 8.--Water-level data collected at the Defense Fuel Supply Point and adjacent properties, Hanahan, S.C., between April 1991 and September 1995--Continued

[ft, feet; ---, data not available; WL, water-level well; EW, extraction well; MW, monitoring well; SW, surface-water site; -, negative depth to water indicates water-level altitude is above measuring point]

\begin{tabular}{|c|c|c|c|c|c|c|c|}
\hline $\begin{array}{c}\text { Site } \\
\text { (plate 1) }\end{array}$ & $\begin{array}{l}\text { Site } \\
\text { type }\end{array}$ & Date & Time & $\begin{array}{c}\text { Measuring-point } \\
\text { altitude } \\
\text { (ft above } \\
\text { sea level) }\end{array}$ & $\begin{array}{l}\text { Depth to water } \\
\text { (ft below } \\
\text { measuring point) }\end{array}$ & $\begin{array}{l}\text { Water-level } \\
\text { altitude } \\
\text { (ft above } \\
\text { sea level) }\end{array}$ & Remarks \\
\hline MWGS-05A & MW & $05 / 06 / 94$ & 1231 & 20.14 & 3.18 & 16.96 & \\
\hline MWGS-05A & MW & $06 / 03 / 94$ & 1202 & 20.14 & 3.80 & 16.34 & \\
\hline MWGS-05A & MW & 07/08/94 & 1240 & 20.14 & 2.48 & 17.66 & \\
\hline MWGS-05A & MW & $08 / 05 / 94$ & 1505 & 20.14 & 1.90 & 18.24 & \\
\hline MWGS-05A & MW & $09 / 07 / 94$ & 1340 & 20.14 & 1.89 & 18.25 & \\
\hline MWGS-05A & MW & $10 / 06 / 94$ & 1332 & 20.14 & .83 & 19.31 & \\
\hline MWGS-05A & MW & $11 / 09 / 94$ & 1403 & 20.14 & 1.12 & 19.02 & \\
\hline MWGS-05A & MW & $12 / 06 / 94$ & 1235 & 20.14 & 1.15 & 18.99 & \\
\hline MWGS-05A & MW & $01 / 10 / 95$ & 1701 & 20.14 & 1.34 & 18.80 & \\
\hline MWGS-05A & MW & $02 / 02 / 95$ & 1431 & 20.14 & 1.35 & 18.79 & \\
\hline MWGS-05A & MW & $03 / 02 / 95$ & 1603 & 20.14 & 1.42 & 18.72 & \\
\hline MWGS-05A & MW & $04 / 05 / 95$ & 1228 & 20.14 & 2.00 & 18.14 & \\
\hline MWGS-05A & MW & $05 / 02 / 95$ & 1349 & 20.14 & 2.38 & 17.76 & \\
\hline MWGS-05A & MW & $06 / 09 / 95$ & 1308 & 20.14 & 2.93 & 17.21 & \\
\hline MWGS-05A & MW & $07 / 06 / 95$ & 1228 & 20.14 & 3.04 & 17.10 & \\
\hline MWGS-05A & MW & $08 / 10 / 95$ & 1315 & 20.14 & 3.01 & 17.13 & \\
\hline MWGS-05A & MW & $09 / 13 / 95$ & 1503 & 20.14 & 1.90 & 18.24 & \\
\hline MWGS-20 & MW & $04 / 30 / 91$ & 1120 & 34.10 & 6.39 & 27.71 & \\
\hline MWGS-20 & MW & $05 / 28 / 91$ & -- & 34.10 & 6.55 & 27.55 & \\
\hline MWGS-20 & MW & $07 / 01 / 91$ & -- & 34.10 & 6.66 & 27.44 & \\
\hline MWGS-20 & MW & $08 / 06 / 91$ & -- & 34.10 & 6.00 & 28.10 & \\
\hline MWGS-20 & MW & $08 / 30 / 91$ & -- & 34.10 & 5.84 & 28.26 & \\
\hline MWGS-20 & MW & $09 / 27 / 91$ & -- & 34.10 & 6.66 & 27.44 & \\
\hline MWGS-20 & MW & $10 / 29 / 91$ & -- & 34.10 & 7.00 & 27.10 & \\
\hline MWGS-20 & MW & $12 / 02 / 91$ & $\cdots$ & 34.10 & 7.16 & 26.94 & \\
\hline MWGS-20 & MW & $12 / 16 / 91$ & -- & 34.10 & 7.30 & 26.80 & \\
\hline MWGS-20 & MW & $01 / 02 / 92$ & -- & 34.10 & 7.36 & 26.74 & \\
\hline MWGS-20 & MW & $02 / 04 / 92$ & $\cdots$ & 34.10 & 6.78 & 27.32 & \\
\hline MWGS-20 & MW & $03 / 06 / 92$ & -- & 34.10 & 6.87 & 27.23 & \\
\hline MWGS-20 & MW & $03 / 31 / 92$ & $\cdots$ & 34.10 & 6.97 & 27.13 & \\
\hline MWGS-20 & MW & $05 / 01 / 92$ & -- & 34.10 & 6.93 & 27.17 & \\
\hline MWGS-20 & MW & $06 / 11 / 92$ & 1504 & 34.10 & 6.11 & 27.99 & \\
\hline MWGS-20 & MW & $07 / 07 / 92$ & 1507 & 34.10 & 6.79 & 27.31 & \\
\hline MWGS-20 & MW & $08 / 07 / 92$ & 1348 & 34.10 & 6.44 & 27.66 & \\
\hline MWGS-20 & MW & $08 / 31 / 92$ & 1217 & 34.10 & 6.00 & 28.10 & \\
\hline MWGS-20 & MW & $10 / 07 / 92$ & 1221 & 34.10 & 5.84 & 28.26 & \\
\hline MWGS-20 & MW & $10 / 28 / 92$ & 1335 & 34.10 & 6.41 & 27.69 & \\
\hline MWGS-20 & MW & $12 / 03 / 92$ & 1218 & 34.10 & 6.22 & 27.88 & \\
\hline MWGS-20 & MW & $01 / 04 / 93$ & 1157 & 34.10 & 6.66 & 27.44 & \\
\hline MWGS-20 & MW & $02 / 04 / 93$ & 1331 & 34.10 & 6.10 & 28.00 & \\
\hline MWGS-20 & MW & $03 / 05 / 93$ & 1205 & 34.10 & 6.11 & 27.99 & \\
\hline MWGS-20 & MW & $03 / 30 / 93$ & 1126 & 34.10 & 6.04 & 28.06 & \\
\hline MWGS-20 & MW & $05 / 06 / 93$ & 1144 & 34.10 & 6.61 & 27.49 & \\
\hline MWGS-20 & MW & $05 / 28 / 93$ & 1136 & 34.10 & 6.81 & 27.29 & \\
\hline
\end{tabular}


Table 8.--Water-level data collected at the Defense Fuel Supply Point and adjacent properties, Hanahan, S.C., between April 1991 and September 1995--Continued

[ft, feet; ---, data not available; WL, water-level well; EW, extraction well; MW, monitoring well; SW, surface-water site; -, negative depth to water indicates water-level altitude is above measuring point]

\begin{tabular}{|c|c|c|c|c|c|c|c|}
\hline $\begin{array}{c}\text { Site } \\
\text { (plate 1) }\end{array}$ & $\begin{array}{l}\text { Site } \\
\text { type }\end{array}$ & Date & Time & $\begin{array}{c}\text { Measuring-point } \\
\text { altitude } \\
\text { (ft above } \\
\text { sea level) }\end{array}$ & $\begin{array}{l}\text { Depth to water } \\
\text { (ft below } \\
\text { measuring point) }\end{array}$ & $\begin{array}{l}\text { Water-level } \\
\text { altitude } \\
\text { (ft above } \\
\text { sea level) }\end{array}$ & Remarks \\
\hline MWGS-20 & MW & $07 / 07 / 93$ & 1125 & 34.10 & 6.78 & 27.32 & \\
\hline MWGS-20 & MW & $08 / 06 / 93$ & 1106 & 34.10 & 6.44 & 27.66 & \\
\hline MWGS-20 & MW & $09 / 03 / 93$ & 1122 & 34.10 & 6.72 & 27.38 & \\
\hline MWGS-20 & MW & $09 / 30 / 93$ & 1012 & 34.10 & 6.56 & 27.54 & \\
\hline MWGS-20 & MW & $10 / 28 / 93$ & 1122 & 34.10 & 7.00 & 27.10 & \\
\hline MWGS-20 & MW & $12 / 01 / 93$ & 1427 & 34.10 & 6.76 & 27.34 & \\
\hline MWGS-20 & MW & $01 / 06 / 94$ & 1143 & 34.10 & 6.43 & 27.67 & \\
\hline MWGS-20 & MW & $02 / 01 / 94$ & 1125 & 34.10 & 5.78 & 28.32 & \\
\hline MWGS-20 & MW & $03 / 04 / 94$ & 1124 & 34.10 & 5.85 & 28.25 & \\
\hline MWGS-20 & MW & $04 / 04 / 94$ & 1102 & 34.10 & 6.72 & 27.38 & \\
\hline MWGS-20 & MW & $05 / 06 / 94$ & 1159 & 34.10 & 7.10 & 27.00 & \\
\hline MWGS-20 & MW & $06 / 03 / 94$ & 1145 & 34.10 & 7.33 & 26.77 & \\
\hline MWGS-20 & MW & $07 / 08 / 94$ & 1158 & 34.10 & 5.87 & 28.23 & \\
\hline MWGS-20 & MW & $08 / 05 / 94$ & 1439 & 34.10 & 6.01 & 28.09 & \\
\hline MWGS-20 & MW & $09 / 07 / 94$ & 1132 & 34.10 & 6.09 & 28.01 & \\
\hline MWGS-20 & MW & $10 / 06 / 94$ & 1215 & 34.10 & 4.74 & 29.36 & \\
\hline MWGS-20 & MW & $11 / 09 / 94$ & 1329 & 34.10 & 5.87 & 28.23 & \\
\hline MWGS-20 & MW & $12 / 06 / 94$ & 1150 & 34.10 & 5.46 & 28.64 & \\
\hline MWGS-20 & MW & $01 / 10 / 95$ & 1625 & 34.10 & 5.90 & 28.20 & \\
\hline MWGS-20 & MW & $02 / 02 / 95$ & 1404 & 34.10 & 5.99 & 28.11 & \\
\hline MWGS-20 & MW & $03 / 02 / 95$ & 1538 & 34.10 & 5.92 & 28.18 & \\
\hline MWGS-20 & MW & $04 / 05 / 95$ & 1148 & 34.10 & 6.48 & 27.62 & \\
\hline MWGS-20 & MW & $05 / 02 / 95$ & 1333 & 34.10 & 6.73 & 27.37 & \\
\hline MWGS-20 & MW & 06/09/95 & 1133 & 34.10 & 6.66 & 27.44 & \\
\hline MWGS-20 & MW & $07 / 06 / 95$ & 1118 & 34.10 & 6.60 & 27.50 & \\
\hline MWGS-20 & MW & $08 / 10 / 95$ & 1253 & 34.10 & 6.69 & 27.41 & \\
\hline MWGS-20 & MW & $09 / 13 / 95$ & 1216 & 34.10 & 5.95 & 28.15 & \\
\hline MWGS-21 & MW & $04 / 30 / 91$ & 1125 & 33.41 & 4.81 & 28.60 & \\
\hline MWGS-21 & MW & $05 / 28 / 91$ & -- & 33.41 & 4.96 & 28.45 & \\
\hline MWGS-21 & MW & $07 / 01 / 91$ & --- & 33.41 & 5.09 & 28.32 & \\
\hline MWGS-21 & MW & $08 / 06 / 91$ & -- & 33.41 & 4.44 & 28.97 & \\
\hline MWGS-21 & MW & $08 / 30 / 91$ & $\ldots$ & 33.41 & 4.25 & 29.16 & \\
\hline MWGS-21 & MW & $09 / 27 / 91$ & -- & 33.41 & 5.07 & 28.34 & \\
\hline MWGS-21 & MW & $10 / 29 / 91$ & --. & 33.41 & 5.44 & 27.97 & \\
\hline MWGS-21 & MW & $12 / 02 / 91$ & $\ldots$ & 33.41 & 5.61 & 27.80 & \\
\hline MWGS-21 & MW & $12 / 16 / 91$ & -- & 33.41 & 5.75 & 27.66 & \\
\hline MWGS-21 & MW & $01 / 02 / 92$ & $\cdots$ & 33.41 & 5.79 & 27.62 & \\
\hline MWGS-21 & MW & $02 / 04 / 92$ & $\ldots$ & 33.41 & 5.20 & 28.21 & \\
\hline MWGS-21 & MW & $03 / 06 / 92$ & $\cdots$ & 33.41 & 5.30 & 28.11 & \\
\hline MWGS-21 & MW & $03 / 31 / 92$ & --. & 33.41 & 5.36 & 28.05 & \\
\hline MWGS-21 & MW & $05 / 01 / 92$ & --- & 33.41 & 5.37 & 28.04 & \\
\hline MWGS-21 & MW & $06 / 11 / 92$ & 1506 & 33.41 & 4.40 & 29.01 & \\
\hline MWGS-21 & MW & $07 / 07 / 92$ & 1505 & 33.41 & 5.14 & 28.27 & \\
\hline MWGS-21 & MW & $08 / 07 / 92$ & 1350 & 33.41 & 4.81 & 28.60 & \\
\hline
\end{tabular}


Table 8.--Water-level data collected at the Defense Fuel Supply Point and adjacent properties, Hanahan, S.C., between April 1991 and September 1995--Continued

[ft, feet; ---, data not available; WL, water-level well; EW, extraction well; MW, monitoring well; SW, surface-water site; -, negative depth to water indicates water-level altitude is above measuring point]

\begin{tabular}{|c|c|c|c|c|c|c|c|}
\hline $\begin{array}{c}\text { Site } \\
\text { (plate 1) }\end{array}$ & $\begin{array}{l}\text { Site } \\
\text { type }\end{array}$ & Date & Time & $\begin{array}{l}\text { Measuring-point } \\
\text { altitude } \\
\text { (ft above } \\
\text { sea level) }\end{array}$ & $\begin{array}{l}\text { Depth to water } \\
\text { (ft below } \\
\text { measuring point) }\end{array}$ & $\begin{array}{l}\text { Water-level } \\
\text { altitude } \\
\text { (ft above } \\
\text { sea level) }\end{array}$ & Remarks \\
\hline MWGS-21 & $\overline{M W}$ & $08 / 31 / 92$ & 1222 & 33.41 & 4.34 & 29.07 & \\
\hline MWGS-21 & MW & $10 / 07 / 92$ & 1223 & 33.41 & 4.17 & 29.24 & \\
\hline MWGS-21 & MW & $10 / 28 / 92$ & 1338 & 33.41 & 4.73 & 28.68 & \\
\hline MWGS-21 & MW & $12 / 03 / 92$ & 1221 & 33.41 & 4.56 & 28.85 & \\
\hline MWGS-21 & MW & $01 / 04 / 93$ & 1203 & 33.41 & 5.01 & 28.40 & \\
\hline MWGS-21 & MW & $02 / 04 / 93$ & 1333 & 33.41 & 4.40 & 29.01 & \\
\hline MWGS-21 & MW & $03 / 05 / 93$ & 1209 & 33.41 & 4.33 & 29.08 & \\
\hline MWGS-21 & MW & $03 / 30 / 93$ & 1128 & 33.41 & 4.38 & 29.03 & \\
\hline MWGS-21 & MW & $05 / 06 / 93$ & 1148 & 33.41 & 4.94 & 28.47 & \\
\hline MWGS-21 & MW & $05 / 28 / 93$ & 1140 & 33.41 & 5.15 & 28.26 & \\
\hline MWGS-21 & MW & $07 / 07 / 93$ & 1128 & 33.41 & 5.20 & 28.21 & \\
\hline MWGS-21 & MW & $08 / 06 / 93$ & 1110 & 33.41 & 4.97 & 28.44 & \\
\hline MWGS-21 & MW & $09 / 03 / 93$ & 1126 & 33.41 & 5.10 & 28.31 & \\
\hline MWGS-21 & MW & $09 / 30 / 93$ & 1153 & 33.41 & 4.95 & 28.46 & \\
\hline MWGS-21 & MW & $10 / 28 / 93$ & 1125 & 33.41 & 5.40 & 28.01 & \\
\hline MWGS-21 & MW & $12 / 01 / 93$ & 1431 & 33.41 & 5.17 & 28.24 & \\
\hline MWGS-21 & MW & $01 / 06 / 94$ & 1146 & 33.41 & 4.90 & 28.51 & \\
\hline MWGS-21 & MW & $02 / 01 / 94$ & 1120 & 33.41 & 4.10 & 29.31 & \\
\hline MWGS-21 & MW & $03 / 04 / 94$ & 1127 & 33.41 & 4.12 & 29.29 & \\
\hline MWGS-21 & MW & $04 / 04 / 94$ & 1104 & 33.41 & 5.14 & 28.27 & \\
\hline MWGS-21 & MW & $05 / 06 / 94$ & 1202 & 33.41 & 5.53 & 27.88 & \\
\hline MWGS-2I & MW & $06 / 03 / 94$ & 1147 & 33.41 & 5.74 & 27.67 & \\
\hline MWGS-21 & MW & $07 / 08 / 94$ & 1200 & 33.41 & 4.22 & 29.19 & \\
\hline MWGS-21 & MW & $08 / 05 / 94$ & 1441 & 33.41 & 4.37 & 29.04 & \\
\hline MWGS-21 & MW & $09 / 07 / 94$ & 1135 & 33.41 & 4.52 & 28.89 & \\
\hline MWGS-21 & MW & $10 / 06 / 94$ & 1226 & 33.41 & 3.30 & 30.11 & \\
\hline MWGS-21 & MW & $11 / 09 / 94$ & 1332 & 33.41 & 4.38 & 29.03 & \\
\hline MWGS-21 & MW & $12 / 06 / 94$ & 1152 & 33.41 & 3.99 & 29.42 & \\
\hline MWGS-21 & MW & $01 / 10 / 95$ & 1628 & 33.41 & 4.50 & 28.91 & \\
\hline MWGS-21 & MW & $02 / 02 / 95$ & 1405 & 33.41 & 4.55 & 28.86 & \\
\hline MWGS-21 & MW & $03 / 02 / 95$ & 1540 & 33.41 & 4.45 & 28.96 & \\
\hline MWGS-21 & MW & $04 / 05 / 95$ & 1151 & 33.41 & 5.06 & 28.35 & \\
\hline MWGS-21 & MW & $05 / 02 / 95$ & 1336 & 33.41 & 5.32 & 28.09 & \\
\hline MWGS-21 & MW & $06 / 09 / 95$ & 1135 & 33.41 & 5.29 & 28.12 & \\
\hline MWGS-21 & MW & $07 / 06 / 95$ & 1121 & 33.41 & 5.21 & 28.20 & \\
\hline MWGS-21 & MW & $08 / 10 / 95$ & 1254 & 33.41 & 5.22 & 28.19 & \\
\hline MWGS-21 & MW & $09 / 13 / 95$ & 1220 & 33.41 & 4.50 & 28.91 & \\
\hline MWGS-22 & MW & $04 / 30 / 91$ & 1040 & 18.71 & 2.61 & 16.10 & \\
\hline MWGS-22 & MW & $05 / 28 / 91$ & 1205 & 18.71 & 2.78 & 15.93 & \\
\hline MWGS-22 & MW & $07 / 01 / 91$ & -- & 18.71 & 2.92 & 15.79 & \\
\hline MWGS-22 & MW & $08 / 06 / 91$ & -- & 18.71 & 2.43 & 16.28 & \\
\hline MWGS-22 & MW & $08 / 30 / 91$ & --- & 18.71 & 2.28 & 16.43 & \\
\hline MWGS-22 & MW & $09 / 27 / 91$ & -- & 18.71 & 2.80 & 15.91 & \\
\hline MWGS-22 & MW & $10 / 29 / 91$ & -- & 18.71 & 3.14 & 15.57 & \\
\hline
\end{tabular}


Table 8.--Water-level data collected at the Defense Fuel Supply Point and adjacent properties, Hanahan, S.C., between April 1991 and Septembe1995--Continued

[ft, feet; --, data not available; WL, water-level well; EW, extraction well; MW, monitoring well; SW, surface-water site; -, negative depth to water indicates water-level altitude is above measuring point]

\begin{tabular}{|c|c|c|c|c|c|c|c|}
\hline $\begin{array}{c}\text { Site } \\
\text { (plate 1) }\end{array}$ & $\begin{array}{l}\text { Site } \\
\text { type }\end{array}$ & Date & Time & $\begin{array}{l}\text { Measuring-point } \\
\text { altitude } \\
\text { (ft above } \\
\text { sea level) }\end{array}$ & $\begin{array}{l}\text { Depth to water } \\
\text { (ft below } \\
\text { measuring point) }\end{array}$ & $\begin{array}{l}\text { Water-level } \\
\text { altitude } \\
\text { (ft above } \\
\text { sea level) }\end{array}$ & Remarks \\
\hline MWGS-22 & $\mathrm{MW}$ & $12 / 02 / 91$ & -- & 18.71 & 3.24 & 15.47 & \\
\hline MWGS-22 & MW & $01 / 02 / 92$ & -- & 18.71 & 3.44 & 15.27 & \\
\hline MWGS-22 & $\mathrm{MW}$ & $02 / 04 / 92$ & -- & 18.71 & 4.84 & 13.87 & \\
\hline MWGS-22 & MW & $03 / 06 / 92$ & $\cdots$ & 18.71 & 4.20 & 14.51 & \\
\hline MWGS-22 & MW & $03 / 31 / 92$ & $\cdots$ & 18.71 & 4.01 & 14.70 & \\
\hline MWGS-22 & MW & $05 / 01 / 92$ & --- & 18.71 & 4.32 & 14.39 & \\
\hline MWGS-22 & MW & $06 / 11 / 92$ & 1703 & 18.71 & 3.90 & 14.81 & \\
\hline MWGS-22 & MW & $07 / 07 / 92$ & 1030 & 18.71 & 4.73 & 13.98 & \\
\hline MWGS-22 & MW & $08 / 31 / 92$ & 1345 & 18.71 & 3.18 & 15.53 & \\
\hline MWGS-22 & MW & $10 / 07 / 92$ & 1506 & 18.71 & 2.70 & 16.01 & \\
\hline MWGS-22 & MW & $10 / 28 / 92$ & 1329 & 18.71 & 2.88 & 15.83 & \\
\hline MWGS-22 & MW & $12 / 03 / 92$ & 1356 & 18.71 & 2.94 & 15.77 & \\
\hline MWGS-22 & MW & $01 / 04 / 93$ & 1330 & 18.71 & 3.49 & 15.22 & \\
\hline MWGS-22 & MW & $02 / 04 / 93$ & 1424 & 18.71 & 2.33 & 16.38 & \\
\hline MWGS-22 & MW & $03 / 05 / 93$ & 1355 & 18.71 & 2.54 & 16.17 & \\
\hline MWGS-22 & MW & $03 / 30 / 93$ & 1256 & 18.71 & 2.77 & 15.94 & \\
\hline MWGS-22 & MW & $05 / 06 / 93$ & 1309 & 18.71 & 3.52 & 15.19 & \\
\hline MWGS-22 & MW & $05 / 28 / 93$ & 1257 & 18.71 & 4.03 & 14.68 & \\
\hline MWGS-22 & MW & $07 / 07 / 93$ & 1315 & 18.71 & 3.99 & 14.72 & \\
\hline MWGS-22 & MW & $08 / 06 / 93$ & 1321 & 18.71 & 3.93 & 14.78 & \\
\hline MWGS-22 & $\mathrm{MW}$ & $09 / 03 / 93$ & 1213 & 18.71 & 4.46 & 14.25 & \\
\hline MWGS-22 & MW & $09 / 30 / 93$ & 1305 & 18.71 & 4.42 & 14.29 & \\
\hline MWGS-22 & MW & $10 / 28 / 93$ & 1250 & 18.71 & 4.36 & 14.35 & \\
\hline MWGS-22 & MW & $12 / 01 / 93$ & 1535 & 18.71 & 4.14 & 14.57 & \\
\hline MWGS-22 & MW & $01 / 06 / 94$ & 1325 & 18.71 & 3.70 & 15.01 & \\
\hline MWGS-22 & MW & $02 / 01 / 94$ & 1255 & 18.71 & 2.93 & 15.78 & \\
\hline MWGS-22 & MW & $03 / 04 / 94$ & 1236 & 18.71 & 2.78 & 15.93 & \\
\hline MWGS-22 & MW & $04 / 04 / 94$ & 1156 & 18.71 & 3.58 & 15.13 & \\
\hline MWGS-22 & $\mathrm{MW}$ & $05 / 06 / 94$ & 1300 & 18.71 & 4.28 & 14.43 & \\
\hline MWGS-22 & MW & $06 / 03 / 94$ & 1233 & 18.71 & 4.67 & 14.04 & \\
\hline MWGS-22 & MW & $07 / 08 / 94$ & 1220 & 18.71 & 3.15 & 15.56 & \\
\hline MWGS-22 & MW & $08 / 05 / 94$ & 1454 & 18.71 & 3.05 & 15.66 & \\
\hline MWGS-22 & MW & $09 / 07 / 94$ & 1332 & 18.71 & 2.84 & 15.87 & \\
\hline MWGS-22 & MW & $10 / 06 / 94$ & 1308 & 18.71 & 1.96 & 16.75 & \\
\hline MWGS-22 & MW & $11 / 09 / 94$ & 1341 & 18.71 & 2.23 & 16.48 & \\
\hline MWGS-22 & MW & $12 / 06 / 94$ & 1208 & 18.71 & 2.13 & 16.58 & \\
\hline MWGS-22 & MW & $01 / 10 / 95$ & 1645 & 18.71 & 2.47 & 16.24 & \\
\hline MWGS-22 & MW & $02 / 02 / 95$ & 1418 & 18.71 & 2.37 & 16.34 & \\
\hline MWGS-22 & MW & $03 / 02 / 95$ & 1554 & 18.71 & 2.42 & 16.29 & \\
\hline MWGS-22 & MW & $04 / 05 / 95$ & 1213 & 18.71 & 3.07 & 15.64 & \\
\hline MWGS-22 & MW & $05 / 02 / 95$ & 1413 & 18.71 & 3.46 & 15.25 & \\
\hline MWGS-22 & MW & $06 / 09 / 95$ & 1300 & 18.71 & 3.63 & 15.08 & \\
\hline MWGS-22 & MW & $07 / 06 / 95$ & 1222 & 18.71 & 3.76 & 14.95 & \\
\hline MWGS-22 & MW & $08 / 10 / 95$ & 1308 & 18.71 & 3.80 & 14.91 & \\
\hline MWGS-22 & MW & $09 / 13 / 95$ & 1440 & 18.71 & 2.60 & 16.11 & \\
\hline
\end{tabular}


Table 8.--Water-level data collected at the Defense Fuel Supply Point and adjacent properties, Hanahan, S.C., between April 1991 and September 1995--Continued

[ft, feet; ---, data not available; WL, water-level well; EW, extraction well; MW, monitoring well; SW, surface-water site; -, negative depth to water indicates water-level altitude is above measuring point]

\begin{tabular}{|c|c|c|c|c|c|c|c|}
\hline $\begin{array}{c}\text { Site } \\
\text { (plate 1) }\end{array}$ & $\begin{array}{l}\text { Site } \\
\text { type }\end{array}$ & Date & Time & $\begin{array}{l}\text { Measuring-point } \\
\text { altitude } \\
\text { (ft above } \\
\text { sea level) }\end{array}$ & $\begin{array}{l}\text { Depth to water } \\
\text { (ft below } \\
\text { measuring point) }\end{array}$ & $\begin{array}{l}\text { Water-level } \\
\text { altitude } \\
\text { (ft above } \\
\text { sea level) }\end{array}$ & Remarks \\
\hline MWGS-27A & MW & $11 / 21 / 91$ & $-\cdots$ & 35.32 & 12.67 & 22.65 & \\
\hline MWGS-27A & MW & $05 / 14 / 92$ & 1142 & 35.32 & 12.43 & 22.89 & \\
\hline MWGS-27A & MW & $06 / 11 / 92$ & 1210 & 35.32 & 12.26 & 23.06 & \\
\hline MWGS-27A & MW & $08 / 07 / 92$ & 1111 & 35.32 & 12.15 & 23.17 & \\
\hline MWGS-27A & MW & $08 / 31 / 92$ & 1054 & 35.32 & 10.39 & 24.93 & \\
\hline MWGS-27A & MW & $10 / 07 / 92$ & 1115 & 35.30 & 10.63 & 24.67 & New measuring point established. \\
\hline MWGS-27A & MW & $12 / 03 / 92$ & 1056 & 35.30 & 11.07 & 24.23 & \\
\hline MWGS-27A & MW & $01 / 04 / 93$ & 1030 & 35.30 & 11.84 & 23.46 & \\
\hline MWGS-27A & MW & $02 / 04 / 93$ & 1129 & 35.30 & 10.53 & 24.77 & \\
\hline MWGS-27A & MW & $03 / 05 / 93$ & 1045 & 35.30 & 11.29 & 24.01 & \\
\hline MWGS-27A & MW & $03 / 30 / 93$ & 1015 & 35.30 & 11.38 & 23.92 & \\
\hline MWGS-27A & MW & $05 / 06 / 93$ & 1025 & 35.30 & 11.74 & 23.56 & \\
\hline MWGS-27A & MW & $05 / 28 / 93$ & 1035 & 35.30 & -- & -- & Well appears dry. \\
\hline MWGS-27A & MW & $07 / 07 / 93$ & 1020 & 35.30 & --- & -- & Well appears dry. \\
\hline MWGS-27A & MW & $08 / 06 / 93$ & 1000 & 35.30 & -- & $\cdots$ & Well appears dry. \\
\hline MWGS-27B & MW & $05 / 14 / 92$ & 1148 & 35.40 & 15.18 & 20.22 & \\
\hline MWGS-27B & MW & $08 / 07 / 92$ & 1116 & 35.40 & --- & -- & Free product present in well. \\
\hline MWGS-27C & MW & $11 / 21 / 91$ & -- & 35.29 & 14.73 & 20.56 & \\
\hline MWGS-27C & MW & $05 / 14 / 92$ & 1145 & 35.29 & 15.39 & 19.90 & \\
\hline MWGS-27C & MW & $06 / 11 / 92$ & 1215 & 35.29 & 16.25 & 19.04 & \\
\hline MWGS-27C & MW & $08 / 07 / 92$ & 1118 & 35.29 & 15.89 & 19.40 & \\
\hline MWGS-27C & MW & $08 / 31 / 92$ & 1053 & 35.29 & 13.10 & 22.19 & \\
\hline MWGS-27C & MW & $10 / 07 / 92$ & 1118 & 35.28 & 12.91 & 22.37 & New measuring point established. \\
\hline MWGS-27C & MW & $10 / 28 / 92$ & 1145 & 35.28 & 13.47 & 21.81 & \\
\hline MWGS-27C & MW & $12 / 03 / 92$ & 1052 & 35.28 & 13.60 & 21.68 & \\
\hline MWGS-27C & MW & $01 / 04 / 93$ & 1024 & 35.28 & 14.50 & 20.78 & \\
\hline MWGS-27C & MW & $02 / 04 / 93$ & 1132 & 35.28 & 12.73 & 22.55 & \\
\hline MWGS-27C & MW & $03 / 05 / 93$ & 1048 & 35.28 & 13.46 & 21.82 & \\
\hline MWGS-27C & MW & $03 / 30 / 93$ & 1017 & 35.28 & 15.52 & 19.76 & \\
\hline MWGS-27C & MW & $05 / 06 / 93$ & 1024 & 35.28 & 15.93 & 19.35 & \\
\hline MWGS-27C & MW & $05 / 28 / 93$ & 1032 & 35.28 & 16.61 & 18.67 & \\
\hline MWGS-27C & MW & $07 / 07 / 93$ & 1019 & 35.28 & 15.67 & 19.61 & \\
\hline MWGS-27C & MW & $08 / 06 / 93$ & 1005 & 35.28 & 16.39 & 18.89 & \\
\hline MWGS-27C & MW & $09 / 03 / 93$ & 1023 & 35.28 & 16.33 & 18.95 & \\
\hline MWGS-27C & MW & $09 / 30 / 93$ & 1116 & 35.28 & 15.86 & 19.42 & \\
\hline MWGS-27C & MW & $10 / 28 / 93$ & 1016 & 35.28 & 16.70 & 18.58 & \\
\hline MWGS-27C & MW & $12 / 01 / 93$ & 1316 & 35.28 & 16.75 & 18.53 & \\
\hline MWGS-27C & MW & $01 / 06 / 94$ & 1037 & 35.28 & 16.87 & 18.41 & \\
\hline MWGS-27C & MW & $02 / 01 / 94$ & 1033 & 35.28 & 15.69 & 19.59 & \\
\hline MWGS-27C & MW & $03 / 04 / 94$ & 1015 & 35.28 & 15.35 & 19.93 & \\
\hline MWGS-27C & MW & $04 / 04 / 94$ & 1001 & 35.28 & 15.85 & 19.43 & \\
\hline MWGS-27C & MW & $05 / 06 / 94$ & 1051 & 35.28 & 16.78 & 18.50 & \\
\hline MWGS-27C & MW & $06 / 03 / 94$ & 1033 & 35.28 & 17.57 & 17.71 & \\
\hline
\end{tabular}


Table 8.--Water-level data collected at the Defense Fuel Supply Point and adjacent properties, Hanahan, S.C., between April 1991 and September 1995--Continued

[ft, feet; ---, data not available; WL, water-level well; EW, extraction well; MW, monitoring well; SW, surface-water site; -, negative depth to water indicates water-level altitude is above measuring point]

\begin{tabular}{|c|c|c|c|c|c|c|c|}
\hline $\begin{array}{c}\text { Site } \\
\text { (plate 1) }\end{array}$ & $\begin{array}{l}\text { Site } \\
\text { type }\end{array}$ & Date & Time & $\begin{array}{l}\text { Measuring-point } \\
\text { altitude } \\
\text { (ft above } \\
\text { sea level) }\end{array}$ & $\begin{array}{l}\text { Depth to water } \\
\text { (ft below } \\
\text { measuring point) }\end{array}$ & $\begin{array}{l}\text { Water-level } \\
\text { altitude } \\
\text { (ft above } \\
\text { sea level) }\end{array}$ & Remarks \\
\hline MWGS-27C & $\overline{M W}$ & $07 / 08 / 94$ & 1037 & 35.28 & 16.08 & 19.20 & \\
\hline MWGS-27C & MW & $08 / 05 / 94$ & 1340 & 35.28 & 14.31 & 20.97 & \\
\hline MWGS-27C & MW & $09 / 07 / 94$ & 1040 & 35.28 & 14.18 & 21.10 & \\
\hline MWGS-27C & MW & $10 / 06 / 94$ & 1107 & 35.28 & 11.71 & 23.57 & \\
\hline MWGS-27C & MW & $11 / 09 / 94$ & 1233 & 35.28 & 13.18 & 22.10 & \\
\hline MWGS-27C & MW & $12 / 06 / 94$ & 1043 & 35.28 & 13.89 & 21.39 & \\
\hline MWGS-27C & MW & $01 / 10 / 95$ & 1632 & 35.28 & 13.83 & 21.45 & \\
\hline MWGS-27C & MW & $02 / 02 / 95$ & 1319 & 35.28 & 13.58 & 21.70 & \\
\hline MWGS-27C & MW & $03 / 02 / 95$ & 1452 & 35.28 & 13.65 & 21.63 & \\
\hline MWGS-27C & MW & $04 / 05 / 95$ & 1028 & 35.28 & 14.56 & 20.72 & \\
\hline MWGS-27C & MW & $05 / 02 / 95$ & 1130 & 35.28 & 15.20 & 20.08 & \\
\hline MWGS-27C & MW & $06 / 09 / 95$ & 1030 & 35.28 & 16.14 & 19.14 & \\
\hline MWGS-27C & MW & $07 / 06 / 95$ & 1134 & 35.28 & 15.93 & 19.35 & \\
\hline MWGS-27C & MW & $08 / 10 / 95$ & 1102 & 35.28 & 15.52 & 19.76 & \\
\hline MWGS-27C & MW & $09 / 13 / 95$ & 1134 & 35.28 & 13.24 & 22.04 & \\
\hline MWGS-28A & MW & $11 / 21 / 91$ & -- & 34.97 & 13.74 & 21.23 & \\
\hline MWGS-28A & MW & $05 / 14 / 92$ & 1129 & 34.97 & 13.44 & 21.53 & \\
\hline MWGS-28A & MW & $06 / 11 / 92$ & 1150 & 34.97 & 13.63 & 21.34 & \\
\hline MWGS-28A & MW & $08 / 07 / 92$ & 1055 & 34.97 & 13.10 & 21.87 & \\
\hline MWGS-28A & MW & $08 / 31 / 92$ & 1107 & 34.97 & 10.78 & 24.19 & \\
\hline MWGS-28A & MW & $10 / 07 / 92$ & 1110 & 34.97 & 10.96 & 24.01 & \\
\hline MWGS-28A & MW & $10 / 28 / 92$ & 1139 & 34.97 & 11.29 & 23.68 & \\
\hline MWGS-28A & MW & $12 / 03 / 92$ & 1030 & 34.97 & 11.37 & 23.60 & \\
\hline MWGS-28A & MW & $01 / 04 / 93$ & 1040 & 34.97 & 12.10 & 22.87 & \\
\hline MWGS-28A & MW & $02 / 04 / 93$ & 1117 & 34.97 & 10.90 & 24.07 & \\
\hline MWGS-28A & MW & $03 / 05 / 93$ & 1055 & 34.97 & 11.57 & 23.40 & \\
\hline MWGS-28A & MW & $03 / 30 / 93$ & 0959 & 34.97 & 11.78 & 23.19 & \\
\hline MWGS-28A & MW & $05 / 06 / 93$ & 1035 & 34.97 & 12.59 & 22.38 & \\
\hline MWGS-28A & MW & $05 / 28 / 93$ & 1029 & 34.97 & 14.32 & 20.65 & \\
\hline MWGS-28A & MW & $07 / 07 / 93$ & 1029 & 34.97 & 14.17 & 20.80 & \\
\hline MWGS-28A & MW & $08 / 06 / 93$ & 1010 & 34.97 & 14.24 & 20.73 & \\
\hline MWGS-28A & MW & $09 / 03 / 93$ & 1025 & 34.97 & 11.98 & 22.99 & \\
\hline MWGS-28A & MW & $09 / 30 / 93$ & 1107 & 34.97 & 11.96 & 23.01 & \\
\hline MWGS-28A & MW & $10 / 28 / 93$ & 1013 & 34.97 & 13.15 & 21.82 & \\
\hline MWGS-28A & MW & $12 / 01 / 93$ & 1322 & 34.97 & 13.85 & 21.12 & \\
\hline MWGS-28A & MW & $01 / 06 / 94$ & 1034 & 34.97 & 14.19 & 20.78 & \\
\hline MWGS-28A & MW & $02 / 01 / 94$ & 1025 & 34.97 & 11.98 & 22.99 & \\
\hline MWGS-28A & MW & $03 / 04 / 94$ & 1011 & 34.97 & 11.75 & 23.22 & \\
\hline MWGS-28A & MW & $04 / 04 / 94$ & 1007 & 34.97 & 12.35 & 22.62 & \\
\hline MWGS-28A & MW & $05 / 06 / 94$ & 1057 & 34.97 & 14.24 & 20.73 & \\
\hline MWGS-28A & MW & $06 / 03 / 94$ & 1026 & 34.97 & 14.67 & 20.30 & \\
\hline MWGS-28A & MW & $07 / 08 / 94$ & 1040 & 34.97 & 11.67 & 23.30 & \\
\hline MWGS-28A & MW & $08 / 05 / 94$ & 1327 & 34.97 & 11.05 & 23.92 & \\
\hline MWGS-28A & MW & $09 / 07 / 94$ & 1055 & 34.97 & 11.24 & 23.73 & \\
\hline
\end{tabular}


Table 8.--Water-level data collected at the Defense Fuel Supply Point and adjacent properties, Hanahan, S.C., between April 1991 and September 1995--Continued

[ft, feet; ---, data not available; WL, water-level well; EW, extraction well; MW, monitoring well; SW, surface-water site; -, negative depth to water indicates water-level altitude is above measuring point]

\begin{tabular}{|c|c|c|c|c|c|c|c|}
\hline $\begin{array}{c}\text { Site } \\
\text { (plate 1) }\end{array}$ & $\begin{array}{l}\text { Site } \\
\text { type }\end{array}$ & Date & Time & $\begin{array}{l}\text { Measuring-point } \\
\text { altitude } \\
\text { (ft above } \\
\text { sea level) }\end{array}$ & $\begin{array}{l}\text { Depth to water } \\
\text { (ft below } \\
\text { measuring point) }\end{array}$ & $\begin{array}{l}\text { Water-level } \\
\text { altitude } \\
\text { (ft above } \\
\text { sea level) }\end{array}$ & Remarks \\
\hline MWGS-28A & MW & $10 / 06 / 94$ & 1105 & 34.97 & 9.56 & 25.41 & \\
\hline MWGS-28A & MW & $11 / 09 / 94$ & 1237 & 34.97 & 10.75 & 24.22 & \\
\hline MWGS-28A & MW & $12 / 06 / 94$ & 1049 & 34.97 & 11.47 & 23.50 & \\
\hline MWGS-28A & MW & $01 / 10 / 95$ & 1254 & 34.97 & 11.37 & 23.60 & \\
\hline MWGS-28A & MW & $02 / 02 / 95$ & -- & 34.97 & -- & --- & Free product present in well. \\
\hline MWGS-28A & MW & $03 / 02 / 95$ & 1434 & 34.97 & 11.60 & 23.37 & \\
\hline MWGS-28A & MW & $04 / 05 / 95$ & 1039 & 34.97 & 14.73 & 20.24 & \\
\hline MWGS-28A & MW & $05 / 02 / 95$ & 1143 & 34.97 & 13.11 & 21.86 & \\
\hline MWGS-28A & MW & $06 / 09 / 95$ & 1035 & 34.97 & 17.19 & 17.78 & \\
\hline MWGS-28A & MW & $07 / 06 / 95$ & 1026 & 34.97 & 13.84 & 21.13 & \\
\hline MWGS-28A & MW & $08 / 10 / 95$ & 1232 & 34.97 & 12.14 & 22.83 & \\
\hline MWGS-28A & MW & $09 / 13 / 95$ & 1139 & 34.97 & 10.78 & 24.19 & \\
\hline MWGS-28B & MW & $11 / 21 / 91$ & -- & 35.17 & 14.77 & 20.40 & \\
\hline MWGS-28B & MW & $05 / 14 / 92$ & 1133 & 35.17 & 15.44 & 19.73 & \\
\hline MWGS-28B & MW & $06 / 11 / 92$ & 1155 & 35.17 & 16.45 & 18.72 & \\
\hline MWGS-28B & MW & $08 / 07 / 92$ & 1104 & 35.17 & 16.06 & 19.11 & \\
\hline MWGS-28B & MW & $08 / 31 / 92$ & 1104 & 35.17 & 13.25 & 21.92 & \\
\hline MWGS-28B & MW & $10 / 07 / 92$ & 1105 & 35.16 & 13.09 & 22.07 & New measuring point established. \\
\hline MWGS-28B & MW & $10 / 28 / 92$ & 1137 & 35.16 & 13.64 & 21.52 & \\
\hline MWGS-28B & MW & $12 / 03 / 92$ & 1048 & 35.16 & 13.77 & 21.39 & \\
\hline MWGS-28B & MW & $01 / 04 / 93$ & 1033 & 35.16 & 14.66 & 20.50 & \\
\hline MWGS-28B & MW & $02 / 04 / 93$ & 1121 & 35.16 & 12.90 & 22.26 & \\
\hline MWGS-28B & MW & $03 / 05 / 93$ & 1058 & 35.16 & 13.63 & 21.53 & \\
\hline MWGS-28B & MW & $03 / 30 / 93$ & 1007 & 35.16 & 16.09 & 19.07 & \\
\hline MWGS-28B & MW & $05 / 06 / 93$ & 1041 & 35.16 & 16.55 & 18.61 & \\
\hline MWGS-28B & MW & $05 / 28 / 93$ & 1024 & 35.16 & 17.19 & 17.97 & \\
\hline MWGS-28B & MW & $07 / 07 / 93$ & 1022 & 35.16 & 15.77 & 19.39 & \\
\hline MWGS-28B & MW & $08 / 06 / 93$ & 1008 & 35.16 & 16.66 & 18.50 & \\
\hline MWGS-28B & MW & $09 / 03 / 93$ & 1028 & 35.16 & 16.77 & 18.39 & \\
\hline MWGS-28B & MW & $09 / 30 / 93$ & 1113 & 35.16 & 16.15 & 19.01 & \\
\hline MWGS-28B & MW & $10 / 28 / 93$ & 1010 & 35.16 & 16.97 & 18.19 & \\
\hline MWGS-28B & MW & $12 / 01 / 93$ & 1324 & 35.16 & 17.04 & 18.12 & \\
\hline MWGS-28B & MW & $01 / 06 / 94$ & 1032 & 35.16 & 17.09 & 18.07 & \\
\hline MWGS-28B & MW & $02 / 01 / 94$ & 1030 & 35.16 & 15.88 & 19.28 & \\
\hline MWGS-28B & MW & $03 / 04 / 94$ & 1013 & 35.16 & 15.59 & 19.57 & \\
\hline MWGS-28B & MW & $04 / 04 / 94$ & 1008 & 35.16 & 16.15 & 19.01 & \\
\hline MWGS-28B & MW & $05 / 06 / 94$ & 1053 & 35.16 & 17.02 & 18.14 & \\
\hline MWGS-28B & MW & $06 / 03 / 94$ & 1025 & 35.16 & 17.86 & 17.30 & \\
\hline MWGS-28B & MW & $07 / 08 / 94$ & 1035 & 35.16 & 16.28 & 18.88 & \\
\hline MWGS-28B & MW & $08 / 05 / 94$ & 1320 & 35.16 & 14.55 & 20.61 & \\
\hline MWGS-28B & MW & $09 / 07 / 94$ & 1050 & 35.16 & 14.43 & 20.73 & \\
\hline MWGS-28B & MW & $10 / 06 / 94$ & - & 35.16 & 11.98 & 23.18 & \\
\hline MWGS-28B & MW & $11 / 09 / 94$ & 1235 & 35.16 & 13.51 & 21.65 & \\
\hline MWGS-28B & MW & $12 / 06 / 94$ & 1048 & 35.16 & 14.32 & 20.84 & \\
\hline
\end{tabular}


Table 8.--Water-level data collected at the Defense Fuel Supply Point and adjacent properties, Hanahan, S.C., between April 1991 and September 1995--Continued

[ft, feet; ---, data not available; WL, water-level well; EW, extraction well; MW, monitoring well; SW, surface-water site; -, negative depth to wa+er indicates water-level altitude is above measuring point]

\begin{tabular}{|c|c|c|c|c|c|c|c|}
\hline $\begin{array}{c}\text { Site } \\
\text { (plate 1) }\end{array}$ & $\begin{array}{l}\text { Site } \\
\text { type }\end{array}$ & Date & Time & $\begin{array}{c}\text { Measuring-point } \\
\text { altitude } \\
\text { (ft above } \\
\text { sea level) }\end{array}$ & $\begin{array}{l}\text { Depth to water } \\
\text { (ft below } \\
\text { measuring point) }\end{array}$ & $\begin{array}{l}\text { Water-level } \\
\text { altitude } \\
\text { (ft above } \\
\text { sea level) }\end{array}$ & Remarks \\
\hline MWGS-28B & MW & $01 / 10 / 95$ & 1256 & 35.16 & 14.16 & 21.00 & \\
\hline MWGS-28B & MW & $02 / 02 / 95$ & 1317 & 35.16 & 13.76 & 21.40 & \\
\hline MWGS-28B & MW & $03 / 02 / 95$ & 1545 & 35.16 & 13.81 & 21.35 & \\
\hline MWGS-28B & MW & $04 / 05 / 95$ & 1157 & 35.16 & 12.19 & 22.97 & \\
\hline MWGS-28B & MW & $05 / 02 / 95$ & 1241 & 35.16 & 15.33 & 19.83 & \\
\hline MWGS-28B & MW & $06 / 09 / 95$ & 1148 & 35.16 & 16.32 & 18.84 & \\
\hline MWGS-28B & MW & $07 / 06 / 95$ & 1130 & 35.16 & 16.09 & 19.07 & \\
\hline MWGS-28B & MW & $08 / 10 / 95$ & 1135 & 35.16 & 15.67 & 19.49 & \\
\hline MWGS-28B & MW & $09 / 13 / 95$ & 1137 & 35.16 & 13.40 & 21.76 & \\
\hline MWGS-28C & MW & $11 / 21 / 91$ & $\cdots$ & 34.70 & 13.49 & 21.21 & \\
\hline MWGS-28C & MW & $05 / 14 / 92$ & 1135 & 34.70 & 13.10 & 21.60 & \\
\hline MWGS-28C & MW & $06 / 11 / 92$ & 1200 & 34.70 & 13.13 & 21.57 & \\
\hline MWGS-28C & MW & $08 / 07 / 92$ & 1057 & 34.70 & 12.96 & 21.74 & \\
\hline MWGS-28C & MW & $08 / 31 / 92$ & 1102 & 34.70 & 11.16 & 23.54 & \\
\hline MWGS-28C & MW & $10 / 07 / 92$ & 1108 & 34.70 & 11.20 & 23.50 & \\
\hline MWGS-28C & MW & $10 / 28 / 92$ & 1132 & 34.70 & 11.58 & 23.12 & \\
\hline MWGS-28C & MW & $12 / 03 / 92$ & 1044 & 34.70 & 11.82 & 22.88 & \\
\hline MWGS-28C & MW & $01 / 04 / 93$ & 1044 & 34.70 & 12.60 & 22.10 & \\
\hline MWGS-28C & MW & $02 / 04 / 93$ & 1114 & 34.70 & 11.26 & 23.44 & \\
\hline MWGS-28C & MW & $03 / 05 / 93$ & 1052 & 34.70 & 11.40 & 23.30 & \\
\hline MWGS-28C & MW & $03 / 30 / 93$ & 1008 & 34.70 & 12.80 & 21.90 & \\
\hline MWGS-28C & MW & $05 / 06 / 93$ & 1030 & 34.70 & 13.24 & 21.46 & \\
\hline MWGS-28C & MW & $07 / 07 / 93$ & 1028 & 34.70 & - & --- & Well appears dry. \\
\hline MWGS-28C & MW & $08 / 06 / 93$ & 1000 & 34.70 & - & -- & Well appears dry. \\
\hline MWGS-28D & MW & $10 / 28 / 93$ & 1019 & 34.73 & 16.41 & 18.32 & \\
\hline MWGS-28D & MW & $12 / 01 / 93$ & 1326 & 34.73 & 16.52 & 18.21 & \\
\hline MWGS-28D & MW & $01 / 06 / 94$ & 1030 & 34.73 & 16.55 & 18.18 & \\
\hline MWGS-28D & MW & $02 / 01 / 94$ & 1023 & 34.73 & 15.39 & 19.34 & \\
\hline MWGS-28D & MW & $03 / 04 / 94$ & 1008 & 34.73 & 15.08 & 19.65 & \\
\hline MWGS-28D & MW & $04 / 04 / 94$ & 1003 & 34.73 & 15.61 & 19.12 & \\
\hline MWGS-28D & MW & $06 / 03 / 94$ & 1022 & 34.73 & 17.29 & 17.44 & \\
\hline MWGS-28D & MW & $07 / 08 / 94$ & 1044 & 34.73 & 15.72 & 19.01 & \\
\hline MWGS-28D & MW & $08 / 05 / 94$ & 1325 & 34.73 & 14.10 & 20.63 & \\
\hline MWGS-28D & MW & $09 / 07 / 94$ & 1052 & 34.73 & 13.95 & 20.78 & \\
\hline MWGS-28D & MW & $10 / 06 / 94$ & 1103 & 34.73 & 11.59 & 23.14 & \\
\hline MWGS-28D & MW & $11 / 09 / 94$ & 1239 & 34.73 & -- & $-\cdots$ & Free product present in well. \\
\hline MWGS-28D & MW & $12 / 06 / 94$ & 1044 & 34.73 & 13.86 & 20.87 & \\
\hline MWGS-28D & MW & $01 / 10 / 95$ & 1258 & 34.73 & 13.68 & 21.05 & \\
\hline MWGS-28D & MW & $02 / 02 / 95$ & 1315 & 34.73 & -- & -- & Free product present in well. \\
\hline MWGS-28D & MW & $03 / 02 / 95$ & -- & 34.73 & -- & -- & Free product present in well. \\
\hline MWGS-28D & MW & $04 / 05 / 95$ & 1029 & 34.73 & 14.20 & 20.53 & \\
\hline MWGS-28D & MW & $05 / 02 / 95$ & 1134 & 34.73 & -- & - & Free product present in well. \\
\hline MWGS-28D & MW & $06 / 09 / 95$ & 1034 & 34.73 & --. & -.- & Free product present in well. \\
\hline
\end{tabular}


Table 8.--Water-level data collected at the Defense Fuel Supply Point and adjacent properties, Hanahan, S.C., between April 1991 and September 1995--Continued

[ft, feet; ---, data not available; WL, water-level well; EW, extraction well; MW, monitoring well; SW, surface-water site; -, negative depth to water indicates water-level altitude is above measuring point]

\begin{tabular}{|c|c|c|c|c|c|c|c|}
\hline $\begin{array}{c}\text { Site } \\
\text { (plate 1) }\end{array}$ & $\begin{array}{l}\text { Site } \\
\text { type }\end{array}$ & Date & Time & $\begin{array}{l}\text { Measuring-point } \\
\text { altitude } \\
\text { (ft above } \\
\text { sea level) }\end{array}$ & $\begin{array}{l}\text { Depth to water } \\
\text { (ft below } \\
\text { measuring point) }\end{array}$ & $\begin{array}{l}\text { Water-level } \\
\text { altitude } \\
\text { (ft above } \\
\text { sea level) }\end{array}$ & Remarks \\
\hline MWGS-28D & MW & $07 / 06 / 95$ & -- & 34.73 & -- & $\cdots$ & Free product present in well. \\
\hline MWGS-28D & MW & $08 / 10 / 95$ & - & 34.73 & -- & --- & Free product present in well. \\
\hline MWGS-29A & MW & $11 / 21 / 91$ & -- & 35.36 & 14.57 & 20.79 & \\
\hline MWGS-29A & MW & $05 / 14 / 92$ & 1115 & 35.36 & 15.32 & 20.04 & \\
\hline MWGS-29A & MW & $06 / 11 / 92$ & 1253 & 35.36 & 16.18 & 19.18 & \\
\hline MWGS-29A & MW & 07/07/92 & 1730 & 35.36 & 15.14 & 20.22 & \\
\hline MWGS-29A & MW & $08 / 07 / 92$ & 1141 & 35.36 & --- & -- & Free product present in well. \\
\hline MWGS-29B & MW & $11 / 21 / 91$ & -- & 35.39 & 14.61 & 20.78 & \\
\hline MWGS-29B & MW & $12 / 16 / 91$ & -- & 35.39 & 15.08 & 20.31 & \\
\hline MWGS-29B & MW & $05 / 14 / 92$ & 1117 & 35.39 & 15.36 & 20.03 & \\
\hline MWGS-29B & MW & $06 / 11 / 92$ & 1245 & 35.39 & 16.25 & 19.14 & \\
\hline MWGS-29B & MW & 07/07/92 & 1735 & 35.39 & 15.73 & 19.66 & \\
\hline MWGS-29B & MW & $08 / 07 / 92$ & 1143 & 35.39 & 15.95 & 19.44 & \\
\hline MWGS-29B & MW & $08 / 31 / 92$ & 1119 & 35.39 & 13.09 & 22.30 & \\
\hline MWGS-29B & MW & $10 / 07 / 92$ & 1124 & 35.39 & 13.03 & 22.36 & \\
\hline MWGS-29B & MW & $10 / 28 / 92$ & 1155 & 35.39 & 13.50 & 21.89 & \\
\hline MWGS-29B & MW & $12 / 03 / 92$ & 1106 & 35.39 & 13.77 & 21.62 & \\
\hline MWGS-29B & MW & $01 / 04 / 93$ & 1053 & 35.39 & 14.66 & 20.73 & \\
\hline MWGS-29B & MW & $02 / 04 / 93$ & 1144 & 35.39 & 13.00 & 22.39 & \\
\hline MWGS-29B & MW & $03 / 05 / 93$ & 1105 & 35.39 & 13.64 & 21.75 & \\
\hline MWGS-29B & MW & $03 / 30 / 93$ & 1027 & 35.39 & 16.13 & 19.26 & \\
\hline MWGS-29B & MW & $05 / 06 / 93$ & 1052 & 35.39 & 16.56 & 18.83 & \\
\hline MWGS-29B & MW & $05 / 28 / 93$ & 1041 & 35.39 & 17.20 & 18.19 & \\
\hline MWGS-29B & MW & $07 / 07 / 93$ & 1039 & 35.39 & 16.50 & 18.89 & \\
\hline MWGS-29B & MW & $08 / 06 / 93$ & 1016 & 35.39 & 16.66 & 18.73 & \\
\hline MWGS-29B & MW & $09 / 03 / 93$ & 1036 & 35.39 & 16.88 & 18.51 & \\
\hline MWGS-29B & MW & $09 / 30 / 93$ & 1055 & 35.39 & 16.34 & 19.05 & \\
\hline MWGS-29B & MW & $10 / 28 / 93$ & 1032 & 35.39 & 17.05 & 18.34 & \\
\hline MWGS-29B & MW & $12 / 01 / 93$ & 1333 & 35.39 & 16.93 & 18.46 & \\
\hline MWGS-29B & MW & $01 / 06 / 94$ & 1041 & 35.39 & 17.27 & 18.12 & \\
\hline MWGS-29B & MW & $02 / 01 / 94$ & 1038 & 35.39 & 15.41 & 19.98 & \\
\hline MWGS-29B & MW & $03 / 04 / 94$ & 1020 & 35.39 & 15.96 & 19.43 & \\
\hline MWGS-29B & MW & $04 / 04 / 94$ & 1015 & 35.39 & 16.42 & 18.97 & \\
\hline MWGS-29B & MW & $05 / 06 / 94$ & 1104 & 35.39 & 17.21 & 18.18 & \\
\hline MWGS-29B & MW & $06 / 03 / 94$ & 1042 & 35.39 & 17.91 & 17.48 & \\
\hline MWGS-29B & MW & $07 / 08 / 94$ & 1051 & 35.39 & 16.55 & 18.84 & \\
\hline MWGS-29B & MW & $08 / 05 / 94$ & 1352 & 35.39 & 14.71 & 20.68 & \\
\hline MWGS-29B & MW & 09/07/94 & 1110 & 35.39 & 14.40 & 20.99 & \\
\hline MWGS-29B & MW & $10 / 06 / 94$ & 1115 & 35.39 & 12.32 & 23.07 & \\
\hline MWGS-29B & MW & $11 / 09 / 94$ & 1246 & 35.39 & 13.94 & 21.45 & \\
\hline MWGS-29B & MW & $12 / 06 / 94$ & 1106 & 35.39 & 14.56 & 20.83 & \\
\hline MWGS-29B & MW & $01 / 10 / 95$ & 1302 & 35.39 & 14.40 & 20.99 & \\
\hline MWGS-29B & MW & $02 / 02 / 95$ & 1323 & 35.39 & 14.23 & 21.16 & \\
\hline
\end{tabular}


Table 8.--Water-level data collected at the Defense Fuel Supply Point and adjacent properties, Hanahan, S.C., between April 1991 and September 1995--Continued

[ft, feet; ---, data not available; WL, water-level well; EW, extraction well; MW, monitoring well; SW, surface-water site; -, negative depth to water indicates water-level altitude is above measuring point]

\begin{tabular}{|c|c|c|c|c|c|c|c|}
\hline $\begin{array}{c}\text { Site } \\
\text { (plate 1) }\end{array}$ & $\begin{array}{l}\text { Site } \\
\text { type }\end{array}$ & Date & Time & $\begin{array}{l}\text { Measuring-point } \\
\text { altitude } \\
\text { (ft above } \\
\text { sea level) }\end{array}$ & $\begin{array}{l}\text { Depth to water } \\
\text { ( } \mathrm{ft} \text { below } \\
\text { measuring point) }\end{array}$ & $\begin{array}{l}\text { Water-level } \\
\text { altitude } \\
\text { (ft above } \\
\text { sea level) }\end{array}$ & Remarks \\
\hline MWGS-29B & MW & $03 / 02 / 95$ & 1449 & 35.39 & 14.05 & 21.34 & \\
\hline MWGS-29B & MW & $04 / 05 / 95$ & 1045 & 35.39 & 14.70 & 20.69 & \\
\hline MWGS-29B & MW & $05 / 02 / 95$ & 1150 & 35.39 & 15.46 & 19.93 & \\
\hline MWGS-29B & MW & $06 / 09 / 95$ & 1038 & 35.39 & 16.32 & 19.07 & \\
\hline MWGS-29B & MW & $07 / 06 / 95$ & 1036 & 35.39 & 15.98 & 19.41 & \\
\hline MWGS-29B & MW & $08 / 10 / 95$ & 1108 & 35.39 & 15.90 & 19.49 & \\
\hline MWGS-29B & MW & $09 / 13 / 95$ & 1145 & 35.39 & 13.53 & 21.86 & \\
\hline MWGS-30A & MW & $11 / 21 / 91$ & --- & 36.22 & 15.18 & 21.04 & \\
\hline MWGS-30A & MW & $05 / 14 / 92$ & 1104 & 36.22 & 15.92 & 20.30 & \\
\hline MWGS-30A & MW & $06 / 11 / 92$ & 1258 & 36.22 & 16.27 & 19.95 & \\
\hline MWGS-30A & MW & $07 / 07 / 92$ & 1740 & 36.22 & 15.63 & 20.59 & \\
\hline MWGS-30A & MW & $08 / 07 / 92$ & 1148 & 36.22 & --- & --- & Free product present in well. \\
\hline MWGS-30B & MW & $11 / 21 / 91$ & -- & 36.14 & 15.09 & 21.05 & \\
\hline MWGS-30B & MW & $12 / 16 / 91$ & -- & 36.14 & 15.57 & 20.57 & \\
\hline MWGS-30B & MW & $05 / 14 / 92$ & 1112 & 36.14 & 15.83 & 20.31 & \\
\hline MWGS-30B & MW & $06 / 11 / 92$ & 1255 & 36.14 & 16.57 & 19.57 & \\
\hline MWGS-30B & MW & $07 / 07 / 92$ & 1742 & 36.14 & 15.90 & 20.24 & \\
\hline MWGS-30B & MW & $08 / 07 / 92$ & 1146 & 36.14 & 16.18 & 19.96 & \\
\hline MWGS-30B & MW & $08 / 31 / 92$ & 1115 & 36.14 & 13.40 & 22.74 & \\
\hline MWGS-30B & MW & $10 / 07 / 92$ & 1128 & 36.14 & 13.37 & 22.77 & \\
\hline MWGS-30B & MW & $10 / 28 / 92$ & 1157 & 36.14 & 13.86 & 22.28 & \\
\hline MWGS-30B & MW & $12 / 03 / 92$ & 1101 & 36.14 & 14.09 & 22.05 & \\
\hline MWGS-30B & MW & $01 / 04 / 93$ & 1050 & 36.14 & 14.97 & 21.17 & \\
\hline MWGS-30B & MW & $02 / 04 / 93$ & 1142 & 36.14 & 13.35 & 22.79 & \\
\hline MWGS-30B & MW & $03 / 05 / 93$ & 1102 & 36.14 & 13.99 & 22.15 & \\
\hline MWGS-30B & MW & $03 / 30 / 93$ & 1024 & 36.14 & 15.93 & 20.21 & \\
\hline MWGS-30B & MW & $05 / 06 / 93$ & 1048 & 36.14 & 16.35 & 19.79 & \\
\hline MWGS-30B & MW & $05 / 28 / 93$ & 1044 & 36.14 & 17.05 & 19.09 & \\
\hline MWGS-30B & MW & $07 / 07 / 93$ & 1036 & 36.14 & 16.55 & 19.59 & \\
\hline MWGS-30B & MW & $08 / 06 / 93$ & 1019 & 36.14 & 16.78 & 19.36 & \\
\hline MWGS-30B & MW & $09 / 03 / 93$ & 1038 & 36.14 & 16.69 & 19.45 & \\
\hline MWGS-30B & MW & $09 / 30 / 93$ & 1054 & 36.14 & 16.25 & 19.89 & \\
\hline MWGS-30B & MW & $10 / 28 / 93$ & 1030 & 36.14 & 17.05 & 19.09 & \\
\hline MWGS-30B & MW & $12 / 01 / 93$ & 1332 & 36.14 & 16.99 & 19.15 & \\
\hline MWGS-30B & MW & $01 / 06 / 94$ & 1043 & 36.14 & 17.32 & 18.82 & \\
\hline MWGS-30B & MW & $02 / 01 / 94$ & 1042 & 36.14 & 15.90 & 20.24 & \\
\hline MWGS-30B & MW & $03 / 04 / 94$ & 1022 & 36.14 & 15.92 & 20.22 & \\
\hline MWGS-30B & MW & $04 / 04 / 94$ & 1019 & 36.14 & 16.33 & 19.81 & \\
\hline MWGS-30B & MW & $05 / 06 / 94$ & 1101 & 36.14 & 17.22 & 18.92 & \\
\hline MWGS-30B & MW & $06 / 03 / 94$ & 1041 & 36.14 & 17.92 & 18.22 & \\
\hline MWGS-30B & MW & $07 / 08 / 94$ & 1055 & 36.14 & 16.56 & 19.58 & \\
\hline MWGS-30B & MW & $08 / 05 / 94$ & 1350 & 36.14 & 14.80 & 21.34 & \\
\hline MWGS-30B & MW & $09 / 07 / 94$ & 1105 & 36.14 & 14.44 & 21.70 & \\
\hline
\end{tabular}


Table 8.--Water-level data collected at the Defense Fuel Supply Point and adjacent properties, Hanahan, S.C., between April 1991 and September 1995--Continued

[ft, feet; --, data not available; WL, water-level well; EW, extraction well; MW, monitoring well; SW, surface-water site; -, negative depth to water indicates water-level altitude is above measuring point]

\begin{tabular}{|c|c|c|c|c|c|c|c|}
\hline $\begin{array}{c}\text { Site } \\
\text { (plate 1) }\end{array}$ & $\begin{array}{l}\text { Site } \\
\text { type }\end{array}$ & Date & Time & $\begin{array}{l}\text { Measuring-point } \\
\text { altitude } \\
\text { (ft above } \\
\text { sea level) }\end{array}$ & $\begin{array}{l}\text { Depth to water } \\
\text { (ft below } \\
\text { measuring point) }\end{array}$ & $\begin{array}{l}\text { Water-level } \\
\text { altitude } \\
\text { (ft above } \\
\text { sea level) }\end{array}$ & Remarks \\
\hline MWGS-30B & MW & $10 / 06 / 94$ & 1120 & 36.14 & 12.23 & 23.91 & \\
\hline MWGS-30B & MW & $11 / 09 / 94$ & 1244 & 36.14 & 13.84 & 22.30 & \\
\hline MWGS-30B & MW & $12 / 06 / 94$ & 1100 & 36.14 & 14.57 & 21.57 & \\
\hline MWGS-30B & MW & $01 / 10 / 95$ & 1305 & 36.14 & 14.47 & 21.67 & \\
\hline MWGS-30B & MW & $02 / 02 / 95$ & 1321 & 36.14 & 14.09 & 22.05 & \\
\hline MWGS-30B & MW & $03 / 02 / 95$ & 1447 & 36.14 & 14.26 & 21.88 & \\
\hline MWGS-30B & MW & $04 / 05 / 95$ & 1050 & 36.14 & 14.99 & 21.15 & \\
\hline MWGS-30B & MW & $05 / 02 / 95$ & 1154 & 36.14 & 15.65 & 20.49 & \\
\hline MWGS-30B & MW & $06 / 09 / 95$ & 1041 & 36.14 & 16.50 & 19.64 & \\
\hline MWGS-30B & MW & $07 / 06 / 95$ & 1034 & 36.14 & 16.22 & 19.92 & \\
\hline MWGS-30B & MW & $08 / 10 / 95$ & 1106 & 36.14 & 16.00 & 20.14 & \\
\hline MWGS-30B & MW & $09 / 13 / 95$ & 1147 & 36.14 & 13.71 & 22.43 & \\
\hline MWGS-31A & MW & $02 / 04 / 92$ & --- & 34.58 & 7.70 & 26.88 & Pre-start-up water levels. \\
\hline MWGS-31A & MW & $02 / 20 / 92$ & 1600 & 34.58 & 7.91 & 26.67 & \\
\hline MWGS-31A & MW & $02 / 25 / 92$ & 0954 & 34.58 & 7.79 & 26.79 & \\
\hline MWGS-31A & MW & $06 / 11 / 92$ & 1443 & 34.58 & 7.27 & 27.31 & \\
\hline MWGS-31A & MW & $07 / 07 / 92$ & 1558 & 34.58 & 7.50 & 27.08 & \\
\hline MWGS-31A & MW & $08 / 07 / 92$ & 1323 & 34.58 & 7.37 & 27.21 & \\
\hline MWGS-31A & MW & $08 / 31 / 92$ & 1159 & 34.58 & 6.08 & 28.50 & \\
\hline MWGS-31A & MW & $10 / 07 / 92$ & 1211 & 34.58 & 5.90 & 28.68 & \\
\hline MWGS-31A & MW & $10 / 28 / 92$ & 1321 & 34.58 & 6.81 & 27.77 & \\
\hline MWGS-31A & MW & $12 / 03 / 92$ & 1206 & 34.58 & 6.57 & 28.01 & \\
\hline MWGS-31A & MW & $01 / 04 / 93$ & 1151 & 34.58 & 7.36 & 27.22 & \\
\hline MWGS-3IA & MW & $02 / 04 / 93$ & 1233 & 34.58 & 6.33 & 28.25 & \\
\hline MWGS-31A & MW & $03 / 05 / 93$ & 1155 & 34.58 & 6.71 & 27.87 & \\
\hline MWGS-31A & MW & $03 / 30 / 93$ & 1114 & 34.58 & 6.29 & 28.29 & \\
\hline MWGS-31A & MW & $05 / 06 / 93$ & 1133 & 34.58 & 7.15 & 27.43 & \\
\hline MWGS-31A & MW & $05 / 28 / 93$ & 1126 & 34.58 & 7.59 & 26.99 & \\
\hline MWGS-31A & MW & $07 / 07 / 93$ & 1116 & 34.58 & 7.48 & 27.10 & \\
\hline MWGS-31A & MW & $08 / 06 / 93$ & 1056 & 34.58 & 7.15 & 27.43 & \\
\hline MWGS-31A & MW & $09 / 03 / 93$ & 1114 & 34.58 & 7.34 & 27.24 & \\
\hline MWGS-31A & MW & $09 / 30 / 93$ & 0957 & 34.58 & 7.13 & 27.45 & \\
\hline MWGS-31A & MW & $10 / 28 / 93$ & 1111 & 34.58 & 8.00 & 26.58 & \\
\hline MWGS-31A & MW & $12 / 01 / 93$ & 1411 & 34.58 & 7.57 & 27.01 & \\
\hline MWGS-31A & MW & $01 / 06 / 94$ & 1129 & 34.58 & 6.97 & 27.61 & \\
\hline MWGS-31A & MW & $02 / 01 / 94$ & 1129 & 34.58 & 6.22 & 28.36 & \\
\hline MWGS-31A & MW & $03 / 04 / 94$ & 1056 & 34.58 & 6.40 & 28.18 & \\
\hline MWGS-31A & MW & $04 / 04 / 94$ & 1054 & 34.58 & 7.55 & 27.03 & \\
\hline MWGS-31A & MW & $05 / 06 / 94$ & 1147 & 34.58 & 8.20 & 26.38 & \\
\hline MWGS-31A & MW & $06 / 03 / 94$ & 1125 & 34.58 & 8.67 & 25.91 & \\
\hline MWGS-31A & MW & $07 / 08 / 94$ & 1145 & 34.58 & 6.17 & 28.41 & \\
\hline MWGS-3IA & MW & $08 / 05 / 94$ & 1428 & 34.58 & 6.18 & 28.40 & \\
\hline MWGS-3IA & MW & $09 / 07 / 94$ & 1245 & 34.58 & 6.38 & 28.20 & \\
\hline MWGS-31A & MW & $10 / 06 / 94$ & 1201 & 34.58 & 4.72 & 29.86 & \\
\hline
\end{tabular}


Table 8.--Water-level data collected at the Defense Fuel Supply Point and adjacent properties, Hanahan, S.C., between April 1991 and September 1995--Continued

[ft, feet; ---, data not available; WL, water-level well; EW, extraction well; MW, monitoring well; SW, surface-water site; -, negative depth to wateindicates water-level altitude is above measuring point]

\begin{tabular}{|c|c|c|c|c|c|c|c|}
\hline $\begin{array}{c}\text { Site } \\
\text { (plate 1) }\end{array}$ & $\begin{array}{l}\text { Site } \\
\text { type }\end{array}$ & Date & Time & $\begin{array}{l}\text { Measuring-point } \\
\text { altitude } \\
\text { (ft above } \\
\text { sea level) }\end{array}$ & $\begin{array}{l}\text { Depth to water } \\
\text { (ft below } \\
\text { measuring point) }\end{array}$ & $\begin{array}{l}\text { Water-level } \\
\text { altitude } \\
\text { (ft above } \\
\text { sea level) }\end{array}$ & Remarks \\
\hline MWGS-31A & MW & $11 / 09 / 94$ & 1321 & 34.58 & 6.28 & 28.30 & \\
\hline MWGS-31A & MW & $12 / 06 / 94$ & 1141 & 34.58 & 6.15 & 28.43 & \\
\hline MWGS-3IA & MW & $01 / 10 / 95$ & 1619 & 34.58 & 6.46 & 28.12 & \\
\hline MWGS-31A & MW & $02 / 02 / 95$ & 1358 & 34.58 & 6.59 & 27.99 & \\
\hline MWGS-31A & MW & $03 / 02 / 95$ & 1528 & 34.58 & 6.58 & 28.00 & \\
\hline MWGS-31A & MW & $04 / 05 / 95$ & 1138 & 34.58 & 7.36 & 27.22 & \\
\hline MWGS-31A & MW & $05 / 02 / 95$ & 1325 & 34.58 & 7.76 & 26.82 & \\
\hline MWGS-31A & MW & $06 / 09 / 95$ & 1121 & 34.58 & 7.62 & 26.96 & \\
\hline MWGS-31A & MW & $07 / 06 / 95$ & 1109 & 34.58 & 7.49 & 27.09 & \\
\hline MWGS-31A & MW & $08 / 10 / 95$ & 1242 & 34.58 & 7.59 & 26.99 & \\
\hline MWGS-31A & MW & $09 / 13 / 95$ & 1207 & 34.58 & 6.49 & 28.09 & \\
\hline MWGS-31B & MW & $02 / 04 / 92$ & --- & 34.47 & 7.67 & 26.80 & Pre-start-up water levels. \\
\hline MWGS-31B & MW & $02 / 20 / 92$ & 1557 & 34.47 & 7.94 & 26.53 & \\
\hline MWGS-31B & MW & $02 / 25 / 92$ & 0956 & 34.47 & 7.78 & 26.69 & \\
\hline MWGS-31B & MW & $06 / 11 / 92$ & 1444 & 34.47 & 7.34 & 27.13 & \\
\hline MWGS-31B & MW & 07/07/92 & 1600 & 34.47 & 7.45 & 27.02 & \\
\hline MWGS-3IB & MW & $08 / 07 / 92$ & 1325 & 34.47 & 7.32 & 27.15 & \\
\hline MWGS-31B & MW & $08 / 31 / 92$ & 1204 & 34.47 & 6.16 & 28.31 & \\
\hline MWGS-31B & MW & $10 / 07 / 92$ & 1212 & 34.47 & 5.89 & 28.58 & \\
\hline MWGS-31B & MW & $10 / 28 / 92$ & 1324 & 34.47 & 6.75 & 27.72 & \\
\hline MWGS-31B & MW & $12 / 03 / 92$ & 1208 & 34.47 & 6.53 & 27.94 & \\
\hline MWGS-31B & MW & $01 / 04 / 93$ & 1148 & 34.47 & 7.30 & 27.17 & \\
\hline MWGS-31B & MW & $02 / 04 / 93$ & 1234 & 34.47 & 6.28 & 28.19 & \\
\hline MWGS-31B & MW & $03 / 05 / 93$ & 1156 & 34.47 & 6.70 & 27.77 & \\
\hline MWGS-31B & MW & $03 / 30 / 93$ & 1116 & 34.47 & 6.34 & 28.13 & \\
\hline MWGS-31B & MW & $05 / 06 / 93$ & 1141 & 34.47 & 7.16 & 27.31 & \\
\hline MWGS-31B & MW & $05 / 28 / 93$ & 1133 & 34.47 & 7.60 & 26.87 & \\
\hline MWGS-31B & MW & $07 / 07 / 93$ & 1117 & 34.47 & 7.48 & 26.99 & \\
\hline MWGS-31B & MW & $08 / 06 / 93$ & 1058 & 34.47 & 7.10 & 27.37 & \\
\hline MWGS-31B & MW & $09 / 03 / 93$ & 1115 & 34.47 & 7.30 & 27.17 & \\
\hline MWGS-31B & MW & $09 / 30 / 93$ & 0958 & 34.47 & 7.12 & 27.35 & \\
\hline MWGS-31B & MW & $10 / 28 / 93$ & 1113 & 34.47 & 8.07 & 26.40 & \\
\hline MWGS-31B & MW & $12 / 01 / 93$ & 1420 & 34.47 & 7.61 & 26.86 & \\
\hline MWGS-31B & MW & $01 / 06 / 94$ & 1132 & 34.47 & 6.98 & 27.49 & \\
\hline MWGS-31B & MW & $02 / 01 / 94$ & 1130 & 34.47 & 6.25 & 28.22 & \\
\hline MWGS-31B & MW & $03 / 04 / 94$ & 1101 & 34.47 & 6.40 & 28.07 & \\
\hline MWGS-3IB & MW & $04 / 04 / 94$ & 1056 & 34.47 & 7.65 & 26.82 & \\
\hline MWGS-31B & MW & $05 / 06 / 94$ & 1149 & 34.47 & 8.22 & 26.25 & \\
\hline MWGS-31B & MW & $06 / 03 / 94$ & 1128 & 34.47 & 8.70 & 25.77 & \\
\hline MWGS-31B & MW & $07 / 08 / 94$ & 1204 & 34.47 & 6.22 & 28.25 & \\
\hline MWGS-31B & MW & $08 / 05 / 94$ & 1436 & 34.47 & 6.31 & 28.16 & \\
\hline MWGS-31B & MW & $09 / 07 / 94$ & 1254 & 34.47 & 6.58 & 27.89 & \\
\hline MWGS-31B & MW & $10 / 06 / 94$ & 1204 & 34.47 & 4.66 & 29.81 & \\
\hline MWGS-31B & MW & $11 / 09 / 94$ & 1322 & 34.47 & 6.27 & 28.20 & \\
\hline
\end{tabular}


Table 8.--Water-level data collected at the Defense Fuel Supply Point and adjacent properties, Hanahan, S.C., between April 1991 and September 1995--Continued

[ft, feet; ---, data not available; WL, water-level well; EW, extraction well; MW, monitoring well; SW, surface-water site; -, negative depth to water indicates water-level altitude is above measuring point]

\begin{tabular}{|c|c|c|c|c|c|c|c|}
\hline $\begin{array}{c}\text { Site } \\
\text { (plate 1) }\end{array}$ & $\begin{array}{l}\text { Site } \\
\text { type }\end{array}$ & Date & Time & $\begin{array}{l}\text { Measuring-point } \\
\text { altitude } \\
\text { (ft above } \\
\text { sea level) }\end{array}$ & $\begin{array}{l}\text { Depth to water } \\
\text { ( } \mathrm{ft} \text { below } \\
\text { measuring point) }\end{array}$ & $\begin{array}{l}\text { Water-level } \\
\text { altitude } \\
\text { (ft above } \\
\text { sea level) }\end{array}$ & Remarks \\
\hline MWGS-31B & MW & $12 / 06 / 94$ & 1154 & 34.47 & 6.23 & 28.24 & \\
\hline MWGS-31B & MW & $01 / 10 / 95$ & 1620 & 34.47 & 6.50 & 27.97 & \\
\hline MWGS-31B & MW & $02 / 02 / 95$ & 1359 & 34.47 & 6.59 & 27.88 & \\
\hline MWGS-31B & MW & $03 / 02 / 95$ & 1536 & 34.47 & 6.71 & 27.76 & \\
\hline MWGS-31B & MW & $04 / 05 / 95$ & 1139 & 34.47 & 7.47 & 27.00 & \\
\hline MWGS-31B & MW & $05 / 02 / 95$ & 1326 & 34.47 & 7.78 & 26.69 & \\
\hline MWGS-31B & MW & 06/09/95 & 1135 & 34.47 & 7.65 & 26.82 & \\
\hline MWGS-31B & MW & $07 / 06 / 95$ & 1124 & 34.47 & 7.56 & 26.91 & \\
\hline MWGS-31B & MW & 08/10/95 & 1246 & 34.47 & 7.54 & 26.93 & \\
\hline MWGS-31B & MW & $09 / 13 / 95$ & 1223 & 34.47 & 6.61 & 27.86 & \\
\hline MWGS-32A & MW & $02 / 04 / 92$ & --- & 35.06 & 8.55 & 26.51 & Pre-start-up water levels. \\
\hline MWGS-32A & MW & $02 / 20 / 92$ & 1604 & 35.06 & 8.84 & 26.22 & \\
\hline MWGS-32A & MW & $02 / 25 / 92$ & 0936 & 35.06 & 8.67 & 26.39 & \\
\hline MWGS-32A & MW & $06 / 11 / 92$ & 1436 & 35.06 & 7.75 & 27.31 & \\
\hline MWGS-32A & MW & $07 / 07 / 92$ & 1620 & 35.06 & 8.32 & 26.74 & \\
\hline MWGS-32A & MW & $08 / 07 / 92$ & 1304 & 35.06 & 7.99 & 27.07 & \\
\hline MWGS-32A & MW & $08 / 31 / 92$ & 1144 & 35.06 & 6.83 & 28.23 & \\
\hline MWGS-32A & MW & $10 / 07 / 92$ & 1155 & 35.06 & 6.47 & 28.59 & \\
\hline MWGS-32A & MW & $10 / 28 / 92$ & 1310 & 35.06 & 7.51 & 27.55 & \\
\hline MWGS-32A & MW & $12 / 03 / 92$ & 1134 & 35.06 & 7.35 & 27.71 & \\
\hline MWGS-32A & MW & $01 / 04 / 93$ & 1135 & 35.06 & 8.18 & 26.88 & \\
\hline MWGS-32A & MW & $02 / 04 / 93$ & 1210 & 35.06 & 7.13 & 27.93 & \\
\hline MWGS-32A & MW & $03 / 05 / 93$ & 1138 & 35.06 & 7.53 & 27.53 & \\
\hline MWGS-32A & MW & $03 / 30 / 93$ & 1050 & 35.06 & 7.17 & 27.89 & \\
\hline MWGS-32A & MW & $05 / 06 / 93$ & 1117 & 35.06 & 7.85 & 27.21 & \\
\hline MWGS-32A & MW & $05 / 28 / 93$ & 1114 & 35.06 & 8.37 & 26.69 & \\
\hline MWGS-32A & MW & $07 / 07 / 93$ & 1103 & 35.06 & 8.07 & 26.99 & \\
\hline MWGS-32A & MW & $08 / 06 / 93$ & 1042 & 35.06 & 7.72 & 27.34 & \\
\hline MWGS-32A & MW & 09/03/93 & 1057 & 35.06 & 8.02 & 27.04 & \\
\hline MWGS-32A & MW & $09 / 30 / 93$ & 1019 & 35.06 & 7.82 & 27.24 & \\
\hline MWGS-32A & MW & $10 / 28 / 93$ & 1052 & 35.06 & 8.65 & 26.41 & \\
\hline MWGS-32A & MW & $12 / 01 / 93$ & 1356 & 35.06 & 7.90 & 27.16 & \\
\hline MWGS-32A & MW & $01 / 06 / 94$ & 1105 & 35.06 & 7.20 & 27.86 & \\
\hline MWGS-32A & MW & $02 / 01 / 94$ & 1115 & 35.06 & 6.52 & 28.54 & \\
\hline MWGS-32A & MW & $03 / 04 / 94$ & 1043 & 35.06 & 6.79 & 28.27 & \\
\hline MWGS-32A & MW & $04 / 04 / 94$ & $104 I$ & 35.06 & 8.16 & 26.90 & \\
\hline MWGS-32A & MW & $05 / 06 / 94$ & 1132 & 35.06 & 8.77 & 26.29 & \\
\hline MWGS-32A & MW & $06 / 03 / 94$ & 1111 & 35.06 & 9.35 & 25.71 & \\
\hline MWGS-32A & MW & $07 / 08 / 94$ & 1121 & 35.06 & 6.15 & 28.91 & \\
\hline MWGS-32A & MW & $08 / 05 / 94$ & 1419 & 35.06 & 6.44 & 28.62 & \\
\hline MWGS-32A & MW & $09 / 07 / 94$ & 1230 & 35.06 & 6.68 & 28.38 & \\
\hline MWGS-32A & MW & $10 / 06 / 94$ & 1142 & 35.06 & 5.20 & 29.86 & \\
\hline MWGS-32A & MW & $11 / 09 / 94$ & 1310 & 35.06 & 6.68 & 28.38 & \\
\hline MWGS-32A & MW & $12 / 06 / 94$ & 1126 & 35.06 & 6.20 & 28.86 & \\
\hline
\end{tabular}


Table 8.--Water-level data collected at the Defense Fuel Supply Point and adjacent properties, Hanahan, S.C., between April 1991 and September 1995--Continued

[ft, feet; --., data not available; WL, water-level well; EW, extraction well; MW, monitoring well; SW, surface-water site; -, negative depth to wateindicates water-level altitude is above measuring point]

\begin{tabular}{|c|c|c|c|c|c|c|c|}
\hline $\begin{array}{c}\text { Site } \\
\text { (plate 1) }\end{array}$ & $\begin{array}{l}\text { Site } \\
\text { type }\end{array}$ & Date & Time & $\begin{array}{l}\text { Measuring-point } \\
\text { altitude } \\
\text { (ft above } \\
\text { sea level) }\end{array}$ & $\begin{array}{l}\text { Depth to water } \\
\text { (ft below } \\
\text { measuring point) }\end{array}$ & $\begin{array}{l}\text { Water-level } \\
\text { altitude } \\
\text { (ft above } \\
\text { sea level) }\end{array}$ & Remarks \\
\hline MWGS-32A & MW & $01 / 10 / 95$ & 1331 & 35.06 & 6.70 & 28.36 & \\
\hline MWGS-32A & MW & $02 / 02 / 95$ & 1344 & 35.06 & 7.02 & 28.04 & \\
\hline MWGS-32A & MW & $03 / 02 / 95$ & 1516 & 35.06 & 7.10 & 27.96 & \\
\hline MWGS-32A & MW & $04 / 05 / 95$ & 1117 & 35.06 & 7.92 & 27.14 & \\
\hline MWGS-32A & MW & $05 / 02 / 95$ & 1301 & 35.06 & 8.30 & 26.76 & \\
\hline MWGS-32A & MW & $06 / 09 / 95$ & 1105 & 35.06 & 7.33 & 27.73 & \\
\hline MWGS-32A & MW & $07 / 06 / 95$ & 1057 & 35.06 & 8.01 & 27.05 & \\
\hline MWGS-32A & MW & $08 / 10 / 95$ & 1129 & 35.06 & 8.17 & 26.89 & \\
\hline MWGS-32A & MW & $09 / 13 / 95$ & 1029 & 35.06 & 6.97 & 28.09 & \\
\hline MWGS-32B & MW & $02 / 04 / 92$ & -- & 35.02 & 8.50 & 26.52 & Pre-start-up water levels. \\
\hline MWGS-32B & MW & $02 / 20 / 92$ & 1602 & 35.02 & 8.79 & 26.23 & \\
\hline MWGS-32B & MW & $02 / 25 / 92$ & 0938 & 35.02 & 8.62 & 26.40 & \\
\hline MWGS-32B & MW & $06 / 11 / 92$ & 1435 & 35.02 & 7.71 & 27.31 & \\
\hline MWGS-32B & MW & $07 / 07 / 92$ & 1625 & 35.02 & 8.27 & 26.75 & \\
\hline MWGS-32B & MW & $08 / 07 / 92$ & 1305 & 35.02 & 7.93 & 27.09 & \\
\hline MWGS-32B & MW & $08 / 31 / 92$ & 1146 & 35.02 & 6.79 & 28.23 & \\
\hline MWGS-32B & MW & $10 / 07 / 92$ & 1158 & 35.02 & 6.46 & 28.56 & \\
\hline MWGS-32B & MW & $10 / 28 / 92$ & 1312 & 35.02 & 7.47 & 27.55 & \\
\hline MWGS-32B & MW & $12 / 03 / 92$ & 1136 & 35.02 & 7.30 & 27.72 & \\
\hline MWGS-32B & MW & $01 / 04 / 93$ & 1132 & 35.02 & 8.13 & 26.89 & \\
\hline MWGS-32B & MW & $02 / 04 / 93$ & 1214 & 35.02 & 7.08 & 27.94 & \\
\hline MWGS-32B & MW & $03 / 05 / 93$ & 1140 & 35.02 & 7.49 & 27.53 & \\
\hline MWGS-32B & MW & $03 / 30 / 93$ & 1051 & 35.02 & 7.13 & 27.89 & \\
\hline MWGS-32B & MW & $05 / 06 / 93$ & 1119 & 35.02 & 7.82 & 27.20 & \\
\hline MWGS-32B & MW & $05 / 28 / 93$ & 1115 & 35.02 & 8.32 & 26.70 & \\
\hline MWGS-32B & MW & $07 / 07 / 93$ & 1105 & 35.02 & 8.03 & 26.99 & \\
\hline MWGS-32B & MW & $08 / 06 / 93$ & 1045 & 35.02 & 7.69 & 27.33 & \\
\hline MWGS-32B & MW & $09 / 03 / 93$ & 1058 & 35.02 & 7.88 & 27.14 & \\
\hline MWGS-32B & MW & $09 / 30 / 93$ & 1021 & 35.02 & 7.78 & 27.24 & \\
\hline MWGS-32B & MW & $10 / 28 / 93$ & 1057 & 35.02 & 8.64 & 26.38 & \\
\hline MWGS-32B & MW & $12 / 01 / 93$ & 1358 & 35.02 & 8.00 & 27.02 & \\
\hline MWGS-32B & MW & $01 / 06 / 94$ & 1110 & 35.02 & 7.31 & 27.71 & \\
\hline MWGS-32B & MW & $02 / 01 / 94$ & 1109 & 35.02 & 6.60 & 28.42 & \\
\hline MWGS-32B & MW & $03 / 04 / 94$ & 1045 & 35.02 & 6.79 & 28.23 & \\
\hline MWGS-32B & MW & $04 / 04 / 94$ & 1043 & 35.02 & 8.12 & 26.90 & \\
\hline MWGS-32B & MW & $05 / 06 / 94$ & 1135 & 35.02 & 8.75 & 26.27 & \\
\hline MWGS-32B & MW & $06 / 03 / 94$ & 1106 & 35.02 & 9.32 & 25.70 & \\
\hline MWGS-32B & MW & $07 / 08 / 94$ & 1123 & 35.02 & 6.28 & 28.74 & \\
\hline MWGS-32B & MW & $08 / 05 / 94$ & 1420 & 35.02 & 6.51 & 28.51 & \\
\hline MWGS-32B & MW & $09 / 07 / 94$ & 1227 & 35.02 & 6.71 & 28.31 & \\
\hline MWGS-32B & MW & $10 / 06 / 94$ & 1144 & 35.02 & 5.30 & 29.72 & \\
\hline MWGS-32B & MW & $11 / 09 / 94$ & 1312 & 35.02 & 6.71 & 28.31 & \\
\hline MWGS-32B & MW & $12 / 06 / 94$ & 1128 & 35.02 & 6.40 & 28.62 & \\
\hline MWGS-32B & MW & $01 / 10 / 95$ & 1334 & 35.02 & 6.82 & 28.20 & \\
\hline
\end{tabular}


Table 8.--Water-level data collected at the Defense Fuel Supply Point and adjacent properties, Hanahan, S.C., between April 1991 and September 1995--Continued

[ft, feet; ---, data not available; WL, water-level well; EW, extraction well; MW, monitoring well; SW, surface-water site; -, negative depth to water indicates water-level altitude is above measuring point]

\begin{tabular}{|c|c|c|c|c|c|c|c|}
\hline $\begin{array}{c}\text { Site } \\
\text { (plate 1) }\end{array}$ & $\begin{array}{l}\text { Site } \\
\text { type }\end{array}$ & Date & Time & $\begin{array}{l}\text { Measuring-point } \\
\text { altitude } \\
\text { (ft above } \\
\text { sea level) }\end{array}$ & $\begin{array}{l}\text { Depth to water } \\
\text { (ft below } \\
\text { measuring point) }\end{array}$ & $\begin{array}{l}\text { Water-level } \\
\text { altitude } \\
\text { (ft above } \\
\text { sea level) }\end{array}$ & Remarks \\
\hline MWGS-32B & MW & $02 / 02 / 95$ & 1346 & 35.02 & 7.06 & 27.96 & \\
\hline MWGS-32B & MW & $03 / 02 / 95$ & 1518 & 35.02 & 7.10 & 27.92 & \\
\hline MWGS-32B & MW & $04 / 05 / 95$ & 1125 & 35.02 & 7.91 & 27.11 & \\
\hline MWGS-32B & MW & $05 / 02 / 95$ & 1311 & 35.02 & 8.32 & 26.70 & \\
\hline MWGS-32B & MW & $06 / 09 / 95$ & 1111 & 35.02 & 8.10 & 26.92 & \\
\hline MWGS-32B & MW & $07 / 06 / 95$ & 1100 & 35.02 & 8.02 & 27.00 & \\
\hline MWGS-32B & MW & $08 / 10 / 95$ & 1132 & 35.02 & 8.16 & 26.86 & \\
\hline MWGS-32B & MW & $09 / 13 / 95$ & 1027 & 35.02 & 7.03 & 27.99 & \\
\hline MWGS-33A & MW & $02 / 04 / 92$ & --- & 37.48 & 11.07 & 26.41 & Pre-start-up water levels. \\
\hline MWGS-33A & MW & $02 / 25 / 92$ & 0937 & 37.48 & 11.20 & 26.28 & \\
\hline MWGS-33A & MW & $02 / 25 / 92$ & 1529 & 37.48 & 11.57 & 25.91 & \\
\hline MWGS-33A & MW & $06 / 11 / 92$ & 1430 & 37.48 & 10.34 & 27.14 & \\
\hline MWGS-33A & MW & $07 / 07 / 92$ & 1648 & 37.48 & 10.88 & 26.60 & \\
\hline MWGS-33A & MW & $08 / 07 / 92$ & 1314 & 37.48 & 10.54 & 26.94 & \\
\hline MWGS-33A & MW & $08 / 31 / 92$ & 1141 & 37.48 & 9.45 & 28.03 & \\
\hline MWGS-33A & MW & $10 / 07 / 92$ & 1149 & 37.48 & 9.14 & 28.34 & \\
\hline MWGS-33A & MW & $10 / 28 / 92$ & 1304 & 37.48 & 10.10 & 27.38 & \\
\hline MWGS-33A & MW & $12 / 03 / 92$ & 1152 & 37.48 & 9.93 & 27.55 & \\
\hline MWGS-33A & MW & $01 / 04 / 93$ & 1120 & 37.48 & 10.73 & 26.75 & \\
\hline MWGS-33A & MW & $02 / 04 / 93$ & 1222 & 37.48 & 9.71 & 27.77 & \\
\hline MWGS-33A & MW & $03 / 05 / 93$ & 1131 & 37.48 & 10.09 & 27.39 & \\
\hline MWGS-33A & MW & $03 / 30 / 93$ & 1048 & 37.48 & 9.75 & 27.73 & \\
\hline MWGS-33A & MW & $05 / 06 / 93$ & 1114 & 37.48 & 10.43 & 27.05 & \\
\hline MWGS-33A & MW & $05 / 28 / 93$ & 1109 & 37.48 & 10.93 & 26.55 & \\
\hline MWGS-33A & MW & $07 / 07 / 93$ & 1059 & 37.48 & 10.65 & 26.83 & \\
\hline MWGS-33A & MW & $08 / 06 / 93$ & 1038 & 37.48 & 10.31 & 27.17 & \\
\hline MWGS-33A & MW & $09 / 03 / 93$ & 1105 & 37.48 & 10.62 & 26.86 & \\
\hline MWGS-33A & MW & $09 / 30 / 93$ & 1017 & 37.48 & 10.40 & 27.08 & \\
\hline MWGS-33A & MW & $10 / 28 / 93$ & 1102 & 37.48 & 11.34 & 26.14 & \\
\hline MWGS-33A & MW & $12 / 01 / 93$ & 1408 & 37.48 & 10.74 & 26.74 & \\
\hline MWGS-33A & MW & $01 / 06 / 94$ & 1100 & 37.48 & 9.96 & 27.52 & \\
\hline MWGS-33A & MW & $02 / 01 / 94$ & 1105 & 37.48 & 9.23 & 28.25 & \\
\hline MWGS-33A & MW & $03 / 04 / 94$ & 1038 & 37.48 & 9.45 & 28.03 & \\
\hline MWGS-33A & MW & $04 / 04 / 94$ & 1040 & 37.48 & 10.72 & 26.76 & \\
\hline MWGS-33A & MW & $05 / 06 / 94$ & 1122 & 37.48 & 11.36 & 26.12 & \\
\hline MWGS-33A & MW & $06 / 03 / 94$ & 1100 & 37.48 & 11.89 & 25.59 & \\
\hline MWGS-33A & MW & $07 / 08 / 94$ & 1115 & 37.48 & 8.99 & 28.49 & \\
\hline MWGS-33A & MW & $08 / 05 / 94$ & 1415 & 37.48 & 9.21 & 28.27 & \\
\hline MWGS-33A & MW & $09 / 07 / 94$ & 1236 & 37.48 & 9.39 & 28.09 & \\
\hline MWGS-33A & MW & $10 / 06 / 94$ & 1149 & 37.48 & 8.04 & 29.44 & \\
\hline MWGS-33A & MW & $11 / 09 / 94$ & 1305 & 37.48 & 9.37 & 28.11 & \\
\hline MWGS-33A & MW & $12 / 06 / 94$ & 1123 & 37.48 & 9.04 & 28.44 & \\
\hline MWGS-33A & MW & $01 / 10 / 95$ & 1337 & 37.48 & 9.47 & 28.01 & \\
\hline MWGS-33A & MW & $02 / 02 / 95$ & 1340 & 37.48 & 9.69 & 27.79 & \\
\hline
\end{tabular}


Table 8.--Water-level data collected at the Defense Fuel Supply Point and adjacent properties, Hanahan, S.C., between April 1991 and September 1995--Continued

[ft, feet; ---, data not available; WL, water-level well; EW, extraction well; MW, monitoring well; SW, surface-water site; -, negative depth to wateindicates water-level altitude is above measuring point]

\begin{tabular}{|c|c|c|c|c|c|c|c|}
\hline $\begin{array}{c}\text { Site } \\
\text { (plate 1) }\end{array}$ & $\begin{array}{l}\text { Site } \\
\text { type }\end{array}$ & Date & Time & $\begin{array}{l}\text { Measuring-point } \\
\text { altitude } \\
\text { (ft above } \\
\text { sea level) }\end{array}$ & $\begin{array}{l}\text { Depth to water } \\
\text { (ft below } \\
\text { measuring point) }\end{array}$ & $\begin{array}{l}\text { Water-level } \\
\text { altitude } \\
\text { (ft above } \\
\text { sea level) }\end{array}$ & Remarks \\
\hline MWGS-33A & MW & $03 / 02 / 95$ & 1510 & 37.48 & 9.73 & 27.75 & \\
\hline MWGS-33A & MW & $04 / 05 / 95$ & 1110 & 37.48 & 10.52 & 26.96 & \\
\hline MWGS-33A & MW & $05 / 02 / 95$ & 1257 & 37.48 & 10.94 & 26.54 & \\
\hline MWGS-33A & MW & $06 / 09 / 95$ & 1101 & 37.48 & 10.72 & 26.76 & \\
\hline MWGS-33A & MW & 07/06/95 & 1053 & 37.48 & 10.64 & 26.84 & \\
\hline MWGS-33A & MW & $08 / 10 / 95$ & 1125 & 37.48 & 10.77 & 26.71 & \\
\hline MWGS-33A & MW & $09 / 13 / 95$ & 1020 & 37.48 & 9.68 & 27.80 & \\
\hline MWGS-33B & MW & $02 / 04 / 92$ & -- & 37.09 & 10.68 & 26.41 & Pre-start-up water levels. \\
\hline MWGS-33B & MW & $02 / 25 / 92$ & 0934 & 37.09 & 10.82 & 26.27 & \\
\hline MWGS-33B & MW & $02 / 25 / 92$ & 1525 & 37.09 & 11.34 & 25.75 & \\
\hline MWGS-33B & MW & $06 / 11 / 92$ & 1432 & 37.09 & 9.96 & 27.13 & \\
\hline MWGS-33B & MW & $07 / 07 / 92$ & 1645 & 37.09 & 10.48 & 26.61 & \\
\hline MWGS-33B & MW & $08 / 07 / 92$ & 1316 & 37.09 & 10.13 & 26.96 & \\
\hline MWGS-33B & MW & $08 / 31 / 92$ & 1138 & 37.09 & 9.05 & 28.04 & \\
\hline MWGS-33B & MW & $10 / 07 / 92$ & 1150 & 37.09 & 8.73 & 28.36 & \\
\hline MWGS-33B & MW & $10 / 28 / 92$ & 1308 & 37.09 & 9.70 & 27.39 & \\
\hline MWGS-33B & MW & $12 / 03 / 92$ & 1154 & 37.09 & 9.53 & 27.56 & \\
\hline MWGS-33B & MW & $01 / 04 / 93$ & 1125 & 37.09 & 10.31 & 26.78 & \\
\hline MWGS-33B & MW & $02 / 04 / 93$ & 1226 & 37.09 & 9.30 & 27.79 & \\
\hline MWGS-33B & MW & $03 / 05 / 93$ & 1134 & 37.09 & 9.67 & 27.42 & \\
\hline MWGS-33B & MW & $03 / 30 / 93$ & 1046 & 37.09 & 9.34 & 27.75 & \\
\hline MWGS-33B & MW & $05 / 06 / 93$ & 1113 & 37.09 & 10.04 & 27.05 & \\
\hline MWGS-33B & MW & $05 / 28 / 93$ & 1112 & 37.09 & 10.53 & 26.56 & \\
\hline MWGS-33B & MW & $07 / 07 / 93$ & 1100 & 37.09 & 10.24 & 26.85 & \\
\hline MWGS-33B & MW & $08 / 06 / 93$ & 1035 & 37.09 & 9.92 & 27.17 & \\
\hline MWGS-33B & MW & $09 / 03 / 93$ & 1104 & 37.09 & 10.22 & 26.87 & \\
\hline MWGS-33B & MW & $09 / 30 / 93$ & 1016 & 37.09 & 10.01 & 27.08 & \\
\hline MWGS-33B & MW & $10 / 28 / 93$ & 1104 & 37.09 & 11.03 & 26.06 & \\
\hline MWGS-33B & MW & $12 / 01 / 93$ & 1405 & 37.09 & 10.41 & 26.68 & \\
\hline MWGS-33B & MW & $01 / 06 / 94$ & 1102 & 37.09 & 9.58 & 27.51 & \\
\hline MWGS-33B & MW & $02 / 01 / 94$ & 1106 & 37.09 & 8.90 & 28.19 & \\
\hline MWGS-33B & MW & $03 / 04 / 94$ & 1039 & 37.09 & 9.07 & 28.02 & \\
\hline MWGS-33B & MW & $04 / 04 / 94$ & 1036 & 37.09 & 10.33 & 26.76 & \\
\hline MWGS-33B & MW & $05 / 06 / 94$ & 1124 & 37.09 & 10.97 & 26.12 & \\
\hline MWGS-33B & MW & $06 / 03 / 94$ & 1104 & 37.09 & 11.50 & 25.59 & \\
\hline MWGS-33B & MW & $07 / 08 / 94$ & 1117 & 37.09 & 8.61 & 28.48 & \\
\hline MWGS-33B & MW & $08 / 05 / 94$ & 1417 & 37.09 & 8.79 & 28.30 & \\
\hline MWGS-33B & MW & $09 / 07 / 94$ & 1238 & 37.09 & 8.97 & 28.12 & \\
\hline MWGS-33B & MW & $10 / 06 / 94$ & 1152 & 37.09 & 7.62 & 29.47 & \\
\hline MWGS-33B & MW & $11 / 09 / 94$ & 1307 & 37.09 & 8.96 & 28.13 & \\
\hline MWGS-33B & MW & $12 / 06 / 94$ & 1124 & 37.09 & 8.64 & 28.45 & \\
\hline MWGS-33B & MW & $01 / 10 / 95$ & 1340 & 37.09 & 9.06 & 28.03 & \\
\hline MWGS-33B & MW & $02 / 02 / 95$ & 1342 & 37.09 & 9.29 & 27.80 & \\
\hline MWGS-33B & MW & $03 / 02 / 95$ & 1513 & 37.09 & 9.32 & 27.77 & \\
\hline
\end{tabular}


Table 8.-Water-level data collected at the Defense Fuel Supply Point and adjacent properties, Hanahan, S.C., between April 1991 and September 1995--Continued

[ft, feet; ---, data not available; WL, water-level well; EW, extraction well; MW, monitoring well; SW, surface-water site; -, negative depth to water indicates water-level altitude is above measuring point]

\begin{tabular}{|c|c|c|c|c|c|c|c|}
\hline $\begin{array}{c}\text { Site } \\
\text { (plate 1) }\end{array}$ & $\begin{array}{l}\text { Site } \\
\text { type }\end{array}$ & Date & Time & $\begin{array}{l}\text { Measuring-point } \\
\text { altitude } \\
\text { (ft above } \\
\text { sea level) }\end{array}$ & $\begin{array}{l}\text { Depth to water } \\
\text { (ft below } \\
\text { measuring point) }\end{array}$ & $\begin{array}{l}\text { Water-level } \\
\text { altitude } \\
\text { (ft above } \\
\text { sea level) }\end{array}$ & Remarks \\
\hline MWGS-33B & MW & $04 / 05 / 95$ & 1112 & 37.09 & 10.12 & 26.97 & \\
\hline MWGS-33B & MW & $05 / 02 / 95$ & 1258 & 37.09 & 10.52 & 26.57 & \\
\hline MWGS-33B & MW & $06 / 09 / 95$ & 1103 & 37.09 & 10.30 & 26.79 & \\
\hline MWGS-33B & MW & $07 / 06 / 95$ & 1055 & 37.09 & 10.23 & 26.86 & \\
\hline MWGS-33B & MW & $08 / 10 / 95$ & 1127 & 37.09 & 10.36 & 26.73 & \\
\hline MWGS-33B & MW & $09 / 13 / 95$ & 1022 & 37.09 & 9.27 & 27.82 & \\
\hline MWGS-34A & MW & $02 / 04 / 92$ & -- & 36.60 & 9.80 & 26.80 & Pre-start-up water levels. \\
\hline MWGS-34A & MW & $02 / 25 / 92$ & 0925 & 36.60 & 9.93 & 26.67 & \\
\hline MWGS-34A & MW & $06 / 11 / 92$ & 1439 & 36.60 & 9.45 & 27.15 & \\
\hline MWGS-34A & MW & $07 / 07 / 92$ & 1617 & 36.60 & 9.65 & 26.95 & \\
\hline MWGS-34A & MW & $08 / 07 / 92$ & 1319 & 36.60 & 9.48 & 27.12 & \\
\hline MWGS-34A & MW & $08 / 31 / 92$ & 1155 & 36.60 & 8.25 & 28.35 & \\
\hline MWGS-34A & MW & $10 / 07 / 92$ & 1206 & 36.60 & 8.07 & 28.53 & \\
\hline MWGS-34A & MW & $10 / 28 / 92$ & 1318 & 36.60 & 8.96 & 27.64 & \\
\hline MWGS-34A & MW & $12 / 03 / 92$ & 1203 & 36.60 & 8.70 & 27.90 & \\
\hline MWGS-34A & MW & $01 / 04 / 93$ & 1142 & 36.60 & 9.47 & 27.13 & \\
\hline MWGS-34A & MW & $02 / 04 / 93$ & 1240 & 36.60 & 8.47 & 28.13 & \\
\hline MWGS-34A & MW & $03 / 05 / 93$ & 1149 & 36.60 & 8.82 & 27.78 & \\
\hline MWGS-34A & MW & 03/30/93 & 1110 & 36.60 & 8.43 & 28.17 & \\
\hline MWGS-34A & MW & $05 / 06 / 93$ & 1127 & 36.60 & 9.28 & 27.32 & \\
\hline MWGS-34A & MW & $05 / 28 / 93$ & 1124 & 36.60 & 9.70 & 26.90 & \\
\hline MWGS-34A & MW & $07 / 07 / 93$ & 1113 & 36.60 & 9.60 & 27.00 & \\
\hline MWGS-34A & MW & $08 / 06 / 93$ & 1051 & 36.60 & 9.26 & 27.34 & \\
\hline MWGS-34A & MW & $09 / 03 / 93$ & 1110 & 36.60 & 9.47 & 27.13 & \\
\hline MWGS-34A & MW & $09 / 30 / 93$ & 0952 & 36.60 & 9.27 & 27.33 & \\
\hline MWGS-34A & MW & $10 / 28 / 93$ & 1105 & 36.60 & 10.16 & 26.44 & \\
\hline MWGS-34A & MW & $12 / 01 / 93$ & 1419 & 36.60 & 9.70 & 26.90 & \\
\hline MWGS-34A & MW & $01 / 06 / 94$ & 1125 & 36.60 & 9.09 & 27.51 & \\
\hline MWGS-34A & MW & $02 / 01 / 94$ & 1124 & 36.60 & 8.34 & 28.26 & \\
\hline MWGS-34A & MW & $03 / 04 / 94$ & 1052 & 36.60 & 8.55 & 28.05 & \\
\hline MWGS-34A & MW & $04 / 04 / 94$ & 1051 & 36.60 & 9.74 & 26.86 & \\
\hline MWGS-34A & MW & $05 / 06 / 94$ & 1143 & 36.60 & 10.35 & 26.25 & \\
\hline MWGS-34A & MW & $06 / 03 / 94$ & 1135 & 36.60 & 10.85 & 25.75 & \\
\hline MWGS-34A & MW & $07 / 08 / 94$ & 1138 & 36.60 & 8.40 & 28.20 & \\
\hline MWGS-34A & MW & $08 / 05 / 94$ & 1425 & 36.60 & 8.42 & 28.18 & \\
\hline MWGS-34A & MW & $09 / 07 / 94$ & 1300 & 36.60 & 8.57 & 28.03 & \\
\hline MWGS-34A & MW & $10 / 06 / 94$ & 1211 & 36.60 & 6.99 & 29.61 & \\
\hline MWGS-34A & MW & $11 / 09 / 94$ & 1317 & 36.60 & 8.50 & 28.10 & \\
\hline MWGS-34A & MW & $12 / 06 / 94$ & 1136 & 36.60 & 8.35 & 28.25 & \\
\hline MWGS-34A & MW & $01 / 10 / 95$ & 1346 & 36.60 & 8.68 & 27.92 & \\
\hline MWGS-34A & MW & $02 / 02 / 95$ & 1355 & 36.60 & 8.81 & 27.79 & \\
\hline MWGS-34A & MW & $03 / 02 / 95$ & 1525 & 36.60 & 8.80 & 27.80 & \\
\hline MWGS-34A & MW & $04 / 05 / 95$ & 1135 & 36.60 & 9.55 & 27.05 & \\
\hline MWGS-34A & MW & $05 / 02 / 95$ & 1318 & 36.60 & 9.94 & 26.66 & \\
\hline
\end{tabular}


Table 8.--Water-level data collected at the Defense Fuel Supply Point and adjacent properties, Hanahan, S.C., between April 1991 and September 1995--Continued

[ft, feet; ---, data not available; WL, water-level well; EW, extraction well; MW, monitoring well; SW, surface-water site; -, negative depth to wateindicates water-level altitude is above measuring point]

\begin{tabular}{|c|c|c|c|c|c|c|c|}
\hline $\begin{array}{c}\text { Site } \\
\text { (plate 1) }\end{array}$ & $\begin{array}{l}\text { Site } \\
\text { type }\end{array}$ & Date & Time & $\begin{array}{l}\text { Measuring-point } \\
\text { altitude } \\
\text { (ft above } \\
\text { sea level) }\end{array}$ & $\begin{array}{l}\text { Depth to water } \\
\text { (ft below } \\
\text { measuring point) }\end{array}$ & $\begin{array}{l}\text { Water-level } \\
\text { altitude } \\
\text { (ft above } \\
\text { sea level) }\end{array}$ & Remarks \\
\hline MWGS-34A & $\overline{M W}$ & $06 / 09 / 95$ & 1118 & 36.60 & 9.79 & 26.81 & \\
\hline MWGS-34A & MW & $07 / 06 / 95$ & 1105 & 36.60 & 9.68 & 26.92 & \\
\hline MWGS-34A & MW & $08 / 10 / 95$ & 1251 & 36.60 & 9.73 & 26.87 & \\
\hline MWGS-34A & MW & $09 / 13 / 95$ & 1204 & 36.60 & 8.74 & 27.86 & \\
\hline MWGS-34B & MW & $02 / 04 / 92$ & -- & 36.56 & 9.77 & 26.79 & Pre-start-up water levels. \\
\hline MWGS-34B & MW & $02 / 25 / 92$ & 0927 & 36.56 & 9.94 & 26.62 & \\
\hline MWGS-34B & MW & $06 / 11 / 92$ & 1441 & 36.56 & 9.41 & 27.15 & \\
\hline MWGS-34B & MW & $07 / 07 / 92$ & 1615 & 36.56 & 9.62 & 26.94 & \\
\hline MWGS-34B & MW & $08 / 07 / 92$ & 1321 & 36.56 & 9.46 & 27.10 & \\
\hline MWGS-34B & MW & $08 / 31 / 92$ & 1158 & 36.56 & 8.24 & 28.32 & \\
\hline MWGS-34B & MW & $10 / 07 / 92$ & 1210 & 36.56 & 8.06 & 28.50 & \\
\hline MWGS-34B & MW & $10 / 28 / 92$ & 1320 & 36.56 & 8.95 & 27.61 & \\
\hline MWGS-34B & MW & $12 / 03 / 92$ & 1201 & 36.56 & 8.69 & 27.87 & \\
\hline MWGS-34B & MW & $01 / 04 / 93$ & 1140 & 36.56 & 9.45 & 27.11 & \\
\hline MWGS-34B & MW & $02 / 04 / 93$ & 1242 & 36.56 & 8.46 & 28.10 & \\
\hline MWGS-34B & MW & $03 / 05 / 93$ & 1152 & 36.56 & 8.80 & 27.76 & \\
\hline MWGS-34B & MW & $03 / 30 / 93$ & 1112 & 36.56 & 8.40 & 28.16 & \\
\hline MWGS-34B & MW & $05 / 06 / 93$ & 1129 & 36.56 & 9.27 & 27.29 & \\
\hline MWGS-34B & MW & $05 / 28 / 93$ & 1125 & 36.56 & 9.68 & 26.88 & \\
\hline MWGS-34B & MW & $07 / 07 / 93$ & 1114 & 36.56 & 9.58 & 26.98 & \\
\hline MWGS-34B & MW & $08 / 06 / 93$ & 1054 & 36.56 & 9.25 & 27.31 & \\
\hline MWGS-34B & MW & $09 / 03 / 93$ & 1112 & 36.56 & 9.46 & 27.10 & \\
\hline MWGS-34B & MW & $09 / 30 / 93$ & 0948 & 36.56 & 9.26 & 27.30 & \\
\hline MWGS-34B & MW & $10 / 28 / 93$ & 1109 & 36.56 & 10.23 & 26.33 & \\
\hline MWGS-34B & MW & $12 / 01 / 93$ & 1417 & 36.56 & 9.76 & 26.80 & \\
\hline MWGS-34B & MW & $01 / 06 / 94$ & 1128 & 36.56 & 9.07 & 27.49 & \\
\hline MWGS-34B & MW & $02 / 01 / 94$ & 1127 & 36.56 & 8.35 & 28.21 & \\
\hline MWGS-34B & MW & $03 / 04 / 94$ & 1054 & 36.56 & 8.60 & 27.96 & \\
\hline MWGS-34B & MW & $04 / 04 / 94$ & 1053 & 36.56 & 9.86 & 26.70 & \\
\hline MWGS-34B & MW & $05 / 06 / 94$ & 1144 & 36.56 & 10.43 & 26.13 & \\
\hline MWGS-34B & MW & $06 / 03 / 94$ & 1137 & 36.56 & 10.90 & 25.66 & \\
\hline MWGS-34B & MW & $07 / 08 / 94$ & 1140 & 36.56 & 8.47 & 28.09 & \\
\hline MWGS-34B & MW & $08 / 05 / 94$ & 1427 & 36.56 & 8.52 & 28.04 & \\
\hline MWGS-34B & MW & $09 / 07 / 94$ & 1302 & 36.56 & 8.69 & 27.87 & \\
\hline MWGS-34B & MW & $10 / 06 / 94$ & 1212 & 36.56 & 7.03 & 29.53 & \\
\hline MWGS-34B & MW & $11 / 09 / 94$ & 1320 & 36.56 & 8.59 & 27.97 & \\
\hline MWGS-34B & MW & $12 / 06 / 94$ & 1140 & 36.56 & 8.48 & 28.08 & \\
\hline MWGS-34B & MW & $01 / 10 / 95$ & 1348 & 36.56 & 8.78 & 27.78 & \\
\hline MWGS-34B & MW & $02 / 02 / 95$ & 1356 & 36.56 & 8.91 & 27.65 & \\
\hline MWGS-34B & MW & $03 / 02 / 95$ & 1526 & 36.56 & 8.92 & 27.64 & \\
\hline MWGS-34B & MW & $04 / 05 / 95$ & 1136 & 36.56 & 9.65 & 26.91 & \\
\hline MWGS-34B & MW & $05 / 02 / 95$ & 1320 & 36.56 & 10.01 & 26.55 & \\
\hline MWGS-34B & MW & $06 / 09 / 95$ & 1120 & 36.56 & 9.85 & 26.71 & \\
\hline MWGS-34B & MW & $07 / 06 / 95$ & 1107 & 36.56 & 9.74 & 26.82 & \\
\hline
\end{tabular}


Table 8.--Water-level data collected at the Defense Fuel Supply Point and adjacent properties, Hanahan, S.C., between April 1991 and September 1995--Continued

[ft, feet; ---, data not available; WL, water-level well; EW, extraction well; MW, monitoring well; SW, surface-water site; -, negative depth to water indicates water-level altitude is above measuring point]

\begin{tabular}{|c|c|c|c|c|c|c|c|}
\hline $\begin{array}{c}\text { Site } \\
\text { (plate 1) }\end{array}$ & $\begin{array}{l}\text { Site } \\
\text { type }\end{array}$ & Date & Time & $\begin{array}{l}\text { Measuring-point } \\
\text { altitude } \\
\text { ( } \mathrm{ft} \text { above } \\
\text { sea level) }\end{array}$ & $\begin{array}{l}\text { Depth to water } \\
\text { (ft below } \\
\text { measuring point) }\end{array}$ & $\begin{array}{l}\text { Water-level } \\
\text { altitude } \\
\text { (ft above } \\
\text { sea level) }\end{array}$ & Remarks \\
\hline MWGS-34B & MW & $08 / 10 / 95$ & 1255 & 36.56 & 9.71 & 26.85 & \\
\hline MWGS-34B & MW & $09 / 13 / 95$ & 1205 & 36.56 & 8.88 & 27.68 & \\
\hline MWGS-35 & MW & $05 / 14 / 92$ & 1005 & 37.04 & 15.41 & 21.63 & \\
\hline MWGS-35 & MW & $06 / 11 / 92$ & 1105 & 37.04 & 16.02 & 21.02 & \\
\hline MWGS-35 & MW & $07 / 07 / 92$ & 1815 & 37.04 & 15.06 & 21.98 & \\
\hline MWGS-35 & MW & $08 / 07 / 92$ & 0949 & 37.04 & 15.47 & 21.57 & Petroleum odor. \\
\hline MWGS-35 & MW & $08 / 31 / 92$ & 1031 & 37.04 & 12.60 & 24.44 & \\
\hline MWGS-35 & MW & $10 / 07 / 92$ & 1038 & 37.04 & 12.49 & 24.55 & \\
\hline MWGS-35 & MW & $10 / 28 / 92$ & 1107 & 37.04 & 13.08 & 23.96 & \\
\hline MWGS-35 & MW & $12 / 03 / 92$ & 0956 & 37.04 & 13.25 & 23.79 & \\
\hline MWGS-35 & MW & $01 / 04 / 93$ & 1225 & 37.04 & 14.12 & 22.92 & \\
\hline MWGS-35 & MW & $02 / 04 / 93$ & 1036 & 37.04 & 12.38 & 24.66 & \\
\hline MWGS-35 & MW & $03 / 05 / 93$ & 1019 & 37.04 & 13.12 & 23.92 & \\
\hline MWGS-35 & MW & $03 / 30 / 93$ & 0934 & 37.04 & 14.27 & 22.77 & \\
\hline MWGS-35 & MW & $05 / 06 / 93$ & 1004 & 37.04 & 14.68 & 22.36 & \\
\hline MWGS-35 & MW & $05 / 28 / 93$ & 1007 & 37.04 & 15.40 & 21.64 & \\
\hline MWGS-35 & MW & $07 / 07 / 93$ & 0956 & 37.04 & 15.38 & 21.66 & \\
\hline MWGS-35 & MW & $08 / 06 / 93$ & 0947 & 37.04 & 15.74 & 21.30 & \\
\hline MWGS-35 & MW & $09 / 03 / 93$ & 1006 & 37.04 & 15.25 & 21.79 & \\
\hline MWGS-35 & MW & $09 / 30 / 93$ & 1131 & 37.04 & 14.95 & 22.09 & \\
\hline MWGS-35 & MW & $10 / 28 / 93$ & 0955 & 37.04 & 15.89 & 21.15 & \\
\hline MWGS-35 & MW & $12 / 01 / 93$ & 0958 & 37.04 & 15.95 & 21.09 & \\
\hline MWGS-35 & MW & $01 / 06 / 94$ & 1010 & 37.04 & 16.27 & 20.77 & \\
\hline MWGS-35 & MW & $02 / 01 / 94$ & 0958 & 37.04 & 15.17 & 21.87 & \\
\hline MWGS-35 & MW & $03 / 04 / 94$ & 0954 & 37.04 & 14.74 & 22.30 & \\
\hline MWGS-35 & MW & $04 / 04 / 94$ & 0945 & 37.04 & 14.98 & 22.06 & \\
\hline MWGS-35 & MW & $05 / 06 / 94$ & 1036 & 37.04 & 15.99 & 21.05 & \\
\hline MWGS-35 & MW & $06 / 03 / 94$ & 1005 & 37.04 & 16.67 & 20.37 & \\
\hline MWGS-35 & MW & $07 / 08 / 94$ & 1007 & 37.04 & 15.49 & 21.55 & \\
\hline MWGS-35 & MW & $08 / 05 / 94$ & 1305 & 37.04 & 13.60 & 23.44 & \\
\hline MWGS-35 & MW & $09 / 07 / 94$ & 1015 & 37.04 & -- & -- & $0.01 \mathrm{ft}$ of free product in well. \\
\hline MWGS-35 & MW & $10 / 06 / 94$ & 1033 & 37.04 & 10.84 & 26.20 & \\
\hline MWGS-35 & MW & $11 / 09 / 94$ & 1212 & 37.04 & 12.32 & 24.72 & \\
\hline MWGS-35 & MW & $12 / 06 / 94$ & 1014 & 37.04 & 13.35 & 23.69 & \\
\hline MWGS-35 & MW & $01 / 10 / 95$ & 1212 & 37.04 & 13.26 & 23.78 & \\
\hline MWGS-35 & MW & $02 / 02 / 95$ & 1253 & 37.04 & 13.07 & 23.97 & \\
\hline MWGS-35 & MW & $03 / 02 / 95$ & 1411 & 37.04 & 13.19 & 23.85 & \\
\hline MWGS-35 & MW & $04 / 05 / 95$ & 1010 & 37.04 & 14.09 & 22.95 & \\
\hline MWGS-35 & MW & $05 / 02 / 95$ & 1110 & 37.04 & 14.72 & 22.32 & \\
\hline MWGS-35 & MW & $06 / 09 / 95$ & 1012 & 37.04 & 15.63 & 21.41 & \\
\hline MWGS-35 & MW & $07 / 06 / 95$ & 1002 & 37.04 & 15.46 & 21.58 & \\
\hline MWGS-35 & MW & $08 / 10 / 95$ & 1040 & 37.04 & 14.94 & 22.10 & \\
\hline MWGS-35 & MW & $09 / 13 / 95$ & -- & 37.04 & --- & -- & Free product present in well. \\
\hline
\end{tabular}


Table 8.--Water-level data collected at the Defense Fuel Supply Point and adjacent properties, Hanahan, S.C., between April 1991 and September 1995--Continued

[ft, feet; ---, data not available; WL, water-level well; EW, extraction well; MW, monitoring well; SW, surface-water site; -, negative depth to wateindicates water-level altitude is above measuring point]

\begin{tabular}{|c|c|c|c|c|c|c|c|}
\hline $\begin{array}{c}\text { Site } \\
\text { (plate 1) }\end{array}$ & $\begin{array}{l}\text { Site } \\
\text { type }\end{array}$ & Date & Time & $\begin{array}{l}\text { Measuring-point } \\
\text { altitude } \\
\text { (ft above } \\
\text { sea level) }\end{array}$ & $\begin{array}{l}\text { Depth to water } \\
\text { (ft below } \\
\text { measuring point) }\end{array}$ & $\begin{array}{l}\text { Water-level } \\
\text { altitude } \\
\text { (ft above } \\
\text { sea level) }\end{array}$ & Remarks \\
\hline MWGS-36 & MW & $05 / 14 / 92$ & 1007 & 37.02 & 14.85 & 22.17 & \\
\hline MWGS-36 & MW & $06 / 11 / 92$ & 1110 & 37.02 & 15.44 & 21.58 & \\
\hline MWGS-36 & MW & $07 / 07 / 92$ & 1810 & 37.02 & 14.43 & 22.59 & \\
\hline MWGS-36 & MW & $08 / 07 / 92$ & 0947 & 37.02 & 14.84 & 22.18 & \\
\hline MWGS-36 & MW & $08 / 31 / 92$ & 1030 & 37.02 & 12.00 & 25.02 & \\
\hline MWGS-36 & MW & $10 / 07 / 92$ & 1033 & 37.02 & 11.86 & 25.16 & \\
\hline MWGS-36 & MW & $10 / 28 / 92$ & 1103 & 37.02 & 12.43 & 24.59 & \\
\hline MWGS-36 & MW & $12 / 03 / 92$ & 0951 & 37.02 & 12.59 & 24.43 & \\
\hline MWGS-36 & MW & $01 / 04 / 93$ & 1006 & 37.02 & 13.55 & 23.47 & \\
\hline MWGS-36 & MW & $02 / 04 / 93$ & 1032 & 37.02 & 11.65 & 25.37 & \\
\hline MWGS-36 & MW & $03 / 05 / 93$ & 1017 & 37.02 & 12.45 & 24.57 & \\
\hline MWGS-36 & MW & $03 / 30 / 93$ & 0931 & 37.02 & 13.33 & 23.69 & \\
\hline MWGS-36 & MW & $05 / 06 / 93$ & 1002 & 37.02 & 13.85 & 23.17 & \\
\hline MWGS-36 & MW & $05 / 28 / 93$ & 1005 & 37.02 & 14.60 & 22.42 & \\
\hline MWGS-36 & MW & 07/07/93 & 0953 & 37.02 & 14.83 & 22.19 & \\
\hline MWGS-36 & MW & $08 / 06 / 93$ & 0943 & 37.02 & 15.20 & 21.82 & \\
\hline MWGS-36 & MW & $09 / 03 / 93$ & 1004 & 37.02 & 14.61 & 22.41 & \\
\hline MWGS-36 & MW & $09 / 30 / 93$ & 1134 & 37.02 & 14.33 & 22.69 & \\
\hline MWGS-36 & MW & $10 / 28 / 93$ & 0951 & 37.02 & 15.29 & 21.73 & \\
\hline MWGS-36 & MW & $12 / 01 / 93$ & 0955 & 37.02 & 15.40 & 21.62 & \\
\hline MWGS-36 & MW & $01 / 06 / 94$ & 1007 & 37.02 & 15.78 & 21.24 & \\
\hline MWGS-36 & MW & $02 / 01 / 94$ & 0954 & 37.02 & 14.68 & 22.34 & \\
\hline MWGS-36 & MW & $03 / 04 / 94$ & 0948 & 37.02 & 14.21 & 22.81 & \\
\hline MWGS-36 & MW & $04 / 04 / 94$ & 0942 & 37.02 & 14.42 & 22.60 & \\
\hline MWGS-36 & MW & $05 / 06 / 94$ & 1033 & 37.02 & 15.46 & 21.56 & \\
\hline MWGS-36 & MW & $06 / 03 / 94$ & 1002 & 37.02 & 16.18 & 20.84 & \\
\hline MWGS-36 & MW & $07 / 08 / 94$ & 1005 & 37.02 & 15.05 & 21.97 & \\
\hline MWGS-36 & MW & $08 / 05 / 94$ & 1302 & 37.02 & 13.06 & 23.96 & \\
\hline MWGS-36 & MW & $09 / 07 / 94$ & 1010 & 37.02 & 12.61 & 24.41 & \\
\hline MWGS-36 & MW & $10 / 06 / 94$ & 1028 & 37.02 & 10.03 & 26.99 & \\
\hline MWGS-36 & MW & $11 / 09 / 94$ & 1210 & 37.02 & --- & --- & Free product present in well. \\
\hline MWGS-36 & MW & $12 / 06 / 94$ & 1010 & 37.02 & -- & --- & Free product present in well. \\
\hline MWGS-36 & MW & $01 / 10 / 95$ & 1210 & 37.02 & 12.56 & 24.46 & \\
\hline MWGS-36 & MW & $02 / 02 / 95$ & 1255 & 37.02 & 12.37 & 24.65 & \\
\hline MWGS-36 & MW & $03 / 02 / 95$ & --- & 37.02 & -- & -- & Sheen on water surface; petroleum odor. \\
\hline MWGS-36 & MW & $04 / 05 / 95$ & 1006 & 37.02 & --- & --- & Free product present in well. \\
\hline MWGS-36 & MW & $05 / 02 / 95$ & 1107 & 37.02 & 14.18 & 22.84 & \\
\hline MWGS-36 & MW & 06/09/95 & 1009 & 37.02 &.-- & --- & Free product present in well. \\
\hline MWGS-36 & MW & $07 / 06 / 95$ & -- & 37.02 & -- & -- & Free product present in well. \\
\hline MWGS-36 & MW & $08 / 10 / 95$ & -- & 37.02 & $\cdots$ & --- & Free product present in well. \\
\hline MWGS-36 & MW & $09 / 13 / 95$ & -- & 37.02 & -- & -- & Free product present in well. \\
\hline MWGS-37 & MW & $05 / 14 / 92$ & 1000 & 37.00 & 16.55 & 20.45 & \\
\hline MWGS-37 & MW & $06 / 11 / 92$ & 1113 & 37.00 & 17.42 & 19.58 & \\
\hline MWGS-37 & MW & 07/07/92 & 1820 & 37.00 & 16.40 & 20.60 & \\
\hline
\end{tabular}


Table 8.--Water-level data collected at the Defense Fuel Supply Point and adjacent properties, Hanahan, S.C., between April 1991 and September 1995--Continued

[ft, feet; ---, data not available; WL, water-level well; EW, extraction well; MW, monitoring well; SW, surface-water site; -, negative depth to water indicates water-level altitude is above measuring point]

\begin{tabular}{|c|c|c|c|c|c|c|c|}
\hline $\begin{array}{c}\text { Site } \\
\text { (plate 1) }\end{array}$ & $\begin{array}{l}\text { Site } \\
\text { type }\end{array}$ & Date & Time & $\begin{array}{l}\text { Measuring-point } \\
\text { altitude } \\
\text { (ft above } \\
\text { sea level) }\end{array}$ & $\begin{array}{l}\text { Depth to water } \\
\text { (ft below } \\
\text { measuring point) }\end{array}$ & $\begin{array}{l}\text { Water-level } \\
\text { altitude } \\
\text { (ft above } \\
\text { sea level) }\end{array}$ & Remarks \\
\hline MWGS-37 & MW & $08 / 07 / 92$ & 0952 & 37.00 & 16.92 & 20.08 & \\
\hline MWGS-37 & MW & $08 / 31 / 92$ & 1034 & 37.00 & 13.73 & 23.27 & \\
\hline MWGS-37 & MW & $10 / 07 / 92$ & 1041 & 37.00 & 13.34 & 23.66 & \\
\hline MWGS-37 & MW & $10 / 28 / 92$ & 1115 & 37.00 & 14.14 & 22.86 & \\
\hline MWGS-37 & MW & $12 / 03 / 92$ & 1007 & 37.00 & 14.25 & 22.75 & \\
\hline MWGS-37 & MW & $01 / 04 / 93$ & 1231 & 37.00 & 15.33 & 21.67 & \\
\hline MWGS-37 & MW & $02 / 04 / 93$ & 1040 & 37.00 & 13.30 & 23.70 & \\
\hline MWGS-37 & MW & $03 / 05 / 93$ & 1025 & 37.00 & 14.17 & 22.83 & \\
\hline MWGS-37 & MW & $03 / 30 / 93$ & 0940 & 37.00 & 15.38 & 21.62 & \\
\hline MWGS-37 & MW & $05 / 06 / 93$ & 1006 & 37.00 & 15.83 & 21.17 & \\
\hline MWGS-37 & MW & $05 / 28 / 93$ & 1009 & 37.00 & 16.60 & 20.40 & \\
\hline MWGS-37 & MW & $07 / 07 / 93$ & 0958 & 37.00 & 16.46 & 20.54 & \\
\hline MWGS-37 & MW & $08 / 06 / 93$ & 0950 & 37.00 & 16.86 & 20.14 & \\
\hline MWGS-37 & MW & $09 / 03 / 93$ & 1009 & 37.00 & 16.39 & 20.61 & \\
\hline MWGS-37 & MW & $09 / 30 / 93$ & 1129 & 37.00 & 16.05 & 20.95 & \\
\hline MWGS-37 & MW & $10 / 28 / 93$ & 0958 & 37.00 & 17.42 & 19.58 & \\
\hline MWGS-37 & MW & $12 / 01 / 93$ & 1001 & 37.00 & 17.20 & 19.80 & \\
\hline MWGS-37 & MW & $01 / 06 / 94$ & 1014 & 37.00 & 17.80 & 19.20 & \\
\hline MWGS-37 & MW & $02 / 01 / 94$ & 1008 & 37.00 & 16.05 & 20.95 & \\
\hline MWGS-37 & MW & $03 / 04 / 94$ & 0956 & 37.00 & 15.83 & 21.17 & \\
\hline MWGS-37 & MW & $04 / 04 / 94$ & 0948 & 37.00 & 16.05 & 20.95 & \\
\hline MWGS-37 & MW & $05 / 06 / 94$ & 1038 & 37.00 & 17.53 & 19.47 & \\
\hline MWGS-37 & MW & $06 / 03 / 94$ & 1008 & 37.00 & 18.26 & 18.74 & \\
\hline MWGS-37 & MW & $07 / 08 / 94$ & 1010 & 37.00 & 15.37 & 21.63 & \\
\hline MWGS-37 & MW & $08 / 05 / 94$ & 1300 & 37.00 & 14.67 & 22.33 & \\
\hline MWGS-37 & MW & $09 / 07 / 94$ & 1020 & 37.00 & 14.45 & 22.55 & \\
\hline MWGS-37 & MW & $10 / 06 / 94$ & 1036 & 37.00 & 10.60 & 26.40 & \\
\hline MWGS-37 & MW & $11 / 09 / 94$ & 1215 & 37.00 & 13.16 & 23.84 & \\
\hline MWGS-37 & MW & $12 / 06 / 94$ & 1016 & 37.00 & 14.52 & 22.48 & \\
\hline MWGS-37 & MW & $01 / 10 / 95$ & 1215 & 37.00 & 14.17 & 22.83 & \\
\hline MWGS-37 & MW & $02 / 02 / 95$ & 1300 & 37.00 & 13.92 & 23.08 & \\
\hline MWGS-37 & MW & $03 / 02 / 95$ & 1415 & 37.00 & 14.15 & 22.85 & \\
\hline MWGS-37 & MW & $04 / 05 / 95$ & 1014 & 37.00 & 15.24 & 21.76 & \\
\hline MWGS-37 & MW & $05 / 02 / 95$ & 1112 & 37.00 & 15.92 & 21.08 & \\
\hline MWGS-37 & MW & $06 / 09 / 95$ & 1015 & 37.00 & 16.97 & 20.03 & \\
\hline MWGS-37 & MW & $07 / 06 / 95$ & 1006 & 37.00 & 15.83 & 21.17 & \\
\hline MWGS-37 & MW & $08 / 10 / 95$ & 1045 & 37.00 & 15.73 & 21.27 & \\
\hline MWGS-37 & MW & $09 / 13 / 95$ & 1123 & 37.00 & 13.50 & 23.50 & \\
\hline MWGS-38 & MW & $05 / 14 / 92$ & 0958 & 34.82 & 15.30 & 19.52 & \\
\hline MWGS-38 & MW & $06 / 11 / 92$ & 1120 & 34.82 & 16.25 & 18.57 & \\
\hline MWGS-38 & MW & $07 / 07 / 92$ & 1857 & 34.82 & 15.29 & 19.53 & \\
\hline MWGS-38 & MW & $08 / 07 / 92$ & 0958 & 34.82 & 15.91 & 18.91 & \\
\hline MWGS-38 & MW & $08 / 31 / 92$ & 1038 & 34.82 & 12.95 & 21.87 & \\
\hline MWGS-38 & MW & $10 / 07 / 92$ & 1045 & 34.82 & 12.52 & 22.30 & \\
\hline
\end{tabular}


Table 8.--Water-level data collected at the Defense Fuel Supply Point and adjacent properties, Hanahan, S.C., between April 1991 and September 1995--Continued

[ft, feet; ---, data not available; WL, water-level well; EW, extraction well; MW, monitoring well; SW, surface-water site; -, negative depth to wateindicates water-level altitude is above measuring point]

\begin{tabular}{|c|c|c|c|c|c|c|c|}
\hline $\begin{array}{c}\text { Site } \\
\text { (plate 1) }\end{array}$ & $\begin{array}{l}\text { Site } \\
\text { type }\end{array}$ & Date & Time & $\begin{array}{l}\text { Measuring-point } \\
\text { altitude } \\
\text { (ft above } \\
\text { sea level) }\end{array}$ & $\begin{array}{l}\text { Depth to water } \\
\text { (ft below } \\
\text { measuring point) }\end{array}$ & $\begin{array}{l}\text { Water-level } \\
\text { altitude } \\
\text { (ft above } \\
\text { sea level) }\end{array}$ & Remarks \\
\hline MWGS-38 & MW & $10 / 28 / 92$ & 1120 & 34.82 & 13.19 & 21.63 & \\
\hline MWGS-38 & MW & $12 / 03 / 92$ & 1016 & 34.82 & 13.27 & 21.55 & \\
\hline MWGS-38 & MW & $01 / 04 / 93$ & 1228 & 34.82 & 14.22 & 20.60 & \\
\hline MWGS-38 & MW & $02 / 04 / 93$ & 1048 & 34.82 & 12.30 & 22.52 & \\
\hline MWGS-38 & MW & $03 / 05 / 93$ & 1031 & 34.82 & 13.12 & 21.70 & \\
\hline MWGS-38 & MW & $03 / 30 / 93$ & 0942 & 34.82 & 14.44 & 20.38 & \\
\hline MWGS-38 & MW & $05 / 06 / 93$ & 1008 & 34.82 & 14.54 & 20.28 & \\
\hline MWGS-38 & MW & $05 / 28 / 93$ & 1012 & 34.82 & 15.27 & 19.55 & \\
\hline MWGS-38 & MW & $07 / 07 / 93$ & 1001 & 34.82 & 15.40 & 19.42 & \\
\hline MWGS-38 & MW & $08 / 06 / 93$ & 0953 & 34.82 & 16.00 & 18.82 & \\
\hline MWGS-38 & MW & $09 / 03 / 93$ & 1013 & 34.82 & 15.80 & 19.02 & \\
\hline MWGS-38 & MW & $09 / 30 / 93$ & 1126 & 34.82 & 15.55 & 19.27 & \\
\hline MWGS-38 & MW & $10 / 28 / 93$ & 1001 & 34.82 & 16.40 & 18.42 & \\
\hline MWGS-38 & MW & $12 / 01 / 93$ & 1004 & 34.82 & 16.46 & 18.36 & \\
\hline MWGS-38 & MW & $01 / 06 / 94$ & 1018 & 34.82 & 16.60 & 18.22 & \\
\hline MWGS-38 & MW & $02 / 01 / 94$ & 1014 & 34.82 & 15.45 & 19.37 & \\
\hline MWGS-38 & MW & $03 / 04 / 94$ & 1000 & 34.82 & 14.92 & 19.90 & \\
\hline MWGS-38 & MW & $04 / 04 / 94$ & 0951 & 34.82 & 15.43 & 19.39 & \\
\hline MWGS-38 & MW & $05 / 06 / 94$ & 1040 & 34.82 & 16.47 & 18.35 & \\
\hline MWGS-38 & MW & $06 / 03 / 94$ & 1010 & 34.82 & 17.15 & 17.67 & \\
\hline MWGS-38 & MW & $07 / 08 / 94$ & 1014 & 34.82 & 15.77 & 19.05 & \\
\hline MWGS-38 & MW & $08 / 05 / 94$ & 1309 & 34.82 & 14.11 & 20.71 & \\
\hline MWGS-38 & MW & $09 / 07 / 94$ & 1025 & 34.82 & 13.75 & 21.07 & \\
\hline MWGS-38 & MW & $10 / 06 / 94$ & 1039 & 34.82 & 11.14 & 23.68 & \\
\hline MWGS-38 & MW & $11 / 09 / 94$ & 1225 & 34.82 & 12.55 & 22.27 & \\
\hline MWGS-38 & MW & $12 / 06 / 94$ & 1028 & 34.82 & 13.61 & 21.21 & \\
\hline MWGS-38 & MW & $01 / 10 / 95$ & 1219 & 34.82 & 13.42 & 21.40 & \\
\hline MWGS-38 & MW & $02 / 02 / 95$ & 1305 & 34.82 & 13.23 & 21.59 & \\
\hline MWGS-38 & MW & $03 / 02 / 95$ & 1419 & 34.82 & 13.36 & 21.46 & \\
\hline MWGS-38 & MW & $04 / 05 / 95$ & 1016 & 34.82 & 14.27 & 20.55 & \\
\hline MWGS-38 & MW & $05 / 02 / 95$ & 1115 & 34.82 & 14.94 & 19.88 & \\
\hline MWGS-38 & MW & $06 / 09 / 95$ & 1018 & 34.82 & 15.78 & 19.04 & \\
\hline MWGS-38 & MW & $07 / 06 / 95$ & 1010 & 34.82 & 15.66 & 19.16 & \\
\hline MWGS-38 & MW & $08 / 10 / 95$ & 1047 & 34.82 & 15.32 & 19.50 & \\
\hline MWGS-38 & MW & $09 / 13 / 95$ & 1126 & 34.82 & 13.07 & 21.75 & \\
\hline MWGS-39 & MW & $05 / 01 / 92$ & 1531 & 38.30 & 17.05 & 21.25 & \\
\hline MWGS-39 & MW & $05 / 01 / 92$ & 1030 & 38.30 & 17.09 & 21.21 & \\
\hline MWGS-39 & MW & $05 / 14 / 92$ & 1203 & 38.30 & 17.31 & 20.99 & \\
\hline MWGS-39 & MW & $06 / 11 / 92$ & 1403 & 38.30 & 17.53 & 20.77 & \\
\hline MWGS-39 & MW & $07 / 07 / 92$ & 1717 & 38.30 & 16.76 & 21.54 & \\
\hline MWGS-39 & MW & $08 / 07 / 92$ & 1244 & 38.30 & 17.25 & 21.05 & \\
\hline MWGS-39 & MW & $08 / 31 / 92$ & 1125 & 38.30 & 14.93 & 23.37 & \\
\hline MWGS-39 & MW & $10 / 07 / 92$ & 1132 & 38.30 & 15.20 & 23.10 & \\
\hline MWGS-39 & MW & $10 / 28 / 92$ & 1250 & 38.30 & 15.52 & 22.78 & \\
\hline
\end{tabular}


Table 8.--Water-level data collected at the Defense Fuel Supply Point and adjacent properties, Hanahan, S.C., between April 1991 and September 1995--Continued

[ft, feet; ---, data not available; WL, water-level well; EW, extraction well; MW, monitoring well; SW, surface-water site; -, negative depth to water indicates water-level altitude is above measuring point]

\begin{tabular}{|c|c|c|c|c|c|c|c|}
\hline $\begin{array}{c}\text { Site } \\
\text { (plate 1) }\end{array}$ & $\begin{array}{l}\text { Site } \\
\text { type }\end{array}$ & Date & Time & $\begin{array}{l}\text { Measuring-point } \\
\text { altitude } \\
\text { (ft above } \\
\text { sea level) }\end{array}$ & $\begin{array}{l}\text { Depth to water } \\
\text { ( } \mathrm{ft} \text { below } \\
\text { measuring point) }\end{array}$ & $\begin{array}{l}\text { Water-level } \\
\text { altitude } \\
\text { (ft above } \\
\text { sea level) }\end{array}$ & Remarks \\
\hline MWGS-39 & MW & $12 / 03 / 92$ & 1111 & 38.30 & 15.72 & 22.58 & \\
\hline MWGS-39 & MW & $01 / 04 / 93$ & 1057 & 38.30 & 16.49 & 21.81 & \\
\hline MWGS-39 & MW & $02 / 04 / 93$ & 1149 & 38.30 & 15.23 & 23.07 & \\
\hline MWGS-39 & MW & $03 / 05 / 93$ & 1111 & 38.30 & 15.74 & 22.56 & \\
\hline MWGS-39 & MW & $03 / 30 / 93$ & 1032 & 38.30 & 16.31 & 21.99 & \\
\hline MWGS-39 & MW & $05 / 06 / 93$ & 1057 & 38.30 & 16.70 & 21.60 & \\
\hline MWGS-39 & MW & $05 / 28 / 93$ & 1051 & 38.30 & 17.25 & 21.05 & \\
\hline MWGS-39 & MW & $07 / 07 / 93$ & 1042 & 38.30 & 17.28 & 21.02 & \\
\hline MWGS-39 & MW & $08 / 06 / 93$ & 1022 & 38.30 & 17.37 & 20.93 & \\
\hline MWGS-39 & MW & $09 / 03 / 93$ & 1042 & 38.30 & 16.85 & 21.45 & \\
\hline MWGS-39 & MW & $09 / 30 / 93$ & 1048 & 38.30 & 16.68 & 21.62 & \\
\hline MWGS-39 & MW & $10 / 28 / 93$ & 1038 & 38.30 & 17.41 & 20.89 & \\
\hline MWGS-39 & MW & $12 / 01 / 93$ & 1337 & 38.30 & 17.35 & 20.95 & \\
\hline MWGS-39 & MW & $01 / 06 / 94$ & 1046 & 38.30 & 17.60 & 20.70 & \\
\hline MWGS-39 & MW & $02 / 01 / 94$ & 1046 & 38.30 & 16.83 & 21.47 & \\
\hline MWGS-39 & MW & $03 / 04 / 94$ & 1027 & 38.30 & 16.56 & 21.74 & \\
\hline MWGS-39 & MW & $04 / 04 / 94$ & 1022 & 38.30 & 16.82 & 21.48 & \\
\hline MWGS-39 & MW & $05 / 06 / 94$ & 1107 & 38.30 & 17.60 & 20.70 & \\
\hline MWGS-39 & MW & $06 / 03 / 94$ & 1046 & 38.30 & 18.08 & 20.22 & \\
\hline MWGS-39 & MW & $07 / 08 / 94$ & 1059 & 38.30 & 16.70 & 21.60 & \\
\hline MWGS-39 & MW & $08 / 05 / 94$ & 1400 & 38.30 & 15.54 & 22.76 & \\
\hline MWGS-39 & MW & $09 / 07 / 94$ & 1115 & 38.30 & 15.24 & 23.06 & \\
\hline MWGS-39 & MW & $10 / 06 / 94$ & 1123 & 38.30 & 13.40 & 24.90 & \\
\hline MWGS-39 & MW & $11 / 09 / 94$ & 1253 & 38.30 & 14.90 & 23.40 & \\
\hline MWGS-39 & MW & $12 / 06 / 94$ & 1109 & 38.30 & 15.51 & 22.79 & \\
\hline MWGS-39 & MW & $01 / 10 / 95$ & 1312 & 38.30 & 15.56 & 22.74 & \\
\hline MWGS-39 & MW & $02 / 02 / 95$ & 1330 & 38.30 & 15.58 & 22.72 & \\
\hline MWGS-39 & MW & $03 / 02 / 95$ & 1457 & 38.30 & 15.67 & 22.63 & \\
\hline MWGS-39 & MW & $04 / 05 / 95$ & 1055 & 38.30 & 16.31 & 21.99 & \\
\hline MWGS-39 & MW & $05 / 02 / 95$ & 1244 & 38.30 & 16.77 & 21.53 & \\
\hline MWGS-39 & MW & $06 / 09 / 95$ & 1046 & 38.30 & 17.30 & 21.00 & \\
\hline MWGS-39 & MW & $07 / 06 / 95$ & 1039 & 38.30 & 17.10 & 21.20 & \\
\hline MWGS-39 & MW & $08 / 10 / 95$ & 1110 & 38.30 & 16.57 & 21.73 & \\
\hline MWGS-39 & MW & $09 / 13 / 95$ & 1150 & 38.30 & 15.02 & 23.28 & \\
\hline MWGS-40A & MW & $07 / 07 / 92$ & 1605 & 34.65 & 7.56 & 27.09 & \\
\hline MWGS-40A & MW & 08/07/92 & 1326 & 34.65 & 7.41 & 27.24 & \\
\hline MWGS-40A & MW & $08 / 31 / 92$ & 1205 & 34.65 & 6.13 & 28.52 & \\
\hline MWGS-40A & MW & $10 / 07 / 92$ & 1213 & 34.65 & 5.97 & 28.68 & \\
\hline MWGS-40A & MW & $10 / 28 / 92$ & 1325 & 34.65 & 6.85 & 27.80 & \\
\hline MWGS-40A & MW & $12 / 03 / 92$ & 1209 & 34.65 & 6.60 & 28.05 & \\
\hline MWGS-40A & MW & $01 / 04 / 93$ & 1146 & 34.65 & 7.38 & 27.27 & \\
\hline MWGS-40A & MW & $02 / 04 / 93$ & 1235 & 34.65 & 6.37 & 28.28 & \\
\hline MWGS-40A & MW & $03 / 05 / 93$ & 1159 & 34.65 & 6.74 & 27.91 & \\
\hline MWGS-40A & MW & $03 / 30 / 93$ & 1118 & 34.65 & 6.33 & 28.32 & \\
\hline
\end{tabular}


Table 8.--Water-level data collected at the Defense Fuel Supply Point and adjacent properties, Hanahan, S.C., between April 1991 and September 1995--Continued

[ft, feet; ---, data not available; WL, water-level well; EW, extraction well; MW, monitoring well; SW, surface-water site; -, negative depth to water indicates water-level altitude is above measuring point]

\begin{tabular}{|c|c|c|c|c|c|c|c|}
\hline $\begin{array}{c}\text { Site } \\
\text { (plate 1) }\end{array}$ & $\begin{array}{l}\text { Site } \\
\text { type }\end{array}$ & Date & Time & $\begin{array}{l}\text { Measuring-point } \\
\text { altitude } \\
\text { (ft above } \\
\text { sea level) }\end{array}$ & $\begin{array}{l}\text { Depth to water } \\
\text { ( } \mathrm{ft} \text { below } \\
\text { measuring point) }\end{array}$ & $\begin{array}{l}\text { Water-level } \\
\text { altitude } \\
\text { (ft above } \\
\text { sea level) }\end{array}$ & Remarks \\
\hline MWGS-40A & MW & $05 / 06 / 93$ & 1135 & 34.65 & 7.20 & 27.45 & \\
\hline MWGS-40A & MW & $05 / 28 / 93$ & 1130 & 34.65 & 7.60 & 27.05 & \\
\hline MWGS-40A & MW & $07 / 07 / 93$ & 1119 & 34.65 & 7.50 & 27.15 & \\
\hline MWGS-40A & MW & $08 / 06 / 93$ & 1059 & 34.65 & 7.18 & 27.47 & \\
\hline MWGS-40A & MW & $09 / 03 / 93$ & 1117 & 34.65 & 7.36 & 27.29 & \\
\hline MWGS-40A & MW & $09 / 30 / 93$ & 1000 & 34.65 & 7.15 & 27.50 & \\
\hline MWGS-40A & MW & $10 / 28 / 93$ & 1115 & 34.65 & 8.00 & 26.65 & \\
\hline MWGS-40A & MW & $12 / 01 / 93$ & 1413 & 34.65 & 7.59 & 27.06 & \\
\hline MWGS-40A & MW & $01 / 06 / 94$ & 1135 & 34.65 & 7.02 & 27.63 & \\
\hline MWGS-40A & MW & $02 / 01 / 94$ & 1129 & 34.65 & 6.27 & 28.38 & \\
\hline MWGS-40A & MW & $03 / 04 / 94$ & 1059 & 34.65 & 6.43 & 28.22 & \\
\hline MWGS-40A & MW & $04 / 04 / 94$ & 1058 & 34.65 & 7.55 & 27.10 & \\
\hline MWGS-40A & MW & $05 / 06 / 94$ & 1152 & 34.65 & 8.18 & 26.47 & \\
\hline MWGS-40A & MW & $06 / 03 / 94$ & 1130 & 34.65 & 8.66 & 25.99 & \\
\hline MWGS-40A & MW & $07 / 08 / 94$ & 1150 & 34.65 & 6.23 & 28.42 & \\
\hline MWGS-40A & MW & $08 / 05 / 94$ & 1430 & 34.65 & 6.22 & 28.43 & \\
\hline MWGS-40A & MW & $09 / 07 / 94$ & 1250 & 34.65 & 6.42 & 28.23 & \\
\hline MWGS-40A & MW & $10 / 06 / 94$ & 1206 & 34.65 & 4.76 & 29.89 & \\
\hline MWGS-40A & MW & $11 / 09 / 94$ & 1324 & 34.65 & 6.31 & 28.34 & \\
\hline MWGS-40A & MW & $12 / 06 / 94$ & 1144 & 34.65 & 6.18 & 28.47 & \\
\hline MWGS-40A & MW & $01 / 10 / 95$ & 1622 & 34.65 & 6.48 & 28.17 & \\
\hline MWGS-40A & MW & $02 / 02 / 95$ & 1359 & 34.65 & 6.61 & 28.04 & \\
\hline MWGS-40A & MW & $03 / 02 / 95$ & 1532 & 34.65 & 6.60 & 28.05 & \\
\hline MWGS-40A & MW & $04 / 05 / 95$ & 1141 & 34.65 & 7.37 & 27.28 & \\
\hline MWGS-40A & MW & $05 / 02 / 95$ & 1328 & 34.65 & 7.77 & 26.88 & \\
\hline MWGS-40A & MW & $06 / 09 / 95$ & 1126 & 34.65 & 7.64 & 27.01 & \\
\hline MWGS-40A & MW & $07 / 06 / 95$ & 1112 & 34.65 & 7.50 & 27.15 & \\
\hline MWGS-40A & MW & $08 / 10 / 95$ & 1248 & 34.65 & 7.61 & 27.04 & \\
\hline MWGS-40A & MW & $09 / 13 / 95$ & 1209 & 34.65 & 6.51 & 28.14 & \\
\hline MWGS-40B & MW & $07 / 07 / 92$ & 1610 & 34.61 & 7.49 & 27.12 & \\
\hline MWGS-40B & MW & $08 / 07 / 92$ & 1328 & 34.61 & 7.36 & 27.25 & \\
\hline MWGS-40B & MW & $08 / 31 / 92$ & 1208 & 34.61 & 6.10 & 28.51 & \\
\hline MWGS-40B & MW & $10 / 07 / 92$ & 1214 & 34.61 & 5.94 & 28.67 & \\
\hline MWGS-40B & MW & $10 / 28 / 92$ & 1327 & 34.61 & 6.81 & 27.80 & \\
\hline MWGS-40B & MW & $12 / 03 / 92$ & 1212 & 34.61 & 6.57 & 28.04 & \\
\hline MWGS-40B & MW & $01 / 04 / 93$ & 1145 & 34.61 & 7.34 & 27.27 & \\
\hline MWGS-40B & MW & $02 / 04 / 93$ & 1237 & 34.61 & 6.34 & 28.27 & \\
\hline MWGS-40B & MW & $03 / 05 / 93$ & 1200 & 34.61 & 6.71 & 27.90 & \\
\hline MWGS-40B & MW & $03 / 30 / 93$ & 1120 & 34.61 & 6.30 & 28.31 & \\
\hline MWGS-40B & MW & $05 / 06 / 93$ & 1137 & 34.61 & 7.15 & 27.46 & \\
\hline MWGS-40B & MW & $05 / 28 / 93$ & 1131 & 34.61 & 7.57 & 27.04 & \\
\hline MWGS-40B & MW & $07 / 07 / 93$ & 1120 & 34.61 & 7.47 & 27.14 & \\
\hline MWGS-40B & MW & $08 / 06 / 93$ & 1101 & 34.61 & 7.15 & 27.46 & \\
\hline MWGS-40B & MW & $09 / 03 / 93$ & 1119 & 34.61 & 7.33 & 27.28 & \\
\hline
\end{tabular}


Table 8.--Water-level data collected at the Defense Fuel Supply Point and adjacent properties, Hanahan, S.C., between April 1991 and September 1995--Continued

[ft, feet; ---, data not available; WL, water-level well; EW, extraction well; $\mathrm{MW}$, monitoring well; SW, surface-water site; -, negative depth to water indicates water-level altitude is above measuring point]

\begin{tabular}{|c|c|c|c|c|c|c|c|}
\hline $\begin{array}{c}\text { Site } \\
\text { (plate 1) }\end{array}$ & $\begin{array}{l}\text { Site } \\
\text { type }\end{array}$ & Date & Time & $\begin{array}{l}\text { Measuring-point } \\
\text { altitude } \\
\text { (ft above } \\
\text { sea level) }\end{array}$ & $\begin{array}{l}\text { Depth to water } \\
\text { (ft below } \\
\text { measuring point) }\end{array}$ & $\begin{array}{l}\text { Water-level } \\
\text { altitude } \\
\text { (ft above } \\
\text { sea level) }\end{array}$ & Remarks \\
\hline MWGS-40B & MW & $09 / 30 / 93$ & 1011 & 34.61 & 7.12 & 27.49 & \\
\hline MWGS-40B & MW & $10 / 28 / 93$ & 1116 & 34.61 & 7.95 & 26.66 & \\
\hline MWGS-40B & MW & $12 / 01 / 93$ & 1414 & 34.61 & 7.55 & 27.06 & \\
\hline MWGS-40B & MW & $01 / 06 / 94$ & 1138 & 34.61 & 6.98 & 27.63 & \\
\hline MWGS-40B & MW & $02 / 01 / 94$ & 1130 & 34.61 & 6.23 & 28.38 & \\
\hline MWGS-40B & MW & $03 / 04 / 94$ & 1100 & 34.61 & 6.40 & 28.21 & \\
\hline MWGS-40B & MW & $04 / 04 / 94$ & 1059 & 34.61 & 7.50 & 27.11 & \\
\hline MWGS-40B & MW & $05 / 06 / 94$ & 1155 & 34.61 & 8.14 & 26.47 & \\
\hline MWGS-40B & MW & $06 / 03 / 94$ & 1133 & 34.61 & 8.61 & 26.00 & \\
\hline MWGS-40B & MW & $07 / 08 / 94$ & 1151 & 34.61 & 6.20 & 28.41 & \\
\hline MWGS-40B & MW & $08 / 05 / 94$ & 1435 & 34.61 & 6.21 & 28.40 & \\
\hline MWGS-40B & MW & $09 / 07 / 94$ & 1252 & 34.61 & 6.40 & 28.21 & \\
\hline MWGS-40B & MW & $10 / 06 / 94$ & 1207 & 34.61 & 4.74 & 29.87 & \\
\hline MWGS-40B & MW & $11 / 09 / 94$ & 1325 & 34.61 & 6.27 & 28.34 & \\
\hline MWGS-40B & MW & $12 / 06 / 94$ & 1156 & 34.61 & 6.13 & 28.48 & \\
\hline MWGS-40B & MW & $01 / 10 / 95$ & 1623 & 34.61 & 6.45 & 28.16 & \\
\hline MWGS-40B & MW & $02 / 02 / 95$ & 1401 & 34.61 & 6.56 & 28.05 & \\
\hline MWGS-40B & MW & $03 / 02 / 95$ & 1534 & 34.61 & 6.56 & 28.05 & \\
\hline MWGS-40B & MW & $04 / 05 / 95$ & 1142 & 34.61 & 7.32 & 27.29 & \\
\hline MWGS-40B & MW & $05 / 02 / 95$ & 1329 & 34.61 & 7.72 & 26.89 & \\
\hline MWGS-40B & MW & $06 / 09 / 95$ & 1127 & 34.61 & 7.59 & 27.02 & \\
\hline MWGS-40B & MW & $07 / 06 / 95$ & 1114 & 34.61 & 7.47 & 27.14 & \\
\hline MWGS-40B & MW & $08 / 10 / 95$ & 1250 & 34.61 & 7.55 & 27.06 & \\
\hline MWGS-40B & MW & $09 / 13 / 95$ & 1210 & 34.61 & 6.46 & 28.15 & \\
\hline MWGS-41A & MW & $07 / 07 / 92$ & 1635 & 34.95 & 8.08 & 26.87 & \\
\hline MWGS-41A & MW & $08 / 07 / 92$ & 1308 & 34.95 & 7.84 & 27.11 & \\
\hline MWGS-41A & MW & $08 / 31 / 92$ & 1150 & 34.95 & 6.64 & 28.31 & \\
\hline MWGS-4IA & MW & $10 / 07 / 92$ & 1200 & 34.95 & 6.30 & 28.65 & \\
\hline MWGS-41A & MW & $10 / 28 / 92$ & 1313 & 34.95 & 7.34 & 27.61 & \\
\hline MWGS-41A & MW & $12 / 03 / 92$ & 1140 & 34.95 & 7.16 & 27.79 & \\
\hline MWGS-41A & MW & $01 / 04 / 93$ & 1130 & 34.95 & 7.99 & 26.96 & \\
\hline MWGS-41A & MW & $02 / 04 / 93$ & 1218 & 34.95 & 6.91 & 28.04 & \\
\hline MWGS-41A & MW & $03 / 05 / 93$ & 1143 & 34.95 & 7.34 & 27.61 & \\
\hline MWGS-41A & MW & $03 / 30 / 93$ & 1052 & 34.95 & 6.95 & 28.00 & \\
\hline MWGS-41A & MW & $05 / 06 / 93$ & 1121 & 34.95 & 7.70 & 27.25 & \\
\hline MWGS-41A & MW & $05 / 28 / 93$ & 1118 & 34.95 & 8.20 & 26.75 & \\
\hline MWGS-41A & MW & $07 / 07 / 93$ & 1106 & 34.95 & 7.93 & 27.02 & \\
\hline MWGS-41A & MW & $08 / 06 / 93$ & 1047 & 34.95 & 7.59 & 27.36 & \\
\hline MWGS-41A & MW & $09 / 03 / 93$ & 1059 & 34.95 & 7.86 & 27.09 & \\
\hline MWGS-41A & MW & $09 / 30 / 93$ & 1024 & 34.95 & 7.66 & 27.29 & \\
\hline MWGS-41A & MW & $10 / 28 / 93$ & 1059 & 34.95 & 8.51 & 26.44 & \\
\hline MWGS-41A & MW & $12 / 01 / 93$ & 1400 & 34.95 & 7.89 & 27.06 & \\
\hline MWGS-41A & MW & $01 / 06 / 94$ & 1113 & 34.95 & 7.22 & 27.73 & \\
\hline MWGS-41A & MW & $02 / 01 / 94$ & 1118 & 34.95 & 6.46 & 28.49 & \\
\hline
\end{tabular}


Table 8.--Water-level data collected at the Defense Fuel Supply Point and adjacent properties, Hanahan, S.C., between April 1991 and September 1995--Continued

[ft, feet; ---, data not available; WL, water-level well; EW, extraction well; MW, monitoring well; SW, surface-water site; -, negative depth to water indicates water-level altitude is above measuring point]

\begin{tabular}{|c|c|c|c|c|c|c|c|}
\hline $\begin{array}{c}\text { Site } \\
\text { (plate 1) }\end{array}$ & $\begin{array}{l}\text { Site } \\
\text { type }\end{array}$ & Date & Time & $\begin{array}{l}\text { Measuring-point } \\
\text { altitude } \\
\text { (ft above } \\
\text { sea level) }\end{array}$ & $\begin{array}{l}\text { Depth to water } \\
\text { ( } \mathrm{ft} \text { below } \\
\text { measuring point) }\end{array}$ & $\begin{array}{l}\text { Water-level } \\
\text { altitude } \\
\text { ( } \mathrm{ft} \text { above } \\
\text { sea level) }\end{array}$ & Remarks \\
\hline MWGS-41A & MW & $03 / 04 / 94$ & 1047 & 34.95 & 6.68 & 28.27 & \\
\hline MWGS-41A & MW & $04 / 04 / 94$ & 1045 & 34.95 & 8.00 & 26.95 & \\
\hline MWGS-41A & MW & $05 / 06 / 94$ & 1137 & 34.95 & 8.65 & 26.30 & \\
\hline MWGS-41A & MW & $06 / 03 / 94$ & 1116 & 34.95 & 9.20 & 25.75 & \\
\hline MWGS-4 IA & MW & $07 / 08 / 94$ & 1125 & 34.95 & 6.13 & 28.82 & \\
\hline MWGS-41A & MW & $08 / 05 / 94$ & 1421 & 34.95 & 6.37 & 28.58 & \\
\hline MWGS-41A & MW & $09 / 07 / 94$ & 1232 & 34.95 & 6.59 & 28.36 & \\
\hline MWGS-41A & MW & $10 / 06 / 94$ & 1145 & 34.95 & 5.05 & 29.90 & \\
\hline MWGS-41A & MW & $11 / 09 / 94$ & 1314 & 34.95 & 6.59 & 28.36 & \\
\hline MWGS-41A & MW & $12 / 06 / 94$ & 1129 & 34.95 & 6.24 & 28.71 & \\
\hline MWGS-41A & MW & $01 / 10 / 95$ & 1335 & 34.95 & 6.70 & 28.25 & \\
\hline MWGS-41A & MW & $02 / 02 / 95$ & 1348 & 34.95 & 6.94 & 28.01 & \\
\hline MWGS-41A & MW & $03 / 02 / 95$ & 1519 & 34.95 & 6.96 & 27.99 & \\
\hline MWGS-41A & MW & $04 / 05 / 95$ & 1127 & 34.95 & 7.78 & 27.17 & \\
\hline MWGS-41A & MW & $05 / 02 / 95$ & 1314 & 34.95 & 8.23 & 26.72 & \\
\hline MWGS-41A & MW & $06 / 09 / 95$ & 1113 & 34.95 & 7.99 & 26.96 & \\
\hline MWGS-41A & MW & $07 / 06 / 95$ & 1102 & 34.95 & 7.90 & 27.05 & \\
\hline MWGS-41A & MW & $08 / 10 / 95$ & 1134 & 34.95 & 8.04 & 26.91 & \\
\hline MWGS-41A & MW & $09 / 13 / 95$ & 1026 & 34.95 & 6.88 & 28.07 & \\
\hline MWGS-41B & MW & $07 / 07 / 92$ & 1638 & 34.65 & 7.79 & 26.86 & \\
\hline MWGS-41B & MW & $08 / 07 / 92$ & 1311 & 34.65 & 7.50 & 27.15 & \\
\hline MWGS-41B & MW & $08 / 31 / 92$ & 1214 & 34.65 & 6.30 & 28.35 & \\
\hline MWGS-41B & MW & $10 / 07 / 92$ & 1201 & 34.65 & 5.99 & 28.66 & \\
\hline MWGS-41B & MW & $10 / 28 / 92$ & 1314 & 34.65 & 7.00 & 27.65 & \\
\hline MWGS-41B & MW & $12 / 03 / 92$ & 1145 & 34.65 & 6.83 & 27.82 & \\
\hline MWGS-4IB & MW & $01 / 04 / 93$ & 1127 & 34.65 & 7.66 & 26.99 & \\
\hline MWGS-41B & MW & $02 / 04 / 93$ & 1220 & 34.65 & 6.58 & 28.07 & \\
\hline MWGS-41B & MW & $03 / 05 / 93$ & 1145 & 34.65 & 6.99 & 27.66 & \\
\hline MWGS-4IB & MW & $03 / 30 / 93$ & 1053 & 34.65 & 6.61 & 28.04 & \\
\hline MWGS-41B & MW & $05 / 06 / 93$ & 1123 & 34.65 & 7.36 & 27.29 & \\
\hline MWGS-41B & MW & $05 / 28 / 93$ & 1120 & 34.65 & 7.83 & 26.82 & \\
\hline MWGS-4IB & MW & $07 / 07 / 93$ & 1108 & 34.65 & 7.60 & 27.05 & \\
\hline MWGS-4IB & MW & $08 / 06 / 93$ & 1048 & 34.65 & 7.25 & 27.40 & \\
\hline MWGS-41B & MW & $09 / 03 / 93$ & 1101 & 34.65 & 7.53 & 27.12 & \\
\hline MWGS-4IB & MW & $09 / 30 / 93$ & 1025 & 34.65 & 7.32 & 27.33 & \\
\hline MWGS-41B & MW & $10 / 28 / 93$ & 1100 & 34.65 & 8.19 & 26.46 & \\
\hline MWGS-41B & MW & $12 / 01 / 93$ & 1404 & 34.65 & 7.61 & 27.04 & \\
\hline MWGS-41B & MW & $01 / 06 / 94$ & 1118 & 34.65 & 6.92 & 27.73 & \\
\hline MWGS-41B & MW & $02 / 01 / 94$ & 1111 & 34.65 & 6.15 & 28.50 & \\
\hline MWGS-41B & MW & $03 / 04 / 94$ & 1049 & 34.65 & 6.36 & 28.29 & \\
\hline MWGS-41B & MW & $04 / 04 / 94$ & 1046 & 34.65 & 7.67 & 26.98 & \\
\hline MWGS-41B & MW & $05 / 06 / 94$ & 1139 & 34.65 & 8.33 & 26.32 & \\
\hline MWGS-41B & MW & $06 / 03 / 94$ & 1109 & 34.65 & 8.87 & 25.78 & \\
\hline MWGS-41B & MW & $07 / 08 / 94$ & 1126 & 34.65 & 5.86 & 28.79 & \\
\hline
\end{tabular}


Table 8.--Water-level data collected at the Defense Fuel Supply Point and adjacent properties, Hanahan, S.C., between April 1991 and September 1995--Continued

[ft, feet; ---, data not available; WL, water-level well; EW, extraction well; MW, monitoring well; SW, surface-water site; -, negative depth to water indicates water-level altitude is above measuring point]

\begin{tabular}{|c|c|c|c|c|c|c|c|}
\hline $\begin{array}{c}\text { Site } \\
\text { (plate 1) }\end{array}$ & $\begin{array}{l}\text { Site } \\
\text { type }\end{array}$ & Date & Time & $\begin{array}{l}\text { Measuring-point } \\
\text { altitude } \\
\text { (ft above } \\
\text { sea level) }\end{array}$ & $\begin{array}{l}\text { Depth to water } \\
\text { (ft below } \\
\text { measuring point) }\end{array}$ & $\begin{array}{l}\text { Water-level } \\
\text { altitude } \\
\text { (ft above } \\
\text { sea level) }\end{array}$ & Remarks \\
\hline MWGS-4IB & MW & $08 / 05 / 94$ & 1423 & 34.65 & 6.06 & 28.59 & \\
\hline MWGS-4IB & MW & $09 / 07 / 94$ & 1235 & 34.65 & 6.27 & 28.38 & \\
\hline MWGS-41B & MW & $10 / 06 / 94$ & 1147 & 34.65 & 4.76 & 29.89 & \\
\hline MWGS-4IB & MW & $11 / 09 / 94$ & 1316 & 34.65 & 6.26 & 28.39 & \\
\hline MWGS-41B & MW & $12 / 06 / 94$ & 1132 & 34.65 & 5.98 & 28.67 & \\
\hline MWGS-41B & MW & $01 / 10 / 95$ & 1336 & 34.65 & 6.39 & 28.26 & \\
\hline MWGS-41B & MW & $02 / 02 / 95$ & 1350 & 34.65 & 6.60 & 28.05 & \\
\hline MWGS-41B & MW & $03 / 02 / 95$ & 1522 & 34.65 & 6.63 & 28.02 & \\
\hline MWGS-41B & MW & $04 / 05 / 95$ & 1130 & 34.65 & 7.45 & 27.20 & \\
\hline MWGS-41B & MW & $05 / 02 / 95$ & 1315 & 34.65 & 7.89 & 26.76 & \\
\hline MWGS-41B & MW & $06 / 09 / 95$ & 1115 & 34.65 & 7.67 & 26.98 & \\
\hline MWGS-41B & MW & $07 / 06 / 95$ & 1103 & 34.65 & 7.59 & 27.06 & \\
\hline MWGS-41B & MW & $08 / 10 / 95$ & 1137 & 34.65 & 7.71 & 26.94 & \\
\hline MWGS-41B & MW & $09 / 13 / 95$ & 1024 & 34.65 & 6.55 & 28.10 & \\
\hline NWS-12-1 & WL & $04 / 30 / 91$ & 1430 & 23.59 & 11.71 & 11.88 & \\
\hline NWS-12-1 & WL & $05 / 28 / 91$ & 1329 & 23.59 & 12.05 & 11.54 & \\
\hline NWS-12-1 & WL & $07 / 01 / 91$ & -- & 23.59 & 12.44 & 11.15 & \\
\hline NWS-12-1 & WL & $08 / 06 / 91$ & -- & 23.59 & 10.68 & 12.91 & \\
\hline NWS-12-1 & WL & $08 / 30 / 91$ & --- & 23.59 & 10.69 & 12.90 & \\
\hline NWS-12-1 & WL & $09 / 27 / 91$ & -- & 23.59 & 11.47 & 12.12 & \\
\hline NWS-12-1 & WL & $10 / 29 / 91$ & --- & 23.59 & 12.49 & 11.10 & \\
\hline NWS-12-1 & WL & $12 / 02 / 91$ & -- & 23.59 & 13.46 & 10.13 & \\
\hline NWS-12-1 & WL & $01 / 02 / 92$ & -- & 23.59 & 14.32 & 9.27 & \\
\hline NWS-12-1 & WL & $02 / 04 / 92$ & --- & 23.59 & 14.52 & 9.07 & \\
\hline NWS-12-1 & WL & $03 / 06 / 92$ & --- & 23.59 & 14.65 & 8.94 & \\
\hline NWS-12-1 & WL & $03 / 31 / 92$ & --- & 23.59 & 14.67 & 8.92 & \\
\hline NWS-12-1 & WL & $05 / 01 / 92$ & 1455 & 23.59 & 14.56 & 9.03 & \\
\hline NWS-12-1 & WL & $06 / 11 / 92$ & 1605 & 23.59 & 14.29 & 9.30 & \\
\hline NWS-12-1 & WL & $07 / 07 / 92$ & 1355 & 23.59 & 13.67 & 9.92 & \\
\hline NWS-12-1 & WL & $08 / 31 / 92$ & 1449 & 23.59 & 10.62 & 12.97 & \\
\hline NWS-12-1 & WL & $10 / 07 / 92$ & 1350 & 23.59 & 10.23 & 13.36 & \\
\hline NWS-12-1 & WL & $10 / 28 / 92$ & 1445 & 23.59 & 10.48 & 13.11 & \\
\hline NWS-12-1 & WL & $12 / 03 / 92$ & 1508 & 23.59 & 10.00 & 13.59 & \\
\hline NWS-12-1 & WL & $01 / 04 / 93$ & 1432 & 23.59 & 10.76 & 12.83 & \\
\hline NWS-12-1 & WL & $02 / 04 / 93$ & 1555 & 23.59 & 8.40 & 15.19 & \\
\hline NWS-12-1 & WL & $03 / 05 / 93$ & 1451 & 23.59 & 8.80 & 14.79 & \\
\hline NWS-12-1 & WL & $03 / 30 / 93$ & 1406 & 23.59 & 8.75 & 14.84 & \\
\hline NWS-12-1 & WL & $05 / 06 / 93$ & 1408 & 23.59 & 9.24 & 14.35 & \\
\hline NWS-12-1 & WL & $05 / 28 / 93$ & 1355 & 23.59 & 10.00 & 13.59 & \\
\hline NWS-12-1 & WL & $07 / 07 / 93$ & 1435 & 23.59 & 11.22 & 12.37 & \\
\hline NWS-12-2 & WL & $04 / 30 / 91$ & 1450 & 23.78 & 11.32 & 12.46 & \\
\hline NWS-12-2 & WL & $05 / 28 / 91$ & 1335 & 23.78 & 11.76 & 12.02 & \\
\hline NWS-12-2 & WL & $07 / 01 / 91$ & --- & 23.78 & 12.20 & 11.58 & \\
\hline
\end{tabular}


Table 8.--Water-level data collected at the Defense Fuel Supply Point and adjacent properties, Hanahan, S.C., between April 1991 and September 1995--Continued

[ft, feet; ---, data not available; WL, water-level well; EW, extraction well; MW, monitoring well; SW, surface-water site; -, negative depth to water indicates water-level altitude is above measuring point]

\begin{tabular}{|c|c|c|c|c|c|c|c|}
\hline $\begin{array}{c}\text { Site } \\
\text { (plate 1) }\end{array}$ & $\begin{array}{l}\text { Site } \\
\text { type }\end{array}$ & Date & Time & $\begin{array}{l}\text { Measuring-point } \\
\text { altitude } \\
\text { (ft above } \\
\text { sea level) }\end{array}$ & $\begin{array}{l}\text { Depth to water } \\
\text { ( } f \text { below } \\
\text { measuring point) }\end{array}$ & $\begin{array}{l}\text { Water-level } \\
\text { altitude } \\
\text { (ft above } \\
\text { sea level) }\end{array}$ & Remarks \\
\hline NWS-12-2 & $\overline{W L}$ & $08 / 06 / 91$ & --- & 23.78 & 10.93 & 12.85 & \\
\hline NWS-12-2 & WL & $08 / 30 / 91$ & -- & 23.78 & 10.61 & 13.17 & \\
\hline NWS-12-2 & WL & $09 / 27 / 91$ & --- & 23.78 & 11.08 & 12.70 & \\
\hline NWS-12-2 & WL & $10 / 29 / 91$ & --- & 23.78 & 11.96 & 11.82 & \\
\hline NWS-12-2 & WL & $12 / 02 / 91$ & -- & 23.78 & 12.90 & 10.88 & \\
\hline NWS-12-2 & WL & $01 / 02 / 92$ & -- & 23.78 & 13.80 & 9.98 & \\
\hline NWS-12-2 & WL & $02 / 04 / 92$ & --- & 23.78 & 14.42 & 9.36 & \\
\hline NWS-12-2 & WL & 03/06/92 & -- & 23.78 & 14.78 & 9.00 & \\
\hline NWS-12-2 & WL & $03 / 31 / 92$ & --- & 23.78 & 14.97 & 8.81 & \\
\hline NWS-12-2 & WL & $05 / 01 / 92$ & 1500 & 23.78 & 15.01 & 8.77 & \\
\hline NWS-12-3 & WL & $04 / 30 / 91$ & 1520 & 27.13 & 4.91 & 22.22 & \\
\hline NWS-12-3 & WL & $05 / 28 / 91$ & 1345 & 27.13 & 5.60 & 21.53 & \\
\hline NWS-12-3 & WL & $07 / 01 / 91$ & -- & 27.13 & 7.04 & 20.09 & \\
\hline NWS-12-3 & WL & $08 / 06 / 91$ & -- & 27.13 & 4.62 & 22.51 & \\
\hline NWS-12-3 & WL & $08 / 30 / 91$ & -- & 27.13 & 4.06 & 23.07 & \\
\hline NWS-12-3 & WL & $09 / 27 / 91$ & --- & 27.13 & 5.95 & 21.18 & \\
\hline NWS-12-3 & WL & $10 / 29 / 91$ & -- & 27.13 & 7.00 & 20.13 & \\
\hline NWS-12-3 & WL & $12 / 02 / 91$ & - & 27.13 & 7.74 & 19.39 & \\
\hline NWS-12-3 & WL & $01 / 02 / 92$ & $\cdots$ & 27.13 & 7.15 & 19.98 & \\
\hline NWS-12-3 & WL & $02 / 04 / 92$ & --- & 27.13 & 7.62 & 19.51 & \\
\hline NWS-12-3 & WL & $03 / 06 / 92$ & --- & 27.13 & 8.08 & 19.05 & \\
\hline NWS-12-3 & WL & $03 / 31 / 92$ & $\cdots$ & 27.13 & 7.72 & 19.41 & \\
\hline NWS-12-3 & WL & $05 / 01 / 92$ & --- & 27.13 & 8.13 & 19.00 & \\
\hline NWS-12-4 & WL & $04 / 30 / 91$ & 1530 & 28.66 & 4.92 & 23.74 & \\
\hline NWS-12-4 & WL & $05 / 28 / 91$ & 1350 & 28.66 & 5.54 & 23.12 & \\
\hline NWS-12-4 & WL & $07 / 01 / 91$ & -- & 28.66 & 6.10 & 22.56 & \\
\hline NWS-12-4 & WL & $08 / 06 / 91$ & -- & 28.66 & 4.47 & 24.19 & \\
\hline NWS-12-4 & WL & $08 / 30 / 91$ & --- & 28.66 & 4.03 & 24.63 & \\
\hline NWS-12-4 & WL & $09 / 27 / 91$ & --- & 28.66 & 5.93 & 22.73 & \\
\hline NWS-12-4 & WL & $10 / 29 / 91$ & -- & 28.66 & 6.93 & 21.73 & \\
\hline NWS-12-4 & WL & $12 / 02 / 91$ & -- & 28.66 & 7.43 & 21.23 & \\
\hline NWS-12-4 & WL & $01 / 02 / 92$ & - & 28.66 & 7.99 & 20.67 & \\
\hline NWS-12-4 & WL & $02 / 04 / 92$ & -- & 28.66 & 6.94 & 21.72 & \\
\hline NWS-12-4 & WL & $03 / 06 / 92$ & -- & 28.66 & 7.31 & 21.35 & \\
\hline NWS-12-4 & WL & $03 / 31 / 92$ & -- & 28.66 & 7.05 & 21.61 & \\
\hline NWS-12-4 & WL & $05 / 01 / 92$ & -- & 28.66 & 7.37 & 21.29 & \\
\hline NWS-12-5 & WL & $04 / 30 / 91$ & 1400 & 30.59 & 7.10 & 23.49 & \\
\hline NWS-12-5 & WL & $05 / 28 / 91$ & 1318 & 30.59 & 7.41 & 23.18 & \\
\hline NWS-12-7 & WL & $04 / 30 / 91$ & 1510 & 30.24 & 22.35 & 7.89 & \\
\hline NWS-12-7 & WL & $05 / 28 / 91$ & 1321 & 30.24 & 22.73 & 7.51 & \\
\hline NWS-12-7 & WL & $07 / 01 / 91$ & -- & 30.24 & 21.04 & 9.20 & \\
\hline
\end{tabular}


Table 8.--Water-level data collected at the Defense Fuel Supply Point and adjacent properties, Hanahan, S.C., between April 1991 and September 1995--Continued

[ft, feet; ---, data not available; WL, water-level well; EW, extraction well; MW, monitoring well; SW, surface-water site; -, negative depth to water indicates water-level altitude is above measuring point]

\begin{tabular}{|c|c|c|c|c|c|c|c|}
\hline $\begin{array}{c}\text { Site } \\
\text { (plate 1) }\end{array}$ & $\begin{array}{l}\text { Site } \\
\text { type }\end{array}$ & Date & Time & $\begin{array}{l}\text { Measuring-point } \\
\text { altitude } \\
\text { (ft above } \\
\text { sea level) }\end{array}$ & $\begin{array}{l}\text { Depth to water } \\
\text { (ft below } \\
\text { measuring point) }\end{array}$ & $\begin{array}{l}\text { Water-level } \\
\text { altitude } \\
\text { (ft above } \\
\text { sea level) }\end{array}$ & Remarks \\
\hline NWS-12-7 & WL & $08 / 06 / 91$ & -- & 30.24 & 22.36 & 7.88 & \\
\hline NWS-12-7 & WL & $08 / 30 / 91$ & -- & 30.24 & 21.97 & 8.27 & \\
\hline NWS-12-7 & WL & $09 / 27 / 91$ & --- & 30.24 & 22.40 & 7.84 & \\
\hline NWS-12-7 & WL & $10 / 29 / 91$ & -- & 30.24 & 23.13 & 7.11 & \\
\hline NWS-12-7 & WL & $12 / 02 / 91$ & -- & 30.24 & 23.82 & 6.42 & \\
\hline NWS-12-7 & WL & $01 / 02 / 92$ & --- & 30.24 & 24.37 & 5.87 & \\
\hline NWS-12-7 & WL & $02 / 04 / 92$ & $\ldots$ & 30.24 & 24.48 & 5.76 & \\
\hline NWS-12-7 & WL & $03 / 06 / 92$ & -- & 30.24 & 24.49 & 5.75 & \\
\hline NWS-12-7 & WL & $03 / 31 / 92$ & --- & 30.24 & 24.51 & 5.73 & \\
\hline NWS-12-7 & WL & $05 / 01 / 92$ & 1447 & 30.24 & 24.31 & 5.93 & \\
\hline NWS-12-7 & WL & $06 / 11 / 92$ & 1601 & 30.24 & 24.25 & 5.99 & \\
\hline NWS-12-7 & WL & $07 / 07 / 92$ & 1347 & 30.24 & 23.75 & 6.49 & \\
\hline NWS-12-7 & WL & $08 / 31 / 92$ & 1441 & 30.24 & 22.45 & 7.79 & \\
\hline NWS-12-7 & WL & $10 / 07 / 92$ & 1340 & 30.24 & 21.86 & 8.38 & \\
\hline NWS-12-7 & WL & $10 / 28 / 92$ & 1437 & 30.24 & 21.55 & 8.69 & \\
\hline NWS-12-7 & WL & $12 / 03 / 92$ & 1500 & 30.24 & 21.70 & 8.54 & \\
\hline NWS-12-7 & WL & $01 / 04 / 93$ & 1425 & 30.24 & 22.14 & 8.10 & \\
\hline NWS-12-7 & WL & $02 / 04 / 93$ & 1550 & 30.24 & 20.25 & 9.99 & \\
\hline NWS-12-7 & WL & $03 / 05 / 93$ & 1443 & 30.24 & 20.60 & 9.64 & \\
\hline NWS-12-7 & WL & $03 / 30 / 93$ & 1400 & 30.24 & 20.76 & 9.48 & \\
\hline NWS-12-7 & WL & $05 / 06 / 93$ & 1401 & 30.24 & 20.83 & 9.41 & \\
\hline NWS-12-7 & WL & $05 / 28 / 93$ & 1350 & 30.24 & 21.77 & 8.47 & \\
\hline NWS-12-7 & WL & $07 / 07 / 93$ & 1424 & 30.24 & 22.83 & 7.41 & \\
\hline PW-01A & MW & $10 / 28 / 92$ & 1130 & 25.08 & 9.53 & 15.55 & \\
\hline PW-01A & MW & $12 / 03 / 92$ & 1424 & 25.08 & 9.06 & 16.02 & \\
\hline PW-01A & MW & $01 / 04 / 93$ & 1350 & 25.08 & 9.48 & 15.60 & \\
\hline PW-01A & MW & $02 / 04 / 93$ & 1442 & 25.08 & 8.75 & 16.33 & \\
\hline PW-01A & MW & $03 / 05 / 93$ & 1410 & 25.08 & 8.67 & 16.41 & \\
\hline PW-0IA & MW & $03 / 30 / 93$ & 1325 & 25.08 & 8.16 & 16.92 & . \\
\hline PW-01A & MW & $05 / 06 / 93$ & 1325 & 25.08 & 8.66 & 16.42 & \\
\hline PW-01A & MW & $05 / 28 / 93$ & 1314 & 25.08 & 9.30 & 15.78 & \\
\hline PW-01A & MW & $07 / 07 / 93$ & 1340 & 25.08 & 9.57 & 15.51 & \\
\hline PW-01A & MW & $08 / 06 / 93$ & 1304 & 25.08 & 9.99 & 15.09 & \\
\hline PW-01A & MW & $09 / 03 / 93$ & 1156 & 25.08 & 10.00 & 15.08 & \\
\hline PW-01A & MW & $09 / 30 / 93$ & 1253 & 25.08 & 9.64 & 15.44 & \\
\hline PW-01A & MW & $10 / 28 / 93$ & 1330 & 25.08 & 9.98 & 15.10 & \\
\hline PW-01A & MW & $12 / 01 / 93$ & 1525 & 25.08 & 9.81 & 15.27 & \\
\hline PW-01A & MW & $01 / 06 / 94$ & 1314 & 25.08 & 9.64 & 15.44 & \\
\hline PW-01A & MW & $02 / 01 / 94$ & 1242 & 25.08 & 8.92 & 16.16 & \\
\hline PW-01A & MW & $03 / 04 / 94$ & 1219 & 25.08 & 8.52 & 16.56 & \\
\hline PW-01A & MW & $04 / 04 / 94$ & 1138 & 25.08 & 9.30 & 15.78 & \\
\hline PW-01A & MW & $05 / 06 / 94$ & 1314 & 25.08 & 8.35 & 16.73 & \\
\hline PW-01A & MW & $06 / 03 / 94$ & 1208 & 25.08 & 10.80 & 14.28 & \\
\hline PW-01A & MW & $07 / 08 / 94$ & 1342 & 25.08 & 8.94 & 16.14 & \\
\hline
\end{tabular}


Table 8.--Water-level data collected at the Defense Fuel Supply Point and adjacent properties, Hanahan, S.C., between April 1991 and September 1995--Continued

[ft, feet; ---, data not available; WL, water-level well; EW, extraction well; MW, monitoring well; SW, surface-water site; -, negative depth to water indicates water-level altitude is above measuring point]

\begin{tabular}{|c|c|c|c|c|c|c|c|}
\hline $\begin{array}{c}\text { Site } \\
\text { (plate 1) }\end{array}$ & $\begin{array}{l}\text { Site } \\
\text { type }\end{array}$ & Date & Time & $\begin{array}{c}\text { Measuring-point } \\
\text { altitude } \\
\text { (ft above } \\
\text { sea level) }\end{array}$ & $\begin{array}{l}\text { Depth to water } \\
\text { ( } \mathrm{ft} \text { below } \\
\text { measuring point) }\end{array}$ & $\begin{array}{l}\text { Water-level } \\
\text { altitude } \\
\text { (ft above } \\
\text { sea level) }\end{array}$ & Remarks \\
\hline PW-0IB & MW & $10 / 28 / 92$ & 1132 & 23.12 & 6.93 & 16.19 & \\
\hline PW-01B & MW & $12 / 03 / 92$ & 1427 & 23.12 & 7.48 & 15.64 & \\
\hline PW-01B & MW & $01 / 04 / 93$ & 1352 & 23.12 & 8.09 & 15.03 & \\
\hline PW-01B & MW & $02 / 04 / 93$ & 1500 & 23.12 & 6.92 & 16.20 & \\
\hline PW-01B & MW & $03 / 05 / 93$ & 1405 & 23.12 & 7.09 & 16.03 & \\
\hline PW-01B & MW & $03 / 30 / 93$ & 1330 & 23.12 & 6.97 & 16.15 & \\
\hline PW-01B & MW & $05 / 06 / 93$ & 1328 & 23.12 & 7.60 & 15.52 & \\
\hline PW-0IB & MW & $05 / 28 / 93$ & 1316 & 23.12 & 8.10 & 15.02 & \\
\hline PW-01B & MW & $07 / 07 / 93$ & 1342 & 23.12 & 8.44 & 14.68 & \\
\hline PW-02 & MW & $05 / 15 / 92$ & 1200 & 26.70 & 8.37 & 18.33 & \\
\hline PW-02 & MW & $06 / 11 / 92$ & 1725 & 26.70 & 8.38 & 18.32 & \\
\hline PW-02 & MW & $08 / 31 / 92$ & 1330 & 26.70 & 6.00 & 20.70 & \\
\hline PW-02 & MW & $10 / 07 / 92$ & 1450 & 26.70 & 5.83 & 20.87 & \\
\hline PW-02 & MW & $10 / 28 / 92$ & 1336 & 26.70 & 6.30 & 20.40 & \\
\hline PW-02 & MW & $12 / 03 / 92$ & 1348 & 26.70 & 6.37 & 20.33 & \\
\hline PW-02 & MW & $01 / 04 / 93$ & 1341 & 26.70 & 7.07 & 19.63 & \\
\hline PW-02 & MW & $02 / 04 / 93$ & 1420 & 26.70 & 5.68 & 21.02 & \\
\hline PW-02 & MW & $03 / 05 / 93$ & 1540 & 26.70 & 6.23 & 20.47 & \\
\hline PW-02 & MW & $03 / 30 / 93$ & 1324 & 26.70 & 7.25 & 19.45 & \\
\hline PW-02 & MW & $07 / 07 / 93$ & 1353 & 26.70 & 8.48 & 18.22 & \\
\hline PW-02 & MW & $08 / 06 / 93$ & 1320 & 26.70 & 8.76 & 17.94 & \\
\hline PW-02 & MW & $09 / 03 / 93$ & 1218 & 26.70 & 8.72 & 17.98 & \\
\hline PW-02 & MW & $09 / 30 / 93$ & 1301 & 26.70 & 8.29 & 18.41 & \\
\hline PW-02 & MW & $10 / 28 / 93$ & 1315 & 26.70 & 9.00 & 17.70 & \\
\hline PW-02 & MW & $12 / 01 / 93$ & 1530 & 26.70 & 8.95 & 17.75 & \\
\hline PW-02 & MW & $01 / 06 / 94$ & 1320 & 26.70 & 8.93 & 17.77 & \\
\hline PW-02 & MW & $02 / 01 / 94$ & 1249 & 26.70 & 7.65 & 19.05 & \\
\hline PW-02 & MW & $03 / 04 / 94$ & 1232 & 26.70 & 7.47 & 19.23 & \\
\hline PW-02 & MW & $04 / 04 / 94$ & 1151 & 26.70 & 8.19 & 18.51 & \\
\hline PW-02 & MW & $05 / 06 / 94$ & 1244 & 26.70 & 9.11 & 17.59 & \\
\hline PW-02 & MW & $06 / 03 / 94$ & 1225 & 26.70 & 9.82 & 16.88 & \\
\hline PW-02 & MW & $07 / 08 / 94$ & 1228 & 26.70 & 8.27 & 18.43 & \\
\hline PW-02 & MW & $10 / 06 / 94$ & 1315 & 26.70 & 4.96 & 21.74 & \\
\hline PW-02 & MW & $11 / 09 / 94$ & 1355 & 26.70 & 6.06 & 20.64 & \\
\hline PW-02 & MW & $12 / 06 / 94$ & 1224 & 26.70 & 6.56 & 20.14 & \\
\hline PW-02 & MW & $01 / 10 / 95$ & 1654 & 26.70 & 6.60 & 20.10 & \\
\hline PW-02 & MW & $02 / 02 / 95$ & 1421 & 26.70 & 6.37 & 20.33 & \\
\hline PW-02 & MW & $03 / 02 / 95$ & 1609 & 26.70 & 6.42 & 20.28 & \\
\hline PW-02 & MW & $04 / 05 / 95$ & 1222 & 26.70 & 7.21 & 19.49 & \\
\hline PW-02 & MW & $05 / 02 / 95$ & 1405 & 26.70 & 7.81 & 18.89 & Well in use. \\
\hline PW-02 & MW & $08 / 10 / 95$ & 1327 & 26.70 & 8.19 & 18.51 & \\
\hline PW-02 & MW & $09 / 13 / 95$ & 1451 & 26.70 & 6.16 & 20.54 & \\
\hline PW-05 & MW & $10 / 28 / 92$ & 1440 & 28.75 & 11.25 & 17.50 & \\
\hline
\end{tabular}


Table 8.--Water-level data collected at the Defense Fuel Supply Point and adjacent properties, Hanahan, S.C., between April 1991 and September 1995--Continued

[ft, feet; ---, data not available; WL, water-level well; EW, extraction well; MW, monitoring well; SW, surface-water site; -, negative depth to water indicates water-level altitude is above measuring point]

\begin{tabular}{|c|c|c|c|c|c|c|c|}
\hline $\begin{array}{c}\text { Site } \\
\text { (plate 1) }\end{array}$ & $\begin{array}{l}\text { Site } \\
\text { type }\end{array}$ & Date & Time & $\begin{array}{l}\text { Measuring-point } \\
\text { altitude } \\
\text { (ft above } \\
\text { sea level) }\end{array}$ & $\begin{array}{l}\text { Depth to water } \\
\text { (ft below } \\
\text { measuring point) }\end{array}$ & $\begin{array}{l}\text { Water-level } \\
\text { altitude } \\
\text { (ft above } \\
\text { sea level) }\end{array}$ & Remarks \\
\hline PW-05 & MW & $12 / 03 / 92$ & 1415 & 28.75 & 11.11 & 17.64 & \\
\hline PW-05 & MW & $01 / 04 / 93$ & 1340 & 28.75 & 11.66 & 17.09 & \\
\hline PW-05 & MW & $02 / 04 / 93$ & 1405 & 28.75 & 10.30 & 18.45 & \\
\hline PW-05 & $\mathbf{M W}$ & $03 / 05 / 93$ & 1335 & 28.75 & 10.67 & 18.08 & \\
\hline PW-05 & $\mathrm{MW}$ & $03 / 30 / 93$ & 1312 & 28.75 & 10.31 & 18.44 & \\
\hline PW-05 & MW & $05 / 06 / 93$ & 1326 & 28.75 & 10.75 & 18.00 & \\
\hline PW-05 & MW & $05 / 28 / 93$ & 1313 & 28.75 & 11.28 & 17.47 & \\
\hline PW-05 & MW & $07 / 07 / 93$ & 1327 & 28.75 & 11.95 & 16.80 & \\
\hline PW-05 & MW & $08 / 06 / 93$ & 1305 & 28.75 & 12.53 & 16.22 & \\
\hline PW-05 & MW & $09 / 03 / 93$ & 1155 & 28.75 & 12.35 & 16.40 & \\
\hline PW-05 & MW & $09 / 30 / 93$ & 1250 & 28.75 & 12.14 & 16.61 & \\
\hline PW-05 & MW & $10 / 28 / 93$ & 1305 & 28.75 & 12.71 & 16.04 & \\
\hline PW-05 & $\mathrm{MW}$ & $12 / 01 / 93$ & 1520 & 28.75 & 12.82 & 15.93 & \\
\hline PW-05 & MW & $01 / 06 / 94$ & 1305 & 28.75 & 12.95 & 15.80 & \\
\hline PW-05 & MW & $02 / 01 / 94$ & 1235 & 28.75 & 12.06 & 16.69 & \\
\hline PW-05 & MW & $03 / 04 / 94$ & 1215 & 28.75 & 11.62 & 17.13 & \\
\hline SW-01 & SW & $06 / 11 / 92$ & -- & 13.38 & .98 & 12.40 & \\
\hline SW-01 & SW & 07/07/92 & 1155 & 13.38 & 1.02 & 12.36 & \\
\hline SW-01 & SW & $08 / 31 / 92$ & 1325 & 13.38 & 1.02 & 12.36 & \\
\hline SW-01 & SW & $10 / 07 / 92$ & 1445 & 13.38 & 1.03 & 12.35 & \\
\hline SW-01 & SW & $10 / 28 / 92$ & 1456 & 13.38 & 1.05 & 12.33 & \\
\hline SW-01 & SW & $12 / 03 / 92$ & 1425 & 13.38 & .91 & 12.47 & \\
\hline SW-01 & SW & $01 / 04 / 93$ & 1345 & 13.38 & .81 & 12.57 & \\
\hline SW-01 & SW & $02 / 04 / 93$ & 1425 & 13.38 & .80 & 12.58 & \\
\hline SW-01 & SW & $03 / 05 / 93$ & 1340 & 13.38 & 1.01 & 12.37 & \\
\hline SW-01 & SW & $03 / 30 / 93$ & 1319 & 13.38 & 1.03 & 12.35 & \\
\hline SW-01 & SW & $05 / 06 / 93$ & 1333 & 13.38 & 1.05 & 12.33 & \\
\hline SW-01 & SW & $05 / 28 / 93$ & 1319 & 13.38 & 1.05 & 12.33 & \\
\hline SW-01 & SW & $07 / 07 / 93$ & 1335 & 13.38 & .90 & 12.48 & \\
\hline SW-0I & SW & $08 / 06 / 93$ & 1328 & 13.38 & .88 & 12.50 & \\
\hline SW-01 & SW & $09 / 03 / 93$ & 1220 & 13.38 & .78 & 12.60 & \\
\hline SW-01 & SW & $09 / 30 / 93$ & 1310 & 13.38 & .85 & 12.53 & \\
\hline SW-01 & SW & $10 / 28 / 93$ & 1335 & 13.38 & .84 & 12.54 & \\
\hline SW-01 & SW & $12 / 01 / 93$ & 1540 & 13.38 & 1.06 & 12.32 & \\
\hline SW-01 & SW & $01 / 06 / 94$ & 1325 & 13.38 & 1.04 & 12.34 & \\
\hline SW-01 & SW & $02 / 01 / 94$ & 1255 & 13.38 & 1.05 & 12.33 & \\
\hline SW-01 & SW & $03 / 04 / 94$ & 1233 & 13.38 & 1.00 & 12.38 & \\
\hline SW-08 & SW & $06 / 11 / 92$ & 1501 & 27.48 & .97 & 26.51 & \\
\hline SW-08 & SW & $07 / 07 / 92$ & 1520 & 27.48 & 1.07 & 26.41 & \\
\hline SW-08 & SW & 08/07/92 & 1346 & 27.48 & 1.04 & 26.44 & \\
\hline SW-08 & SW & $08 / 31 / 92$ & 1110 & 27.48 & 1.14 & 26.34 & \\
\hline SW-08 & SW & $10 / 07 / 92$ & 1100 & 27.48 & 1.11 & 26.37 & \\
\hline SW-08 & SW & $10 / 28 / 92$ & 1602 & 27.48 & 1.12 & 26.36 & \\
\hline
\end{tabular}


Table 8.--Water-level data collected at the Defense Fuel Supply Point and adjacent properties, Hanahan, S.C., between April 1991 and September 1995--Continued

[ft, feet; ---, data not available; WL, water-level well; EW, extraction well; MW, monitoring well; SW, surface-water site; -, negative depth to water indicates water-level altitude is above measuring point]

\begin{tabular}{|c|c|c|c|c|c|c|c|}
\hline $\begin{array}{c}\text { Site } \\
\text { (plate 1) }\end{array}$ & $\begin{array}{l}\text { Site } \\
\text { type }\end{array}$ & Date & Time & $\begin{array}{l}\text { Measuring-point } \\
\text { altitude } \\
\text { (ft above } \\
\text { sea level) }\end{array}$ & $\begin{array}{l}\text { Depth to water } \\
\text { (ft below } \\
\text { measuring point) }\end{array}$ & $\begin{array}{l}\text { Water-level } \\
\text { altitude } \\
\text { (ft above } \\
\text { sea level) }\end{array}$ & Remarks \\
\hline SW-08 & SW & $12 / 03 / 92$ & 1055 & 27.48 & 1.08 & 26.40 & \\
\hline SW-08 & sw & $01 / 04 / 93$ & 1035 & 27.48 & .99 & 26.49 & \\
\hline SW-08 & SW & $02 / 04 / 93$ & 1107 & 27.48 & 1.04 & 26.44 & \\
\hline SW-08 & sw & $03 / 05 / 93$ & 1035 & 27.48 & 1.10 & 26.38 & \\
\hline SW-08 & SW & $03 / 30 / 93$ & 1025 & 27.48 & 1.07 & 26.41 & \\
\hline SW-08 & sw & $05 / 06 / 93$ & 1046 & 27.48 & 1.13 & 26.35 & \\
\hline SW-08 & sw & $05 / 28 / 93$ & 1038 & 27.48 & .97 & 26.51 & \\
\hline SW-08 & SW & $07 / 07 / 93$ & 1052 & 27.48 & 1.13 & 26.35 & \\
\hline SW-08 & sw & $08 / 06 / 93$ & 1033 & 27.48 & 1.11 & 26.37 & \\
\hline SW-08 & SW & $09 / 03 / 93$ & 1045 & 27.48 & 1.10 & 26.38 & \\
\hline SW-08 & sw & $09 / 30 / 93$ & 1035 & 27.48 & 1.01 & 26.47 & \\
\hline SW-08 & sw & $10 / 28 / 93$ & 1037 & 27.48 & 1.08 & 26.40 & \\
\hline SW-08 & SW & $12 / 01 / 93$ & 1340 & 27.48 & 1.01 & 26.47 & \\
\hline SW-08 & sw & $01 / 06 / 94$ & 1112 & 27.48 & .94 & 26.54 & \\
\hline SW-08 & sw & $02 / 01 / 94$ & 1010 & 27.48 & .80 & 26.68 & \\
\hline SW-08 & sw & $03 / 04 / 94$ & 1010 & 27.48 & .65 & 26.83 & Dewatering upstream. \\
\hline SW-13 & SW & $10 / 07 / 92$ & 1115 & 32.35 & 3.73 & 28.62 & \\
\hline SW-13 & SW & $10 / 28 / 92$ & 1509 & 32.35 & 1.77 & 30.58 & \\
\hline SW-13 & SW & $12 / 03 / 92$ & 1112 & 32.35 & 3.70 & 28.65 & \\
\hline SW-13 & sw & $01 / 04 / 93$ & 1045 & 32.35 & 3.72 & 28.63 & \\
\hline SW-13 & Sw & $02 / 04 / 93$ & 1137 & 32.35 & 3.59 & 28.76 & \\
\hline SW-13 & SW & $03 / 05 / 93$ & 1105 & 32.35 & 3.65 & 28.70 & \\
\hline SW-13 & sw & $03 / 30 / 93$ & 1036 & 32.35 & 3.59 & 28.76 & \\
\hline SW-13 & sW & $05 / 06 / 93$ & 1055 & 32.35 & 3.70 & 28.65 & \\
\hline SW-13 & SW & $07 / 07 / 93$ & 1102 & 32.35 & 3.89 & 28.46 & \\
\hline SW-13 & SW & $08 / 06 / 93$ & 1044 & 32.35 & 3.86 & 28.49 & \\
\hline SW-13 & sw & $09 / 03 / 93$ & 1050 & 32.35 & 3.79 & 28.56 & \\
\hline SW-13 & SW & $09 / 30 / 93$ & 1049 & 32.35 & 3.74 & 28.61 & \\
\hline SW-13 & SW & $10 / 28 / 93$ & 1044 & 32.35 & 3.84 & 28.51 & \\
\hline SW-13 & sw & $12 / 01 / 93$ & 1400 & 32.35 & 3.84 & 28.51 & \\
\hline SW-13 & SW & $01 / 06 / 94$ & 1130 & 32.35 & 3.80 & 28.55 & \\
\hline SW-13 & sw & $02 / 01 / 94$ & 1019 & 32.35 & 1.70 & 30.65 & \\
\hline SW-13 & sw & $03 / 04 / 94$ & 1020 & 32.35 & 2.84 & 29.51 & Dam gate open. \\
\hline SW-14 & sw & $10 / 07 / 92$ & 1120 & 31.63 & 1.76 & 29.87 & \\
\hline SW-14 & sw & $10 / 28 / 92$ & 1510 & 31.63 & 3.83 & 27.80 & \\
\hline SW-14 & SW & $12 / 03 / 92$ & 1110 & 31.63 & 1.78 & 29.85 & \\
\hline SW-14 & SW & $01 / 04 / 93$ & 1047 & 31.63 & 1.79 & 29.84 & \\
\hline SW-14 & SW & $02 / 04 / 93$ & 1125 & 31.63 & 1.77 & 29.86 & \\
\hline SW-14 & sw & $03 / 05 / 93$ & 1106 & 31.63 & 1.75 & 29.88 & \\
\hline SW-14 & SW & $03 / 30 / 93$ & 1035 & 31.63 & 1.76 & 29.87 & \\
\hline SW-14 & SW & $05 / 06 / 93$ & 1057 & 31.63 & 1.77 & 29.86 & \\
\hline SW-14 & sw & $07 / 07 / 93$ & 1102 & 31.63 & 1.80 & 29.83 & \\
\hline SW-14 & sw & $08 / 06 / 93$ & 1045 & 31.63 & 1.79 & 29.84 & \\
\hline
\end{tabular}


Table 8.--Water-level data collected at the Defense Fuel Supply Point and adjacent properties, Hanahan, S.C., between April 1991 and September 1995--Continued

[ft, feet; ---, data not available; WL, water-level well; EW, extraction well; MW, monitoring well; SW, surface-water site; -, negative depth to water indicates water-level altitude is above measuring point]

\begin{tabular}{|c|c|c|c|c|c|c|c|}
\hline $\begin{array}{c}\text { Site } \\
\text { (plate 1) }\end{array}$ & $\begin{array}{l}\text { Site } \\
\text { type }\end{array}$ & Date & Time & $\begin{array}{l}\text { Measuring-point } \\
\text { altitude } \\
\text { (ft above } \\
\text { sea level) }\end{array}$ & $\begin{array}{l}\text { Depth to water } \\
\text { (ft below } \\
\text { measuring point) }\end{array}$ & $\begin{array}{l}\text { Water-level } \\
\text { altitude } \\
\text { (ft above } \\
\text { sea level) }\end{array}$ & Remarks \\
\hline SW-14 & SW & $09 / 03 / 93$ & 1055 & 31.63 & 1.79 & 29.84 & \\
\hline SW-14 & SW & $09 / 30 / 93$ & 1050 & 31.63 & 1.76 & 29.87 & \\
\hline SW-14 & sw & $10 / 28 / 93$ & 1045 & 31.63 & 1.83 & 29.80 & \\
\hline SW-14 & SW & $12 / 01 / 93$ & 1401 & 31.63 & 1.78 & 29.85 & \\
\hline SW-14 & sw & $01 / 06 / 94$ & 1131 & 31.63 & 1.78 & 29.85 & \\
\hline SW-14 & SW & $02 / 01 / 94$ & 1020 & 31.63 & 3.66 & 27.97 & \\
\hline SW-14 & SW & $03 / 04 / 94$ & 1021 & 31.63 & 3.75 & 27.88 & \\
\hline W-001 & MW & $04 / 30 / 91$ & 1041 & 16.81 & .60 & 16.21 & \\
\hline W-001 & MW & $05 / 28 / 91$ & 1211 & 16.81 & .74 & 16.07 & \\
\hline W-001 & MW & $07 / 01 / 91$ & -- & 16.81 & 1.22 & 15.59 & \\
\hline W-001 & MW & $08 / 06 / 91$ & - & 16.81 & .50 & 16.31 & \\
\hline W-001 & MW & $08 / 30 / 91$ & - & 16.81 & .29 & 16.52 & \\
\hline W-001 & MW & $09 / 27 / 91$ & - & 16.81 & .78 & 16.03 & \\
\hline W-001 & MW & $10 / 29 / 91$ & -- & 16.81 & 1.07 & 15.74 & \\
\hline W-001 & MW & $12 / 02 / 91$ & -- & 16.81 & 1.30 & i 5.51 & \\
\hline W-001 & MW & $01 / 02 / 92$ & $\cdots$ & 16.81 & 1.47 & 15.34 & \\
\hline W-001 & MW & $02 / 04 / 92$ & $\cdots$ & 16.81 & 1.69 & 15.12 & \\
\hline W-001 & MW & $03 / 06 / 92$ & $--\cdot$ & 16.81 & 1.94 & 14.87 & \\
\hline W-00I & MW & $03 / 31 / 92$ & -.. & 16.81 & 1.50 & 15.31 & \\
\hline W-00l & MW & $05 / 01 / 92$ & 1413 & 16.81 & 2.18 & 14.63 & \\
\hline W-001 & MW & $06 / 11 / 92$ & 1713 & 16.81 & 1.85 & 14.96 & \\
\hline W-001 & MW & $07 / 07 / 92$ & 1038 & 16.81 & 2.06 & 14.75 & \\
\hline W-001 & MW & $08 / 31 / 92$ & 1320 & 16.81 & .98 & 15.83 & \\
\hline W-001 & MW & $10 / 07 / 92$ & 1433 & 16.81 & .43 & 16.38 & \\
\hline W-001 & MW & $10 / 28 / 92$ & 1342 & 16.81 & .70 & 16.11 & \\
\hline W-001 & MW & $12 / 03 / 92$ & 1409 & 16.81 & .70 & 16.11 & \\
\hline W-00I & MW & $01 / 04 / 93$ & 1321 & 16.81 & 1.00 & 15.81 & \\
\hline W-001 & MW & $02 / 04 / 93$ & 1408 & 16.81 & .30 & 16.51 & \\
\hline W- -01 & MW & $03 / 05 / 93$ & 1345 & 16.81 & .50 & 16.31 & \\
\hline W-001 & MW & $03 / 30 / 93$ & 1302 & 16.81 & .78 & 16.03 & \\
\hline W-001 & MW & $05 / 06 / 93$ & 1316 & 16.93 & 1.38 & 15.55 & \\
\hline W-001 & MW & $05 / 28 / 93$ & 1302 & 16.93 & 1.79 & 15.14 & \\
\hline W-001 & MW & $07 / 07 / 93$ & 1325 & 16.81 & 1.90 & 14.91 & \\
\hline W-001 & MW & $08 / 06 / 93$ & 1316 & 16.81 & 1.87 & 14.94 & \\
\hline W-001 & MW & $09 / 03 / 93$ & 1204 & 16.81 & 2.04 & 14.77 & \\
\hline W-001 & MW & 09/30/93 & 1255 & 16.81 & 1.58 & 15.23 & \\
\hline W-001 & MW & $10 / 28 / 93$ & 1255 & 16.81 & 2.07 & 14.74 & \\
\hline W-001 & MW & $12 / 01 / 93$ & 1530 & 16.81 & 1.80 & 15.01 & \\
\hline W-001 & MW & $01 / 06 / 94$ & 1315 & 16.81 & 1.57 & 15.24 & \\
\hline W-001 & MW & $02 / 01 / 94$ & 1250 & 16.81 & 1.05 & 15.76 & \\
\hline W-001 & MW & $03 / 04 / 94$ & 1222 & 16.81 & .80 & 16.01 & \\
\hline W-001 & MW & $04 / 04 / 94$ & 1142 & 16.81 & 1.47 & 15.34 & \\
\hline W-001 & MW & $05 / 06 / 94$ & 1239 & 16.81 & 2.44 & 14.37 & \\
\hline W-001 & MW & $06 / 03 / 94$ & 1215 & 16.81 & 2.84 & 13.97 & \\
\hline
\end{tabular}


Table 8.--Water-level data collected at the Defense Fuel Supply Point and adjacent properties, Hanahan, S.C., between April 1991 and September 1995--Continued

[ft, feet; ---, data not available; WL, water-leve] well; EW, extraction well; MW, monitoring well; SW, surface-water site; -, negative depth to wateindicates water-level altitude is above measuring point]

\begin{tabular}{|c|c|c|c|c|c|c|c|}
\hline $\begin{array}{c}\text { Site } \\
\text { (plate 1) }\end{array}$ & $\begin{array}{l}\text { Site } \\
\text { type }\end{array}$ & Date & Time & $\begin{array}{l}\text { Measuring-point } \\
\text { altitude } \\
\text { (ft above } \\
\text { sea level) }\end{array}$ & $\begin{array}{l}\text { Depth to water } \\
\text { (ft below } \\
\text { measuring point) }\end{array}$ & $\begin{array}{l}\text { Water-level } \\
\text { altitude } \\
\text { (ft above } \\
\text { sea level) }\end{array}$ & Remarks \\
\hline W-001 & $\mathrm{MW}$ & $07 / 08 / 94$ & 1224 & 16.81 & 1.58 & 15.23 & \\
\hline W-001 & MW & $08 / 05 / 94$ & 1500 & 16.81 & 1.27 & 15.54 & \\
\hline W-001 & MW & $09 / 07 / 94$ & 1320 & 16.81 & 1.01 & 15.80 & \\
\hline W-001 & MW & $10 / 06 / 94$ & 1327 & 16.81 & .00 & 16.81 & $\begin{array}{l}\text { Water-table elevation greater than or } \\
\text { equal to measuring point. }\end{array}$ \\
\hline W-001 & MW & $11 / 09 / 94$ & 1345 & 16.81 & .42 & 16.39 & \\
\hline W-001 & MW & $12 / 06 / 94$ & 1216 & 16.81 & .39 & 16.42 & \\
\hline W-001 & MW & $01 / 10 / 95$ & 1651 & 16.81 & .60 & 16.21 & \\
\hline W-001 & MW & $02 / 02 / 95$ & 1425 & 16.81 & .62 & 16.19 & \\
\hline W-001 & MW & $03 / 02 / 95$ & 1558 & 16.81 & .54 & 16.27 & \\
\hline W-001 & MW & $04 / 05 / 95$ & 1217 & 16.81 & 1.06 & 15.75 & \\
\hline W-001 & MW & $05 / 02 / 95$ & 1352 & 16.81 & 1.62 & 15.19 & \\
\hline W-001 & MW & $06 / 09 / 95$ & 1313 & 16.81 & 1.90 & 14.91 & \\
\hline W-001 & MW & $07 / 06 / 95$ & 1232 & 16.81 & 1.88 & 14.93 & \\
\hline W-001 & MW & $08 / 10 / 95$ & 1318 & 16.81 & 1.94 & 14.87 & \\
\hline W-001 & MW & $09 / 13 / 95$ & 1447 & 16.81 & .80 & 16.01 & \\
\hline W-002 & MW & $04 / 30 / 91$ & 1030 & 16.26 & 6.92 & 9.34 & \\
\hline W-002 & MW & $05 / 28 / 91$ & 1200 & 16.26 & 7.02 & 9.24 & \\
\hline W-002 & MW & 07/01/91 & --- & 16.26 & 7.29 & 8.97 & \\
\hline W-002 & MW & $08 / 06 / 91$ & -- & 16.26 & 6.67 & 9.59 & \\
\hline W-002 & MW & $08 / 30 / 91$ & --- & 16.26 & 6.46 & 9.80 & \\
\hline W-002 & MW & $09 / 27 / 91$ & -- & 16.26 & 7.03 & 9.23 & \\
\hline W-002 & MW & $10 / 29 / 91$ & -- & 16.26 & 7.40 & 8.86 & \\
\hline W-002 & MW & $12 / 02 / 91$ & --- & 16.26 & 7.59 & 8.67 & \\
\hline W-002 & MW & $01 / 02 / 92$ & --- & 16.26 & 7.71 & 8.55 & \\
\hline W-002 & MW & $02 / 04 / 92$ & --- & 16.26 & 7.25 & 9.01 & \\
\hline W-002 & MW & $03 / 06 / 92$ & -- & 16.26 & 7.43 & 8.83 & \\
\hline W-002 & MW & $03 / 31 / 92$ & -- & 16.26 & 7.31 & 8.95 & \\
\hline W-002 & MW & $05 / 01 / 92$ & $\cdots$ & 16.26 & 7.43 & 8.83 & \\
\hline W-002 & MW & $06 / 11 / 92$ & 1650 & 16.26 & 7.03 & 9.23 & \\
\hline W-002 & MW & $07 / 07 / 92$ & 1115 & 16.26 & 7.35 & 8.91 & \\
\hline W-002 & MW & $08 / 31 / 92$ & 1255 & 16.26 & 6.83 & 9.43 & \\
\hline W-002 & MW & $10 / 07 / 92$ & 1400 & 16.26 & 6.56 & 9.70 & \\
\hline W-002 & MW & $10 / 28 / 92$ & 1626 & 16.26 & 6.88 & 9.38 & \\
\hline W-002 & MW & $12 / 03 / 92$ & 1240 & 16.26 & 6.70 & 9.56 & \\
\hline W-002 & MW & $01 / 04 / 93$ & 1205 & 16.26 & 7.09 & 9.17 & \\
\hline W-002 & MW & $02 / 04 / 93$ & 1240 & 16.26 & 6.55 & 9.71 & \\
\hline W-002 & MW & $03 / 05 / 93$ & 1215 & 16.26 & 6.69 & 9.57 & \\
\hline W-002 & MW & $03 / 30 / 93$ & 1138 & 16.26 & 6.60 & 9.66 & \\
\hline W-002 & MW & $05 / 06 / 93$ & 1205 & 16.26 & 7.10 & 9.16 & \\
\hline W-002 & MW & $05 / 28 / 93$ & 1159 & 16.26 & 7.39 & 8.87 & \\
\hline W-002 & MW & $07 / 07 / 93$ & 1225 & 16.26 & 7.73 & 8.53 & \\
\hline W-002 & MW & $08 / 06 / 93$ & 1210 & 16.26 & 7.80 & 8.46 & \\
\hline
\end{tabular}


Table 8.--Water-level data collected at the Defense Fuel Supply Point and adjacent properties, Hanahan, S.C., between April 1991 and September 1995--Continued

[ft, feet; ---, data not available; WL, water-leve] well; EW, extraction well; MW, monitoring well; SW, surface-water site; -, negative depth to water indicates water-level altitude is above measuring point]

\begin{tabular}{|c|c|c|c|c|c|c|c|}
\hline $\begin{array}{c}\text { Site } \\
\text { (plate 1) }\end{array}$ & $\begin{array}{l}\text { Site } \\
\text { type }\end{array}$ & Date & Time & $\begin{array}{l}\text { Measuring-point } \\
\text { altitude } \\
\text { (ft above } \\
\text { sea level) }\end{array}$ & $\begin{array}{l}\text { Depth to water } \\
\text { (ft below } \\
\text { measuring point) }\end{array}$ & $\begin{array}{l}\text { Water-level } \\
\text { altitude } \\
\text { (ft above } \\
\text { sea level) }\end{array}$ & Remarks \\
\hline W-003 & MW & $04 / 30 / 91$ & 1026 & 32.95 & 15.20 & 17.75 & \\
\hline W-003 & MW & $05 / 28 / 91$ & 1145 & 32.95 & 15.52 & 17.43 & \\
\hline W-003 & MW & $07 / 01 / 91$ & $\cdots$ & 32.95 & 16.07 & 16.88 & \\
\hline W-003 & MW & $08 / 06 / 91$ & --- & 32.95 & 15.02 & 17.93 & \\
\hline W-003 & MW & $08 / 30 / 91$ & -- & 32.95 & 14.67 & 18.28 & \\
\hline W-003 & MW & $09 / 27 / 91$ & -- & 32.95 & 15.36 & 17.59 & \\
\hline W-003 & MW & $10 / 29 / 91$ & -- & 32.95 & 16.04 & 16.91 & \\
\hline W-003 & MW & $12 / 02 / 91$ & - & 32.95 & 16.39 & 16.56 & \\
\hline W-003 & MW & $01 / 02 / 92$ & -- & 32.95 & 16.70 & 16.25 & \\
\hline W-003 & MW & $02 / 04 / 92$ & - & 32.95 & 16.28 & 16.67 & \\
\hline W-003 & MW & $03 / 06 / 92$ & - & 32.95 & 16.32 & 16.63 & \\
\hline W-003 & MW & $03 / 31 / 92$ & -- & 32.95 & 16.18 & 16.77 & \\
\hline W-003 & MW & $05 / 01 / 92$ & 1356 & 32.95 & 16.50 & 16.45 & \\
\hline W-003 & MW & $06 / 11 / 92$ & 1645 & 32.95 & 16.43 & 16.52 & \\
\hline W-003 & MW & $07 / 07 / 92$ & 1120 & 32.95 & 16.48 & 16.47 & \\
\hline W-003 & MW & $08 / 31 / 92$ & 1200 & 32.95 & 15.63 & 17.32 & \\
\hline W-003 & MW & $10 / 07 / 92$ & 1210 & 32.95 & 15.04 & 17.91 & \\
\hline W-003 & MW & $10 / 28 / 92$ & 1529 & 32.95 & 15.19 & 17.76 & \\
\hline W-003 & MW & $12 / 03 / 92$ & 1215 & 32.95 & 14.81 & 18.14 & \\
\hline W-003 & MW & $01 / 04 / 93$ & 1140 & 32.95 & 15.30 & 17.65 & \\
\hline W-003 & MW & $02 / 04 / 93$ & 1215 & 32.95 & 14.10 & 18.85 & \\
\hline W-003 & MW & $03 / 05 / 93$ & 1155 & 32.95 & 14.08 & 18.87 & \\
\hline W-003 & MW & $03 / 30 / 93$ & 1114 & 32.95 & 13.97 & 18.98 & \\
\hline W-003 & MW & $05 / 06 / 93$ & 1145 & 32.95 & 14.83 & 18.12 & \\
\hline W-003 & MW & $05 / 28 / 93$ & 1132 & 32.95 & 15.48 & 17.47 & \\
\hline W-003 & MW & $07 / 07 / 93$ & 1155 & 32.95 & 16.24 & 16.71 & \\
\hline W-003 & MW & $08 / 06 / 93$ & 1142 & 32.95 & 16.52 & 16.43 & \\
\hline W-103 & MW & $04 / 30 / 91$ & 1010 & 37.05 & 15.14 & 21.91 & \\
\hline W-103 & MW & $05 / 28 / 91$ & --- & 37.05 & 15.35 & 21.70 & \\
\hline W-103 & MW & $07 / 01 / 91$ & $\cdots$ & 37.05 & 15.88 & 21.17 & \\
\hline W-103 & MW & $08 / 06 / 91$ & -- & 37.05 & 12.74 & 24.31 & \\
\hline W-103 & MW & $08 / 30 / 91$ & --- & 37.05 & 12.23 & 24.82 & \\
\hline W-103 & MW & $09 / 27 / 91$ & - & 37.05 & 14.65 & 22.40 & \\
\hline W-103 & MW & $10 / 29 / 91$ & - & 37.05 & 15.97 & 21.08 & \\
\hline W-103 & MW & $12 / 02 / 91$ & -- & 37.05 & 16.60 & 20.45 & \\
\hline W-103 & MW & $01 / 02 / 92$ & -- & 37.05 & 17.13 & 19.92 & \\
\hline W-103 & MW & $02 / 04 / 92$ & -- & 37.05 & 17.04 & 20.01 & \\
\hline W-103 & MW & $03 / 06 / 92$ & -- & 37.05 & 16.77 & 20.28 & \\
\hline W-103 & MW & $03 / 31 / 92$ & - & 37.05 & 16.68 & 20.37 & \\
\hline W-103 & MW & $05 / 01 / 92$ & -- & 37.05 & 16.78 & 20.27 & \\
\hline W-103 & MW & $06 / 11 / 92$ & 1230 & 37.05 & 17.94 & 19.11 & \\
\hline W-103 & MW & $07 / 07 / 92$ & 1727 & 37.05 & 17.23 & 19.82 & \\
\hline W-103 & MW & $08 / 07 / 92$ & 1010 & 37.05 & 17.64 & 19.41 & \\
\hline W-103 & MW & $08 / 31 / 92$ & 1030 & 37.05 & 13.13 & 23.92 & \\
\hline
\end{tabular}


Table 8.--Water-level data collected at the Defense Fuel Supply Point and adjacent properties, Hanahan, S.C., between April 1991 and September 1995--Continued

[ft, feet; ---, data not available; WL, water-level well; EW, extraction well; MW, monitoring well; SW, surface-water site; -, negative depth to wat ’r indicates water-level altitude is above measuring point]

\begin{tabular}{|c|c|c|c|c|c|c|c|}
\hline $\begin{array}{c}\text { Site } \\
\text { (plate 1) }\end{array}$ & $\begin{array}{l}\text { Site } \\
\text { type }\end{array}$ & Date & Time & $\begin{array}{l}\text { Measuring-point } \\
\text { altitude } \\
\text { (ft above } \\
\text { sea level) }\end{array}$ & $\begin{array}{l}\text { Depth to water } \\
\text { (ft below } \\
\text { measuring point) }\end{array}$ & $\begin{array}{l}\text { Water-level } \\
\text { altitude } \\
\text { (ft above } \\
\text { sea level) }\end{array}$ & Remarks \\
\hline $\mathrm{W}-103$ & $\overline{M W}$ & $10 / 07 / 92$ & 1030 & 37.05 & 13.10 & 23.95 & \\
\hline W-103 & MW & $12 / 03 / 92$ & 0955 & 37.05 & 14.56 & 22.49 & \\
\hline W-103 & MW & $01 / 04 / 93$ & 1005 & 37.05 & 16.33 & 20.72 & \\
\hline W-103 & MW & $02 / 04 / 93$ & 1030 & 37.05 & 12.98 & 24.07 & \\
\hline W-103 & MW & $03 / 05 / 93$ & 1015 & 37.05 & 14.94 & 22.11 & \\
\hline W-103 & MW & $03 / 30 / 93$ & 0933 & 37.05 & 17.25 & 19.80 & \\
\hline W-103 & MW & $05 / 06 / 93$ & 1009 & 37.05 & 18.06 & 18.99 & \\
\hline W-103 & MW & $05 / 28 / 93$ & 1010 & 37.05 & 18.98 & 18.07 & \\
\hline W-103 & MW & $07 / 07 / 93$ & 1000 & 37.05 & -- & -- & Free product present in well. \\
\hline W-103 & MW & $08 / 06 / 93$ & 0945 & 37.05 & 18.25 & 18.80 & \\
\hline W-103 & MW & $09 / 03 / 93$ & 1005 & 37.05 & 18.19 & 18.86 & \\
\hline W-103 & MW & $09 / 30 / 93$ & 1005 & 37.05 & 17.44 & 19.61 & \\
\hline W-103 & MW & $10 / 28 / 93$ & 0955 & 37.05 & 18.50 & 18.55 & Petroleum odor. \\
\hline W-103 & MW & $01 / 06 / 94$ & 1015 & 37.05 & -- & --- & $2.55 \mathrm{ft}$ of free product in well. \\
\hline W-105 & MW & $04 / 30 / 91$ & 1020 & 36.45 & 12.78 & 23.67 & \\
\hline W-105 & MW & $05 / 28 / 91$ & -- & 36.45 & 12.80 & 23.65 & \\
\hline W-105 & MW & $08 / 30 / 91$ & -- & 36.43 & 11.75 & 24.68 & New measuring point established \\
\hline W-105 & MW & $09 / 27 / 91$ & -- & 36.43 & 12.58 & 23.85 & \\
\hline W-105 & MW & $10 / 29 / 91$ & -- & 36.43 & 13.13 & 23.30 & \\
\hline W-105 & MW & $12 / 02 / 91$ & -- & 36.43 & 13.44 & 22.99 & \\
\hline W-105 & MW & $01 / 02 / 92$ & -- & 36.43 & 13.75 & 22.68 & \\
\hline W-105 & MW & $02 / 04 / 92$ & --- & 36.43 & 12.82 & 23.61 & \\
\hline W-105 & MW & $03 / 06 / 92$ & --- & 36.43 & 13.56 & 22.87 & \\
\hline W-105 & MW & $03 / 31 / 92$ & -- & 36.43 & 13.71 & 22.72 & \\
\hline W-105 & MW & $05 / 01 / 92$ & 1540 & 36.43 & 13.62 & 22.81 & \\
\hline W-105 & MW & $05 / 01 / 92$ & 1030 & 36.43 & 13.70 & 22.73 & \\
\hline W-105 & MW & $06 / 11 / 92$ & 1406 & 36.43 & 13.73 & 22.70 & \\
\hline W-105 & MW & $07 / 07 / 92$ & 1714 & 36.43 & 13.55 & 22.88 & \\
\hline W-105 & MW & $08 / 31 / 92$ & 1128 & 36.43 & 12.43 & 24.00 & \\
\hline W-105 & MW & $10 / 07 / 92$ & 1138 & 36.43 & 12.40 & 24.03 & \\
\hline W-105 & MW & $10 / 28 / 92$ & 1254 & 36.43 & 12.66 & 23.77 & \\
\hline W-105 & MW & $12 / 03 / 92$ & 1116 & 36.43 & 12.70 & 23.73 & \\
\hline W-105 & MW & $01 / 04 / 93$ & 1105 & 36.43 & 13.40 & 23.03 & \\
\hline W-105 & MW & $02 / 04 / 93$ & 1154 & 36.43 & 12.78 & 23.65 & \\
\hline W-105 & MW & $03 / 05 / 93$ & 1121 & 36.43 & 13.05 & 23.38 & \\
\hline W-105 & MW & $03 / 30 / 93$ & 1037 & 36.43 & 13.00 & 23.43 & \\
\hline W-105 & MW & $05 / 06 / 93$ & 1103 & 36.43 & 12.97 & 23.46 & \\
\hline W-105 & MW & $05 / 28 / 93$ & 1056 & 39.26 & 16.00 & 23.26 & New measuring point established \\
\hline W-105 & MW & 07/07/93 & 1046 & 39.26 & 15.82 & 23.44 & \\
\hline W-105 & MW & $08 / 06 / 93$ & 1024 & 39.26 & 16.18 & 23.08 & \\
\hline W-105 & MW & 09/03/93 & 1045 & 39.26 & 16.03 & 23.23 & \\
\hline W-105 & MW & $09 / 30 / 93$ & 1040 & 39.26 & 15.61 & 23.65 & \\
\hline W-105 & MW & $10 / 28 / 93$ & 1042 & 39.26 & 16.04 & 23.22 & \\
\hline W-105 & MW & $12 / 01 / 93$ & 1341 & 39.26 & 15.73 & 23.53 & \\
\hline
\end{tabular}


Table 8.--Water-level data collected at the Defense Fuel Supply Point and adjacent properties, Hanahan, S.C., between April 1991 and September 1995--Continued

[ft, feet; ---, data not available; WL, water-level well; EW, extraction well; MW, monitoring well; SW, surface-water site; -, negative depth to water indicates water-level altitude is above measuring point]

\begin{tabular}{|c|c|c|c|c|c|c|c|}
\hline $\begin{array}{c}\text { Site } \\
\text { (plate 1) }\end{array}$ & $\begin{array}{l}\text { Site } \\
\text { type }\end{array}$ & Date & Time & $\begin{array}{l}\text { Measuring-point } \\
\text { altitude } \\
\text { (ft above } \\
\text { sea level) }\end{array}$ & $\begin{array}{l}\text { Depth to water } \\
\text { (ft below } \\
\text { measuring point) }\end{array}$ & $\begin{array}{l}\text { Water-level } \\
\text { altitude } \\
\text { (ft above } \\
\text { sea level) }\end{array}$ & Remarks \\
\hline$W-105$ & MW & $01 / 06 / 94$ & 1051 & 39.26 & 15.69 & 23.57 & \\
\hline W-105 & MW & $02 / 01 / 94$ & 1050 & 39.26 & 15.12 & 24.14 & \\
\hline W-105 & MW & $03 / 04 / 94$ & 1030 & 39.26 & 15.17 & 24.09 & \\
\hline W-105 & MW & $04 / 04 / 94$ & 1025 & 39.26 & 15.38 & 23.88 & \\
\hline W-105 & MW & $05 / 06 / 94$ & 1111 & 39.26 & 15.93 & 23.33 & \\
\hline W-105 & MW & $06 / 03 / 94$ & 1054 & 39.26 & 16.41 & 22.85 & \\
\hline W-105 & MW & $07 / 08 / 94$ & 1102 & 39.26 & 14.69 & 24.57 & \\
\hline W-105 & MW & $08 / 05 / 94$ & 1405 & 39.26 & 14.11 & 25.15 & \\
\hline W-105 & MW & $09 / 07 / 94$ & 1120 & 39.26 & 14.39 & 24.87 & \\
\hline W-105 & MW & $10 / 06 / 94$ & 1131 & 39.26 & 13.39 & 25.87 & \\
\hline W-105 & MW & $11 / 09 / 94$ & 1255 & 39.26 & 14.44 & 24.82 & \\
\hline W-105 & MW & $12 / 06 / 94$ & 1113 & 39.26 & 14.65 & $24.6 \mathrm{I}$ & \\
\hline W-105 & MW & $01 / 10 / 95$ & 1320 & 39.26 & 14.80 & 24.46 & \\
\hline$W-105$ & MW & $02 / 02 / 95$ & 1332 & 39.26 & 14.81 & 24.45 & \\
\hline W-105 & MW & $03 / 02 / 95$ & 1500 & 39.26 & 14.90 & 24.36 & \\
\hline W-I05 & $M W$ & $04 / 05 / 95$ & 1058 & 39.26 & 15.37 & 23.89 & \\
\hline W-105 & MW & $05 / 02 / 95$ & 1248 & 39.26 & 15.68 & 23.58 & \\
\hline W-I05 & MW & $06 / 09 / 95$ & 1049 & 39.26 & 15.87 & 23.39 & \\
\hline W-105 & MW & $07 / 06 / 95$ & 1042 & 39.26 & 15.67 & 23.59 & \\
\hline W-105 & MW & $08 / 10 / 95$ & 1115 & 39.26 & 15.45 & 23.81 & \\
\hline W-105 & MW & $09 / 13 / 95$ & 1153 & 39.26 & 14.88 & 24.38 & \\
\hline W-107 & MW & $04 / 30 / 91$ & 1212 & 34.98 & 7.61 & 27.37 & \\
\hline W-107 & MW & $05 / 28 / 91$ & -- & 34.98 & 7.84 & 27.14 & \\
\hline W-107 & MW & $07 / 01 / 91$ & -- & 34.98 & 8.04 & 26.94 & \\
\hline W-107 & MW & $08 / 06 / 91$ & -- & 34.38 & 5.10 & 29.28 & New measuring point established. \\
\hline W-107 & MW & $08 / 30 / 91$ & --- & 34.38 & 5.80 & 28.58 & \\
\hline W-107 & MW & $09 / 27 / 91$ & --- & 34.38 & 7.35 & 27.03 & \\
\hline W-107 & MW & $10 / 29 / 91$ & $-\cdots$ & 34.38 & 8.05 & 26.33 & \\
\hline W-107 & MW & $12 / 02 / 91$ & -- & 34.38 & 8.40 & 25.98 & \\
\hline W-107 & MW & $02 / 04 / 92$ & --- & 34.38 & 8.40 & 25.98 & \\
\hline W-107 & MW & $03 / 06 / 92$ & --- & 34.38 & 8.64 & 25.74 & \\
\hline W-107 & MW & $03 / 31 / 92$ & --- & 34.38 & 8.57 & 25.81 & \\
\hline W-107 & MW & $05 / 01 / 92$ & -- & 34.38 & 8.09 & 26.29 & \\
\hline W-108 & MW & $04 / 30 / 91$ & 1040 & 37.03 & 9.54 & 27.49 & \\
\hline W-108 & MW & $05 / 28 / 91$ & -- & 37.03 & 9.77 & 27.26 & \\
\hline W-108 & MW & $07 / 01 / 91$ & --- & 37.03 & 9.97 & 27.06 & \\
\hline W-108 & MW & $08 / 06 / 91$ & -- & 37.03 & 8.41 & 28.62 & \\
\hline W-108 & MW & $08 / 30 / 91$ & --- & 37.03 & 8.22 & 28.81 & \\
\hline W-108 & MW & $09 / 27 / 91$ & -- & 37.03 & 9.90 & 27.13 & \\
\hline W-108 & MW & $10 / 29 / 91$ & $\cdots$ & 37.03 & 10.67 & 26.36 & \\
\hline W-108 & MW & $12 / 02 / 91$ & - & 37.03 & 11.07 & 25.96 & \\
\hline W-108 & MW & $01 / 02 / 92$ & --- & 37.03 & 11.45 & 25.58 & \\
\hline W-108 & MW & $02 / 04 / 92$ & -- & 37.03 & 10.55 & 26.48 & Pre-start-up water levels. \\
\hline
\end{tabular}


Table 8.--Water-level data collected at the Defense Fuel Supply Point and adjacent properties, Hanahan, S.C., between April 1991 and September 1995--Continued

[ft, feet; ---, data not available; WL, water-level well; EW, extraction well; MW, monitoring well; SW, surface-water site; -, negative depth to water indicates water-level altitude is above measuring point]

\begin{tabular}{|c|c|c|c|c|c|c|c|}
\hline $\begin{array}{c}\text { Site } \\
\text { (plate 1) }\end{array}$ & $\begin{array}{l}\text { Site } \\
\text { type }\end{array}$ & Date & Time & $\begin{array}{l}\text { Measuring-point } \\
\text { altitude } \\
\text { ( } \mathrm{ft} \text { above } \\
\text { sea level) }\end{array}$ & $\begin{array}{l}\text { Depth to water } \\
\text { (ft below } \\
\text { measuring point) }\end{array}$ & $\begin{array}{l}\text { Water-level } \\
\text { altitude } \\
\text { (ft above } \\
\text { sea level) }\end{array}$ & Remarks \\
\hline W-108 & MW & $03 / 06 / 92$ & $\cdots$ & 37.03 & 11.63 & 25.40 & \\
\hline W-108 & MW & $03 / 31 / 92$ & --- & 37.03 & 10.62 & 26.41 & \\
\hline W-108 & MW & $05 / 01 / 92$ & --- & 37.03 & 10.61 & 26.42 & \\
\hline W-108 & MW & $06 / 11 / 92$ & 1420 & 37.03 & 9.67 & 27.36 & \\
\hline W-108 & MW & $07 / 07 / 92$ & 1655 & 37.03 & 10.16 & 26.87 & \\
\hline W-108 & MW & $08 / 07 / 92$ & 1034 & 37.03 & 9.91 & 27.12 & \\
\hline W-108 & MW & $08 / 31 / 92$ & 1045 & 37.03 & 8.63 & 28.40 & \\
\hline W-108 & MW & $10 / 07 / 92$ & 1040 & 37.03 & 8.30 & 28.73 & \\
\hline W-108 & MW & $12 / 03 / 92$ & 1020 & 37.03 & 9.15 & 27.88 & \\
\hline W-108 & MW & $01 / 04 / 93$ & 1010 & 37.03 & 10.07 & 26.96 & \\
\hline W-108 & MW & $02 / 04 / 93$ & 1045 & 37.03 & 8.96 & 28.07 & \\
\hline W-108 & MW & $03 / 05 / 93$ & 1025 & 37.03 & 9.31 & 27.72 & \\
\hline W-108 & MW & $03 / 30 / 93$ & 0953 & 37.03 & 8.97 & 28.06 & \\
\hline W-108 & MW & $05 / 06 / 93$ & 1023 & 37.03 & 9.75 & 27.28 & \\
\hline W-108 & MW & $05 / 28 / 93$ & 1019 & 37.03 & 10.23 & 26.80 & \\
\hline W-108 & MW & $07 / 07 / 93$ & 1010 & 37.03 & 9.98 & 27.05 & \\
\hline W-108 & MW & $08 / 06 / 93$ & 1000 & 37.03 & 9.59 & 27.44 & \\
\hline W-108 & MW & $09 / 03 / 93$ & 1020 & 37.03 & 9.89 & 27.14 & \\
\hline W-108 & MW & $09 / 30 / 93$ & 1020 & 37.03 & 9.68 & 27.35 & \\
\hline W-108 & MW & $10 / 28 / 93$ & 1010 & 37.03 & 10.64 & 26.39 & \\
\hline WT-01 & WL & $04 / 30 / 91$ & 1001 & 29.97 & 4.41 & 25.56 & \\
\hline WT-01 & WL & $05 / 28 / 91$ & 1100 & 29.97 & 4.79 & 25.18 & \\
\hline WT-01 & WL & $07 / 01 / 91$ & --- & 29.97 & 5.18 & 24.79 & \\
\hline WT-0I & WL & 08/06/91 & -- & 29.97 & 4.52 & 25.45 & \\
\hline WT-01 & WL & $08 / 30 / 91$ & --- & 29.97 & 3.98 & 25.99 & \\
\hline WT-01 & WL & $09 / 27 / 91$ & $\cdots$ & 29.97 & 5.29 & 24.68 & \\
\hline WT-01 & WL & $10 / 29 / 91$ & -- & 29.97 & 5.82 & 24.15 & \\
\hline WT-01 & WL & $12 / 02 / 91$ & --- & 29.97 & 5.96 & 24.01 & \\
\hline WT-01 & WL & $01 / 02 / 92$ & --- & 29.97 & 5.88 & 24.09 & \\
\hline WT-01 & WL & $02 / 13 / 92$ & -- & 29.97 & 5.02 & 24.95 & \\
\hline WT-01 & WL & $03 / 06 / 92$ & --- & 29.97 & 5.20 & 24.77 & \\
\hline WT-01 & WL & $03 / 31 / 92$ & --- & 29.97 & 4.65 & 25.32 & \\
\hline WT-01 & WL & $05 / 01 / 92$ & - & 29.97 & 5.30 & 24.67 & \\
\hline WT-01 & WL & $06 / 11 / 92$ & 1622 & 29.97 & 3.93 & 26.04 & \\
\hline WT-01 & WL & $07 / 07 / 92$ & 1320 & 29.97 & 5.29 & 24.68 & \\
\hline WT-01 & WL & $08 / 07 / 92$ & 1446 & 29.97 & 4.70 & 25.27 & \\
\hline WT-01 & WL & $08 / 31 / 92$ & 1135 & 29.97 & 4.47 & 25.50 & \\
\hline WT-01 & WL & $10 / 07 / 92$ & 1145 & 29.97 & 3.87 & 26.10 & \\
\hline WT-01 & WL & $10 / 28 / 92$ & 1507 & 29.97 & 4.87 & 25.10 & \\
\hline WT-01 & WL & $12 / 03 / 92$ & 1145 & 29.97 & 4.18 & 25.79 & \\
\hline WT-01 & WL & $01 / 04 / 93$ & 1105 & 29.97 & 4.77 & 25.20 & \\
\hline WT-01 & WL & $02 / 04 / 93$ & 1155 & 29.97 & 4.12 & 25.85 & \\
\hline WT-01 & WL & $03 / 05 / 93$ & 1138 & 29.97 & 3.65 & 26.32 & \\
\hline WT-01 & WL & $03 / 30 / 93$ & 1058 & 29.97 & 3.90 & 26.07 & \\
\hline
\end{tabular}


Table 8.--Water-level data collected at the Defense Fuel Supply Point and adjacent properties, Hanahan, S.C., between April 1991 and September 1995--Continued

[ft, feet; ---, data not available; WL, water-level well; EW, extraction well; MW, monitoring well; SW, surface-water site; -, negative depth to water indicates water-level altitude is above measuring point]

\begin{tabular}{|c|c|c|c|c|c|c|c|}
\hline $\begin{array}{c}\text { Site } \\
\text { (plate 1) }\end{array}$ & $\begin{array}{l}\text { Site } \\
\text { type }\end{array}$ & Date & Time & $\begin{array}{l}\text { Measuring-point } \\
\text { altitude } \\
\text { (ft above } \\
\text { sea level) }\end{array}$ & $\begin{array}{l}\text { Depth to water } \\
\text { (ft below } \\
\text { measuring point) }\end{array}$ & $\begin{array}{l}\text { Water-level } \\
\text { altitude } \\
\text { (ft above } \\
\text { sea level) }\end{array}$ & Remarks \\
\hline WT-01 & $\overline{\mathrm{WL}}$ & $05 / 06 / 93$ & 1124 & 29.97 & 4.90 & 25.07 & \\
\hline WT-01 & WL & $05 / 28 / 93$ & 1115 & 29.97 & 5.59 & 24.38 & \\
\hline WT-01 & WL & $07 / 07 / 93$ & 1125 & 29.97 & 5.37 & 24.60 & \\
\hline WT-01 & WL & $08 / 06 / 93$ & 1115 & 29.97 & 4.97 & 25.00 & \\
\hline WT-02 & WL & $04 / 30 / 91$ & 0921 & 33.65 & 6.74 & 26.91 & \\
\hline WT-02 & WL & $05 / 28 / 91$ & 1125 & 33.65 & 6.96 & 26.69 & \\
\hline WT-02 & WL & $07 / 01 / 91$ & --- & 33.65 & 7.46 & 26.19 & \\
\hline WT-02 & WL & $08 / 06 / 91$ & $\ldots$ & 33.65 & 6.16 & 27.49 & \\
\hline WT-02 & WL & $08 / 30 / 91$ & $\ldots$ & 33.65 & 5.39 & 28.26 & \\
\hline WT-02 & WL & $09 / 27 / 91$ & --- & 33.65 & 7.15 & 26.50 & \\
\hline WT-02 & WL & $10 / 29 / 91$ & -- & 33.65 & 8.01 & 25.64 & \\
\hline WT-02 & WL & $12 / 02 / 91$ & - & 33.65 & 8.53 & 25.12 & \\
\hline WT-02 & WL & $01 / 02 / 92$ & - & 33.65 & 8.84 & 24.81 & \\
\hline WT-02 & WL & $02 / 13 / 92$ & - & 33.65 & 7.55 & 26.10 & \\
\hline WT-02 & WL & $03 / 06 / 92$ & --- & 33.65 & 7.56 & 26.09 & \\
\hline WT-02 & WL & $03 / 31 / 92$ & -- & 33.65 & 7.14 & 26.51 & \\
\hline WT-02 & WL & $05 / 01 / 92$ & - & 33.65 & 7.64 & 26.01 & \\
\hline WT-02 & WL & $06 / 11 / 92$ & 1625 & 33.65 & 6.43 & 27.22 & \\
\hline WT-02 & WL & $07 / 07 / 92$ & 1313 & 33.65 & 7.51 & 26.14 & \\
\hline WT-02 & WL & $08 / 07 / 92$ & 1449 & 33.65 & 7.45 & 26.20 & \\
\hline WT-02 & WL & $08 / 31 / 92$ & 1140 & 33.65 & 6.13 & 27.52 & \\
\hline WT-02 & WL & $10 / 07 / 92$ & 1150 & 33.65 & 5.30 & 28.35 & \\
\hline WT-02 & WL & $10 / 28 / 92$ & 1516 & 33.65 & 6.58 & 27.07 & \\
\hline WT-02 & WL & $12 / 03 / 92$ & 1147 & 33.65 & 5.95 & 27.70 & \\
\hline WT-02 & WL & $01 / 04 / 93$ & 1120 & 33.65 & 6.85 & 26.80 & \\
\hline WT-02 & WL & $02 / 04 / 93$ & 1200 & 33.65 & 5.61 & 28.04 & \\
\hline WT-02 & WL & $03 / 05 / 93$ & 1142 & 33.65 & 5.29 & 28.36 & \\
\hline WT-02 & WL & $03 / 30 / 93$ & 1100 & 33.65 & 5.32 & 28.33 & \\
\hline WT-02 & WL & $05 / 06 / 93$ & 1128 & 33.65 & 6.83 & 26.82 & \\
\hline WT-02 & WL & $05 / 28 / 93$ & 1118 & 33.65 & 7.67 & 25.98 & \\
\hline WT-02 & WL & $07 / 07 / 93$ & 1135 & 33.65 & 7.94 & 25.71 & \\
\hline WT-02 & WL & $08 / 06 / 93$ & 1120 & 33.65 & 7.70 & 25.95 & \\
\hline WT-03 & WL & $04 / 30 / 91$ & 1001 & 30.49 & 5.70 & 24.79 & \\
\hline WT-03 & WL & $05 / 28 / 91$ & 1135 & 30.49 & 5.94 & 24.55 & \\
\hline WT-03 & WL & $07 / 01 / 91$ & -- & 30.49 & 6.28 & 24.21 & \\
\hline WT-03 & WL & 08/06/91 & --- & 30.49 & 5.40 & 25.09 & \\
\hline WT-03 & WL & $08 / 30 / 91$ & - & 30.49 & 4.82 & 25.67 & \\
\hline WT-03 & WL & $09 / 27 / 91$ & $\cdots$ & 30.49 & 6.00 & 24.49 & \\
\hline WT-03 & WL & $10 / 29 / 91$ & - & 30.49 & 6.70 & 23.79 & \\
\hline WT-03 & WL & $12 / 02 / 91$ & -- & 30.49 & 7.14 & 23.35 & \\
\hline WT-03 & WL & $01 / 02 / 92$ & --- & 30.49 & 7.50 & 22.99 & \\
\hline WT-03 & WL & $02 / 04 / 92$ & -- & 30.49 & 6.90 & 23.59 & \\
\hline WT-03 & WL & $03 / 06 / 92$ & $\ldots$ & 30.49 & 6.98 & 23.51 & \\
\hline
\end{tabular}


Table 8.--Water-level data collected at the Defense Fuel Supply Point and adjacent properties, Hanahan, S.C., between April 1991 and September 1995--Continued

[ft, feet; ---, data not available; WL, water-level well; EW, extraction well; MW, monitoring well; SW, surface-water site; -, negative depth to water indicates water-level altitude is above measuring point]

\begin{tabular}{|c|c|c|c|c|c|c|c|}
\hline $\begin{array}{c}\text { Site } \\
\text { (plate 1) }\end{array}$ & $\begin{array}{l}\text { Site } \\
\text { type }\end{array}$ & Date & Time & $\begin{array}{l}\text { Measuring-point } \\
\text { altitude } \\
\text { (ft above } \\
\text { sea level) }\end{array}$ & $\begin{array}{l}\text { Depth to water } \\
\text { (ft below } \\
\text { measuring point) }\end{array}$ & $\begin{array}{l}\text { Water-level } \\
\text { altitude } \\
\text { (ft above } \\
\text { sea level) }\end{array}$ & Remarks \\
\hline WT-03 & WL & $03 / 31 / 92$ & --- & 30.49 & 6.86 & 23.63 & \\
\hline WT-03 & WL & $05 / 01 / 92$ & 1343 & 30.49 & 6.97 & 23.52 & \\
\hline WT-03 & WL & $06 / 11 / 92$ & 1637 & 30.49 & 6.25 & 24.24 & \\
\hline WT-03 & WL & $07 / 07 / 92$ & 1140 & 30.49 & 6.82 & 23.67 & \\
\hline WT-03 & WL & $08 / 31 / 92$ & 1205 & 30.49 & 5.86 & 24.63 & \\
\hline WT-03 & WL & $10 / 07 / 92$ & 1220 & 30.49 & 5.30 & 25.19 & \\
\hline WT-03 & WL & $10 / 28 / 92$ & 1548 & 30.49 & 5.88 & 24.61 & \\
\hline WT-03 & WL & $12 / 03 / 92$ & 1220 & 30.49 & 5.63 & 24.86 & \\
\hline WT-03 & WL & $01 / 04 / 93$ & 1145 & 30.49 & 6.18 & 24.31 & \\
\hline WT-03 & WL & $02 / 04 / 93$ & 1220 & 30.49 & 5.27 & 25.22 & \\
\hline WT-03 & WL & $03 / 05 / 93$ & 1200 & 30.49 & 4.94 & 25.55 & \\
\hline WT-03 & WL & $03 / 30 / 93$ & 1121 & 30.49 & 5.22 & 25.27 & \\
\hline WT-03 & WL & $05 / 06 / 93$ & 1150 & 30.49 & 6.10 & 24.39 & \\
\hline WT-03 & WL & $05 / 28 / 93$ & 1140 & 30.49 & 6.64 & 23.85 & \\
\hline WT-03 & WL & $07 / 07 / 93$ & 1207 & 30.49 & 6.86 & 23.63 & \\
\hline WT-03 & WL & $08 / 06 / 93$ & 1150 & 30.49 & 6.74 & 23.75 & \\
\hline WT-03 & WL & $09 / 03 / 93$ & 1115 & 30.49 & 6.98 & 23.51 & \\
\hline WT-03 & WL & $09 / 30 / 93$ & 1115 & 30.49 & 6.69 & 23.80 & \\
\hline WT-03 & WL & $10 / 28 / 93$ & 1110 & 30.49 & 7.28 & 23.21 & \\
\hline WT-03 & WL & $12 / 01 / 93$ & 1420 & 30.49 & 7.09 & 23.40 & \\
\hline WT-03 & WL & $01 / 06 / 94$ & 1150 & 30.49 & 6.83 & 23.66 & \\
\hline WT-03 & WL & $02 / 01 / 94$ & 1040 & 30.49 & 6.07 & 24.42 & \\
\hline WT-03 & WL & 03/04/94 & 1050 & 30.49 & 5.99 & 24.50 & \\
\hline WT-04 & WL & $04 / 30 / 91$ & 1009 & 32.89 & 7.67 & 25.22 & \\
\hline WT-04 & WL & $05 / 28 / 91$ & 1135 & 32.89 & 7.76 & 25.13 & \\
\hline WT-04 & WL & $07 / 01 / 91$ & -- & 32.89 & 8.26 & 24.63 & \\
\hline WT-04 & WL & $08 / 06 / 91$ & $\cdots$ & 32.89 & 7.08 & 25.81 & \\
\hline WT-04 & WL & $08 / 30 / 91$ & --- & 32.89 & 6.29 & 26.60 & \\
\hline WT-04 & WL & $09 / 27 / 91$ & - & 32.89 & 7.45 & 25.44 & \\
\hline WT-04 & WL & $10 / 29 / 91$ & - & 32.89 & 8.32 & 24.57 & \\
\hline WT-04 & WL & $12 / 02 / 91$ & - & 32.89 & 8.98 & 23.91 & \\
\hline WT-04 & WL & $01 / 02 / 92$ & --- & 32.89 & 9.54 & 23.35 & \\
\hline WT-04 & WL & $03 / 06 / 92$ & --- & 32.89 & 8.95 & 23.94 & \\
\hline WT-04 & WL & $03 / 31 / 92$ & -- & 32.89 & 8.96 & 23.93 & \\
\hline WT-04 & WL & $05 / 01 / 92$ & 1346 & 32.89 & 8.91 & 23.98 & \\
\hline WT-04 & WL & $06 / 11 / 92$ & 1635 & 32.89 & 9.17 & 23.72 & \\
\hline WT-04 & WL & $07 / 07 / 92$ & 1138 & 32.89 & 8.67 & 24.22 & \\
\hline WT-04 & WL & $08 / 31 / 92$ & 1210 & 32.89 & 7.42 & 25.47 & \\
\hline WT-04 & WL & $10 / 07 / 92$ & 1225 & 32.89 & 6.90 & 25.99 & \\
\hline WT-04 & WL & $10 / 28 / 92$ & 1544 & 32.89 & 7.31 & 25.58 & \\
\hline WT-04 & WL & $12 / 03 / 92$ & 1225 & 32.89 & 7.12 & 25.77 & \\
\hline WT-04 & WL & $01 / 04 / 93$ & 1148 & 32.89 & 7.84 & 25.05 & \\
\hline WT-04 & WL & $02 / 04 / 93$ & 1225 & 32.89 & 6.42 & 26.47 & \\
\hline WT-04 & WL & $03 / 05 / 93$ & 1205 & 32.89 & 6.68 & 26.21 & \\
\hline
\end{tabular}


Table 8.--Water-level data collected at the Defense Fuel Supply Point and adjacent properties, Hanahan, S.C., between April 1991 and September 1995--Continued

[ft, feet; ---, data not available; WL, water-level well; EW, extraction well; MW, monitoring well; SW, surface-water site; -, negative depth to water indicates water-level altitude is above measuring point]

\begin{tabular}{|c|c|c|c|c|c|c|c|}
\hline $\begin{array}{c}\text { Site } \\
\text { (plate 1) }\end{array}$ & $\begin{array}{l}\text { Site } \\
\text { type }\end{array}$ & Date & Time & $\begin{array}{l}\text { Measuring-point } \\
\text { altitude } \\
\text { (ft above } \\
\text { sea level) }\end{array}$ & $\begin{array}{l}\text { Depth to water } \\
\text { (ft below } \\
\text { measuring point) }\end{array}$ & $\begin{array}{l}\text { Water-level } \\
\text { altitude } \\
\text { (ft above } \\
\text { sea level) }\end{array}$ & Remarks \\
\hline WT-04 & WL & $03 / 30 / 93$ & 1124 & 32.89 & 6.85 & 26.04 & \\
\hline WT-04 & WL & $05 / 06 / 93$ & 1154 & 32.89 & 7.74 & 25.15 & \\
\hline WT-04 & WL & $05 / 28 / 93$ & 1145 & 32.89 & 8.45 & 24.44 & \\
\hline WT-04 & WL & $07 / 07 / 93$ & 1210 & 32.89 & 8.99 & 23.90 & \\
\hline WT-04 & WL & $08 / 06 / 93$ & 1153 & 32.89 & 9.17 & 23.72 & \\
\hline WT-04 & WL & $09 / 03 / 93$ & 1120 & 32.89 & 8.86 & 24.03 & \\
\hline WT-04 & WL & $09 / 30 / 93$ & 1120 & 32.89 & 8.60 & 24.29 & \\
\hline WT-04 & WL & $10 / 28 / 93$ & 1112 & 32.89 & 9.30 & 23.59 & \\
\hline WT-04 & WL & $12 / 01 / 93$ & 1425 & 32.89 & 9.29 & 23.60 & \\
\hline WT-04 & WL & $01 / 06 / 94$ & 1155 & 32.89 & 9.50 & 23.39 & \\
\hline WT-04 & WL & $02 / 01 / 94$ & 1045 & 32.89 & 8.70 & 24.19 & \\
\hline WT-04 & WL & $03 / 04 / 94$ & 1055 & 32.89 & 8.26 & 24.63 & \\
\hline WT-05 & WL & $04 / 30 / 91$ & 1015 & 32.65 & 10.12 & 22.53 & \\
\hline WT-05 & WL & $05 / 28 / 91$ & 1130 & 32.65 & 10.15 & 22.50 & \\
\hline WT-05 & WL & $07 / 01 / 91$ & -- & 32.65 & 10.59 & 22.06 & \\
\hline WT-05 & WL & $08 / 06 / 91$ & --- & 32.65 & 9.88 & 22.77 & \\
\hline WT-05 & WL & $08 / 30 / 91$ & --- & 32.65 & 9.41 & 23.24 & \\
\hline WT-05 & WL & $09 / 27 / 91$ & $\ldots$ & 32.65 & 9.96 & 22.69 & \\
\hline WT-05 & WL & $10 / 29 / 91$ & - & 32.65 & 10.61 & 22.04 & \\
\hline WT-05 & WL & $12 / 02 / 91$ & -- & 32.65 & 11.15 & 21.50 & \\
\hline WT-05 & WL & $01 / 02 / 92$ & -- & 32.65 & 11.66 & 20.99 & \\
\hline WT-05 & WL & $02 / 04 / 92$ & -- & 32.65 & 11.34 & 21.31 & \\
\hline WT-05 & WL & $03 / 06 / 92$ & -- & 32.65 & 11.31 & 21.34 & \\
\hline WT-05 & WL & $03 / 31 / 92$ & -- & 32.65 & 11.34 & 21.31 & \\
\hline WT-05 & WL & $05 / 01 / 92$ & 1351 & 32.65 & 11.34 & 21.31 & \\
\hline WT-05 & WL & $06 / 11 / 92$ & 1633 & 32.65 & 11.54 & 21.11 & \\
\hline WT-05 & WL & $07 / 07 / 92$ & 1134 & 32.65 & 11.08 & 21.57 & \\
\hline WT-05 & WL & $08 / 31 / 92$ & 1215 & 32.65 & 10.38 & 22.27 & \\
\hline WT-05 & WL & $10 / 07 / 92$ & 1230 & 32.65 & 9.92 & 22.73 & \\
\hline WT-05 & WL & $10 / 28 / 92$ & 1536 & 32.65 & 9.84 & 22.81 & \\
\hline WT-05 & WL & $12 / 03 / 92$ & 1230 & 32.65 & 9.76 & 22.89 & \\
\hline WT-05 & WL & $01 / 04 / 93$ & 1150 & 32.65 & 10.29 & 22.36 & \\
\hline WT-05 & WL & $02 / 04 / 93$ & 1230 & 32.65 & 9.15 & 23.50 & \\
\hline WT-05 & WL & $03 / 05 / 93$ & 1207 & 32.65 & 9.39 & 23.26 & \\
\hline WT-05 & WL & $03 / 30 / 93$ & 1127 & 32.65 & 9.35 & 23.30 & \\
\hline WT-05 & WL & $05 / 06 / 93$ & 1157 & 32.65 & 9.96 & 22.69 & \\
\hline WT-05 & WL & $05 / 28 / 93$ & 1150 & 32.65 & 10.49 & 22.16 & \\
\hline WT-05 & WL & $07 / 07 / 93$ & 1215 & 32.65 & 11.12 & 21.53 & \\
\hline WT-05 & WL & $08 / 06 / 93$ & 1157 & 32.65 & 11.40 & 21.25 & \\
\hline WT-05 & WL & $09 / 03 / 93$ & 1125 & 32.65 & 11.28 & 21.37 & \\
\hline WT-05 & WL & $09 / 30 / 93$ & 1122 & 32.65 & 11.06 & 21.59 & \\
\hline WT-05 & WL & $10 / 28 / 93$ & 1114 & 32.65 & 11.57 & 21.08 & \\
\hline WT-05 & WL & $12 / 01 / 93$ & 1430 & 32.65 & 11.64 & 21.01 & \\
\hline WT-05 & WL & $01 / 06 / 94$ & 1200 & 32.65 & 11.78 & 20.87 & \\
\hline
\end{tabular}


Table 8.--Water-level data collected at the Defense Fuel Supply Point and adjacent properties, Hanahan, S.C., between April 1991 and September 1995--Continued

[ft, feet; ---, data not available; WL, water-level well; EW, extraction well; MW, monitoring well; SW, surface-water site; -, negative depth to water indicates water-level altitude is above measuring point]

\begin{tabular}{|c|c|c|c|c|c|c|c|}
\hline $\begin{array}{c}\text { Site } \\
\text { (plate 1) }\end{array}$ & $\begin{array}{l}\text { Site } \\
\text { type }\end{array}$ & Date & Time & $\begin{array}{l}\text { Measuring-point } \\
\text { altitude } \\
\text { (ft above } \\
\text { sea level) }\end{array}$ & $\begin{array}{l}\text { Depth to water } \\
\text { (ft below } \\
\text { measuring point) }\end{array}$ & $\begin{array}{l}\text { Water-level } \\
\text { altitude } \\
\text { (ft above } \\
\text { sea level) }\end{array}$ & Remarks \\
\hline WT-05 & WL & $02 / 01 / 94$ & 1050 & 32.65 & 11.16 & 21.49 & \\
\hline WT-05 & WL & $03 / 04 / 94$ & 1100 & 32.65 & 10.79 & 21.86 & \\
\hline WT-06 & WL & $04 / 30 / 91$ & 0954 & 33.15 & 6.61 & 26.54 & \\
\hline WT-06 & WL & $05 / 28 / 91$ & 1055 & 33.15 & 7.11 & 26.04 & \\
\hline WT-06 & WL & $07 / 01 / 91$ & -- & 33.15 & 7.71 & 25.44 & \\
\hline WT-06 & WL & $08 / 06 / 91$ & -.. & 33.15 & 6.39 & 26.76 & \\
\hline WT-06 & WL & $08 / 30 / 91$ & $\ldots$ & 33.15 & 5.50 & 27.65 & \\
\hline WT-06 & WL & $09 / 27 / 91$ & --- & 33.15 & 7.44 & 25.71 & \\
\hline WT-06 & WL & $10 / 29 / 91$ & --- & 33.15 & 8.17 & 24.98 & \\
\hline WT-06 & WL & $12 / 02 / 91$ & $\ldots$ & 33.15 & 8.46 & 24.69 & \\
\hline WT-06 & WL & $01 / 02 / 92$ & --- & 33.15 & 8.69 & 24.46 & \\
\hline WT-06 & WL & $02 / 13 / 92$ & -- & 33.15 & 7.38 & 25.77 & \\
\hline WT-06 & WL & $03 / 06 / 92$ & -- & 33.15 & 7.40 & 25.75 & \\
\hline WT-06 & WL & $03 / 31 / 92$ & $\ldots$ & 33.15 & 6.74 & 26.41 & \\
\hline WT-06 & WL & $05 / 01 / 92$ & 1332 & 33.15 & 7.52 & 25.63 & \\
\hline WT-06 & WL & $06 / 11 / 92$ & 1618 & 33.15 & 6.38 & 26.77 & \\
\hline WT-06 & WL & $07 / 07 / 92$ & 1317 & 33.15 & 7.51 & 25.64 & \\
\hline WT-06 & WL & $08 / 07 / 92$ & 1443 & 33.15 & 7.67 & 25.48 & \\
\hline WT-06 & WL & $08 / 31 / 92$ & 1130 & 33.15 & 6.33 & 26.82 & \\
\hline WT-06 & WL & $10 / 07 / 92$ & 1140 & 33.15 & 5.39 & 27.76 & \\
\hline WT-06 & WL & $10 / 28 / 92$ & 1512 & 33.15 & 6.92 & 26.23 & \\
\hline WT-06 & WL & $12 / 03 / 92$ & 1140 & 33.15 & 6.06 & 27.09 & \\
\hline WT-06 & WL & $01 / 04 / 93$ & 1110 & 33.15 & 7.00 & 26.15 & \\
\hline WT-06 & WL & $02 / 04 / 93$ & 1150 & 33.15 & 6.00 & 27.15 & \\
\hline WT-06 & WL & $03 / 05 / 93$ & 1135 & 33.15 & 5.25 & 27.90 & \\
\hline WT-06 & WL & $03 / 30 / 93$ & 1055 & 33.15 & 5.38 & 27.77 & \\
\hline WT-06 & WL & $05 / 06 / 93$ & 1114 & 33.15 & 7.08 & 26.07 & \\
\hline WT-06 & WL & $05 / 28 / 93$ & 1106 & 33.15 & 7.75 & 25.40 & \\
\hline WT-06 & WL & $07 / 07 / 93$ & 1130 & 33.15 & 8.08 & 25.07 & \\
\hline WT-06 & WL & $08 / 06 / 93$ & 1110 & 33.15 & 7.69 & 25.46 & \\
\hline WT-07 & WL & $04 / 30 / 91$ & 0956 & 38.43 & 5.40 & 33.03 & \\
\hline WT-07 & WL & $05 / 28 / 91$ & 1056 & 38.43 & 6.00 & 32.43 & \\
\hline WT-07 & WL & $07 / 01 / 91$ & --. & 38.43 & 6.72 & 31.71 & \\
\hline WT-07 & WL & $08 / 06 / 91$ & --. & 38.43 & 5.05 & 33.38 & \\
\hline WT-07 & WL & $08 / 30 / 91$ & - & 38.43 & 4.34 & 34.09 & \\
\hline WT-07 & WL & $09 / 27 / 91$ & $\cdots$ & 38.43 & 6.67 & 31.76 & \\
\hline WT-07 & WL & $10 / 29 / 91$ & $\cdots$ & 38.43 & 8.36 & 30.07 & \\
\hline WT-07 & WL & $12 / 02 / 91$ & $\ldots$ & 38.43 & 8.35 & 30.08 & \\
\hline WT-07 & WL & $01 / 02 / 92$ & --- & 38.43 & 8.31 & 30.12 & \\
\hline WT-07 & WL & $02 / 04 / 92$ &.-- & 38.43 & 5.80 & 32.63 & \\
\hline WT-07 & WL & $03 / 06 / 92$ & -- & 38.43 & 6.47 & 31.96 & \\
\hline WT-07 & WL & $03 / 31 / 92$ & -.. & 38.43 & 5.63 & 32.80 & \\
\hline WT-07 & WL & $05 / 01 / 92$ & 1325 & 38.43 & 6.55 & 31.88 & \\
\hline
\end{tabular}


Table 8.--Water-level data collected at the Defense Fuel Supply Point and adjacent properties, Hanahan, S.C., between April 1991 and September 1995--Continued

[ft, feet; ---, data not available; WL, water-level well; EW, extraction well; MW, monitoring well; SW, surface-water site; -, negative depth to water indicates water-level altitude is above measuring point]

\begin{tabular}{|c|c|c|c|c|c|c|c|}
\hline $\begin{array}{c}\text { Site } \\
\text { (plate 1) }\end{array}$ & $\begin{array}{l}\text { Site } \\
\text { type }\end{array}$ & Date & Time & $\begin{array}{l}\text { Measuring-point } \\
\text { altitude } \\
\text { (ft above } \\
\text { sea level) }\end{array}$ & $\begin{array}{l}\text { Depth to water } \\
\text { (ft below } \\
\text { measuring point) }\end{array}$ & $\begin{array}{l}\text { Water-level } \\
\text { altitude } \\
\text { (ft above } \\
\text { sea level) }\end{array}$ & Remarks \\
\hline WT-07 & WL & $06 / 11 / 92$ & 1545 & 38.43 & 4.21 & 34.22 & \\
\hline WT-07 & WL & $07 / 07 / 92$ & 1325 & 38.43 & 6.57 & 31.86 & \\
\hline WT-07 & WL & $08 / 07 / 92$ & 1434 & 38.43 & 6.47 & 31.96 & \\
\hline WT-07 & WL & $08 / 31 / 92$ & 1120 & 38.43 & 5.23 & 33.20 & \\
\hline WT-07 & WL & $10 / 07 / 92$ & 1130 & 38.43 & 4.31 & 34.12 & \\
\hline WT-07 & WL & $10 / 28 / 92$ & 1458 & 38.43 & 6.10 & 32.33 & \\
\hline WT-07 & WL & $12 / 03 / 92$ & 1125 & 38.43 & 4.88 & 33.55 & \\
\hline WT-07 & WL & $01 / 04 / 93$ & 1115 & 38.43 & 6.13 & 32.30 & \\
\hline WT-07 & WL & $02 / 04 / 93$ & 1145 & 38.43 & 5.17 & 33.26 & \\
\hline WT-07 & WL & $03 / 05 / 93$ & 1125 & 38.43 & 4.19 & 34.24 & \\
\hline WT-07 & WL & $03 / 30 / 93$ & 1046 & 38.43 & 4.33 & 34.10 & \\
\hline WT-07 & WL & $05 / 06 / 93$ & 1120 & 38.43 & 6.38 & 32.05 & \\
\hline WT-07 & WL & $05 / 28 / 93$ & 1110 & 38.43 & 7.70 & 30.73 & \\
\hline WT-07 & WL & $07 / 07 / 93$ & 1118 & 38.43 & 8.27 & 30.16 & \\
\hline WT-07 & WL & $08 / 06 / 93$ & 1105 & 38.43 & 7.64 & 30.79 & \\
\hline WT-08 & WL & $04 / 30 / 91$ & 1200 & 36.33 & 3.09 & 33.24 & \\
\hline WT-08 & WL & $05 / 28 / 91$ & -- & 36.33 & 3.54 & 32.79 & \\
\hline WT-08 & WL & $07 / 01 / 91$ & -- & 36.33 & 3.24 & 33.09 & \\
\hline WT-08 & WL & $08 / 06 / 91$ & -- & 36.33 & 2.97 & 33.36 & \\
\hline WT-08 & WL & $08 / 30 / 91$ & - & 36.33 & 2.76 & 33.57 & \\
\hline WT-08 & WL & $09 / 27 / 91$ & --- & 36.33 & 4.50 & 31.83 & \\
\hline WT-08 & WL & $10 / 29 / 91$ & -- & 36.33 & 5.32 & 31.01 & \\
\hline WT-08 & WL & $12 / 02 / 91$ & - & 36.33 & 6.12 & 30.21 & \\
\hline WT-08 & WL & $01 / 02 / 92$ & --- & 36.33 & 5.86 & 30.47 & \\
\hline WT-08 & WL & $02 / 04 / 92$ & -- & 36.33 & 3.24 & 33.09 & \\
\hline WT-08 & WL & $03 / 06 / 92$ & -- & 36.33 & 4.18 & 32.15 & \\
\hline WT-08 & WL & $03 / 31 / 92$ & -- & 36.33 & 3.29 & 33.04 & \\
\hline WT-08 & WL & $05 / 01 / 92$ & --- & 36.33 & 4.58 & 31.75 & \\
\hline WT-08 & WL & $06 / 11 / 92$ & 1522 & 36.33 & 2.80 & 33.53 & \\
\hline WT-08 & WL & $07 / 07 / 92$ & 1436 & 36.33 & 4.39 & 31.94 & \\
\hline WT-08 & WL & $08 / 07 / 92$ & 1409 & 36.33 & 3.34 & 32.99 & \\
\hline WT-08 & WL & $08 / 31 / 92$ & 1250 & 36.33 & 2.99 & 33.34 & \\
\hline WT-08 & WL & $10 / 07 / 92$ & 1350 & 36.33 & 2.72 & 33.61 & \\
\hline WT-08 & WL & $10 / 28 / 92$ & 1401 & 36.33 & 3.62 & 32.71 & \\
\hline WT-08 & WL & $12 / 03 / 92$ & 1247 & 36.33 & 2.94 & 33.39 & \\
\hline WT-08 & WL & $01 / 04 / 93$ & 1225 & 36.33 & 3.79 & 32.54 & \\
\hline WT-08 & WL & $02 / 04 / 93$ & 1345 & 36.33 & 2.97 & 33.36 & \\
\hline WT-08 & WL & $03 / 05 / 93$ & 1226 & 36.33 & 2.81 & 33.52 & \\
\hline WT-08 & WL & $03 / 30 / 93$ & 1152 & 36.33 & 2.80 & 33.53 & \\
\hline WT-08 & WL & $05 / 06 / 93$ & 1201 & 36.33 & 3.53 & 32.80 & \\
\hline WT-08 & WL & $05 / 28 / 93$ & 1159 & 36.33 & 4.90 & 31.43 & \\
\hline WT-08 & WL & $07 / 07 / 93$ & 1151 & 36.33 & 4.14 & 32.19 & \\
\hline WT-08 & WL & $08 / 06 / 93$ & 1127 & 36.33 & 3.56 & 32.77 & \\
\hline WT-08 & WL & $09 / 03 / 93$ & 1138 & 36.33 & 4.83 & 31.50 & \\
\hline
\end{tabular}


Table 8.--Water-level data collected at the Defense Fuel Supply Point and adjacent properties, Hanahan, S.C., between April 1991 and September 1995--Continued

[ft, feet; ---, data not available; WL, water-level well; EW, extraction well; MW, monitoring well; SW, surface-water site; -, negative depth to water indicates water-level altitude is above measuring point]

\begin{tabular}{|c|c|c|c|c|c|c|c|}
\hline $\begin{array}{c}\text { Site } \\
\text { (plate 1) }\end{array}$ & $\begin{array}{l}\text { Site } \\
\text { type }\end{array}$ & Date & Time & $\begin{array}{l}\text { Measuring-point } \\
\text { altitude } \\
\text { (ft above } \\
\text { sea level) }\end{array}$ & $\begin{array}{l}\text { Depth to water } \\
\text { (ft below } \\
\text { measuring point) }\end{array}$ & $\begin{array}{l}\text { Water-level } \\
\text { aititude } \\
\text { (ft above } \\
\text { sea level) }\end{array}$ & Remarks \\
\hline WT-08 & WL & $09 / 30 / 93$ & 1145 & 36.33 & 4.16 & 32.17 & \\
\hline WT-08 & WL & $10 / 28 / 93$ & 1145 & 36.33 & 5.06 & 31.27 & \\
\hline WT-08 & WL & $12 / 01 / 93$ & 1455 & 36.33 & 3.95 & 32.38 & \\
\hline WT-08 & WL & $01 / 06 / 94$ & 1206 & 36.33 & 3.01 & 33.32 & \\
\hline WT-08 & WL & $02 / 01 / 94$ & 1105 & 36.33 & 2.68 & 33.65 & \\
\hline WT-08 & WL & $03 / 04 / 94$ & 1120 & 36.33 & 2.80 & 33.53 & \\
\hline WT-09 & WL & $04 / 30 / 91$ & 1140 & 34.99 & 4.64 & 30.35 & \\
\hline WT-09 & WL & $05 / 28 / 91$ & --- & 34.99 & 4.64 & 30.35 & \\
\hline WT-09 & WL & $07 / 01 / 91$ & -- & 34.99 & 4.71 & 30.28 & \\
\hline WT-09 & WL & $08 / 06 / 91$ & - & 34.99 & 4.58 & 30.41 & \\
\hline WT-09 & WL & $08 / 30 / 91$ & -- & 34.99 & 4.54 & 30.45 & \\
\hline WT-09 & WL & $09 / 27 / 91$ & $\cdots$ & 34.99 & 4.74 & 30.25 & \\
\hline WT-09 & WL & $10 / 29 / 91$ & -- & 34.99 & 4.87 & 30.12 & \\
\hline WT-09 & WL & $12 / 02 / 91$ & -- & 34.99 & 4.84 & 30.15 & \\
\hline WT-09 & WL & $01 / 02 / 92$ & -- & 34.99 & 4.90 & 30.09 & \\
\hline WT-09 & WL & $02 / 04 / 92$ & -- & 34.99 & 4.70 & 30.29 & \\
\hline WT-09 & WL & $03 / 06 / 92$ & $\cdots$ & 34.99 & 4.78 & 30.21 & \\
\hline WT-09 & WL & $03 / 31 / 92$ & --- & 34.99 & 4.75 & 30.24 & \\
\hline WT-09 & WL & $05 / 01 / 92$ & --. & 34.99 & 4.83 & 30.16 & \\
\hline WT-09 & WL & $06 / 11 / 92$ & 1513 & 34.99 & 4.51 & 30.48 & \\
\hline WT-09 & WL & $07 / 07 / 92$ & 1453 & 34.99 & 4.83 & 30.16 & \\
\hline WT-09 & WL & $08 / 07 / 92$ & 1358 & 34.99 & 4.62 & 30.37 & \\
\hline WT-09 & WL & $08 / 31 / 92$ & 1233 & 34.99 & 4.65 & 30.34 & \\
\hline WT-09 & WL & $10 / 07 / 92$ & 1232 & 34.99 & 4.57 & 30.42 & \\
\hline WT-09 & WL & $10 / 28 / 92$ & 1346 & 34.99 & 4.69 & 30.30 & \\
\hline WT-09 & WL & $12 / 03 / 92$ & 1232 & 34.99 & 4.63 & 30.36 & \\
\hline WT-09 & WL & $01 / 04 / 93$ & 1040 & 34.99 & 4.70 & 30.29 & \\
\hline WT-09 & WL & $02 / 04 / 93$ & 1115 & 34.99 & 4.63 & 30.36 & \\
\hline WT-09 & WL & $03 / 05 / 93$ & 1100 & 34.99 & 4.58 & 30.41 & \\
\hline WT-09 & WL & $03 / 30 / 93$ & 1040 & 34.99 & 4.60 & 30.39 & \\
\hline WT-09 & WL & $05 / 06 / 93$ & 1050 & 34.99 & 4.73 & 30.26 & \\
\hline WT-09 & WL & $05 / 28 / 93$ & 1044 & 34.99 & 4.84 & 30.15 & \\
\hline WT-09 & WL & $07 / 07 / 93$ & 1057 & 34.99 & 4.76 & 30.23 & \\
\hline WT-09 & WL & $08 / 06 / 93$ & 1050 & 34.99 & 5.56 & 29.43 & May be affected by dewatering. \\
\hline WT-09 & WL & $09 / 03 / 93$ & 1057 & 34.99 & 4.88 & 30.11 & \\
\hline WT-09 & WL & $09 / 30 / 93$ & 1055 & 34.99 & 4.73 & 30.26 & \\
\hline WT-09 & WL & $10 / 28 / 93$ & 1050 & 34.99 & 4.73 & 30.26 & \\
\hline WT-09 & WL & $12 / 01 / 93$ & 1405 & 34.99 & 4.60 & 30.39 & \\
\hline WT-09 & WL & $01 / 06 / 94$ & 1125 & 34.99 & 4.49 & 30.50 & \\
\hline WT-09 & WL & $02 / 01 / 94$ & 1015 & 34.99 & 4.34 & 30.65 & \\
\hline WT-09 & WL & $03 / 04 / 94$ & 1015 & 34.99 & 4.47 & 30.52 & \\
\hline WT-10 & WL & $04 / 30 / 91$ & 1100 & 31.81 & 5.00 & 26.81 & \\
\hline WT-10 & WL & $05 / 28 / 91$ & -- & 31.81 & 5.36 & 26.45 & \\
\hline
\end{tabular}


Table 8.--Water-level data collected at the Defense Fuel Supply Point and adjacent properties, Hanahan, S.C., between April 1991 and September 1995--Continued

[ft, feet; ---, data not available; WL, water-level well; EW, extraction well; MW, monitoring well; SW, surface-water site; -, negative depth to water indicates water-level altitude is above measuring point]

\begin{tabular}{|c|c|c|c|c|c|c|c|}
\hline $\begin{array}{c}\text { Site } \\
\text { (plate 1) }\end{array}$ & $\begin{array}{l}\text { Site } \\
\text { type }\end{array}$ & Date & Time & $\begin{array}{l}\text { Measuring-point } \\
\text { altitude } \\
\text { (ft above } \\
\text { sea level) }\end{array}$ & $\begin{array}{l}\text { Depth to water } \\
\text { (ft below } \\
\text { measuring point) }\end{array}$ & $\begin{array}{l}\text { Water-level } \\
\text { altitude } \\
\text { (ft above } \\
\text { sea level) }\end{array}$ & Remarks \\
\hline WT-10 & $\overline{W L}$ & $07 / 01 / 91$ & $-\cdots$ & 31.81 & 5.54 & 26.27 & \\
\hline WT-10 & WL & $08 / 06 / 91$ & -- & 31.81 & 4.92 & 26.89 & \\
\hline WT-10 & WL & $08 / 30 / 91$ & --- & 31.81 & 4.69 & 27.12 & \\
\hline WT-10 & WL & $09 / 27 / 91$ & --- & 31.81 & 5.84 & 25.97 & \\
\hline WT-10 & WL & $10 / 29 / 91$ & --- & 31.81 & 6.21 & 25.60 & \\
\hline WT-10 & WL & $12 / 02 / 91$ & -- & 31.81 & 6.43 & 25.38 & \\
\hline WT-10 & WL & $01 / 02 / 92$ & -- & 31.81 & 6.44 & 25.37 & \\
\hline WT-10 & WL & $02 / 04 / 92$ & $\ldots$ & 31.81 & 5.70 & 26.11 & \\
\hline WT-10 & WL & $03 / 06 / 92$ &.-- & 31.81 & 5.89 & 25.92 & \\
\hline WT-10 & WL & $03 / 31 / 92$ & --- & 31.81 & 5.64 & 26.17 & \\
\hline WT-10 & WL & $05 / 01 / 92$ & $-\cdots$ & 31.81 & 5.94 & 25.87 & \\
\hline WT-10 & WL & $06 / 11 / 92$ & 1457 & 31.81 & 4.28 & 27.53 & \\
\hline WT-10 & WL & $07 / 07 / 92$ & 1530 & 31.81 & 5.92 & 25.89 & \\
\hline WT-10 & WL & $08 / 07 / 92$ & 1342 & 31.81 & 5.15 & 26.66 & \\
\hline WT-10 & WL & $08 / 31 / 92$ & 1105 & 31.81 & 5.03 & 26.78 & \\
\hline WT-10 & WL & $10 / 07 / 92$ & 1104 & 31.81 & 4.38 & 27.43 & \\
\hline WT-10 & WL & $10 / 28 / 92$ & 1608 & 31.81 & 5.40 & 26.41 & \\
\hline WT-10 & WL & $12 / 03 / 92$ & 1050 & 31.81 & 4.92 & 26.89 & \\
\hline WT-10 & WL & $01 / 04 / 93$ & 1030 & 31.81 & 5.41 & 26.40 & \\
\hline WT-10 & WL & $02 / 04 / 93$ & 1105 & 31.81 & 4.94 & 26.87 & \\
\hline WT-10 & WL & $03 / 05 / 93$ & 1045 & 31.81 & 4.41 & 27.40 & \\
\hline WT-10 & WL & $03 / 30 / 93$ & 1018 & 31.81 & 4.51 & 27.30 & \\
\hline WT-10 & WL & $05 / 06 / 93$ & 1043 & 31.81 & 5.41 & 26.40 & \\
\hline WT-10 & WL & $05 / 28 / 93$ & 1035 & 31.81 & 5.68 & 26.13 & \\
\hline WT-10 & WL & $07 / 07 / 93$ & 1048 & 31.81 & 5.63 & 26.18 & \\
\hline WT-10 & WL & $08 / 06 / 93$ & 1036 & 31.81 & 4.29 & 27.52 & \\
\hline WT-10 & WL & $09 / 03 / 93$ & 1040 & 31.81 & 5.76 & 26.05 & \\
\hline WT-10 & WL & $09 / 30 / 93$ & 1040 & 31.81 & 5.58 & 26.23 & \\
\hline WT-10 & WL & $10 / 28 / 93$ & 1032 & 31.81 & 5.97 & 25.84 & \\
\hline WT-10 & WL & $12 / 01 / 93$ & 1345 & 31.81 & 5.61 & 26.20 & \\
\hline WT-10 & WL & $01 / 06 / 94$ & 1110 & 31.81 & 5.26 & 26.55 & \\
\hline WT-10 & WL & $02 / 01 / 94$ & 1005 & 31.81 & 4.23 & 27.58 & \\
\hline WT-10 & WL & $03 / 04 / 94$ & 1005 & 31.81 & 4.29 & 27.52 & \\
\hline WT-11 & WL & $09 / 27 / 91$ & -- & 25.66 & 5.64 & 20.02 & \\
\hline WT-11 & WL & $10 / 29 / 91$ & -- & 25.66 & 6.17 & 19.49 & \\
\hline WT-11 & WL & $12 / 02 / 91$ & -- & 25.66 & 6.49 & 19.17 & \\
\hline WT-11 & WL & $01 / 02 / 92$ & -- & 25.66 & 6.74 & 18.92 & \\
\hline WT-11 & WL & $02 / 04 / 92$ & -- & 25.66 & 6.16 & 19.50 & \\
\hline WT-11 & WL & $03 / 06 / 92$ & -- & 25.66 & 6.17 & 19.49 & \\
\hline WT-11 & WL & $03 / 31 / 92$ & -- & 25.66 & 6.25 & 19.41 & \\
\hline WT-11 & WL & $05 / 01 / 92$ & $\cdots$ & 25.66 & 6.30 & 19.36 & \\
\hline WT-11 & WL & $06 / 11 / 92$ & 1626 & 25.66 & 6.06 & 19.60 & \\
\hline WT-11 & WL & $07 / 07 / 92$ & 1300 & 25.66 & 6.09 & 19.57 & \\
\hline WT-11 & WL & $08 / 31 / 92$ & 1150 & 25.66 & 5.60 & 20.06 & \\
\hline
\end{tabular}


Table 8.--Water-level data collected at the Defense Fuel Supply Point and adjacent properties, Hanahan, S.C., between April 1991 and September 1995--Continued

[ft, feet; ---, data not available; WL, water-level well; EW, extraction well; MW, monitoring well; SW, surface-water site; -, negative depth to water indicates water-level altitude is above measuring point]

\begin{tabular}{|c|c|c|c|c|c|c|c|}
\hline $\begin{array}{c}\text { Site } \\
\text { (plate 1) }\end{array}$ & $\begin{array}{l}\text { Site } \\
\text { type }\end{array}$ & Date & Time & $\begin{array}{c}\text { Measuring-point } \\
\text { altitude } \\
\text { (ft above } \\
\text { sea level) }\end{array}$ & $\begin{array}{l}\text { Depth to water } \\
\text { (ft below } \\
\text { measuring point) }\end{array}$ & $\begin{array}{l}\text { Water-level } \\
\text { altitude } \\
\text { (ft above } \\
\text { sea level) }\end{array}$ & Remarks \\
\hline WT-11 & WL & $10 / 07 / 92$ & 1205 & 25.66 & 5.41 & 20.25 & \\
\hline WT-11 & WL & $10 / 28 / 92$ & 1525 & 25.66 & 5.65 & 20.01 & \\
\hline WT-11 & WL & $12 / 03 / 92$ & 1205 & 25.66 & 5.45 & 20.21 & \\
\hline WT-11 & WL & $01 / 04 / 93$ & 1130 & 25.66 & 5.85 & 19.81 & \\
\hline WT-11 & WL & $02 / 04 / 93$ & 1210 & 25.66 & 5.13 & 20.53 & \\
\hline WT-11 & WL & $03 / 05 / 93$ & 1150 & 25.66 & 5.17 & 20.49 & \\
\hline WT-11 & WL & $03 / 30 / 93$ & 1108 & 25.66 & 4.95 & 20.71 & \\
\hline WT-11 & WL & $05 / 06 / 93$ & 1135 & 25.66 & 5.45 & 20.21 & \\
\hline WT-11 & WL & $05 / 28 / 93$ & 1126 & 25.66 & 5.82 & 19.84 & \\
\hline WT-11 & WL & $07 / 07 / 93$ & 1145 & 25.66 & 6.07 & 19.59 & \\
\hline WT-11 & WL & $08 / 06 / 93$ & 1136 & 25.66 & 6.27 & 19.39 & \\
\hline 36-inch recovery well & WL & $04 / 30 / 91$ & 0940 & 34.93 & 13.15 & 21.78 & \\
\hline 36-inch recovery well & WL & $05 / 28 / 91$ & --- & 34.93 & 13.47 & 21.46 & \\
\hline 36-inch recovery well & WL & $08 / 06 / 91$ & --- & 34.93 & 11.80 & 23.13 & \\
\hline 36-inch recovery well & WL & $08 / 30 / 91$ & -- & 34.93 & 11.33 & 23.60 & \\
\hline 36-inch recovery well & WL & $09 / 27 / 91$ & --- & 34.93 & 12.98 & 21.95 & \\
\hline 36-inch recovery well & WL & $10 / 29 / 91$ & -- & 34.93 & 14.08 & 20.85 & \\
\hline 36-inch recovery well & WL & $12 / 02 / 91$ & - & 34.93 & 14.79 & 20.14 & \\
\hline 36-inch recovery well & WL & $01 / 02 / 92$ & --- & 34.93 & 14.82 & 20.11 & \\
\hline 36-inch recovery well & WL & $02 / 04 / 92$ & -- & 34.93 & 15.14 & 19.79 & \\
\hline 36-inch recovery well & WL & $03 / 06 / 92$ & -- & 34.93 & 14.97 & 19.96 & \\
\hline 36-inch recovery well & WL & $03 / 31 / 92$ & -- & 34.93 & 14.86 & 20.07 & \\
\hline 36-inch recovery well & WL & $05 / 01 / 92$ & $\ldots$ & 34.93 & 14.92 & 20.01 & \\
\hline 36-inch recovery well & WL & $05 / 14 / 92$ & 1048 & 34.93 & 15.16 & 19.77 & \\
\hline
\end{tabular}


Table 9.--Analytical results for volatile organics, extractable organics, and metals detected in lake-bottomsediment samples collected from Gold Cup Springs Lake, Hanahan, S.C., February 8, 1994

[ $\mu \mathrm{g} / \mathrm{kg}$, microgram per kilogram; mg/kg, milligram per kilogram]

\begin{tabular}{lcccc}
\hline $\begin{array}{c}\text { Sample location } \\
\text { (plate 1) }\end{array}$ & $\begin{array}{c}\text { Methylene } \\
\text { chloride } \\
(\mu \mathrm{g} / \mathrm{kg})\end{array}$ & $\begin{array}{c}\text { Chromium } \\
(\mathrm{mg} / \mathrm{kg})\end{array}$ & $\begin{array}{c}\text { Lead } \\
(\mathrm{mg} / \mathrm{kg})\end{array}$ & $\begin{array}{c}\text { Zinc } \\
(\mathrm{mg} / \mathrm{kg})\end{array}$ \\
\hline Outfall-1 & 17 & 4.0 & 54 & 14 \\
Outfall-1R & 19 & 7.8 & 3.5 & 5.4 \\
Input-2 & 15 & 2.5 & 3.9 & 14 \\
Lake Center & 12 & 3.5 & 6.5 & 5.9 \\
\hline
\end{tabular}




\begin{abstract}
APPENDIX
Lithologic descriptions of sediment recovered from boreholes at the Defense Fuel Supp'y Point property, Hanahan, S.C.
\end{abstract}


Appendix--Lithologic descriptions of sediment recovered from boreholes at the Defense Fuel Supply Point property, Hanahan, S.C.

\section{Borehole DV-4}

[Approximate land-surface altitude at borehole DV-4 is 34.9 feet above sea level. All depths are reported in feet below land surface. Borehole location is shown in plate 1.]

\begin{tabular}{cl}
\hline $\begin{array}{c}\text { Depth } \\
\text { (feet) }\end{array}$ & \multicolumn{1}{c}{ Lithologic description } \\
\hline $0.0-0.3$ & Gravel \\
$0.3-1.6$ & Sand, brown to grayish brown \\
$1.6-2.1$ & Sand, light gray \\
$2.1-2.6$ & Sand, dark gray \\
$2.6-3.8$ & Sand, light gray; becoming browner near base of interval \\
$3.8-4.8$ & Sand, brownish gray \\
$4.8-5.9$ & Sand, gray \\
$5.9-6.4$ & Clayey sand, gray \\
$6.4-6.9$ & Sandy clay, gray \\
$6.9-7.6$ & Clay, gray \\
$7.6-9.3$ & Sand, gray, becoming whiter near base of interval \\
$9.3-9.7$ & Clay, gray \\
$9.7-10.0$ & Sandy clay, gray \\
$10.0-14.0$ & Sand, gray \\
\hline
\end{tabular}


Borehole 56-E

[Approximate land-surface altitude at borehole 56-E is 32.5 feet above sea level. All depths are reported in feet below land surface. Borehole location is shown in plate 1.]

\begin{aligned} & \hline $\begin{array}{c}\text { Depth } \\ \text { (feet) }\end{array} \\ &$\hline 0.0-10.0 Not sampled \\ & 10.0-10.8 Sand, light grayish brown; fine-grained; mottled with brown sand \\ & $10.8-11.4$ Sand, light gray; fine-grained; mottled with reddish-brown sand \\ & $11.4-12.0$ Sand, grayish brown; fine-grained; mottled with reddish-brown sand \\ & $12.0-12.7$ Sand, gray; fine-grained, and clayey sand, brownish gray; with discrete areas of black-stained \\ & sand \\ & $12.7-13.3$ Silty sand, grayish brown; fine-grained \\ & $13.3-13.9$ Clayey sand, light to dark gray; fine-grained; mottled with brown clayey sand \\ & $13.9-14.5$ Sandy clay \\ & $14.5-15.0$ Sandy clay, gray to grayish brown sand; fine-grained \\ & $15.0-15.5$ Sand, grayish brown; fine-grained \\ & $15.5-16.7$ Sand, brownish gray; fine-grained; with silty matrix \\ & $16.7-17.3$ Silty sand, fine- to medium-grained; with patches of loose sand \\ & $17.3-18.0$ Sand, gray; fine-grained; loose \\ & $18.0-18.5$ Sand, gray; fine- to medium-grained; loose \\ & $18.5-19.1$ Sand, gray; fine- to medium-grained; abundant mica; gray and black minerals present \\ & $19.1-21.6$ Sand, gray; fine-grained; loose; gray and black minerals present \\ & $21.6-23.0$ Sand, gray; medium-grained; loose; gray and black minerals present \\ & \hline\end{aligned}


Borehole 56-F

[Approximate land-surface altitude at borehole 56-F is 35.6 feet above sea level. All depths are reported in feet below land surface. Borehole location is shown in plate 1.]

\begin{aligned} & \hline $\begin{array}{r}\text { Depth } \\ \text { (feet) }\end{array} \\ &$\hline $0.0-10.0$ Not sampled \\ & $10.0-10.8$ Sand, grayish brown; fine-grained; loose; dark minerals present \\ & $10.8-11.4$ Sand, grayish brown; medium-grained; loose; dark minerals present \\ & $11.4-12.0$ Sand, brownish gray; fine-grained; loose \\ & $12.0-12.9$ Clayey sand, medium gray; fine-grained \\ & $12.9-13.5$ Clay, gray \\ & $13.5-14.0$ Clay, gray; to sand, gray \\ & \hline\end{aligned}




\section{Borehole 56-I}

[Approximate land-surface altitude at borehole $56-1$ is 36.2 feet above sea level. All depths are reported in feet below land surface. Borehole location is shown in plate 1.]

\begin{aligned} \hline $\begin{array}{c}\text { Depth } \\ \text { (feet) }\end{array} & \\$\hline $0.0-10.0 &$ Not sampled \\ $10.0-10.2 &$ Sand, grayish brown; fine-grained \\ $10.2-10.8 &$ Sand, grayish brown; fine- to medium-grained \\ $10.8-12.0 &$ No recovery \\ $12.0-12.8 &$ Sandy clay to clay, grayish brown \\ $12.8-13.4 &$ Clay, grayish brown \\ $13.4-14.0 &$ Clay, gray; to clayey sand, gray \\ $14.0-14.6 &$ Sand, gray; medium-grained; loose \\ $14.6-15.2 &$ Sand, gray; very fine- to fine-grained \\ $15.2-15.8 &$ Sand, gray; fine- to medium-grained; slightly silty \\ $15.8-16.4 &$ Sand, gray; fine-grained \\ $16.4-17.0 &$ Sand, gray; fine-grained; with some clay \\ $17.0-17.3 &$ Sand, gray; fine- to medium-grained \\ $17.3-18.6 &$ Sand, gray; fine-grained \\ $18.6-20.0 &$ No recovery \\ $20.0-20.6 &$ Sand, gray; fine- to medium-grained \\ $20.6-21.3 &$ Sand, gray; fine-grained \\ $21.3-22.0 &$ Sand, gray; fine- to medium-grained \\ $22.0-22.8 &$ Sand, gray; fine-grained \\ \hline & \end{aligned}




\section{Borehole 56-J}

[Approximate land-surface altitude at borehole $56-J$ is 35.5 feet above sea level. All depths are reported in feet below land surface. Borehole location is shown in plate 1.]

\begin{aligned} & \hline $\begin{array}{r}\text { Depth } \\ \text { (feet) }\end{array} \\ &$\hline 0.0-10.0 Not sampled \\ & $10.0-10.6 \begin{array}{l}\text { Sand, grayish brown; fine-grained; with discrete patches of orangish-brown } \\ \text { staining }\end{array} \\ & \multicolumn{1}{c}{$ Sand, gray; medium-grained; with discrete patches of orangish-brown staining } \\ & $10.6-13.0$ Silty sand, gray; fine-grained \\ & $13.0-13.5$ Sandy clay to clay, gray \\ & $13.5-14.0$ Clay to sandy clay, gray \\ & $14.0-14.2$ Clay, gray \\ & $14.2-14.7$ Clay to sandy clay, gray \\ & $14.7-15.2$ Silty sand, gray; fine-grained \\ & $15.2-15.8$ Sandy clay, gray \\ & $15.8-16.4$ Sandy clay, gray; to clayey sand, gray; fine- to medium-grained \\ & $16.4-17.0$ Silty sand, gray; very fine-grained \\ & $17.0-17.3$ Sand, gray; fine- to medium-grained \\ & $17.3-17.9$ Sand, grayish brown; fine-grained \\ & $17.9-19.3$ Sand, grayish brown; fine- to medium-grained; abundant dark minerals \\ & $19.3-20.0$ Sand, gray; fine-grained \\ & $20.0-21.0$ Sand, light gray; fine-grained \\ & \hline $21.0-22.5 \\ &$\hline\end{aligned}


Well MWGS-27C

[Approximate land-surface altitude at well MWGS-27C is 35.6 feet above sea level. All depths are repored in feet below land surface. Well location is shown in plate 1.]

Depth

(feet)

\section{Lithologic description}

0.0-0.5 Not sampled

0.5-1.5 Sand, orange brown; fine-grained; partially cemented

1.5-1.9 Sand, light brown; fined-grained; less cemented than above

1.9-2.0 Sand, dark brown; fine-grained; loose

2.0-2.3 Sand, very light brown; fined-grained; with a sharp bottom contact

2.3-3.0 Sand, dark brown; fine-grained; with thin layers of lighter and darker sand lenses

3.0-3.2 Sand, light brown; fine-grained; partially cemented

3.2-4.2 Sand, light reddish brown; fine-grained; loose

4.2-4.5 Sand, fine-grained; with very dark brown stains

4.5-5.5 Sand, light brown; fine-grained; becomes paler with depth

5.5-5.6 Sand, fine-grained; with very dark brown stains

5.6-5.9 Sand, pale brown; fine-grained

5.9-6.8 Sand, pale brown; fine-grained; loose

6.8-7.0 Clayey sand, gray; fine-grained

7.0-7.4 Sand, light brown; fine-grained; partially cemented

7.4-8.4 Sand, brown; fine-grained; loose

8.4-8.7 Sand, very dark brown; fine-grained

8.7-9.2 Sand, white; fine-grained; loose

9.2-9.5 Sand, dark brown to white; fine-grained; partially cemented white sand at base

9.5-10.5 Sand, white; fine-grained; with discrete patches of orangish-brown stained sand; loose

10.5-11.0 Sand, white; fine- to medium-grained; loose

Note: 11.0-13.0 feet is missing 6 inches, so depths may be slightly off for that interval.

11.0-12.5 Sand, white; fine-grained; with diffuse and discrete patches of orangish-brown stained sand

12.5-13.0 Clayey sand, grayish brown

13.0-13.3 Sand, brownish gray; fine-grained

13.3-14.0 Sandy clay, gray; increasing clay content with depth

14.0-14.9 Clay, gray 
Well MWGS-27C--Continued

[Approximate land-surface altitude at well MWGS-27C is 35.6 feet above sea level. All depths are reported in feet below land surface. Well location is shown in plate 1.]

\begin{tabular}{cl}
\hline $\begin{array}{c}\text { Depth } \\
\text { (feet) }\end{array}$ & \multicolumn{1}{c}{ Lithologic description } \\
\hline $14.9-16.1$ & Sand, grayish brown; fine-grained; loose \\
$16.1-16.9$ & Clayey sand, gray; very fine-grained \\
$16.9-17.2$ & Sand, gray; fine-grained \\
$17.2-17.3$ & Clay, gray \\
$17.3-25.0$ & Sand, gray; fine-grained; becomes darker with depth \\
\hline
\end{tabular}


Well MWGS-28E

[Approximate land-surface altitude at well MWGS-28E is 35.2 feet above sea level. All depths are report?d in feet below land surface. Well location is shown in plate 1.]

\begin{aligned} & \hline $\begin{array}{c}\text { Depth } \\ \text { (feet) }\end{array} \\ &$\hline 0.0-10.0 Not sampled \\ & $10.0-10.6$ Sand, light brown; fine- to medium-grained \\ & $10.6-12.0$ Sand, grayish brown; fine- to medium-grained; with discrete patches of reddish-brown \\ & stained sand \\ & $12.0-12.7$ Sand, gray; fine-grained; with patches of black stained sand \\ & $12.7-13.5$ Clayey sand \\ & $13.5-14.3$ Sand, brownish gray; fine-grained; light silty matrix \\ & $14.3-15.1$ Sand, brownish gray; fine-grained \\ & $15.1-16.0$ Silty sand, brownish gray; fine-grained \\ & $16.0-16.7$ Sandy clay, grayish brown \\ & $16.7-17.3$ Clay, grayish brown \\ & $17.3-17.9$ Sandy clay, gray \\ & $17.9-18.5$ Silty sand, brownish gray; fine-grained \\ & $18.5-19.0$ Clayey sand, brownish gray; fine- to medium-grained \\ & $19.0-19.4$ Sand, grayish brown; silty matrix \\ & $19.4-20.1$ Clayey sand, grayish brown; very fine-grained \\ & $20.1-20.8$ Sand, grayish brown; medium-grained \\ & $20.8-21.5$ Sand, grayish brown; very fine- to fine-grained \\ & $21.5-22.0$ No recovery \\ & $22.0-23.0$ Sand, grayish brown; medium-grained; loose \\ & $23.0-25.0$ Sand, grayish brown; fine-grained; loose \\ & \hline \\ & \hline\end{aligned}


Well MWGS-33B

[Approximate land-surface altitude at well MWGS-33B is 34.9 feet above sea level. All depths are reportein feet below land surface. Well location is shown in plate 1.]

\begin{tabular}{|c|c|}
\hline $\begin{array}{l}\text { Depth } \\
\text { (feet) }\end{array}$ & Lithologic description \\
\hline $0.0-2.0$ & Not sampled \\
\hline $2.0-2.3$ & Sand, dark brown \\
\hline $2.3-4.0$ & Sand, $\tan$ \\
\hline $4.0-4.6$ & Sand, dark brown \\
\hline 4.6-5.2 & Sand, $\tan$ \\
\hline $5.2-6.0$ & Silty sand, reddish brown; with a sharp upper contact \\
\hline $6.0-18.4$ & Sand, gray; with a greenish tint from 13.0 to 13.1 feet \\
\hline 18.4-20.0 & Sand, gray; with 1-inch thick interspersed bands of brown sand \\
\hline $20.0-21.3$ & Sand, gray \\
\hline $21.3-22.0$ & Sandy clay, gray; sharp contact with above sand \\
\hline $22.0-22.2$ & No recovery \\
\hline 22.2-22.4 & Sand, brown \\
\hline 22.4-23.3 & Sand, gray \\
\hline 23.3-23.5 & Sandy clay, reddish gray \\
\hline $23.5-24.0$ & Clayey sand, gray \\
\hline $24.0-24.3$ & Clayey sand, brown \\
\hline 24.3-26.0 & Slightly clayey to clayey sand, gray; with gray clay layer from 25.7 to 25.8 feet \\
\hline $26.0-26.5$ & Silty sand, gray \\
\hline 26.5-26.7 & Silty sand, brown \\
\hline 26.7-27.7 & Sand, gray \\
\hline 27.7-27.8 & Clayey sand, gray \\
\hline 27.8-28.0 & Sand, brown \\
\hline 28.0-28.8 & Clayey sand, grayish brown \\
\hline 28.8-28.9 & Clay, gray \\
\hline 28.9-30.3 & Sand, gray \\
\hline 30.3-30.8 & Sand, very coarse; with some black staining \\
\hline $30.8-32.0$ & Clayey sand, gray; with shells and pebbles \\
\hline
\end{tabular}


Well MWGS-33B--Continued

[Approximate land-surface altitude at well MWGS-33B is 34.9 feet above sea level. All depths are report ?d in feet below land surface. Well location is shown in plate 1.]

Depth

(feet)

Lithologic description

32.0-33.6 Clayey sand; poorly sorted

33.6-36.0 Clayey silt, gray green 
Well MWGS-34A

[Approximate land-surface altitude at well MWGS-34A is 34.5 feet above sea level. All depths are reporte-1 in feet below land surface. Well location is shown in plate 1.]

\begin{tabular}{|c|c|}
\hline $\begin{array}{l}\text { Depth } \\
\text { (feet) }\end{array}$ & Lithologic description \\
\hline $0.0-0.7$ & Sand, reddish brown \\
\hline $0.7-1.0$ & Clayey sand, orange brown \\
\hline $1.0-2.3$ & Sand, reddish brown to dark brown \\
\hline $2.3-4.0$ & Sand, $\tan$ \\
\hline $4.0-5.0$ & Sand, gray \\
\hline $5.0-6.0$ & No recovery \\
\hline $6.0-8.4$ & Sand, gray; loose \\
\hline $8.4-10.0$ & No recovery \\
\hline $10.0-17.7$ & Sand, gray; loose \\
\hline $17.7-17.8$ & Sand, brown \\
\hline $17.8-19.3$ & Sand, gray \\
\hline 19.3-19.7 & Sand, light brown; with sharp upper contact \\
\hline $19.7-20.0$ & Sand, reddish brown; with very thin layers of gray clay \\
\hline $20.0-21.4$ & Sand, gray \\
\hline $21.4-22.0$ & Sand, brown \\
\hline $22.0-22.8$ & Sand, brown; slightly clayey \\
\hline $22.8-23.0$ & Sand, pinkish; with very thin layers of gray clay \\
\hline $23.0-24.0$ & No recovery \\
\hline $24.0-25.4$ & Sand, pinkish; with very thin lenses of gray clayey sand \\
\hline 25.4-26.0 & $\begin{array}{l}\text { Sand, reddish brown; with gray sand lens between } 25.5 \text { and } 25.55 \text { feet and black staining } \\
\text { between } 25.6 \text { and } 25.7 \text { feet }\end{array}$ \\
\hline $26.0-28.5$ & Sand, reddish brown; with patches of gray clay \\
\hline 28.5-28.6 & Clay, brownish gray \\
\hline $28.6-28.8$ & Ironstone, brownish red; with shells and fossils \\
\hline $28.8-29.0$ & Sand, yellowish brown; partially cemented \\
\hline $29.0-30.0$ & Sand, gray; with black cobbles and white shell fragments \\
\hline $30.0-31.3$ & Sand, gray; with pebbles \\
\hline $31.3-32.0$ & Clayey silt, gray green \\
\hline
\end{tabular}


Well MWGS-34B

[Approximate land-surface altitude at well MWGS-34B is 34.5 feet above sea level. All depths are reported in feet below land surface. Well location is shown in plate 1.]

\begin{aligned} & \hline $\begin{array}{c}\text { Depth } \\ \text { (feet) }\end{array} \\ &$\hline $0.0-4.0$ Not sampled \\ & $4.0-4.2$ Sand, dark brown \\ & $4.2-8.0$ Sand, tan \\ & $8.0-20.9$ Sand, gray to dark gray \\ & $20.9-22.0$ Sand, brown. \\ & $22.0-23.1$ Sand, grayish brown \\ & $23.1-24.0$ Sand, reddish brown; some silt matrix and slight iron cementation \\ & $24.0-24.9$ Sand, grayish brown \\ & $24.9-26.0$ Clayey sand, reddish brown \\ & \hline\end{aligned}

Well MWGS-35

[Approximate land-surface altitude at well MWGS-35 is 34.4 feet above sea level. All depths are reportein feet below land surface. Well location is shown in plate 1.]

\begin{aligned} & \hline $\begin{array}{r}\text { Depth } \\ \text { (feet) }\end{array} \\ &$\hline $0.0-9.0$ Not sampled \\ & $9.0-10.4$ Sand, gray \\ & $10.4-11.0$ Sand, brown \\ & $11.0-11.6$ Sandy clay, gray \\ & $11.6-12.6$ Clay, gray \\ & $12.6-13.3$ Sandy clay, gray \\ & $13.3-14.3$ Sand, gray \\ & $14.3-14.9$ Sandy clay, gray \\ & $14.9-15.5$ Sand and clay, gray \\ & $15.5-16.2$ Clay, gray \\ & $16.2-19.2$ Sand, gray, with gray clay layers from 18.6 to 18.7 feet and 19.0 to 19.2 feet \\ & $19.2-21.0$ Sand, grayish tan \\ & \hline\end{aligned}


Well MWGS-36

[Approximate land-surface altitude at well MWGS-36 is 34.8 feet above sea level. All depths are reporte-1 in feet below land surface. Well location is shown in plate 1.]

\begin{aligned} & \hline $\begin{array}{r}\text { Depth } \\ \text { (feet) }\end{array} \\ &$\hline $0.0-8.5$ Not sampled \\ & $8.5-9.1$ Sand, gray \\ & $9.1-9.3$ Clay, gray \\ & $9.3-9.5$ Sand, gray \\ & $9.5-10.0$ Clay, gray \\ & $10.0-10.9$ Sand, gray \\ & $10.9-11.5$ Clay, gray \\ & $11.5-12.5$ Sand, gray \\ & $12.5-19.5$ Sand, gray; with a greenish sand lens from 14.0 to 14.2 feet and a dark gray silt layer from \\ & 17.9 to 18.0 feet \\ & $19.5-20.0$ Clayey sand, reddish brown \\ & $20.0-20.5$ Clay, very dark brown \\ & $20.5-22.8$ Sand, gray; with a dark brown sand lens from 21.2 to 21.3 feet \\ & $22.8-24.5$ Clay, dark brown to gray \\ & $24.5-25.8$ Clay, dark gray, plastic \\ & $25.8-26.5$ Sand, light grayish brown to gray \\ & \hline\end{aligned}




\section{Well MWGS-37}

[Approximate land-surface altitude at well MWGS-37 is 34.4 feet above sea level. All depths are reported in feet below land surface. Well location is shown in plate 1.]

\begin{aligned} & $\begin{array}{r}\text { Depth } \\ \text { (feet) }\end{array} \\ &$\hline $0.0-8.0$ Not sampled \\ & $8.0-8.8$ Sand, light brown \\ & $8.8-9.3$ Sand, gray; with a gradational upper contact \\ & $9.3-10.0$ Clay, gray; tight and plastic \\ & $10.0-11.0$ Sand, gray; with a clay lens from 10.3 to 10.4 feet \\ & $11.0-11.3$ Clay, gray \\ & $11.3-11.4$ Sand, gray \\ & $11.4-12.0$ Clay, gray \\ & $12.0-12.1$ Sand, gray \\ & $12.1-12.4$ Clayey sand, gray; with basal layer of brown gravel \\ & $12.4-17.1$ Sand, light gray to gray; loose; with green staining at 13.3 and 13.5 feet; brown staining and \\ & clay nodule at 16.6 feet \\ & \hline $17.1-17.3$ Sand, gray; loose; with thin layers of clay interspersed \\ & $17.3-18.0$ Sand, grayish tan to grayish brown \\ & $18.0-19.2$ Sand, grayish brown \\ & $19.2-19.4$ Sand, brown \\ & $19.4-20.0$ Sand, gray \\ & \hline\end{aligned}


Well MWGS-38

[Approximate land-surface altitude at well MWGS-38 is 34.0 feet above sea level. All depths are reportec in feet below land surface. Well location is shown in plate 1.]

\begin{aligned} & $\begin{array}{r}\text { Depth } \\ \text { (feet) }\end{array} \\ &$\hline $0.0-8.0$ Not sampled \\ & $8.0-8.9$ Sand, reddish brown \\ & $8.9-10.6$ Sand, tan \\ & $10.6-10.8$ Clayey sand, grayish brown \\ & $10.8-11.0$ Sand, grayish brown \\ & $11.0-11.1$ Clayey sand, gray \\ & $11.1-11.9$ Clay, gray \\ & $11.9-12.0$ Clayey sand, gray \\ & $12.0-12.2$ Clay, gray \\ & $12.2-12.3$ Sand, gray \\ & $12.3-12.7$ Sandy clay, gray \\ & $12.7-13.6$ Sand, gray \\ & $13.6-14.2$ Clay, gray \\ & $14.2-24.0$ Sand, gray \\ & \hline\end{aligned}


Well MWGS-39

[Approximate land-surface altitude at well MWGS-39 is 36.3 feet above sea level. All depths are reporter in feet below land surface. Well location is shown in plate 1.]

\begin{aligned} & \hline $\begin{array}{r}\text { Depth } \\ \text { (feet) }\end{array} \\ &$\hline $0.0-6.0$ Not sampled \\ & $6.0-8.0$ Sand, tan \\ & $8.0-9.0$ Sand, brown; with black staining between 8.9 and 9.0 feet \\ & 9.0-10.0 Sand, tan; silty from 9.7 to 9.8 feet \\ & $10.0-11.1$ Sand, brown to tan \\ & $11.1-11.7$ Sand, tan \\ & $11.7-11.8$ Sandy clay, reddish brown \\ & $11.8-12.0$ Sandy clay, gray and red \\ & $12.0-12.6$ Clay, maroon \\ & $12.6-13.1$ Sandy clay, gray with brown patches \\ & $13.1-14.0$ Sand, gray; loose \\ & $14.0-14.4$ Sandy clay, brown \\ & $14.4-14.6$ Sand, gray \\ & $14.6-15.1$ Sand, grayish brown to gray; slight clay matrix \\ & $15.1-15.6$ Sand, tan; slight clay matrix \\ & $15.6-20.0$ Sand, gray; loose \\ & $20.0-24.0$ Sand, grayish brown to gray \\ & $24.0-25.1$ Sand, gray to black \\ & $25.1-25.7$ Clay, gray to brown \\ & $25.7-26.0$ Sandy clay, gray \\ & \hline\end{aligned}


Well MWGS-44

[Approximate land-surface altitude at well MWGS-44 is 34.3 feet above sea level. All depths are reported in feet below land surface. Well location is shown in plate 1.]

\begin{tabular}{|c|c|}
\hline $\begin{array}{l}\text { Depth } \\
\text { (feet) }\end{array}$ & Lithologic description \\
\hline $0.0-2.0$ & Not sampled \\
\hline $2.0-3.2$ & Sand, gray to brown; fine-grained; mottled; with clayey sand nodules \\
\hline $3.2-3.6$ & Sand, black; fine-grained \\
\hline $3.6-4.0$ & No recovery \\
\hline $4.0-5.0$ & Sand, light brown; fine-grained; with clay nodules located at the top \\
\hline $5.0-5.5$ & Sand, gray; fine-grained \\
\hline $5.5-6.0$ & No recovery \\
\hline $6.0-7.0$ & Sand, light gray; fine-grained \\
\hline $7.0-7.9$ & Silty sand, gray \\
\hline $7.9-8.0$ & No recovery \\
\hline $8.0-8.9$ & Silty sand, dark to very dark gray; mottled \\
\hline $8.9-9.3$ & Sand, tan; very fine-grained \\
\hline $9.3-10.0$ & No recovery \\
\hline $10.0-10.3$ & Sand, tan; very fine-grained \\
\hline $10.3-11.3$ & Silty sand, dark gray \\
\hline $11.3-11.8$ & Silty clay, dark grading to light gray; very tight \\
\hline $11.8-12.0$ & No recovery \\
\hline $12.0-12.2$ & Clayey silt, light gray \\
\hline $12.2-12.8$ & Clay, light gray; dense \\
\hline $12.8-13.0$ & Silty sand, gray \\
\hline $13.0-13.2$ & Clay, light gray; dense \\
\hline $13.2-13.6$ & Sand, light gray; very fine-grained \\
\hline $13.6-14.0$ & No recovery \\
\hline $14.0-14.4$ & Silty sand, gray \\
\hline 14.4-14.6 & Sand, gray; fine-grained \\
\hline $14.6-15.3$ & Clay, gray; dense; with a trace of silt \\
\hline
\end{tabular}


Well MWGS-44-Continued

[Approximate land-surface altitude at well MWGS-44 is 34.3 feet above sea level. All depths are reporte] in feet below land surface. Well location is shown in plate 1.]

\begin{tabular}{cl}
\hline $\begin{array}{c}\text { Depth } \\
\text { (feet) }\end{array}$ & \multicolumn{1}{c}{ Lithologic description } \\
\hline $15.3-19.8$ & $\begin{array}{c}\text { Sand, light gray; fine- to very fine-grained; with a clayey sand layer between } 18.7 \text { and } 18.8 \\
\text { feet }\end{array}$ \\
$19.8-20.0$ & No recovery \\
$20.0-21.9$ & Sand, light gray; very fine-grained \\
$22.0-22.3$ & Sand, tan; very fine-grained \\
$22.3-24.0$ & Sand, light gray; very fine-grained \\
\hline
\end{tabular}


Well MWGS-51

[Approximate land-surface altitude at well MWGS-51 is 34.2 feet above sea level. All depths are reported in feet below land surface. Well location is shown in plate 1.]

\begin{aligned} & \hline $\begin{array}{r}\text { Depth } \\ \text { (feet) }\end{array} \\ &$\hline $0.0-8.0$ Not sampled \\ & $8.0-9.3$ Sand, dark to medium gray; fine-grained \\ & $9.3-10.0$ No recovery \\ & $10.0-11.4$ Sand, dark gray to brown; very fine-grained; mottled; with some silt \\ & $11.4-12.0$ No recovery \\ & $12.0-12.3$ Silty sand, very dark gray \\ & $12.3-13.4$ Clay, dark to light gray; mottled \\ & $13.4-13.8$ Clayey sand, gray \\ & $13.8-14.0$ No recovery \\ & $14.0-14.1$ Silty sand, grayish brown \\ & $14.1-14.7$ Sand, light gray \\ & $14.7-16.0$ Sandy to silty clay; with a small sand lens at 15.3 feet \\ & $16.0-16.2$ Silty sand, dark gray \\ & $16.2-17.7$ Sand, light gray; fine-grained \\ & $17.7-18.0$ No recovery \\ & $18.0-19.8$ Sand, light to medium gray; fine-grained; with intermittent clay bands at bottom \\ & \hline\end{aligned}


Well MWGS-55

[Approximate land-surface altitude at well MWGS-55 is 34.5 feet above sea level. All depths are reported in feet below land surface. Well location is shown in plate 1.]

\begin{aligned} & \hline $\begin{array}{r}\text { Depth } \\ \text { (feet) }\end{array} \\ &$\hline $0.0-4.0$ Not sampled \\ & $4.0-5.8$ Sand, gray to brownish gray; some mottling \\ & $5.8-6.0$ No recovery \\ & $6.0-7.5$ Sand, light gray; bottom 0.5 feet is darker \\ & $7.5-8.0$ No recovery \\ & $8.0-8.4$ Sand, medium to light gray; fine-grained \\ & $8.4-10.5$ Silty sand, dark to very dark gray; with a trace of clay between 10.0 to 10.5 feet \\ & $10.5-11.5$ Clayey sand, light gray; some organic material intermixed \\ & $11.5-11.9$ Clay, very tight; with a trace of sand \\ & $11.9-12.0$ No recovery \\ & $12.0-12.3$ Silty sand, dark gray \\ & $12.3-12.6$ Clayey sand, light gray \\ & $12.6-13.0$ Sand, light gray \\ & $13.0-13.5$ Clayey sand, light gray; with clay lens at bottom \\ & $13.5-14.0$ No recovery \\ & $14.0-14.2$ Sand, light gray \\ & $14.2-14.5$ Clay; tight; with a trace of sand \\ & $14.5-14.8$ Sandy clay \\ & $14.8-15.8$ Sand, light gray; some mottling \\ & \hline\end{aligned}


Well MWGS-62

[Approximate land-surface altitude at well MWGS-62 is 34.4 feet above sea level. All depths are reported in feet below land surface. Well location is shown in plate 1.]

\begin{tabular}{|c|c|}
\hline $\begin{array}{l}\text { Depth } \\
\text { (feet) }\end{array}$ & Lithologic descriptlon \\
\hline $0.0-2.0$ & Not sampled \\
\hline $2.0-3.8$ & Sand, gray to brownish; medium- to fine-grained; mottled \\
\hline $3.8-4.0$ & No recovery \\
\hline $4.0-5.7$ & Sand, gray to light gray; very fine-grained; some organic material \\
\hline $5.7-6.0$ & No recovery \\
\hline $6.0-6.4$ & Sand, gray; very fine-grained; intermixed with small patches of brown sand \\
\hline $6.4-7.0$ & Sand, brown \\
\hline $7.0-7.5$ & Sand, very dark gray; with some silt \\
\hline $7.5-7.8$ & Sand, dark gray \\
\hline $7.8-8.0$ & No recovery \\
\hline $8.0-8.3$ & Sand, brown \\
\hline $8.3-9.6$ & Sand, dark to very dark gray \\
\hline 9.6-10.0 & No recovery \\
\hline $10.0-10.5$ & Silty sand, dark gray; intermixed with very dark silt \\
\hline $10.5-11.8$ & Clay; tight; plastic; intermixed with organic material; very fine-grained, light gray sand at bottom \\
\hline $11.8-12.0$ & No recovery \\
\hline $12.0-12.6$ & Sand, light gray; very fine-grained \\
\hline $12.6-14.0$ & Clayey sand; intermixed with thin lenses of sand \\
\hline $14.0-15.6$ & Sand, gray to light gray \\
\hline $15.6-16.0$ & No recovery \\
\hline $16.0-17.5$ & Sand, very light gray \\
\hline
\end{tabular}

